\title{
The Duration, Frequency, and Volume of Exclusive Human Milk and/or Infant Formula Consumption and Overweight and Obesity: A Systematic Review
}

2020 Dietary Guidelines Advisory Committee, Birth to 24 Months Subcommittee Published date: July 15, 2020

Nutrition Evidence Systematic Review Center for Nutrition Policy and Promotion

Food and Nutrition Service

U.S. Department of Agriculture Braddock Metro Center II 1320 Braddock Place Alexandria, Virginia 22314 
This systematic review was conducted by the 2020 Dietary Guidelines Advisory Committee in collaboration with the Nutrition Evidence Systematic Review (NESR) team at the Center for Nutrition Policy and Promotion, Food and Nutrition Service, U.S. Department of Agriculture (USDA). All systematic reviews from the 2020 Advisory Committee Project are available on the NESR website: https://nesr.usda.gov/2020-dietary-guidelines-advisory-committee-systematic-reviews.

Conclusion statements drawn as part of this systematic review describe the state of science related to the specific question examined. Conclusion statements do not draw implications, and should not be interpreted as dietary guidance. This portfolio provides the complete documentation for this systematic review. A summary of this review is included in the 2020 Advisory Committee's Scientific Report available at www.DietaryGuidelines.gov.

The contents of this document may be used and reprinted without permission. Endorsements by NESR, the Center for Nutrition Policy and Promotion, the Food and Nutrition Service, or the USDA of derivative products developed from this work may not be stated or implied.

Suggested citation for this systematic review: 2020 Dietary Guidelines Advisory Committee and Nutrition Evidence Systematic Review Team. The Duration, Frequency, and Volume of Exclusive Human Milk and/or Infant Formula Consumption and Overweight and Obesity: A Systematic Review. 2020 Dietary Guidelines Advisory Committee Project. Alexandria, VA: U.S. Department of Agriculture, Food and Nutrition Service, Center for Nutrition Policy and Promotion, July 2020. Available at: https://nesr.usda.gov/2020-dietary-guidelines-advisory-committee-systematic-reviews.

Related citation: Dietary Guidelines Advisory Committee. 2020. Scientific Report of the 2020 Dietary Guidelines Advisory Committee: Advisory Report to the Secretary of Agriculture and the Secretary of Health and Human Services. U.S. Department of Agriculture, Agricultural Research Service, Washington, DC.

In accordance with Federal civil rights law and USDA civil rights regulations and policies, the USDA, its Agencies, offices, and employees, and institutions participating in or administering USDA programs are prohibited from discriminating based on race, color, national origin, religion, sex, gender identity (including gender expression), sexual orientation, disability, age, marital status, family/parental status, income derived from a public assistance program, political beliefs, or reprisal or retaliation for prior civil rights activity, in any program or activity conducted or funded by USDA (not all bases apply to all programs). Remedies and complaint filing deadlines vary by program or incident.

Persons with disabilities who require alternative means of communication for program information (e.g., Braille, large print, audiotape, American Sign Language, etc.) should contact the responsible Agency or USDA's TARGET Center at (202) 720-2600 (voice and TTY) or contact USDA through the Federal Relay Service at (800) 877-8339. Additionally, program information may be made available in languages other than English.

To file a program discrimination complaint, complete the USDA Program Discrimination Complaint Form, AD-3027, found online at How to File a Program Discrimination Complaint and at any USDA office or write a letter addressed to USDA and provide in the letter all of the information requested in the form. To request a copy of the complaint form, call (866) 6329992. Submit your completed form or letter to USDA by: (1) mail: U.S. Department of Agriculture, Office of the Assistant Secretary for Civil Rights, 1400 Independence Avenue, SW, Washington, D.C. 20250-9410; (2) fax: (202) 690-7442; or (3) email: program.intake@usda.gov.

USDA is an equal opportunity provider, employer, and lender. 


\section{ACKNOWLEDGEMENTS}

\section{Birth to 24 Months Subcommittee:}

- Kathryn Dewey, PhD, University of California, Davis, Subcommittee Chair

- Lydia Bazzano, MD, PhD, Tulane University and Ochsner Health System

- Teresa Davis, PhD, Baylor College of Medicine

- Sharon Donovan, PhD, RD, University of Illinois, Urbana-Champaign

- Elsie Taveras, MD, MPH, Massachusetts General Hospital, Harvard Medical School, and Harvard T.H. Chan School of Public Health

- Ronald Kleinman, MD, Massachusetts General Hospital and Harvard Medical School, Vice-Chair of the 2020 Dietary Guidelines Advisory Committee

\section{Nutrition Evidence Systematic Review (NESR) Team:}

- Darcy Güngör, MS, Analyst, Panum Group ${ }^{i}$

- Emily Madan, PhD, Analyst, Panum Groupi

- Sudha Venkatramanan, PhD, Analyst, Panum Groupi

- Nancy Terry, MS, MLS, Biomedical Librarian, National Institutes of Health (NIH), U.S. Department of Health and Human Services (HHS)

- Gisela Butera, MLIS, MEd, Systematic Review Librarian, Panum Group ${ }^{i}$

- Julie Obbagy, PhD, RD, Project Lead, Office of Nutrition Guidance and Analysis (ONGA), Center for Nutrition Policy and Promotion (CNPP), Food and Nutrition Service (FNS), U.S. Department of Agriculture (USDA)

\section{Federal Liaisons:}

- Cria Perrine, PhD, Division of Nutrition, Physical Activity, and Obesity, Centers for Disease Control and Prevention, HHS

- Jennifer Lerman, MPH, RD, National Cancer Institute, NIH, HHS

- Kelley Scanlon, PhD, RD, Special Nutrition Research and Analysis Division, Office of Policy Support, FNS, USDA

\section{Project Leadership:}

- Eve Essery Stoody, PhD, Designated Federal Officer and Director, ONGA, CNPP, FNS, USDA

- Janet de Jesus, MS, RD, Nutrition Advisor, Office of Disease Prevention and Health Promotion, Office of the Assistant Secretary for Health, HHS

USDA and HHS implemented a process to identify topics and scientific questions to be examined by the 2020 Dietary Guidelines Advisory Committee. The Committee conducted its review of evidence in subcommittees for discussion by the full Committee during its public meetings. The role of the Committee members involved establishing all aspects of the protocol, which presented the plan for how they would examine the scientific evidence, including the inclusion and exclusion criteria; reviewing all studies that met the criteria they set; deliberating

' Under contract with the Food and Nutrition Service, United States Department of Agriculture. 
on the body of evidence for each question; and writing and grading the conclusion statements to be included in the scientific report the 2020 Committee submitted to USDA and HHS. The NESR team with assistance from Federal Liaisons and Project Leadership, supported the Committee by facilitating, executing, and documenting the work necessary to ensure the reviews were completed in accordance with NESR methodology. More information about the 2020 Dietary Guidelines Advisory Committee, including the process used to identify topics and questions, can be found at www. DietaryGuidelines.gov. More information about NESR can be found at NESR.usda.gov.

The Committee and NESR staff thank USDA's Agricultural Research Service for coordinating the peer review of this systematic review, and the Federal scientist peer reviewers for their time and expertise.

FUNDING SOURCE: United States Department of Agriculture, Food and Nutrition Service, Center for Nutrition Policy and Promotion, Alexandria, VA 


\section{TABLE OF CONTENTS}

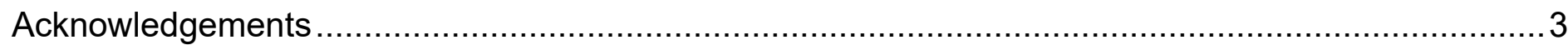

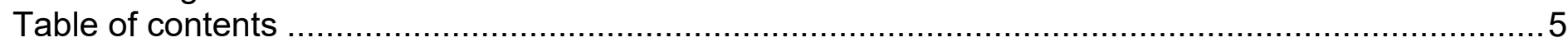

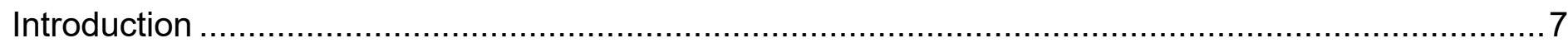

What is the relationship between the duration, frequency, and volume of exclusive human milk and/or

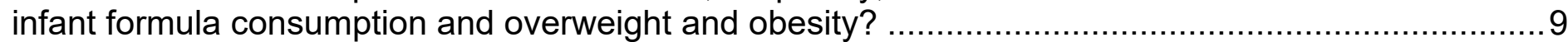

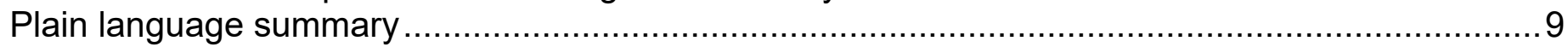

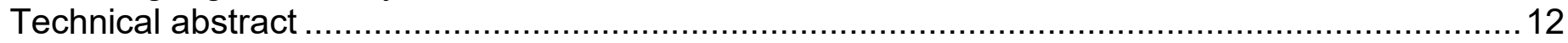

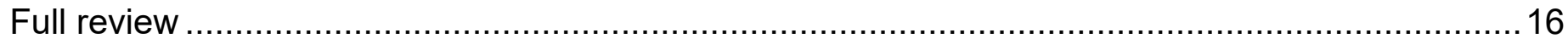

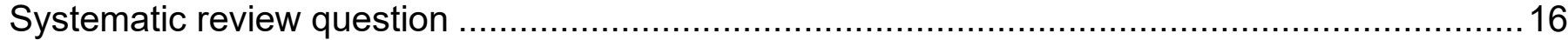

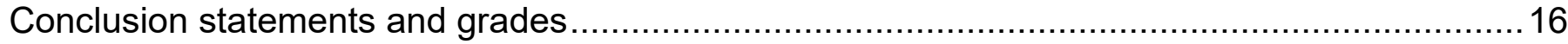

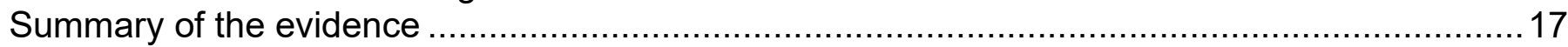

Ever, compared with never, consuming human milk ..................................................... 18

Duration of any human milk consumption among infants fed human milk ................................64

Duration of exclusive human milk consumption before the introduction of infant formula .............93

Intensity, proportion, or amount of human milk consumed by mixed-fed infants.........................95

Intensity, proportion, or amount of human milk consumed at the breast versus by bottle.............95

Consuming human milk or infant formula (i.e., a single substance) during as single feeding

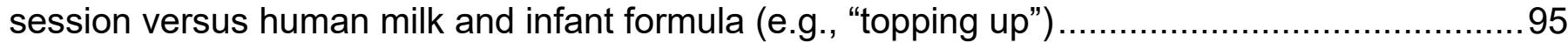

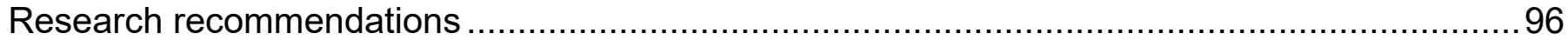

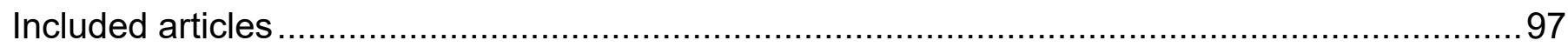

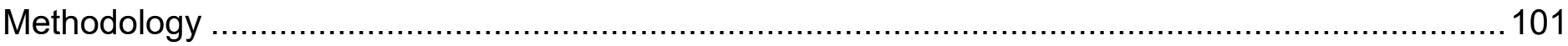

Analytic framework

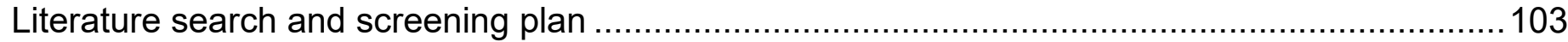

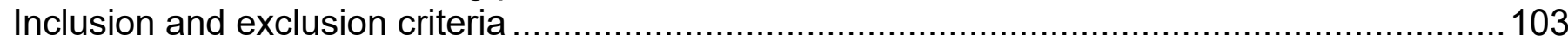

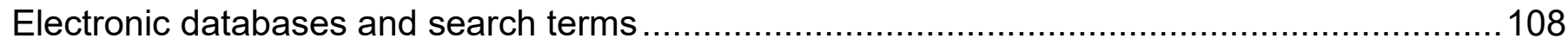

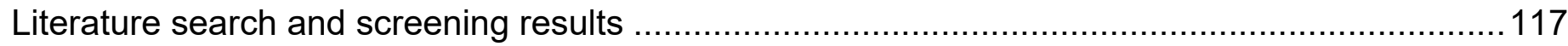

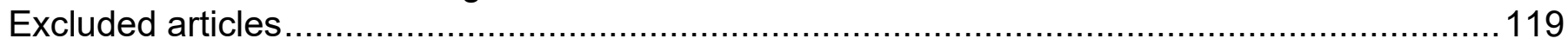

Table 1. Evidence examining the relationship between ever, compared with never, consuming human milk and body composition, including obesity, from studies that conducted within-family comparisons

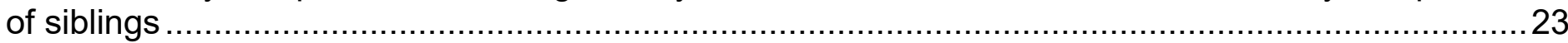

Table 2. Evidence examining the relationship between ever, compared with never, consuming human

milk and overweight and/or obesity at 2 years of age and older

Table 3. Risk of bias for the non-randomized controlled trial examining the relationship between ever

vs never consuming human milk and overweight and obesity

Table 4. Risk of bias for observational studies examining the relationship between ever vs never consuming human milk and overweight and obesity....

Table 5. Evidence examining the relationship between the duration of any human milk consumption, among infants fed human milk, and body composition, including obesity, from studies that conducted within-family comparisons of siblings

Table 6. Evidence examining the relationship between the duration of any human milk consumption, among infants fed human milk, and overweight and/or obesity at 2 years of age and older

Table 7. Risk of bias for the randomized controlled trial examining the relationship between the duration of any human milk consumption among infants fed human milk and overweight and obesity

Table 8. Risk of bias for observational studies examining the relationship between the duration of any 
human milk consumption among infants fed human milk and overweight and obesity

Table 9. Evidence examining the relationship between the duration of exclusive human milk consumption before to the introduction of infant formula and and overweight and/or obesity at 2 years

of age and older 94

Table 10. Risk of bias for observational studies examining the relationship between the duration of exclusive human milk consumption before the introduction of infant formula and overweight and obesity .94

Table 11. Inclusion and exclusion criteria 103

Table 12. Full-text exclusions from the Pregnancy and Birth to 24 Months Project literature search 119 Table 13. Full-text exclusions from the update to the Pregnancy and Birth to 24 Months Project literature search

Figure 1. Analytic framework

Figure 2. Pregnancy and Birth to 24 Months Project literature search

Figure 3. Update to the Pregnancy and Birth to 24 Months Project literature search 


\section{INTRODUCTION}

This document describes a systematic review conducted to answer the following question: What is the relationship between the duration, frequency, and volume of exclusive human milk and/or infant formula consumption and overweight and obesity? This systematic review was conducted by the 2020 Dietary Guidelines Advisory Committee, supported by USDA's Nutrition Evidence Systematic Review (NESR).

More information about the 2020 Dietary Guidelines Advisory Committee is available at the following website: www.DietaryGuidelines.gov.

NESR specializes in conducting food- and nutrition-related systematic reviews using a rigorous, protocol-driven methodology. More information about NESR is available at the following website: NESR.usda.gov.

NESR's systematic review methodology involves developing a protocol, searching for and selecting studies, extracting data from and assessing the risk of bias of each included study, synthesizing the evidence, developing conclusion statements, grading the evidence underlying the conclusion statements, and recommending future research. A detailed description of the systematic reviews conducted for the 2020 Dietary Guidelines Advisory Committee, including information about methodology, is available on the NESR website: https://nesr.usda.gov/2020-dietary-guidelinesadvisory-committee-systematic-reviews. In addition, starting on page 101, this document describes the final protocol as it was applied in the systematic review. A description of and rationale for the modifications made to the protocol can be found in the 2020 Dietary Guidelines Advisory Committee Report, Chapter 4. Duration, Frequency, and Volume of Exclusive Human Milk and/or Infant Formula Feeding. 


\section{List of abbreviations}

\begin{tabular}{ll}
\hline Abbreviation & Full name \\
\hline BMI & Body mass index \\
\hline CDC & Centers for Disease Control and Prevention \\
\hline CNPP & Center for Nutrition Policy and Promotion \\
\hline FDA & U.S. Food and Drug Administration \\
\hline FNS & USDA Food and Nutrition Service \\
\hline HHS & U.S. Department of Health and Human Services \\
\hline IOTF & International Obesity Task Force \\
\hline NESR & Nutrition Evidence Systematic Review \\
\hline NHANES & National Health and Nutrition Examination Survey \\
\hline NIH & National Institutes of Health \\
\hline ONGA & Office of Nutrition Guidance and Analysis \\
\hline SES & Socio-economic status \\
\hline USDA & U.S. Department of Agriculture \\
\hline WIC & Supplemental Nutrition Program for Women, Infants and Children \\
\hline WFL & Weight-for-length \\
\hline WHO & World Health Organization \\
\hline
\end{tabular}




\section{WHAT IS THE RELATIONSHIP BETWEEN THE DURATION, FREQUENCY, AND VOLUME OF EXCLUSIVE HUMAN MILK AND/OR INFANT FORMULA CONSUMPTION AND OVERWEIGHT AND OBESITY?}

\section{PLAIN LANGUAGE SUMMARY}

\section{What is the question?}

- The question is: What is the relationship between the duration, frequency, and volume of exclusive human milk and/or infant formula consumption and overweight and obesity?

\section{What is the answer to the question?}

- Moderate evidence from observational studies indicates that ever, compared with never, consuming human milk is associated with lower risk of overweight and obesity at 2 years of age and older, particularly if the duration of human milk consumption is 6 months or longer.

- Insufficient evidence is available to determine the relationship between the duration of any human milk consumption, among infants fed human milk, and overweight and obesity at 2 years of age and older; the available evidence was inconsistent.

- Insufficient evidence is available to determine the relationship between the duration of exclusive human milk consumption prior to the introduction of infant formula and overweight and obesity at 2 years of age and older.

- No evidence is available to determine the relationship between the intensity, proportion, or amount of human milk consumed by mixed-fed infants and overweight and obesity at 2 years of age and older.

- No evidence is available to determine the relationship between the intensity, proportion, or amount of human milk consumed at the breast vs by bottle in infants fed human milk as their only source of milk and overweight and obesity at 2 years of age and older.

- No evidence is available to determine the relationship between consuming human milk or infant formula (i.e., a single substance) vs human milk and infant formula (i.e., both substances, e.g., "topping up") during a single feeding session and overweight and obesity at 2 years of age and older.

\section{Why was this question asked?}

- This important public health question was identified by the U.S. Departments of Agriculture (USDA) and Health and Human Services (HHS) to be examined by the 2020 Dietary Guidelines Advisory Committee.

\section{How was this question answered?}

- The 2020 Dietary Guidelines Advisory Committee, Birth to 24 Months Subcommittee conducted a systematic review to answer this question with support from the Nutrition Evidence Systematic Review (NESR) team. 


\section{What is the population of interest?}

- The systematic review studied 2 different populations. The first was generally healthy infants and toddlers (birth to 24 months) who were fed human milk and/or infant formula and who had overweight and obesity outcomes examined at 2 years of age or older. The second was generally healthy siblings who were fed differently during infancy or who had different outcomes, or both, and who had rapid weight gain examined between birth and 24 months and/or BMI, body composition, overweight or obesity examined at 2 years of age or older.

\section{What evidence was found?}

- This review includes 42 articles.

- The review examined available evidence about:

- Infants who ever consumed milk (i.e., any amount of human milk) compared with infants who never consumed human milk,

- Infants who consumed human milk (i.e., any amount of human milk) for different durations,

- Infants who consumed human milk exclusively for different durations before infant formula was added to their diets, and

- Mixed-fed infants (i.e., consuming both human milk and infant formula, but not complementary foods and beverages) who consumed different amounts of human milk.

- Infants who consumed human milk as their only source of milk and who consumed different intensities, proportions, or amounts of human milk at the breast versus by bottle, and

- Mixed-fed infants who consumed a single substance at a single feeding session (i.e., either human milk or infant formula) with mixed-fed infants who consumed both substances at a single feeding session (e.g., "topping up").

- These articles examined available evidence related to overweight and obesity at 2 years of age and older and, for siblings, about rapid weight gain from birth to 24 months, BMI, and body composition at 2 years of age and older.

- Most evidence examined overweight and obesity in infants who ever consumed human milk compared to infants who never consumed human milk, or in infants who consumed human milk for different durations.

- Infants who ever consume human milk, and especially infants who consume human milk for 6 months or longer, may have lower risk of overweight and obesity throughout life than infants who never consume human milk. The findings may be due, in part, to factors other than infant feeding that also impact overweight and obesity.

- It is not clear whether infants who consume human milk for different durations have different risk of overweight and obesity at 2 years of age and older. The evidence was inconsistent.

- There was not a lot of evidence about infants who consumed human milk exclusively for different durations before infant formula was added to their diets. There was no evidence about infants who consumed human milk at the breast compared to by bottle, or about mixed-fed infants who consumed different amounts of human milk or who consumed human milk and infant formula during the same feeding session compared to during different feeding sessions. 


\section{How up-to-date is this systematic review?}

- This review searched for studies published between January 2011 and September 2019 and additional sibling studies published between January 1980 and September 2019. 


\section{TECHNICAL ABSTRACT}

\section{Background}

- This important public health question was identified by the U.S. Departments of Agriculture (USDA) and Health and Human Services (HHS) to be examined by the 2020 Dietary Guidelines Advisory Committee.

- The 2020 Dietary Guidelines Advisory Committee, Birth to 24 Months Subcommittee, conducted a systematic review to answer this question with support from the Nutrition Evidence Systematic Review (NESR) team.

- The goal of this systematic review was to examine the following question: What is the relationship between the duration, frequency, and volume of exclusive human milk and/or infant formula consumption and overweight and obesity?

\section{Conclusion statements and grades}

- Ever vs never consuming human milk

- Moderate evidence from observational studies indicates that ever, compared with never, consuming human milk is associated with lower risk of overweight and obesity at ages 2 years and older, particularly if the duration of human milk consumption is 6 months or longer. (Grade: Moderate)

\section{- Duration of any human milk consumption among infants fed human milk}

- Insufficient evidence is available to determine the relationship between the duration of any human milk consumption, among infants fed human milk, and overweight and obesity at ages 2 years and older; the available evidence was inconsistent. (Grade: Grade not assignable)

- Duration of exclusive human milk consumption before the introduction of infant formula

- Insufficient evidence is available to determine the relationship between the duration of exclusive human milk consumption before the introduction of infant formula and overweight and obesity at ages 2 years and older. (Grade: Grade not assignable)

- Intensity, proportion, or amount of human milk consumed by mixed-fed infants

- No evidence is available to determine the relationship between the intensity, proportion, or amount of human milk consumed by mixed-fed infants and overweight and obesity at ages 2 years and older. (Grade: Grade not assignable)

- Intensity, proportion, or amount of human milk consumed at the breast vs by bottle in infants fed human milk as their only source of milk

- No evidence is available to determine the relationship between the intensity, proportion, or amount of human milk consumed at the breast vs by bottle in infants fed human milk as their only source of milk and overweight and obesity at ages 2 years and older. (Grade: Grade not assignable)

- Consuming human milk or infant formula (i.e., a single substance) vs human milk and infant formula (i.e., both substances) during a single feeding session

- No evidence is available to determine the relationship between consuming human milk or infant formula (i.e., a single substance) vs human milk and infant formula (i.e., both 
substances, e.g., "topping up") during a single feeding session and overweight and obesity at ages 2 years and older. (Grade: Grade not assignable)

\section{Methods}

- Two literature searches were conducted to identify articles published from January 1980 to March 2016, and from March 2016 to September 2019. The searches used 4 databases (PubMed, Embase, CINAHL, and Cochrane). A manual search was conducted to identify articles that may not have been included in the electronic databases searched.

- Articles were screened by 2 NESR analysts independently for inclusion based on predetermined criteria. Articles published between 2011 and 2019 were included if they met the pre-determined criteria and examined human milk and infant formula exposures from birth to 24 months and the outcomes of overweight and obesity at 2 years of age and older. Additional articles, published between 1980 and 2011, were included if they met the pre-determined criteria and compared siblings within the same family. Sibling studies are unique because they reduce the risk of bias from confounding from genetic and environmental factors that siblings share.

- Data extraction and risk of bias assessment were conducted for each included study, and both were checked for accuracy.

- The Committee qualitatively synthesized the body of evidence to inform development of a conclusion statement, and graded the strength of evidence using pre-established criteria for risk of bias, consistency, directness, precision, and generalizability.

\section{Summary of the evidence}

- This systematic review examined the relationship between the duration, frequency, and volume of exclusive human milk and/or infant formula consumption and overweight and obesity. Specifically, this systematic review examined available evidence that compared:

- Infants who ever consumed milk (i.e., any amount of human milk) with infants who never consumed human milk,

○ Infants who consumed human milk (i.e., any amount of human milk) for different durations,

- Infants who consumed human milk exclusively for different durations before the introduction of infant formula,

- Mixed-fed infants (i.e., consuming both human milk and infant formula, but not complementary foods and beverages) who consumed different intensities, proportions, or amounts of human milk,

- Infants who consumed human milk as their only source of milk and who consumed different intensities, proportions, or amounts of human milk at the breast versus by bottle, and

- Mixed-fed infants who consumed a single substance at a single feeding session (i.e., either human milk or infant formula) with mixed-fed infants who consumed both substances at a single feeding session (e.g., "topping up").

- Human milk refers to mother's own milk provided at the breast (i.e., nursing) or expressed and fed fresh or after refrigeration or freezing. Examinations of donor milk are not included in this review. Exclusive human milk consumption refers to consuming human milk alone and not in combination with infant formula or complementary foods and beverages. Infant formula refers to commercially prepared infant formula meeting the FDA and/or Codex Alimentarius 
international food standards. Complementary foods and beverages are foods and beverages other than human milk or infant formula (liquids, semisolids, and solids) provided to an infant or young child to provide nutrients and energy.

- The outcomes of interest were overweight and obesity at ages 2 years and older. Available evidence about rapid weight gain from birth to 24 months and BMI and body composition at ages 2 years and older were also examined from studies that conducted within-famliy analyses of discordant siblings (i.e., siblings fed differently during infancy, siblings with differences in outcome status, or both).

- This review identified 42 articles that met the inclusion criteria. Thirty of the 42 articles presented evidence about ever, compared with never, consuming human milk, and 21 of the 42 articles presented evidence about different durations of any human milk consumption (i.e. some articles presented evidence about both exposures).

- The 30 articles that examined the relationship between ever, compared with never, consuming human milk, and overweight and/or obesity at ages 2 years and older presented evidence from 21 independent cohorts.

- The evidence had strong consistency. Fourteen of the 21 studies found significant associations and all of them showed that ever, compared with never, consuming human milk is associated with lower risk of overweight and/or obesity at ages 2 years and older. One study showed a marginal association in the same direction, and some of the remaining studies were underpowered.

- The evidence available from 5 of 7 studies that compared infants who consumed human milk for different durations with infants who never consumed human milk suggested that a longer duration of human milk consumption (e.g., $\geq 6$ months) is most likely to be associated with reduced risk of overweight or obesity, compared to never consuming human milk.

- In 4 studies, the investigators conducted within-family analyses of siblings, which are designed to reduce bias due to confounding from genetic and environmental factors (factors that siblings share). Some of these analyses showed an attenuation of the significant associations found in full-sample analyses, suggesting that confounding may explain some of the association between ever, compared with never, consuming human milk and overweight and/or obesity at ages 2 years and older.

- The ability to draw stronger conclusions was primarily limited by the potential for confounding in a body of evidence made up of observational studies, and some concerns about the generalizability of the evidence from the studies conducted outside the United States (because U.S. populations may have higher risk of overweight and obesity than do the populations sampled for the non-U.S. studies).

- The 21 articles that examined the relationship between the duration of any human milk consumption, among infants fed human milk, and overweight and/or obesity at ages 2 years and older presented evidence from 1 cluster randomized controlled trial and 18 independent cohorts.

- The evidence was inconclusive. Nine of the 19 studies reported significant associations, but they were inconsistent in direction. In addition, potential bias from confounding and the limited generalizability of the evidence from the non-U.S. studies raised concerns (in particular because the prevalence of obesity among the participants of the cluster randomized controlled trial, conducted in Belarus, was much lower than the prevalence among youth in the United States). 
- Evidence available from 2 studies was insufficient to determine the relationship between the duration of exclusive human milk consumption before the introduction of infant formula and overweight and/or obesity at ages 2 years and older.

- No studies were identified that examined the intensity, proportion, or amount of human milk consumed by mixed-fed infants, the intensity, proportion, or amount of human milk consumed at the breast versus by bottle, or consuming a single substance (i.e., either human milk or infant formula) versus both human milk and infant formula during a single feeding session. 


\section{FULL REVIEW}

\section{Systematic review question}

What is the relationship between the duration, frequency, and volume of exclusive human milk and/or infant formula consumption and overweight and obesity?

\section{Conclusion statements and grades}

\section{Ever vs never consuming human milk}

Moderate evidence from observational studies indicates that ever, compared with never, consuming human milk is associated with lower risk of overweight and obesity at age 2 years and older, particularly if the duration of human milk consumption is 6 months or longer. (Grade: Moderate)

\section{Duration of any human milk consumption among infants fed human milk}

Insufficient evidence is available to determine the relationship between the duration of any human milk consumption, among infants fed human milk, and overweight and obesity at age 2 years and older; the available evidence was inconsistent. (Grade: Grade not assignable)

\section{Duration of exclusive human milk consumption before the introduction of infant formula} Insufficient evidence is available to determine the relationship between the duration of exclusive human milk consumption before the introduction of infant formula and overweight and obesity at age 2 years and older. (Grade: Grade not assignable)

\section{Intensity, proportion, or amount of human milk consumed by mixed-fed infants}

No evidence is available to determine the relationship between the intensity, proportion, or amount of human milk consumed by mixed-fed infants and overweight and obesity at age 2 years and older. (Grade: Grade not assignable)

Intensity, proportion, or amount of human milk consumed at the breast vs by bottle in infants fed human milk as their only source of milk

No evidence is available to determine the relationship between the intensity, proportion, or amount of human milk consumed at the breast vs by bottle in infants fed human milk as their only source of milk and overweight and obesity at age 2 years and older. (Grade: Grade not assignable)

Consuming human milk or infant formula (i.e., a single substance) vs human milk and infant formula (i.e., both substances) during a single feeding session

No evidence is available to determine the relationship between consuming human milk or infant formula (i.e., a single substance) vs human milk and infant formula (i.e., both substances, e.g., "topping up") during a single feeding session and overweight and obesity at age 2 years and older. (Grade: Grade not assignable) 


\section{Summary of the evidence}

- This systematic review examined the relationship between the duration, frequency, and volume of exclusive human milk and/or infant formula consumption and overweight and obesity. Specifically, this systematic review examined available evidence that compared:

- Infants who ever consumed milk (i.e., any amount of human milk) with infants who never consumed human milk,

- Infants who consumed human milk (i.e., any amount of human milk) for different durations,

- Infants who consumed human milk exclusively for different durations before the introduction of infant formula,

- Mixed-fed infants (i.e., consuming both human milk and infant formula, but not complementary foods and beverages) who consumed different intensities, proportions, or amounts of human milk,

- Infants who consumed human milk as their only source of milk and who consumed different intensities, proportions, or amounts of human milk at the breast versus by bottle, and

- Mixed-fed infants who consumed a single substance at a single feeding session (i.e., either human milk or infant formula) with mixed-fed infants who consumed both substances at a single feeding session (e.g., "topping up").

- Human milk refers to mother's own milk provided at the breast (i.e., nursing) or expressed and fed fresh or after refrigeration or freezing. Examinations of donor milk are not included in this review. Exclusive human milk consumption refers to consuming human milk alone and not in combination with infant formula or complementary foods and beverages. Infant formula refers to commercially prepared infant formula meeting the FDA and/or Codex Alimentarius international food standards. Complementary foods and beverages are foods and beverages other than human milk or infant formula (liquids, semisolids, and solids) provided to an infant or young child to provide nutrients and energy.

- The outcomes of interest were overweight and obesity at ages 2 years and older. Available evidence about rapid weight gain from birth to 24 months and BMI and body composition at ages 2 years and older were also examined from studies that conducted within-famliy analyses of discordant siblings (i.e., siblings fed differently during infancy, siblings with differences in outcome status, or both).

- This review identified 42 articles that met the inclusion criteria. ${ }^{1-42}$ Thirty of the 42 articles presented evidence about ever, compared with never, consuming human milk, and 21 of the 42 articles presented evidence about different durations of any human milk consumption (i.e. some articles presented evidence about both exposures).

- The 30 articles that examined the relationship between ever, compared with never, consuming human milk, and overweight and/or obesity at ages 2 years and older presented evidence from 21 independent cohorts.

- The evidence had strong consistency. Fourteen of the 21 studies found significant associations and all of them showed that ever, compared with never, consuming human milk is associated with lower risk of overweight and/or obesity at ages 2 years and older. One study showed a marginal association in the same direction, and some of the remaining studies were underpowered.

- The evidence available from 5 of 7 studies that compared infants who consumed human milk for different durations with infants who never consumed human milk suggested that a 
longer duration of human milk consumption (e.g., $\geq 6$ months) is most likely to be associated with reduced risk of overweight or obesity, compared to never consuming human milk.

- In 4 studies, the investigators conducted within-family analyses of siblings, which are designed to reduce bias due to confounding from genetic and environmental factors (factors that siblings share). Some of these analyses showed an attenuation of the significant associations found in full-sample analyses, suggesting that confounding may explain some of the association between ever, compared with never, consuming human milk and overweight and/or obesity at ages 2 years and older.

- The ability to draw stronger conclusions was primarily limited by the potential for confounding in a body of evidence made up of observational studies, and some concerns about the generalizability of the evidence from the studies conducted outside the United States (because U.S. populations may have higher risk of overweight and obesity than do the populations sampled for the non-U.S. studies).

- The 21 articles that examined the relationship between the duration of any human milk consumption, among infants fed human milk, and overweight and/or obesity at ages 2 years and older presented evidence from 1 cluster randomized controlled trial and 18 independent cohorts.

- The evidence was inconclusive. Nine of the 19 studies reported significant associations, but they were inconsistent in direction. In addition, potential bias from confounding and the limited generalizability of the evidence from the non-U.S. studies raised concerns (in particular because the prevalence of obesity among the participants of the cluster randomized controlled trial, conducted in Belarus, was much lower than the prevalence among youth in the United States).

- Evidence available from 2 studies was insufficient to determine the relationship between the duration of exclusive human milk consumption before the introduction of infant formula and overweight and/or obesity at ages 2 years and older.

- No studies were identified that examined the intensity, proportion, or amount of human milk consumed by mixed-fed infants, the intensity, proportion, or amount of human milk consumed at the breast versus by bottle, or consuming a single substance (i.e., either human milk or infant formula) versus both human milk and infant formula during a single feeding session.

\section{Ever, compared with never, consuming human milk}

\section{Description of the evidence examining the relationship between ever, compared with never, consuming human milk and body composition, including obesity, from studies that conducted within-family comparisons of siblings (Table 1)}

Six articles, published between 2003 and 2019, conducted within-family analyses that compared siblings who ever and never consumed human milk and their BMI or overweight and/or obesity status. ${ }^{3,6,10,14,27,32}$ These articles presented evidence from 4 independent U.S. cohorts, which are described below.

\section{Linked CENTURY Study}

Hawkins et al ${ }^{14}$ examined a sample of singleton children from the Linked CENTURY Study in the United States. Participants were from an existing clinical database of well-child visits at Atrius Health in Eastern Massachusetts. The authors examined initiation of human milk feeding as a dichotomous variable, which was obtained from hospital records at birth. Height 
and weight were measured by medical assistants at 2 and 5 years of age. The outcomes of interest were BMI z-score as a continuous variable and obesity (i.e., BMI $\geq 95^{\text {th }}$ percentile) as a dichotomous variable (both based on CDC growth curves). The authors conducted analyses in the full sample ( $N=55,058$ at 2 years and 43,893 at 5 years), in a subsample consisting of siblings ( $\mathrm{N}=21,455$ at 2 years and 17,083 at 5 years) and in a subsample of siblings with discordant outcomes ( $\mathrm{N}=2260$ at 2 years and 3249 at 5 years).

- In the full sample, initiating, compared with not initiating, human milk feeding was associated with a significantly lower BMI z-score at 2 and at 5 years of age $(\beta[95 \% \mathrm{Cl}]=$ $-0.06[-0.09,-0.04]$ and $-0.09[-0.11,-0.07]$, respectively). In the subsample of families with more than 1 sibling, the associations were nearly identical $(\beta[95 \% \mathrm{Cl}]=-0.06$ $[-0.10,-0.02]$ and $-0.09[-0.13,-0.06])$. In the subsample of siblings with discordant outcomes, initiating human milk feeding was associated with a significantly lower BMI zscore at 5 years of age $(\beta=-0.07[95 \% \mathrm{Cl}-0.13,-0.01]$; the association at 2 years of age was in the same direction but lacked statistical significance $(\beta=-0.04[95 \% \mathrm{Cl}-0.10$, 0.03]).

- In the full sample, initiating, compared with not initiating, human milk feeding was associated with significantly lower odds of obesity at both 2 and 5 years of age (OR [95\% $\mathrm{Cl}]=0.80[0.73,0.87]$ and 0.77 [0.72, 0.83], respectively). In the subsample of families with more than 1 sibling, the odds were the same with slightly wider confidence intervals $(\mathrm{OR}[95 \% \mathrm{Cl}]=0.80[0.69,0.92]$ and $0.77[0.69,0.87]$, respectively). In the subsample of siblings with discordant outcomes, initiating human milk feeding was not significantly associated with obesity at 2 or 5 years of age, and the odds ratios were closer to the null $(\mathrm{OR}[95 \% \mathrm{Cl}]=0.97[0.72,1.32]$ and $0.94[0.74,1.20]$, respectively $)$.

\section{Children of the National Longitudinal Survey on Youth 1979 Cohort (Children of NLSY79)}

Two articles ${ }^{3,6}$ examined samples of singleton children and adolescents whose mothers were part of the NLSY79 cohort from the United States. Anderson et al $(2003)^{3}$ examined offspring who were 3 to 11 years of age between 1986 and 1996, whereas Colen and Ramey 6 examined offspring who were 4 to 14 years of age between 1986 and 2010 and born after the year 1978 . Colen and Ramey ${ }^{6}$ reported that about $75 \%$ of participants were non-Hispanic white.

Both articles examined ever versus never consuming human milk as a dichotomous variable. Infant-feeding data were obtained by maternal report at biannual survey rounds starting in 1986.

Both articles used height and weight data, which were measured for most participants (i.e., in the article by Anderson et al (2003), ${ }^{3} 15 \%$ of children's height and $22 \%$ of children's weight were reported by mothers and in the article by Colen and Ramey, ${ }^{6}$ approximately $36 \%$ of child and adolescent height and weight data were reported by mothers or participants). Both articles examined obesity (i.e., BMI $\geq 95^{\text {th }}$ percentile based on CDC growth curves) as a dichotomous variable, and Colen and Ramey ${ }^{6}$ also examined BMI as a continuous variable.

Anderson et al (2003) $)^{3}$ conducted analyses on the full sample $(N=16,650$ observations using a probit model and $\mathrm{N}=15,050$ observations using an instrumental variable model), and on subsamples of siblings measured at the same point in time (mean 5.9 and 9.2 years of age for the younger and older siblings, respectively; $\mathrm{N}=7919$ observations), and at the same age (mean age of 6.6 years; $\mathrm{N}=4471$ observations).

- In the full sample, children who were ever fed human milk had an approximately $1.8 \%$ 
decrease in the likelihood of being obese relative to children never fed human milk at 3 to 11 years of age $(\beta=-0.018 \pm 0.008$ and $-0.016 \pm 0.010$, from probit and instrumental variable models, respectively; $p$-values were not reported, but the instrumental variable model was described as non-signficant).

- In both sibling subsamples (i.e., with outcomes measured at the same age and at the same time point), being fed human milk was not significantly associated with the likelihood of being obese at 3 to 11 years of age $(\beta=-0.021 \pm 0.023$ and $0.012 \pm 0.017$, respectively).

Colen and Ramey ${ }^{6}$ conducted analyses on the full sample ( $N=8237$ participants), on a subsample of siblings ( $N=7319$ participants), and on a second subsample of siblings with discordant infant feeding (i.e., siblings fed differently during infancy; $\mathrm{N}=1773$ participants).

- In the full sample and sibling subsample, ever compared with never consuming human milk was associated with a significantly lower BMI at 4 to 14 years of age $(\beta=-0.449 \pm$ 0.094 and $-0.413 \pm 0.101$, respectively; $p<0.001$ for both). In the discordant sibling subsample, the association was in the same direction but was smaller and lacked statistical significance $(\beta=-0.141 \pm 0.101)$.

- Likewise, in the full sample and sibling subsample, ever compared with never consuming human milk was associated with a significantly lower log odds of obesity at 4 to 14 years of age ( $\beta=-0.342 \pm 0.066$ and $-0.369 \pm 0.074$, respectively; $p<0.001$ for both). In the discordant sibling subsample, the association was in the same direction but was smaller and lacked statistical significance $(\beta=-0.173 \pm 0.164)$.

\section{National Longitudinal Study of Adolescent Health (Add Health)}

Two articles ${ }^{10,32}$ presented evidence from the Add Health study from the United States. This cohort is a nationally representative sample of 10 - to 18-year-olds in grades 7 to 12 during the 1994-1995 school year (baseline). Low-income, African-American, and Hispanic youth were oversampled. Nelson et $\mathrm{al}^{32}$ excluded members of the cohort if they had severe disabilities or pregnancies, were Native Americans, or had graduated from high school, and reported that the sample was $72 \%$ non-Hispanic white, $13 \%$ non-Hispanic black, $11 \%$ Hispanic and 3\% Asian.

Both articles examined ever versus never consuming human milk as a dichotomous variable. In addition, Nelson et $\mathrm{al}^{32}$ examined dichotomous comparisons of participants who were never fed human milk with participants who were fed human milk for different durations (i.e., $\geq 3$ months, $<3$ months, 3-5.99 months, 6-8.99 months and $\geq 9$ months). Infant-feeding data were obtained by parent recall at 10 to 18 years (i.e., baseline).

Evenhouse and Reilly ${ }^{10}$ examined BMI as a continuous variable and overweight/obesity (i.e., $\mathrm{BMI} \geq 85^{\text {th }}$ percentile based on CDC growth curves) and obesity (i.e., BMI $\geq 95^{\text {th }}$ percentile based on CDC growth curves) as dichotomous variables at baseline (i.e., 10 to 18 years of age). Nelson et al ${ }^{32}$ examined BMI z-score (based on CDC growth curves) as a continuous variable and overweight/obesity (i.e., $\mathrm{BMI} \geq 85^{\text {th }}$ percentile based on $\mathrm{CDC}$ growth curves) as a dichotomous variable at 12 to 21 years of age. Neither of the articles reported the methods for anthropometric data collection.

Evenhouse and Reilly ${ }^{10}$ conducted analyses on the full sample $(\mathrm{N}=16,903)$, on a subsample of siblings $(\mathrm{N}=3773)$ and on a second subsample of siblings with discordant infant feeding (i.e., sibships in which 1 sibling was ever fed human milk and the other was never fed human milk; $\mathrm{N}=576$ ). 
- In the full sample, the sibling subsample, and the discordant sibling subsample, ever compared with never consuming human milk was not significantly associated with $\mathrm{BMI}$ at 10 to 18 years of age $(\beta=-0.141 \pm 0.101,-0.34 \pm 0.16$, and $0.40 \pm 0.33$, respectively; $p<0.10$ for the full-sample and sibling-subsample analyses)

- Likewise, in the full sample, the sibling subsample, and the discordant sibling subsample, ever compared with never consuming human milk was not significantly associated with odds of overweight/obesity (OR $=0.79 \pm 0.03,0.87 \pm 0.08,1.32 \pm 0.21$, respectively; $\mathrm{p}<0.10$ for the full-sample and discordant-sibling subsample analyses), or obesity (OR = $0.77 \pm 0.04,0.88 \pm 0.11,1.17 \pm 0.25$, respectively; $p<0.10$ for the full-sample analysis) at 10 to 18 years of age.

Nelson et al $^{32}$ conducted analyses on the full sample stratified by sex ( $\mathrm{N}=5929$ males, 6069 females), on a subsample of siblings ( $\mathrm{N}=1700)$, and on subsamples of siblings with discordant infant feeding and outcomes (i.e., in which the overweight sibling had been fed human milk and the non-overweight sibling had not, and in which the overweight sibling had not been fed human milk and the non-overweight sibling had; $\mathrm{N}=224$ discordant siblings).

- In the full sample, ever compared with never consuming human milk was associated with significantly lower odds of overweight/obesity at 12 to 21 years of age in females (OR $0.83[95 \% \mathrm{Cl} 0.72,0.95]$ ); however, the association in males was closer to the null and nonsignificant (OR 0.90 [95\% Cl 0.76, 1.05]). In the subsample of siblings, ever compared with never consuming human milk was also associated with significantly lower odds of overweight/obesity at 12 to 21 years of age (OR 0.78 [95\% Cl 0.63, 0.97]). The withinfamily sibling analyses did not suggest any significant associations between ever, compared with never, consuming human milk and overweight/obesity at 12 to 21 years of age.

- In the full sample, there were no significant associations between consuming human milk for durations of $<3,3-5.99$, or 6-8.99 months, in comparison to never consuming human milk, and odds of overweight/obesity in males or in females at 12 to 21 years of age (OR [95\% Cl] 1.03 [0.85, 1.24], 0.81 [0.63, 1.04], and 0.86 [0.63, 1.17], respectively, in males, and $0.90[0.74,1.09], 0.82[0.64,1.05]$, and 0.73 [0.54, 1.00], respectively, in females). There was a significant association between consuming human milk for $\geq 9$ months, compared with never, and odds of overweight/obesity at 12 to 21 years of age in females but not in males (OR [95\% Cl] $0.78[0.64,0.96]$ and 0.83 [0.67, 1.04], respectively). These analyses were not reported for the subsamples of siblings or discordant siblings.

- In the subsample of siblings, there was a significant association between consuming human milk for $\geq 3$ months, compared with never consuming human milk, and lower odds of overweight/obesity at 12 to 21 years of age (OR 0.69 [95\% Cl $0.54,0.87]$. This analysis was not reported for the full sample or the subsample of discordant siblings.

- In a subsample of siblings with discordant exposures, there was no significant association between ever compared with never consuming human milk and difference in BMI z-score at 12 to 21 years of age when the lighter sibling was the one fed human milk ( $\beta=-0.004 \pm$ 0.11 ) or when the heavier sibling was the one fed human milk ( $\beta=-0.09 \pm 0.11)$. This analysis was not reported for the full sample or the subsample of siblings.

\section{Child Development Supplement of the Panel Study of Income Dynamics (CDS)}

Metzger and McDade ${ }^{27}$ examined a sample of children from the CDS in the United States. The sample consisted of children born between 1984 and 1993 with complete data on both infant feeding and BMI. The authors examined ever versus never consuming human milk as 
a dichotomous variable, which was obtained by recall from the primary caregiver when participants were between 4 and 13 years of age. Height and weight data were collected by study staff, but the specific methods were not reported. The outcomes of interest were BMI zscore as a continuous variable, and BMI considered as 2 separate dichotomous variables (1) $50^{\text {th }}$ percentile as a cut-off and (2) overweight/obesity (i.e., BMI $\geq 85^{\text {th }}$ percentile based on CDC growth curves). The authors conducted analyses in the full sample at 9 to 19 years of age $(\mathrm{N}=2591)$ using both an ordinary least squares model and a fixed-effects model, in a sibling subsample ( $N=976$, including 118 siblings with discordant exposures), and in a subsample of siblings with differences in both feeding and BMI status ( $N=30$ to 44).

- In the full sample, ever compared with never consuming human milk was significantly associated with a $0.150 \pm 0.065(p<0.05)$ unit lower BMl z-score. In the sibling subsample, ever compared with never consuming human milk was significantly associated with a $0.397 \pm 0.176(p<0.05)$ unit lower BMI $z$-score in the fixed-effects models, but with a non-significantly $0.152 \pm 0.106$ unit lower BMI z-score in the ordinary least squares model.

- In the sub-sample of participants with differences in both feeding and BMI status, ever compared with never consuming human milk was not significantly associated with the odds of having a BMI $>50^{\text {th }}$ percentile (OR $\sim 1.00$ and $\sim 1.30$ from fixed effects and ordinary least squares regression models, respectively).

- In the sub-sample of participants with differences in both feeding and BMI status, ever compared with never consuming human milk was significantly associated with a lower odds of overweight/obesity (OR $\sim 0.60, p<0.01$, and $\sim 0.40, p<0.05$, respectively from fixed effects and ordinary least squares regression models). 
Table 1. Evidence examining the relationship between ever, compared with never, consuming human milk and body composition, including obesity, from studies that conducted within-family comparisons of siblings ${ }^{a}$

\begin{tabular}{|c|c|c|c|c|}
\hline $\begin{array}{l}\text { Article } \\
\text { Study design (Study } \\
\text { name) } \\
\text { Country }\end{array}$ & $\begin{array}{l}\text { Ever vs never consuming human } \\
\text { milk exposure }{ }^{\text {b }}\end{array}$ & Outcome ${ }^{c}$ & Significant findings & $\begin{array}{l}\text { Nonsignificant } \\
\text { findings }\end{array}$ \\
\hline \multirow{17}{*}{$\begin{array}{l}\text { Hawkins 2019 } \\
\text { RC (Linked CENTURY } \\
\text { Study) } \\
\text { U.S. }\end{array}$} & Initiated BF vs Did not initiate BF & $\mathrm{BMIz}^{*}(\beta[95 \% \mathrm{Cl}])$ & & \\
\hline & & @ 2 y & & \\
\hline & & In the full sample $(\mathrm{N}=55,058)$ & $-0.06[-0.09,-0.04]$ & \\
\hline & & In subsample of siblings $(\mathrm{N}=21,455)$ & $-0.06[-0.10,-0.02]$ & \\
\hline & & $\begin{array}{l}\text { In the subsample of siblings with discordant } \\
\text { outcomes }(N=2260)\end{array}$ & & $-0.04[-0.10,0.03]$ \\
\hline & & @ 5 y & & \\
\hline & & In the full sample $(\mathrm{N}=43,893)$ & $-0.09[-0.11,-0.07]$ & \\
\hline & & In the subsample of siblings $(\mathrm{N}=17,083)$ & $-0.09[-0.13,-0.06]$ & \\
\hline & & $\begin{array}{l}\text { In the subsample of siblings with discordant } \\
\text { outcomes }(N=3249)\end{array}$ & $-0.07[-0.13,-0.01]$ & \\
\hline & & $\mathrm{OB}^{*}(\mathrm{OR}[95 \% \mathrm{Cl}])$ & & \\
\hline & & @ 2 y & & \\
\hline & & In the full sample $(\mathrm{N}=55,058)$ & $0.80[0.73,0.87]$ & \\
\hline & & In the subsample of siblings $(\mathrm{N}=21,445)$ & $0.80[0.69,0.92]$ & \\
\hline & & $\begin{array}{l}\text { In the subsample of siblings with discordant } \\
\text { outcomes }(N=2260)\end{array}$ & & $0.97[0.72,1.32]$ \\
\hline & & $@ 5$ y & & \\
\hline & & In the full sample $(\mathrm{N}=43,893)$ & $0.77[0.72,0.83]$ & \\
\hline & & In the subsample of siblings $(\mathrm{N}=17,081)$ & $0.77[0.69,0.87]$ & \\
\hline
\end{tabular}




\section{Article}

Study design (Study

name)

Ever vs never consuming human

milk exposure ${ }^{b}$

Outcome $^{c}$

Nonsignificant

findings

\section{Anderson $2003^{3}$}

Ever BF vs Never BF (ref)

PC (Children of NLSY79)

U.S.

Never BF (ref)

\begin{tabular}{|c|c|c|c|c|}
\hline \multicolumn{4}{|c|}{ Instrumental variable model ( $\mathrm{N}=15,050$ observations) } & \multirow[t]{2}{*}{$-0.016 \pm 0.010$} \\
\hline & & In the subsample of siblings & & \\
\hline & & $\begin{array}{l}\text { at the same point in time (mean } 5.9 \text { y and } 9.2 \text { y for } \\
\text { younger and older siblings, respectively) } \\
\text { ( } N=7919 \text { observations) }\end{array}$ & & $0.012 \pm 0.017 ; \mathrm{NS}$ \\
\hline & & $\begin{array}{l}\text { at the same age (mean } 6.6 \mathrm{y}) \\
(\mathrm{N}=4471 \text { observations) }\end{array}$ & & $-0.021 \pm 0.023 ; \mathrm{NS}$ \\
\hline \multirow{7}{*}{$\begin{array}{l}\text { Colen } 2014^{6} \\
\text { PC (Children of NLSY79) } \\
\text { U.S. }\end{array}$} & Ever BF vs Never BF (ref) & BMI ( $\beta \pm \mathrm{SE}, \mathrm{kg} / \mathrm{m} 2) @ 4-14$ y & & \\
\hline & & In the full sample $(\mathrm{N}=8,237)$ & $\begin{array}{l}-0.449 \pm 0.094 \\
p<0.001\end{array}$ & \\
\hline & & In the subsample of siblings $(\mathrm{N}=7,319)$ & $\begin{array}{l}-0.413 \pm 0.101 \\
p<0.001\end{array}$ & \\
\hline & & $\begin{array}{l}\text { In the subsample of siblings with discordant exposures } \\
(\mathrm{N}=1,773)\end{array}$ & & $-0.141 \pm 0.188 ; \mathrm{NS}$ \\
\hline & & $\mathrm{OB}^{*}(\beta \pm \mathrm{SE}) @ 4-14$ y & & \\
\hline & & In the full sample $(N=8,237)$ & $\begin{array}{l}-0.342 \pm 0.066 \\
p<0.001\end{array}$ & \\
\hline & & In the subsample of siblings $(\mathrm{N}=7,319)$ & $\begin{array}{l}-0.369 \pm 0.074 \\
p<0.001\end{array}$ & \\
\hline
\end{tabular}

\begin{tabular}{|c|c|c|c|}
\hline & & $\begin{array}{l}\text { In subsample of siblings with discordant exposures } \\
(\mathrm{N}=1,773)\end{array}$ & $-0.173 \pm 0.164 ; \mathrm{NS}$ \\
\hline \multirow{3}{*}{$\begin{array}{l}\text { Evenhouse } 2005^{10} \\
\text { CS (Add Health) } \\
\text { U.S. }\end{array}$} & Ever BF vs Never BF (ref) & BMI ( $\beta \pm$ SE, kg/m2) @ 10-18 y & \\
\hline & & In the full sample $(\mathrm{N}=7417,9486)$ & $-0.41 \pm 0.07 ; p<0.10$ \\
\hline & & In the subsample of siblings $(\mathrm{N}=1607,2166)$ & $-0.34 \pm 0.16 ; p<0.10$ \\
\hline
\end{tabular}




\section{Article}

Study design (Study

name)

Ever vs never consuming human

milk exposure

Outcome $^{c}$

Nonsignificant

findings

In the subsample of siblings with discordant exposures

$0.40 \pm 0.33 ; \mathrm{NS}$

$(\mathrm{N}=288,288)$

OWOB* (OR \pm SE from logit) @ 10-18 y

In the full sample $(\mathrm{N}=7417,9486)$

$0.79 \pm 0.03 ; p<0.10$

In the subsample of siblings $(\mathrm{N}=1607,2166)$

$0.87 \pm 0.08 ; N S$

In the subsample of siblings with discordant exposures

$(\mathrm{N}=288,288)$

$1.32 \pm 0.21 ; p<0.10$

$\mathrm{OB}^{*}(\mathrm{OR} \pm \mathrm{SE}$ from logit) @ 10-18 y

In the full sample $(\mathrm{N}=7417,9486)$

$0.77 \pm 0.04 ; p<0.10$

In the subsample of siblings $(\mathrm{N}=1607,2166)$

$0.88 \pm 0.11 ; \mathrm{NS}$

In the subsample of siblings with discordant exposures

$1.17 \pm 0.25 ; \mathrm{NS}$

$(\mathrm{N}=288,288)$

\section{Nelson $2005^{32}$}

PC (Add Health)

U.S.

Ever BF vs Never BF (ref)

In the full sample

In males $(\mathrm{N}=2787,3142)$

$0.90[0.76,1.05]$

In females $(\mathrm{N}=2975,3094)$

$0.83[0.72,0.95]$

In the subsample of siblings $(\mathrm{N}=860,840)$

$0.78[0.63,0.97]$

In the subsample of siblings with discordant exposures and outcomes $(\mathrm{N}=224)$

Odds that the non-OW sibling was ever BF and the

OW sibling was never BF vs both siblings OW and

never BF (ref)

Odds that the non-OW sibling was ever BF and the

OW sibling was never BF vs both siblings non-OW

and never BF (ref)

Odds that the OW sibling was ever BF and the non-

OW sibling was never BF vs both siblings OW and

never BF (ref) 


\section{Article}

Study design (Study

name)

Ever vs never consuming human

milk exposure ${ }^{b}$

Outcome $^{c}$

Nonsignificant

findings

Country

Odds that the OW sibling was ever BF and the non-

OW sibling was never BF vs both siblings non-OW

$1.27[0.65,2.50]$

and never BF (ref)

$\mathrm{BMIZ}^{*}$ difference ( $\left.\beta \pm \mathrm{SE}\right) @ 12-21$ y

In the subsample of siblings with discordant exposures

Lighter sibling $B F(N=224=112$ pairs $) \quad-0.004 \pm 0.11$

Heavier sibling $B F(N=224=112$ pairs $)$

$-0.09 \pm 0.11$

$\mathrm{BF} \geq 3 \mathrm{mo}$ vs Never BF (ref) $\quad \mathrm{OWOB}^{*}(\mathrm{OR}[95 \% \mathrm{Cl}]) @ 12-21 \mathrm{y}$ in the subsample of $0.69[0.54,0.87]$

siblings

$\mathrm{BF}<3$ mo vs Never BF (ref) $\quad \mathrm{OWOB}^{*}(\mathrm{OR}[95 \% \mathrm{Cl}]) @ 12-21$ y

In the full sample

In males $(\mathrm{N}=889,3142)$

$1.03[0.85,1.24]$

In females $(\mathrm{N}=910,3094)$

$0.90[0.74,1.09]$

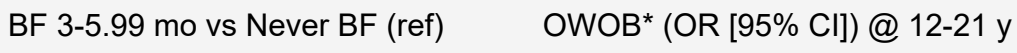

In the full sample

In males $(\mathrm{N}=593,3142)$

$0.81[0.63,1.04]$

In females $(\mathrm{N}=728,3094)$

$0.82[0.64,1.05]$

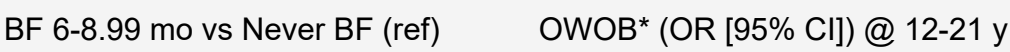

In the full sample

In males $(\mathrm{N}=474,3142)$

$0.86[0.63,1.17]$

In females $(\mathrm{N}=425,3094)$

$0.73[0.54,1.00]$

$B F \geq 9$ mo vs Never BF (ref)

OWOB* (OR [95\% Cl]) @ 12-21 y

In the full sample

In males $(\mathrm{N}=830,3142)$

$0.83[0.67,1.04]$

In females $(\mathrm{N}=607,3094)$

$0.78[0.64,0.96]$ 


\section{Article}

Study design (Study

name)

Ever vs never consuming human

milk exposure ${ }^{b}$

Significant findings

Nonsignificant

Country

\section{Metzger 200927}

PC (CDS)

U.S.
Ever BF vs Never BF (ref)

In full sample $(\mathrm{N}=2591)$

$-0.150 \pm 0.065 ; p<0.05$

In sibling subsample ( $\mathrm{N}=976$, including 118 siblings

with discordant exposures)

Ordinary least squares model

Fixed-effects model

$-0.397 \pm 0.176 ; p<0.05$

BMI $>50^{\text {th }} \%$ ile* vs $<50^{\text {th }} \%$ ile* $($ ref $)(O R) @ 9-19 y$ in

sibling subsample ( $\mathrm{N}=30$ to 44 with differences in both

feeding and BMI status)

Ordinary least squares model

Fixed-effects mode

$\sim 1.30 ;$ NS

OWOB* vs Not OWOB (ref) (OR) @ 9-19 y in sibling

subsample ( $\mathrm{N}=30$ to 44 with differences in both feeding and BMI status)

Ordinary least squares model

$\sim 0.60 ; p<0.01$

Fixed-effects model

$\sim 0.40 ; p<0.05$

$\mathrm{OB}^{*}$ vs Not $\mathrm{OB}^{*}$ (ref) (OR) @ 9-19 y in sibling subsample

$(\mathrm{N}=30$ to 44 with differences in both feeding and BM status)

Ordinary least squares model

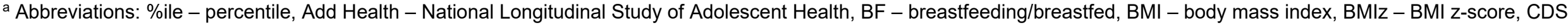

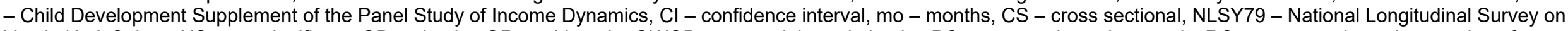

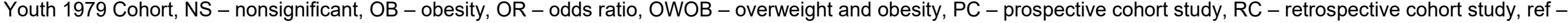
reference, SE - standard error, U.S. - United States of America, y - years

${ }^{b}$ Exposures, from the articles included in the body of evidence, which address ever vs never consuming human milk, or vice versa

c Notations used for growth references: ${ }^{*} \mathrm{CDC}$, †WHO, ‡lOTF, §Country-specific

${ }^{d}$ Anderson et al (2003) did not explicitly state that this finding was significant; however the text of the article implied that it was significant 
Description of the evidence examining the relationship between ever, compared with never, consuming human milk and overweight and/or obesity at 2 years of age and older (Table 2)

Thirty articles, published between 2003 and 2019, were identified that met the inclusion criteria and examined ever, compared with never, consuming human milk and overweight and/or obesity. ${ }^{1-11,14-17,19-22,26,27,30-32,35-37,39-41}$ They presented evidence from 21 independent studies. Four of the 21 studies were also described in the section about evidence from siblings (i.e., Linked CENTURY Study, Children of NLSY79, Add Health, and CDS). A description of the studies is presented below by age group (i.e., 2 to 5 years, 6 to 11 years, 12 to 19 years, and 20 years and older).

\section{Studies with evidence in multiple age groups}

\section{Generation Rotterdam (Generation R) Study}

Durmus et al (2011), ${ }^{9}$ Heppe et al, ${ }^{16}$ and Durmus et al (2014) ${ }^{8}$ examined samples of children who participated in the Generation R Study conducted in the Netherlands. All samples consisted of singleton infants who had available information on infant feeding. Heppe et al ${ }^{16}$ additionally restricted the sample to include only Caucasian infants. Durmus et al $(2011)^{9}$ examined 2 dichotomous variables: never consuming human milk compared with (1) consuming human milk for $>6$ months, and (2) consuming human milk exclusively until 4 months. Heppe et $\mathrm{al}^{16}$ and Durmus et al $(2014)^{8}$ examined never consuming human milk compared with ever consuming human milk (irrespective of duration) as a dichotomous variable. Infant-feeding data were obtained from clinical delivery reports at birth, and by maternal report at 2, 6 and 12 months of age. The outcome of interest in all 3 studies was overweight and obesity (combined). Durmus et al (2011) 9 defined overweight/obesity based on the Dutch growth reference charts (BMI $>1$ SDS, corresponding to an approximate adult BMI of $\left.>25 \mathrm{~kg} / \mathrm{m}^{2}\right)$ at 2 and 3 years of age ( $\mathrm{N}=5074$ at both ages). Heppe et $\mathrm{al}^{16}$ and Durmus et al $(2014)^{8}$ defined overweight/obesity based on the cut-offs provided by the International Obesity Taskforce (IOTF) at approximately 4 years $(\mathrm{N}=3610)$, and 6 years of age $(\mathrm{N}=5063)$, respectively. Anthropometric data were measured by trained research staff using standardized protocols. Standing height, rather than recumbent lengths, were measured after 14 months of age.

- Durmus et al $(2011)^{9}$ found no significant associations between never consuming human, compared with consuming human milk for $>6$ months and the odds of overweight/obesity at 2 or at 3 years of age (OR $[95 \% \mathrm{Cl}] \sim 1.5[\sim 0.9, \sim 2]$ and $\sim 0.9[\sim 0.8, \sim 2]$, respectively). Likewise, the authors found no significant associations between never consuming human milk, compared with consuming human milk exclusively until 4 months of age, and the odds of overweight/obesity at 2 or at 3 years of age (OR $[95 \% \mathrm{Cl}] \sim 1.5[\sim 0.8, \sim 2]$ and $\sim 1.5[\sim 0.8,2]$, respectively).

- Heppe et $\mathrm{al}^{16}$ and Durmus et al $(2014)^{8}$ found no significant associations between never consuming human milk compared with ever consuming human milk and the odds of overweight/obesity at 4 and at 6 years of age $(\mathrm{OR}[95 \% \mathrm{Cl}] 0.77[0.47,1.28]$ and $\sim 0.8$ $[\sim 0.6, \sim 1.3]$, respectively).

2. Lurbe et $\mathrm{al}^{22}$ examined a sample of children enrolled in a prospective cohort study conducted in Spain. The sample included singleton term infants ( $\geq 37$ weeks gestational age) born via vaginal delivery at the Hospital General at the University of Valencia. The sample 
was $100 \%$ white Spanish and approximately $70 \%$ of the sample were considered appropriate weight for gestational age. The authors compared infants who were fed human milk at birth with infants who were not fed human milk at birth, as reported by the mother at birth. The outcome of interest was obesity (i.e., BMI z-score >2 SD based on the 2007 WHO growth standard) at 5 and 10 years of age ( $\mathrm{N}=145$ and 100 and 5 and 10 years of age, respectively). Weight and height data were measured by study staff.

- Lurbe et $\mathrm{al}^{22}$ found that ever consuming human milk was associated with a significantly lower relative risk of obesity compared with never consuming human milk ( $\beta-0.190$, $p=0.022$ ) at 5 years of age. The association was, however, no longer significant at 10 years of age $(\beta-0.032, p=0.760)$.

3. Moschonis et $\mathbf{a l}^{30}$ presented analyses conducted on 4 European cohorts: Avon Longitudinal Study of Parents and Children (ALSPAC), Etude des Déterminants pré et post natals de la santé et du développement de l'Enfant (EDEN), EuroPrevall, and Generation XXI, conducted in the United Kingdom, France, Greece, and Portugal, respectively. Infant-feeding data were collected by parent report via questionnaire at 6 and 15 months of age in ALSPAC, at 4, 8, and 12 months of age in EDEN, at 15 months of age in Generation XXI, and at 12, 24, and 30 months of age in EuroPrevall. The authors examined never consuming human milk, compared with consuming human milk for $\geq 6$ months, as a dichotomous variable. Height and weight were measured at each study site and used to identify participants who were overweight and obese (combined, using IOTF cutoffs) at 4 $(\mathrm{N}=6522), 9(\mathrm{~N}=7540)$, and 13 years of age $(\mathrm{N}=6124)$ in the ALSPAC cohort, at 4 years of age in the EuroPrevall $(\mathrm{N}=309)$ and Generation $X X I(N=3387)$ cohorts, and at 5 years of age $(\mathrm{N}=1070)$ in EDEN cohort.

- Moschonis et $\mathrm{al}^{30}$ found no significant associations between never consuming human milk, compared with consuming milk for $\geq 6$ months, and the odds of overweight/obesity at 4,9 , or 13 years of age (OR 0.8, 1.2, and $\sim 1.1$, respectively; Cls not reported) in ALSPAC, at 4 years of age in EuroPrevall and Generation XXI (OR 1.5 and $\sim 1.2$, respectively; Cls not reported), and at 5 years of age in the EDEN cohort (OR $\sim 1.0 ; \mathrm{Cl}$ not reported).

\section{Millennium Cohort Study (MCS)}

Weng et $\mathrm{al}^{41}$ and Massion et $\mathrm{al}^{26}$ examined samples of children who participated in the MCS, a nationally representative sample of children born in the United Kingdom between September 2000 and January 2002. Children living in disadvantaged areas and ethnic minorities were oversampled to adequately represent hard-to-reach populations. Weng et $\mathrm{al}^{41}$ restricted their sample to infants who were singletons born at term. Weng et al ${ }^{41}$ examined ever (irrespective of duration) compared with never consuming human milk as a dichotomous variable, while Massion et al ${ }^{26}$ examined consuming human milk for $>4$ months versus never consuming human milk as a dichotomous variable. Infant-feeding data were obtained by parent report at the first interview when infants were between 6 and 12 months of age (mean age of 9.2 months). The outcome of interest was overweight and obesity (combined; defined using IOTF cutoffs) at 3 years of age $(\mathrm{N}=10,810$ in the study by Weng et $\left.\mathrm{al}^{41}\right)$ and 11 years of age $\left(\mathrm{N}=11,764\right.$ in the study by Massion $\left.\mathrm{et}^{\mathrm{al}^{26}}\right)$. Children's heights and weights were measured by trained research staff using standardized protocols.

- Weng et al ${ }^{41}$ found a significant association between never consuming human milk, compared with ever consuming human milk, and higher odds of overweight/obesity at 3 years of age (OR 1.25 [95\% Cl 1.09, 1.42]).

- Massion et $\mathrm{al}^{26}$ found a significant association between never consuming human milk, 
compared with consuming human milk for $>4$ months, and a higher relative risk of overweight/obesity at 11 years of age $(\mathrm{RR} \sim 1.1[95 \% \mathrm{Cl} \sim 1, \sim 1.25], \mathrm{p}<0.05)$.

\section{Children of the National Longitudinal Survey on Youth 1979 Cohort (Children of NLSY79)}

Anderson et al (2003), ${ }^{3}$ Colen and Ramey, ${ }^{6}$ and Huang et al ${ }^{17}$ examined samples of children and adolescents whose mothers were part of the National Longitudinal Survey on Youth 1979 Cohort from the United States. Anderson et al $(2003)^{3}$ examined offspring who were 3 to 11 years of age between 1986 and 1996, Colen and Ramey ${ }^{6}$ examined offspring who were 4 to 14 years of age between 1986 and 2010 and born after the year 1978, and Huang et a $\left.\right|^{17}$ examined children who had outcome data at 10 years of age and who had completed at least 8 waves of biannual data collection between 1986 and 2008. Colen and Ramey ${ }^{6}$ reported that about $75 \%$ of participants were non-Hispanic white, and Huang et al ${ }^{17}$ reported that about $42 \%$ were white, $35 \%$ were African American and $22 \%$ were Latino.

All of the articles examined ever versus never consuming human milk as a dichotomous variable. Infant-feeding data were obtained by maternal report at biannual survey rounds starting in 1986.

All of the articles used height and weight data. In the articles by Anderson et al (2003) and Colen and Ramey, ${ }^{6}$ height and weight were measured for most participants (i.e., in the article by Anderson et al (2003), $15 \%$ of children's height and $22 \%$ of children's weight were reported by mothers; in the article by Colen and Ramey, approximately $36 \%$ of child and adolescent height and weight data were reported by mothers or participants). In the article by Huang et al, ${ }^{17}$ height and weight were self-reported. All of the articles examined obesity (i.e., $\mathrm{BMI} \geq 95^{\text {th }}$ percentile based on CDC growth curves) as a dichotomous variable.

Anderson et al (2003) ${ }^{3}$ conducted analyses on the full sample $(\mathrm{N}=16,650$ observations using a probit model and $\mathrm{N}=15,050$ observations using an instrumental variable model), and on subsamples of siblings measured at the same point in time (mean 5.9 and 9.2 years of age for the younger and older siblings, respectively; $\mathrm{N}=7919$ observations), and at the same age (mean age of 6.6 years; $\mathrm{N}=4471$ observations).

- In the full sample, children who were ever fed human milk had an approximately $1.8 \%$ decrease in the likelihood of being obese relative to children never fed human milk at 3 to 11 years of age $(\beta=-0.018 \pm 0.008$ and $-0.016 \pm 0.010$, from probit and instrumental variable models, respectively; $p$-values were not reported, but the instrumental variable model was described as non-signficant).

- In both sibling subsamples (i.e., with outcomes measured at the same age and at the same time point), being fed human milk was not significantly associated with the likelihood of being obese at 3 to 11 years of age $(\beta=-0.021 \pm 0.023$ and $0.012 \pm 0.017$, respectively).

Colen and Ramey ${ }^{6}$ conducted analyses on the full sample ( $N=8237$ participants), on a subsample of siblings ( $N=7319$ participants), and on a second subsample of siblings with discordant infant feeding (i.e., siblings fed differently during infancy; $\mathrm{N}=1773$ participants).

- In the full sample and sibling subsample, ever compared with never consuming human milk was associated with a significantly lower log odds of obesity at 4 to 14 years of age ( $\beta=-0.342 \pm 0.066$ and $-0.369 \pm 0.074$, respectively; $p<0.001$ for both). In the discordant sibling subsample, the association was in the same direction but was smaller and lacked statistical significance $(\beta=-0.173 \pm 0.164)$. 
Huang et al ${ }^{17}$ conducted analyses on the full sample $(\mathrm{N}=5156)$.

- Huang et al ${ }^{17}$ found that ever, compared with never, consuming human milk was associated with significantly lower odds of obesity at 10 years (OR: $0.79, p<0.05 ; \mathrm{Cl}$ not reported).

\section{Prevention and Incidence of Asthma and Mite Allergy (PIAMA) cohort}

Ruijsbroek et $\mathbf{a l}^{37}$ and Pluyman et $\mathbf{a} \mathbf{l}^{36}$ examined samples of children that participated in the PIAMA study from the Netherlands, which was a birth cohort study and prospective follow-up of (1) participants in an intervention study of mite-impermeable mattress and pillow covers, and (2) participants in a natural-history study in which no intervention was applied. The sample for the intervention arm of the study consisted of children of allergic mothers (mothers with at least 1 of the following: asthma ever, pet allergy, house dust mite allergy or nasal allergy such as hay fever). The sample for the natural-history study consisted of children born to both allergic and non-allergic mothers. Ruijsbroek et $\mathrm{al}^{37}$ examined never consuming human milk, compared with consuming human milk for $>16$ weeks, as a dichotomous variable. Pluyman et $\mathrm{a}^{36}$ examined consuming human milk (irrespective of duration) and consuming human milk for 3 different durations ( $<3$ months, 3 to $<6$ months and $\geq 6$ months) compared to never consuming human milk. Infant-feeding data were collected prospectively by parental report at 3 months of infant age. In the article by Ruijsbroek et al, ${ }^{37}$ the outcomes of interest were overweight and obesity (combined) and obesity, defined using IOTF cut-offs, at 3 to 8 years of age $(\mathrm{N}=3963)$. From 3 to 8 years of age, anthropometric data were collected via parental recall (based on doctor or nurse report if measured within the last 3 months, or measured by parents), but specific methods were not reported. In the article by Pluyman et al, ${ }^{36}$ the outcome of interest was overweight and obesity (combined), defined using the IOTF cutoff, at 12 years of age $(\mathrm{N}=2787)$. At 12 years of age, height and weight were measured by study staff.

- Ruijsbroek et $\mathrm{al}^{37}$ found no significant associations between never consuming human milk, compared with consuming human milk for $>16$ weeks, and the odds of overweight/obesity or obesity at 3 to 8 years of age (OR $[95 \% \mathrm{Cl}] 1.08[0.91,1.29]$ and $1.44[0.95,2.18]$, respectively); however the wide confidence interval around the odds ratio for obesity may indicate an insufficient sample size to detect the outcome.

- In contrast, Pluyman et al, ${ }^{36}$ found that ever consuming human milk (irrespective of duration), compared with never consuming human milk, was significantly associated with lower odds of overweight/obesity at 12 years of age (OR 0.61 [95\% Cl $0.38,0.97]$ ). When the authors divided the group ever fed human milk into 3 categories of duration (i.e., $<3$ months, $3-<6$ months, $\geq 6$ months), the odds ratios were similar, but the confidence intervals were wider so the associations lacked statistical significance (OR $[95 \% \mathrm{Cl}] 0.63$ $[0.37,1.08], 0.55$ [0.30, 1.00], and 0.61 [0.36, 1.06], respectively).

\section{National Longitudinal Study of Adolescent Health (Add Health)}

Evenhouse and Reilly, ${ }^{10}$ Bohr et al,${ }^{5}$ and Nelson et al ${ }^{32}$ presented evidence from the Add Health study from the United States. This cohort is a nationally representative sample of 10to 18-year-olds in grades 7 to 12 during the 1994-1995 school year (baseline). Low-income, African-American, and Hispanic youth were oversampled. Nelson et al ${ }^{32}$ excluded members of the cohort if they had severe disabilities or pregnancies, were Native Americans, or had graduated from high school, and reported that the sample was $72 \%$ non-Hispanic white, $13 \%$ non-Hispanic black, $11 \%$ Hispanic and $3 \%$ Asian.

All 3 articles examined ever versus never consuming human milk as a dichotomous variable. 
In addition, Nelson et al ${ }^{32}$ examined dichotomous comparisons of participants who were never fed human milk with participants who were fed human milk for different durations (i.e., $\geq 3$ months, $<3$ months, 3-5.99 months, 6-8.99 months and $\geq 9$ months). Infant-feeding data were obtained by parent recall at 10 to 18 years (i.e., baseline).

The articles looked at outcomes at baseline (i.e., 10 to 18 years), 2 years later (i.e.,12 to 21 years), and 14 years later (i.e., 24 to 33 years). Evenhouse and Reilly ${ }^{10}$ examined overweight/obesity (i.e., BMI $\geq 85^{\text {th }}$ percentile based on CDC growth curves) and obesity (i.e., $\mathrm{BMI} \geq 95^{\text {th }}$ percentile based on CDC growth curves) as dichotomous variables at 10 to 18 years of age. Nelson et al ${ }^{32}$ examined overweight/obesity (i.e., BMI $\geq 85^{\text {th }}$ percentile based on CDC growth curves) as a dichotomous variable at 12 to 21 years of age. Bohr et al ${ }^{5}$ examined obesity (i.e., BMI $\geq 30 \mathrm{~kg} / \mathrm{m}^{2}$ ) as a dichotomous variable when members of the cohort were 24 to 33 years of age. None of the articles reported the methods for anthropometric data collection.

Evenhouse and Reilly ${ }^{10}$ conducted analyses on the full sample $(\mathrm{N}=16,903)$, on a subsample of siblings $(\mathrm{N}=3773)$ and on a second subsample of siblings with discordant infant feeding (i.e., sibships in which 1 sibling was ever fed human milk and the other was never fed human milk; $\mathrm{N}=576$ ).

- In the full sample, the sibling subsample, and the discordant sibling subsample, ever compared with never consuming human milk was not significantly associated with odds of overweight/obesity $(\mathrm{OR}=0.79 \pm 0.03,0.87 \pm 0.08,1.32 \pm 0.21$, respectively; $p<0.10$ for the full-sample and discordant-sibling subsample analyses), or obesity (OR $=0.77 \pm 0.04$, $0.88 \pm 0.11,1.17 \pm 0.25$, respectively; $p<0.10$ for the full-sample analysis) at 10 to 18 years of age.

Nelson et al $^{32}$ conducted analyses on the full sample stratified by sex ( $\mathrm{N}=5929$ males, 6069 females), on a subsample of siblings ( $\mathrm{N}=1700)$, and on subsamples of siblings with discordant infant feeding and outcomes (i.e., in which the overweight sibling had been fed human milk and the non-overweight sibling had not, and in which the overweight sibling had not been fed human milk and the non-overweight sibling had; $\mathrm{N}=224$ discordant siblings).

- In the full sample, ever compared with never consuming human milk was associated with significantly lower odds of overweight/obesity at 12 to 21 years of age in females (OR $0.83[95 \% \mathrm{Cl} 0.72,0.95]$ ); however, the association in males was closer to the null and nonsignificant (OR 0.90 [95\% Cl 0.76, 1.05]). In the subsample of siblings, ever compared with never consuming human milk was also associated with significantly lower odds of overweight/obesity at 12 to 21 years of age (OR 0.78 [95\% Cl 0.63, 0.97]). The withinfamily sibling analyses did not suggest any significant associations between ever, compared with never, consuming human milk and overweight/obesity at 12 to 21 years of age.

- In the full sample, there were no significant associations between consuming human milk for durations of $<3,3-5.99$, or 6-8.99, in comparison to never consuming human milk, and odds of overweight/obesity in males or in females at 12 to 21 years of age $(\mathrm{OR}$ [95\% Cl] 1.03 [0.85, 1.24], $0.81[0.63,1.04]$, and $0.86[0.63,1.17]$, respectively, in males, and 0.90 $[0.74,1.09], 0.82[0.64,1.05]$, and 0.73 [0.54, 1.00], respectively in females). There was a significant association between consuming human milk for $\geq 9$ months, compared with never, and odds of overweight/obesity at 12 to 21 years of age in females but not in males (OR [95\% Cl] $0.78[0.64,0.96]$ and 0.83 [0.67, 1.04], respectively). These analyses were not reported for the subsamples of siblings or discordant siblings.

- In the subsample of siblings, there was a significant association between consuming 
human milk for $\geq 3$ months, compared with never consuming human milk, and lower odds of overweight/obesity at 12 to 21 years of age (OR 0.69 [95\% Cl $0.54,0.87]$. This analysis was not reported for the full sample or the subsample of discordant siblings.

Bohr et al ${ }^{5}$ conducted analyses in an unrelated sample $(\mathrm{N}=4648)$ and a sibling pair sample $(\mathrm{N}=1179)$.

- In the unrelated sample, the odds of being obese at 24 to 33 years were significantly lower in those ever compared with never fed human milk (OR [95\% Cl] 0.854 [0.75, 0.97], $p=0.018)$. In the sibling pair sample, the direction and magnitude of effect was the same, but the association was not significant $(0.854$ [0.64, 1.13], $p=0.277)$.

\section{Child Development Supplement of the Panel Study of Income Dynamics (CDS)}

Metzger and McDade ${ }^{27}$ examined a sample of children from the U.S.-based CDS. The sample consisted of children born between 1984 and 1993 with complete data on both infant feeding and BMI. The authors examined ever versus never consuming human milk as a dichotomous variable, which was obtained by recall from the primary caregiver when participants were between 4 and 13 years of age. Height and weight data were collected by study staff, but the specific methods were not reported. The outcome of interest was BMI considered as 2 separate dichotomous variables (1) $50^{\text {th }}$ percentile as a cut-off and (2) overweight/obesity (i.e., BMI $\geq 85^{\text {th }}$ percentile based on CDC growth curves). The authors conducted analyses in the full sample at 9 to 19 years of age $(\mathrm{N}=2591)$ using both an ordinary least squares model and a fixed-effects model, in a sibling subsample ( $N=976$, including 118 siblings with discordant exposures), and in a subsample off siblings with differences in both feeding and BMI status ( $\mathrm{N}=30$ to 44$)$.

- In the sub-sample of participants with differences in both feeding and BMI status, ever compared with never consuming human milk was not significantly associated with the odds of having a BMI $>50^{\text {th }}$ percentile (OR $~ 1.00$ and $\sim 1.30$ from fixed effects and ordinary least squares regression models, respectively).

- In the sub-sample of participants with differences in both feeding and BMI status, ever compared with never consuming human milk was significantly associated with a lower odds of overweight/obesity $(O R \sim 0.60(p<0.01)$ and $\sim 0.40(p<0.05)$, respectively from fixed effects and ordinary least squares regression models, respectively).

\section{Studies with evidence from 2 to 5 years of age}

\section{Growing Up in Scotland (GUS)}

Abraham et al ${ }^{1}$ examined data from the U.K.-based prospective cohort study GUS, which had a representative sample of Scottish infants from 130 geographically clustered areas across Scotland. Infants were born between June 2004 and May 2005 and were selected at random from child benefit records. The authors examined ever consuming human milk (irrespective of duration) compared to never consuming human milk. Infant-feeding data were reported by the primary caregiver (typically mothers) when the child was between 9 and 12 months of age. The outcome of interest was overweight/obesity (combined) at $45-48$ months of age (i.e., BMI z-score $\geq 1.04$ based on the 1990 U.K. growth reference curves; $N=3515$ ). Height and weight were measured by study staff, but specific methods were not reported.

- Abraham et al ${ }^{1}$ found a nonsignificant association between ever, compared with never, consuming human milk and lower odds of overweight/obesity at 45-48 months (OR 0.81 $[95 \% \mathrm{Cl} 0.64,1.01])$. 
Anderson et al (2003) ${ }^{3}$ : See number 5, above

10. Anderson et al (2014) ${ }^{2}$ examined data from a cohort of infants born between 2005 and 2009 who participated in the Supplemental Nutrition Program for Women, Infants and Children (WIC) in Hawaii, United States. The majority of the participants were of Native Hawaiian $(62 \%)$ and Asian $(25 \%)$ ancestry. The authors examined dichotomous comparisons of infants who consumed human milk for $<1$ month $(\mathrm{N}=3769), 1-2$ months $(\mathrm{N}=4864), 3-5$ months $(\mathrm{N}=3119)$, or $\geq 6$ months $(\mathrm{N}=2192)$ with infants who never consumed human milk $(\mathrm{N}=1197)$. Infant-feeding data were collected from mothers by WIC staff. The outcome of interest was overweight and obesity (combined; i.e., BMI $\geq 85^{\text {th }}$ percentile based on CDC growth curves) at 2 years of age. Height and weight were measured by WIC staff, but the methods used were not reported.

- There was a significant association between consuming human milk for $\geq 6$ months, compared with never consuming human milk, and a lower prevalence ratio of overweight/obesity at 2 years of age (prevalence ratio 0.82 [95\% $\mathrm{Cl} 0.72,0.94], \mathrm{p}<0.05$ ). The associations between consuming shorter durations of human milk (i.e., $<1,1-2$, and 3-5 months), compared with never consuming human milk, and the odds of overweight and obesity at 2 years of age were closer to the null and nonsignificant $(\mathrm{OR}[95 \% \mathrm{Cl}] 0.97$ [0.86, 1.09], 0.96 [0.85, 1.08], and 0.99 [0.89, 1.12], respectively).

Colen and Ramey 6 : See number 5 , above

Durmus et al (2011) ${ }^{9}$ : See number 1 , above

11. Feldman-Winter et al $^{11}$ examined a cohort of infants born in an urban Baby-Friendly hospital in the United States that primarily served racial and ethnic minority groups and families with a low SES. Participants were singletons, born adequate weight for gestational age, about $70 \%$ Hispanic, $20 \%$ black, and $5 \%$ white, and about $85 \%$ were WIC participants. The authors compared infants who consumed infant formula exclusively for the first week of life $(\mathrm{N}=12)$ with infants who consumed human milk exclusively for the first week of life $(\mathrm{N}=71)$. Infantfeeding data were collected from post-delivery medical records and from maternal report during a home visit when infants were 7 days old. The outcome of interest was overweight and obesity (combined; i.e., BMI $\geq 85$ th percentile based on $\mathrm{WHO}$ growth curves) at 2 years of age.

- The authors found no significant association between consuming infant formula exclusively for the first week of life, compared with consuming human milk exclusively for the first week of life, and odds of overweight/obesity at 2 years of life (OR $0.7[95 \% \mathrm{Cl}$ $0.1,7.0], p=0.79$ ). The study was underpowered, because at this Baby-Friendly hospital, there were few infants who were exclusively fed infant formula.

\section{Linked CENTURY Study}

Hawkins et al ${ }^{14}$ examined a sample of singleton children from the U.S.-based Linked CENTURY Study. Participants were from an existing clinical database of well-child visits at Atrius Health in Eastern Massachusetts. The authors examined initiation of human milk feeding as a dichotomous variable, which was obtained from hospital records at birth. Height and weight were measured by medical assistants at 2 and 5 years of age. The outcome of interest was obesity (i.e., BMI $\geq 95^{\text {th }}$ percentile based on CDC growth curves) as a dichotomous variable. The authors conducted analyses in the full sample ( $N=55,058$ at 2 years and 43,893 at 5 years), in a subsample consisting of siblings $(\mathrm{N}=21,455$ at 2 years and 17,083 at 5 years) and in a subsample of siblings with discordant outcomes ( $N=2260$ at 2 years and 3249 at 5 years). 
- In the full sample, initiating, compared with not initiating, human milk feeding was associated with significantly lower odds of obesity at both 2 and 5 years of age (OR [95\% $\mathrm{Cl}]=0.80[0.73,0.87]$ and $0.77[0.72,0.83]$, respectively). In the subsample of families with more than 1 sibling, the odds were the same with slightly wider confidence intervals (OR $[95 \% \mathrm{Cl}]=0.80[0.69,0.92]$ and $0.77[0.69,0.87]$, respectively). In the subsample of siblings with discordant outcomes, initiating human milk feeding was not significantly associated with obesity at 2 or 5 years of age, and the odds ratios were closer to the null $(\mathrm{OR}[95 \% \mathrm{Cl}]=0.97[0.72,1.32]$ and $0.94[0.74,1.20]$, respectively).

Heppe et al ${ }^{16}$ : See number 1 , above

13. Layte et al ${ }^{19}$ presented evidence from a nationally representative sample of infants in Ireland who were 9 months old in 2008-2009. Participants were predominantly of Irish nationality $(86 \%)$. The authors compared infants who consumed human milk for $<3$ months ( $N=386$ ), $<6$ months $(\mathrm{N}=543)$, and $\geq 6$ months $(\mathrm{N}=870)$ with infants who never consumed human milk $(\mathrm{N}=4743)$. Authors did not indicate whether the $<6$ month variable included or excluded infants fed human milk $<3$ months. Infant-feeding data were collected by maternal reports via interview at 9 months. Study staff measured participant height and weight at 3 years of age, which were used to identify participants who were obese using the IOTF threshold.

- Layte et al ${ }^{19}$ found a significant association between consuming human milk for $\geq 6$ months, compared with never consuming human milk, and lower odds of obesity at 3 years of age (OR 0.51, $\mathrm{p}<0.001 ; \mathrm{Cl}$ not reported). The associations between consuming human milk for shorter durations (i.e., $<3$ and $<6$ months), compared with never consuming human milk, were closer to the null and nonsignificant (OR $0.96, p=0.79$ and $0.97, p=0.87$, respectively; Cls not reported).

14. Lee et $\mathrm{al}^{20}$ retrospectively examined a cohort of 38,049 infants born in Korea in 2008 who had completed 7 routine medical check-ups at 4 to 6,9 to 12,18 to 24,30 to 36,42 to 48,54 to 60 , and 66 to 72 months of age. The authors compared infants who were mixed-fed from birth to 4-6 months and infants who consumed human milk exclusively from birth to 4-6 months with infants who consumed infant formula exclusively from birth to 4-6 months. Infant-feeding data were reported by parents during the 4-6 month medical check-up and extracted from the National Health Insurance Service database. The outcome of interest was overweight/obesity (combined; i.e., BMI $>90^{\text {th }}$ percentile based on the Korean National Growth Charts) at 30-36, 42-48, 54-60, and 66-72 months of age. Height and weight were measured during the medical check-ups. The authors stratified the analyses by birthweight category (i.e., normal birth weight, high birthweight).

- In the high-birthweight subsample, there were significant associations between being mixed-fed from birth to 4 to 6 months, compared with being exclusively formula fed from birth to 4 to 6 months, and lower odds of overweight/obesity at 30-36 months and 42-48 months (OR [95\% Cl] $0.66[0.48,0.92]$ and 0.69 [0.50, 0.97], respectively). The association at 54-60 months was in the same direction but was slightly attenuated and lacked statistical significance (OR $0.75[95 \% \mathrm{Cl} 0.54,1.03]$ ). The association at 66 to 72 months was close to the null (OR 1.02 [95\% Cl 0.72, 1.45]).

- In the normal-birthweight subsample, there were no significant associations between being mixed-fed from birth to 4 to 6 months, compared with being exclusively formula fed from birth to 4 to 6 months, and the odds of overweight/obesity at 30-36, 42-48, 54-60, or 66-72 months of age (OR [95\% Cl] 0.94 [0.86, 1.02]), 0.97 [0.89, 1.05]), $0.92[0.84,1.00]$, and $0.95[0.88,1.04]$, respectively).

- In the high-birthweight subsample, there were significant associations between being fed 
human milk exclusively from birth to 4-6 months, compared with being fed infant formula exclusively from birth to 4-6 months, and lower odds of overweight/obesity at 30-36 and $54-60$ months of age $(\mathrm{OR}[95 \% \mathrm{Cl}] 0.73[0.56,0.96]$ and $0.69[0.53,0.90]$, respectively). The association at 42-48 months was in the same direction but the confidence interval included the null $(\mathrm{OR} 0.76[95 \% \mathrm{Cl} 0.58,1.00]$. The association at $66-72$ months was in the opposite direction, but was nonsignificant (OR 1.24 [95\% Cl 0.92, 1.66].

- In the normal-birthweight subsample, there were significant associations between being fed human milk exclusively from birth to 4-6 months, compared with being fed infant formula exclusively from birth to 4-6 months, and lower odds of overweight/obesity at 30$36,42-48,54-60$, and $66-72$ months of age (OR [95\% Cl] 0.75 [0.70, 0.81], 0.85 [0.79, 0.91 ], 0.87 [0.81, 0.93], and 0.87 [0.81, 0.93], respectively).

\section{Early Childhood Longitudinal Study-Birth (ECLS-B)}

Li et $\mathrm{al}^{21}$ and Moss and Yeaton ${ }^{31}$ examined samples from the ECLS-B cohort from the United States. Participants were a nationally representative sample born in the United States in 2001 to mothers who were at least 15 or 16 years of age, respectively. ${ }^{21,31}$ Moss and Yeaton ${ }^{31}$ restricted the sample to participants with a birthweight $\geq 1500 \mathrm{~g}$. Li et $\mathrm{al}^{21}$ reported that $42 \%$ of participants were non-Hispanic white, $16 \%$ were non-Hispanic black, $20 \%$ were Hispanic, $11 \%$ were Asian, and $11 \%$ had other racial/ethnic backgrounds; Moss and Yeaton ${ }^{31}$ did not report similar information. Both articles examined ever consuming human milk, compared with never consuming human milk, as a dichotomous variable. Moss and Yeaton ${ }^{31}$ secondarily stratified infants by their timing of introduction to complementary foods. Infant-feeding data were collected by maternal interview at 9 months and 4 years. Li et $\mathrm{al}^{21}$ assessed obesity (i.e., BMI $\geq 95^{\text {th }}$ percentile based on CDC growth curves) at 4 years $(\mathrm{N}=8150)$. Moss and Yeaton ${ }^{31}$ assessed obesity as well as overweight (i.e., BMI $\geq 85^{\text {th }}-94^{\text {th }}$ percentile based on CDC growth curves) and healthy weight (i.e., BMI $<85^{\text {th }}$ percentile based on CDC growth curves, including underweight infants) at 2 years (total analytic $\mathrm{N}=7200$ ) and 4 years (total analytic $\mathrm{N}=6950$ ). Height and weight were measured by trained research staff.

- Li et al ${ }^{21}$ found that ever consuming human milk, compared with never consuming human milk, was associated with significantly lower odds of obesity at 2 years of age (OR 0.76 [95\% Cl 0.63, 0.92]).

- Likewise, Moss and Yeaton ${ }^{31}$ found that ever consuming human milk, compared with never consuming human milk, was associated with significantly lower odds of obesity (compared with overweight) at 2 and at 4 years of age (OR [95\% Cl] $0.64[0.51,0.80]$, $p<0.001$, and $0.67[0.53,0.85], p<0.01$ respectively). The associations with normal weight (compared with overweight) at 2 and at 4 years were closer to the null and nonsignificant (OR [95\% Cl] $1.08[0.89,1.31]$ and $0.94[0.75,1.18]$ respectively).

- In secondary analyses that incorporated the timing of participants' introduction to complementary foods:

- When all infants were introduced to complementary foods $<4$ months, there was a significant association between ever consuming human milk, compared with never consuming human milk, and lower odds of obesity (compared with healthy weight) at 2 years of age (OR 0.64 [95\% Cl 0.47, 0.86], p<0.01). However the association with obesity at 4 years of age was slightly attenuated and nonsignificant (OR $0.72[95 \% \mathrm{Cl}$ $0.49,1.06], p<0.10)$. The associations with overweight (compared with healthy weight) at 2 and at 4 years were nonsignificant $(\mathrm{OR}[95 \% \mathrm{Cl}] 0.89[0.62,1.29]$ and $0.91[0.62$, $1.34]$ respectively). 
- When infants ever fed human milk were introduced to complementary foods at 4-5 months and infants never fed human milk were introduced to complementary foods at $<4$ months, there were significant associations between ever consuming human milk, compared with never consuming human milk, and lower odds of obesity (compared with health weight) at 2 and at 4 years of age (OR [95\% Cl] 0.38 [0.29, 0.51], $p<0.001$ and $0.45[0.33,0.61], p<0.001$, respectively). The associations with overweight (compared with healthy weight) at 2 and at 4 years were nonsignificant $(\mathrm{OR}[95 \% \mathrm{Cl}]$ $0.92[0.67,1.28]$ and $0.80[0.55,1.16]$, respectively).

- Likewise, when infants ever fed human milk were introduced to complementary foods at $\geq 6$ months and infants never fed human milk were introduced to complementary foods at $<4$ months, there were significant associations between ever consuming human milk, compared with never consuming human milk, and lower odds of obesity (compared with health weight) at 2 and at 4 years of age $(\mathrm{OR}[95 \% \mathrm{Cl}] 0.38[0.27$, $0.53], p<0.001$ and 0.51 [0.36, 0.72], $p<0.001$, respectively). The associations with overweight (compared with healthy weight) at 2 and at 4 years were nonsignificant (OR $[95 \% \mathrm{Cl}] 0.82[0.58,1.15]$ and $0.81[0.54,1.22]$ respectively).

Lurbe et al ${ }^{22}$ : See number 2, above

Moschonis et al ${ }^{30}$ : See number 3 , above

Moss and Yeaton ${ }^{31}$ : See number 15, above

\section{First Baby Study (FBS)}

Pattison et $\mathbf{a l}^{35}$ conducted a secondary analysis of a sample of children enrolled in the FBS in the United States. Children included in the analyses were first-born, primarily white (approximately $84 \%$ of sample) and had available data on human milk consumption. The authors compared ever consuming human milk (irrespective of duration), as well as consuming human milk for 2 different durations (0-6 months and $<6$ months) with never consuming human milk. Infant-feeding data were collected prospectively via maternal interviews at 1,6 , and 12 months of age. The outcomes of interest were overweight/obesity (combined; i.e., $\mathrm{BMI} \geq 85^{\text {th }}$ percentile using $\mathrm{CDC}$ growth curves) and obesity (i.e., BMI $\geq 95^{\text {th }}$ percentile using CDC growth curves) at 36 months of age ( $N=1626,867$, and 870 for analyses comparing ever, 0-6 months, and $>6$ months with never consuming human milk, respectively). Height and weight were measured and reported by mothers.

- Pattison et $\mathrm{al}^{35}$ found that ever consuming human milk was associated with a significantly lower odds of overweight/obesity at 36 months compared with never consuming human milk (OR [95\% Cl] $0.63[0.41,0.96])$. Likewise, the authors found that consuming human milk for $>6$ months was associated with a significantly lower odds of overweight/obesity at 36 months of age compared with never consuming human milk (OR 0.54 [95\% $\mathrm{Cl} 0.34$, $0.85]$ ). The association between consuming human milk for 0-6 months and the odds of overweight/obesity was in the same direction and of similar magnitude, but was not statistically significant (OR $0.68[95 \% \mathrm{Cl} 0.44,1.05])$.

- Ever consuming human milk (irrespective of duration), consuming human milk for 0-6 months, and consuming human milk for $>6$ months were all significantly associated with a lower odds of obesity at 36 months compared to never consuming human milk (OR [95\% $\mathrm{CI}] 0.51$ [0.30, 0.86], 0.54 [0.32, 0.94] and 0.43 [0.24, 0.78], respectively).

Ruijsbroek et al ${ }^{37}$ : See number 6 , above

17. Thorland et al ${ }^{39}$ examined first-born children from low-income families enrolled in the Nurse- 
Family Partnership in the United States between 2007 and 2010. The authors examined dichotomous comparisons of infants who consumed human milk for 1-3, 4-8, 8-12, or >20 weeks with infants who never consumed human milk. Infant-feeding data were collected by parent report. The outcome of interest was high weight-for-length (WFL) status (i.e., WFL $\geq 97.7^{\text {th }}$ percentile based on the WHO growth curves) at 24 months of age $(\mathrm{N}=6,383)$. Length and weight were measured by Nurse-Family Partnership staff during home visits using NHANES guidelines.

- There was a significant association between consuming human milk for $>20$ weeks, compared with never consuming human milk, and lower odds of high WFL status at 24 months of age (OR 0.60 [95\% Cl $0.41,0.88], p<0.05$ ). The associations between shorter durations of human milk consumption (i.e., 1-3, 4-8, and 8-20 weeks), compared with never consuming human milk, and high WFL status at 24 months of age were in the same direction but were attenuated and nonsignificant (OR [95\% Cl] $0.82[0.60,1.12]), 0.95$ [0.71-1.28]), and 0.91 [0.66-1.27], respectively).

\section{Project Viva}

van Rossem et al ${ }^{40}$ presented evidence from Project Viva, conducted in the United States. Participants were born $\geq 34$ weeks gestational age, and had mothers who attended a prenatal visit at 1 of 8 affiliated medical offices in eastern Massachusetts between 1999-2002. Participants were predominantly Caucasian $(74 \%)$ from highly educated families. The authors examined dichotomous comparisons of infants who (a) consumed human milk for $<6$ months, (b) consumed human milk for 6 months, and (c) consumed human milk exclusively for 6 months with infants who never consumed human milk. Infant-feeding data were collected from maternal interview at 6 months. The outcome of interest was obesity (i.e., BMI $\geq 95^{\text {th }}$ percentile based on CDC growth curves) at 3 years of age $(\mathrm{N}=713)$. Height and weight were measured by trained research staff using standardized protocols. The authors used analyses without and with adjustment for change in weight-for-age z-score from birth to 6 months, in order to determine the mediating effects of infant weight gain.

- In the analyses that did not control for infant weight gain, there was a significant association between consuming human milk exclusively for 6 months, compared with never consuming human milk, and lower odds of obesity at 3 years of age (OR 0.21 [95\% $\mathrm{Cl} 0.07,0.68]$ ). Consuming human milk non-exclusively for $<6$ or 6 months, compared with never consuming human milk were not significantly associated with the odds of obesity at 3 years; the odds were closer to the null (OR $[95 \% \mathrm{Cl}] 0.90[0.40,2.03]$ and $0.88[0.37,2.10]$, respectively).

- In the analyses that did control for infant weight gain, there were no significant associations between consuming any human milk for $<6$ or 6 months or consuming human milk exclusively for 6 months and the odds of obesity at 3 years of age (OR [95\% $\mathrm{Cl} 0.85[0.33,2.18], 1.44[0.53,3.96]$, and $0.29[0.08,1.05]$, respectively).

Weng et al ${ }^{41}$ : See number 4 , above

\section{Studies with evidence from 6 to 11 years of age}

Anderson et al (2003) ${ }^{3}$ : See number 5 , above

19. Bjertnaes et $\mathrm{a}^{4}$ examined a sample of children enrolled in a prospective cohort study conducted in Norway. Children included in the cohort started school in Oppland County, a predominantly rural county, in 2007 . The authors examined consuming human milk 
(irrespective of duration), compared to never consuming human milk, as a dichotomous variable. Infant-feeding data were recalled by parents at routine school entry health assessments when children were 5-6 years of age. The outcome of interest was overweight/obesity (combined) at approximately 8 years of age ( $N=951)$ using BMI cut-offs provided by the IOTF. Height and weight were measured by school nurses, but specific methods were not reported.

- Bjertnaes et al ${ }^{4}$ found no significant association between ever, compared with never, consuming human milk and the odds of overweight/obesity at 8 years of age (OR 0.99 [95\% Cl 0.48, 2.02], $\mathrm{p}=0.97$ )

Colen and Ramey 6 : See number 5, above

\section{Groningen Long-Chain Polyunsaturated Fatty Acid (LCPUFA) Study}

de Jong et al $^{7}$ conducted a randomized controlled trial, the Groningen LCPUFA Study, in the Netherlands of infants born between 37 and 42 weeks of gestational age. Infants whose mothers chose to formula feed were randomly assigned to receive either a control formula from birth to 6 months of age, or an AA- and DHA-supplemented formula from birth to 3 months of age followed by a control formula from 3 to 6 months of age. A human milk-fed reference group was also enrolled in the study, and infants in the reference group were given AA- and DHA-supplemented formula until $3 \mathrm{mo}$ of age, and control formula from 3 to 6 mo of age if human milk feeding stopped $<2$ months. The authors compared the reference group fed human milk with each of the randomized formula-fed groups. Mothers reported infantfeeding data prospectively in daily diaries until 8 months of infant age. The outcome of interest was overweight/obesity (combined; defined using IOTF BMI cut-offs) at 9 years of age $(\mathrm{N}=218-250)$. Height and weight were measured by study staff using standardized protocols.

- de Jong et $\mathrm{al}^{7}$ found no significant associations between a nonrandomized group ever fed human milk and randomized groups fed either a standard infant formula or an AA- and DHA-supplemented infant formula, and the risk of overweight/obesity at 9 years (data NR).

Durmus et al (2014): See number 1 , above

Evenhouse and Reilly ${ }^{10}$ : See number 7, above

\section{Growing Right onto Wellness (GROW)}

Heerman et al ${ }^{15}$ examined a prospective cohort from the GROW study conducted in the United States. Participants were from low-income families (e.g., 100\% qualified for services for underserved populations such as Medicaid or WIC, $43 \%$ were food insecure), mostly Hispanic (i.e., 63\% Hispanic Mexican, 28\% Hispanic non-Mexican, 9\% non-Hispanic), and considered to be at high risk for developing obesity at baseline when they were 3 to 5 years of age (i.e., BMI $\geq 50^{\text {th }}$ and $<95^{\text {th }}$ percentile based on CDC growth curves). Infant-feeding data were collected by parent recall at baseline, and the authors examined a dichotomous comparison of human milk consumption for $\geq 6$ months compared to never consuming human milk. Trained study staff measured height and weight. The outcome of interest was obesity (i.e., $\mathrm{BMI} \geq 95^{\text {th }}$ percentile based on CDC growth curves) when participants were a mean of about 7 years of age $(\mathrm{N}=356)$.

- In this high-risk cohort, consuming human milk for $\geq 6$ months, compared with never consuming human milk, was not significantly associated with the odds of obesity at approximately 7 years of age (OR 0.88 [95\% Cl 0.48, 1.60]). 
Huang et al ${ }^{15}$ : See number 5 , above

Lurbe et al ${ }^{22}$ : See number 2, above

Massion et al ${ }^{26}$ : See number 4 , above

Metzger and McDade ${ }^{27}$ : See number 8, above

Moschonis et al $^{30}$ : See number 3 , above

Ruijsbroek et $\mathbf{a}^{37}$ : See number 6 , above

\section{Studies with evidence from 12 to 19 years of age}

Colen and Ramey ${ }^{6}$ : See number 5, above

Evenhouse and Reilly ${ }^{10}$ : See number 7, above

Metzger and McDade ${ }^{27}$ : See number 8, above

Moschonis et al $^{30}$ : See number 3 , above

Nelson et al ${ }^{32}$ : See number 7 , above

Pluyman et al ${ }^{36}$ : See number 6 , above

\section{Studies with evidence at $\mathbf{2 0}$ years of age or later}

Bohr et al ${ }^{5}$ : See number 7 , above

Nelson et al ${ }^{32}$ : See number 7 , above

\section{Synthesis and assessment ${ }^{\text {ii }}$ of the evidence examining the relationship between ever, compared with never, consuming human milk and overweight and/or obesity at 2 years of age and older (Table 2)}

Thirty articles presented evidence from 21 independent studies about the relationship between ever, compared with never, consuming human milk and overweight and/or obesity at 2 years of age and older. ${ }^{1-11,14-17,19-22,26,27,30-32,35-37,39-41} \mathrm{~A}$ synthesis and assessment of the evidence follows.

Consistency: The evidence had strong consistency.

Fourteen of the 21 studies (18 of 30 articles) found significant associations between ever, compared with never, consuming human milk and overweight and/or obesity. $2,5,6,14,17,19-$

$22,26,27,31,32,35,36,39-41$ The significant associations were consistent in direction. They were all in the direction of ever consuming human milk being associated with lower risk of overweight and/or obesity across a wide range ages from childhood into adulthood:

- 2 years of age $2,14,31,39$

\footnotetext{
ii A detailed description of the methodology used for grading the strength of the evidence is available on the NESR website: https://nesr.usda.gov/2020-dietary-guidelines-advisory-committee-systematic-reviews and in Part C of the following reference: Dietary Guidelines Advisory Committee. 2020. Scientific Report of the 2020 Dietary Guidelines Advisory Committee: Advisory Report to the Secretary of Agriculture and the Secretary of Health and Human Services. U.S. Department of Agriculture, Agricultural Research Service, Washington, DC.
} 
- 3 years of age ${ }^{19,20,35,40,41}$

- 4 years of age $\mathrm{e}^{20,21,31}$

- 5 years of age ${ }^{14,20,22}$

- 6 years of age $\mathrm{a}^{20}$

- 10 years of age ${ }^{17}$

- 11 years of age ${ }^{26}$

- 12 years of age ${ }^{36}$

- 4 to 14 years of age ${ }^{6}$

- 9 to 19 years of age $^{27}$

- 12 to 21 years of age ${ }^{32}$

- 24 to 33 years of age ${ }^{5}$

The remaining 7 studies found no significant associations between ever, compared with never, consuming human milk and overweight and/or obesity. One of the 7 studies ${ }^{1}$ reported a marginal association between ever, compared with never, consuming human milk and lower odds of overweight/obesity at $45-48$ months (OR $0.81[95 \% \mathrm{Cl} 0.64,1.01])$. The remaining 6 studies may have had insufficient statistical power for some of their analyses, which may account for the lack of statistical significance (see Precision, below). ${ }^{4,7,9,11,15,30}$

A closer examination of the evidence from 7 studies (which divided participants into more than 1 group of infants ever fed human milk, based on how long infants consumed human milk, and compared these infants with infants never fed human milk) revealed a trend in the data. Five of the 7 articles found that a longer duration of human milk consumption (e.g., >6 months), compared to never consuming human milk, was significantly associated with lower risk of overweight and/or obesity, but that a shorter duration (e.g., $<6$ months) was not:

- Anderson et al $(2014)^{2}$ found a significant association between consuming human milk for $\geq 6$ months, compared with never consuming human milk, and a lower prevalence ratio of overweight/obesity at 2 years of age. However, associations between shorter durations of human milk consumption (i.e., $<1,1-2$, and 3-5 months) and overweight/obesity at 2 years of age were not statisitically significant.

- Layte et $\mathrm{al}^{19}$ found a significant association between consuming human milk for $\geq 6$ months, compared with never consuming human milk, and lower odds of obesity at 3 years of age. However, associations between shorter durations of human milk consumption (i.e., $<3$ and $<6$ months) and obesity at 3 years of age were not statistically significant.

- Pattison et $\mathrm{al}^{35}$ found significant associations between consuming human milk for $>6$ months, compared with never consuming human milk, and lower odds of overweight/obesity at 36 months of age and obesity at 36 months of age, and between consuming human milk for 0-6 months, compared with never consuming human milk, and lower odds of obesity at 36 months. However, the association between the shorter duration of 0-6 months of human milk consumption and overweight/obesity at 36 months was nonsignificant.

- Thorland et al ${ }^{39}$ found a significant association between consuming human milk for $>20$ weeks, compared with never consuming human milk, and lower odds of high weight-forlength status (defined as $\geq 97.7^{\text {th }}$ percentile based on WHO growth curves) at 24 months of 
age. However, associations between shorter durations of human milk consumption (i.e., 1-3, 4-8, and 8-20 weeks) and high weight-for-length status at 24 months of age were not statistically significant.

- Nelson et al ${ }^{32}$ found a significant association between consuming human milk for $\geq 9$ months, compared with never consuming human milk, and lower odds of overweight/obesity at 12-21 years of age in females (but not males). However, associations between shorter durations of human milk consumption (i.e., <3, 3-5.99, and 6-8.99 months) and overweight/obesity at 1221 years of age were not statistically significant in either sex.

The remaining 2 articles did not find a similar trend. Pluyman et $\mathrm{al}^{36}$ reported marginal associations for all durations of human milk consumption that were compared to never consuming human milk (OR [95\% Cl]: 0.63 [0.37, 1.08], 0.55 [0.30, 1.00], and $0.61[0.36,1.06]$ for durations of $<3,3-6$, and $\geq 6$ months, respectively). In the article by van Rossem et $a^{40}$, the associations between consuming human milk for $<6$ and $\geq 6$ months, compared with never consuming human milk, had wide confidence intervals (i.e., 0.40 to 2.03 and 0.37 to 2.10, respectively), suggesting insufficient statistical power.

The evidence from 4 studies was of particular interest because the studies conducted withinfamily analyses of siblings to reduce confounding from genetic and environmental factors (factors that siblings share) (see Risk of Bias, below). The evidence had some inconsistencies.

- Metzger and McDade ${ }^{27}$ reported significant associations from within-family sibling analyses between ever, compared with never, consuming human and lower odds of overweight/ obesity at 9 to 19 years of age.

- The other 3 studies did not find significant associations from within-family sibling analyses between ever, compared with never, consuming human milk and overweight and/or obesity. $3,6,10,14,32$ Rather, they tended to find that the significant associations found in the fullsample analyses were no longer significant. This may be due, in part, to the much smaller sample size available for the within-family analyses; however, in 2 articles it was clear that there was an attenuation of the estimated association that had been seen in the full-sample analyses. ${ }^{6,14}$ These findings suggest that some of the association between ever consuming human milk and lower risk of overweight and/or obesity is explained by confounding. Unfortunately, none of these studies had within-family analyses that compared infants who consumed human milk for different durations with infants who never consumed human milk. Therefore, it is not possible to comment on whether the trend described above (i.e., that longer durations of human milk consumption may be important) is true for sibling analyses.

- We also examined BMI (or BMI z-score) as a continuous variable in 3 studies that had both full-sample and within-family analyses. ${ }^{6,10,14}$ The findings were inconsistent. One study did not find significant associations between ever, compared with never, consuming human milk and $\mathrm{BMI}$ at 10 to 18 years of age in either analysis. ${ }^{10}$ One study found a significant association between ever, compared with never, consuming human milk and lower BMI at 4 to 14 years of age in the full-sample analysis that was attenuated and nonsignificant in the within-family analysis. ${ }^{6}$ One study found significant associations between initiating, compared with not initiating, human milk feeding and lower BMI z-score at 2 and at 5 years of age in the full sample, and a slight attenuation of the estimated associations in the within-family analyses so that the association at 5 years of age was still statistically significant. ${ }^{14}$ 
Precision: The evidence had strong precision.

The body of evidence had 21 studies, and 14 of them had sufficient statistical power to detect significant associations. Of the remaining 7 studies, 1 reported a marginal association in the same direction as, and with a similar magnitude to, the associations with statistical significance, ${ }^{1}$ and 6 may have had insufficient statistical power for some of their analyses, which may account for the lack of statistical significance. For example, Feldman-Winter et al ${ }^{11}$ compared infants fed infant formula exclusively with infants fed human milk exclusively for their first week of life following birth in a Baby-Friendly hospital, where the proportion of infants never fed human milk was low. As a result, the confidence interval around the odds ratio was very wide (i.e., 0.1 to 7.0). Associations reported by 3 other studies had confidence intervals greater than 1 , which suggests insufficient statistical power. $4,9,15$ The final 2 studies did not report confidence intervals ${ }^{7,30}$; however, de Jong et $\mathrm{al}^{7}$ had fewer participants than many of the samples in the body of evidence (about 250 participants), and Moschonis et al ${ }^{30}$ suggested that analyses of their sample from the EuroPrevall cohort (about 300 participants) in their multi-cohort study, in particular, may have been underpowered.

Directness: The evidence had strong directness.

Most of the articles reported study objectives that included the examination of human milk consumption and overweight and/or obesity.

Generalizability: The evidence had moderate generalizability.

Eleven of the 21 studies (16 of 30 articles) were conducted in the United

States. ${ }^{2,3,5,6,10,11,14,15,17,21,27,31,32,35,39,40}$ The studies included nationally representative samples, samples with racial and ethnic diversity, and samples from families with low income.

One study was conducted in Korea, ${ }^{20}$ and the remaining 9 studies (13 of 30 articles) were conducted in European countries. 1,4,7-9,16,19,22,26,30,36,37,41 There may be some concerns about the generalizability of the evidence from these studies to U.S. populations, which may have higher risk of overweight and obesity than the populations from which participants were sampled in the non-U.S. studies.

The generalizability of the evidence may also be limited because few studies examined the outcomes past childhood. Only 5 studies examined outcomes in adolescents, $, 10,27,30,32,36$ and only 1 examined outcomes in adults. ${ }^{5,32}$

Risk of bias: There is a moderate likelihood that the design and conduct of the studies have prevented or minimized bias.

Infant-feeding decisions can be strongly socially patterned; therefore, in this entirely observational body of evidence, the risk of bias from confounding is of particular concern. Four studies conducted within-family analyses of siblings to reduce confounding from shared genetic and environmental factors. $3,6,10,14,27,32$ However, these studies were prone to other risks of bias. For example, the Add Health and CDS studies asked mothers to recall how they fed their offspring during infancy when those offspring were between 4 and 18 years of age..$^{10,27,32}$ In addition, in the Children of NLSY79 study some participants reported their own height and weight, 3,6 and in the Add Health study the methods used to collect outcome data were not reported. ${ }^{10,32}$

None of the remaining 17 studies controlled for all of the key confounders identified on the 
analytic framework. In particular, studies tended not to account for complementary feeding practices and childhood diet. In addition, the studies had other risks of bias, which varied between studies (Tables 3 and 4).

\section{Publication bias:}

In addition to the graded elements of the assessment of the evidence, above, it is important to give consideration to publication bias, which may be present given the consistency of the evidence.

Conclusion: Given this synthesis and assessment of the evidence, we conclude that moderate evidence from observational studies indicates that ever, compared with never, consuming human milk is associated with lower risk of overweight and obesity at 2 years of age and older, particularly if the duration of human milk consumption is 6 months or longer. 
Table 2. Evidence examining the relationship between ever, compared with never, consuming human milk and overweight and/or obesity at 2 years of age and older ${ }^{a}$

\begin{tabular}{|c|c|c|c|c|}
\hline $\begin{array}{l}\text { Article } \\
\text { Study design (Study } \\
\text { name) } \\
\text { Country }\end{array}$ & $\begin{array}{l}\text { Ever vs never consuming human } \\
\text { milk exposure }\end{array}$ & Outcome $^{c}$ & Significant findings & $\begin{array}{l}\text { Nonsignificant } \\
\text { findings }\end{array}$ \\
\hline \multicolumn{5}{|c|}{ Outcomes from 2 to 5 years } \\
\hline $\begin{array}{l}\text { Abraham } 2012^{1} \\
\text { PC (GUS) } \\
\text { U.K. }\end{array}$ & Ever BF vs Never BF (ref) & $\begin{array}{l}\text { OWOB§ (OR [95\% Cl] @ 45-48 mo } \\
\text { (N=1913 BF/not OWOB; } 231 \text { BF/OWOB; } 1176 \text { Not } \\
\text { BF/not OWOB; } 195 \text { Not BF/OWOB) }\end{array}$ & & $0.81[0.64,1.01]$ \\
\hline \multirow{8}{*}{$\begin{array}{l}\text { Anderson } \mathbf{2 0 0 3}^{3} \\
\text { PC (Children of NLSY79) } \\
\text { U.S. }\end{array}$} & Ever BF vs Never BF (ref) & $\mathrm{OB}^{*}$ vs Not OB* (ref) $(\beta \pm \mathrm{SE}) @$ 3-11 y & & \\
\hline & & Note: this analysis is across 2 age groups & & \\
\hline & & In the full sample & & \\
\hline & & Probit model (N=16,650 observations) & $-0.018 \pm 0.008^{d}$ & \\
\hline & & $\begin{array}{l}\text { Instrumental variable model }(\mathrm{N}=15,050 \\
\text { observations) }\end{array}$ & & $-0.016 \pm 0.010$ \\
\hline & & In the subsample of siblings & & \\
\hline & & $\begin{array}{l}\text { at the same point in time (mean } 5.9 \text { y and } 9.2 \text { y } \\
\text { for younger and older siblings, respectively) } \\
\text { ( } N=7919 \text { observations) }\end{array}$ & & $0.012 \pm 0.017 ; \mathrm{NS}$ \\
\hline & & $\begin{array}{l}\text { at the same age (mean } 6.6 \mathrm{y}) \\
(\mathrm{N}=4471 \text { observations) }\end{array}$ & & $-0.021 \pm 0.023 ; \mathrm{NS}$ \\
\hline \multirow{4}{*}{$\begin{array}{l}\text { Anderson } 2014^{2} \\
\text { RC } \\
\text { U.S. }\end{array}$} & BF $<1$ mo vs Never BF (ref) & $\begin{array}{l}\text { OWOB (prevalence ratio }[95 \% \mathrm{Cl}]) @ 2 \text { y } \\
(\mathrm{N}=3769,1197)\end{array}$ & & $0.97[0.86,1.09]$ \\
\hline & BF 1-2 mo vs Never BF (ref) & $\begin{array}{l}\text { OWOB (prevalence ratio }[95 \% \mathrm{Cl}]) @ 2 \text { y } \\
(\mathrm{N}=4864,1197)\end{array}$ & & $0.96[0.85,1.08]$ \\
\hline & BF 3-5 mo vs Never BF (ref) & $\begin{array}{l}\text { OWOB (prevalence ratio }[95 \% \mathrm{Cl}]) @ 2 \text { y } \\
(\mathrm{N}=3119,1197)\end{array}$ & & $0.99[0.89,1.12]$ \\
\hline & BF $\geq 6$ mo vs Never BF (ref) & $\begin{array}{l}\text { OWOB (prevalence ratio }[95 \% \mathrm{CI}]) @ 2 \text { y } \\
(\mathrm{N}=2192,1197)\end{array}$ & $\begin{array}{l}0.82[0.72,0.94] \\
p<0.05\end{array}$ & \\
\hline
\end{tabular}




\begin{tabular}{|c|c|c|c|c|}
\hline $\begin{array}{l}\text { Article } \\
\text { Study design (Study } \\
\text { name) } \\
\text { Country }\end{array}$ & $\begin{array}{l}\text { Ever vs never consuming human } \\
\text { milk exposure }{ }^{b}\end{array}$ & Outcome $^{c}$ & Significant findings & $\begin{array}{l}\text { Nonsignificant } \\
\text { findings }\end{array}$ \\
\hline \multirow{5}{*}{$\begin{array}{l}\text { Colen } 2014^{6} \\
\text { PC (Children of NLSY79) } \\
\text { U.S. }\end{array}$} & Ever BF vs Never BF (ref) & $\mathrm{OB}^{*}(\beta \pm \mathrm{SE}) @ 4-14$ y & & \\
\hline & & Note: this analysis is across 3 age groups & & \\
\hline & & In Full Sample $(\mathrm{N}=8,237)$ & $\begin{array}{l}-0.342 \pm 0.066 \\
p<0.001\end{array}$ & \\
\hline & & In sibling sample $(\mathrm{N}=7,319)$ & $\begin{array}{l}-0.369 \pm 0.074 \\
p<0.001\end{array}$ & \\
\hline & & In discordant sibling sample $(\mathrm{N}=1,773)$ & & $-0.173 \pm 0.164 ;$ NS \\
\hline \multirow{4}{*}{$\begin{array}{l}\text { Durmus } 2011^{9} \\
\text { PC (Generation R) } \\
\text { Netherlands }\end{array}$} & Never BF vs BF >6 mo (ref) & $\begin{array}{l}\text { OWOB§ (OR [95\% Cl]) @ } 2 \text { y } \\
\text { (Total analytic N=5074) }\end{array}$ & & $\sim 1.5[\sim 0.9, \sim 2]$ \\
\hline & & $\begin{array}{l}\text { OWOB§ (OR [95\% Cl]) @ } 3 \text { y } \\
\text { (Total analytic } \mathrm{N}=5074)\end{array}$ & & $\sim 0.9[\sim 0.8, \sim 2]$ \\
\hline & Never BF vs EBF until 4 mo (ref) & $\begin{array}{l}\text { OWOB§ (OR [95\% Cl]) @ } 2 \text { y } \\
\text { (Total analytic N=5074) }\end{array}$ & & $\sim 1.5[\sim 0.8,2]$ \\
\hline & & $\begin{array}{l}\text { OWOB§ (OR }[95 \% \mathrm{Cl}]) @ 3 \text { y } \\
\text { (Total analytic } \mathrm{N}=5074)\end{array}$ & & $\sim 1.5[\sim 0.8,2]$ \\
\hline $\begin{array}{l}\text { Feldman-Winter } \mathbf{2 0 1 8 ^ { 1 1 }} \\
\text { PC } \\
\text { U.S. }\end{array}$ & $\begin{array}{l}\text { Exclusive FF 1st week vs EBF 1st wk } \\
\text { (ref) }\end{array}$ & $\begin{array}{l}\text { OWOB† (OR }[95 \% \mathrm{Cl}]) @ 2 \text { y } \\
\text { (N=58 EBF/not OWOB; } 13 \text { EBF/OWOB; } 11 \text { Exclusive } \\
\text { FF/not OWOB; } 1 \text { Exclusive FF/OWOB) }\end{array}$ & & $0.7[0.1,7.0] ; p=0.79$ \\
\hline \multirow{7}{*}{$\begin{array}{l}\text { Hawkins } 2019^{14} \\
\text { RC (Linked CENTURY } \\
\text { Study) } \\
\text { U.S. }\end{array}$} & Initiated BF vs Did not initiate BF (ref) & $\mathrm{OB}^{*}(\mathrm{OR}[95 \% \mathrm{Cl}])$ & & \\
\hline & & @ 2 y & & \\
\hline & & In full sample (Total analytic $N=55,058$ ) & $0.80[0.73,0.87]$ & \\
\hline & & $\begin{array}{l}\text { In subsample of families with more than } 1 \text { sibling } \\
\text { (Total analytic } N=21,445 \text { ) }\end{array}$ & $0.80[0.69,0.92]$ & \\
\hline & & $\begin{array}{l}\text { In subsample of siblings with discordant } \\
\text { outcomes (Total analytic } N=2260 \text { ) }\end{array}$ & & $0.97[0.72,1.32]$ \\
\hline & & @ 5 y & & \\
\hline & & In full sample (Total analytic $\mathrm{N}=43,893$ ) & $0.77[0.72,0.83]$ & \\
\hline
\end{tabular}




\section{Article}

Study design (Study

name)

Country
Ever vs never consuming human milk exposure ${ }^{b}$

Outcome ${ }^{c}$
Nonsignificant

findings

\begin{tabular}{|c|c|c|c|c|}
\hline & & $\begin{array}{l}\text { In subsample of families with more than } 1 \text { sibling } \\
\text { (Total analytic } N=17,081 \text { ) }\end{array}$ & $0.77[0.69,0.87]$ & \\
\hline & & $\begin{array}{l}\text { In subsample of siblings with discordant } \\
\text { outcomes (Total analytic } \mathrm{N}=3249 \text { ) }\end{array}$ & & $0.94[0.74,1.20]$ \\
\hline $\begin{array}{l}\text { Heppe } 2013^{16} \\
\text { PC (Generation R) } \\
\text { Netherlands }\end{array}$ & No BF vs BF (ref) & $\begin{array}{l}\text { OWOB } \ddagger \text { (OR }[95 \% \mathrm{Cl}]) @ \sim 4 \text { y } \\
\text { (Total analytic N=335 OWOB; } 3275 \text { Not OWOB) }\end{array}$ & & $0.77[0.47,1.28]$ \\
\hline \multirow{3}{*}{$\begin{array}{l}\text { Layte } 2014^{19} \\
\text { PC } \\
\text { Ireland }\end{array}$} & BF <3 mo vs Never BF (ref) & OB $\ddagger(\mathrm{OR}) @ 3$ y $(\mathrm{N}=386,4743)$ & & $0.96 ; p=0.79$ \\
\hline & BF $<6$ mo vs Never BF (ref) & OB $\ddagger(\mathrm{OR}) @ 3$ y $(\mathrm{N}=543,4743)$ & & $0.97 ; p=0.87$ \\
\hline & $B F \geq 6$ mo vs Never BF (ref) & OB $\ddagger(\mathrm{OR}) @ 3$ y $(\mathrm{N}=870,4743)$ & $0.51 ; p<0.001$ & \\
\hline \multirow[t]{8}{*}{$\begin{array}{l}\text { Lee } 2019^{20} \\
\text { RC } \\
\text { Korea }\end{array}$} & $\begin{array}{l}\text { Mixed-fed from birth to } 4-6 \text { mo vs FF } \\
\text { from birth to 4-6 mo (ref) }\end{array}$ & $\begin{array}{l}\text { OWOB§ (OR }[95 \% \mathrm{Cl}]) \\
\qquad @ 30-36 \text { mo }\end{array}$ & & \\
\hline & & $\begin{array}{l}\text { In normal birthweight subsample } \\
(\mathrm{N}=6,816 \mathrm{NBW} \text { MF, } 10,780 \mathrm{NBW} F \mathrm{~F})\end{array}$ & . & $0.94[0.86,1.02]$ \\
\hline & & $\begin{array}{l}\text { In high birthweight subsample } \\
(\mathrm{N}=303 \mathrm{HBW} \mathrm{MF}, 416 \mathrm{HBW} F \mathrm{~F})\end{array}$ & $0.66[0.48,0.92]$ & \\
\hline & & @ 42-48 mo & & \\
\hline & & $\begin{array}{l}\text { In normal birthweight subsample } \\
(\mathrm{N}=6,816 \mathrm{NBW} \text { MF, } 10,780 \mathrm{NBW} F \mathrm{~F})\end{array}$ & . & $0.97[0.89,1.05]$ \\
\hline & & $\begin{array}{l}\text { In high birthweight subsample } \\
(\mathrm{N}=303 \mathrm{HBW} \text { MF, } 416 \mathrm{HBW} \text { FF) }\end{array}$ & $0.69[0.50,0.97]$ & \\
\hline & & @ 54-60 mo & & \\
\hline & & $\begin{array}{l}\text { In normal birthweight subsample } \\
(\mathrm{N}=6,816 \mathrm{NBW} \text { MF, } 10,780 \mathrm{NBW} F \mathrm{~F})\end{array}$ & . & $0.92[0.84,1.00]$ \\
\hline
\end{tabular}




\section{Article}

Study design (Study

name)

Ever vs never consuming human

milk exposure ${ }^{b}$

Outcome

Significant findings

Nonsignificant

findings

\begin{tabular}{|c|c|c|c|c|}
\hline \multicolumn{5}{|c|}{ @ 66-72 mo } \\
\hline & & $\begin{array}{l}\text { In normal birthweight subsample } \\
\text { ( } N=6,816 \text { NBW MF, 10,780 NBW FF) }\end{array}$ & . & $0.95[0.88,1.04]$ \\
\hline & & $\begin{array}{l}\text { In high birthweight subsample } \\
\text { (N=303 HBW MF, } 416 \text { HBW FF) }\end{array}$ & & $1.02[0.72,1.45]$ \\
\hline & $\begin{array}{l}\text { EBF from birth to } 4-6 \text { mo vs FF from } \\
\text { birth to } 4-6 \text { mo (ref) }\end{array}$ & $\begin{array}{l}\text { OWOB§ (OR }[95 \% \mathrm{Cl}]) \\
\qquad \text { @ 30-36 mo }\end{array}$ & & \\
\hline & & $\begin{array}{l}\text { In normal birthweight subsample } \\
(\mathrm{N}=16,487 \text { NBW EBF, } 10,780 \text { NBW FF) }\end{array}$ & $0.75[0.70,0.81]$ & . \\
\hline & & $\begin{array}{l}\text { In high birthweight subsample } \\
\text { ( } N=673 \text { HBW EBF, } 416 \text { HBW FF) }\end{array}$ & $0.73[0.56,0.96]$ & . \\
\hline \multicolumn{5}{|c|}{ @ 42-48 mo } \\
\hline & & $\begin{array}{l}\text { In normal birthweight subsample } \\
(\mathrm{N}=16,487 \text { NBW EBF, 10,780 NBW FF) }\end{array}$ & $0.85[0.79,0.91]$ & . \\
\hline & & $\begin{array}{l}\text { In high birthweight subsample } \\
\text { (N=673 HBW EBF, } 416 \text { HBW FF) }\end{array}$ & . & $0.76[0.58,1.00]$ \\
\hline \multicolumn{5}{|c|}{ @ 54-60 mo } \\
\hline & & $\begin{array}{l}\text { In normal birthweight subsample } \\
(\mathrm{N}=16,487 \text { NBW EBF, } 10,780 \mathrm{NBW} F \mathrm{~F})\end{array}$ & $0.87[0.81,0.93]$ & . \\
\hline & & $\begin{array}{l}\text { In high birthweight subsample } \\
\text { (N=673 HBW EBF, } 416 \text { HBW FF) }\end{array}$ & $0.69[0.53,0.90]$ & . \\
\hline \multicolumn{5}{|c|}{ @ 66-72 mo } \\
\hline & & $\begin{array}{l}\text { In normal birthweight subsample } \\
(\mathrm{N}=16,487 \mathrm{NBW} \text { EBF, } 10,780 \text { NBW FF) }\end{array}$ & $0.87[0.81,0.93]$ & . \\
\hline & & $\begin{array}{l}\text { In high birthweight subsample } \\
\text { ( } N=673 \text { HBW EBF, } 416 \text { HBW FF) }\end{array}$ & . & $1.24[0.92,1.66]$ \\
\hline $\begin{array}{l}\text { Li } \mathbf{2 0 1 1 ^ { 2 1 }} \\
\text { PC (ECLS-B) } \\
\text { U.S. }\end{array}$ & Ever BF vs Never BF (ref) & $\mathrm{OB}^{*}(\mathrm{OR}[95 \% \mathrm{Cl}]) @ 4$ y $(\mathrm{N}=5600,2550)$ & $0.76[0.63,0.92]$ & \\
\hline
\end{tabular}




\begin{tabular}{|c|c|c|c|c|}
\hline $\begin{array}{l}\text { Article } \\
\text { Study design (Study } \\
\text { name) } \\
\text { Country }\end{array}$ & $\begin{array}{l}\text { Ever vs never consuming human } \\
\text { milk exposure }\end{array}$ & Outcome $^{c}$ & Significant findings & $\begin{array}{l}\text { Nonsignificant } \\
\text { findings }\end{array}$ \\
\hline $\begin{array}{l}\text { Lurbe } 2018^{22} \\
\text { PC } \\
\text { Spain }\end{array}$ & BF at birth vs Not BF at birth (ref) & OB $(\beta, R R) @ 5$ y $(N=145)$ & $-0.190 ; p=0.022$ & \\
\hline \multirow{4}{*}{$\begin{array}{l}\text { Moschonis } \mathbf{2 0 1 7 ^ { 3 0 }} \\
\text { RC (ALSPAC, EDEN, } \\
\text { EuroPrevall, Generation } \\
\text { XXI) } \\
\text { U.K., France, Greece, } \\
\text { Portugal }\end{array}$} & Never BF vs BF $\geq 6$ mo (ref) & $\begin{array}{l}\text { OWOB† (OR) } \\
\text { @ } 4 \text { y } \\
\quad \text { in ALSPAC cohort }(\mathrm{N}=6522)\end{array}$ & & $\sim 0.8$ : NS \\
\hline & & in EuroPrevall cohort $(\mathrm{N}=309)$ & & $\sim 1.5 ; \mathrm{NS}$ \\
\hline & & in Generation XXI cohort $(\mathrm{N}=3387)$ & & $\sim 1.2 ; \mathrm{NS}$ \\
\hline & & @ 5 y in EDEN cohort $(\mathrm{N}=1070)$ & & $\sim 1.0 ;$ NS \\
\hline \multirow{11}{*}{$\begin{array}{l}\text { Moss 2014 } \\
\text { PC (ECLS-B) } \\
\text { U.S. }\end{array}$} & Ever BF vs Never BF (ref) & $\mathrm{OB}^{*}(\mathrm{OR}[95 \% \mathrm{Cl}])$ vs OW (ref) & & \\
\hline & & @ 2 y (Total analytic $N=7200$ ) & $\begin{array}{l}0.64[0.51,0.80] \\
p<0.001\end{array}$ & \\
\hline & & @ 4 y (Total analytic N=6950) & $\begin{array}{l}0.67[0.53,0.85] \\
p<0.01\end{array}$ & \\
\hline & & Healthy weight status* (OR [95\% Cl]) vs OW (ref) & & $1.08[0.89,1.31]$ \\
\hline & & @ 2 y (Total analytic N=7200) & & \\
\hline & & @ 4 y & & $0.94[0.75,1.18]$ \\
\hline & & (Total analytic $\mathrm{N}=6950$ ) & & \\
\hline & $\begin{array}{l}\text { Ever BF, CF }<4 \text { mo vs Never BF, CF }<4 \\
\text { mo (ref) }\end{array}$ & $\begin{array}{l}\text { OW* (OR }[95 \% \mathrm{Cl}]) \text { vs healthy weight status (ref) } \\
\text { @ } 2 \text { y (Total analytic } \mathrm{N}=7200)\end{array}$ & & $0.89[0.62,1.29]$ \\
\hline & & @ 4 y (Total analytic N=6950) & & $0.91[0.62,1.34]$ \\
\hline & & $\begin{array}{l}\mathrm{OB}^{*}(\mathrm{OR}[95 \% \mathrm{Cl}]) \text { vs healthy weight status (ref) } \\
\text { @ } 2 \text { y (Total analytic } \mathrm{N}=7200)\end{array}$ & $\begin{array}{l}0.64[0.47,0.86] \\
p<0.01\end{array}$ & \\
\hline & & @ 4 y (Total analytic N=6950) & & $\begin{array}{l}0.72[0.49,1.06] \\
p<0.10\end{array}$ \\
\hline
\end{tabular}




\begin{tabular}{|c|c|c|c|c|}
\hline $\begin{array}{l}\text { Article } \\
\text { Study design (Study } \\
\text { name) } \\
\text { Country }\end{array}$ & $\begin{array}{l}\text { Ever vs never consuming human } \\
\text { milk exposure }{ }^{\mathrm{b}}\end{array}$ & Outcome $^{c}$ & Significant findings & $\begin{array}{l}\text { Nonsignificant } \\
\text { findings }\end{array}$ \\
\hline & $\begin{array}{l}\text { Ever BF, CF } 4-5 \text { mo vs Never BF, CF } \\
<4 \text { mo (ref) }\end{array}$ & $\begin{array}{l}\text { OW* (OR }[95 \% \mathrm{CI}]) \text { vs healthy weight status (ref) } \\
\text { @ } 2 \text { y (Total analytic N=7200) }\end{array}$ & & $0.92[0.67,1.28]$ \\
\hline & & @ 4 y (Total analytic N=6950) & & $0.80[0.55,1.16]$ \\
\hline & & $\begin{array}{l}\mathrm{OB}^{*}(\mathrm{OR}[95 \% \mathrm{Cl}]) \text { vs healthy weight status (ref) } \\
\text { @ } 2 \text { y (Total analytic } \mathrm{N}=7200)\end{array}$ & $\begin{array}{l}0.38[0.29,0.51] \\
p<0.001\end{array}$ & \\
\hline & & @ 4 y (Total analytic N=6950) & $\begin{array}{l}0.45[0.33,0.61] \\
p<0.001\end{array}$ & \\
\hline & $\begin{array}{l}\text { Ever BF, CF } \geq 6 \text { mo vs Never BF, CF }<4 \\
\text { mo (ref) }\end{array}$ & $\begin{array}{l}\text { OW* (OR [95\% Cl]) vs healthy weight status (ref) } \\
\text { @ } 2 \text { y (Total analytic N=7200) }\end{array}$ & & $0.82[0.58,1.15]$ \\
\hline & & @ 4 y (Total analytic N=6950) & & $0.81[0.54,1.22]$ \\
\hline & & $\begin{array}{l}\left.\text { OB* }^{*} \text { OR }[95 \% \mathrm{Cl}]\right) \text { vs healthy weight status (ref) } \\
\text { @ } 2 \text { y (Total analytic } \mathrm{N}=7200)\end{array}$ & $\begin{array}{l}0.38[0.27,0.53] \\
p<0.001\end{array}$ & \\
\hline & & @ 4 y (Total analytic N=6950) & $\begin{array}{l}0.51[0.36,0.72] \\
p<0.001\end{array}$ & \\
\hline \multirow[t]{5}{*}{$\begin{array}{l}\text { Pattison } 2019^{35} \\
\text { RC (FBS) } \\
\text { U.S. }\end{array}$} & Ever BF vs Never BF (ref) & $\begin{array}{l}\text { OWOB vs no OWOB (OR [95\% Cl]) @ } 36 \text { mo } \\
\text { (N=356 ever BF/OWOB, } 42 \text { never BF/OWOB, } 1159 \\
\text { ever BF/not OWOB, } 69 \text { never BF/not OWOB) }\end{array}$ & $0.63[0.41,0.96]$ & \\
\hline & & $\begin{array}{l}\text { OB vs No OB }(\mathrm{OR}[95 \% \mathrm{Cl}]) @ 36 \text { mo } \\
(\mathrm{N}=138 \text { ever } \mathrm{BF} / \mathrm{OB}, 24 \text { never } \mathrm{BF} / \mathrm{OB}, 1377 \text { ever } \\
\mathrm{BF} / \text { No OB, } 87 \text { never } \mathrm{B} / \text { No OB })\end{array}$ & $0.51[0.30,0.86]$ & \\
\hline & BF 0-6 mo vs Never BF (ref) & $\begin{array}{l}\text { OWOB vs No OWOB (OR }[95 \% \mathrm{Cl}]) @ 36 \text { mo } \\
\text { (N=203 BF 0-6 mo/OWOB, } 42 \text { never BF/OWOB, } 69 \\
553 \text { BF 0-6 mo/No OWOB, never BF/no OWOB) }\end{array}$ & & $0.68[0.44,1.05]$ \\
\hline & & $\begin{array}{l}\text { OB vs No OB (OR }[95 \% \text { Cl]) @ } 36 \text { mo } \\
(\mathrm{N}=83 \text { BF 0-6 mo/OB, } 24 \text { never BF/OB, } 673 \text { BF 0-6 } \\
\text { mo/No OB, } 87 \text { never BF/No OB) }\end{array}$ & $0.54[0.32,0.94]$ & \\
\hline & BF >6 mo vs Never BF (ref) & $\begin{array}{l}\text { OWOB vs No OWOB }(\mathrm{OR}[95 \% \mathrm{Cl}]) @ 36 \mathrm{mo} \\
(\mathrm{N}=153 \mathrm{BF}>6 \mathrm{mo} / \mathrm{OWOB}, 42 \text { never BF/OWOB, } 606 \\
\mathrm{BF}>6 \mathrm{mo} / \mathrm{No} \text { OWOB, } 69 \text { never BF/No OWOB) }\end{array}$ & $0.54[0.34,0.85]$ & \\
\hline
\end{tabular}




\section{Article}

Study design (Study

name)

Ever vs never consuming human

milk exposure

Outcome $^{\mathrm{c}}$

Significant findings

Nonsignificant

findings

OB vs No OB (OR [95\% Cl]) @ $36 \mathrm{mo}$

$(\mathrm{N}=55 \mathrm{BF}>6 \mathrm{mo} / \mathrm{OB}, 24$ never $\mathrm{BF} / \mathrm{OB}, 704 \mathrm{BF}>6$

mo/No OB, 87 never BF/No OB)

Ruijsbroek $2011^{37}$

PC (PIAMA)

Netherlands

Never BF vs BF >16 wk (ref)

OWOB $\ddagger$ (OR [95\% Cl]) in childhood (3-8 y)

(Total analytic $\mathrm{N}=3963$ )

$1.08[0.91,1.29]$

Note: this analysis is across 2 age groups

OB $\ddagger$ (OR $[95 \% \mathrm{Cl}])$ in childhood (3-8 y) (N=NR)

$1.44[0.95,2.18]$

Note: this analysis is across 2 age groups

High WFL status ( $\geq 97.7 \%$ ile †) (OR [95\% Cl]) @ 24

mo $(\mathrm{N}=6,383)$

$0.82[0.60,1.12]$

PC

BF 1-3 wk vs Never BF (ref)

BF 4-8 wk vs Never BF (ref)

High WFL status ( $\geq 97.7 \%$ ile †) (OR [95\% Cl]) @ 24

$0.95[0.71,1.28]$

mo $(\mathrm{N}=6,383)$

BF 8-20 wk vs Never BF (ref)

High WFL status ( $\geq 97.7 \%$ ile †) (OR [95\% Cl]) @ 24 mo $(\mathrm{N}=6,383)$

BF >20 wk vs Never BF (ref)

BF $<6$ mo vs Never BF (ref)

High WFL status ( $\geq 97.7 \%$ ile †) (OR [95\% Cl]) @ 24 $\mathrm{mo}(\mathrm{N}=6,383)$

$0.91[0.66,1.27]$

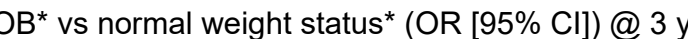

$(\mathrm{N}=713)$

In final model

$0.90[0.40,2.03]$

In final model, adjusted for change in infant WAZ

from birth to $6 \mathrm{mo}$

$0.85[0.33,2.18]$

BF 6 mo vs Never BF (ref)

$\mathrm{OB}^{*}$ vs normal weight status* (OR $\left.[95 \% \mathrm{Cl}]\right) @ 3$ y

$(\mathrm{N}=713)$

In final model

$0.88[0.37,2.10]$

In final model, adjusted for change in infant WAZ

from birth to $6 \mathrm{mo}$ 


\section{Article}

Study design (Study

name)

Ever vs never consuming human

milk exposure ${ }^{b}$

Outcome $^{\mathrm{c}}$

Significant findings

Nonsignificant

Country

EBF 6 mo vs Never BF (ref)

$\mathrm{OB}^{*}$ vs normal weight status* $(\mathrm{OR}[95 \% \mathrm{Cl}]) @ 3 \mathrm{y}$

$(\mathrm{N}=713)$

In final model

$0.21[0.07,0.68]$

In final model, adjusted for change in infant WAZ

from birth to $6 \mathrm{mo}$

$\begin{array}{lll}\text { Weng 201341 } & \text { Never BF vs Ever BF (ref) } & \text { OWOB } ¥ \text { (OR [95\% Cl]) @ } 3 \text { y }(\mathrm{N}=10810) \\ \text { PC (MCS) } & \end{array}$

\section{Outcomes from 6 to 11 years}

Anderson $\mathbf{2 0 0 3}^{\mathbf{3}} \quad$ Ever BF vs Never BF (ref)

PC (Children of NLSY79)

U.S.

$\mathrm{OB}^{*}$ vs Not OB* (ref) ( $\left.\beta \pm \mathrm{SE}\right) @$ 3-11 y

Note: this analysis is across 2 age groups

In the full sample

Probit model ( $\mathrm{N}=16,650$ observations)

$-0.018 \pm 0.008^{4}$

Instrumental variable model $(\mathrm{N}=15,050$

$-0.016 \pm 0.010$

observations)

In the subsample of siblings

at the same point in time (mean $5.9 \mathrm{y}$ and $9.2 \mathrm{y}$

for younger and older siblings, respectively)

( $\mathrm{N}=7919$ observations)

at the same age (mean $6.6 \mathrm{y}$ )

( $N=4471$ observations)

\section{Bjertnaes 20194}

PC

Ever BF vs Never BF (ref)

Norway

OWOB $¥$ (OR [95\% Cl]) @ 8 y

(Total analytic N 200 OWOB, $~ 751$ Not OWOB)

$0.99[0.48,2.02]$

$\mathrm{p}=0.97$

\section{Colen 2014}

PC (Children of NLSY79)

U.S.

Ever BF vs Never BF (ref)

$\mathrm{OB}^{*}(\beta \pm \mathrm{SE}) @ 4-14$ y

Note: this analysis is across 3 age groups

In Full Sample $(\mathrm{N}=8,237)$

In sibling sample $(\mathrm{N}=7,319)$
$-0.021 \pm 0.023 ; N S$

$0.012 \pm 0.017 ; \mathrm{NS}$ 


\section{Article}

Study design (Study

name)

Ever vs never consuming human

milk exposure ${ }^{b}$

Outcome $^{\mathrm{c}}$

Nonsignificant

findings

untry

In discordant sibling sample $(\mathrm{N}=1,773)$

$-0.173 \pm 0.164 ;$ NS

de Jong $2011^{7}$

NRCT (Groningen

LCPUFA Study)

Normal (vs OWOB $\ddagger$ ) BMI @ 9 y

(Total analytic $\mathrm{N}=250$ )

NS; data NR

Netherlands

\begin{tabular}{|c|c|c|c|}
\hline & $\begin{array}{l}\text { Ever BF vs AA- and DHA-supplemented } \\
\text { FF 0-6 mo }\end{array}$ & $\begin{array}{l}\text { Normal } \ddagger \text { (vs OW } \ddagger \text { ) BMI @ } 9 \text { y } \\
\text { (Total analytic } \mathrm{N}=218 \text { ) }\end{array}$ & NS; data NR \\
\hline $\begin{array}{l}\text { Durmus } 2014^{8} \\
\text { PC (Generation R) } \\
\text { Netherlands }\end{array}$ & Never BF vs Ever BF (ref) & OWOB $\ddagger($ OR $[95 \% \mathrm{Cl}]) @ 6$ y $(\mathrm{N}=385,4678)$ & $\sim 0.8[\sim 0.6, \sim 1.3]$ \\
\hline \multirow{5}{*}{$\begin{array}{l}\text { Evenhouse } 2005^{10} \\
\text { CS (Add Health) } \\
\text { U.S. }\end{array}$} & Ever BF vs Never BF (ref) & OWOB* $^{*}$ OR \pm SE from logit) @ 10-18 y & \\
\hline & & Note: this analysis is across 2 age groups & \\
\hline & & In the full sample $(\mathrm{N}=7417,9486)$ & $0.79 \pm 0.03 ; p<0.10$ \\
\hline & & In the subsample of siblings $(\mathrm{N}=1607,2166)$ & $0.87 \pm 0.08 ; \mathrm{NS}$ \\
\hline & & $\begin{array}{l}\text { In the subsample of siblings with discordant } \\
\text { exposures }(N=288,288)\end{array}$ & $1.32 \pm 0.21 ; p<0.10$ \\
\hline
\end{tabular}

OB* (OR \pm SE from logit) @ 10-18 y

Note: this analysis is across 2 age groups

In the full sample $(\mathrm{N}=7417,9486)$

$0.77 \pm 0.04 ; p<0.10$

\begin{tabular}{|c|c|c|c|c|}
\hline & & In the subsample of siblings $(\mathrm{N}=1607,2166)$ & & $0.88 \pm 0.11 ; \mathrm{NS}$ \\
\hline & & $\begin{array}{l}\text { In the subsample of siblings with discordant } \\
\text { exposures }(N=288,288)\end{array}$ & & $1.17 \pm 0.25 ; \mathrm{NS}$ \\
\hline $\begin{array}{l}\text { Heerman } 2019^{15} \\
\text { PC (GROW) } \\
\text { U.S. }\end{array}$ & No $B F$ vs $B F \geq 6$ mo (ref) & $\begin{array}{l}\mathrm{OB}^{*}(\mathrm{OR}[95 \% \mathrm{Cl}]) @ \sim 7 \mathrm{y} \\
(\mathrm{N}=62 \mathrm{No} B F / \mathrm{No} \text { OB, } 29 \text { No BF/OB, } 167 \text { BF/No OB, } \\
98 \mathrm{BF} / \mathrm{OB})\end{array}$ & & $0.88[0.48,1.60]$ \\
\hline $\begin{array}{l}\text { Huang } \mathbf{2 0 1 4}^{\mathbf{1 5}} \\
\text { PC (Children of NLSY79) } \\
\text { U.S. }\end{array}$ & Ever BF vs Never BF (ref) & $\mathrm{OB}^{*}(\mathrm{OR}) @ 10$ y (Total analytic N=5156) & $\begin{array}{l}0.79 ; p<0.05(95 \% \mathrm{Cl} \\
\text { NR) }\end{array}$ & \\
\hline
\end{tabular}




\begin{tabular}{|c|c|c|c|c|}
\hline $\begin{array}{l}\text { Article } \\
\text { Study design (Study } \\
\text { name) } \\
\text { Country }\end{array}$ & $\begin{array}{l}\text { Ever vs never consuming human } \\
\text { milk exposure }{ }^{\text {b }}\end{array}$ & Outcome $^{c}$ & Significant findings & $\begin{array}{l}\text { Nonsignificant } \\
\text { findings }\end{array}$ \\
\hline $\begin{array}{l}\text { Lurbe } 2018^{22} \\
\text { PC } \\
\text { Spain }\end{array}$ & BF at birth vs Not BF at birth (ref) & OB $(\beta, R R) @ 10$ y $(N=100)$ & & $-0.032 ; p=0.760$ \\
\hline $\begin{array}{l}\text { Massion } 2016^{26} \\
\text { PC (MCS) } \\
\text { U.K. }\end{array}$ & Never BF vs Any BF >4 mo (ref) & $\begin{array}{l}\text { OWOB } \ddagger(\text { RR }[95 \% \text { Cl] }) @ 11 \text { y } \\
\text { (Total analytic } N=3445 \text { OWOB, } 8319 \text { Not OWOB) }\end{array}$ & $\begin{array}{l}\sim 1.1[\sim 1, \sim 1.25] \\
p<0.05\end{array}$ & \\
\hline \multirow[t]{12}{*}{$\begin{array}{l}\text { Metzger } 2009^{27} \\
\text { PC (CDS) } \\
\text { U.S. }\end{array}$} & Ever BF vs Never BF (ref) & $\begin{array}{l}\text { BMI }>50^{\text {th }} \% \text { ile* } v s<50^{\text {th }} \% \text { ile* }(\text { ref })(O R) @ 9-19 \text { y in } \\
\text { sibling subsample } \\
\text { ( } N=30 \text { to } 44 \text { with differences in both feeding and BMI } \\
\text { status) }\end{array}$ & & $\sim 1.00 ; \mathrm{NS}$ \\
\hline & & Note: this analysis is across 2 age groups & & \\
\hline & & Ordinary least squares model & & \\
\hline & & Fixed-effects model & & 1.30; NS \\
\hline & & $\begin{array}{l}\text { OWOB* vs Not OWOB (ref) (OR) @ 9-19 y in sibling } \\
\text { subsample } \\
\text { ( } N=30 \text { to } 44 \text { with differences in both feeding and BMI } \\
\text { status) }\end{array}$ & $\sim 0.60 ; p<0.01$ & \\
\hline & & Note: this analysis is across 2 age groups & & \\
\hline & & Ordinary least squares model & & \\
\hline & & Fixed-effects model & $\sim 0.40 ; p<0.05$ & \\
\hline & & $\begin{array}{l}\mathrm{OB}^{*} \text { vs Not } \mathrm{OB}^{*} \text { (ref) }(\mathrm{OR}) @ 9-19 \text { y in sibling } \\
\text { subsample } \\
(\mathrm{N}=30 \text { to } 44 \text { with differences in both feeding and BMI } \\
\text { status) }\end{array}$ & & $\sim 0.70 ; \mathrm{NS}$ \\
\hline & & Note: this analysis is across 2 age groups & & \\
\hline & & Ordinary least squares model & & \\
\hline & & Fixed-effects model & $\sim 0.20 ; p<0.01$ & \\
\hline
\end{tabular}




\section{Article}

Study design (Study

name)

Ever vs never consuming human

milk exposure

Outcome $^{\mathrm{c}}$

Significant findings

Nonsignificant

Country

Moschonis $2017^{30}$

Never BF vs BF $\geq 6$ mo (ref)

OWOB十‡ (OR) @ 9 y in ALSPAC cohort $(N=7540)$

findings

RC (ALSPAC, EDEN

EuroPrevall, Generation

$\mathrm{XXI)}$

U.K., France, Greece

Portugal

\section{Ruijsbroek 201137}

PC (PIAMA)

Never BF vs BF >16 wk (ref)

Netherlands

(Total analytic $\mathrm{N}=3963$ )

$1.08[0.91,1.29]$

Note: this analysis is across 2 age groups

OB $\ddagger(\mathrm{OR}[95 \% \mathrm{Cl}])$ in childhood (3-8 y) $(\mathrm{N}=\mathrm{NR})$

$1.44[0.95,2.18]$

Note: this analysis is across 2 age groups

\section{Outcomes from 12 to 19 years}

\begin{tabular}{|c|c|c|c|}
\hline \multirow{3}{*}{$\begin{array}{l}\text { Colen } \mathbf{2 0 1 4 ^ { 6 }} \\
\text { PC (Children of NLSY79) } \\
\text { U.S. }\end{array}$} & \multirow[t]{3}{*}{ Ever BF vs Never BF (ref) } & $\mathrm{OB}^{*}(\beta \pm \mathrm{SE}) @ 4-14$ y & \multirow{3}{*}{$\begin{array}{l}-0.342 \pm 0.066 \\
p<0.001\end{array}$} \\
\hline & & Note: this analysis is across 3 age groups & \\
\hline & & In Full Sample $(\mathrm{N}=8,237)$ & \\
\hline
\end{tabular}

\begin{tabular}{|c|c|c|c|}
\hline & & In sibling sample $(\mathrm{N}=7,319)$ & $\begin{array}{l}-0.369 \pm 0.074 \\
p<0.001\end{array}$ \\
\hline & & In discordant sibling sample $(\mathrm{N}=1,773)$ & $-0.173 \pm 0.164 ; \mathrm{NS}$ \\
\hline \multirow{9}{*}{$\begin{array}{l}\text { Evenhouse } 2005^{10} \\
\text { CS (Add Health) } \\
\text { U.S. }\end{array}$} & Ever BF vs Never BF (ref) & OWOB* (OR × SE from logit) @ 10-18 y & \\
\hline & & Note: this analysis is across 2 age groups & \\
\hline & & In the full sample $(\mathrm{N}=7417,9486)$ & $0.79 \pm 0.03 ; p<0.10$ \\
\hline & & In the subsample of siblings $(\mathrm{N}=1607,2166)$ & $0.87 \pm 0.08 ; \mathrm{NS}$ \\
\hline & & $\begin{array}{l}\text { In the subsample of siblings with discordant } \\
\text { exposures }(\mathrm{N}=288,288)\end{array}$ & $1.32 \pm 0.21 ; p<0.10$ \\
\hline & & $\mathrm{OB}^{*}(\mathrm{OR} \pm \mathrm{SE}$ from logit) @ 10-18 y & \\
\hline & & Note: this analysis is across 2 age groups & \\
\hline & & In the full sample $(\mathrm{N}=7417,9486)$ & $0.77 \pm 0.04 ; p<0.10$ \\
\hline & & In the subsample of siblings $(\mathrm{N}=1607,2166)$ & $0.88 \pm 0.11 ; \mathrm{NS}$ \\
\hline
\end{tabular}




\section{Article}

Study design (Study

name)

Ever vs never consuming human

milk exposure ${ }^{b}$

Outcome $^{c}$

Significant findings

Nonsignificant

findings

In the subsample of siblings with discordant

$1.17 \pm 0.25 ; \mathrm{NS}$

exposures $(\mathrm{N}=288,288)$

Metzger 200927 Ever BF vs Never BF (ref)

PC (CDS)

BMI $>50^{\text {th }} \%$ ile* $v s<50^{\text {th }} \%$ ile* $($ ref) $(O R) @ 9-19$ y in

sibling subsample

$(\mathrm{N}=30$ to 44 with differences in both feeding and $\mathrm{BMI}$

status)

Note: this analysis is across 2 age groups

Ordinary least squares model

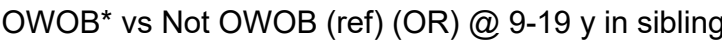

subsample

$(\mathrm{N}=30$ to 44 with differences in both feeding and BMI

$\sim 0.60 ; p<0.01$

status)

Note: this analysis is across 2 age groups

Ordinary least squares model

$\mathrm{OB}^{*}$ vs Not $\mathrm{OB}^{*}$ (ref) (OR) @ 9-19 y in sibling

subsample

$(\mathrm{N}=30$ to 44 with differences in both feeding and BMI

status)

Note: this analysis is across 2 age groups

Ordinary least squares model

\begin{tabular}{|c|c|c|c|}
\hline & & Fixed-effects model & $\sim 0.20 ; p<0.01$ \\
\hline $\begin{array}{l}\text { Moschonis } \mathbf{2 0 1 7 ^ { 3 0 }} \\
\text { RC (ALSPAC, EDEN, } \\
\text { EuroPrevall, Generation } \\
\text { XXI) } \\
\text { U.K., France, Greece, } \\
\text { Portugal }\end{array}$ & Never BF vs BF $\geq 6$ mo (ref) & OWOB †‡ (OR) @ 13 y in ALSPAC cohort (N=6124) & 1.1; NS \\
\hline
\end{tabular}




\section{Article}

Study design (Study

name)

Ever vs never consuming human

milk exposure ${ }^{b}$

Outcome $^{c}$

Significant findings

Nonsignificant

findings

\section{Nelson $2005^{32}$}

PC (Add Health)

Ever BF vs Never BF (ref)

OWOB* (OR [95\% Cl]) @ 12-21 y

Note: this analysis is across 2 age groups

In the full sample

In males $(\mathrm{N}=2787,3142)$

$0.90[0.76,1.05]$

In females $(\mathrm{N}=2975,3094)$

$0.83[0.72,0.95]$

In the subsample of siblings $(\mathrm{N}=860,840)$

$0.78[0.63,0.97]$

In the subsample of siblings with discordant

exposures and outcomes $(\mathrm{N}=224)$

Odds that the non-OW sibling was ever BF and

the OW sibling was never BF vs both siblings

$0.52[95 \% \mathrm{Cl} 0.22$

OW and never BF (ref)

Odds that the non-OW sibling was ever BF and

the OW sibling was never BF vs both siblings

non-OW and never BF (ref)

Odds that the OW sibling was ever BF and the

non-OW sibling was never BF vs both siblings

OW and never BF (ref)

Odds that the OW sibling was ever BF and the

non-OW sibling was never BF vs both siblings

non-OW and never BF (ref)

BF $\geq 3$ mo vs Never BF (ref)

BF $<3$ mo vs Never BF (ref)
OWOB* (OR [95\% Cl]) @ 12-21 y in the subsample of siblings

Note: this analysis is across 2 age groups

OWOB* (OR [95\% CI]) @ 12-21 y

Note: this analysis is across 2 age groups

In the full sample

In males $(\mathrm{N}=889,3142)$

$1.03[0.85,1.24]$

In females $(\mathrm{N}=910,3094)$
$1.22[95 \% \mathrm{Cl} 0.64$,

2.32]

$2.03[95 \% \mathrm{Cl} 0.64$ 6.43

$1.27[95 \% \mathrm{Cl} 0.65$

2.50] 


\begin{tabular}{|c|c|c|c|c|}
\hline $\begin{array}{l}\text { Article } \\
\text { Study design (Study } \\
\text { name) } \\
\text { Country }\end{array}$ & $\begin{array}{l}\text { Ever vs never consuming human } \\
\text { milk exposure }{ }^{b}\end{array}$ & Outcome $^{c}$ & Significant findings & $\begin{array}{l}\text { Nonsignificant } \\
\text { findings }\end{array}$ \\
\hline & \multirow[t]{3}{*}{ BF 3-5.99 mo vs Never BF (ref) } & OWOB* (OR [95\% Cl]) @ 12-21 y & & \\
\hline & & Note: this analysis is across 2 age groups & & \\
\hline & & In the full sample & & \\
\hline & & In females $(N=728,3094)$ & & $0.82[0.64,1.05]$ \\
\hline & \multirow[t]{4}{*}{ BF 6-8.99 mo vs Never BF (ref) } & 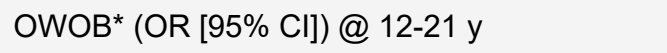 & & \\
\hline & & Note: this analysis is across 2 age groups & & \\
\hline & & In the full sample & & \\
\hline & & In males $(\mathrm{N}=474,3142)$ & & $0.86[0.63,1.17]$ \\
\hline & & In females $(N=425,3094)$ & & $0.73[0.54,1.00]$ \\
\hline & \multirow[t]{5}{*}{$B F \geq 9$ mo vs Never BF (ref) } & OWOB* (OR [95\% Cl]) @ 12-21 y & & \\
\hline & & Note: this analysis is across 2 age groups & & \\
\hline & & In the full sample & & \\
\hline & & In males $(\mathrm{N}=830,3142)$ & & $0.83[0.67,1.04]$ \\
\hline & & In females $(\mathrm{N}=607,3094)$ & $0.78[0.64,0.96]$ & \\
\hline \multirow{4}{*}{$\begin{array}{l}\text { Pluyman 201936 } \\
\text { PC (PIAMA) } \\
\text { Netherlands }\end{array}$} & Ever BF vs Never BF (ref) & OWOB $\ddagger(\mathrm{OR}[95 \% \mathrm{Cl}]) @ 12$ y $(\mathrm{N}=1288,211)$ & $0.61[0.38,0.97]$ & \\
\hline & BF $<3$ mo vs Never BF (ref) & \multicolumn{2}{|l|}{ OWOB $\neq($ OR $[95 \% \mathrm{Cl}]) @ 12$ y $(\mathrm{N}=511,211)$} & $0.63[0.37,1.08]$ \\
\hline & BF 3 to $<6$ mo vs Never BF (ref) & \multicolumn{2}{|l|}{ OWOB } & $0.55[0.30,1.00]$ \\
\hline & BF $\geq 6$ mo vs Never BF (ref) & \multicolumn{2}{|l|}{ OWOB $\ddagger($ OR $[95 \% \mathrm{Cl}]) @ 12$ y $(\mathrm{N}=439,211)$} & $0.61[0.36,1.06]$ \\
\hline \multicolumn{5}{|c|}{ Outcomes at 20 years or later } \\
\hline \multirow{2}{*}{$\begin{array}{l}\text { Bohr } 2015^{5} \\
\text { PC (Add Health) } \\
\text { U.S. }\end{array}$} & \multirow[t]{2}{*}{ Ever vs Never BF (ref) } & \multicolumn{2}{|l|}{ OB (OR [95\% Cl]) @ 24-33 y } & \\
\hline & & In unrelated sample $(\mathrm{N}=4648)$ & $\begin{array}{l}0.854[0.75,0.97] \\
p=0.018\end{array}$ & \\
\hline
\end{tabular}




\section{Article}

Study design (Study

name)

Ever vs never consuming human

milk exposure

Outcome $^{\mathrm{c}}$

Significant findings

Nonsignificant

findings

untry

In sibling pair sample $(\mathrm{N}=1179)$

$0.854[0.64,1.13]$

$p=0.277$

\section{Nelson $2005^{32}$}

PC (Add Health)

Ever BF vs Never BF (ref)

OWOB* (OR [95\% Cl]) @ 12-21 y

U.S.

Note: this analysis is across 2 age groups

In the full sample

In males $(\mathrm{N}=2787,3142)$

$0.90[0.76,1.05]$

\begin{tabular}{|c|c|c|c|}
\hline & In females $(\mathrm{N}=2975,3094)$ & $0.83[0.72,0.95]$ & \\
\hline & In the subsample of siblings $(\mathrm{N}=860,840)$ & $0.78[0.63,0.97]$ & \\
\hline & \multicolumn{2}{|l|}{$\begin{array}{l}\text { In the subsample of siblings with discordant } \\
\text { exposures and outcomes }(\mathrm{N}=224)\end{array}$} & \\
\hline & \multicolumn{2}{|l|}{$\begin{array}{l}\text { Odds that the non-OW sibling was ever BF and } \\
\text { the OW sibling was never BF vs both siblings } \\
\text { OW and never BF (ref) }\end{array}$} & $\begin{array}{l}0.52[95 \% \mathrm{Cl} 0.22 \\
1.24]\end{array}$ \\
\hline & \multicolumn{2}{|l|}{$\begin{array}{l}\text { Odds that the non-OW sibling was ever BF and } \\
\text { the OW sibling was never BF vs both siblings } \\
\text { non-OW and never BF (ref) }\end{array}$} & $\begin{array}{l}1.22[95 \% \mathrm{Cl} 0.64 \\
2.32]\end{array}$ \\
\hline & \multicolumn{2}{|l|}{$\begin{array}{l}\text { Odds that the OW sibling was ever BF and the } \\
\text { non-OW sibling was never BF vs both siblings } \\
\text { OW and never BF (ref) }\end{array}$} & $\begin{array}{l}2.03[95 \% \mathrm{Cl} 0.64 \\
6.43]\end{array}$ \\
\hline & \multicolumn{2}{|l|}{$\begin{array}{l}\text { Odds that the OW sibling was ever BF and the } \\
\text { non-OW sibling was never BF vs both siblings } \\
\text { non-OW and never BF (ref) }\end{array}$} & $\begin{array}{l}1.27[95 \% \mathrm{Cl} 0.65 \\
2.50]\end{array}$ \\
\hline \multirow[t]{2}{*}{$B F \geq 3$ mo vs Never BF (ref) } & $\begin{array}{l}\text { OWOB* (OR }[95 \% \mathrm{CI}]) @ 12-21 \text { y in the subsample } \\
\text { of siblings }\end{array}$ & \multirow[t]{2}{*}{$0.69[0.54,0.87]$} & \\
\hline & Note: this analysis is across 2 age groups & & \\
\hline
\end{tabular}

BF $<3$ mo vs Never BF (ref)

OWOB* (OR [95\% Cl]) @ 12-21 y

Note: this analysis is across 2 age groups

In the full sample 


\section{Article}

Study design (Study

name)

Ever vs never consuming human

milk exposure ${ }^{\mathrm{b}}$

Outcome $^{\mathrm{c}}$

Significant findings

Nonsignificant

findings

Country

In females $(\mathrm{N}=910,3094)$

$0.90[0.74,1.09]$

BF 3-5.99 mo vs Never BF (ref) OWOB* (OR [95\% Cl]) @ 12-21 y

Note: this analysis is across 2 age groups

In the full sample

In males $(\mathrm{N}=593,3142)$

$0.81[0.63,1.04]$

In females $(\mathrm{N}=728,3094)$

$0.82[0.64,1.05]$

BF 6-8.99 mo vs Never BF (ref)

OWOB* (OR [95\% Cl]) @ 12-21 y

Note: this analysis is across 2 age groups

In the full sample

In males $(\mathrm{N}=474,3142)$

$0.86[0.63,1.17]$

In females $(\mathrm{N}=425,3094)$

$0.73[0.54,1.00]$

BF $\geq 9$ mo vs Never BF (ref)

OWOB* (OR [95\% Cl]) @ 12-21 y

Note: this analysis is across 2 age groups

In the full sample

In males $(\mathrm{N}=830,3142)$

$0.83[0.67,1.04]$

In females $(\mathrm{N}=607,3094)$

$0.78[0.64,0.96]$

a Abbreviations: \%ile - percentile, ALSPAC - Avon Longitudinal Study of Parents and Children, Add Health - National Longitudinal Study of Adolescent Health, BF -

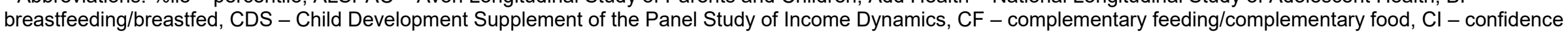

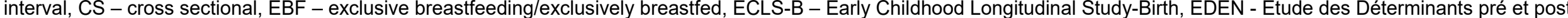

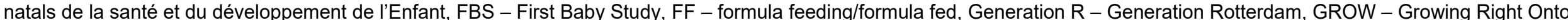

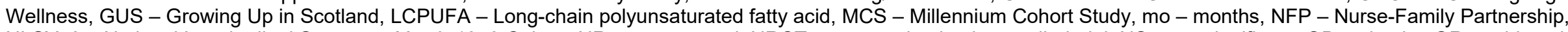

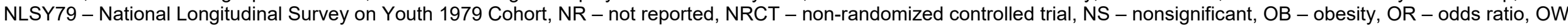

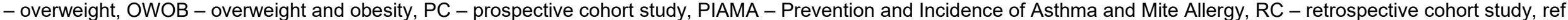

- reference, SE - standard error, UK - United Kingdom, U.S. - United States of America, WFL - weight-for-length, wk - weeks, y - years

${ }^{b}$ Exposures, from the articles included in the body of evidence, which address ever vs never consuming human milk, or vice versa

${ }^{c}$ Notations used for growth references: * $\mathrm{CDC}$, †WHO, ‡IOTF, §Country-specific

${ }^{d}$ Anderson et al 2003 did not explicitly state that this finding was significant; however the text of the article implied that it was significant 
Table 3. Risk of bias for the non-randomized controlled trial examining the relationship between ever vs never consuming human milk and overweight and obesity ${ }^{a b}$

\begin{tabular}{|c|c|c|c|c|c|c|c|}
\hline Study & Confounding & Selection of participants & $\begin{array}{l}\text { Classification of } \\
\text { interventions }\end{array}$ & $\begin{array}{l}\text { Deviations from } \\
\text { intended } \\
\text { interventions }\end{array}$ & $\begin{array}{c}\text { Outcome } \\
\text { measurement }\end{array}$ & Missing data & $\begin{array}{l}\text { Selection of the } \\
\text { reported result }\end{array}$ \\
\hline de Jong, $2011^{7}$ & Serious & Moderate & Low & Low & Moderate & Moderate & Low \\
\hline
\end{tabular}

${ }^{a}$ A detailed description of the methodology used for assessing risk of bias is available on the NESR website: https://nesr.usda.gov/2020-dietary-guidelinesadvisory-committee-systematic-reviews and in Part C of the following reference: Dietary Guidelines Advisory Committee. 2020. Scientific Report of the 2020 Dietary Guidelines Advisory Committee: Advisory Report to the Secretary of Agriculture and the Secretary of Health and Human Services. U.S. Department of Agriculture, Agricultural Research Service, Washington, DC.

b Possible ratings of low, moderate, serious, critical, or no information determined using the "Risk of Bias in Non-randomized Studies of Interventions (ROBINS-I) tool" (Sterne JAC, Hernán MA, Reeves BC, Savović J, Berkman ND, Viswanathan M, Henry D, Altman DG, Ansari MT, Boutron I, Carpenter JR, Chan AW, Churchill R, Deeks JJ, Hróbjartsson A, Kirkham J, Jüni P, Loke YK, Pigott TD, Ramsay CR, Regidor D, Rothstein HR, Sandhu L, Santaguida PL, Schünemann HJ, Shea B, Shrier I, Tugwell P, Turner L, Valentine JC, Waddington H, Waters E, Wells GA, Whiting PF, Higgins JPT. ROBINS-I: a tool for assessing risk of bias in non-randomized studies of interventions. BMJ 2016; 355; i4919; doi: 10.1136/bmj.i4919.) 
Table 4. Risk of bias for observational studies examining the relationship between ever vs never consuming human milk and overweight and obesity ${ }^{\text {ab }}$

\begin{tabular}{|c|c|c|c|c|c|c|c|}
\hline Study & Confounding & Selection of participants & $\begin{array}{c}\text { Classification of } \\
\text { exposures }\end{array}$ & $\begin{array}{l}\text { Deviations from } \\
\text { intended } \\
\text { exposures }\end{array}$ & $\begin{array}{c}\text { Outcome } \\
\text { measurement }\end{array}$ & $\begin{array}{l}\text { Missing } \\
\text { outcome data }\end{array}$ & $\begin{array}{l}\text { Selection of the } \\
\text { reported result }\end{array}$ \\
\hline $\begin{array}{l}\text { Abraham, } \\
\text { 2012 }^{1}\end{array}$ & Serious & Moderate & Moderate & Low & Serious & Low & Low \\
\hline $\begin{array}{l}\text { Anderson, } \\
2003^{3}\end{array}$ & Serious & Serious & Serious & Low & Serious & Low & Low \\
\hline $\begin{array}{l}\text { Anderson, } \\
2014^{2}\end{array}$ & Serious & Serious & Serious & Low & Serious & Low & Low \\
\hline $\begin{array}{l}\text { Bjertnaes, } \\
2019^{4}\end{array}$ & Serious & Serious & Serious & Low & Serious & Low & Low \\
\hline Bohr, $2015^{5}$ & Serious & Serious & Serious & Low & Serious & Serious & Low \\
\hline Colen, $2014^{6}$ & Serious & Serious & Serious & Low & Serious & Low & Low \\
\hline Durmus, $2011^{9}$ & Serious & Moderate & Moderate & Low & Serious & Moderate & Low \\
\hline Durmus, $2014^{8}$ & Serious & Serious & Moderate & Low & Moderate & Moderate & Low \\
\hline $\begin{array}{l}\text { Evenhouse, } \\
2005^{10}\end{array}$ & Serious & Serious & Serious & Low & Serious & No information & Low \\
\hline $\begin{array}{l}\text { Feldman- } \\
\text { Winter, 2018 }\end{array}$ & Serious & Serious & Low & Low & Moderate & Moderate & Low \\
\hline $\begin{array}{l}\text { Hawkins, } \\
2019^{14}\end{array}$ & Serious & Serious & Low & Low & Moderate & Serious & Low \\
\hline $\begin{array}{l}\text { Heerman, } \\
2019^{15}\end{array}$ & Serious & Low & Moderate & Moderate & Moderate & Low & Low \\
\hline Heppe, $2013^{16}$ & Serious & Serious & Serious & Low & Serious & Low & Low \\
\hline Huang, 2014 ${ }^{17}$ & Serious & Serious & Serious & Low & Serious & Low & Low \\
\hline Layte, $2014^{19}$ & Serious & Serious & Moderate & Low & Moderate & Low & Low \\
\hline Lee, $\mathbf{2 0 1 9}^{20}$ & Serious & Serious & Moderate & Low & Serious & Low & Low \\
\hline Li, 201121 & Serious & Serious & Serious & Low & Moderate & Low & Low \\
\hline Lurbe, $2018^{22}$ & Serious & Serious & Serious & Low & Moderate & Low & Low \\
\hline $\begin{array}{l}\text { Massion, } \\
2016^{26}\end{array}$ & Serious & Moderate & Moderate & Low & Serious & Serious & Low \\
\hline $\begin{array}{l}\text { Metzger, } \\
2009^{27}\end{array}$ & Serious & Serious & Serious & Low & Moderate & Low & Low \\
\hline $\begin{array}{l}\text { Moschonis, } \\
2017^{30}\end{array}$ & Serious & No information & Moderate & Low & Serious & No information & Low \\
\hline Moss, $2014^{31}$ & Serious & Moderate & Moderate & Low & Moderate & No information & Low \\
\hline Nelson, $2005^{32}$ & Serious & Moderate & Serious & Low & Serious & Moderate & Low \\
\hline
\end{tabular}




\begin{tabular}{|c|c|c|c|c|c|c|c|}
\hline Study & Confounding & Selection of participants & $\begin{array}{l}\text { Classification of } \\
\text { exposures }\end{array}$ & $\begin{array}{c}\text { Deviations from } \\
\text { intended } \\
\text { exposures }\end{array}$ & $\begin{array}{c}\text { Outcome } \\
\text { measurement }\end{array}$ & $\begin{array}{c}\text { Missing } \\
\text { outcome data }\end{array}$ & $\begin{array}{l}\text { Selection of the } \\
\text { reported result }\end{array}$ \\
\hline $\begin{array}{l}\text { Pattison, } \\
2019^{35}\end{array}$ & Serious & Moderate & Moderate & Low & Serious & Moderate & Low \\
\hline $\begin{array}{l}\text { Pluyman, } \\
2018^{36}\end{array}$ & Serious & Moderate & Moderate & Low & Moderate & Moderate & Low \\
\hline $\begin{array}{l}\text { Ruijsbroek, } \\
\text { 2011 }^{37}\end{array}$ & Serious & Low & Moderate & Low & Serious & Moderate & Low \\
\hline $\begin{array}{l}\text { Thorland, } \\
2017^{39}\end{array}$ & Serious & Serious & Serious & Low & Serious & Moderate & Low \\
\hline $\begin{array}{l}\text { van Rossem, } \\
2011^{40}\end{array}$ & Serious & Serious & Moderate & Low & Low & Serious & Low \\
\hline Weng, $2013^{41}$ & Serious & Serious & Moderate & Low & Serious & Low & Low \\
\hline
\end{tabular}

${ }^{a}$ A detailed description of the methodology used for assessing risk of bias is available on the NESR website: https://nesr.usda.gov/2020-dietary-guidelinesadvisory-committee-systematic-reviews and in Part C of the following reference: Dietary Guidelines Advisory Committee. 2020. Scientific Report of the 2020 Dietary Guidelines Advisory Committee: Advisory Report to the Secretary of Agriculture and the Secretary of Health and Human Services. U.S. Department of Agriculture, Agricultural Research Service, Washington, DC.

b Possible ratings of low, moderate, serious, critical, or no information determined using the "Risk of Bias for Nutrition Observational Studies" tool (RoB-NObs) (Dietary Guidelines Advisory Committee. 2020. Scientific Report of the 2020 Dietary Guidelines Advisory Committee: Advisory Report to the Secretary of Agriculture and the Secretary of Health and Human Services. U.S. Department of Agriculture, Agricultural Research Service, Washington, DC.) 


\title{
Duration of any human milk consumption among infants fed human milk
}

\author{
Description of the evidence examining the relationship between the duration of any human \\ milk consumption, among infants fed human milk, and body composition, including \\ obesity, from studies that conducted within-family comparisons of siblings (Table 5)
}

Four articles, published between 2005 and 2014, conducted within-family analyses comparing the duration of any human milk consumption between siblings and the siblings' BMI, body fat percentage, or overweight and/or obesity status. ${ }^{6,10,12,34}$ The articles presented evidence from 4 independent cohorts from the United States $(\mathrm{N}=3)$ and Finland $(\mathrm{N}=1)$, described below.

\section{Children of the National Longitudinal Survey on Youth 1979 Cohort (Children of NLSY79)}

Colen and Ramey ${ }^{6}$ examined a sample of children and adolescents whose mothers were part of the NLSY79 cohort from the United States. The study sample consisted of the subset of offspring who were singletons, born after the year 1978, and 4 to 14 years of age between 1986 and 2010 . About $75 \%$ of participants were non-Hispanic white. Infant-feeding data were collected by maternal report at biannual survey rounds starting in 1986. The outcomes of interest were BMI and obesity (i.e., BMI $\geq 95^{\text {th }}$ percentile based on CDC growth curves) at 4 to 14 years of age. Approximately $64 \%$ of height and weight data were collected by study staff, and the remainder were from maternal- or self-report. The authors conducted analyses on the full sample $(\mathrm{N}=8237)$, on a subsample consisting of participants with siblings in the study $(\mathrm{N}=7319)$, and on a second subsample consisting of siblings with discordant infant feeding (i.e., siblings fed differently during infancy; $N=1773$ ).

- In the full sample and sibling subsample, there were small, but statistically significant, inverse associations between weeks of human milk consumption and BMI at 4 to 14 years ( $\beta=-0.007 \pm 0.002$ and $\beta=-0.007 \pm 0.003$, respectively; $p<0.01$ for both). In the discordant sibling subsample, there was a small positive association that was nonsignificant $(\beta=0.005 \pm 0.003)$.

- Likewise, in the full sample and sibling subsample, there were small, but statistically significant, inverse associations between weeks of human milk consumption and obesity at 4 to 14 years $(\beta=-0.007 \pm 0.002, p<0.01$ and $\beta=-0.006 \pm 0.002, p<0.05$, respectively). In the discordant sibling subsample, there was no significant association ( $\beta$ $=0.001 \pm 0.004$ ).

\section{National Longitudinal Study of Adolescent Health (Add Health)}

Evenhouse and Reilly ${ }^{10}$ studied the Add Health cohort from the United States. This cohort is a nationally representative sample of 10- to 18-year-olds in grades 7 to 12 during the 19941995 school year (baseline). Low-income, African-American, and Hispanic youth were oversampled. The duration of any human milk consumption (i.e., 0-3, 3-6, 6-9, 9-12, 12-24, or $>24$ months) among participants fed human milk ( $\mathrm{N}=7417$ ) was collected by parent recall at baseline. The outcomes of interest were BMI, overweight/obesity (i.e., BMI $\geq 85^{\text {th }}$ percentile based on CDC growth curves) and obesity (i.e., BMI $\geq 95^{\text {th }}$ percentile based on CDC growth curves) at 10 to 18 years of age. The method used to collect anthropometric data was not reported. The authors conducted analyses on the full sample, on a subsample consisting of participants with a sibling in the study $(\mathrm{N}=1607)$, and on a second subsample consisting of siblings with discordant infant feeding (i.e., siblings fed human milk for different durations; $\mathrm{N}=470)$. 
- In the full sample, sibling subsample, and discordant sibling subsample, there were no significant associations between the duration of any human milk consumption (as a quasicontinuous measure using the midpoints of the categorical durations) and BMI, overweight/obesity, or obesity at 10 to 18 years. The nonsignificant associations for the full sample and sibling subsample were small inverse associations (BMI $\beta=-0.03 \pm 0.006$ and $-0.03 \pm 0.01$, overweight/obesity $\mathrm{OR}=0.98 \pm 0.00$ and $0.98 \pm 0.01$, obesity OR 0.98 \pm 0.01 and $0.99 \pm 0.01$, respectively). The nonsignificant associations for the discordant sibling subsample were closer to the null or at the null (BMI $\beta=0.01 \pm 0.03$, overweight/obesity OR $1.01 \pm 0.01$, obesity OR $1.00 \pm 0.02$ ).

\section{Growing Up Today Study (GUTS)}

Gillman et al ${ }^{12}$ examined a sample of children and adolescents in the U.S.-based GUTS cohort, whose mothers were part of the Nurses' Health Study II. The study sample consisted of the subset of offspring who were (a) singletons, (b) born at 34 completed weeks of gestation or later, (c) 9 to 14 years of age at baseline, and (d) from sibships in which siblings shared the same biological father (based on maternal report of father's height). Almost all participants were white (95\%). Participants self-reported their height and weight at baseline, and infant-feeding data were collected by maternal recall a year later (when participants were 10 to 15 years of age). The authors assessed the association between the duration of human milk consumption and overweight/obesity (i.e., BMI $\geq 85^{\text {th }}$ percentile using CDC growth curves) at 9 to 14 years in the full sample of siblings ( $N=5614$ participants from 2709 families), and the subsample of siblings with discordant infant feeding (i.e., siblings fed human milk for different durations, $\mathrm{N}=2372$ ).

- In the sample of siblings, each additional 3.7-month increase in the duration of any human milk consumption (i.e., the mean difference in duration for discordant siblings) was associated with significantly lower odds of overweight/obesity at 9 to 14 years when applying the same statistical adjustments for confounders as the discordant sibling analysis (i.e., age, sex, tanner stage, menarcheal status for girls, birthweight, birth order, inactivity, physical activity, and energy intake; OR [95\% Cl] $=0.88[0.82,0.94])$. When the model was additionally adjusted for maternal BMI and smoking, and household income, the magnitude of the association was slightly attenuated and the confidence interval included the null $(\mathrm{OR}[95 \% \mathrm{Cl}]=0.94[0.88,1.00])$.

- In the discordant sibling analysis, there was no significant association between consuming human milk for a duration longer than the mean family duration, compared with a duration shorter than the mean family duration, and odds of overweight/obesity at 9 to 14 years of age. The odds ratio was of similar magnitude to the odds ratios in the sibling analyses above, but the confidence interval was wider and included the null (OR $[95 \% \mathrm{Cl}]=0.92[0.76,1.11])$.

\section{Helsinki Birth Cohort}

O'Tierney et $\mathbf{a l}^{34}$ studied the Helsinki Birth Cohort from Finland. Participants were born between 1934 and 1944 in Helsinki, Finland. The study sample consisted of members of the cohort who (a) were fed human milk, (b) had a sibling in the cohort, and (c) provided followup data in the year 2000, along with their sibling. Pediatric clinicians recorded infant feeding prospectively (participants had an average of 8 pediatric visits, which were free, from birth to 2 years of age). The outcomes of interest were BMI and overweight (i.e., BMI $25-30 \mathrm{~kg} / \mathrm{m}^{2}$ ) from self-reported height and weight via questionnaire $(\mathrm{N}=831)$, and $\mathrm{BMI}$ and percent body fat from clinical measurement $(\mathrm{N}=129)$ at about 62 years of age. The analyses compared siblings with discordant infant feeding (i.e., siblings fed human milk for different durations). 
- There were no significant differences in BMI at about 62 years of age between participants fed human milk for $<2,3-4,5-7$ and $\geq 8$ months. It may be important to note that when BMI was based on clinical measure (but not self-report) the quadratic trend approached significance $(p=0.08)$. The $U$-shaped association between duration and BMI measured in the clinic is also somewhat evident from the following analyses. In comparison to a duration of 5 to 7 months, durations of $<2$ months and $\geq 8$ months have nonsignificant associations with higher BMl:

(a) <2 compared with $5-7$ months: mean difference $2.3 \mathrm{~kg} / \mathrm{m}^{2}[95 \% \mathrm{Cl}-0.1,4.7]$ for clinical measure $(0.3[-0.5,1.1]$ for self-report)

(b) 3-4 compared with 5-7 months: mean difference $-0.4 \mathrm{~kg} / \mathrm{m}^{2}[95 \% \mathrm{Cl}-2.7,1.8]$ for clinical measure $(0.3[-0.5,1.1]$ for self-report $)$

(c) $\geq 8$ compared with $5-7$ months: mean difference $1.2 \mathrm{~kg} / \mathrm{m}^{2}[95 \% \mathrm{Cl}-0.9,3.2]$ for clinical measure $(0.2[-0.5,1.0]$ for self-report)

- There were no significant differences in the percent of participants who were overweight at about 62 years of age between participants fed human milk for $<2,3-4,5-7$ and $\geq 8$ months. Likewise, there were no significant differences in the percent of participants who were overweight between participants fed human milk for:

(a) <2 compared with 5-7 months: mean difference 1.6\% [95\% $\mathrm{Cl}-7.7,11.0]$

(b) 3-4 compared with 5-7 months: mean difference $1.9 \%[95 \% \mathrm{Cl}-8.0,11.8]$

(c) $\geq 8$ compared with 5-7 months: mean difference $8.8 \%[95 \% \mathrm{Cl}-0.3,18.0]$

- There was a significant quadratic trend $(p=0.03)$ in the percent body fat of participants fed human milk for $<2,3-4,5-7$ and $\geq 8$ months. The U-shaped association between duration and percent body fat is also evident from the following analyses:

(a) <2 compared with 5-7 months: mean difference 3.9\% [95\% Cl 0.3, 7.4]; $p<0.05$

(b) 3-4 compared with 5-7 months: mean difference $0.3 \%[95 \% \mathrm{Cl}-3.1,3.7]$

(c) $\geq 8$ compared with 5-7 months: mean difference 1.3\% [95\% Cl -1.7, 4.3] 
Table 5. Evidence examining the relationship between the duration of any human milk consumption, among infants fed human milk, and body composition, including obesity, from studies that conducted within-family comparisons of siblings ${ }^{a}$

\section{Article}

Study design (Study Duration of any human milk

name) exposure $^{b}$

Outcome $^{c}$

Significant findings

Nonsignificant findings

Country

$\begin{array}{ll}\text { Colen } 2014^{6} & \text { BF duration (wk) }\end{array}$

PC (Children of NLSY79)

U.S.

BMI ( $\beta \pm$ SE, kg/m2) @ 4-14 y

In the full sample $(\mathrm{N}=8,237)$

$-0.007 \pm 0.002 ; p<0.01$

In the subsample of siblings $(N=7,319) \quad-0.007 \pm 0.003 ; p<0.01$

In the subsample of siblings with discordant exposures $(\mathrm{N}=1,773)$

$\mathrm{OB}^{*}(\beta \pm \mathrm{SE}) @ 4-14 \mathrm{y}$

In the full sample $(\mathrm{N}=8,237)$

$-0.007 \pm 0.002 ; p<0.01$

In the subsample of siblings $(\mathrm{N}=7,319)$

$-0.006 \pm 0.002 ; p<0.05$

In the subsample of siblings with discordant exposures $(\mathrm{N}=1,773)$
Evenhouse 2005 ${ }^{10}$

CS (Add Health)

U.S.

$24,>24 \mathrm{mo}$ )

Duration (mo)
BMI ( $\beta \pm$ SE, kg/m2) @ 10-18 y

In the full sample $(\mathrm{N}=7417)$

$0.03 \pm 0.006 ; p<0.10$
$0.005 \pm 0.003 ;$ NS

$0.001 \pm 0.004 ;$ NS

(quasi-continuous using midpoint

In the subsample of siblings $(\mathrm{N}=1607)$

$-0.03 \pm 0.01 ; p<0.10$

In the subsample of siblings with

discordant exposures $(\mathrm{N}=470)$

$0.01 \pm 0.03 ; \mathrm{NS}$

OWOB* (OR \pm SE from logit) @ 10-18 y

In the full sample $(\mathrm{N}=7417)$

$0.98 \pm 0.00 ; p<0.10$

In the subsample of siblings $(\mathrm{N}=1607)$

$0.98 \pm 0.01 ; p<0.10$

In the subsample of siblings with

discordant exposures $(\mathrm{N}=470)$

$1.01 \pm 0.01 ;$ NS

$\mathrm{OB}^{*}(\mathrm{OR} \pm \mathrm{SE}$ from logit) @ 10-18 y

In the full sample $(\mathrm{N}=7417)$

$0.98 \pm 0.01 ; p<0.10$ 


\section{Article}

Study design (Study

name)

Duration of any human milk

exposure $^{b}$

Outcome $^{c}$

Significant findings

Nonsignificant findings

Country

In the subsample of siblings $(\mathrm{N}=1607)$

$0.99 \pm 0.01 ; \mathrm{NS}$

In the subsample of siblings with

discordant exposures $(\mathrm{N}=470)$

\begin{tabular}{|c|c|c|c|c|}
\hline $\begin{array}{l}\text { Gillman } 2006^{12} \\
\text { CS (GUTS) } \\
\text { U.S. }\end{array}$ & $\begin{array}{l}\text { BF duration (per } 3.7 \mathrm{mo} \text {, which } \\
\text { was the mean difference in } \mathrm{BF} \\
\text { duration for discordant siblings) }\end{array}$ & $\begin{array}{l}\text { OWOB* }(\mathrm{OR}[95 \% \mathrm{Cl}]) @ 9-14 \text { y in the } \\
\text { sample of siblings }(\mathrm{N}=5614)\end{array}$ & $\begin{array}{l}0.88[0.82,0.94] \\
\text { (Adjusted for same confounders } \\
\text { as discordant sibling analysis) }\end{array}$ & $\begin{array}{l}0.94[0.88,1.00] \\
\text { (Also adjusted for maternal BMI } \\
\text { and smoking, household income) }\end{array}$ \\
\hline & $\begin{array}{l}\text { BF longer than the mean BF } \\
\text { duration of his/her participating } \\
\text { sibship vs shorter }\end{array}$ & $\begin{array}{l}\text { OWOB* }(\mathrm{OR}[95 \% \mathrm{Cl}]) @ 9-14 \text { y in the } \\
\text { subsample of siblings with discordant } \\
\text { exposures }(\mathrm{N}=2372)\end{array}$ & & $0.92[0.76,1.11]$ \\
\hline \multirow[t]{8}{*}{$\begin{array}{l}\text { O'Tierney } 2009^{34} \\
\text { PC (Helsinki Birth Cohort) } \\
\text { Finland }\end{array}$} & $\begin{array}{l}\text { Trend across the BF duration } \\
\text { categories }<2,3-4,5-7, \geq 8 \text { mo }\end{array}$ & $\begin{array}{l}\text { BMI (mean } \pm \mathrm{SD}, \mathrm{kg} / \mathrm{m} 2) \text { at } \sim 62 \mathrm{y} \text { in the } \\
\text { sample of siblings with discordant } \\
\text { exposures }\end{array}$ & & \\
\hline & & $\begin{array}{l}\text { from self-reported height and weight } \\
(N=206,168,233,224)\end{array}$ & & $\begin{array}{l}26.6 \pm 4.3 \text { vs } 26.3 \pm 4.3 \text { vs } 26.2 \pm \\
4.2 \text { vs } 26.5 \pm 4.0\end{array}$ \\
\hline & & & & $p=0.80$ (linear), 0.90 (quadratic) \\
\hline & & $\begin{array}{l}\text { from clinical measurement } \\
(\mathrm{N}=22,30,38,39)\end{array}$ & & $\begin{array}{l}29.5 \pm 4.3 \text { vs } 26.8 \pm 5.2 \text { vs } 26.9 \pm \\
4.2 \text { vs } 28.2 \pm 4.9\end{array}$ \\
\hline & & & & $p=0.80$ (linear), 0.08 (quadratic) \\
\hline & & $\begin{array}{l}\text { OW }(\% \text { BMI } 25-30 \mathrm{~kg} / \mathrm{m} 2) \text { from self- } \\
\text { reported height and weight at } \sim 62 \text { y in a } \\
\text { sample of siblings with discordant } \\
\text { exposures }(\mathrm{N}=206,168,233,224)\end{array}$ & & $\begin{array}{l}42.7 \text { vs } 42.9 \text { vs } 41.2 \text { vs } 50.0 \\
p=0.08 \text { (linear), } 0.50 \text { (quadratic) }\end{array}$ \\
\hline & & $\begin{array}{l}\% \mathrm{FM} \text { (mean } \pm \mathrm{SD}, \%) \text { at } \sim 62 \text { y in a } \\
\text { sibling sample }(N=21,26,36,38)\end{array}$ & $\begin{array}{l}32.6 \pm 9.7 \text { vs } 27.6 \pm 8.5 \text { vs } 28.4 \pm \\
6.6 \text { vs } 30.8 \pm 8.0\end{array}$ & $\mathrm{p}=0.50$ (linear) \\
\hline & & & $p=0.03$ (quadratic) & \\
\hline
\end{tabular}

BF $<2$ mo vs BF 5-7 mo (ref)

BMI (mean difference [95\% Cl], kg/m2)

at $\sim 62 \mathrm{y}$ in a sample of siblings with

discordant exposures

from self-reported height and weight

$(\mathrm{N}=206,233)$

$0.3[-0.5,1.1]$

from clinical measurement $(\mathrm{N}=22,38$

$2.3[-0.1,4.7]$ 


\section{Article}

Study design (Study

name)

Duration of any human milk

exposure $^{b}$

Outcome

Significant findings

Nonsignificant findings

ountry

OW (difference, \% BMI 25-30 kg/m2)

from self-reported height and weight at

$\sim 62 \mathrm{y}$ in a sample of siblings with

discordant exposures ( $\mathrm{N}=206,233)$

$\% F M$ (difference, \%) at $\sim 62$ y in a

sample of siblings with discordant

$3.9[0.3,7.4]$

exposures $(\mathrm{N}=21,36)$

\begin{tabular}{|c|c|c|}
\hline BF 3-4 mo vs BF 5-7 mo (ref) & $\begin{array}{l}\text { BMI (mean difference }[95 \% \mathrm{Cl}], \mathrm{kg} / \mathrm{m} 2 \text { ) } \\
\text { at } \sim 62 \text { in a sample of siblings with } \\
\text { discordant exposures }\end{array}$ & \\
\hline & $\begin{array}{l}\text { from self-reported height and weight } \\
(\mathrm{N}=168,233)\end{array}$ & $0.3[-0.5,1.1]$ \\
\hline & from clinical measurement $(\mathrm{N}=30,38)$ & $-0.4[-2.7,1.8]$ \\
\hline & $\begin{array}{l}\text { OW (difference, } \% \text { BMI } 25-30 \mathrm{~kg} / \mathrm{m} 2) \\
\text { from self-reported height and weight at } \\
\sim 62 \text { y in a sample of siblings with } \\
\text { discordant exposures }(\mathrm{N}=168,233)\end{array}$ & $1.9[-8.0,11.8]$ \\
\hline & $\begin{array}{l}\% \mathrm{FM} \text { (difference, } \%) \text { at } \sim 62 \text { y in a } \\
\text { sample of siblings with discordant } \\
\text { exposures }(N=26,36)\end{array}$ & $0.3[-3.1,3.7]$ \\
\hline $\mathrm{BF} \geq 8 \mathrm{mo}$ vs BF $5-7$ mo (ref) & $\begin{array}{l}\text { BMI (mean difference }[95 \% \mathrm{Cl}], \mathrm{kg} / \mathrm{m} 2 \text { ) } \\
\text { at } \sim 62 \text { in a sample of siblings with } \\
\text { discordant exposures }\end{array}$ & \\
\hline & $\begin{array}{l}\text { from self-reported height and weight } \\
(\mathrm{N}=224,233)\end{array}$ & $0.2[-0.5,1.0]$ \\
\hline & from clinical measurement $(\mathrm{N}=39,38)$ & $1.2[-0.9,3.2]$ \\
\hline & $\begin{array}{l}\text { OW (difference, } \% \text { BMI } 25-30 \mathrm{~kg} / \mathrm{m} 2) \\
\text { from self-reported height and weight at } \\
\sim 62 \text { y in a sample of siblings with } \\
\text { discordant exposures }(\mathrm{N}=224,233)\end{array}$ & $8.8[-0.3,18.0]$ \\
\hline & $\begin{array}{l}\% \mathrm{FM} \text { (difference, } \% \text { ) at } \sim 62 \text { y in a } \\
\text { sample of siblings with discordant } \\
\text { exposures }(N=38,36)\end{array}$ & $1.3[-1.7,4.3]$ \\
\hline
\end{tabular}




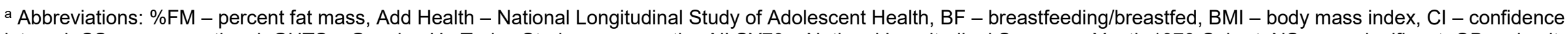

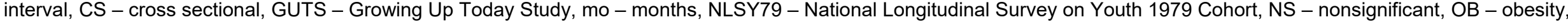

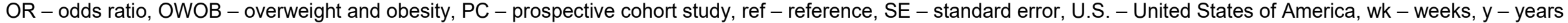

${ }^{b}$ Exposures, from the articles included in the body of evidence, which address the duration of any human milk consumption among infants fed human milk

${ }^{c}$ Notations used for growth references: *CDC, +WHO, ‡IOTF, §Country-specific 
Description of the evidence examining the relationship between the duration of any human milk consumption, among infants fed human milk, and overweight and/or obesity at 2 years of age and older (Table 6 )

Twenty-one articles, published between 2005 and 2019, were identified that met the inclusion criteria and examined the duration of any human milk consumption, among infants fed human milk, and overweight and/or obesity. 4,6,8-10,12,13,15,18,23-26,28-30,33,34,37,38,42 They presented evidence from 19 independent studies. Four of the 19 studies were also described in the section about evidence from siblings. ${ }^{6,10,12,34} \mathrm{~A}$ description of the studies is presented below by age group (i.e., 2 to 5 years, 6 to 11 years, 12 to 19 years, and 20 years and older).

\section{Studies with evidence in multiple age groups}

\section{Generation Rotterdam (Generation R) Study}

Durmus et al (2011) ${ }^{9}$ and Durmus et al (2014) ${ }^{8}$ presented evidence from the Generation $\mathrm{R}$ Study, which was a prospective cohort study conducted in the Netherlands. Both samples consisted of singleton infants who had available information on infant feeding. Durmus et al $(2011)^{9}$ examined dichotomous comparisons of 0-3 months and 3-6 months of human milk consumption with $>6$ months of human milk consumption. Durmus et al $(2014)^{8}$ examined dichotomous comparisons of $>0-1.99,2-3.00$, and $4-5.99$ months of human milk consumption with $\geq 6$ months of human milk consumption, and also examined the trend across the 4 duration categories. Infant-feeding data were obtained from clinical delivery reports at birth and by maternal report at 2, 6, and 12 months of age. The outcome of interest in both articles was overweight and obesity (combined).

- Durmus et al (2011) $)^{9}$ found significant inverse associations between the duration of any human milk consumption and odds of overweight/obesity at 2, but not at 3 years of age. Consuming human milk for shorter durations of 0-3 months or 3-6 months, in comparison to a longer duration of $>6$ months, was associated with significantly higher odds of overweight/obesity at 2 years (OR [95\% Cl] $\sim 2[\sim 1.5, \sim 3], p<0.01$ and $\sim 2[\sim 1, \sim 3], p<0.05$, respectively). However, at 3 years, the associations were nonsignificant and slightly below the null (OR $[95 \% \mathrm{Cl}] \sim 0.9[\sim 0.8, \sim 2]$ and $\sim 0.8[\sim 0.7, \sim 2]$, respectively).

- Durmus et al $(2014)^{8}$ found some significant positive associations between the duration of any human milk consumption and odds of overweight/obesity at 6 years of age. There was a significant trend across 4 duration categories (>0-1.9, 2-3.9, 4-5.9, and $\geq 6$ months). In addition, consuming human milk for the shorter duration of $>0-1.9$ months, in comparison to the longer duration of $\geq 6$ months, was associated with significantly lower odds of overweight/obesity at 6 years of age (OR $\sim 0.7$ with the top error bar just below 1.0). However, the association was nonsignificant when durations of 2-3.9 and 4-5.9 months were compared with $\geq 6$ months $(\mathrm{OR}[95 \% \mathrm{Cl}] \sim 0.8[\sim 0.7, \sim 1.1]$ and $\sim 1.0[\sim 0.8$, $\sim 1.3$ ], respectively).

2. Moschonis et $\mathbf{a l}^{30}$ presented analyses of 4 European cohorts: Avon Longitudinal Study of Parents and Children (ALSPAC), Etude des Déterminants pré et post natals de la santé et du développement de l'Enfant (EDEN), EuroPrevall, and Generation XXI, conducted in the United Kingdom, France, Greece, and Portugal, respectively. Infant-feeding data were collected by parent report via questionnaire at 6 and 15 months of age in ALSPAC, at 4, 8, and 12 months of age in EDEN, at 15 months of age in Generation XXI, and at 12, 24, and 30 months of age in EuroPrevall. The authors examined dichotomous comparisons of $<1$ 
month, 1-3 months, and 3-6 months, compared with $\geq 6$ months of human milk consumption. Height and weight were measured at each study site and used to identify participants who were overweight and obese (combined, using IOTF cutoffs) at 4 ( $N=6522), 9(\mathrm{~N}=7540)$, and 13 years of age $(\mathrm{N}=6124)$ in the ALSPAC cohort, at 4 years of age in the EuroPrevall $(\mathrm{N}=309)$ and Generation XXI $(\mathrm{N}=3387)$ cohorts, and at 5 years of age $(\mathrm{N}=1070)$ in EDEN cohort.

- In the ALSPAC cohort, consuming human milk for 3-6 months, compared with $\geq 6$ months, was associated with significantly lower odds of overweight/obesity at 4 and at 13 years of age (ORs $\sim 0.7, p=0.008$, and $\sim 0.8, p=0.031$, respectively; Cls not reported), but not at 9 years of age (OR $\sim 1.1, \mathrm{Cl}$ not reported). There were no significant associations between consuming human milk for $<1$ or $1-3$ months, compared with $\geq 6$ months, and overweight/obesity at 4,9 , or 13 years of age in the ALSPAC cohort (ORs 1.0 and $\sim 0.9$, respectively, at 4 years of age, $\sim 1.1$ and $\sim 1.1$, respectively, at 9 years of age, and $\sim 1.3$ and $\sim 1.0$, respectively, at 13 years of age).

- In the EuroPrevall and Generation XXI cohorts, there were no significant associations between consuming human milk for $<1,1-3$, or 3-6 months, compared with $\geq 6$ months, and the odds of overweight/obesity at 4 years of age $(\mathrm{OR} \sim 0.6, \sim 0.7$, and $\sim 1.3$, respectively, in the EuroPrevall cohort and $\sim 0.9, \sim 1.0$, and $\sim 1.1$, respectively, in the Generation XXI cohort; Cls not reported). Likewise, in the EDEN cohort, there were no significant associations between consuming human milk for $<1,1-3$, or 3-6 months, compared with $\geq 6$ months, and the odds of overweight/obesity at 5 years of age (OR $\sim 0.8, \sim 0.8$, and $\sim 0.6$, respectively; Cls not reported).

\section{Promotion of Breastfeeding Intervention Trial (PROBIT)}

Martin et al (2013) ${ }^{25}$ and Martin et al (2017) ${ }^{24}$ presented evidence from the PROBIT study conducted in Belarus. This was a cluster randomized controlled trial of an intervention to promote prolonged duration and exclusivity of human milk feeding among mothers who chose to feed human milk. The study enrolled 17,046 infants at birth and followed 13,879 children to 11.5 years of age ${ }^{25}$ and 13,557 adolescents to 16 years of age ${ }^{24}$. Study pediatricians collected human milk-feeding data at well-baby medical appointments, and the intervention group had higher rates of any human milk consumption measured at 3, 6, 9, and 12 months. The outcomes, overweight/obesity (i.e., BMI $\geq 85^{\text {th }}$ percentile based on CDC growth curves) and obesity (i.e., BMI $\geq 95^{\text {th }}$ percentile based on CDC growth curves), were secondary outcomes of the study iii and were measured by study staff. The authors used an intention-to-treat analysis that compared the intervention group with the control group.

- At 11.5 years of age, the intervention group had significantly higher odds of overweight/obesity (compared with $\mathrm{BMI}<85^{\text {th }}$ percentile) than the control group (OR 1.18 $[95 \% \mathrm{Cl}: 1.01,1.39])$. The odds of obesity (also compared with $\mathrm{BMI}<85^{\text {th }}$ percentile) were similar, but the confidence interval was wider and included the null (OR 1.17 [0.97, 1.41]).

- Likewise, at 16 years of age, the intervention group had significantly higher odds of overweight/obesity (compared with BMI $<85^{\text {th }}$ percentile) than the control group (OR 1.14 [95\% Cl: $1.02,1.28]$ ). The odds of obesity (in contrast to the 11.5 year analysis, this was compared with BMI $<95^{\text {th }}$ percentile) were slightly lower and had a wider confidence interval that included the null (OR 1.09 [0.92, 1.29]).

iii U.S. National Library of Medicine. Clinicaltrials.gov. Version 10 October 2013. Internet: https://clinicaltrials.gov/ct2/show/NCT01561612\#wrapper 


\section{Prevention and Incidence of Asthma and Mite Allergy (PIAMA)}

Ruijsbroek et al ${ }^{37}$ presented evidence from the PIAMA study in the Netherlands, which was a birth cohort study and prospective follow-up of (1) participants in an intervention study of mite-impermeable mattress and pillow covers, and (2) participants in a natural-history study in which no intervention was applied. The sample for the intervention arm of the study consisted of children of allergic mothers (mothers with at least 1 of the following: asthma ever, pet allergy, house dust mite allergy or nasal allergy such as hay fever). The sample for the natural-history study consisted of children born to both allergic and non-allergic mothers. The authors examined consuming human milk for 1-16 weeks, compared with $>16$ weeks, as a dichotomous variable. Infant-feeding data were collected prospectively by parental report at 3 and 12 months of age. The outcomes of interest were overweight and obesity (combined) and obesity, defined using IOTF cut-offs, at 3 to 8 years of age $(\mathrm{N}=3963)$. Anthropometric data were collected via parental recall (based on doctor or nurse report if measured within the last 3 months, or measured by parents), but specific methods were not reported.

- There was a significant association between consuming human milk for 1-16 weeks, compared with $>16$ weeks, and higher odds of obesity at 3 to 8 years of age (OR 1.81 $[95 \% \mathrm{Cl} 1.07,3.04])$. The odds of overweight/obesity were in the same direction but were not statistically significant (OR 1.25 [95\% Cl 0.98, 1.59]).

\section{Children of the National Longitudinal Survey on Youth 1979 Cohort (Children of NLSY79)}

Colen and Ramey ${ }^{6}$ examined a sample of children and adolescents whose mothers were part of the National Longitudinal Survey on Youth 1979 Cohort from the United States. The study sample consisted of the subset of offspring who were singletons, born after the year 1978, and 4 to 14 years of age between 1986 and 2010. About $75 \%$ of participants were non-Hispanic white. Infant-feeding data were collected by maternal report at biannual survey rounds starting in 1986. The outcome of interest was obesity (i.e., BMI $\geq 95^{\text {th }}$ percentile based on CDC growth curves) at 4 to 14 years of age. Approximately $64 \%$ of height and weight data were from measurements by study staff, and the remainder were from maternal- or selfreport. The authors conducted analyses on the full sample $(\mathrm{N}=8237)$, on a subsample consisting of participants with siblings in the study $(\mathrm{N}=7319)$, and on a second subsample consisting of siblings with discordant infant feeding (i.e., siblings fed differently during infancy; $\mathrm{N}=1773$ ).

- In the full sample and sibling subsample, there were small, but statistically significant, inverse associations between weeks of human milk consumption and obesity at 4 to 14 years $(\beta=-0.007 \pm 0.002, p<0.01$ and $\beta=-0.006 \pm 0.002, p<0.05$, respectively). In the discordant sibling subsample, there was no significant association $(\beta=0.001 \pm 0.004)$.

\section{National Longitudinal Study of Adolescent Health (Add Health)}

Evenhouse and Reilly ${ }^{10}$ studied the Add Health cohort from the United States. This cohort is a nationally representative sample of 10- to 18-year-olds in grades 7 to 12 during the 19941995 school year (baseline). Low-income, African-American, and Hispanic youth were oversampled. The duration of any human milk consumption (i.e., 0-3, 3-6, 6-9, 9-12, 12-24, or $>24$ months) among participants fed human milk ( $\mathrm{N}=7417$ ) was collected by parent recall at baseline. The outcomes of interest were overweight/obesity (i.e., BMI $\geq 85^{\text {th }}$ percentile based on CDC growth curves) and obesity (i.e., BMI $\geq 95^{\text {th }}$ percentile based on CDC growth curves) at 10 to 18 years of age. The method used to collect anthropometric data was not reported. The authors conducted analyses on the full sample, on a subsample consisting of participants with a sibling in the study $(\mathrm{N}=1607)$, and on a second subsample consisting of 
siblings with discordant infant feeding (i.e., siblings fed human milk for different durations; $\mathrm{N}=470$ ).

- In the full sample, sibling subsample, and discordant sibling subsample, there were no significant associations between the duration of any human milk consumption (as a quasicontinuous measure using the midpoints of the categorical durations) and overweight/obesity or obesity at 10 to 18 years. The nonsignificant associations for the full sample and sibling subsample were small inverse associations (overweight/obesity OR = $0.98 \pm 0.00$ and $0.98 \pm 0.01$, obesity OR $0.98 \pm 0.01$ and $0.99 \pm 0.01$, respectively). The nonsignificant associations for the discordant sibling subsample were closer to the null or at the null (overweight/obesity OR $1.01 \pm 0.01$, obesity OR $1.00 \pm 0.02$ ).

\section{Growing Up Today Study (GUTS)}

Gillman et al ${ }^{12}$ examined a sample of children and adolescents in the U.S.-based GUTS cohort, whose mothers were part of the Nurses' Health Study II. The study sample consisted of the subset of offspring who were (a) singletons, (b) born at 34 completed weeks of gestation or later, (c) 9 to 14 years of age at baseline, and (d) from sibships in which siblings shared the same biological father (based on maternal report of father's height). Almost all participants were white (95\%). Participants self-reported their height and weight at baseline, and infant-feeding data were collected by maternal recall a year later (when participants were 10 to 15 years of age). The authors assessed the association between the duration of human milk consumption and overweight/obesity (i.e., BMI $\geq 85^{\text {th }}$ percentile using CDC growth curves) at 9 to 14 years in the full sample of siblings ( $N=5614$ participants from 2709 families), and the subsample of siblings with discordant infant feeding (i.e., siblings fed human milk for different durations, $\mathrm{N}=2372$ ).

- In the sample of siblings, each additional 3.7-month increase in the duration of any human milk consumption (i.e., the mean difference in duration for discordant siblings) was associated with significantly lower odds of overweight/obesity at 9 to 14 years when applying the same statistical adjustments for confounders as the discordant sibling analysis (i.e., age, sex, tanner stage, menarcheal status for girls, birthweight, birth order, inactivity, physical activity, and energy intake; OR $[95 \% \mathrm{Cl}]=0.88[0.82,0.94])$. When the model was additionally adjusted for maternal BMI and smoking, and household income, the magnitude of the association was slightly attenuated and the confidence interval included the null $(\mathrm{OR}[95 \% \mathrm{Cl}]=0.94[0.88,1.00])$.

- In the discordant sibling analysis, there was no significant association between consuming human milk for a duration longer than the mean family duration, compared with a duration shorter than the mean family duration, and odds of overweight/obesity at 9 to 14 years of age. The odds ratio was of similar magnitude to the odds ratios in the sibling analyses above, but the confidence interval was wider and included the null (OR $[95 \% \mathrm{Cl}]=0.92[0.76,1.11])$.

\section{Child Development Supplement of the Panel Study of Income Dynamics (CDS)}

Jiang and Foster ${ }^{18}$ examined a sample of randomly-selected children born between 1984 and 1997 from the U.S.-based CDS. The sample was $47 \%$ white, $40 \%$ black, and $13 \%$ of of other racial or ethnic backgrounds. The authors examined the number of months participants consumed human milk as a continuous variable, which was truncated at 12 months for those who consumed human milk beyond the first year $(\mathrm{N}=268)$. Infant-feeding data were reported by mothers in 1997 when participants were between 4 months and 13 years of age. The outcome of interest was obesity at 5 to 18 years of age (i.e., BMI $\geq 95^{\text {th }}$ percentile based on CDC growth curves; $N=1512$ ). Height and weight were measured by study staff for $99 \%$ of 
participants using methods that were not reported. For the remaining $1 \%$ of participants, height and weight were reported by parents from participants' last doctor's visit.

- There was no significant association between the number of months participants consumed human milk and obesity at 5-18 years of age (data not reported).

\section{Studies with evidence from 2 to 5 years of age}

Colen and Ramey 6 : See number 5, above

Durmus et al (2011) ${ }^{9}$ : See number 1 , above

\section{Child, Parents, and Health: Lifestyle and Genetic Constitution (KOALA)}

Gubbels et al $^{13}$ assessed participants in the prospective KOALA birth cohort study from the Netherlands. The sample consisted of infants born to mothers with "conventional lifestyles" (about $83 \%$ of the baseline sample) and "alternative lifestyles" with regard to child-rearing practices, dietary habits (e.g., organic, vegetarian), vaccination schemes, and/or use of antibiotics. Infant-feeding data were collected by parent report via questionnaires at 3,7 , and 12 months of age, and the authors examined the duration of any human milk consumption, in months, as a continuous variable. Height and weight data were also reported by parents when participants were 2 and 4 years of age. The outcome of interest was overweight/obesity (combined; i.e., BMI $z$-score $\geq 85^{\text {th }}$ percentile according to a national reference).

- There was no association between the number of months participants consumed human milk and the odds of overweight/obesity at 2 or 4 years of age (i.e., the odds were at the null; OR [95\% CI] 1.01 [0.97, 1.05] and 1.00 [0.96, 1.05], respectively).

Jiang and Foster ${ }^{18}$ : See number 8 , above

\section{Steps to Healthy Development (STEPS)}

Makela et a ${ }^{23}$ examined participants in the STEPS study, a prospective cohort study conducted in Finland. The sample was 100\% Finnish. Infant-feeding data were reported prospectively by parents using a diary, and the study authors assessed the duration of any human milk consumption, in months, as a continuous variable. Health-care professionals measured weight and length ( $<24$ months) or height ( $>24$ months) of participants and identified individuals who were overweight or obese at 2 years of age by plotting BMI on national growth references.

- There was no significant association between the number of months participants consumed human milk and the odds of overweight or obesity at 2 years of age (OR 0.96, $\mathrm{p}=0.157$ and $0.93, \mathrm{p}=0.141$, respectively; Cls not reported).

\section{Oregon Pregnancy Risk Assessment Monitoring System (Oregon PRAMS)}

Modrek et al ${ }^{28}$ assessed data collected through Oregon PRAMS, a state-based data collection initiative of the Centers for Disease Control and Prevention in the United States. The sample was born in 2009, and had mothers (selected via PRAMS by stratified random sampling of birth certificates) who completed PRAMS surveys when their child was 2 to 6 months and 2 years of age. Oregon oversampled mothers from racial and ethnic minorities; however the sample was still predominantly non-Hispanic white (i.e., $76 \%$ of mothers; child race and ethnicity were not reported). The exposure of interest was the duration of any human milk consumption, in weeks, as a continuous variable. The authors used an instrumental variable analysis that used variation on whether a mother fed human milk to her 
infant exclusively in the hospital after birth (data collected at the first survey) as an instrumental variable to examine the relationship between the duration of any human milk consumption (data collected in the second survey) and the outcome of interest. The outcomes of interest were overweight at 2 years of age and obesity at 2 years of age, applying IOTF thresholds. Weight and height at 2 years of age were reported by mothers during the second survey.

- There was a significant inverse association between the duration of any human milk consumption and obesity at 2 years of age (instrumental variable estimate -0.0082 [SE $0.018,-0.001] ; p<0.05)$. The association with overweight was similar but was not statistically significant (instrumental variable estimate -0.008 [SE -0.024, 0.004]; $p<0.1$ ).

Moschonis et al ${ }^{30}$ : See number 2, above

Ruijsbroek et al ${ }^{37}$ : See number 4 , above

\section{Alaska Pregnancy Risk Assessment Monitoring System (Alaska PRAMS)}

Wojcicki et al ${ }^{42}$ assessed data collected through Alaska PRAMS. The sample was born in 2005-2006, and had mothers (selected via PRAMS by stratified random sampling of birth certificates) who completed PRAMS surveys when their children were 2 to 6 months and 3 years of age. Alaska conducted stratification according to maternal Alaska Native race and infant birth weight. The exposure of interest was the duration of any human milk consumption, in months, as a continuous variable. Weight and height at 3 years of age were reported by mothers during the second survey. The outcome of interest was obesity at 3 years of age (i.e., BMI $\geq 95^{\text {th }}$ percentile; the growth reference used was not reported). The authors conducted analyses in Alaska non-Native $(\mathrm{N}=474)$ and Alaska Native $(\mathrm{N}=226)$ children.

- In Alaska non-Native participants, there was a significant inverse association between the number of months of human milk consumption and odds of obesity at 3 years of age (OR 0.95 [95\% Cl 0.91, 0.995], $\mathrm{p}=0.03$ ).

- In Alaska Native participants, the association was attenuated and nonsignificant (OR 0.99 $[95 \% \mathrm{Cl} 0.96,1.02], p=0.44)$.

\section{Studies with evidence from 6 to 11 years of age}

13. Bjertnaes et al ${ }^{4}$ examined a sample of children enrolled in a prospective cohort study conducted in Norway (no cohort name provided). Children included in the cohort started school in Oppland County, a predominantly rural county, in 2007. The authors examined the number of months participants consumed human milk as a continuous variable. Infantfeeding data were recalled by parents at routine school entry health assessments when children were 5-6 years of age. The outcome of interest was overweight/obesity (combined) at approximately 8 years of age $(\mathrm{N}=951)$ using BMI cut-offs provided by the IOTF. Height and weight were measured by school nurses, but specific methods were not reported.

- There was no significant association between the number of months participants consumed human milk and the odds of overweight/obesity at 8 years of age (OR 1.02 $[95 \% \mathrm{Cl} 0.99,1.06], p=0.25)$

Colen and Ramey 6 : See number 5 , above

Durmus et al (2014) ${ }^{8}$ : See number 1 , above

Evenhouse and Reilly ${ }^{10}$ : See number 6, above 
Gillman et al ${ }^{12}$ : See number 7 , above

\section{Growing Right Onto Wellness (GROW)}

Heerman et al ${ }^{15}$ examined data from a prospective cohort from the GROW study conducted in the United States. Participants were from low-income families (e.g., 100\% qualified for services for underserved populations such as Medicaid or WIC, $43 \%$ were food insecure), mostly Hispanic (i.e., 63\% Hispanic Mexican, 28\% Hispanic non-Mexican, 9\% non-Hispanic), and considered to be at high risk for developing obesity at baseline when they were 3 to 5 years of age (i.e., BMI $\geq 50^{\text {th }}$ and $<95^{\text {th }}$ percentile based on CDC growth curves). Infantfeeding data were collected by parent recall at baseline, and the authors examined a dichotomous comparison of $<6$ months with $\geq 6$ months of human milk consumption. Trained study staff measured height and weight. The outcome of interest was obesity (i.e., BMI $\geq 95^{\text {th }}$ percentile based on CDC growth curves) when participants were a mean of about 7 years of age.

- In this high-risk U.S. cohort, there was no significant association between consuming human milk for $<6$ months, compared with $\geq 6$ months, and the odds of obesity at about 7

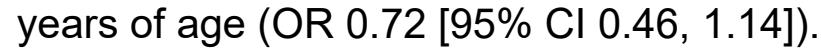

Jiang and Foster ${ }^{18}$ : See number 8 , above

Martin et al (2013) ${ }^{25}$ : See number 3, above

\section{Millennium Cohort Study (MCS)}

Massion et $\mathrm{al}^{26}$ examined samples of children who participated in the MCS, a nationally representative sample of children born in the United Kingdom between September 2000 and January 2002. Children living in disadvantaged areas and ethnic minorities were oversampled to adequately represent hard-to-reach populations. The authors examined consuming human milk for $<4$ months, compared with $>4$ months, as a dichotomous variable. Infant-feeding data were obtained by parent report at the first interview when infants were between 6 and 12 months of age (mean age of 9.2 months). The outcome of interest was overweight and obesity (combined; defined using IOTF cutoffs) at 11 years of age $(\mathrm{N}=11,764)$. Children's heights and weights were measured by trained research staff using standardized protocols.

- The authors reported no significant association between consuming human milk for $<4$ months, compared with $>4$ months, and the relative risk of overweight/obesity at 11 years of age (RR $\sim 1.1[95 \% \mathrm{Cl} \sim 0.9, \sim 1.2])$.

\section{Danish National Birth Cohort (DNBC)}

Morgen et $\mathrm{al}^{29}$ examined a sample from the DNBC. Participants were born full term between 1996 and 2002, and did not have any siblings in the cohort. Infant-feeding data were reported prospectively by mothers during interviews at 6 and 18 months of infant age. The number of weeks infants consumed human milk was assessed using dichotomous comparisons of 20.0-31.9 weeks and 32.0-39.9 weeks with $\geq 40$ weeks. At 7 years of age, height and weight were measured and reported by mothers for the majority of participants $(67 \%)$ or by school doctors, public-health nurses, or general practitioners. Values reported by mothers were compared with values measured by school doctors or public-health nurses for a subgroup of participants $(\mathrm{N}=1122)$, and the study authors found no systematic differences. At 11 years of age, height and weight were only reported by parents. The outcome of interest was overweight/obesity (based on IOTF references) as a dichotomous variable at 7 and 11 years of age. 
- There was a significant association between consuming human milk for 20.0-31.9 weeks, compared with $\geq 40$ weeks, and lower odds of overweight/obesity at both 7 and 11 years of age $(\mathrm{OR}[95 \% \mathrm{Cl}] 0.77[0.64,0.93], p=0.006$ and $0.58[0.43,0.78], p<0.0001$, respectively).

- The associations between consuming human milk for 32.0-39.9 weeks, compared with $\geq 40$ weeks, and odds of overweight/obesity at 7 and 11 years of age were in the same direction, but were attenuated and no longer statistically significant $(\mathrm{OR}[95 \% \mathrm{Cl}] 0.86$ $[0.73,1.01], p=0.7$ and 0.82 [0.64, 1.04], $p=0.1$, respectively).

Moschonis et al ${ }^{30}$ : See number 2 , above

\section{Medio Ambiente y Lactancia Materna (MALAMA)}

Ortega-Garcia et al ${ }^{33}$ presented evidence from the MALAMA study in Spain. Participants were born full term (i.e., gestational age $>37$ weeks) with a birthweight $>2500 \mathrm{~g}$, had 1 - and 5minute Apgar scores of $\geq 7$ and $\geq 8$, respectively, and had infant-feeding and anthropometric data for the analyses $(\mathrm{N}=231)$. Infant-feeding data were reported prospectively by parents during interviews with trained nurses at birth and at 1,3,6, and 12 months of infant age. The study examined the number of weeks infants consumed any human milk as a continuous variable. Weight and height at 6 years of age were measured by pediatricians during wellchild primary care visits, and the authors assessed overweight/obesity (i.e., BMI >1 SD based on WHO growth curves) at 6 years of age.

- The authors found no significant association between the number of weeks participants consumed human milk and overweight/obesity at 6 years of age $(\beta=0.015[95 \% \mathrm{Cl}-0.003$, 0.032], $p=0.11$ ).

Ruijsbroek et $\mathbf{a l}^{37}$ : see number 4 , above

18. Skledar and Milosevic ${ }^{38}$ conducted a retrospective cohort study of Caucasian children who were in their first year of primary school in Croatia between 2002 and 2006. All children in the cohort were born full term (i.e., 37 to 42 weeks gestational age), and had complete data on infant feeding, anthropometry, and the confounding variables used in the analyses $(\mathrm{N}=302)$. Infant-feeding data, height, and weight were recorded in medical charts prospectively by primary care providers and obtained retrospectively for the study from a medical chart review. The authors examined the number of months infants consumed any human milk as a continuous variable, and the odds of overweight/obesity at 6 to 7 years of age (i.e., BMI $\geq 85^{\text {th }}$ percentile based on CDC growth curves).

- There was a nonsignificant positive association between the number of months participants consumed human milk and the odds of overweight/obesity at 6 to 7 years of age; the wide confidence interval included the null (OR 1.45 [95\% $\mathrm{Cl} 0.95,2.20]$, $\mathrm{p}=0.080$ ).

\section{Studies with evidence from 12 to 19 years of age}

Colen and Ramey ${ }^{6}$ : See number 5, above

Evenhouse and Reilly ${ }^{10}$ : See number 6, above

Gillman et al ${ }^{12}$ : See number 7 , above

Jiang and Foster ${ }^{18}$ : See number 8 , above

Martin et al (2017) $)^{24}$ : See number 3 , above 
Moschonis et al ${ }^{30}$ : See number 2 , above

\section{Studies with evidence at 20 years of age and older}

\section{Helsinki Birth Cohort}

O'Tierney et al ${ }^{34}$ studied the Helsinki Birth Cohort from Finland. Participants were born between 1934 and 1944 in Helsinki, Finland. The study sample consisted of members of the cohort who (a) were fed human milk, (b) had a sibling in the cohort, and (c) provided followup data in the year 2000, along with their sibling. Pediatric clinicians recorded infant feeding prospectively (participants had an average of 8 pediatric visits, which were free, from birth to 2 years of age). The outcome of interest was overweight (i.e., BMl $25-30 \mathrm{~kg} / \mathrm{m}^{2}$ ) from selfreported height and weight via questionnaire $(\mathrm{N}=831)$ at about 62 years of age. The analyses compared siblings with discordant infant feeding (i.e., siblings fed human milk for different durations).

- There were no significant differences in the percent of participants who were overweight at about 62 years of age between participants fed human milk for $<2,3-4,5-7$ and $\geq 8$ months. Likewise, there were no significant differences in the percent of participants who were overweight between participants fed human milk for:
(a) <2 compared with 5-7 months: mean difference 1.6\% [95\% Cl -7.7, 11.0]
(b) 3-4 compared with 5-7 months: mean difference $1.9 \%[95 \% \mathrm{Cl}-8.0,11.8]$
(c) $\geq 8$ compared with 5-7 months: mean difference $8.8 \%[95 \% \mathrm{Cl}-0.3,18.0]$

\section{Synthesis and assessment ${ }^{\text {iv }}$ of the evidence examining the relationship between the duration of any human milk consumption, among infants fed human milk, and overweight and/or obesity at 2 years of age and older (Table 6)}

Twenty-one articles presented evidence from 19 independent studies about the relationship between the duration of any human milk consumption, among infants fed human milk, and overweight and/or obesity at 2 years of age and older. $4,6,8-10,12,13,15,18,23-26,28-30,33,34,37,38,42 \mathrm{~A}$ synthesis and assessment of the evidence follows.

\section{Consistency:}

Nine of the 19 studies (11 of 21 articles) found significant associations between the duration of any human milk consumption and overweight and/or obesity. The significant associations were inconsistent in direction:

- Five of the 9 studies found significant inverse associations between the duration of human milk consumption and overweight/obesity at 2 years of age, ${ }^{28} 3$ years of age, ${ }^{42} 3$ to 8 years of age, ${ }^{37} 4$ to 14 years of age,${ }^{6}$ and 9 to 14 years of age. ${ }^{12}$ Four of these studies were from the United States $6,12,28,42$ and 1 was from the Netherlands. ${ }^{37}$

- Three of the 9 studies found significant positive associations between the duration of human

\footnotetext{
iv A detailed description of the methodology used for grading the strength of the evidence is available on the NESR website: https://nesr.usda.gov/2020-dietary-guidelines-advisory-committee-systematic-reviews and in Part C of the following reference: Dietary Guidelines Advisory Committee. 2020. Scientific Report of the 2020 Dietary Guidelines Advisory Committee: Advisory Report to the Secretary of Agriculture and the Secretary of Health and Human Services. U.S. Department of Agriculture, Agricultural Research Service, Washington, DC.
} 
milk consumption and overweight/obesity at 4 and 13 years of age, ${ }^{30} 7$ and 11 years of age, ${ }^{29}$ and 11.5 and 16 years of age ${ }^{24,25}$ All 3 studies were from Europe (Belarus, Denmark, and the U.K. arm of a multi-cohort study).

- The last of the 9 studies, also from Europe (the Netherlands), reported significant associations, but in opposite directions, in different articles. ${ }^{8,9}$ Shorter, compared with longer, durations of human milk consumption were associated with higher risk of overweight/obesity at 2 years of age and lower risk of overweight/obesity at 6 years of age.

The remaining 10 studies found no significant associations between the duration of any human milk consumption and overweight and/or obesity at ages between 2 and 62 years of age. $4,10,13,15,18,23,26,33,34,38$

Five studies were of particular interest because they used analyses intended to help address bias from confounding, which may pervade infant-feeding research (see Risk of Bias, below). One study was a cluster randomized trial to promote prolonged duration and exclusivity of human milk feeding, which resulted in an intervention group with significantly higher rates of any human milk consumption measured at 3, 6, 9, and 12 months of age. Study authors, therefore, were able to conduct intention-to-treat analyses of the intervention versus control group to examine the effect of the intervention on overweight/obesity and obesity. ${ }^{24,25}$ There were also 4 observational studies that conducted within-family analyses of siblings fed differently in infancy, reducing confounding from genetic and environmental factors that siblings share. $6,10,12,34$ The evidence from these 5 studies was also inconsistent:

- The intention-to-treat analyses found that the intervention group had a higher risk of overweight and/or obesity at 11.5 and 16 years of age. ${ }^{24,25}$

- None of the within-family analyses of siblings found significant associations between the duration of any human milk consumption and risk of overweight and/or obesity; point estimates were at or close to the null.

- We also examined BMI in 2 studies that had both full-sample and within-family analyses. ${ }^{6,10}$ One study did not find significant associations between the duration of human milk consumption and BMI at 10 to 18 years of age in either analysis. ${ }^{10}$ One study found a significant association between the duration of human milk consumption and lower BMI at 4 to 14 years of age in the full-sample analysis that was nonsignificant and in the opposite direction in the within-family analysis. ${ }^{6}$ One study assessed percent body fat, and found a significant association between consuming human milk for $<2$ months, compared with 5-7 months, and higher percent body fat at 62 years of age; however, there was not a full-sample analysis with which to compare the within-family analysis.

In summary, evidence for an association between the duration of any human milk consumption, among infants fed human milk, and overweight and/or obesity was inconsistent. One trend in the evidence emerged, which may help explain some of the inconsistencies in the findings between the studies. The direction of the statistically significant associations between the duration of human milk consumption and overweight and/or obesity differed somewhat between studies conducted in the United States and studies conducted in Europe. Four of the 5 studies reporting significant inverse associations between duration and overweight/obesity were from the United States, $6,12,28,42$ and all 3 studies reporting significant positive associations were from Europe. ${ }^{24,25,29,30}$ It is possible that the association between the duration of any human milk consumption and overweight and/or obesity differs between the United States and other 
populations (see Generalizability, below).

\section{Precision:}

Nine of the 19 studies in this body of evidence had sufficient statistical power to detect significant associations. Of the 10 remaining studies, only 2 had wide confidence intervals suggestive of suboptimal statistical power ${ }^{34,38}$ The remaining studies with nonsignificant findings were likely to have sufficient statistical power; in several cases they examined the duration of any human milk consumption as a continuous variable using a sizeable analytic sample $(\mathrm{N}=231$ to 7417 ), and in other cases (i.e., when using categorical variables for duration of human milk consumption) the lack of significance was not attributable to a wide confidence interval but rather to the fact that the point estimates were close to the null. $4,10,13,18,23,26,33$

\section{Directness:}

Most of the articles reported study objectives that included the examination of human milk consumption and overweight and/or obesity.

\section{Generalizability:}

Seven of the 19 studies were conducted in the United States. ${ }^{6,10,12,15,18,28,42}$ The rest were from European countries, and there are some concerns about the generalizability of this evidence to U.S. populations, which may have higher risk of overweight and obesity than the populations from which participants were sampled in the European studies. For example, the authors of the cluster randomized trial in Belarus noted the much lower prevalence of obesity (i.e., about $5 \%$ of the sample at 16 years of age) in comparison to youth in the United States..$^{24,25}$

Another limitation to the generalizability of the evidence is that the majority of evidence examined outcomes in childhood. Only 6 studies examined outcomes in adolescents, ${ }^{6,10,12,18,24,30}$ and only 1 study examined outcomes in adults. ${ }^{34}$

\section{Risk of bias:}

Infant-feeding decisions can be strongly socially patterned, and in this largely observational body of evidence, 5 studies used approaches that were likely to reduce bias from confounding. First, there was 1 cluster randomized trial, and randomization mitigates confounding. ${ }^{24,25} \mathrm{Next}$, there were 4 observational studies that conducted within-family analyses of siblings fed differently in infancy. ${ }^{6,10,12,34}$ Such analyses reduce confounding from genetic and environmental factors, which siblings share; however, these studies had other risks of bias. For example, in 2 studies, mothers were asked to recall infant-feeding data when participants were between 10 and 18 years of age..$^{10,12}$ In addition, in 2 studies, all participants reported their own height and weight, ${ }^{12,34}$ in a $3^{\text {rd }}$ study, some participants reported their own height and weight, ${ }^{6}$ and in the $4^{\text {th }}$ study, the method of anthropometric data collection was not reported. ${ }^{10}$

None of the remaining 14 studies controlled for all of the key confounders identified on the analytic framework, and the studies had other risks of bias, which varied between studies (Tables 7 and 8).

\section{Publication bias:}


In addition to the graded elements of the assessment of the evidence, above, it is important to give consideration to publication bias. There is not a strong indication of publication bias because there are several published studies and they present evidence of associations that are both significant and nonsignificant, and inconsistent in direction.

\section{Conclusion:}

Given this synthesis and assessment of the evidence, we conclude that insufficient evidence is available to determine the relationship between the duration of any human milk consumption among infants fed human milk and overweight and obesity at 2 years of age and older, because the available evidence is inconsistent. 
Table 6. Evidence examining the relationship between the duration of any human milk consumption, among infants fed human milk, and overweight and/or obesity at 2 years of age and older ${ }^{a}$

\section{Article}

Study design (Study Duration of any human milk

name)

exposure $^{b}$

Outcome $^{c}$

Significant findings

Nonsignificant findings

(1)

Outcomes from 2 to 5 years

Colen $2014^{6} \quad$ BF duration (wk)

PC (Children of NLSY79)

U.S

Note: this analysis is across 3 age

groups

\begin{tabular}{ll} 
In the full sample $(\mathrm{N}=8,237)$ & $-0.007 \pm 0.002 ; \mathrm{p}<0.01$ \\
\hline In the subsample of siblings $(\mathrm{N}=7,319)$ & $-0.006 \pm 0.002 ; \mathrm{p}<0.05$
\end{tabular}

In the subsample of siblings with

discordant exposures $(\mathrm{N}=1,773)$

$0.001 \pm 0.004 ;$ NS

\begin{tabular}{lr}
\hline $\begin{array}{l}\text { Durmus } 2011^{9} \\
\text { PC (Generation R) }\end{array}$ & OWOB 0-3 mo vs BF $>6$ mo ( ref) \\
Netherlands & @ 2 y (Total analytic N=5074) \\
\hline
\end{tabular}

@ 3 y (Total analytic $N=5074)$

$\sim 0.9[\sim 0.8, \sim 2]$

BF 3-6 mo vs BF >6 mo (ref) OWOB§ (OR $[95 \% \mathrm{Cl}])$

$\sim 2[\sim 1, \sim 3] ; p<0.05$

@ 2 y (Total analytic N=5074)

@ 3 y (Total analytic $\mathrm{N}=5074$ )

$\sim 0.8[\sim 0.7, \sim 2]$

Gubbels $2011^{13}$

PC (KOALA)

OWOB§ (OR [95\% Cl])

$1.01[0.97,1.05]$

Netherlands

@2 y $(\mathrm{N}=1592)$

@ 4 y $(\mathrm{N}=1733)$

$1.00[0.96,1.05]$

Jiang $2013^{18}$

RC (CDS)

U.S.

BF duration (mo)

$\mathrm{OB}^{*}$ (effect $\left.\pm \mathrm{SE}\right) @$ 5-18 y $(\mathrm{N}=1512)$

NS; data NR

Note: this analysis is across 3 age

groups

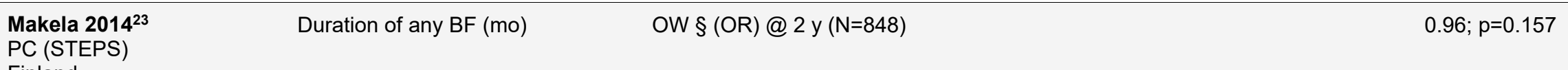

Finland 


\section{Article}

Study design (Study

Duration of any human milk

name)

exposure

Outcome

Significant findings

Nonsignificant findings

Country

\begin{tabular}{|c|c|c|c|c|}
\hline & & OB $\S(\mathrm{OR}) @ 2$ y $(\mathrm{N}=848)$ & & $0.93 ; p=0.141$ \\
\hline \multirow[t]{2}{*}{$\begin{array}{l}\text { Modrek } 2017^{28} \\
\text { RC (Oregon PRAMS) } \\
\text { U.S. }\end{array}$} & BF duration (wk) & $\begin{array}{l}\text { OW } \ddagger @ 2 \text { y instrumental variable } \\
\text { estimate [weighted SE clustered at the } \\
\text { county level] }(N=447)\end{array}$ & & $-0.008[-0.024,0.004] ; p<0.1$ \\
\hline & & $\begin{array}{l}\mathrm{OB} \mp @ 2 \text { y instrumental variable } \\
\text { estimate [weighted SE clustered at the } \\
\text { county level] }(\mathrm{N}=441)\end{array}$ & $-0.0082[-0.018,-0.001] ; p<0.05$ & \\
\hline \multirow{13}{*}{$\begin{array}{l}\text { Moschonis } \mathbf{2 0 1 7 ^ { 3 0 }} \\
\text { RC (ALSPAC, EDEN, } \\
\text { EuroPrevall, Generation } \\
\text { XXI) } \\
\text { U.K., France, Greece, } \\
\text { Portugal }\end{array}$} & $\mathrm{BF}<1 \mathrm{mo}$ vs $\mathrm{BF} \geq 6$ mo (ref) & $\begin{array}{l}\text { OWOB †‡(OR) } \\
\qquad \begin{array}{l}\text { @ } \\
\text { in ALSPAC cohort }(\mathrm{N}=6522)\end{array}\end{array}$ & & $\sim 1.0 ; \mathrm{NS}$ \\
\hline & & in EuroPrevall cohort $(\mathrm{N}=309)$ & & $\sim 0.6$; NS \\
\hline & & @ 5 y in EDEN cohort $(\mathrm{N}=1070)$ & & $\sim 0.8 ; \mathrm{NS}$ \\
\hline & BF $1-3$ mo vs $B F \geq 6$ mo (ref) & OWOB †‡ (OR) & & \\
\hline & & @ 4 y & & \\
\hline & & in ALSPAC cohort $(\mathrm{N}=6522)$ & & $\sim 0.9 ; \mathrm{NS}$ \\
\hline & & in EuroPrevall cohort $(\mathrm{N}=309)$ & & $\sim 0.7 ; \mathrm{NS}$ \\
\hline & & in Generation XXI cohort $(\mathrm{N}=3387)$ & & $\sim 1.0 ;$ NS \\
\hline & & @ 5 y in EDEN cohort $(\mathrm{N}=1070)$ & & $\sim 0.8$; NS \\
\hline & BF $3-6$ mo vs $B F \geq 6$ mo (ref) & OWOB †‡ (OR) & & \\
\hline & & @ 4 y & & \\
\hline & & in ALSPAC cohort $(\mathrm{N}=6522)$ & $\sim 0.7 ; p=0.008$ & \\
\hline & & in Generation XXI cohort $(\mathrm{N}=3387)$ & & $\sim 1.1 ; \mathrm{NS}$ \\
\hline
\end{tabular}




\section{Article}

Study design (Study

Duration of any human milk

exposure $^{b}$

Outcome $^{c}$

\begin{tabular}{|c|c|c|c|}
\hline & & @ 5 y in EDEN cohort $(\mathrm{N}=1070)$ & $\sim 0.6 ; \mathrm{NS}$ \\
\hline \multirow{3}{*}{$\begin{array}{l}\text { Ruijsbroek 201137 } \\
\text { PC (PIAMA) } \\
\text { Netherlands }\end{array}$} & \multirow[t]{3}{*}{ BF 1-16 wk vs BF >16 wk (ref) } & $\begin{array}{l}\text { OWOB } \ddagger(\text { OR }[95 \% \mathrm{Cl}]) \text { in childhood }(3-8 \\
\text { y) }\end{array}$ & \multirow[t]{3}{*}{$1.25[0.98,1.59]$} \\
\hline & & (Total analytic $\mathrm{N}=3963$ ) & \\
\hline & & $\begin{array}{l}\text { Note: this analysis is across } 2 \text { age } \\
\text { groups }\end{array}$ & \\
\hline
\end{tabular}

OB $\ddagger(\mathrm{OR}[95 \% \mathrm{Cl}])$ in childhood (3-8 y) 1.81 [1.07, 3.04]

(Total analytic $\mathrm{N}=3963$ )

Note: this analysis is across 2 age

groups

$\begin{array}{lrr}\text { Wojcicki } 2015^{42} & \text { BF duration }(\mathrm{mo}) & \mathrm{OB}^{\mathrm{d}}(\mathrm{OR}[95 \% \mathrm{Cl}]) @ 3 \text { y } \\ \text { RC (Alaska PRAMS) } & \text { In Alaska non-Native children }(\mathrm{N}=474) & 0.95[0.91,0.995] ; p=0.03 \\ \text { U.S. } & \end{array}$

In Alaska Native children $(\mathrm{N}=226)$

$0.99[0.96,1.02] ; p=0.44$

\section{Outcomes from 6 to 11 years}

\begin{tabular}{|c|c|c|c|c|}
\hline $\begin{array}{l}\text { Bjertnaes } 2019^{4} \\
\text { PC } \\
\text { Norway }\end{array}$ & Duration of partial BF (mo) & $\begin{array}{l}\text { OWOB } ¥ \text { (OR [95\% Cl] @ } 8 \text { y } \\
(\mathrm{N} \sim 181 \text { OWOB, } 770 \text { Not OWOB) }\end{array}$ & & $1.02[0.99,1.06] ; p=0.25$ \\
\hline \multirow{5}{*}{$\begin{array}{l}\text { Colen } 2014^{6} \\
\text { PC (Children of NLSY79) } \\
\text { U.S. }\end{array}$} & BF duration (wk) & $\mathrm{OB}^{*}(\beta \pm \mathrm{SE}) @ 4-14$ y & & \\
\hline & & $\begin{array}{l}\text { Note: this analysis is across } 3 \text { age } \\
\text { groups }\end{array}$ & & \\
\hline & & In the full sample $(\mathrm{N}=8,237)$ & $-0.007 \pm 0.002 ; p<0.01$ & \\
\hline & & In the subsample of siblings $(\mathrm{N}=7,319)$ & $-0.006 \pm 0.002 ; p<0.05$ & \\
\hline & & $\begin{array}{l}\text { In the subsample of siblings with } \\
\text { discordant exposures }(\mathrm{N}=1,773)\end{array}$ & & $0.001 \pm 0.004 ; \mathrm{NS}$ \\
\hline $\begin{array}{l}\text { Durmus } \mathbf{2 0 1 4}^{\mathbf{8}} \\
\text { PC (Generation R) } \\
\text { Netherlands }\end{array}$ & $\begin{array}{l}\text { BF duration trend (categories }>0 \text { - } \\
1.9,2-3.9,4-5.9, \geq 6 \mathrm{mo} \text { ) }\end{array}$ & OWOB $\ddagger($ OR $[95 \%$ Cl] $) @ 6$ y $(N=5063)$ & $p=0.02$ & \\
\hline
\end{tabular}




\begin{tabular}{|c|c|c|c|c|}
\hline $\begin{array}{l}\text { Article } \\
\text { Study design (Study } \\
\text { name) } \\
\text { Country }\end{array}$ & $\begin{array}{l}\text { Duration of any human milk } \\
\text { exposure }^{\text {b }}\end{array}$ & Outcome $^{c}$ & Significant findings & Nonsignificant findings \\
\hline & $\mathrm{BF}>0-1.9 \mathrm{mo}$ vs $\mathrm{BF} \geq 6 \mathrm{mo}$ (ref) & $\begin{array}{l}\text { OWOB } \ddagger(\text { OR }[95 \% \mathrm{Cl}]) @ 6 \text { y }(\mathrm{N}=1000 \\
1258)\end{array}$ & $\begin{array}{l}\sim 0.7[\sim 0.6, \sim 1.0] \text { (error bar just } \\
\text { below } 1.0)\end{array}$ & \\
\hline & BF 2-3.9 mo vs $B F \geq 6$ mo (ref) & $\begin{array}{l}\text { OWOB } \neq(\mathrm{OR}[95 \% \mathrm{Cl}]) @ 6 \text { y }(\mathrm{N}=836 \\
1258)\end{array}$ & & $\sim 0.8[\sim 0.7, \sim 1.1]$ \\
\hline & BF $4-5.9$ mo vs $B F \geq 6$ mo (ref) & $\begin{array}{l}\text { OWOB } ¥ \text { (OR }[95 \% \mathrm{Cl}]) @ 6 \text { y }(\mathrm{N}=479 \\
\text { 1258) }\end{array}$ & & $\sim 1.0[\sim 0.8, \sim 1.3]$ \\
\hline \multirow[t]{9}{*}{$\begin{array}{l}\text { Evenhouse } 2005^{10} \\
\text { CS (Add Health) } \\
\text { U.S. }\end{array}$} & \multirow[t]{2}{*}{$\begin{array}{l}\text { Duration (mo) } \\
\text { (quasi-continuous using midpoint } \\
\text { of ranges 0-3, 3-6, 6-9, 9-12, 12- } \\
24,>24 \mathrm{mo} \text { ) }\end{array}$} & \multirow{2}{*}{\multicolumn{2}{|c|}{$\begin{array}{l}\text { OWOB* (OR } \pm \text { SE from logit) @ } 10-18 \text { y } \\
\text { Note: this analysis is across } 2 \text { age } \\
\text { groups } \\
\quad \text { In the full sample }(\mathrm{N}=7417)\end{array}$}} & \\
\hline & & & & $0.98 \pm 0.00 ; p<0.10$ \\
\hline & & \multicolumn{2}{|l|}{ In the subsample of siblings $(\mathrm{N}=1607)$} & $0.98 \pm 0.01 ; p<0.10$ \\
\hline & & \multicolumn{2}{|l|}{$\begin{array}{l}\text { In the subsample of siblings with } \\
\text { discordant exposures }(\mathrm{N}=470)\end{array}$} & $1.01 \pm 0.01 ; \mathrm{NS}$ \\
\hline & & \multicolumn{2}{|l|}{$\mathrm{OB}^{*}(\mathrm{OR} \pm \mathrm{SE}$ from logit) @ 10-18 y } & \\
\hline & & \multicolumn{2}{|l|}{$\begin{array}{l}\text { Note: this analysis is across } 2 \text { age } \\
\text { groups }\end{array}$} & $098+001 \cdot n<010$ \\
\hline & & \multicolumn{2}{|l|}{ In the full sample $(\mathrm{N}=7417)$} & \\
\hline & & \multicolumn{2}{|l|}{ In the subsample of siblings $(\mathrm{N}=1607)$} & $0.99 \pm 0.01 ; \mathrm{NS}$ \\
\hline & & \multicolumn{2}{|l|}{$\begin{array}{l}\text { In the subsample of siblings with } \\
\text { discordant exposures }(\mathrm{N}=470)\end{array}$} & $1.00 \pm 0.02 ; \mathrm{NS}$ \\
\hline \multirow{3}{*}{$\begin{array}{l}\text { Gillman } 2006^{12} \\
\text { CS (GUTS) } \\
\text { U.S. }\end{array}$} & $\begin{array}{l}\text { BF duration (per } 3.7 \text { mo, which } \\
\text { was the mean difference in BF } \\
\text { duration for discordant siblings) }\end{array}$ & $\begin{array}{l}\text { OWOB* }^{*}(\mathrm{OR}[95 \% \mathrm{CI}]) @ 9-14 \mathrm{y} \text { in the } \\
\text { sample of siblings }(\mathrm{N}=5614)\end{array}$ & $\begin{array}{l}0.88[0.82,0.94] \\
\text { (Adjusted for same confounders } \\
\text { as discordant sibling analysis) }\end{array}$ & $\begin{array}{l}0.94[0.88,1.00] \\
\text { (Also adjusted for maternal } \\
\text { BMI and smoking, household } \\
\text { income) }\end{array}$ \\
\hline & \multirow[t]{2}{*}{$\begin{array}{l}\text { BF longer than the mean BF } \\
\text { duration of his/her participating } \\
\text { sibship vs shorter }\end{array}$} & $\begin{array}{l}\text { OWOB* }(\mathrm{OR}[95 \% \mathrm{CI}]) @ 9-14 \text { y in the } \\
\text { subsample of siblings with discordant } \\
\text { exposures }(\mathrm{N}=2372)\end{array}$ & & \multirow[t]{2}{*}{$0.92[0.76,1.11]$} \\
\hline & & $\begin{array}{l}\text { Note: this analysis is across } 2 \text { age } \\
\text { groups }\end{array}$ & & \\
\hline
\end{tabular}




\section{Article}

Study design (Study

name)

Duration of any human milk

exposure $^{b}$

Outcome $^{c}$

Heerman $2019^{15}$

PC (GROW)

U.S.

\section{Jiang $2013^{18}$}

$\mathrm{RC}$ (CDS)

U.S.

\section{Martin 201325 \\ $\mathrm{RCT}^{\mathrm{e}}$ (PROBIT)}

Belarus

(a)

\section{Massion $2016^{26}$}

PC (MCS)

U.K.

\section{Morgen 2018 29}

PC (DNBC)

Denmark

$\begin{aligned} \text { BF any to } 5 \text { mo vs } B F \geq 6 \text { mo (ref) } & \mathrm{OB}^{*}(\mathrm{OR}[95 \% \mathrm{Cl}]) @ \sim 7 \text { y } \\ & (\mathrm{N}=128 \leq 5 / \mathrm{No} \mathrm{OB}, 60 \leq 5 / \mathrm{OB}, 167 \\ & \geq 6 / \mathrm{No} \mathrm{OB}, 98 \geq 6 / \mathrm{OB})\end{aligned}$

$\mathrm{OB}^{*}$ (effect $\left.\pm \mathrm{SE}\right) @$ 5-18 y $(\mathrm{N}=1512)$

NS; data NR

Note: this analysis is across 3 age

groups

Intervention (higher rates of any
BF measured @ $3,6,9$, and 12

OWOB* (OR [95\% Cl]) @ 11.5 y

$1.18[1.01,1.39]$

mo) vs Control

$\mathrm{OB}^{*}(\mathrm{OR}[95 \% \mathrm{CI}]) @ 11.5 \mathrm{y} \quad 1.17[0.97,1.41]$

Any BF <4 mo vs Any BF >4 mo OWOB † (RR [95\% Cl]) @ 11 y $~ \sim 1.1[\sim 0.9, \sim 1.2]$

$(\mathrm{N}=11,764)$

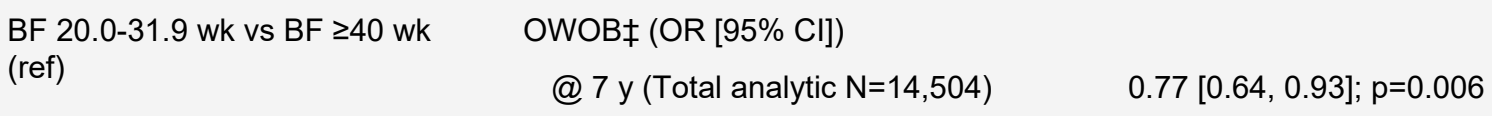

@ 11 y (Total analytic $N=8571) \quad 0.58$ [0.43, 0.78]; $p<0.0001$

\begin{tabular}{|c|c|c|c|}
\hline & $\begin{array}{l}\text { BF } 32.0-39.9 w k \text { vs } B F \geq 40 w k \\
\text { (ref) }\end{array}$ & $\begin{array}{l}\text { OWOB } \ddagger \text { (OR }[95 \% \mathrm{Cl}]) \\
\text { @ } 7 \text { y (Total analytic } \mathrm{N}=14,504)\end{array}$ & $0.86[0.73,1.01] ; p=0.7$ \\
\hline & & @ 11 y (Total analytic $\mathrm{N}=8571$ ) & $0.82[0.64,1.04] ; p=0.1$ \\
\hline \multirow{3}{*}{$\begin{array}{l}\text { Moschonis } \mathbf{2 0 1 7} \mathbf{7}^{30} \\
\text { RC (ALSPAC, EDEN, } \\
\text { EuroPrevall, Generation } \\
\text { XXI) } \\
\text { U.K., France, Greece, } \\
\text { Portugal }\end{array}$} & $\mathrm{BF}<1 \mathrm{mo}$ vs $\mathrm{BF} \geq 6 \mathrm{mo}$ (ref) & $\begin{array}{l}\text { OWOB †‡(OR) @ } 9 \text { y in ALSPAC } \\
\text { cohort }(N=7540)\end{array}$ & $\sim 1.1 ; \mathrm{NS}$ \\
\hline & BF $1-3$ mo vs $B F \geq 6$ mo (ref) & $\begin{array}{l}\text { OWOB †‡ (OR) @ } 9 \text { y in ALSPAC } \\
\text { cohort }(N=7540)\end{array}$ & $\sim 1.1 ; \mathrm{NS}$ \\
\hline & BF $3-6$ mo vs $B F \geq 6$ mo (ref) & $\begin{array}{l}\text { OWOB †‡(OR) @ } 9 \text { y in ALSPAC } \\
\text { cohort }(N=7540)\end{array}$ & $\sim 1.1 ; \mathrm{NS}$ \\
\hline
\end{tabular}

RC (ALSPAC, EDEN,

EuroPrevall, Generation

, France, Greece,

Portugal 


\section{Article}

Study design (Study Duration of any human milk

name)

exposure $^{b}$

Outcome $^{\mathrm{c}}$

Significant findings

Nonsignificant findings

Ortega-Garcia $2018^{33}$

Duration of any BF (wk)

OWOB† ( $\beta[95 \% \mathrm{Cl}]) @ 6 \mathrm{y}(\mathrm{N}=231)$

$0.015[-0.003,0.032] ; p=0.11$

PC (MALAMA)

Spain

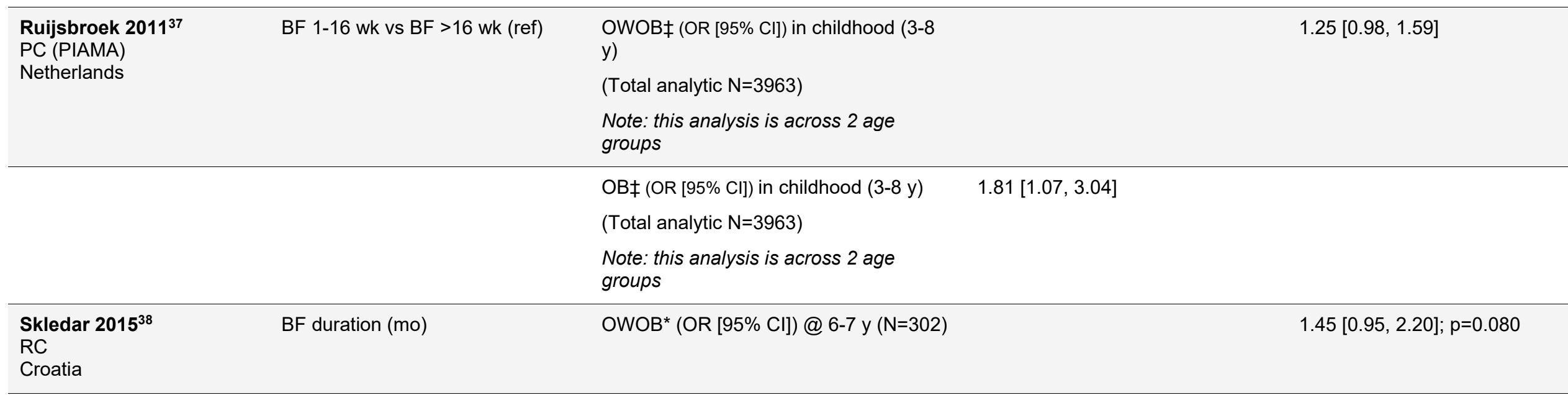

\section{Outcomes from 12 to 19 years}

\begin{tabular}{|c|c|c|c|c|}
\hline \multirow{5}{*}{$\begin{array}{l}\text { Colen } 2014^{6} \\
\text { PC (Children of NLSY79) } \\
\text { U.S. }\end{array}$} & \multirow[t]{5}{*}{ BF duration (wk) } & $\mathrm{OB}^{*}(\beta \pm \mathrm{SE}) @ 4-14$ y & \multirow[b]{3}{*}{$-0.007 \pm 0.002 ; p<0.01$} & \\
\hline & & $\begin{array}{l}\text { Note: this analysis is across } 3 \text { age } \\
\text { groups }\end{array}$ & & \\
\hline & & In the full sample $(\mathrm{N}=8,237)$ & & \\
\hline & & In the subsample of siblings $(\mathrm{N}=7,319)$ & $-0.006 \pm 0.002 ; p<0.05$ & \\
\hline & & $\begin{array}{l}\text { In the subsample of siblings with } \\
\text { discordant exposures }(\mathrm{N}=1,773)\end{array}$ & & $0.001 \pm 0.004 ; \mathrm{NS}$ \\
\hline \multirow{4}{*}{$\begin{array}{l}\text { Evenhouse } 2005^{10} \\
\text { CS (Add Health) } \\
\text { U.S. }\end{array}$} & \multirow{3}{*}{$\begin{array}{l}\text { Duration (mo) } \\
\text { (quasi-continuous using midpoint } \\
\text { of ranges } 0-3,3-6,6-9,9-12,12- \\
24,>24 \mathrm{mo} \text { ) }\end{array}$} & 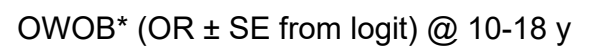 & & \\
\hline & & $\begin{array}{l}\text { Note: this analysis is across } 2 \text { age } \\
\text { groups }\end{array}$ & & \\
\hline & & In the full sample $(\mathrm{N}=7417)$ & & $0.98 \pm 0.00 ; p<0.10$ \\
\hline & & In the subsample of siblings $(\mathrm{N}=1607)$ & & $0.98 \pm 0.01 ; p<0.10$ \\
\hline
\end{tabular}




\section{Article}

Study design (Study

name)

Duration of any human milk

exposure $^{b}$

Outcome $^{c}$

Significant findings

Nonsignificant findings

Country

In the subsample of siblings with

$1.01 \pm 0.01 ;$ NS

discordant exposures $(\mathrm{N}=470)$

$\mathrm{OB}^{*}$ (OR \pm SE from logit) @ 10-18 y

Note: this analysis is across 2 age

groups

$0.98 \pm 0.01 ; p<0.10$

In the full sample $(\mathrm{N}=7417)$

In the subsample of siblings $(\mathrm{N}=1607)$

$0.99 \pm 0.01 ;$ NS

In the subsample of siblings with

discordant exposures $(\mathrm{N}=470)$

$1.00 \pm 0.02 ; N S$

\section{Gillman 2006 12}

CS (GUTS)

U.S.

BF duration (per 3.7 mo, which was the mean difference in BF duration for discordant siblings) BF longer than the mean BF
duration of his/her participating sibship vs shorter

Jiang 2013
RC (CDS)

U.S.

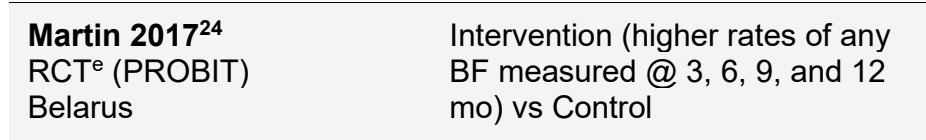

rar

OWOB* (OR [95\% Cl]) @ 9-14 y in the sample of siblings $(\mathrm{N}=5614)$

Note: this analysis is across 2 age groups
OWOB* (OR [95\% Cl]) @ 9-14 y in the

subsample of siblings with discordant

exposures $(\mathrm{N}=2372)$

Note: this analysis is across 2 age

groups

\section{$0.88[0.82,0.94]$ \\ (Adjusted for same confounders \\ as discordant sibling analysis)}

$0.94[0.88,1.00]$

Also adjusted for maternal

BMI and smoking, household ncome)

\section{$0.92[0.76,1.11]$}

$\mathrm{OB}^{*}($ effect $\pm \mathrm{SE}) @$ 5-18 y $(\mathrm{N}=1512)$

Note: this analysis is across 3 age

groups
OWOB* $^{*}$ OR [95\% CI]) @ 16 y 1.14 [1.02-1.28]; p=0.03

$(\mathrm{N}=7064,6493)$
$\mathrm{OB}^{*}(\mathrm{OR}[95 \% \mathrm{Cl}]) @ 16 \mathrm{y}$

$(\mathrm{N}=7064,6493)$

\begin{tabular}{|c|c|c|c|}
\hline $\begin{array}{l}\text { Moschonis } 2017^{30} \\
\text { RC (ALSPAC, EDEN, }\end{array}$ & $\mathrm{BF}<1 \mathrm{mo}$ vs $\mathrm{BF} \geq 6 \mathrm{mo}$ (ref) & $\begin{array}{l}\text { OWOB † (OR) @ } 13 \text { y in ALSPAC } \\
\text { cohort }(N=6124)\end{array}$ & $\sim 1.3 ; \mathrm{NS}$ \\
\hline
\end{tabular}

NS; data NR

EuroPrevall,

$\mathrm{XXI})$

U.K., France, Greece,

Portugal 


\section{Article}

Study design (Study

name)

Duration of any human milk

exposure $^{b}$

Country

BF 1-3 mo vs $B F \geq 6$ mo (ref)

OWOB †‡ (OR) @ 13 y in ALSPAC

$\sim 1.0 ; \mathrm{NS}$ cohort $(\mathrm{N}=6124)$

BF $3-6$ mo vs $B F \geq 6$ mo (ref)

OWOB †‡(OR) @ 13 y in ALSPAC

cohort $(\mathrm{N}=6124)$

\section{Outcomes at 20 years and older}

\begin{tabular}{|c|c|c|}
\hline $\begin{array}{l}\text { O'Tierney } 2009^{34} \\
\text { PC (Helsinki Birth Cohort) } \\
\text { Finland }\end{array}$ & $\begin{array}{l}\text { Trend across the BF duration } \\
\text { categories }<2,3-4,5-7, \geq 8 \text { mo }\end{array}$ & $\begin{array}{l}\text { OW }(\% \text { BMI } 25-30 \mathrm{~kg} / \mathrm{m} 2) \text { from self- } \\
\text { reported height and weight at } \sim 62 \text { y in } \\
\text { sample of siblings with discordant } \\
\text { exposures }(\mathrm{N}=206,168,233,224)\end{array}$ \\
\hline
\end{tabular}

42.7 vs 42.9 vs 41.2 vs 50.0

exposures $(\mathrm{N}=206,168,233,224)$

$p=0.08$ (linear), 0.50

(quadratic)

BF $<2$ mo vs BF 5-7 mo (ref)

OW (difference, \% BMI 25-30 kg/m2)

from self-reported height and weight at

$1.6[-7.7,11.0]$

$\sim 62 \mathrm{y}$ in a sample of siblings with

discordant exposures $(\mathrm{N}=206,233)$

BF 3-4 mo vs BF 5-7 mo (ref)

OW (difference, \% BMI 25-30 kg/m2)

from self-reported height and weight at

$\sim 62 \mathrm{y}$ in a sample of siblings with

discordant exposures $(\mathrm{N}=168,233)$

$\mathrm{BF} \geq 8 \mathrm{mo}$ vs BF $5-7 \mathrm{mo}$ (ref)

OW (difference, \% BMI 25-30 kg/m2)

from self-reported height and weight at

62 y in a sample of siblings with

discordant exposures $(\mathrm{N}=224,233)$

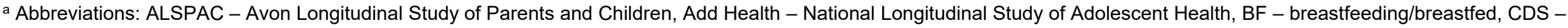

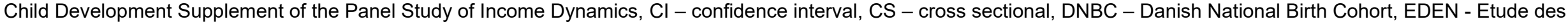

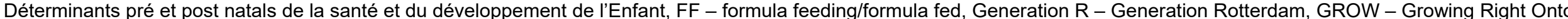

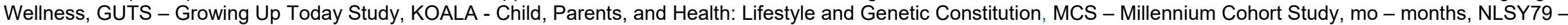

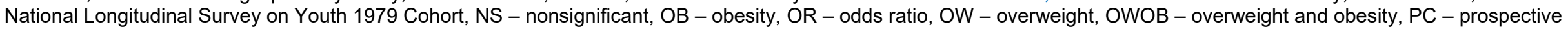

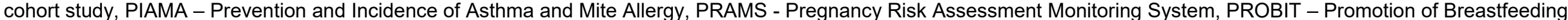

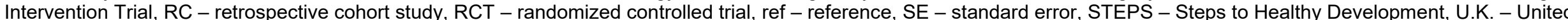

Kingdom, U.S. - United States of America, wk - weeks, y - years

${ }^{b}$ Exposures, from the articles included in the body of evidence, which address the duration of any human milk consumption among infants fed human milk

${ }^{c}$ Notations used for growth references: ${ }^{*} \mathrm{CDC},+\mathrm{WHO}, \ddagger \mathrm{IOTF}, \S$ Country-specific

d This study did not report the growth reference used

e RCT of an intervention to promote prolonged duration and exclusivity of breastfeeding rather than an RCT of breastfeeding, per se 
Table 7. Risk of bias for the randomized controlled trial examining the relationship between the duration of any human milk consumption among infants fed human milk and overweight and obesity ${ }^{\text {ab }}$

\begin{tabular}{|c|c|c|c|c|c|c|}
\hline & Randomzation & $\begin{array}{c}\text { Identification of } \\
\text { participants - } \\
\text { randomization }\end{array}$ & $\begin{array}{l}\text { Deviations from } \\
\text { intended interventions }\end{array}$ & Outcome measurement & $\begin{array}{c}\text { Missing } \\
\text { outcome data }\end{array}$ & $\begin{array}{l}\text { Selection of the } \\
\text { reported results }\end{array}$ \\
\hline Martin $2013^{25}$ & Low & Some concerns & Low & Some concerns & Low & Low \\
\hline Martin $2017^{24}$ & Low & Some concerns & Low & Some concerns & Low & Low \\
\hline
\end{tabular}

${ }^{a}$ A detailed description of the methodology used for assessing risk of bias is available on the NESR website: https://nesr.usda.gov/2020-dietary-guidelinesadvisory-committee-systematic-reviews and in Part C of the following reference: Dietary Guidelines Advisory Committee. 2020. Scientific Report of the 2020 Dietary Guidelines Advisory Committee: Advisory Report to the Secretary of Agriculture and the Secretary of Health and Human Services. U.S. Department of Agriculture, Agricultural Research Service, Washington, DC.

b Possible ratings of low, some concerns, or high determined using the "Cochrane Risk-of-bias 2.0" (RoB 2.0) (August 2016 version)" (Higgins JPT, Sterne JAC, Savović J, Page MJ, Hróbjartsson A, Boutron I, Reeves B, Eldridge S. A revised tool for assessing risk of bias in randomized trials In: Chandler J, McKenzie J, Boutron I, Welch V (editors). Cochrane Methods. Cochrane Database of Systematic Reviews 2016, Issue 10 (Suppl 1). dx.doi.org/10.1002/14651858.CD201601.)

Table 8. Risk of bias for observational studies examining the relationship between the duration of any human milk consumption among infants fed human milk and overweight and obesity ${ }^{\text {ab }}$

\begin{tabular}{|c|c|c|c|c|c|c|c|}
\hline & Confounding & $\begin{array}{l}\text { Selection of } \\
\text { participants }\end{array}$ & $\begin{array}{l}\text { Classification of } \\
\text { exposures }\end{array}$ & $\begin{array}{l}\text { Deviations } \\
\text { from intended } \\
\text { exposures }\end{array}$ & $\begin{array}{c}\text { Outcome } \\
\text { measurement }\end{array}$ & Missing data & $\begin{array}{l}\text { Selection of } \\
\text { the reported } \\
\text { results }\end{array}$ \\
\hline Bjertnaes $2019^{4}$ & Serious & Serious & Serious & Low & Serious & Low & Low \\
\hline Colen $2014^{6}$ & Serious & Serious & Serious & Low & Serious & Low & Low \\
\hline Durmus $2014^{8}$ & Serious & Serious & Moderate & Low & Moderate & Moderate & Low \\
\hline Durmus $2011^{9}$ & Serious & Moderate & Moderate & Low & Serious & Moderate & Low \\
\hline $\begin{array}{l}\text { Evenhouse } \\
2005^{10}\end{array}$ & Serious & Serious & Serious & Low & Serious & No information & Low \\
\hline Gillman $2006^{12}$ & Serious & Serious & Serious & Low & Serious & Low & Low \\
\hline Gubbels $2011^{13}$ & Serious & Serious & Moderate & Low & Serious & Serious & Low \\
\hline Heerman $2019^{15}$ & Serious & Low & Moderate & Moderate & Moderate & Low & Low \\
\hline Jiang $2013^{18}$ & Serious & Serious & Serious & Low & Serious & Low & Low \\
\hline Makela $2014^{23}$ & Serious & Serious & Moderate & Low & Moderate & No information & Low \\
\hline
\end{tabular}




\begin{tabular}{|c|c|c|c|c|c|c|c|}
\hline & Confounding & $\begin{array}{l}\text { Selection of } \\
\text { participants }\end{array}$ & $\begin{array}{l}\text { Classification of } \\
\text { exposures }\end{array}$ & $\begin{array}{l}\text { Deviations } \\
\text { from intended } \\
\text { exposures }\end{array}$ & $\begin{array}{c}\text { Outcome } \\
\text { measurement }\end{array}$ & Missing data & $\begin{array}{l}\text { Selection of } \\
\text { the reported } \\
\text { results }\end{array}$ \\
\hline Massion $2016^{26}$ & Serious & Moderate & Moderate & Low & Serious & Serious & Low \\
\hline Modrek $2017^{28}$ & Serious & Moderate & Moderate & Low & Serious & Moderate & Low \\
\hline Morgen $2018^{29}$ & Serious & Serious & Moderate & Low & Serious & Serious & Low \\
\hline $\begin{array}{l}\text { Moschonis } \\
2017^{30}\end{array}$ & Serious & No information & Moderate & Low & Serious & No information & Low \\
\hline $\begin{array}{l}\text { O'Tierney } \\
2009^{34}\end{array}$ & Serious & Serious & Low & Low & Serious & Low & Low \\
\hline $\begin{array}{l}\text { Ortega-Garcia } \\
2018^{33}\end{array}$ & Serious & Low & Moderate & Low & Moderate & Serious & Low \\
\hline $\begin{array}{l}\text { Ruijsbroek } \\
2011^{37}\end{array}$ & Serious & Low & Moderate & Low & Serious & Moderate & Low \\
\hline Skledar, $2015^{38}$ & Serious & Serious & Serious & Low & Moderate & Low & Low \\
\hline Wojcicki, $2015^{42}$ & Serious & Moderate & Serious & Low & Serious & Serious & Low \\
\hline
\end{tabular}

${ }^{a}$ A detailed description of the methodology used for assessing risk of bias is available on the NESR website: https://nesr.usda.gov/2020-dietary-guidelinesadvisory-committee-systematic-reviews and in Part C of the following reference: Dietary Guidelines Advisory Committee. 2020. Scientific Report of the 2020 Dietary Guidelines Advisory Committee: Advisory Report to the Secretary of Agriculture and the Secretary of Health and Human Services. U.S. Department of Agriculture, Agricultural Research Service, Washington, DC.

b Possible ratings of low, moderate, serious, critical, or no information determined using the "Risk of Bias for Nutrition Observational Studies" tool (RoB-NObs) (Dietary Guidelines Advisory Committee. 2020. Scientific Report of the 2020 Dietary Guidelines Advisory Committee: Advisory Report to the Secretary of Agriculture and the Secretary of Health and Human Services. U.S. Department of Agriculture, Agricultural Research Service, Washington, DC.) 


\section{Duration of exclusive human milk consumption before the introduction of infant formula}

Two articles, published in 2015 and 2018, were identified that met the inclusion criteria and examined the relationship between the duration of exclusive human milk consumption before the introduction of infant formula and overweight and/or obesity at 2 years of age and older. ${ }^{33,38}$

Ortega-Garcia et $\mathrm{al}^{33}$ presented evidence from the Medio Ambiente y Lactancia Materna (MALAMA) study in Spain. Participants were born full term (i.e., gestational age $>37$ weeks) with a birthweight $>2500 \mathrm{~g}$, had 1- and 5-minute Apgar scores of $\geq 7$ and $\geq 8$, respectively, and had infant-feeding and anthropometric data for the analyses $(\mathrm{N}=231)$. Infant-feeding data were reported prospectively by parents during interviews with trained nurses at birth and at $1,3,6$, and 12 months of infant age. The study examined the number of weeks infants consumed human milk exclusively, before bottle feeding was introduced, as a continuous variable. Weight and height at 6 years of age were measured by pediatricians during well-child primary care visits, and the authors assessed overweight/obesity (i.e., BMI >1 SD based on WHO growth curves) at 6 years of age.

- The authors found a significant inverse association between the number of weeks participants consumed human milk and overweight/obesity at 6 years of age $(\beta=-0.052$ [95\% Cl -0.10, -0.003], $p=0.04)$.

Skledar and Milosevic ${ }^{38}$ conducted a retrospective cohort study of Caucasian children who were in their first year of primary school in Croatia between 2002 and 2006 . All children in the cohort were born full term (i.e., 37 to 42 weeks gestational age), and had complete data on infant feeding, anthropometry, and the confounding variables used in the analyses $(\mathrm{N}=302)$. Infantfeeding data, height, and weight were recorded in medical charts prospectively by primary care providers and obtained retrospectively for the study from a medical chart review. The authors compared infants who consumed human milk without additional formula with infants who consumed human milk with infant formula added to the diet before 3 months of age, and examined the odds of overweight/obesity at 6 to 7 years of age (i.e., BMI $\geq 85^{\text {th }}$ percentile based on CDC growth curves).

- A shorter duration of exclusive human milk consumption prior to the introduction of infant formula (i.e., <3 months), compared with a longer duration (i.e., $\geq 3$ months), was associated with significantly higher odds of overweight/obesity at 6 to 7 years of age (OR $3.35[95 \% \mathrm{Cl}$ 1.31, 8.60], $p=0.012$ ).

The 2 studies presented evidence that was consistent in direction, showing that shorter durations of exclusive human milk consumption, prior to the introduction of infant formula, are associated with higher risk of overweight and obesity at 6 to 7 years of age. However, the evidence from these 2 observational studies is insufficient to determine the relationship between the duration of exclusive human milk consumption prior to the introduction of infant formula and overweight and/or obesity at 2 years of age and older. ${ }^{v}$

\footnotetext{
${ }^{v}$ A detailed description of the methodology used for grading the strength of the evidence is available on the NESR website: https://nesr.usda.gov/2020-dietary-guidelines-advisory-committee-systematic-reviews and in Part C of the following reference: Dietary Guidelines Advisory Committee. 2020. Scientific Report of the 2020 Dietary Guidelines Advisory Committee: Advisory Report to the Secretary of Agriculture and the Secretary of Health and Human Services. U.S. Department of Agriculture, Agricultural Research Service, Washington, DC.
} 
Table 9. Evidence examining the relationship between the duration of exclusive human milk consumption before to the introduction of infant formula and and overweight and/or obesity at 2 years of age and older ${ }^{a}$

\begin{tabular}{|c|c|c|c|c|}
\hline $\begin{array}{l}\text { Article } \\
\text { Study design } \\
\text { (Study name) } \\
\text { Country }\end{array}$ & $\begin{array}{l}\text { Duration of exclusive human milk } \\
\text { exposure }^{\mathrm{b}}\end{array}$ & Outcome $^{c}$ & $\begin{array}{l}\text { Significant } \\
\text { findings }\end{array}$ & $\begin{array}{l}\text { Nonsignificant } \\
\text { findings }\end{array}$ \\
\hline $\begin{array}{l}\text { Ortega-Garcia } \\
\text { 2018 } \\
\text { PC (MALAMA) } \\
\text { Spain }\end{array}$ & $\begin{array}{l}\text { Duration of full BF before bottle feeding } \\
\text { first introduced (wk) }\end{array}$ & $\begin{array}{l}\text { OWOB }(\beta[95 \% \mathrm{Cl}]) \\
@ 6 \mathrm{y} \\
(\mathrm{N}=231)\end{array}$ & $\begin{array}{l}-0.052[-0.10,- \\
0.003] ; p=0.04\end{array}$ & \\
\hline $\begin{array}{l}\text { Skledar } \mathbf{2 0 1 5}^{\mathbf{3 8}} \\
\text { RC } \\
\text { Croatia }\end{array}$ & $\begin{array}{l}\text { Addition of formula }<3 \text { mo vs BF without } \\
\text { additional formula (ref) }\end{array}$ & $\begin{array}{l}\text { OWOB }^{*}(\text { OR }[95 \% \\
\text { Cl]) @ 6-7 y } \\
\text { (Total analytic } \\
N=302)\end{array}$ & $\begin{array}{l}3.35[1.31,8.60] \\
p=0.012\end{array}$ & \\
\hline
\end{tabular}

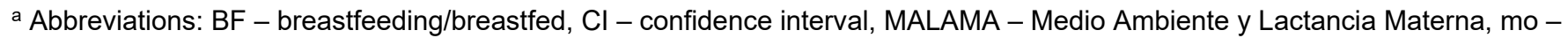
months, OR - odds ratio, OWOB - overweight and obesity, PC - prospective cohort study, RC - retrospective cohort study, ref reference, wk - week(s), y-year(s)

${ }^{b}$ Exposures, from the articles included in the body of evidence, which address the duration of exclusive human milk consumption prior to the introduction of infant formula

${ }^{c}$ Notations used for growth references: ${ }^{*} \mathrm{CDC}$, †WHO, ‡lOTF, §Country-specific
}

\section{Table 10. Risk of bias for observational studies examining the relationship between the duration of exclusive human milk consumption before the introduction of infant formula and overweight and obesity ${ }^{a b}$}

\begin{tabular}{|l|c|c|c|c|c|c|c|}
\hline & Confounding & $\begin{array}{c}\text { Selection of } \\
\text { participants }\end{array}$ & $\begin{array}{c}\text { Classification } \\
\text { of exposures }\end{array}$ & $\begin{array}{c}\text { Deviations } \\
\text { from } \\
\text { intended } \\
\text { exposures }\end{array}$ & $\begin{array}{c}\text { Outcome } \\
\text { measurement }\end{array}$ & $\begin{array}{c}\text { Melection } \\
\text { of the } \\
\text { reported } \\
\text { results }\end{array}$ \\
\hline $\begin{array}{l}\text { Ortega- } \\
\text { Garcia } \\
2018^{33}\end{array}$ & Serious & Low & Moderate & Low & Moderate & Serious & Low \\
\hline $\begin{array}{l}\text { Skledar, } \\
2015^{38}\end{array}$ & Serious & Serious & Serious & Low & Moderate & Low & Low \\
\hline
\end{tabular}

${ }^{a}$ A detailed description of the methodology used for assessing risk of bias is available on the NESR website: https://nesr.usda.gov/2020-dietary-guidelines-advisory-committee-systematic-reviews and in Part C of the following reference: Dietary Guidelines Advisory Committee. 2020. Scientific Report of the 2020 Dietary Guidelines Advisory Committee: Advisory Report to the Secretary of Agriculture and the Secretary of Health and Human Services. U.S. Department of Agriculture, Agricultural Research Service, Washington, DC.

${ }^{b}$ Possible ratings of low, moderate, serious, critical, or no information determined using the "Risk of Bias for Nutrition Observational Studies" tool (RoB-NObs) (Dietary Guidelines Advisory Committee. 2020. Scientific Report of the 2020 Dietary Guidelines Advisory Committee: Advisory Report to the Secretary of Agriculture and the Secretary of Health and Human Services. U.S. Department of Agriculture, Agricultural Research Service, Washington, DC.) 
Intensity, proportion, or amount of human milk consumed by mixed-fed infants

No articles were identified that examined the relationship between the intensity, proportion, or amount of human milk consumed by mixed-fed infants and overweight and/or obesity at 2 years of age and older.

\section{Intensity, proportion, or amount of human milk consumed at the breast versus by bottle}

No articles were identified that examined the relationship between the intensity, proportion, or amount of human milk consumed at the breast versus by bottle and overweight and/or obesity at 2 years of age and older.

Consuming human milk or infant formula (i.e., a single substance) during as single feeding session versus human milk and infant formula (e.g., "topping up")

No articles were identified that examined the relationship between consuming human milk or infant formula (i.e., a single substance) during as single feeding session versus human milk and infant formula (e.g., "topping up") and overweight and/or obesity at 2 years of age and older. 


\section{Research recommendations}

Conduct studies that are designed to reduce bias from confounding factors as much as possible. Sibling-pair studies are one example of this type of study design, but there are few such studies and they tend to have much smaller sample sizes than other types of observational studies.

There is a need for larger sibling-pair studies, and for such studies to examine siblings that differ in terms of the duration of human milk consumption (e.g., $<6 \mathrm{mo}, \geq 6 \mathrm{mo}$ ), not just with respect to ever vs. never consuming human milk.

Another way to approach these questions is with randomized trials of breastfeeding promotion, as was done in the PROBIT trial in Belarus ${ }^{\text {vi }}$. If the trial achieves substantial differences in duration or exclusivity of breastfeeding between intervention groups, this provides an opportunity to examine effects on subsequent overweight or obesity (and many other outcomes).

Observational studies that make use of large datasets, especially those that follow participants longitudinally and in particular, link children with siblings and parents, would also be very useful for more robustly assessing associations and providing more confidence in conclusions regarding causality. This could be achieved by linking surveillance systems that collect data about infant feeding and health outcomes (including overweight and obesity), and making use of emerging electronic medical record data. In general, observational studies need to pay careful attention to minimizing confounding, taking into account all of the key confounders in the analytical framework for this review including aspects of the child's diet (complementary feeding and later dietary patterns). The use of instrumental variables such as Mendelian randomization approaches can also be helpful in minimizing confounding.

In both observational and intervention studies, researchers should consider effect modification in their study design whenever possible (e.g., child sex, parental obesity, socioeconomic status, child diets, child activity levels) to examine the impact of infant feeding on these outcomes within key subgroups.

\footnotetext{
vi Kramer, M. S., Chalmers, B., Hodnett, E. D., Sevkovskaya, Z., Dzikovich, I., Shapiro, S., ... \& Shishko, G. (2001). Promotion of Breastfeeding Intervention Trial (PROBIT): a randomized trial in the Republic of Belarus. JAMA, 285(4), 413-420.
} 


\section{Included articles}

1. Abraham EC, Godwin J, Sherriff A, Armstrong J. Infant feeding in relation to eating patterns in the second year of life and weight status in the fourth year. Public Health Nutr. 2012;15(9):1705-1714.doi: 10.1017/s1368980012002686.

2. Anderson J, Hayes D, Chock L. Characteristics of overweight and obesity at age two and the association with breastfeeding in Hawai'i Women, Infants, and Children (WIC) participants. Matern Child Health J. 2014;18(10):2323-2331.doi: 10.1007/s10995-013-1392-9.

3. Anderson PM, Butcher KF, Levine PB. Maternal employment and overweight children. J Health Econ. 2003;22(3):477-504 .doi: 10.1016/s0167-6296(03)00022-5.

4. Bjertnaes AA, Grundt JH, Donkor HM, et al. No significant associations between breastfeeding practices and overweight in 8-year-old children. Acta Paediatr. 2020;109(1):109-114.doi: 10.1111/apa.14937.

5. Bohr AD, Boardman JD, Domingue BW, McQueen MB. Breastfeeding is associated with waist-to-height ratio in young adults. BMC Public Health. 2015;15:1281.doi: 10.1186/s12889-015-2611-7.

6. Colen CG, Ramey DM. Is breast truly best? Estimating the effects of breastfeeding on long-term child health and wellbeing in the United States using sibling comparisons. Soc Sci Med. 2014;109:55-65.doi: 10.1016/j.socscimed.2014.01.027.

7. de Jong C, Boehm G, Kikkert HK, Hadders-Algra M. The Groningen LCPUFA study: No effect of short-term postnatal long-chain polyunsaturated fatty acids in healthy term infants on cardiovascular and anthropometric development at 9 years. Pediatr Res. 2011;70(4):411-416.doi: 10.1203/PDR.0b013e31822a5ee0.

8. Durmus B, Heppe DH, Gishti O, et al. General and abdominal fat outcomes in school-age children associated with infant breastfeeding patterns. Am J Clin Nutr. 2014;99(6):1351-1358.doi: 10.3945/ajcn.113.075937.

9. Durmus $B$, van Rossem L, Duijts $L$, et al. Breast-feeding and growth in children until the age of 3 years: the Generation R Study. Br J Nutr. 2011;105(11):17041711.doi: $10.1017 / \mathrm{s} 0007114510005374$.

10. Evenhouse E, Reilly S. Improved estimates of the benefits of breastfeeding using sibling comparisons to reduce selection bias. Health Serv Res. 2005;40(6 Pt 1):1781-1802.doi: 10.1111/j.1475-6773.2005.00453.x.

11. Feldman-Winter L, Burnham L, Grossman X, Matlak S, Chen N, Merewood A. Weight gain in the first week of life predicts overweight at 2 years: A prospective cohort study. Matern Child Nutr. 2018;14(1).doi: 10.1111/mcn.12472.

12. Gillman MW, Rifas-Shiman SL, Berkey CS, et al. Breast-feeding and overweight in adolescence: within-family analysis [corrected]. Epidemiology. 2006;17(1):112114.doi: 10.1097/01.ede.0000181629.59452.95.

13. Gubbels JS, Thijs C, Stafleu A, van Buuren S, Kremers SP. Association of breastfeeding and feeding on demand with child weight status up to 4 years. Int J Pediatr Obes. 2011;6(2-2):e515-522.doi: 10.3109/17477166.2010.514343. 
14. Hawkins SS, Baum CF, Rifas-Shiman SL, Oken E, Taveras EM. Examining Associations between Perinatal and Postnatal Risk Factors for Childhood Obesity Using Sibling Comparisons. Child Obes. 2019;15(4):254-261.doi:

10.1089/chi.2018.0335.

15. Heerman WJ, Sommer EC, Slaughter JC, Samuels LR, Martin NC, Barkin SL. Predicting Early Emergence of Childhood Obesity in Underserved Preschoolers. J Pediatr. 2019;213:115-120.doi: 10.1016/j.jpeds.2019.06.031.

16. Heppe DH, Kiefte-de Jong JC, Durmus B, et al. Parental, fetal, and infant risk factors for preschool overweight: the Generation R Study. Pediatr Res. 2013;73(1):120-127.doi: 10.1038/pr.2012.145.

17. Huang DY, Lanza HI, Anglin MD. Trajectory of Adolescent Obesity: Exploring the Impact of Prenatal to Childhood Experiences. J Child Fam Stud. 2014;23(6):10901101.doi: 10.1007/s10826-013-9766-6.

18. Jiang M, Foster EM. Duration of breastfeeding and childhood obesity: a generalized propensity score approach. Health Serv Res. 2013;48(2 Pt 1):628651.doi: 10.1111/j.1475-6773.2012.01456.x.

19. Layte R, Bennett A, McCrory C, Kearney J. Social class variation in the predictors of rapid growth in infancy and obesity at age 3 years. Int $\mathrm{J}$ Obes (Lond). 2014;38(1):82-90.doi: 10.1038/ijo.2013.160.

20. Lee JW, Lee M, Lee J, Kim YJ, Ha E, Kim HS. The Protective Effect of Exclusive Breastfeeding on Overweight/Obesity in Children with High Birth Weight. J Korean Med Sci. 2019;34(10):e85.doi: 10.3346/jkms.2019.34.e85.

21. Li N, Strobino D, Ahmed S, Minkovitz CS. Is there a healthy foreign born effect for childhood obesity in the United States? Matern Child Health J. 2011;15(3):310323.doi: 10.1007/s10995-010-0588-5.

22. Lurbe E, Aguilar F, Alvarez J, Redon P, Torro MI, Redon J. Determinants of Cardiometabolic Risk Factors in the First Decade of Life: A Longitudinal Study Starting at Birth. Hypertension. 2018;71(3):437-443.doi:

10.1161/hypertensionaha.117.10529.

23. Makela J, Vaarno J, Kaljonen A, Niinikoski H, Lagstrom H. Maternal overweight impacts infant feeding patterns--the STEPS Study. Eur J Clin Nutr. 2014;68(1):4349.doi: 10.1038/ejen.2013.229.

24. Martin RM, Kramer MS, Patel R, et al. Effects of Promoting Long-term, Exclusive Breastfeeding on Adolescent Adiposity, Blood Pressure, and Growth Trajectories: A Secondary Analysis of a Randomized Clinical Trial. JAMA Pediatr. 2017;171(7):e170698.doi: 10.1001/jamapediatrics.2017.0698.

25. Martin RM, Patel R, Kramer MS, et al. Effects of promoting longer-term and exclusive breastfeeding on adiposity and insulin-like growth factor-I at age 11.5 years: a randomized trial. Jama. 2013;309(10):1005-1013.doi: 10.1001/jama.2013.167.

26. Massion S, Wickham S, Pearce A, Barr B, Law C, Taylor-Robinson D. Exploring the impact of early life factors on inequalities in risk of overweight in UK children: 
findings from the UK Millennium Cohort Study. Arch Dis Child. 2016;101(8):724730.doi: 10.1136/archdischild-2015-309465.

27. Metzger MW, McDade TW. Breastfeeding as obesity prevention in the United States: a sibling difference model. Am J Hum Biol. 2010;22(3):291-296.doi: 10.1002/ajhb.20982.

28. Modrek S, Basu S, Harding M, et al. Does breastfeeding duration decrease child obesity? An instrumental variables analysis. Pediatr Obes. 2017;12(4):304-311.doi: 10.1111/ijpo.12143.

29. Morgen CS, Angquist L, Baker JL, Andersen AN, Sorensen TIA, Michaelsen KF. Breastfeeding and complementary feeding in relation to body mass index and overweight at ages 7 and $11 \mathrm{y}$ : a path analysis within the Danish National Birth Cohort. Am J Clin Nutr. 2018;107(3):313-322.doi: 10.1093/ajen/nqx058.

30. Moschonis G, de Lauzon-Guillain B, Jones L, et al. The effect of early feeding practices on growth indices and obesity at preschool children from four European countries and UK schoolchildren and adolescents. Eur J Pediatr. 2017;176(9):1181-1192.doi: 10.1007/s00431-017-2961-5.

31. Moss BG, Yeaton WH. Early childhood healthy and obese weight status: potentially protective benefits of breastfeeding and delaying solid foods. Matern Child Health J. 2014;18(5):1224-1232.doi: 10.1007/s10995-013-1357-z.

32. Nelson MC, Gordon-Larsen P, Adair LS. Are adolescents who were breast-fed less likely to be overweight? Analyses of sibling pairs to reduce confounding. Epidemiology. 2005;16(2):247-253.doi: 10.1097/01.ede.0000152900.81355.00.

33. Ortega-Garcia JA, Kloosterman N, Alvarez L, et al. Full Breastfeeding and Obesity in Children: A Prospective Study from Birth to 6 Years. Child Obes. 2018;14(5):327337.doi: 10.1089/chi.2017.0335.

34. O'Tierney PF, Barker DJ, Osmond C, Kajantie E, Eriksson JG. Duration of breastfeeding and adiposity in adult life. J Nutr. 2009;139(2):422s-425s.doi: 10.3945/jn.108.097089.

35. Pattison KL, Kraschnewski JL, Lehman E, et al. Breastfeeding initiation and duration and child health outcomes in the first baby study. Prev Med. 2019;118:16.doi: 10.1016/j.ypmed.2018.09.020.

36. Pluymen LPM, Wijga AH, Gehring U, Koppelman GH, Smit HA, van Rossem L. Breastfeeding and cardiometabolic markers at age 12: a population-based birth cohort study. Int J Obes (Lond). 2019;43(8):1568-1577.doi: 10.1038/s41366-0180317-5.

37. Ruijsbroek A, Wijga AH, Kerkhof M, Koppelman GH, Smit HA, Droomers M. The development of socio-economic health differences in childhood: results of the Dutch longitudinal PIAMA birth cohort. BMC Public Health. 2011;11:225.doi: 10.1186/1471-2458-11-225.

38. Skledar MT, Milosevic M. Breastfeeding and time of complementary food introduction as predictors of obesity in children. Cent Eur J Public Health. 2015;23(1):26-31.doi: 10.21101/cejph.a3956. 
39. Thorland W, Currie D, Colangelo C. Status of High Body Weight Among NurseFamily Partnership Children. MCN Am J Matern Child Nurs. 2017;42(6):352357.doi: $10.1097 / \mathrm{nmc} .0000000000000369$.

40. van Rossem L, Taveras EM, Gillman MW, et al. Is the association of breastfeeding with child obesity explained by infant weight change? Int J Pediatr Obes. 2011;6(22):e415-422.doi: 10.3109/17477166.2010.524700.

41. Weng SF, Redsell SA, Nathan D, Swift JA, Yang M, Glazebrook C. Estimating overweight risk in childhood from predictors during infancy. Pediatrics. 2013;132(2):e414-421.doi: 10.1542/peds.2012-3858.

42. Wojcicki JM, Young MB, Perham-Hester KA, de Schweinitz P, Gessner BD. Risk factors for obesity at age 3 in Alaskan children, including the role of beverage consumption: results from Alaska PRAMS 2005-2006 and its three-year follow-up survey, CUBS, 2008-2009. PLoS One. 2015;10(3):e0118711.doi:

10.1371/journal.pone.0118711. 


\section{METHODOLOGY}

The NESR team used its rigorous, protocol-driven methodology to support the 2020 Dietary Guidelines Advisory Committee in conducting this systematic review.

NESR's systematic review methodology involves:

- Developing a protocol,

- Searching for and selecting studies,

- Extracting data from and assessing the risk of bias of each included study,

- Synthesizing the evidence,

- Developing conclusion statements,

- Grading the evidence underlying the conclusion statement, and

- Recommending future research.

A detailed description of the methodology used in conducting this systematic review is available on the NESR website: https://nesr.usda.gov/2020-dietary-guidelines-advisory-committee-systematicreviews, and can be found in the 2020 Dietary Guidelines Advisory Committee Report, Part C: Methodology. vii This systematic review was peer reviewed by Federal scientists, and information about the peer review process can also be found in the Committee's Report, Part C. Methodology. Additional information about this systematic review, including a description of and rationale for any modifications made to the protocol can be found in the 2020 Dietary Guidelines Advisory Committee Report, Chapter 4. Duration, Frequency, and Volume of Exclusive Human Milk and/or Infant Formula Feeding.

Below are details of the final protocol for the systematic review described herein, including the:

- Analytic framework

- Literature search and screening plan

- Literature search and screening results

vii Dietary Guidelines Advisory Committee. 2020. Scientific Report of the 2020 Dietary Guidelines Advisory Committee: Advisory Report to the Secretary of Agriculture and the Secretary of Health and Human Services. U.S. Department of Agriculture, Agricultural Research Service, Washington, DC. 


\section{ANALYTIC FRAMEWORK}

The analytic framework (Figure 1) illustrates the overall scope of the systematic review, including the population, the interventions and/or exposures, comparators, and outcomes of interest. It also includes definitions of key terms and identifies key confounders considered in the systematic review. The inclusion and exclusion criteria that follow provide additional information about how parts of the analytic framework were defined and operationalized for the review.

\section{Figure 1. Analytic framework}

Systematic review question: What is the relationship between the duration, frequency, and volume of exclusive human milk and/or infant formula consumption and overweight and obesity?

\begin{tabular}{|c|c|c|c|}
\hline & Interventions/exposures & Comparators & Outcomes: all studies \\
\hline \multirow{2}{*}{\multicolumn{2}{|c|}{$\begin{array}{l}\text { Duration of exclusive human milk and/or } \\
\text { infant formula consumption: } \\
\text { a) Ever consuming human milk (i.e., any } \\
\text { amount of human milk feeding) } \\
\text { b) Duration of any human milk } \\
\text { consumption among infants fed human } \\
\text { milk } \\
\text { c) Duration of exclusive human milk } \\
\text { consumption prior to the introduction of } \\
\text { infant formula }\end{array}$}} & $\begin{array}{l}\text { Different durations of exclusive human milk } \\
\text { and/or infant formula consumption: } \\
\text { a) Never consuming human milk } \\
\text { b) Different durations of any human milk } \\
\text { consumption among infants fed human milk } \\
\text { c) Different durations of exclusive human milk }\end{array}$ & $\begin{array}{l}\text { - Overweight } \\
\text { - Obesity } \\
\text { Population: } \\
\text { Children through older adults } \\
\text { (ages } 2 \text { years and older) }\end{array}$ \\
\hline & & $\begin{array}{l}\text { consumption prior to the introduction of infant } \\
\text { formula }\end{array}$ & vithin-family studies \\
\hline \multicolumn{2}{|r|}{$\begin{array}{l}\text { Frequency and volume of human milk } \\
\text { and/or infant formula consumption } \\
\text { a) Intensity/proportion/amount of human } \\
\text { milk consumed by mixed-fed infants } \\
\text { (i.e., both at a given point in time and } \\
\text { over a period of time) }\end{array}$} & $\begin{array}{l}\text { Different frequencies and volumes of human milk } \\
\text { and/or infant formula consumption } \\
\text { a) Different intensities/proportions/amounts of } \\
\text { human milk consumed by mixed-fed infants } \\
\text { (i.e., both at a given point in time and over a } \\
\text { period of time) }\end{array}$ & $\begin{array}{l}\text { of siblings, only } \\
\text { - Rapid weight gain } \\
\text { Population: } \\
\text { Birth to } 24 \text { months }\end{array}$ \\
\hline & $\begin{array}{l}\text { Intensity/proportion/amount of human } \\
\text { milk consumed at the breast versus by } \\
\text { bottle in infants fed human milk as their } \\
\text { only source of milk (i.e., both at a given } \\
\text { point in time and over a period of time) } \\
\text { Consuming human milk or infant } \\
\text { formula (i.e., a single substance) during }\end{array}$ & $\begin{array}{l}\text { b) Different intensities/proportions/amounts of } \\
\text { human milk consumed at the breast versus } \\
\text { by bottle in infants fed human milk as their } \\
\text { only source of milk (i.e., both at a given point } \\
\text { in time and over a period of time) } \\
\text { c) Consuming human milk and infant formula } \\
\text { (i.e., both substances) during a single }\end{array}$ & $\begin{array}{l}\text { Body composition (e.g., \% fat mass, } \\
\text { \% fat-free mass, waist-to-hip ratio) } \\
\text { - BMI, BMl z-score, weight-for-length } \\
\text { Population: } \\
\text { Children through older adults } \\
\text { (ages } 2 \text { years and older) }\end{array}$ \\
\hline \multicolumn{3}{|c|}{$\begin{array}{l}\text { Population: Infants and toddlers (birth to } 24 \text { months); full-term, healthy and/or at risk for chronic } \\
\text { disease }\end{array}$} & \\
\hline
\end{tabular}

Key definitions

Human milk - Mother's own milk provided at the breast (i.e., nursing) or expressed and fed fresh or after refrigeration/ freezing; donor milk is not examined in this review

Human milk feeding - Feeding human milk alone or in combination with infant formula and/or CFB such as cow's milk

Exclusive human milk feeding - Feeding human milk alone and not in combination with infant formula and/or CFB such as cow's milk; inclusive of WHO definitions of "exclusive" and "predominant" breastfeeding, which permit limited quantities of drops or syrups containing vitamins, minerals, or medicines; water and water-based drinks such as sweetened water and teas; fruit juice; oral rehydration salts solution; and ritual fluids

Infant formula - Commercially prepared infant formula meeting FDA and/or Codex Alimentarius international food standards Mixed feeding - Feeding human milk and infant formula but not CFB such as cow's milk

Complementary foods and beverages (CFB) - Foods and beverages other than human milk or infant formula (liquids, semisolids, and solids) provided to an infant or young child to provide nutrients and energy

Topping up - Feeding infant formula after human milk during a single feeding session

Within-family studies of siblings - Studies that compare biological siblings, within the same family, who had discordant exposures or outcomes or both (i.e., siblings fed differently during infancy or with differences in outcome status or both)
Legend

$\longrightarrow$ The relationship of interest in the systematic review

$\ldots \ldots \rightarrow$ Factors that may impact the relationship of interest in the systematic review 


\section{LITERATURE SEARCH AND SCREENING PLAN}

\section{Inclusion and exclusion criteria}

This table provides the inclusion and exclusion criteria for the systematic review. The inclusion and exclusion criteria are a set of characteristics that were used to determine which articles identified in the literature search were included in or excluded from the systematic review.

Table 11. Inclusion and exclusion criteria

\begin{tabular}{|c|c|c|}
\hline Category & Inclusion Criteria & Exclusion Criteria \\
\hline \multirow[t]{3}{*}{ Study participants } & Human participants & \multirow{3}{*}{$\begin{array}{l}\text { Non-human participants (e.g., animal } \\
\text { and in-vitro studies) }\end{array}$} \\
\hline & Males & \\
\hline & Females & \\
\hline $\begin{array}{l}\text { Additional criteria for } \\
\text { study participants for } \\
\text { within-family studies of } \\
\text { siblings viii }\end{array}$ & $\begin{array}{l}\text { Siblings with discordant expsoures or } \\
\text { outcomes or both (i.e., siblings fed } \\
\text { differently during infancy or with } \\
\text { differences in outcome status or both), } \\
\text { who were compared using within-family } \\
\text { analyses }\end{array}$ & $\begin{array}{l}\text { Siblings who were not compared } \\
\text { using within-family analyses }\end{array}$ \\
\hline \multirow{8}{*}{$\begin{array}{l}\text { Health status of study } \\
\text { participants }\end{array}$} & Studies that enroll participants: & \multirow{8}{*}{$\begin{array}{l}\text { Studies that exclusively enroll } \\
\text { participants: } \\
\text { - born preterm (gestational age }<37 \\
\text { weeks and } 0 / 7 \text { days), with low birth } \\
\text { weight ( }<2500 \mathrm{~g}) \text {, or small for } \\
\text { gestational age } \\
\text { - with failure to thrive/underweight, } \\
\text { stunting, or wasting } \\
\text { - diagnosed with a disease or } \\
\text { hospitalized with an illness or injury }\end{array}$} \\
\hline & - born full-term ( $\geq 37$ weeks and $0 / 7$ & \\
\hline & days gestational age) & \\
\hline & $\begin{array}{l}\text { - who are healthy and/or at risk for } \\
\text { chronic disease }\end{array}$ & \\
\hline & Studies that enroll some participants: & \\
\hline & - born preterm (gestational age $<37$ & \\
\hline & $\begin{array}{l}\text { weight }(<2500 \mathrm{~g}) \text {, or small for } \\
\text { gestational age }\end{array}$ & \\
\hline & $\begin{array}{l}\text { - with failure to thrive/underweight, } \\
\text { stunting, or wasting }\end{array}$ & \\
\hline
\end{tabular}

viiilnfant-feeding research is mostly observational (due to ethical concerns about randomizing infants to be fed less or no human milk) and is prone to confounding because infant-feeding decisions are strongly socially patterned. Wtihin-family analyses of siblings help address confounding from genetic and environmental factors (factors that siblings share). Therefore, the systematic review gave particular attention to the results from within-famly analyses of siblings, and there are additional inclusion and exclusion criteira that applied to these studies. 


\begin{tabular}{|c|c|c|}
\hline Category & Inclusion Criteria & Exclusion Criteria \\
\hline \multirow[t]{8}{*}{ Interventions/ exposuresix } & $\begin{array}{l}\text { 1. Duration of exclusive human milk } \\
\text { and/or infant formula consumption: }\end{array}$ & \\
\hline & $\begin{array}{l}\text { a) Ever consuming human milk (i.e., } \\
\text { any amount of human milk } \\
\text { feeding) }\end{array}$ & \\
\hline & $\begin{array}{l}\text { b) Duration of any human milk } \\
\text { consumption among infants fed } \\
\text { human milk }\end{array}$ & $\begin{array}{l}\text { 1b) Variables that include infants who } \\
\text { were never fed human milk }\end{array}$ \\
\hline & $\begin{array}{l}\text { c) Duration of exclusive human milk } \\
\text { consumption prior to the } \\
\text { introduction of infant formula }\end{array}$ & $\begin{array}{l}\text { 1c) Duration of exclusive human milk } \\
\text { consumption prior to the introduction } \\
\text { of complementary foods and } \\
\text { beverages or the concurrent } \\
\text { introduction of complementary foods } \\
\text { and beverages and infant formula } \\
\text { (including when a study does not } \\
\text { specify what follows exclusive human } \\
\text { milk feeding) }\end{array}$ \\
\hline & $\begin{array}{l}\text { 2. Frequency and volume of human } \\
\text { milk and/or infant formula } \\
\text { consumption }\end{array}$ & \\
\hline & $\begin{array}{l}\text { a) Intensity/proportion/amount of } \\
\text { human milk consumed by mixed- } \\
\text { fed infants }\end{array}$ & $\begin{array}{l}\text { 2a) Variables that include infants fed } \\
\text { complementary foods and beverages }\end{array}$ \\
\hline & $\begin{array}{l}\text { b) Intensity/proportion/amount of } \\
\text { human milk consumed at the } \\
\text { breast versus by bottle in infants } \\
\text { fed human milk as their only } \\
\text { source of milk }\end{array}$ & \\
\hline & $\begin{array}{l}\text { c) Consuming human milk or infant } \\
\text { formula (i.e., a single substance) } \\
\text { during a single feeding session }\end{array}$ & \\
\hline
\end{tabular}

ix Articles that did not report sufficient information to determine that they examine the interventions/exposures of interest were excluded. 


\begin{tabular}{ll}
\hline Category & Inclusion Criteria \\
\hline Comparators & $\begin{array}{l}\text { 1. Different durations of exclusive } \\
\text { human milk and/or infant formula } \\
\text { consumption: }\end{array}$
\end{tabular}

a) Never consuming human milk

b) Different durations of any human milk consumption among infants fed human milk

c) Different durations of exclusive human milk consumption prior to the introduction of infant formula

\section{Exclusion Criteria}

1a) Variables that include any amount of human milk feeding (e.g., very short-term or token) or the feeding of infant formula that does not meet the definition below

1b) Variables that include infants who were never fed human milk

1c) Durations of exclusive human milk consumption prior to the introduction of complementary foods and beverages or the concurrent introduction of complementary foods and beverages and infant formula (including when a study does not specify what follows exclusive human milk feeding)

2. Different frequencies and volumes of human milk and/or infant formula consumption

a) Different intensities/proportions/ amounts of human milk consumed by mixed-fed infants

b) Different intensities/proportions/ amounts of human milk consumed at the breast versus by bottle in infants fed human milk as their only source of milk

c) Consuming human milk and infant formula (i.e., both substances) during a single feeding session (e.g., "topping up")

Sources of foods, beverages, or nutrients
Human milk: Mother's own milk (MOM), that is, human milk fed at the breast (i.e., nursing) or expressed and fed fresh or after refrigeration/freezing

Infant formula: commercially prepared infant formula meeting FDA ${ }^{x}$ and/or Codex Alimentarius ${ }^{\mathrm{xi}}$ international food standards
Human milk from third parties (e.g., banked/donor milk)

Infant formulas that are not commercially prepared or that do not meet FDA and/or Codex Alimentarius international food standards

2a) Variables that include infants fed complementary foods and beverages 


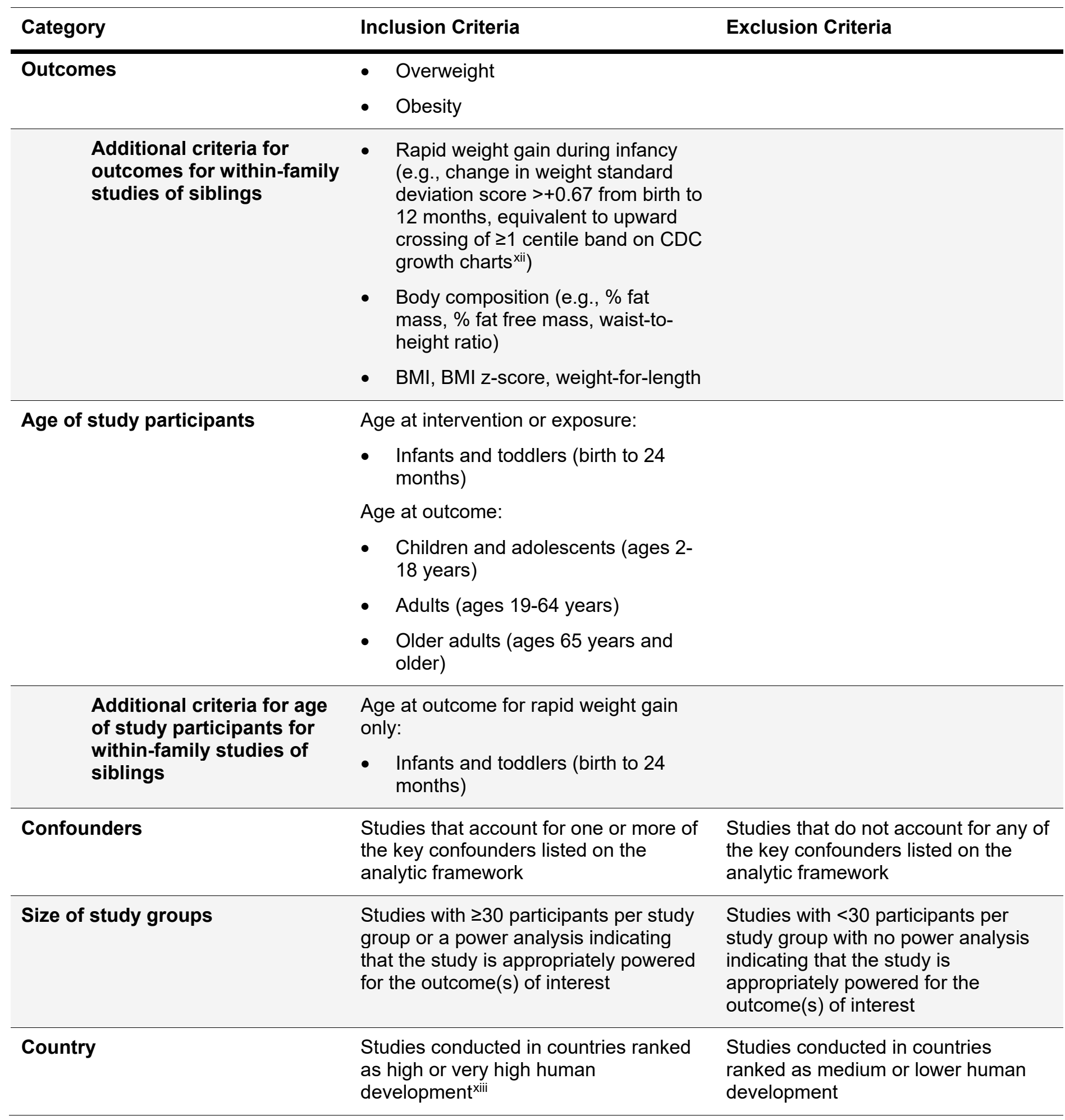

\footnotetext{
xii Ong, K.K. and Loos, R.J., 2006. Rapid infancy weight gain and subsequent obesity: systematic reviews and hopeful suggestions. Acta paediatrica, 95(8), pp.904-908.

xiii The human development classification from the Pregnancy and Birth to 24 Months (P/B-24) Project, which was used to screen growth, size, and body composition literature from the original literature search, was applied to the updated literature search for consistency. During the P/B-24 Project, the human development classification was the Human Development Index (HDI) ranking from the most recent Human Development Report (United Nations Development Programme. Human Development Report 2014. New York, 2014.) 


\begin{tabular}{|c|c|c|}
\hline Category & Inclusion Criteria & Exclusion Criteria \\
\hline \multirow[t]{7}{*}{ Study design } & Randomized controlled trials & Uncontrolled trials \\
\hline & \multirow{2}{*}{$\begin{array}{l}\text { Non-randomized controlled trials, } \\
\text { including quasi-experimental and } \\
\text { controlled before-and-after studies }\end{array}$} & Case-control studies \\
\hline & & Cross-sectional studies \\
\hline & Prospective cohort studies & Uncontrolled before-and-after studies \\
\hline & Retrospective cohort studies & Narrative reviews \\
\hline & \multirow[t]{2}{*}{ Nested case-control studies } & Systematic reviews \\
\hline & & Meta-analyses \\
\hline $\begin{array}{l}\text { Additional criteria for } \\
\text { study design for within- } \\
\text { family studies of siblings }\end{array}$ & Cross-sectional studies & \\
\hline Publication status & Articles that have been peer-reviewed & $\begin{array}{l}\text { Articles that have not been peer- } \\
\text { reviewed and are not published in } \\
\text { peer-reviewed journals, including } \\
\text { unpublished data, manuscripts, } \\
\text { reports, abstracts, and conference } \\
\text { proceedings }\end{array}$ \\
\hline \multirow[t]{2}{*}{ Date of publication } & January 2011 - September 2019 & $\begin{array}{l}\text { Articles published prior to January } \\
2011 \text { when they did not include } \\
\text { within-family analyses of siblings } \\
\text { (see next row) }\end{array}$ \\
\hline & & $\begin{array}{l}\text { Articles published after September } \\
2019\end{array}$ \\
\hline $\begin{array}{l}\text { Additional criteria for date } \\
\text { of publication for within- } \\
\text { family studies of siblings }\end{array}$ & January 1980-January 2011 & $\begin{array}{l}\text { Articles published prior to January } \\
1980\end{array}$ \\
\hline Language of publication & Articles published in English & $\begin{array}{l}\text { Articles published in languages other } \\
\text { than English }\end{array}$ \\
\hline
\end{tabular}




\section{Electronic databases and search terms}

\section{Pregnancy and Birth to 24 Months Project literature search ${ }^{\text {xiv }}$ \\ PubMed}

- Provider: U.S. National Library of Medicine

- Date(s) Searched: Dec 4, 2015 and March 28, 2016 to refine/limit search terms and remove pub type indexing

- Date range searched: January 1, 1980-March 28, 2016

- Search terms:

(breast feeding[mh] OR breastfeeding[tiab] OR breast feeding*[tiab] OR breastfeeding*[tiab] OR breastfed[tiab] OR breast-fed[tiab] OR breastfeed*[tiab] OR "breast feed"[tiab]) OR (Milk, human[mh] OR "breast milk"[tiab] OR breast-milk[tiab] OR "human milk"[tiab] OR "mother's milk"[tiab] OR breastmilk[tiab]) OR (Bottle feeding[mh] OR bottle feeding*[tiab] OR "bottle feeding"[tiab] OR bottle-feeding*[tiab] OR bottle-fed[tiab] OR "bottle fed"[tiab])

NOT ((aids[ti] AND "Acquired Immunodeficiency Syndrome"[Mesh]) OR hiv[ti] OR HIVIAIDS[ti] OR human immunodefic*[ti] OR Acquired Immunodefic*[ti] OR "low birth weight"[ti] OR Ibw[ti] OR vlbw[ti] OR elbw[ti] OR pcb[ti] OR pcbs[ti] OR Polychlorinated Biphenyl*[ti] OR Polychlorobiphenyl Compound*[ti] OR dioxin*[ti] OR (breast[ti] AND (tumor*[ti] OR tumour*[ti] OR cancer*[ti] OR carcinoma*[ti] OR disease*[ti]))) NOT (breastfeed $^{*}[\mathrm{ti}]$ OR breastfed ${ }^{*}[\mathrm{ti}]$ OR feed ${ }^{*}[\mathrm{ti}]$ OR fed[ti] OR milk[ti])

NOT (editorial[ptyp] OR comment[ptyp] OR news[ptyp] OR letter[ptyp] OR review[ptyp] OR systematic[sb])

Filters: Publication date from 1980/01/01 to 2016/03/28, Humans, English

\section{Cochrane Central Register of Controlled Trials (CENTRAL)}

- Provider: John Wiley \& Sons

- Date(s) searched: Dec 8, 2015

- Date range searched: January 1, 1980-December 8, 2015

- Search terms:

"Breast Feeding"OR breast-fed OR "breast fed" OR breastfeeding OR "breast feeding" OR "breast feed" OR "breast feeds" OR breast-feed OR breast-feeds OR (breast NEAR/3 feed") OR "human milk" OR "breast milk" OR breastmilk OR "mother's milk" OR "maternal milk" OR ((mother* OR maternal OR donor* OR donate*) NEAR/3 milk) OR "Bottle feeding" OR "bottle feedings" OR "bottle-feeding" OR "bottle-feedings" OR (bottle NEAR/3 feed $\left.^{*}\right)$

xiv During the Pregnancy and Birth to 24 Months (P/B-24) Project, systematic review questions were defined to examine the relationships between human milk and infant formula consumption and several outcomes, and NESR used a single literature search to identify potential studies for the family of reviews (https://nesr.usda.gov/infant-milk-feeding-practicestechnical-expert-collaborative). Some of the intended reviews, including overweigh and obesity, were not completed before the end of the Project. The 2020 Dietary Guidelines Advisory Committee, Birth to 24 Months Subcommittee, used and updated the literature search and screening underway from the P/B-24 Project according to the inclusion and exclusion criteria described herein. 


\section{Embase}

- Provider: Elsevier

- Date(s) Searched: Dec 5, 2015

- Date range searched: January 1, 1980-December 5, 2015

- Search terms ${ }^{\mathrm{xv}}$ :

'bottle feeding'/exp OR 'bottle feeding':ab,ti OR 'bottle feedings':ab,ti OR 'bottle fed':ab,ti OR bottle* NEAR/3 feed* AND [english]/lim AND [humans]/lim AND [1980-2015]/py OR 'breast milk'/exp OR 'human milk':ab,ti OR 'breast milk':ab,ti OR breastmilk:ab,ti OR mother* NEAR/2 milk OR 'maternal milk':ab,ti AND [english]/lim AND [humans]/lim AND [1980-2015]/py OR 'breast feeding'/exp OR breastfeed":ab,ti OR 'breast feed':ab,ti OR 'breast feeding':ab,ti OR breastfed:ab,ti OR 'breast fed':ab,ti OR feeding NEAR/3 breast AND [english]/lim AND [humans]/lim AND [1980-2015]/py

\section{Cumulative Index of Nursing and Allied Health Literature (CINAHL Plus)}

- Provider: EBSCOhost

- Date(s) searched: Dec 8, 2015

- Date range searched: January 1, 1980-December 8, 2015

- Search terms:

(MH "Breast Feeding+" OR breast-fed OR "breast fed" OR breastfeeding OR breast feeding OR breast-fed) OR MH "Milk, Human" OR "Human Milk" OR "Breast Milk" OR Breastmilk OR breast-milk OR ((maternal OR mother*) n3 milk) OR (MH "Bottle Feeding") OR "bottle feeding" OR (bottle $n 3$ feed") OR bottle-feeding OR bottle-feedings OR "bottle fed" OR "bottle-fed")

\section{Update to the Pregnancy and Birth to 24 Months Project literature search}

\section{PubMed}

- Provider: U.S. National Library of Medicine

- Date(s) Searched: September 5, 2019

- Date range searched: January 1, 2016 - September 31, 2019

- Search terms:

\#1 - Breast feeding[mh] OR breast fed[tiab] OR breast feed*[tiab] OR bottle feed*[tiab] OR breastfeed $^{*}\left[\right.$ tiab] OR bottle fed ${ }^{*}[$ tiab] OR breastfed[tiab] OR breastfeed*[tiab] OR breast feed[tiab] OR Milk, human[mh] OR "breast milk"[tiab] OR "human milk"[tiab] OR "mother's milk"[tiab] OR mothers' milk[tiab] OR mother's own milk[tiab] OR mothers' own milk[tiab] OR "maternal milk"[tiab] OR breastmilk[tiab] OR Bottle feeding[mh] OR infant formula[mh] OR "infant formula"[tiab] OR "milk formula"[tiab]

\#2 - (("Allergy and Immunology"[Mesh:NoExp] OR allergy[tiab] OR allergies[tiab] OR allergic[tiab] OR allergen* OR Hypersensitivit*[tiab] OR atopic[tiab]) AND (food OR foods OR peanut* OR nut OR nuts OR egg OR eggs OR milk OR shellfish OR fish OR wheat OR gluten* OR dairy)) OR "Food Hypersensitivity"[Mesh] OR asthma*[tiab] OR asthma[mh] OR "Rhinitis, Allergic"[Mesh] OR (allergic[tiab] AND rhiniti*[tiab]) OR

${ }^{x v}$ After conducting the search, the librarian used the citation manager to screen out search results from Embase, CINAHL, and Cochrane with the following keywords in the title: (aids AND "Acquired Immunodeficiency Syndrome") OR hiv OR HIVIAIDS OR human immunodefic* OR Acquired Immunodefic* OR "low birth weight" OR Ibw OR vlbw OR elbw OR pcb OR pcbs OR Polychlorinated Biphenyl* OR Polychlorobiphenyl Compound* OR dioxin* OR (breast AND (tumor* OR tumour* OR cancer* OR carcinoma* OR disease* $\left.{ }^{\star}\right)$ OR preterm OR premature 
"Dermatitis, Atopic"[Mesh] OR ((Dermatiti*[tiab] OR eczema[tiab]) AND Atopic[tiab]) OR eczema[mh] OR "Immunoglobulin E"[Mesh] OR "Immunoglobulin E"[tiab]

\#3 - "Body Weights and Measures"[Mesh] OR "Body Weight"[Mesh] OR obesity[tiab] OR obese[tiab] OR overweight[tiab] OR body mass index[tiab] OR BMI[tiab] OR underweight[tiab] OR wasting[tiab] OR healthy weight[tiab] OR "Body Composition"[Mesh] OR body composition[tiab] OR body fat[tiab] OR fat mass[tiab] OR fat free mass[tiab] OR stunting[tiab] OR stunted[tiab] OR "Growth Charts"[Mesh] OR growth chart*[tiab] OR waist circumference[tiab] OR head circumference[tiab] OR arm circumference[tiab] OR thigh circumference[tiab] OR neck circumference[tiab] OR

Anthropometry[Mesh:NoExp]OR Growth[Mesh:NoExp] OR Overnutrition[Mesh] OR failure to thrive[mh] OR anthropometr*[tiab] OR adiposity[tiab] OR calf circumference[tiab] OR failure to thrive[tiab] OR skin fold*[tiab] OR skin fold*[tiab] OR normal weight[tiab] OR weight for age[tiab] OR height for age[tiab] OR recumbent length[tiab] OR length for age[tiab] OR weight for length[tiab]

\#4 - "Mental Disorders"[Mesh] OR mental disorder*[tiab] OR "Cognition"[Mesh] OR cognition[tiab] OR cognitive[tiab] OR metacognition[tiab] OR neurocognitive[tiab] OR neurodevelop*[tiab] OR neurological[tiab] OR "Cognitive Dysfunction"[Mesh] OR "Depressive Disorder"[Mesh] OR "Depression"[Mesh] OR depression[tiab] OR anxiety[tiab] OR "Psychomotor Performance"[Mesh] OR motor skill*[tiab] OR "Executive Function"[Mesh] OR executive function* OR "Attention Deficit and Disruptive Behavior Disorders"[Mesh] OR attention deficit disorder*[tiab] OR ADHD[tiab] OR "Child Behavior Disorders"[Mesh] OR developmental disorder*[tiab] OR "Autism Spectrum Disorder"[Mesh] OR Autism[tiab] OR Asperger[tiab] OR language processing[tiab] OR language delay* OR "Child Development"[Mesh] OR child develop*[tiab] OR "Developmental Disabilities"[Mesh] OR developmental delay[tiab] OR developmental disabilit*[tiab] OR "Motor Skills Disorders"[Mesh] OR motor skill"[tiab] OR "Problem Solving"[Mesh] OR developmental domain* OR academic performance[tiab] OR academic achievement[tiab] OR academic failure[tiab] OR academic success*[tiab]

\#5 - Micronutrients[mh] OR micronutrient*[tiab] OR "Anemia"[Mesh] OR "Anemia, IronDeficiency"[Mesh] OR anemia[tiab] OR anemic[tiab] OR rickets[tiab] OR hematocrit[tiab] OR 25 hydroxyvitamin d[tiab] OR "25(oh)d"[tiab] OR cobalamin[tiab] OR holo-tc[tiab] OR holotranscobalamin[tiab] OR "Zinc"[Mesh] OR zinc[tiab] OR "lodine"[Mesh] OR iodine[tiab] OR "Iron"[Mesh] OR iron[tiab] OR hemoglobin*[tiab] OR ferritin*[tiab] OR transferrin*[tiab] OR "Vitamin B 12"[Mesh] OR "Vitamin B 12 Deficiency"[Mesh] OR "vitamin B"[tiab] OR "Vitamin D"[Mesh] OR "Vitamin D Deficiency"[Mesh] OR vitamin $D^{*}[$ tiab] OR "Fatty Acids"[Mesh:NoExp] OR fatty acid* OR saturated fat* OR "Fatty Acids, Monounsaturated"[Mesh] OR monounsaturated fat* OR mono-unsaturated fat* OR polyunsaturated fat ${ }^{*}$ OR poly-unsaturated fat ${ }^{*} \mathrm{OR}$ unsaturated fat ${ }^{*} \mathrm{OR}$ unsaturated fatty acid* OR "Fatty Acids, Omega-3"[Mesh] OR omega-3[tiab] OR N-3 fatty acid* OR "Fatty Acids, Omega-6"[Mesh] OR omega-6 OR N-6 fatty acid* OR MUFA* OR PUFA* OR alpha-linolenic acid $^{*}$ OR eicosapentaenoic acid $^{*}$ OR docosahexaenoic acid ${ }^{*}$ OR linoleic acid* OR alpha-linolenic acid* OR arachidonic acid* OR "Fats, Unsaturated"[Mesh] OR (((fat[tiab] OR fatty[tiab]) AND (saturat* OR unsatur* OR monounsatur* OR polyunsatur* OR poly-unsatur* OR linolenic acid*)))))

\#6 - Diabetes Mellitus[mh:noexp] OR Diabetes Mellitus, Type 2[Mesh] OR Type 2 diabetes[tiab] OR T2D[tiab] OR T1D[tiab] OR homa-ir[tiab] OR blood pressure[mh] OR hypertension[mh] OR hyperlipidemias[mh] OR hyperlipidemia*[tiab] OR thrombosis[mh] OR "blood pressure"[tiab] OR hdl[tiab] OR Idl[tiab] OR Diabetes Mellitus, Type 1[mesh] 
OR Type 1 diabetes[tiab] OR Prediabetic State[Mesh] OR prediabet*[tiab] OR pre diabet* OR Insulin Resistance[Mesh] OR insulin resistance[tiab] OR Glucose Intolerance[Mesh] OR glucose intolerance[tiab] OR glucose tolerance[tiab] OR Glycated Hemoglobin A[Mesh] OR hemoglobin A1c[ti] OR ((impaired fasting[tiab] OR Diabetes

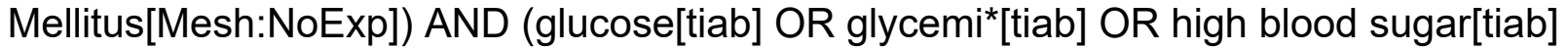
OR low blood sugar[tiab] OR hyperglycemia[mh] OR hypoglycemia[mh] OR hyperglycem*[tiab] OR hypoglycem*[tiab])) OR ((Cardiovascular Diseases[Mesh:noexp] OR cardiovascular disease*[tiab] OR coronary artery disease[tiab] OR heart disease*[tiab] OR Heart Failure[Mesh] OR heart failure[tiab] OR myocardial infarction*[tiab] OR Myocardial Ischemia[Mesh] OR Myocardial Ischemia*[tiab] OR Stroke[Mesh] OR stroke[tiab] OR heart attack[tiab] OR venous thrombosis[tiab] OR hypertension[tiab] OR Lipids/blood[Mesh] OR total cholesterol[tiab] OR Triglycerides[Mesh] OR triglycerides[tiab] OR arterial occlusive diseases[mh]))

\#7 - \#2 OR \#3 OR \#4 OR \#5 OR \#6

\#8 - \#1 AND \#7

\#9 - \#8 NOT ("Animals"[Mesh] NOT ("Animals"[Mesh] AND "Humans"[Mesh])) NOT editorial[ptyp] OR comment[ptyp] OR news[ptyp] OR letter[ptyp] OR review[ptyp] OR systematic review[ptyp] OR systematic review[ti] OR meta-analysis[ptyp] OR metaanalysis[ti] OR meta-analyses[ti] OR retracted publication[ptyp] OR retraction of publication[ptyp] OR retraction of publication[tiab] OR retraction notice[ti]

Filters: Publication date from 2016/01/01 to 2019/09/31; English

\section{Cochrane Central Register of Controlled Trials (CENTRAL)}

- Provider: John Wiley \& Sons

- Date(s) Searched: September 5, 2019

- Date range searched: January 1, 2016 - September 31, 2019

- Search terms:

\#1 - [mh "breast feeding"] OR "breast fed" OR "breast feed" OR "bottle feed"” OR breastfeed* OR "bottle fed"” OR breastfed OR breastfeed" OR "breast feed" OR [mh "milk, human"] OR "breast milk" OR "human milk" OR "mother's milk" OR "mothers' milk" OR "mother's own milk" OR "mothers' own milk" OR "maternal milk" OR breastmilk OR [mh "Bottle feeding"] OR [mh "infant formula"] OR "infant formula" OR "milk formula"

\#2 - [mh ^"Allergy and Immunology"] OR ((allerg*:ti,ab OR Hypersensitivit*:ti,ab) NEAR/4 (food ${ }^{*}$ OR peanut* OR nut OR nuts OR egg* OR milk OR shellfish OR wheat OR dairy OR fish)) OR [mh "Food Hypersensitivity"] OR asthma* OR [mh "Rhinitis, Allergic"] OR (allerg* NEAR/4 Rhiniti*) OR [mh "Dermatitis, Atopic"] OR ((Dermatiti* OR eczema) NEAR/4 Atopic) OR (Infant* NEAR/4 Eczema) OR [mh "Immunoglobulin E"] OR "Immunoglobulin E"

\#3 - [mh "Body Weights and Measures"] OR [mh "Body Weight") OR obesity OR obese OR overweight OR "body mass index" OR BMI OR underweight OR wasting OR "healthy weight" OR [mh "Body Composition"] OR "body composition" OR "body fat" OR "fat mass" OR "fat free mass" OR stunting OR stunted OR [mh "Growth Charts"] OR growth chart* OR "waist circumference" OR "head circumference" OR "arm circumference" OR "thigh circumference" OR "neck circumference" OR [mh ^"Anthropometry"] OR [mh ^"Growth"] OR [mh "Overnutrition"] OR [mh "failure to thrive"] OR anthropometr* OR adiposity OR "calf circumference" OR "failure to thrive" OR "skin fold"” OR "skin fold" OR "normal weight" OR "weight for age" OR "height for age" OR "recumbent length" OR "length for 
age" OR "weight for length"

\#4 - [mh "Mental Disorders"] OR [mh "Cognition"] OR [mh "Cognitive Dysfunction"] OR [mh "Depressive Disorder"] OR [mh "Depression"] OR [mh "Psychomotor Performance"] OR [mh "Executive Function"] OR [mh "Attention Deficit and Disruptive Behavior Disorders"] OR [mh "Child Behavior Disorders"] OR [mh "Autism Spectrum Disorder"] OR [mh "Child Development"] OR [mh "Developmental Disabilities"] OR [mh "Motor Skills Disorders"] OR [mh "Problem Solving"] OR ("mental disorder*" OR cognition OR cognitive OR metacognition OR neurocognitive OR neurodevelop* OR neurological OR depression OR anxiety OR "motor skill*" OR "executive function*" OR "attention deficit disorder" OR ADHD OR "developmental disorder" OR Autism OR Asperger OR "language processing" OR "language delay" OR "child develop*” OR "developmental delay" OR "developmental disabilit*" OR "motor skill"” OR "developmental domain*" OR "academic performance" Or "academic achievement" OR "academic failure" OR "academic success"”):ti,ab,kw

\#5 - [mh Micronutrients] OR micronutrient* OR [mh "Anemia"] OR [mh "Anemia, IronDeficiency"] OR anemia OR anemic OR rickets OR hematocrit OR "25 hydroxyvitamin d" OR "25(oh)d" OR cobalamin OR holo-tc OR holotranscobalamin OR [mh "Zinc"] OR zinc OR [mh "lodine"] OR iodine OR [mh "Iron"] OR iron OR hemoglobin* OR ferritin* OR transferrin* OR [mh "Vitamin B 12"] OR [mh "Vitamin B 12 Deficiency"] OR "vitamin B" OR [mh "Vitamin D"] OR [mh "Vitamin D Deficiency"] OR "vitamin D*" OR [mh ^"Fatty Acids"] OR "fatty acid"” OR "saturated fat"” OR [mh "Fatty Acids, Monounsaturated"] OR "monounsaturated fat" OR "mono-unsaturated fat" OR "polyunsaturated fat" OR "polyunsaturated fat*" OR "unsaturated fat*" OR "unsaturated fatty acid"” OR [mh "Fatty Acids, Omega-3"] OR omega-3 OR "N-3 fatty acid" OR [mh "Fatty Acids, Omega-6"] OR omega-6 OR "N-6 fatty acid*" OR MUFA* OR PUFA* OR "alpha-linolenic acid"” OR "eicosapentaenoic acid" OR "docosahexaenoic acid" OR "linoleic acid"” OR "alphalinolenic acid" OR "arachidonic acid" OR [mh "Fats, Unsaturated"] OR ((fat OR fatty) NEAR/4 (saturat ${ }^{*}$ OR unsatur* OR monounsatur* OR polyunsatur* OR poly-unsatur* OR linolenic acid $\left.^{*}\right)$ )

\#6 - [mh ^"Diabetes Mellitus"] OR [mh "Diabetes Mellitus, Type 2"] OR "Type 2 diabetes" OR T2D OR T1D OR homa-ir OR [mh "blood pressure"] OR [mh "hypertension"] OR [mh "hyperlipidemias"] OR hyperlipidemia* OR [mh "thrombosis"] OR "blood pressure" OR hdl OR Idl OR [mh "Diabetes Mellitus, Type 1"] OR "Type 1 diabetes" OR [mh "Prediabetic State"] OR prediabet* OR "pre diabet" OR [mh "Insulin Resistance"] OR "insulin resistance" OR [mh "Glucose Intolerance"] OR "glucose intolerance" OR "glucose tolerance" OR [mh "Glycated Hemoglobin A"] OR "hemoglobin A1c" OR ("“impaired fasting" OR [mh “"Diabetes Mellitus"]) NEAR/4 (glucose OR glycemi" OR "high blood sugar" OR "low blood sugar" OR [mh hyperglycemia] OR [mh hypoglycemia] OR hyperglycem* OR hypoglycem*)) OR [mh "Cardiovascular Diseases"] OR "cardiovascular disease*" OR "coronary artery disease" OR "heart disease *" OR [mh "Heart Failure"] OR "heart failure" OR "myocardial infarct*" OR [mh "Myocardial Ischemia"] OR "Myocardial Ischemia*" OR [mh Stroke] OR stroke OR "heart attack" OR "venous thrombosis" OR hypertension OR [mh Lipids/BL] OR "total cholesterol" OR [mh Triglycerides] OR triglycerides OR [mh "arterial occlusive diseases"]

\#7 - \#2 OR \#3 OR \#4 OR \#5 OR \#6

\#8 - \#1 AND \#7

Filters - Date limited 2016 to 2019, Trials 


\section{Embase}

- Provider: Elsevier

- Date(s) Searched: September 5, 2019

- Date range searched: January 1, 2016 - September 31, 2019

- Search terms:

\#1 - breast AND 'feeding'/exp OR 'breast fed':ti,ab OR 'bottle feed':'ti,ab OR 'bottle fed $d^{* \prime}: t i, a b$ OR breastfed:ti,ab OR breastfeed":ti,ab OR 'breast feed ${ }^{*}:$ ti,ab OR 'breast milk'/exp OR 'breast milk':ti,ab OR 'human milk':ti,ab OR 'mothers milk':ti,ab OR 'mothers own milk':ti,ab OR 'maternal milk':ti,ab OR breastmilk:ti,ab OR 'bottle feeding'/exp OR 'artificial milk'/exp OR 'infant formula':ti,ab OR 'milk formula':ti,ab

\#2 - 'allergic asthma'/exp OR 'food allergy'/exp OR 'allergic rhinitis'/exp OR 'dermatitis'/exp OR 'eczema'/exp OR 'skin allergy'/exp OR ((allerg* OR hypersensitivity*) NEAR/4 (food OR peanut* OR nut OR nuts OR egg OR eggs OR milk OR shellfish OR wheat OR fish OR dairy)) OR 'immunoglobulin e'/exp OR 'immunoglobulin e':ti,ab

\#3 - 'morphometry'/exp OR obesity:ti,ab OR obese:ti,ab OR overweight:ti,ab OR 'body mass index':ti,ab OR bmi:ti,ab OR underweight:ti,ab OR wasting:ti,ab OR 'healthy weight':ti,ab OR 'body composition':ti,ab OR 'body fat':ti,ab OR 'fat mass':ti,ab OR 'fat free mass':ti,ab OR stunting:ti,ab OR stunted:ti,ab OR 'growth chart*':ti,ab OR 'waist circumference':ti,ab OR 'head circumference':ti,ab OR 'arm circumference':ti,ab OR 'thigh circumference':ti,ab OR 'neck circumference':ti, ab OR anthropometr*:ti,ab OR adiposity:ti,ab OR 'calf circumference':ti,ab OR 'failure to thrive':ti,ab OR 'skin fold":ti,ab OR skinfold*:ti,ab OR 'normal weight':ti,ab OR 'weight for age':ti,ab OR 'height for age':ti,ab OR 'recumbent length':ti,ab OR 'length for age':ti,ab OR 'weight for length':ti,ab OR 'body composition'/mj OR 'waist circumference'/de OR 'body height'/de OR 'growth chart'/de OR 'body weight'/de OR 'anthropometry'/exp OR 'body growth'/de OR 'growth'/de OR 'overnutrition'/de OR 'failure to thrive'/exp OR 'weight for age'/exp OR 'height for age'/exp OR 'length for age'/exp

\#4 - ((micronutrient*:ti,ab OR anemia:ti,ab OR anemic:ti,ab OR rickets:ti,ab OR hematocrit:ti,ab OR '25 hydroxyvitamin d':ti,ab OR '25(oh)d':ti,ab OR cobalamin:ti,ab OR 'holo tc':ti,ab OR holotranscobalamin:ti,ab OR zinc:ti,ab OR iodine:ti,ab OR iron:ti,ab OR hemoglobin*:ti,ab OR ferritin*:ti,ab OR transferrin*:ti,ab OR 'vitamin b':ti,ab OR 'vitamin

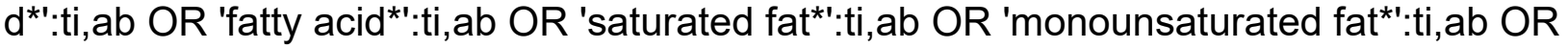
mono-unsaturated) AND fat ${ }^{*}: t i, a b$ OR 'polyunsaturated fat ${ }^{\star \prime}: t i, a b$ OR poly-unsaturated) AND fat ${ }^{*}$ OR 'unsaturated fat ${ }^{* \prime}:$ ti, ab OR 'unsaturated fatty acid*':ti,ab OR 'omega $3^{\prime}:$ ti, ab OR 'n-3 fatty acid*':ti,ab OR 'omega 6':ti,ab OR 'n-6 fatty acid': ti, ab OR mufa*:ti,ab OR pufa*:ti,ab OR 'eicosapentaenoic acid ${ }^{*}:$ ti, ab OR 'docosahexaenoic acid': $:$ ti,ab OR 'linoleic acid':ti,ab OR 'alpha-linolenic acid ${ }^{* \prime}: t i, a b$ OR 'arachidonic acid"':ti,ab OR ((fat:ti,ab OR fatty:ti,ab) AND (saturat* OR unsatur* OR monounsatur* OR polyunsatur* OR polyunsatur* OR linolenic) AND acid*) OR 'trace element'/exp OR 'anemia'/exp OR 'zinc'/exp OR 'iodine'/exp OR 'iron'/exp OR 'cyanocobalamin'/exp OR 'b12 deficiency'/exp OR 'vitamin d'/exp OR 'vitamin d deficiency'/exp OR 'fatty acid'/de OR 'docosahexaenoic acid'/exp OR 'icosapentaenoic acid'/exp OR 'linoleic acid'/exp OR 'linolenic acid'/exp OR 'arachidonic acid'/exp OR 'holotranscobalamin'/exp OR 'hemoglobin'/exp OR 'ferritin'/exp OR 'transferrin'/exp

\#5 - 'type 2 diabetes':ti,ab OR t2d:ti,ab OR t1d:ti,ab OR 'homa ir':ti,ab OR hyperlipidemia*:ti,ab OR 'blood pressure':ti,ab OR hdl:ti,ab OR Idl:ti,ab OR 'type 1 diabetes':ti,ab OR prediabet*:ti,ab OR 'pre diabet':ti,ab OR 'insulin resistance':ti,ab OR 
'glucose intolerance':ti,ab OR 'glucose tolerance':ti,ab OR 'hemoglobin a1c':ti,ab OR (('impaired fasting':ti,ab OR 'diabetes mellitus'/de) AND (glucose:ti,ab OR glycemi*:ti,ab OR 'high blood sugar':ti,ab OR 'low blood sugar':ti,ab OR 'hyperglycemia'/exp OR 'hypoglycemia'/exp OR hyperglycem*:ti,ab OR hypoglycem*:ti,ab)) OR 'cardiovascular disease ${ }^{* \prime}: t i, a b$ OR 'coronary artery disease':ti, ab OR 'heart disease ${ }^{* \prime}: t i, a b$ OR 'heart failure':ti,ab OR 'myocardial infarction*':ti,ab OR 'myocardial ischemia*':ti,ab OR stroke:ti,ab OR 'heart attack':ti,ab OR 'venous thrombosis':ti,ab OR hypertension:ti,ab OR 'total cholesterol':ti,ab OR triglycerides:ti,ab OR 'diabetes mellitus'/de OR 'non insulin dependent diabetes mellitus'/exp OR 'blood pressure'/exp OR 'hypertension'/exp OR 'hyperlipidemia'/exp OR 'thrombosis'/exp OR 'insulin dependent diabetes mellitus'/exp OR 'impaired glucose tolerance'/exp OR 'insulin resistance'/exp OR 'glucose intolerance'/exp OR 'glycosylated hemoglobin'/exp OR 'cardiovascular disease'/de OR 'heart failure'/exp OR 'heart muscle ischemia'/exp OR 'heart infarction'/exp OR 'cerebrovascular accident'/exp OR 'blood lipids'/exp OR 'triacylglycerol'/exp OR 'peripheral occlusive artery disease'/exp

\#6 - 'mental disease'/exp OR 'cognition'/exp OR 'cognitive defect'/exp OR 'depression'/exp OR 'psychomotor performance'/de OR 'executive function'/de OR 'attention deficit disorder'/de OR 'autism'/exp OR 'child development'/de OR 'developmental disorder'/exp OR 'psychomotor disorder'/de OR 'problem solving'/de OR 'mental disorder':ab,ti OR cognition:ab,ti OR cognitive:ab,ti OR metacognition:ab,ti OR neurocognitive:ab,ti OR neurodevelop*:ab,ti OR neurological:ab,ti OR depression:ab,ti OR anxiety:ab,ti OR 'executive function ${ }^{* \prime}: a b, t i$ OR 'attention deficit disorder':ab,ti OR adhd:ab,ti OR 'developmental disorder':ab,ti OR autism:ab,ti OR asperger:ab,ti OR 'language processing':ab,ti OR 'language delay':ab,ti OR 'child develop ${ }^{* 1}: a b, t i$ OR 'developmental delay':ab,ti OR 'developmental disabilit*':ab,ti OR 'motor skill*':ab,ti OR 'developmental domain*':ab,ti OR 'academic performance':ab,ti OR 'academic achievement':ab,ti OR 'academic failure':ab,ti OR 'academic success' ${ }^{* \prime} \cdot a b, t i$

\section{\#7 - \#2 OR \#3 OR \#4 OR \#5 OR \#6}

\#8 - \#1 AND \#7

\#9 - \#8 AND ([article]/lim OR [article in press]/lim) AND [humans]/lim AND [english]/lim AND [2016-2019]/py NOT ([conference abstract]/lim OR [conference paper]/lim OR [editorial]/lim OR [erratum]/lim OR [letter]/lim OR [note]/lim OR [review]/lim OR [systematic review]/lim OR [meta analysis]/lim)

\section{Cumulative Index of Nursing and Allied Health Literature (CINAHL PIus)}

- Provider: EBSCOhost

- Date(s) Searched: September 5, 2019

- Date range searched: January 1, 2016 - September 31, 2019

- Search terms:

\#1 - [mh "breast feeding"] OR "breast fed" OR "breast feed"” OR "bottle feed"” OR breastfeed* OR "bottle fed" OR breastfed OR breastfeed" OR "breast feed" OR [mh "milk, human"] OR "breast milk" OR "human milk" OR "mother's milk" OR "mothers' milk" OR "mother's own milk" OR "mothers' own milk" OR "maternal milk" OR breastmilk OR [mh "Bottle feeding"] OR [mh "infant formula"] OR "infant formula" OR "milk formula"

\#2 - (mh "Allergy and Immunology") OR ((allerg* OR Hypersensitivit ${ }^{*}$ N4 (food* OR peanut* OR nut OR nuts OR egg* OR milk OR shellfish OR wheat OR dairy OR fish)) OR (mh "Food Hypersensitivity+") OR asthma* OR (mh "Rhinitis, Allergic") OR (allerg* N4 
Rhiniti*) OR (mh "Dermatitis, Atopic") OR ((Dermatiti* OR eczema) N4 Atopic)) OR (Infant* N5 Eczema) OR (mh "Immunoglobulin E") OR "Immunoglobulin E"

\#3 - (mh "Body Weights and Measures") OR (mh "Body Weight") OR obesity OR obese OR overweight OR "body mass index" OR BMI OR underweight OR wasting OR "healthy weight" OR (mh "Body Composition") OR "body composition" OR "body fat" OR "fat mass" OR "fat free mass" OR stunting OR stunted OR (mh "Growth Charts") OR growth chart* OR "waist circumference" OR "head circumference" OR "arm circumference" OR "thigh circumference" OR "neck circumference" OR (mh "Anthropometry") OR (mh "Growth") OR (mh "Overnutrition") OR (mh "failure to thrive") OR anthropometr* OR adiposity OR "calf circumference" OR "failure to thrive" OR "skin fold" OR "skin fold*" OR "normal weight" OR "weight for age" OR "height for age" OR "recumbent length" OR "length for age" OR "weight for length"

\#4 - (mh Micronutrients) OR micronutrient* OR (mh Anemia) OR (mh "Anemia, IronDeficiency") OR anemia OR anemic OR rickets OR hematocrit OR "25 hydroxyvitamin d" OR "25(oh)d" OR cobalamin OR holo-tc OR holotranscobalamin OR (mh Zinc) OR zinc OR (mh lodine) OR iodine OR (mh Iron) OR iron OR hemoglobin* OR ferritin* OR transferrin* OR (mh "Vitamin B 12") OR (mh "Vitamin B 12 Deficiency") OR "vitamin B" OR "vitamin b12" OR "vitamin b 12" OR (mh "Vitamin D") OR "vitamin d" OR (mh "Vitamin D Deficiency") OR "vitamin D"” OR (mh "Fatty Acids") OR fatty acid* OR saturated fat" OR (mh "Fatty Acids, Monounsaturated") OR monounsaturated fat* OR monounsaturated fat ${ }^{\star}$ OR polyunsaturated fat ${ }^{*}$ OR poly-unsaturated fat ${ }^{*}$ OR unsaturated fat ${ }^{*}$ OR unsaturated fatty acid* OR (mh "Fatty Acids, Omega-3") OR omega-3* OR N-3 fatty acid* OR (mh "Fatty Acids, Omega-6") OR omega-6* OR N-6 fatty acid* OR MUFA* OR PUFA* OR "alpha-linolenic acid" OR "eicosapentaenoic acid"” OR "docosahexaenoic acid*" OR "linoleic acid"” OR "alpha-linolenic acid"” OR "arachidonic acid"” OR (mh "Fats, Unsaturated") OR (((fat OR fatty) N4 (saturat ${ }^{\star}$ OR unsatur* OR monounsatur* OR polyunsatur* OR poly-unsatur* OR linolenic acid*)))))

\#5 - (mh "Diabetes Mellitus") OR (mh "Diabetes Mellitus, Type 2") OR "Type 2 diabetes" OR T2D OR T1D OR homa-ir OR ( $\mathrm{mh}$ "blood pressure") OR (mh hypertension) OR ( $\mathrm{mh}$ hyperlipidemias) OR hyperlipidemia* OR (mh thrombosis) OR "blood pressure" OR hdl OR Idl OR (mh "Diabetes Mellitus, Type 1") OR "Type 1 diabetes" OR (mh "Prediabetic State") OR prediabet* OR "pre diabet" OR (mh "Insulin Resistance") OR "insulin resistance" OR (mh "Glucose Intolerance") OR "glucose intolerance" OR "glucose tolerance" OR (mh "Glycated Hemoglobin A") OR "hemoglobin A1c" OR (("impaired fasting" OR (mh "Diabetes Mellitus")) N4 (glucose OR glycemi* OR "high blood sugar" OR "low blood sugar" OR (mh hyperglycemia) OR (mh hypoglycemia) OR hyperglycem* OR hypoglycem*)) OR (mh "Cardiovascular Diseases") OR cardiovascular disease* OR coronary artery disease* OR heart disease* OR(mh "Heart Failure") OR "heart failure" OR "myocardial infarction" OR (mh "Myocardial Ischemia") OR "Myocardial Ischemia" OR (mh Stroke) OR stroke OR "heart attack" OR "venous thrombosis" OR hypertens ${ }^{*}$ OR (mh "Lipids/BL") OR "total cholesterol" OR (mh Triglycerides) OR triglycerides OR (mh "arterial occlusive diseases")

\#6 - (MH "Mental Disorders+") OR "mental disorder*" OR (MH "Cognition+") OR cognition OR cognitive OR metacognition OR neurocognitive OR neurodevelop* OR neurological OR "cognitive dysfunction" OR "depressive disorders OR (MH "Depression") OR depression OR (MH "Anxiety") OR anxiety OR (MH "Psychomotor Performance") OR motor skill* OR (MH "Executive Function") OR executive function* OR (MH "Attention Deficit Hyperactivity Disorder") OR attention deficit disorder* OR ADHD OR (MH "Child 
Behavior Disorders") OR developmental disorder* OR (MH "Autistic Disorder") OR autism OR Asperger OR "language processing" OR language delay* OR (MH "Child Development") OR child develop* OR (MH "Developmental Disabilities") OR developmental delay* OR developmental disabilit* OR (MH "Motor Skills Disorders") OR motor skill* OR (MH "Problem Solving") OR developmental domain* OR "academic performance" OR "academic achievement" OR "academic failure" OR academic success*

\#7 - \#2 OR \#3 OR \#4 OR \#5 OR \#6

\#8 - \#1 AND \#7

\#9 - \#8 NOT ( MH "Literature Review" OR MH "Meta Analysis" OR MH "Systematic Review" OR MH "News" OR MH "Retracted Publication" OR MH "Retraction of Publication ) 


\section{LITERATURE SEARCH AND SCREENING RESULTS}

\section{Figure 2. Pregnancy and Birth to 24 Months Project literature search ${ }^{x v i}$}

The flow chart below illustrates the search and screening results for the first literature search. The results of the electronic database searches, after the removal of duplicates ${ }^{\text {xvii }}$, were screened independently by 2 NESR analysts using a step-wise process by reviewing titles and abstracts together, followed by full-texts, to determine which articles met the inclusion criteria. Refer to Table 12 for the rationale for exclusion for each excluded full-text article. A manual search was done to find articles that were not identified when searching the electronic databases. Manually identified articles were also screened to determine whether they met criteria for inclusion.

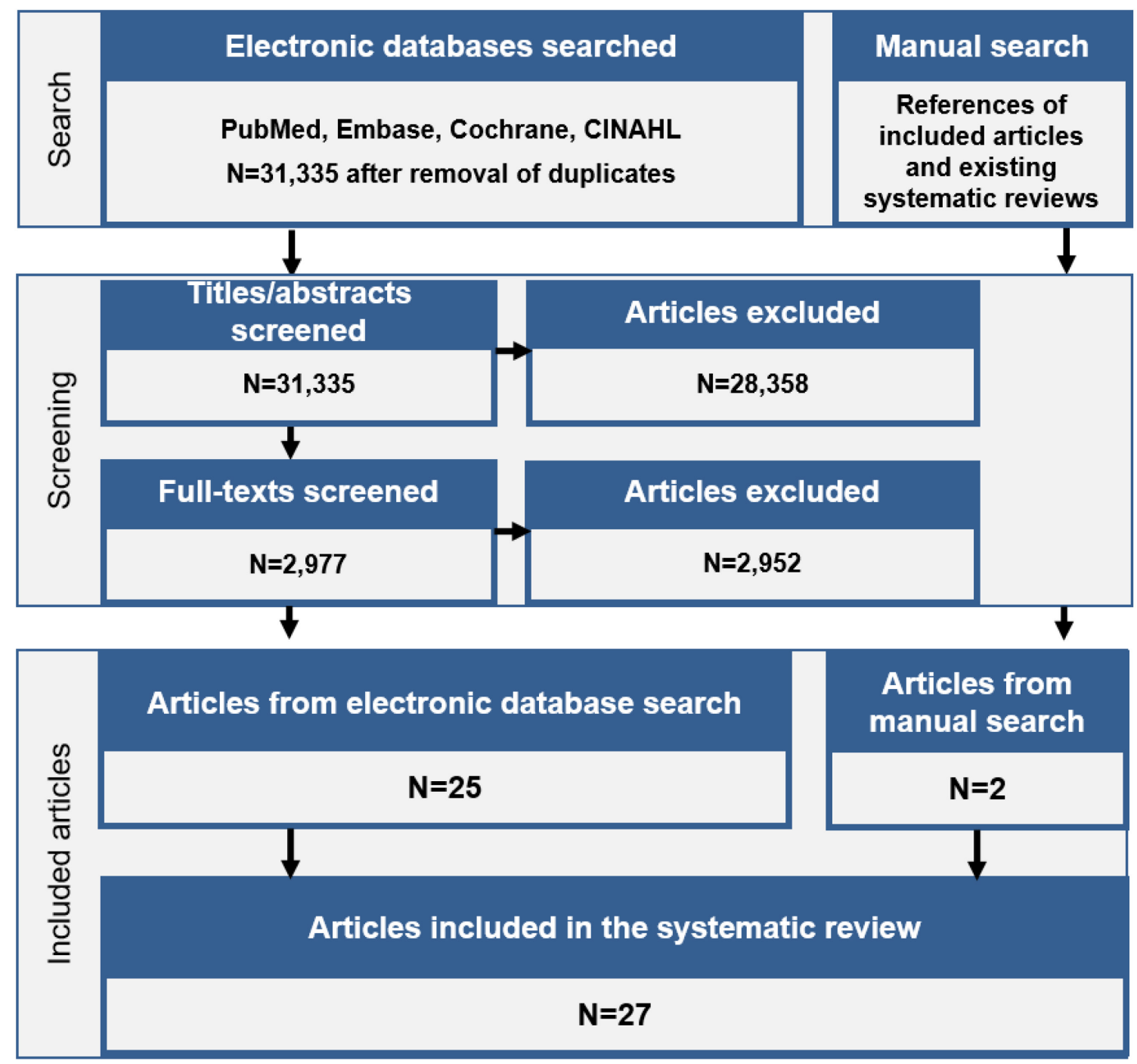

xvi During the Pregnancy and Birth to 24 Months (P/B-24) Project, systematic review questions were defined to examine the relationships between human milk and infant formula consumption and several outcomes, and NESR used a single literature search to identify potential studies for the family of reviews (https://nesr.usda.gov/infant-milk-feeding-practicestechnical-expert-collaborative). Some of the intended reviews, including micronutrient status, were not completed before the end of the Project. The 2020 Dietary Guidelines Advisory Committee, Birth to 24 Months Subcommittee, used and updated the literature search and screening underway from the P/B-24 Project according to the inclusion and exclusion criteria described herein.

xvii The number of records the literature yielded prior to the removal of duplicates is unavailable. 


\section{Figure 3. Update to the Pregnancy and Birth to 24 Months Project literature search}

The flow chart below illustrates the search and screening results for the second literature search. The results of the electronic database searches, after removal of duplicates, were screened independently by 2 NESR analysts using a step-wise process by reviewing titles, followed by abstracts, followed by full-texts, to determine which articles met the inclusion criteria. Refer to Table 13 for the rationale for exclusion for each excluded full-text article. A manual search was done to find articles that were not identified when searching the electronic databases. Manually identified articles were also screened to determine whether they met criteria for inclusion.

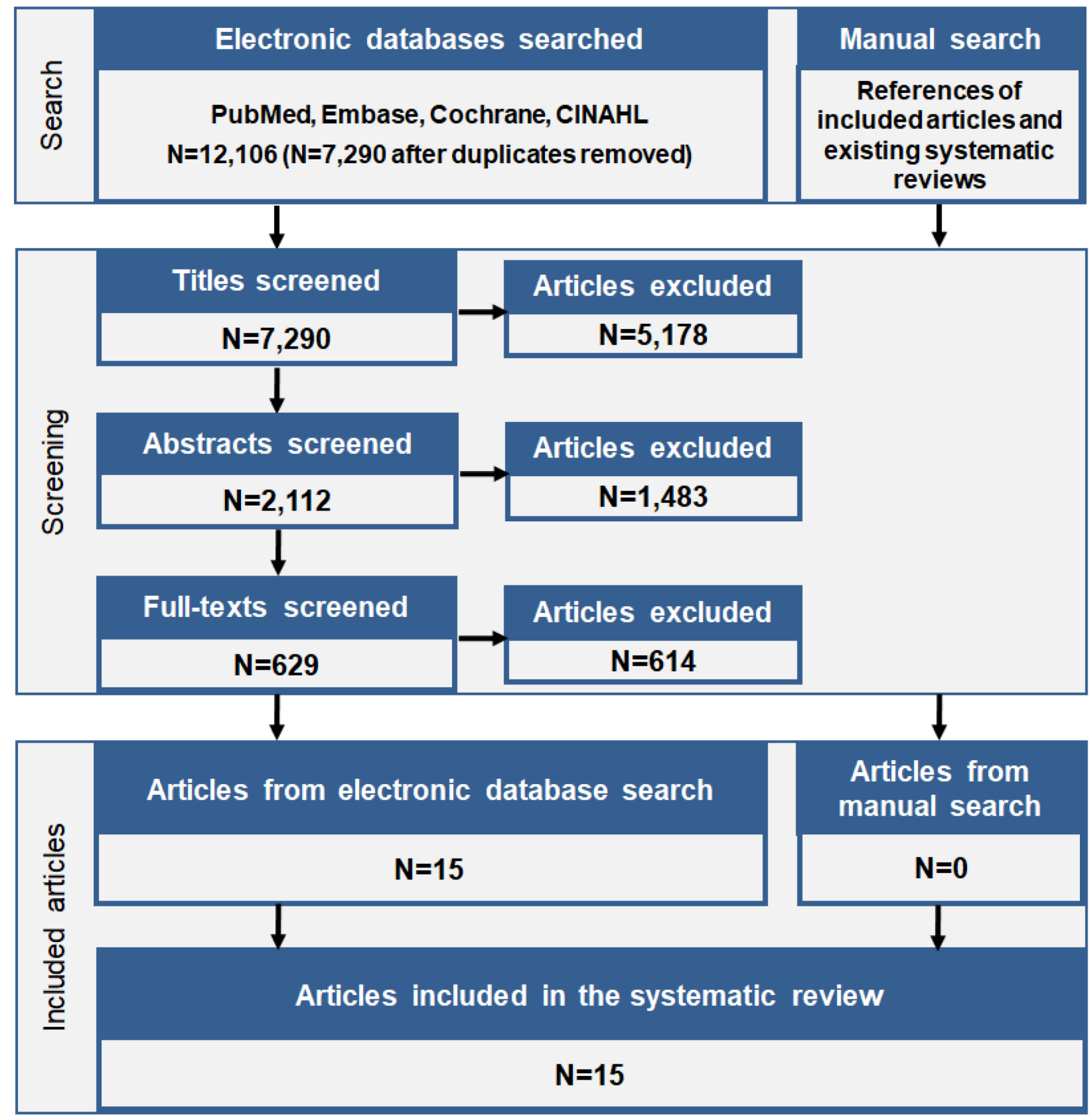




\section{Excluded articles}

\section{Table 12. Full-text exclusions from the Pregnancy and Birth to 24 Months Project literature search ${ }^{x v i i i}$}

The table below lists the articles excluded after full-text screening of the Pregnancy and Birth to 24 Months Project literature search. At least one reason for exclusion is provided for each article, though this may not reflect all possible reasons for exclusion. Information about articles excluded after title and abstract screening is available upon request.

\section{Full-text article screened}

1 Aarts, C.,Kylberg, E.,Hofvander, Y.,Gebre-Medhin, M. (2003). Growth under privileged conditions of healthy Swedish infants exclusively breastfed from birth to 4-6 months: a longitudinal prospective study based on daily records of feeding Acta Paediatr, 92(2), $145-51$

2 Abarin, T.,Yan Wu, Y.,Warrington, N.,Lye, S.,Pennell, C.,Briollais, L. (2012). The impact of breastfeeding on FTO-related BMI growth trajectories: an application to the Raine pregnancy cohort study Int J Epidemiol, 41(6), 1650-60

Abdel-Hafeez, E. H.,Belal, U. S.,Abdellatif, M. Z. M.,Naoi, K.,Norose, K. (2013). Breast-feeding protects infantile diarrhea caused by intestinal protozoan infections Korean Journal of Parasitology, 51(5), 519-524 Abdoll, G. S. (2001). Report on the nursing bottle caries campaign launched by the Free State Oral Health Services Sadj, 56(1), 32- Study design
3

5 Abdulmoneim, I.,Al-Ghamdi, S. A. (2001). Relationship between breast-feeding duration and acute respiratory infections in infants Saudi Med J, 22(4), 347-50

6 Abdul-Razzak, K. K.,Ajlony, M. J.,Khoursheed, A. M.,Obeidat, B. A. (2011). Vitamin D deficiency among healthy infants and toddlers: a prospective study from Irbid, Jordan Pediatr Int, 53(6), 839-45

\section{Reason for exclusion}

Size of study groups, Intervention/exposure

Intervention/exposure

Participant health

Study design, Participant health

Study design,

Intervention/exposure

7

Aberg, N.,Engstrom, I.,Lindberg, U. (1989). Allergic diseases in Swedish school children Acta Paediatr Scand, 78(2), 246-52

Study design

Abuekteish, F.,Alwash, R.,Hassan, M.,Daoud, A. S. (1996). Prevalence of asthma and wheeze in primary school children in northern Study design Jordan Ann Trop Paediatr, 16(3), 227-31

Abusaad, Fawzia E.,El-Gilany, Abdel-Hady (2011). Exclusive breastfeeding and infant morbidity in Sakaka City, Saudi Arabia Middle Intervention/exposure, Outcome East Journal of Nursing, 5(6), 3-8 6p

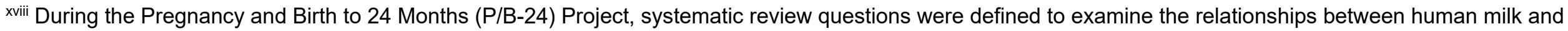
infant formula consumption and several outcomes, and NESR used a single literature search to identify potential studies for the family of reviews

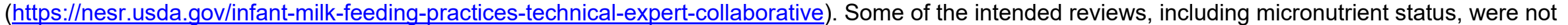

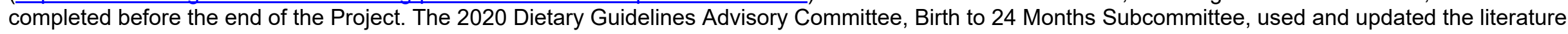
search and screening underway from the P/B-24 Project according to the inclusion and exclusion criteria described herein. 
10 Adgent, M. A.,Hoffman, K.,Goldman, B. D.,Sjodin, A.,Daniels, J. L. (2014). Brominated flame retardants in breast milk and behavioural and cognitive development at 36 months Paediatr Perinat Epidemiol, 28(1), 48-57

11 Adlakha, A. L.,Suchindran, C. M. (1985). Factors affecting infant and child mortality J Biosoc Sci, 17(4), 481-96

Study design

12 Agache, I.,Ciobanu, C. (2010). Risk factors and asthma phenotypes in children and adults with seasonal allergic rhinitis Phys Sportsmed, 38(4), 81-6

Study design, Size of study groups

13 Agarwal, D. K.,Agarwal, K. N.,Khare, B. B. (1985). Study on current status of infant and childhood feeding practices Indian Pediatr 22(9), 716

14 Agostoni, C. (2001). Breast-feeding, human milk, long-chain polyunsaturated fatty acids and development Dev Med Child Neurol Suppl, 86(\#issue\#), 8-9

15 Agostoni, C.,Fiocchi, A.,Riva, E.,Terracciano, L.,Sarratud, T.,Martelli, A.,Lodi, F.,D'Auria, E.,Zuccotti, G.,Giovannini, M. (2007) Growth of infants with IgE-mediated cow's milk allergy fed different formulas in the complementary feeding period Pediatr Allergy Immunol, 18(7), 599-606

16 Agostoni, C.,Grandi, F.,Gianni, M. L.,Silano, M.,Torcoletti, M.,Giovannini, M.,Riva, E. (1999). Growth patterns of breast fed and formula fed infants in the first 12 months of life: an Italian study Arch Dis Child, 81(5), 395-9

Country, Study design

Study design

Participant health Intervention/exposure

Publication date for a non-sibling study

17 Agostoni, C.,Grandi, F.,Scaglioni, S.,Gianni, M. L.,Torcoletti, M.,Radaelli, G.,Fiocchi, A.,Riva, E. (2000). Growth pattern of breastfed Intervention/exposure and nonbreastfed infants with atopic dermatitis in the first year of life Pediatrics, 106(5), E73

18 Agostoni, C.,Marangoni, F.,Giovannini, M.,Galli, C.,Riva, E. (2001). Prolonged breast-feeding (six months or more) and milk fat content at six months are associated with higher developmental scores at one year of age within a breast-fed population Adv Exp Med Biol, 501(\#issue\#), 137-41

19 Agostoni, C.,Marangoni, F.,Lammardo, A. M.,Giovannini, M.,Riva, E.,Galli, C. (2001). Breastfeeding duration, milk fat composition and developmental indices at 1 year of life among breastfed infants Prostaglandins Leukot Essent Fatty Acids, 64(2), 105-9

20 Agostoni, C.,Riva, E.,Bellu, R.,Trojan, S.,Luotti, D.,Giovannini, M. (1994). Effects of diet on the lipid and fatty acid status of full-term Size of study groups infants at 4 months J Am Coll Nutr, 13(6), 658-64

21 Agostoni, C., Trojan, S.,Bellu, R.,Riva, E.,Giovannini, M. (1995). Neurodevelopmental quotient of healthy term infants at 4 months and feeding practice: the role of long-chain polyunsaturated fatty acids Pediatr Res, 38(2), 262-6

Agras, W. S.,Kraemer, Pediatr, 116(5), 805-9

23 Agras, W. S.,Kraemer, H. C.,Berkowitz, R. I.,Korner, A. F.,Hammer, L. D. (1987). Does a vigorous feeding style influence early development of adiposity? J Pediatr, 110(5), 799-804

24

Agre, F. (1985). The relationship of mode of infant feeding and location of care to frequency of infection Am J Dis Child, 139(8), $809-11$

Size of study groups

Outcome

Outcome

Size of study groups

Intervention/exposure

Intervention/exposure 
26 Ahn, S. K.,Kam, S.,Chun, B. Y. (2014). Incidence of and factors for self-reported fragility fractures among middle-aged and elderly women in rural Korea: An 11-year follow-up study Journal of Preventive Medicine and Public Health, 47(6), 289-297

Participant age

Ajetunmobi, O. M.,Whyte, B.,Chalmers, J.,Tappin, D. M.,Wolfson, L.,Fleming, M.,MacDonald, A.,Wood, R.,Stockton, D. L. (2015). Breastfeeding is associated with reduced childhood hospitalization: evidence from a Scottish Birth Cohort (1997-2009) J Pediatr, 166(3), $620-5$ e4

28 Ajrouche, R.,Rudant, J.,Orsi, L.,Petit, A.,Baruchel, A.,Lambilliotte, A.,Gambart, M.,Michel, G.,Bertrand, Y.,Ducassou, S.,Gandemer, V.,Paillard, C.,Saumet, L.,Blin, N.,Hemon, D.,Clavel, J. (2015). Childhood acute lymphoblastic leukaemia and indicators of early immune stimulation: the Estelle study (SFCE) Br J Cancer, 112(6), 1017-26

29 Akeson, P. K.,Axelsson, I. E.,Raiha, N. C.,Warm, A.,Minoli, I.,Moro, G. (2000). Fat intake and metabolism in Swedish and Italian infants Acta Paediatr, 89(1), 28-33

$30 \quad$ Akeson, P. M.,Axelsson, I. E.,Raiha, N. C. (1998). Growth and nutrient intake in three- to twelve-month-old infants fed human milk or

formulas with varying protein concentrations J Pediatr Gastroenterol Nutr, 26(1), 1-8

Intervention/exposure

Intervention/exposure, Size of study groups

31 Akeson, P. M.,Axelsson, I. E.,Raiha, N. C. (1999). Plasma lipids and apolipoproteins in breastfed and formula-fed Swedish infants Acta Paediatr, 88(1), 1-6

32 Akkus, Z.,Camdeviren, H.,Celik, F.,Gur, A.,Nas, K. (2005). Determination of osteoporosis risk factors using a mutiple logistic regression model in postmenopausal Turkish women Saudi Medical Journal, 26(9), 1351-1359

Outcome

33 Al Mamun, A.,O'Callaghan, M. J.,Williams, G. M.,Najman, J. M.,Callaway, L.,Mclntyre, H. D. (2015). Breastfeeding is protective to Outcome diabetes risk in young adults: a longitudinal study Acta Diabetol, 52(5), 837-44 Al-Abbad, A. A.,Bella, H. (1990). Diarrhoea in the under-fives in a Saudi semiurban community Tropical and Geographical Medicine,
42(3), 233-237 al-Ali, F. M.,Hossain, M. M.,Pugh, R. N. (1997). The associations between feeding modes and diarrhoea among urban children in a newly developed country Public Health, 111(4), 239-43

36 Alaluusua, S.,Lukinmaa, P. L.,Koskimies, M.,Pirinen, S.,Holtta, P.,Kallio, M.,Holttinen, T.,Salmenpera, L. (1996). Developmental dental defects associated with long breast feeding Eur J Oral Sci, 104(5-6), 493-7

37 Alaluusua, S.,Myllarniemi, S.,Kallio, M.,Salmenpera, L.,Tainio, V. M. (1990). Prevalence of caries and salivary levels of mutans streptococci in 5-year-old children in relation to duration of breast feeding Scand J Dent Res, 98(3), 193-6

Outcome

Intervention/exposure

Participant age

Study design

Intervention/exposure

Size of study groups

Outcome

(1)

38 Alam, S.,Ahmad, S. A.,Kumar, S. (2001). Dietary regimen for persistent diarrhea in infants under four months Indian Pediatr, 38(4), Country 396-400 

Saudi J Kidney Dis Transpl, 20(2), 260-5

40 Alati, R.,Van Dooren, K.,Najman, J. M.,Williams, G. M.,Clavarino, A. (2009). Early weaning and alcohol disorders in offspring: biological effect, mediating factors or residual confounding? Addiction, 104(8), 1324-32

41 Albert, R. J.,Cantin, R. Y.,Cross, H. G.,Castaldi, C. R. (1988). Nursing caries in the Inuit children of the Keewatin J Can Dent Assoc, $54(10), 751-8$ al-Dashti, A. A.,Williams, S. A.,Curzon, M. E. (1995). Breast feeding, bottle feeding and dental caries in Kuwait, a country with lowfluoride levels in the water supply Community Dent Health, 12(1), 42-7

43 Alderete, T. L.,Autran, C.,Brekke, B. E.,Knight, R.,Bode, L., Goran, M. I.,Fields, D. A. (2015). Associations between human milk oligosaccharides and infant body composition in the first 6 mo of life Am J Clin Nutr, 102(6), 1381-8

44 Alexander, D. A. (2003). Breastfeeding study needs to be viewed in context...'Breastfeeding may increase the risk of asthma and allergies' (Specialty News Bulletin, December 2002) RN, 66(4), 10-10 1p

45 Alexander, E. S.,Martin, L. J.,Collins, M. H.,Kottyan, L. C.,Sucharew, H.,He, H.,Mukkada, V. A.,Succop, P. A.,Abonia, J. P.,Foote, H.,Eby, M. D.,Grotjan, T. M.,Greenler, A. J.,Dellon, E. S.,Demain, J. G.,Furuta, G. T.,Gurian, L. E.,Harley, J. B.,Hopp, R. J.,Kagalwalla, A.,Kaul, A.,Nadeau, K. C.,Noel, R. J.,Putnam, P. E.,von Tiehl, K. F.,Rothenberg, M. E. (2014). Twin and family studies reveal strong environmental and weaker genetic cues explaining heritability of eosinophilic esophagitis $\mathrm{J}$ Allergy Clin Immunol, 134(5), 1084-1092 e1 Alexy, U.,Kersting, M.,Sichert-Hellert, W.,Manz, F.,Schoch, G. (1998). Energy intake and growth of 3- to 36-month-old German infants and children Ann Nutr Metab, 42(2), 68-74 Effect of suboptimal breast-feeding on occurrence of autism: a case-control study Nutrition, 28(7-8), e27-32

48 Alho, O. P.,Koivu, M.,Sorri, M.,Rantakallio, P. (1990). Risk factors for recurrent acute otitis media and respiratory infection in infancy Outcome Int J Pediatr Otorhinolaryngol, 19(2), 151-61

49 Alho, O. P.,Laara, E.,Oja, H. (1996). Public health impact of various risk factors for acute otitis media in northern Finland Am J Epidemiol, 143(11), 1149-56

50 Alho, O. P.,Laara,,Oja, H. (1996). How should relative risk estimates for acute otitis media in children aged less than 2 years be perceived? J Clin Epidemiol, 49(1), 9-14

51 Ali, M. B.,Ghenghesh, K. S.,Aissa, R. B.,Abuhelfaia, A.,Dufani, M. (2005). Etiology of childhood diarrhea in Zliten, Libya Saudi Med J, 26(11), 1759-65

52 Al-Jassir, M. S.,El-Bashir, B. M.,Moizuddin, S. K. (2004). Surveillance of infant feeding practices in Riyadh city Ann Saudi Med, 24(2), $136-40$

Intervention/exposure

Publication status

\section{Study design, Outcome}

Outcome

Intervention/exposure

Study design, Participant health

Study design, Outcome

Study design 
55 Allen, N. B.,Lewinsohn, P. M.,Seeley, J. R. (1998). Prenatal and perinatal influences on risk for psychopathology in childhood and adolescence Dev Psychopathol, 10(3), 513-29

56 Alliet, P.,Scholtens, P.,Raes, M.,Hensen, K.,Jongen, H.,Rummens, J. L.,Boehm, G.,Vandenplas, Y. (2007). Effect of prebiotic galacto-oligosaccharide, long-chain fructo-oligosaccharide infant formula on serum cholesterol and triacylglycerol levels Nutrition, 23(10), $719-23$ Alm, B.,Aberg, N.,Erdes, L.,Mollborg, P.,Pettersson, R.,Norvenius, S. G.,Goksor, E.,Wennergren, G. (2009). Early introduction of fish decreases the risk of eczema in infants Arch Dis Child, 94(1), 11-5

58 Alm, B.,Erdes, L.,Mollborg, P.,Pettersson, R.,Norvenius, S. G.,Aberg, N.,Wennergren, G. (2008). Neonatal antibiotic treatment is a Outcome risk factor for early wheezing Pediatrics, 121(4), 697-702

59 Alm, B.,Norvenius, S. G.,Wennergren, G.,Lagercrantz, H.,Helweg-Larsen, K.,Irgens, L. M. (2000). Living conditions in early infancy Study design in Denmark, Norway and Sweden 1992-95: results from the Nordic Epidemiological SIDS study Acta Paediatr, 89(2), 208-14

60 Alm, B.,Wennergren, G.,Norvenius, S. G.,Skjaerven, R.,Lagercrantz, H.,Helweg-Larsen, K.,Irgens, L. M. (2002). Breast feeding and the sudden infant death syndrome in Scandinavia, 1992-95 Arch Dis Child, 86(6), 400-2

61 Almeida, R. M.,De Marins, V. M.,Valle, J. (1999). Breastfeeding, socio-economic conditions and nutritional status of children younger than 12 months in Brazil Ann Trop Paediatr, 19(3), 257-62

62 Al-Mousawi, M. S.,Lovel, H.,Behbehani, N.,Arifhodzic, N.,Woodcock, A.,Custovic, A. (2004). Asthma and sensitization in a community with low indoor allergen levels and low pet-keeping frequency J Allergy Clin Immunol, 114(6), 1389-94

Outcome

Study design

Outcome

Almquist-Tangen, G.,Dahlgren, J.,Roswall, J.,Bergman, S.,Alm, B. (2013). Milk cereal drink increases BMI risk at 12 and 18 months, Intervention/exposure but formula does not Acta Paediatr, 102(12), 1174-9

Al-Mustafa, Z. H.,Al-Madan, M.,Al-Majid, H. J.,Al-Muslem, S.,Al-Ateeq, S.,Al-Ali, A. K. (2007). Vitamin D deficiency and rickets in the
Eastern Province of Saudi Arabia Ann Trop Paediatr, 27(1), 63-7

Alper, C. M.,Winther, B.,Hendley, J. O.,Doyle, W. J. (2009). Cytokine polymorphisms predict the frequency of otitis media as a

\section{Outcome}

Outcome complication of rhinovirus and RSV infections in children Eur Arch Otorhinolaryngol, 266(2), 199-205

66

Alper, C. M.,Winther, B.,Mandel, E. M.,Hendley, J. O.,Doyle, W. J. (2009). Rate of concurrent otitis media in upper respiratory tract

Study design infections with specific viruses Arch Otolaryngol Head Neck Surg, 135(1), 17-21

Al-Qaoud, N.,Prakash, P. (2009). Breastfeeding and obesity among Kuwaiti preschool children Medical Principles and Practice, 18(2), 111-117

Al-Qaoud, N.,Prakash, P. (2009). 'Can breastfeeding and its duration determine the overweight status of Kuwaiti children at the age of 3-6 years?' Eur J Clin Nutr, 63(8), 1041-3 
Al-Shehri, M. A.,Sadeq, A.,Quli, K. (2005). Bronchiolitis in Abha, Southwest Saudi Arabia: viral etiology and predictors for hospital admission West Afr J Med, 24(4), 299-304

70 Al-Shehri, S. S.,Knox, C. L.,Liley, H. G.,Cowley, D. M.,Wright, J. R.,Henman, M. G.,Hewavitharana, A. K.,Charles, B. G.,Shaw, P. N.,Sweeney, E. L.,Duley, J. A. (2015). Breastmilk-Saliva Interactions Boost Innate Immunity by Regulating the Oral Microbiome in Early Infancy PLoS One, 10(9), e0135047

71 Althaus, B. W. (1999). Growth patterns of Hispanic and Caucasian children \#journal\#, Ph.D.(\#issue\#), 105 p-105 p 1p

Publication status

72 Altinkaynak, S.,Selimoglu, M. A.,Turgut, A.,Kilicaslan, B.,Ertekin, V. (2006). Breast-feeding duration and childhood acute leukemia and lymphomas in a sample of Turkish children Journal of Pediatric Gastroenterology and Nutrition, 42(5), 568-572

73 Altucher, K.,Rasmussen, K. M.,Barden, E. M.,Habicht, J. P. (2005). Predictors of improvement in hemoglobin concentration among toddlers enrolled in the Massachusetts WIC Program J Am Diet Assoc, 105(5), 709-15

74 Alvarado, B. E.,Zunzunegui, M. V.,Delisle, H.,Osorno, J. (2005). Growth trajectories are influenced by breast-feeding and infant health in an afro-colombian community $\mathrm{J}$ Nutr, 135(9), 2171-8

75 Alvarado, R.,Zepeda, A.,Rivero, S.,Rico, N.,Lopez, S.,Diaz, S. (1999). Integrated maternal and infant health care in the postpartum period in a poor neighborhood in Santiago, Chile Stud Fam Plann, 30(2), 133-41

Study design,

Intervention/exposure

Intervention/exposure

Alves, 484-7 Alves, J. G.,Figueiroa, J. N.,Meneses, J.,Alves, G. V. (2012). Breastfeeding protects against type 1 diabetes mellitus: a case-sibling study Breastfeed Med, 7(1), 25-8

78 Amador, M.,Hermelo, M. P.,Canetti, J. E.,Consuegra, E. (1992). Adolescent mothers: do they breast-feed less? Acta Paediatr Hung, Study design 32(3), 269-85

79 Amador-Licona, N.,Martinez-Cordero, C., Guizar-Mendoza, J. M.,Malacara, J. M.,Hernandez, J.,Alcala, J. F. (2007). Catch-up growth Study design in infants born small for gestational age--a longitudinal study J Pediatr Endocrinol Metab, 20(3), 379-86

80 Amaratunge, A.,Ekanayake, S. L. (1984). Rampant caries in Sri Lankan children. A pilot study Odontostomatol Trop, 7(3), 133-8

81 Amigo, H.,Bustos, P.,Leone, C.,Radrigán, M. E. (2001). Community and international nutrition: Growth deficits in Chilean schoo Intervention/exposure children Journal of Nutrition, 131(2), 251-254 Amorim Rde, J.,Coelho, A. F.,de Lira, P. I.,Lima Mde, C. (2014). Is breastfeeding protective for blood pressure in schoolchildren? A Outcome cohort study in northeast Brazil Breastfeed Med, 9(3), 149-56

83 Ananthakrishnan, S.,Bhat, B. V.,Puri, R. K.,Srinivasan, S. (1992). Loose stools in the early neonatal period Indian Pediatr, 29(8), 1005-9

Ancona, J.,Shaker, C. S.,Puhe J Nurs Care Qual, 12(5), 1-4 

and offspring dietary patterns at 9 months of age Eur $\mathrm{J}$ Clin Nutr, 69(6), 668-75

87 Anderson, G. H.,Morson-Pasut, L. A.,Bryan, H.,Cleghorn, G.,Tanaka, P.,Yeung, D.,Zimmerman, B. (1985). Age of introduction of Study design cow's milk to infants J Pediatr Gastroenterol Nutr, 4(5), 692-8

88 Anderson, J. E.,Marks, J. S.,Park, T. K. (1984). Breast-feeding, birth interval, and infant health Pediatrics, 74(4 Pt 2), 695-701

Study design

89 Anderson, K. (2001). The sweet and sour of pediatric caries CDS Rev, 94(7), 16-9

Study design

90 Anderson, L. J.,Parker, R. A.,Strikas, R. A.,Farrar, J. A.,Gangarosa, E. J.,Keyserling, H. L.,Sikes, R. K. (1988). Day-care center attendance and hospitalization for lower respiratory tract illness Pediatrics, 82(3), 300-308

91 Anderson, P. O.,Valdes, V. (2015). Variation of milk intake over time: clinical and pharmacokinetic implications Breastfeed Med, 10(3), $142-4$

92 Andersson, M.,Aeberli, I.,Wust, N.,Piacenza, A. M.,Bucher, T.,Henschen, I.,Haldimann, M.,Zimmermann, M. B. (2010). The Swiss iodized salt program provides adequate iodine for school children and pregnant women, but weaning infants not receiving iodinecontaining complementary foods as well as their mothers are iodine deficient J Clin Endocrinol Metab, 95(12), 5217-24

93 Andreev, A.,Arjas, E. (1998). Acute middle ear infection in small children: a Bayesian analysis using multiple time scales Lifetime Data Anal, 4(2), 121-37

94 Andres, A.,Casey, P. H.,Cleves, M. A.,Badger, T. M. (2013). Body fat and bone mineral content of infants fed breast milk, cow's milk formula, or soy formula during the first year of life J Pediatr, 163(1), 49-54

95 Andres, A.,Cleves, M. A.,Bellando, J. B.,Pivik, R. T.,Casey, P. H.,Badger, T. M. (2012). Developmental status of 1-year-old infants fed breast milk, cow's milk formula, or soy formula Pediatrics, 129(6), 1134-40

96 Anfield, L. (1985). Nutrition in the first year Midwife Health Visit Community Nurse, 21(5), 161-4

Study design

97 Angelsen, N. K.,Vik, T.,Jacobsen, G.,Bakketeig, L. S. (2001). Breast feeding and cognitive development at age 1 and 5 years Arch Outcome Dis Child, 85(3), 183-8

98 Angulo, N.,de Szarvas, S. B.,Guevara, H.,Mathison, Y.,González, D.,Hernández, A. (2014). Lifestyle of a group of obese children located in Valencia Salus, 18(1), 25-31

99 Angurana, S. K.,Angurana, R. S.,Mahajan, G.,Kumar, N.,Mahajan, V. (2014). Prevalence of vitamin D deficiency in apparently healthy children in north India J Pediatr Endocrinol Metab, 27(11-12), 1151-6

100 Anholm, P. C. (1986). Breastfeeding: a preventive approach to health care in infancy Issues Compr Pediatr Nurs, 9(1), 1-10

101 Aniansson, G.,Alm, B.,Andersson, B.,Hakansson, A.,Larsson, P.,Nylen, O.,Peterson, H.,Rigner, P.,Svanborg, M.,Sabharwal, H.,et

Outcome

al., (1994). A prospective cohort study on breast-feeding and otitis media in Swedish infants Pediatr Infect Dis J, 13(3), 183-8 
102 Annamalay, A. A.,Khoo, S. K.,Jacoby, P.,Bizzintino, J.,Zhang, G.,Chidlow, G.,Lee, W. M.,Moore, H. C.,Harnett, G. B.,Smith, D. W.,Gern, J. E.,LeSouef, P. N.,Laing, I. A.,Lehmann, D. (2012). Prevalence of and risk factors for human rhinovirus infection in healthy aboriginal and non-aboriginal Western Australian children Pediatr Infect Dis J, 31(7), 673-9 of factors associated with infant mortality in South-East of Iran J Pak Med Assoc, 64(10), 1123-6

104 Apostolopoulos, K.,Xenelis, J.,Tzagaroulakis, A.,Kandiloros, D.,Yiotakis, J.,Papafragou, K. (1998). The point prevalence of otitis media with effusion among school children in Greece International Journal of Pediatric Otorhinolaryngology, 44(3), $207-214$

105 Apps, J. R.,Beattie, R. M. (2009). Cow's milk allergy in children BMJ, 339(\#issue\#), b2275

Study design

106 Araujo, C. L., Victora, C. G.,Hallal, P. C.,Gigante, D. P. (2006). Breastfeeding and overweight in childhood: evidence from the Pelotas 1993 birth cohort study Int J Obes (Lond), 30(3), 500-6

107 Araujo, D. S.,Marquezin, M. C.,Barbosa, T. S., Gaviao, M. B.,Castelo, P. M. (2015). Evaluation of masticatory parameters in overweight and obese children Eur J Orthod, \#volume\#(\#issue\#), \#Pages\#

108 Arica, S.,Arica, V.,Dag, H.,Kaya, A.,Hatipoglu, S.,Fenercioglu, A.,Karatekin, G. (2011). Serum zinc levels in children of 0-24 months diagnosed with pneumonia admitted to our clinic International Journal of Clinical and Experimental Medicine, 4(3), 227-233

Outcome

Study design

Publication date for a non-sibling study

Study design

Participant health, Intervention/exposure, Size of study groups

Study design

109 Arimond, M.,Daelmans, B.,Dewey, K. (2008). Indicators for feeding practices in children Lancet, 371(9612), 541-2

110 Aris, I. M.,Soh, S. E.,Tint, M. T.,Saw, S. M.,Rajadurai, V. S., Godfrey, K. M.,Gluckman, P. D.,Yap, F.,Chong, Y. S.,Lee, Y. S. (2015).

Intervention/exposure

Associations of infant milk feed type on early postnatal growth of offspring exposed and unexposed to gestational diabetes in utero Eur J Nutr, \#volume\#(\#issue\#), \#Pages\#

111 Arlette, J. P. (1982). Zinc deficiency in children Int J Dermatol, 21(8), 447-8

Study design

112 Armstrong, J.,Reilly, J. J. (2002). Breastfeeding and lowering the risk of childhood obesity Lancet, 359(9322), 2003-4

Publication date for a non-sibling study

113 Arnon, S. S.,Damus, K.,Thompson, B.,Midura, T. F.,Chin, J. (1982). Protective role of human milk against sudden death from infant botulism J Pediatr, 100(4), 568-73

Size of study groups, Intervention/exposure

114 Aronsson, C. A.,Lee, H. S.,Koletzko, S.,Uusitalo, U.,Yang, J.,Virtanen, S. M.,Liu, E.,Lernmark, A.,Norris, J. M.,Agardh, D. (2015) Effects of Gluten Intake on Risk of Celiac Disease: A Case-Control Study on a Swedish Birth Cohort Clin Gastroenterol Hepatol, \#volume\#(\#issue\#), \#Pages\#

115 Arora, N. K.,Bhan, M. K. (1991). Nutritional management of acute diarrhea Indian J Pediatr, 58(6), 763-7

116 Arshad, S. H.,Bateman, B.,Matthews, S. M. (2003). Primary prevention of asthma and atopy during childhood by allergen avoidance Intervention/exposure in infancy: a randomised controlled study Thorax, 58(6), 489-93 
117 Arshad, S. H.,Bateman, B.,Sadeghnejad, A., Gant, C.,Matthews, S. M. (2007). Prevention of allergic disease during childhood by allergen avoidance: the Isle of Wight prevention study J Allergy Clin Immunol, 119(2), 307-13

118 Arton M (1985). Breast feeding--a life-saver in the Third World Midwives Chron, 98(\#issue\#), 200-1

Intervention/exposure

Study design

119 Aryayev, N.,Kukushkin, V. (2002). The perinatal risk factors of sudden infant death syndrome Perinatology, 4(3), 125-133

Publication status

120 Aryayev, N.,Kukushkin, V.,Nepomyashcha, V. (2001). The significance of ante- and perinatal periods for formation of risk of sudden infant death syndrome Ginekologia polska, 72(12), 931-939

121 Asaka, A.,Imaizumi, Y.,Inouye, E. (1981). Analysis of multiple births in Japan. V. Effects of gestational age, maternal age and other factors on growth rate of weight in twins Jinrui Idengaku Zasshi, 26(2), 83-90

122 Ascher, H.,Krantz, I.,Rydberg, L.,Nordin, P.,Kristiansson, B. (1997). Influence of infant feeding and gluten intake on coeliac disease Size of study groups Arch Dis Child, 76(2), 113-7

123 Asha Bai, P. V.,Leela, M.,Subramaniam, V. R. (1980). Adequacy of breast milk for optimal growth of infants Trop Geogr Med, 32(2), Country $158-62$

124 Ashraf, A. P.,Eason, N. B.,Kabagambe, E. K.,Haritha, J.,Meleth, S.,McCormick, K. L. (2010). Dietary iron intake in the first 4 mont of infancy and the development of type 1 diabetes: A pilot study Diabetology and Metabolic Syndrome, 2(1), \#Pages\#

125 Askie, L.,Martin, A.,Espinoza, D.,Campbell, K.,Daniels, L. A.,Hesketh, K.,Margarey, A.,Rissel, C.,Taylor, B., Taylor, R.,Wen, L. M.,Baur, L. A. (2014). What does the EPOCH (early prevention of obesity in childhood) prospective meta-analysis tell us about early life obesity prevention? Obesity research \& clinical practice, 8(\#issue\#), 3-4

126 Assuncao, M. L.,Ferreira, H. S., Coutinho, S. B.,Santos, L. M.,Horta, B. L. (2015). Protective effect of breastfeeding against overweight can be detected as early as the second year of life: a study of children from one of the most socially-deprived areas of Brazil J Health Popul Nutr, 33(1), 85-91

127 Astarita, C., Harris, R. I.,de Fusco, R.,Franzese, A.,Biscardi, D.,Mazzacca, F. R.,Altucci, P. (1988). An epidemiological study of atopy Study design in children Clin Allergy, 18(4), 341-50 from the Melbourne Infant Feeding, Activity and Nutrition Trial (InFANT) Program Br J Nutr, \#volume\#(\#issue\#), 1-9

129 Atladottir, H.,Thorsdottir, I. (2000). Energy intake and growth of infants in Iceland-a population with high frequency of breast-feeding Intervention/exposure and high birth weight Eur J Clin Nutr, 54(9), 695-701

130 Auerbach, K. G.,Renfrew, M. J.,Minchin, M. (1991). Infant feeding comparisons: a hazard to infant health? J Hum Lact, 7(2), 63-8

Study design

131 Auestad, N.,Halter, R.,Hall, R. T.,Blatter, M.,Bogle, M. L.,Burks, W.,Erickson, J. R.,Fitzgerald, K. M.,Dobson, V.,Innis, S. M.,Singer,

Intervention/exposure L. T.,Montalto, M. B.,Jacobs, J. R.,Quu, W.,Bornstein, M. H. (2001). Growth and development in term infants fed long-chain polyunsaturated fatty acids: a double-masked, randomized, parallel, prospective, multivariate study Pediatrics, 108(2), 372-81 
132 Auestad, N.,Montalto, M. B.,Hall, R. T.,Fitzgerald, K. M.,Wheeler, R. E.,Connor, W. E.,Neuringer, M.,Connor, S. L., Taylor, J.

A.,Hartmann, E. E. (1997). Visual acuity, erythrocyte fatty acid composition, and growth in term infants fed formulas with long chain polyunsaturated fatty acids for one year. Ross Pediatric Lipid Study Pediatr Res, 41(1), 1-10

133 Auestad, N.,Scott, D. T.,Janowsky, J. S.,Jacobsen, C.,Carroll, R. E.,Montalto, M. B.,Halter, R., Quu, W.,Jacobs, J. R.,Connor, W. E.,Connor, S. L.,Taylor, J. A.,Neuringer, M.,Fitzgerald, K. M.,Hall, R. T. (2003). Visual, cognitive, and language assessments at 39 months: a follow-up study of children fed formulas containing long-chain polyunsaturated fatty acids to 1 year of age Pediatrics, 112(3 Pt 1), e177-83

134 Auricchio, S.,Follo, D.,de Ritis, G.,Giunta, A.,Marzorati, D.,Prampolini, L.,Ansaldi, N.,Levi, P.,Dall'Olio, D.,Bossi, A.,et al., (1983). Does breast feeding protect against the development of clinical symptoms of celiac disease in children? J Pediatr Gastroenterol Nutr, 2(3), 428-33

135 Avoa, A.,Fischer, P. R. (1990). The influence of perinatal instruction about breast-feeding on neonatal weight loss Pediatrics, 86(2), 313-5

137 Axelsson, I. E.,Ivarsson, S. A.,Raiha, N. C. (1989). Protein intake in early infancy: effects on plasma amino acid concentrations, insulin metabolism, and growth Pediatr Res, 26(6), 614-7

138 Axelsson, I.,Borulf, S.,Righard, L.,Raiha, N. (1987). Protein and energy intake during weaning: I. Effects on growth Acta Paediatr Scand, 76(2), 321-7

139 Ayatollahi, S. M.,Sharafi, Z.,Haem, E. (2015). Child Weight Growth Chart and Its Associated Factors in Birth Cohort of Maku Using a Size of study groups Growth Curve Model and LMS Method Glob J Health Sci, 7(6), 44045

140 Aydemir, G.,Ozkurt, F. E. (2011). Otitis media with effusion in primary schools in Princes' Islands, Istanbul: Prevalence and risk factors Journal of International Medical Research, 39(3), 866-872

141 Ayer, J. G.,Belousova, E.,Harmer, J. A.,David, C.,Marks, G. B.,Celermajer, D. S. (2011). Maternal cigarette smoking is associated with reduced high-density lipoprotein cholesterol in healthy 8-year-old children Eur Heart J, 32(19), 2446-53

142 Azizi, B. H.,Zulkifli, H. I.,Kasim, M. S. (1995). Protective and risk factors for acute respiratory infections in hospitalized urban Malaysian children: a case control study Southeast Asian J Trop Med Public Health, 26(2), 280-5

Country

Size of study groups, Intervention/exposure

Size of study groups

143 Babeely, K.,Kaste, L. M.,Husain, J.,Behbehani, J.,al-Za'abi, F.,Maher, T. C.,Tavares, M.,Soparkar, P.,DePaola, P. (1989). Severity of nursing-bottle syndrome and feeding patterns in Kuwait Community Dent Oral Epidemiol, 17(5), 237-9

144 Backon, J. (1984). Prolonged breast feeding as a prophylaxis for recurrent otitis media: relevance of prostaglandins Med Hypotheses, 13(2), 161

145 Bacopoulou, F., Veltsista, A.,Vassi, I.,Gika, A.,Lekea, V.,Priftis, K.,Bakoula, C. (2009). Can we be optimistic about asthma in childhood? A Greek cohort study J Asthma, 46(2), 171-4

\title{
Study design
}

Intervention/exposure

Study design, Size of study groups

Study design, Intervention/exposure

Publication status Intervention/exposure 
147 Badger, Thomas M. (2014). STUDY SUGGESTS SOY FORMULA MAY BE GOOD CHOICE FOR SOME INFANTS JAAPA: Journal

Publication status of the American Academy of Physician Assistants (Lippincott Williams \& Wilkins), 27(5), 1-3 3p

148 Bagnoli, F.,Casucci, M.,Toti, S.,Cecchi, S.,Iurato, C.,Coriolani, G.,Tiezzi, M.,Vispi, L. (2013). Is vitamin D supplementation necessary in healthy full-term breastfed infants? A follow-up study of bone mineralization in healthy full-term infants with and without supplemental vitamin D Minerva Pediatr, 65(3), 253-60

149 Baheiraei, A.,Ardsetani, N.,Ghazizadeh, Sh (2001). Effects of progestogen-only contraceptives on breast-feeding and infant growth International Journal of Gynecology and Obstetrics, 74(2), 203-205

150 Bahl, R.,Frost, C.,Kirkwood, B. R.,Edmond, K.,Martines, J.,Bhandari, N.,Arthur, P. (2005). Infant feeding patterns and risks of death Outcome and hospitalization in the first half of infancy: multicentre cohort study Bull World Health Organ, 83(6), 418-26

151 Bai, K. I.,Sastry, V. N.,Reddy, C. C. (1981). A comparative study of feeding pattern of infants in rural and urban areas Indian $J$ Pediatr, 48(392), 277-80

152 Bailey W (1981). Malnutrition among babies born to adolescent mothers West Indian Med J, 30(\#issue\#), 72-6

153 Bailey, P.,Tsui, A. O.,Janowitz, B.,Dominik, R.,Araujo, L. (1990). A study of infant mortality and causes of death in a rural north-east Brazilian community J Biosoc Sci, 22(3), 349-63

154 Bailey, W. (1981). Clinical undernutrition in the Kingston/St Andrew metropolitan area: 1967-1976 Soc Sci Med D, 15(4), 471-7

Study design, Outcome

155 Bainbridge, J. (2008). Higher IQs for breastfed babies British Journal of Midwifery, 16(6), 394-394 1p 156 Bainbridge, J. (2009). Breastfed babies less likely to become overweight children British Journal of Midwifery, 17(6), 393-393 1p Study design

Study design

157 Baird, J.,Poole, J.,Robinson, S.,Marriott, L.,Godfrey, K.,Cooper, C.,Inskip, H.,Law, C. (2008). Milk feeding and dietary patterns predict weight and fat gains in infancy Paediatr Perinat Epidemiol, 22(6), 575-86

Publication date for a non-sibling study, Confounding

158 Baker, D. Taylor, H.,Henderson, J. (1998). Inequality in infant morbidity: Causes and consequences in England in the 1990s Journal of Epidemiology and Community Health, 52(7), 451-458

159 Baker, D.,Taylor, H.,Henderson, J. (1998). Inequality in infant morbidity: causes and consequences in England in the 1990s ALSPAC Study Team. Avon Longitudinal Study of Pregnancy and Childhood J Epidemiol Community Health, 52(7), 451-8

160 Baker, J. L.,Michaelsen, K. F.,Rasmussen, K. M.,Sorensen, T. I. (2004). Maternal prepregnant body mass index, duration of

breastfeeding, and timing of complementary food introduction are associated with infant weight gain Am J Clin Nutr, 80(6), 1579-88
161 Baker, R. J.,Hertz-Picciotto, I.,Dostal, M.,Keller, J. A.,Nozicka, J.,Kotesovec, F.,Dejmek, J.,Loomis, D.,Sram, R. J. (2006). Coal

Outcome

Outcome home heating and environmental tobacco smoke in relation to lower respiratory illness in Czech children, from birth to 3 years of age Environ Health Perspect, 114(7), 1126-32

Outcome, Publication date for non-sibling study

Outcome 
163 Balaban, G.,Motta, M. E.,Silva, G. A. (2010). Early weaning and other potential risk factors for overweight among preschool children Study design Clinics (Sao Paulo), 65(2), 181-7

164 Balasubramanian S (2011). Vitamin D deficiency in breastfed infants \& the need for routine vitamin D supplementation Indian J Med Study design Res, 133(\#issue\#), 250-2

165 Ball, T. M.,Wright, A. L. (1999). Health care costs of formula-feeding in the first year of life Pediatrics, 103(4 Pt 2), 870-6

Outcome

166 Bammann, K.,Peplies, J.,De Henauw, S.,Hunsberger, M.,Molnar, D.,Moreno, L. A., Tornaritis, M.,Veidebaum, T.,Ahrens, W.,Siani, A. Study design (2014). Early life course risk factors for childhood obesity: the IDEFICS case-control study PLoS One, 9(2), e86914 167 Bandara, T.,Hettiarachchi, M.,Liyanage, C.,Amarasena, S. (2015). Current infant feeding practices and impact on growth in babies
during the second half of infancy J Hum Nutr Diet, 28(4), 366-74 design

168 Bandoli, G.,von Ehrenstein, O. S.,Flores, M. E.,Ritz, B. (2015). Breastfeeding and Asthmatic Symptoms in The Offspring of Latinas: Study design The Role of Maternal Nativity J Immigr Minor Health, 17(6), 1739-45

169 Bandurska-Stankiewicz, E.,Rutkowska, J. (2008). Environmental risk factors for type 1 diabetes in the north of Poland Diabetologia Study design Doswiadczalna i Kliniczna, 8(2), 81-84

170 Banerji, A., Greenberg, D.,White, L. F.,Macdonald, W. A.,Saxton, A., Thomas, E.,Sage, D.,Mamdani, M.,Lanctot, K. L.,Mahony, J. B.,Dingle, M.,Roberts, A. (2009). Risk factors and viruses associated with hospitalization due to lower respiratory tract infections in Canadian Inuit children : a case-control study Pediatr Infect Dis J, 28(8), 697-701

171 Bankel, M.,Robertson, A.,Kohler, B. (2011). Carious lesions and caries risk predictors in a group of Swedish children 2 to 3 years of age. One year observation Eur J Paediatr Dent, 12(4), 215-9

172 Baranowski, T.,Bryan, G. T.,Harrison, J. A.,Rassin, D. K.,Greaves, K. A.,Baranowski, J. H. (1992). Height, infant-feeding practices and cardiovascular functioning among 3 or 4 year old children in three ethnic groups J Clin Epidemiol, 45(5), 513-8

173 Baranowski, T.,Bryan, G. T.,Rassin, D. K.,Harrison, J. A.,Henske, J. C. (1990). Ethnicity, infant-feeding practices, and childhood adiposity J Dev Behav Pediatr, 11(5), 234-9

174 Barge, K. (2007). Breast-feeding doesn't contribute to dental caries J Dent Hyg, 81(4), 69

Outcome

Study design, Size of study groups

175 Barness LA (1983). Impact of breast feeding--obviating problems J Fla Med Assoc, 70(\#issue\#), 831-2

Study design

Study design

176 Baron, S.,Turck, D.,Leplat, C.,Merle, V.,Gower-Rousseau, C.,Marti, R.,Yzet, T.,Lerebours, E.,Dupas, J. L.,Debeugny, S.,Salomez, J. Outcome L.,Cortot, A., Colombel, J. F. (2005). Environmental risk factors in paediatric inflammatory bowel diseases: a population based case control study Gut, 54(3), 357-63

177 Barreto, B. A Sole D. (2014). Prevalence of asthma and associated factors in adolescents living in Belem (Amazon region), Para, Brazil Allergol Immunopathol (Madr), 42(5), 427-32 
178 Barros, F. C.,Rossello, J. L.,Matijasevich, A.,Dumith, S. C.,Barros, A. J.,dos Santos, I. S.,Mota, D.,Victora, C. G. (2012). Gestational Intervention/exposure age at birth and morbidity, mortality, and growth in the first 4 years of life: findings from three birth cohorts in Southern Brazil BMC Pediatr, 12(\#issue\#), 169

179 Barros, F. C.,Semer, T. C., Tonioli Filho, S.,Tomasi, E., Victora, C. G. (1995). The impact of lactation centres on breastfeeding patterns, morbidity and growth: a birth cohort study Acta Paediatr, 84(11), 1221-6

180 Barros, F. C.,Victora, C. G.,Morris, S. S.,Halpern, R.,Horta, B. L.,Tomasi, E. (1997). Breast feeding, pacifier use and infant development at 12 months of age: a birth cohort study in Brazil Paediatr Perinat Epidemiol, 11(4), 441-50

181 Barros, F. C.,Victora, C. G.,Vaughan, J. P.,Tomasi, E.,Horta, B. L.,Cesar, J. A.,Menezes, M. B.,Halpern, R.,Post, C. L.,del Mar Garcia, M. (2001). The epidemiological transition in maternal and child health in a Brazilian city, 1982-93: a comparison of two population-based cohorts Paediatr Perinat Epidemiol, 15(1), 4-11

182 Barroso, C. S.,Roncancio, A.,Hinojosa, M. B.,Reifsnider, E. (2012). The association between early childhood overweight and maternal factors Child Obes, 8(5), 449-54

Publication date for a non-sibling study

Outcome

Outcome

Study design, Size of study groups

Outcome acquired pneumonia in hospitalised children and adolescents aged 6 months to 13 years old Eur J Pediatr, 172(4), 493-9

184 Bartels, M.,van Beijsterveldt, C. E.,Boomsma, D. I. (2009). Breastfeeding, maternal education and cognitive function: a prospective Outcome study in twins Behav Genet, 39(6), 616-22

185 Bartok, C. J. (2011). Babies fed breastmilk by breast versus by bottle: a pilot study evaluating early growth patterns Breastfeed Med, Size of study groups $6(3), 117-24$

186 Barton, S. J.,Howard, P. K.,Rayens, M. K. (2002). The effects of infant feeding decisions on infant growth J Spec Pediatr Nurs, 7(2), Size of study groups $64-70$

187 Basheer, R. (1988). Breast is best Nurs J India, 79(7), 180, 190

188 Bassal, R.,Reisfeld, A.,Nissan, I.,Agmon, V.,Taran, D.,Schemberg, B.,Cohen, D.,Shohat, T. (2014). Risk factors for sporadic infection with Salmonella Infantis: a matched case-control study Epidemiol Infect, 142(4), 820-5

189 Batstra, L.,Neeleman, J.,Hadders-Algra, M. (2003). Can breast feeding modify the adverse effects of smoking during pregnancy on Study design the child's cognitive development? J Epidemiol Community Health, 57(6), 403-4

190 Bauer, G.,Ewald, L. S.,Hoffman, J.,Dubanoski, R. (1991). Breastfeeding and cognitive development of three-year-old children Psychol Rep, 68(3 Pt 2), 1218

191 Baumgartner, C. (1984). Psychomotor and social development of breast-fed and bottle-fed babies during their first year of life Acta Paediatr Hung, 25(4), 409-17

192 Baur, L. A.,O'Connor, J.,Pan, D. A.,Kriketos, A. D.,Storlien, L. H. (1998). The fatty acid composition of skeletal muscle membrane phospholipid: its relationship with the type of feeding and plasma glucose levels in young children Metabolism, 47(1), 106-12
Study design

Size of study groups, Outcome

Study design

Size of study groups

Size of study groups Intervention/exposure 
193 Baur, L. A.,O'Connor, J.,Pan, D. A.,Wu, B. J.,O'Connor, M. J.,Storlien, L. H. (2000). Relationships between the fatty acid composition of muscle and erythrocyte membrane phospholipid in young children and the effect of type of infant feeding Lipids, $35(1), 77-82$

194 Baxter-Jones, A. D.,Cardy, A. H.,Helms, P. J.,Phillips, D. O.,Smith, W. C. (1999). Influence of socioeconomic conditions on growth in infancy: the 1921 Aberdeen birth cohort Arch Dis Child, 81(1), 5-9

195 Bayley, T. M.,Alasmi, M.,Thorkelson, T.,Jones, P. J.,Corcoran, J.,Krug-Wispe, S.,Tsang, R. C. (2002). Longer term effects of early dietary cholesterol level on synthesis and circulating cholesterol concentrations in human infants Metabolism, 51(1), 25-33

196 Bayley, T. M.,Alasmi, M.,Thorkelson, T.,Krug-Wispe, S.,Jones, P. J.,Bulani, J. L.,Tsang, R. C. (1998). Influence of formula versus breast milk on cholesterol synthesis rates in four-month-old infants Pediatr Res, 44(1), 60-7

197 Baylis, J. M.,Leeds, A. R.,Challacombe, D. N. (1983). Persistent nausea and food aversions in pregnancy. A possible association with cow's milk allergy in infants Clin Allergy, 13(3), 263-9 Bayraktar, S.,Bayraktar, S. T.,Selcuk, N.,Emiroglu, H.,Elevli, M. (2006). Lipid and lipoprotein profile of breast fed, formula fed or mixed-fed 0-6-month-old babies International Pediatrics, 21(2), 84-90

199 Beath, K. J. (2007). Infant growth modelling using a shape invariant model with random effects Stat Med, 26(12), 2547-64

Beauchamp, J. N.,Gaboury, I.,Ni, A.,Boland, M. P.,Mac, K. D. R. (2011). Solid-food introduction in infants diagnosed as having a cow's-milk protein-induced enterocolitis Journal of Pediatric Gastroenterology and Nutrition, 52(5), 639-643

201 Beaudry, M.,Dufour, R.,Marcoux, S. (1995). Reaction between infant feeding and infections during the first six months of life Journa of Pediatrics, 126(2), 191-197

202 Beaudry, M.,Dufour, R.,Marcoux, S. (1995). Relation between infant feeding and infections during the first six months of life J Pediatr, 126(2), 191-7

203 Beaver, K. M.,Vaughn, M. G.,DeLisi, M.,Higgins, G. E. (2010). The biosocial correlates of neuropsychological deficits: results from the national longitudinal study of adolescent health Int J Offender Ther Comp Criminol, 54(6), 878-94

204 Becher, J. C.,Bhushan, S. S.,Lyon, A. J. (2012). Unexpected collapse in apparently healthy newborns--a prospective national study Study design of a missing cohort of neonatal deaths and near-death events Arch Dis Child Fetal Neonatal Ed, 97(1), F30-4

205 Beebe, D. W.,Rausch, J.,Byars, K. C.,Lanphear, B.,Yolton, K. (2012). Persistent snoring in preschool children: predictors and behavioral and developmental correlates Pediatrics, 130(3), 382-9

206 Beentjes VE,Weerheijm KL,Groen HJ (2002). Factors involved in the aetiology of molar-incisor hypomineralisation (MIH) Eur $\mathrm{J}$ Paediatr Dent, 3(\#issue\#), 9-13

207 Beilin, L.,Huang, R. C. (2008). Childhood obesity, hypertension, the metabolic syndrome and adult cardiovascular disease Clin Exp Pharmacol Physiol, 35(4), 409-11
Size of study groups, Intervention/exposure

Publication date for a non-sibling study

Size of study groups

Size of study groups

Size of study groups

Study design

Outcome, Publication date for a non-sibling study

Participant health, Intervention/exposure

Study design

Study design

Outcome

Intervention/exposure, Outcome

Study design, Size of study groups

Study design 
208 Bekkers, M. B.,Brunekreef, B.,Smit, H. A.,Kerkhof, M.,Koppelman, G. H.,Oldenwening, M.,Wijga, A. H. (2011). Early-life determinants of total and HDL cholesterol concentrations in 8-year-old children; the PIAMA birth cohort study PLoS One, 6(9), e25533

209 Belfort, M. B.,Rifas-Shiman, S. L.,Kleinman, K. P.,Guthrie, L. B.,Bellinger, D. C.,Taveras, E. M.,,Gillman, M. W.,Oken, E. (2013) Infant feeding and childhood cognition at ages 3 and 7 years: Effects of breastfeeding duration and exclusivity JAMA Pediatr, 167(9), $836-44$

210 Belfort, M. B.,Rifas-Shiman, S. L.,Rich-Edwards, J. W.,Kleinman, K. P.,Oken, E.,Gillman, M. W. (2008). Infant growth and child cognition at 3 years of age Pediatrics, 122(3), e689-95

211 Ben, X. M.,Zhou, X. Y.,Zhao, W. H.,Yu, W. L.,Pan, W.,Zhang, W. L.,Wu, S. M.,Van Beusekom, C. M.,Schaafsma, A. (2004). Growth and development of term infants fed with milk with long-chain polyunsaturated fatty acid supplementation Chinese Medical Journal, 117(8), $1268-1270$

212 Bener, A.,Alsaied, A.,Al-Ali, M.,Al-Kubaisi, A.,Basha, B.,Abraham, A.,Guiter, G.,Mian, M. (2009). High prevalence of vitamin D deficiency in type 1 diabetes mellitus and healthy children Acta Diabetol, 46(3), 183-9

213 Bener, A.,Denic, S.,Galadari, S. (2001). Longer breast-feeding and protection against childhood leukaemia and lymphomas Eur J Cancer, 37(2), 234-8

214 Bener, A.,Hoffmann, G. F.,Afify, Z.,Rasul, K.,Tewfik, I. (2008). Does prolonged breastfeeding reduce the risk for childhood leukemia and lymphomas? Minerva Pediatr, 60(2), 155-61

215 Benn, C. S.,Wohlfahrt, J.,Aaby, P.,Westergaard, T.,Benfeldt, E.,Michaelsen, K. F.,Bjorksten, B.,Melbye, M. (2004). Breastfeeding and risk of atopic dermatitis, by parental history of allergy, during the first 18 months of life Am J Epidemiol, 160(3), 217-23

\section{Outcome}

\section{Intervention/exposure}

Size of study groups, Intervention/exposure

\section{Study design}

Intervention/exposure

Outcome

Intervention/exposure

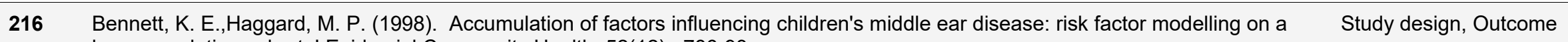
large population cohort J Epidemiol Community Health, 52(12), 786-93

217 Berger, R.,Hadziselimovic, F.,Just, M.,Reigel, P. (1983). Effect of feeding human milk on nosocomial rotavirus infections in an infants ward Dev Biol Stand, 53(\#issue\#), 219-28

218 Bergmann, K. E.,Bergmann, R. L., Von Kries, R.,Bohm, O.,Richter, R.,Dudenhausen, J. W.,Wahn, U. (2003). Early determinants of childhood overweight and adiposity in a birth cohort study: role of breast-feeding Int J Obes Relat Metab Disord, 27(2), 162-72

219 Bergmann, R. L.,Bergler, H.,Moshoudis, E.,Bergmann, K. E.,Lachmann, E. (1988). Prevention of iron deficiency of breast-fed babies Language by using suitable additional food, a prospective, controlled study Monatsschrift fur Kinderheilkunde, 136(\#issue\#), 491

220 Bergmann, R. L.,Bergmann, K. E.,Lau-Schadensdorf, S.,Luck, W.,Dannemann, A.,Bauer, C. P.,Dorsch, W.,Forster, J.,Schmidt, E.,Schulz, J.,et al., (1994). Atopic diseases in infancy. The German multicenter atopy study (MAS-90) Pediatr Allergy Immunol, 5(6 Suppl), $19-25$

221 Bergmann, R. L.,Diepgen, T. L.,Kuss, O.,Bergmann, K. E.,Kujat, J.,Dudenhausen, J. W.,Wahn, U. (2002). Breastfeeding duration is a risk factor for atopic eczema Clin Exp Allergy, 32(2), 205-9 
222 Bergmann, R. L.,Edenharter, G.,Bergmann, K. E.,Lau, S.,Wahn, U. (2000). Socioeconomic status is a risk factor for allergy in parents but not in their children Clin Exp Allergy, 30(12), 1740-5

223 Bergmann, R. L.,Haschke-Becher, E.,Klassen-Wigger, P.,Bergmann, K. E.,Richter, R.,Dudenhausen, J. W.,Grathwohl, D.,Haschke, Intervention/exposure F. (2008). Supplementation with $200 \mathrm{mg} /$ day docosahexaenoic acid from mid-pregnancy through lactation improves the docosahexaenoic acid status of mothers with a habitually low fish intake and of their infants Ann Nutr Metab, 52(2), 157-66

224 Bergstrand, O.,Hellers, G. (1983). Breast-feeding during infancy in patients who later develop Crohn's disease Scand J Gastroenterol, 18(7), 903-6

225 Bergstrom, A.,Skov, T. H.,Bahl, M. I.,Roager, H. M.,Christensen, L. B.,Ejlerskov, K. T.,Molgaard, C.,Michaelsen, K. F.,Licht, T. R. (2014). Establishment of intestinal microbiota during early life: a longitudinal, explorative study of a large cohort of Danish infants Appl Environ Microbiol, 80(9), 2889-900

226 Bergstrom, E.,Hernell, O.,Persson, L. A.,Vessby, B. (1995). Serum lipid values in adolescents are related to family history, infant feeding, and physical growth Atherosclerosis, 117(1), 1-13

227 Beristain-Manterola, R.,Pasquetti-Ceccatelli, A.,Meléndez-Mier, G.,Sánchez-Escobar, O. A.,Cuevas-Covarrubias, S. A. (2010) Evaluation of iron status in healthy six-month-old infants in Mexican population: Evidence of a high prevalence of iron deficiency eSPEN, 5(1), e37-e39

228 Berkowitz, C. D.,Uchiyama, N.,Tully, S. B.,Marble, R. D.,Spencer, M.,Stein, M. T.,Orr, D. P. (1985). Fever in infants less than two months of age: spectrum of disease and predictors of outcome Pediatr Emerg Care, 1(3), 128-35

229 Berkowitz, R. J. (1985). Streptococcus mutans and dental caries in infants Compend Contin Educ Dent, 6(6), 463-6

230 Bernard, A.,Nickmilder, M. (2013). Association of breastfeeding with higher serum inhibin B level at adolescence JAMA Pediatr, 167(9), 869-70

231 Bernard, J. Y.,Armand, M.,Garcia, C.,Forhan, A.,De Agostini, M.,Charles, M. A.,Heude, B. (2015). The association between linoleic acid levels in colostrum and child cognition at 2 and $3 y$ in the EDEN cohort Pediatr Res, 77(6), 829-35

232 Bernard, J. Y.,De Agostini, M.,Forhan, A.,Alfaiate, T.,Bonet, M.,Champion, V.,Kaminski, M.,de Lauzon-Guillain, B.,Charles, M. A.,Heude, B. (2013). Breastfeeding duration and cognitive development at 2 and 3 years of age in the EDEN mother-child cohort $J$ Pediatr, 163(1), 36-42 e1

233 Bernard, J. Y.,De Agostini, M.,Forhan, A.,de Lauzon-Guillain, B.,Charles, M. A.,Heude, B. (2013). The dietary n6:n3 fatty acid ratio during pregnancy is inversely associated with child neurodevelopment in the EDEN mother-child cohort J Nutr, 143(9), 1481-8

234 Bernardi, J. R.,Gama, C. M.,Vitolo, M. R. (2011). An infant feeding update program at healthcare centers and its impact on breastfeeding and morbidity Cadernos de Saude Publica, 27(6), 1213-1222

Study design, Participant health

Study design

Outcome

Outcome

Intervention/exposure

Study design

Study design, Outcome

Outcome

Outcome

Outcome

Language 
235 Berseth, C. L.,Mitmesser, S. H.,Birch, E.,Khoury, J.,Bean, J.,Harris, C.,Scalabrin, D. (2011). Intake of DHA/ARA via breast milk or formula supplementation during infancy can affect the incidence and recurrence of allergic manifestations in young children Journal of Pediatric Gastroenterology and Nutrition. Conference: European Society for Paediatric Gastroenterology, Hepatology, and Nutrition Annual Meeting 2011 Sorrento Italy. Conference Start: 20110525 Conference End: 20110528. Conference Publication: (var.pagings), 52(Suppl 2), E61

236 Betoko, A.,Charles, M. A.,Hankard, R.,Forhan, A.,Bonet, M.,Regnault, N.,Botton, J.,Saurel-Cubizolles, M. J.,de Lauzon-Guillain, B. (2014). Determinants of infant formula use and relation with growth in the first 4 months Matern Child Nutr, 10(2), 267-79

237 Betran, A. P.,de Onis, M.,Lauer, J. A.,Villar, J. (2001). Ecological study of effect of breast feeding on infant mortality in Latin America Study design Bmj, 323(7308), 303-6

238 Beyerlein, A.,Fahrmeir, L.,Mansmann, U.,Toschke, A. M. (2008). Alternative regression models to assess increase in childhood BMI Study design BMC Med Res Methodol, 8(\#issue\#), 59

239 Bhan, M. K.,Arora, N. K.,Singh, K. D. (1991). Management of persistent diarrhea during infancy in clinical practice Indian J Pediat 58(6), 769-74

240 Bhatia, B. D.,Banerjee, D.,Agarwal, D. K.,Agarwal, K. N. (1983). Exterogestate growth: relationship with maternal body size and dietary intakes Indian J Pediatr, 50(404), 241-6

241 Bianchi, C.,Brambilla, P.,Cella, D.,Ragogna, F.,Tettamanti, C.,Del Puppo, M.,Kienle, M. G.,Chiumello, G.,Ruotolo, G. (1997) Influence of breast- and formula-feeding on plasma cholesterol precursor sterols throughout the first year of life J Pediatr, 131(6), 928-31

242 Biering-Sorensen F,Hilden J,Biering-Sorensen K (1983). Breast-feeding and infant health in Copenhagen 1941-1972 Dan Med Bull, 30(\#issue\#), 36-41

243 Biesbroek, G.,Bosch, A. A.,Wang, X.,Keijser, B. J.,Veenhoven, R. H.,Sanders, E. A.,Bogaert, D. (2014). The impact of breastfeeding Outcome on nasopharyngeal microbial communities in infants Am J Respir Crit Care Med, 190(3), 298-308

244 Biesbroek, G.,Tsivtsivadze, E.,Sanders, E. A.,Montijn, R., Veenhoven, R. H.,Keijser, B. J.,Bogaert, D. (2014). Early respiratory microbiota composition determines bacterial succession patterns and respiratory health in children Am J Respir Crit Care Med 190(11), 1283-92

245 Bilenko, N.,Fraser, D.,Naggan, L. (1999). Maternal knowledge and environmental factors associated with risk of diarrhea in Israeli Bedouin children Eur J Epidemiol, 15(10), 907-12 
249 Birch, E. E.,Carlson, S. E.,Hoffman, D. R.,Fitzgerald-Gustafson, K. M.,Fu, V. L.,Drover, J. R.,Castaneda, Y. S.,Minns, L.,Wheaton, D. Intervention/exposure K.,Mundy, D.,Marunycz, J.,Diersen-Schade, D. A. (2010). The DIAMOND (DHA Intake And Measurement Of Neural Development) Study: a double-masked, randomized controlled clinical trial of the maturation of infant visual acuity as a function of the dietary level of docosahexaenoic acid Am J Clin Nutr, 91(4), 848-59

250 Birch, E. E.,Garfield, S.,Castaneda, Y.,Hughbanks-Wheaton, D.,Uauy, R.,Hoffman, D. (2007). Visual acuity and cognitive outcomes at 4 years of age in a double-blind, randomized trial of long-chain polyunsaturated fatty acid-supplemented infant formula Early Hum Dev, 83(5), 279-84

251 Birch, E. E.,Hoffman, D. R.,Castaneda, Y. S.,Fawcett, S. L.,Birch, D. G.,Uauy, R. D. (2002). A randomized controlled trial of longchain polyunsaturated fatty acid supplementation of formula in term infants after weaning at 6 wk of age Am J Clin Nutr, 75(3), 570-

252 Birch, E. E.,Hoffman, D. R.,Uauy, R.,Birch, D. G.,Prestidge, C. (1998). Visual acuity and the essentiality of docosahexaenoic acid and arachidonic acid in the diet of term infants Pediatr Res, 44(2), 201-9

253 Birch, E.,Birch, D.,Hoffman, D.,Hale, L.,Everett, M.,Uauy, R. (1993). Breast-feeding and optimal visual development J Pediatr Ophthalmol Strabismus, 30(1), 33-8

254 Birkbeck JA,Scott HF (1980). 25-Hydroxycholecalciferol serum levels in breast-fed infants Arch Dis Child, 55(\#issue\#), 691-5

study groups Intervention/exposure

255 Birkbeck, J. A.,Buckfield, P. M.,Silva, P. A. (1985). Lack of long-term effect of the method of infant feeding on growth Hum Nutr Clin Nutr, 39(1), 39-44

256 Birkett, D. (2005). On bottle versus breast Health Serv J, 115(5957), 19

Intervention/exposure

Intervention/exposure

Study design

257 Bisgaard, H.,Halkjær, L. B.,Hinge, R.,Giwercman, C.,Palmer, C.,Silveira, L.,Strand, M. (2009). Risk analysis of early childhood eczema Journal of Allergy and Clinical Immunology, 123(6), 1355-1360.e5

258 Bishara, S. E.,Nowak, A. J.,Kohout, F. J.,Heckert, D. A.,Hogan, M. M. (1987). Influence of feeding and non-nutritive sucking methods on the development of the dental arches: longitudinal study of the first 18 months of life Pediatr Dent, 9(1), 13-21

Intervention/exposure

259 Bishara, S. E.,Warren, J. J.,Broffitt, B.,Levy, S. M. (2006). Changes in the prevalence of nonnutritive sucking patterns in the first 8

Size of study groups Intervention/exposure years of life Am J Orthod Dentofacial Orthop, 130(1), 31-6

260 Bishop, W. S. (1985). Weaning the breast-fed toddler or preschooler Pediatr Nurs, 11(3), 211-4

Study design

261 Bjorke-Monsen, A. L. (2014). Is exclusive breastfeeding ensuring an optimal micronutrient status and psychomotor development in infants? Clin Biochem, 47(9), 714

262 Bjorksten, B.,Ait-Khaled, N.,Innes Asher, M.,Clayton, T. O.,Robertson, C. (2011). Global analysis of breast feeding and risk of symptoms of asthma, rhinoconjunctivitis and eczema in 6-7 year old children: ISAAC Phase Three Allergol Immunopathol (Madr), $39(6), 318-25$ 
264 Blattner, C. M.,Murase, J. E. (2014). A practice gap in pediatric dermatology: does breast-feeding prevent the development of infantile atopic dermatitis? J Am Acad Dermatol, 71(2), 405-6

265 Blom, L.,Dahlquist, G.,Nystrom, L.,Sandstrom, A.,Wall, S. (1989). The Swedish childhood diabetes study--social and perinatal determinants for diabetes in childhood Diabetologia, 32(1), 7-13

266 Bloom, K.,Goldbloom, R. B.,Robinson, S. C.,Stevens, F. E. (1982). Breast versus formula feeding Acta Paediatr Scand Suppl, 300(\#issue\#), 1-26

267 Bly, E.,Huntington, J.,Harper, A. L.,Vincent, E. C. (2013). What is the best age to start vitamin D supplementation to prevent rickets in breastfed newborns? Journal of Family Practice, 62(12), 755+763

268 Bocca, B.,Alimonti, A., Giglio, L.,Di Pasquale, M.,Caroli, S.,Ambruzzi, M. A.,Bocca, A. P.,Coni, E. (2000). Nutritive significance of element speciation in breast milk. The case of calcium, copper, iron, magnesium, manganese, and zinc Adv Exp Med Biol, 478(\#issue\#), 385-6

269 Boccolini, C. S.,Carvalho, M. L.,Oliveira, M. I.,Boccolini Pde, M. (2011). Breastfeeding can prevent hospitalization for pneumonia among children under 1 year old J Pediatr (Rio J), 87(5), 399-404

270 Boccolini, C. S.,Carvalho, M. L.,Oliveira, M. I.,Perez-Escamilla, R. (2013). Breastfeeding during the first hour of life and neonata mortality J Pediatr (Rio J), 89(2), 131-6

271 Bodington, M. J.,McNally, P. G.,Burden, A. C. (1994). Cow's milk and type 1 childhood diabetes: no increase in risk Diabet Med, $11(7), 663-5$

272 Boediman, D.,Murakami, R.,Nakamura, H.,Matsuo, T. (1989). Plasma apolipoprotein and lipid profiles in infants in the first year of life Kobe J Med Sci, 35(3), 165-76

273 Boerma, J. T.,Bicego, G. T. (1992). Preceding birth intervals and child survival: searching for pathways of influence Stud Fam Plann, 23(4), 243-56

274 Bogen, D. L.,Hanusa, B. H.,Whitaker, R. C. (2004). The effect of breast-feeding with and without formula use on the risk of obesity at 4 years of age Obes Res, 12(9), 1527-35

275 Bognetti, E.,Meschi, F.,Malavasi, C.,Pastore, M. R.,Sergi, A.,Illeni, M. T.,Maffeis, C.,Pinelli, L.,Chiumello, G. (1992). HLA-antigens in Italian type 1 diabetic patients: role of DR3/DR4 antigens and breast feeding in the onset of the disease Acta Diabetol, 28(3-4), 22932

276 Bohles, H.,Aschenbrenner, M.,Roth, M.,von Loewenich, V.,Ball, F.,Usadel, K. H. (1993). Development of thyroid gland volume during the first 3 months of life in breast-fed versus iodine-supplemented and iodine-free formula-fed infants Clin Investig, 71(1), 1320

277 Bolanos, A. V.,Caire, G.,Valencia, M. E.,Casanueva, E.,Roman Perez, R.,Calderon de la Barca, A. M. (2000). Energy intake and growth of breast-fed infants in two regions of Mexico Adv Exp Med Biol, 478(\#issue\#), 371-2 
278 Bond, S. (2008). Randomized trial provides strong evidence that prolonged, exclusive breastfeeding enhances cognitive development in children Journal of Midwifery \& Women's Health, 53(5), 472-473 $2 p$

279 Bonuck, K. A.,Freeman, K.,Trombley, M. (2006). Randomized controlled trial of a prenatal and postnatal lactation consultant intervention on infant health care use Arch Pediatr Adolesc Med, 160(9), 953-60

280 Bonuck, K.,Avraham, S. B.,Lo, Y.,Kahn, R.,Hyden, C. (2014). Bottle-weaning intervention and toddler overweight J Pediatr, 164(2), 306-12 e1-2

281 Boonyaratavej, N.,Suriyawongpaisal, P.,Takkinsatien, A.,Wanvarie, S., Rajatanavin, R.,Apiyasawat, P. (2001). Physical activity and risk factors for hip fractures in Thai women Osteoporos Int, 12(3), 244-8

282 Borch-Johnsen K,Joner G,Mandrup-Poulsen T,Christy M,Zachau-Christiansen B,Kastrup K,Nerup J (1984). Relation between breast-feeding and incidence rates of insulin-dependent diabetes mellitus. A hypothesis Lancet, 2(\#issue\#), 1083-6 Bordeaux
$145-50$

284 Borgnolo, G.,Barbone, F.,Scornavacca, G.,Franco, D.,Vinci, A.,luculano, F. (1996). A case-control study of Salmonella gastrointestinal infection in Italian children Acta Paediatr, 85(7), 804-8

285 Bornhorst, C.,Siani, A.,Russo, P.,Kourides, Y.,Sion, I.,Molnar, D.,Moreno, L. A., Rodriguez, G.,Ben-Shlomo, Y.,Howe, L., Lissner, L.,Mehlig, K.,Regber, S.,Bammann, K.,Foraita, R.,Ahrens, W.,Tilling, K. (2016). Early Life Factors and Inter-Country Heterogeneity in BMI Growth Trajectories of European Children: The IDEFICS Study PLoS One, 11(2), e0149268

286 Bortolini, G. A.,Vitolo, M. R. (2012). The impact of systematic dietary counseling during the first year of life on prevalence rates of anemia and iron deficiency at 12-16 months J Pediatr (Rio J), 88(1), 33-9

287 Boshuizen, H. C.,Verkerk, P. H.,Reerink, J. D.,Herngreen, W. P.,Zaadstra, B. M.,Verloove-Vanhorick, S. P. (1998). Maternal smoking during lactation: relation to growth during the first year of life in a Dutch birth cohort Am J Epidemiol, 147(2), 117-26

\section{Outcome}

Intervention/exposure, Outcome

Participant age,

Intervention/exposure

Outcome

Study design

Participant health

Duplicate from the othe

literature search

Intervention/exposure

Intervention/exposure

288 Boskabadi, H.,Ramazanzadeh, M.,Zakerihamidi, M.,Omran, F. R. (2014). Risk factors of breast problems in mothers and its effects

Intervention/exposure, Outcome on newborns Iranian Red Crescent Medical Journal, 16(6), \#Pages\#

289 Boulton, J. (1981). Nutrition in childhood and its relationships to early somatic growth, body fat, blood pressure, and physical fitness Publication status Acta Paediatr Scand Suppl, 284(\#issue\#), 1-85

290 Boutwell, B. B.,Beaver, K. M.,Barnes, J. C. (2012). Role of breastfeeding in childhood cognitive development: a propensity score matching analysis J Paediatr Child Health, 48(9), 840-5

291 Bouwstra, H. Boersma, E. R. Boehm, G. Dijck-Brouwer, D. A Muskiet, F. A . Hadders-Algra, M. (2003). Exclusive breastfeeding of healthy term infants for at least 6 weeks improves neurological condition J Nutr, 133(12), 4243-5

292 Bouwstra, H.,Dijck-Brouwer, D. A.,Boehm, G.,Boersma, E. R.,Muskiet, F. A.,Hadders-Algra, M. (2005). Long-chain polyunsaturated fatty acids and neurological developmental outcome at 18 months in healthy term infants Acta Paediatr, 94(1), 26-32 
293 Bouwstra, H.,Dijck-Brouwer, D. A.,Wildeman, J. A.,Tjoonk, H. M.,van der Heide, J. C.,Boersma, E. R.,Muskiet, F. A.,Hadders-Algra, M. (2003). Long-chain polyunsaturated fatty acids have a positive effect on the quality of general movements of healthy term infants Am J Clin Nutr, 78(2), 313-8

294 Bouwstra, H.,Dijck-Brouwer, J.,Decsi, T.,Boehm, G.,Boersma, E. R.,Muskiet, F. A.,Hadders-Algra, M. (2006). Neurologic condition of healthy term infants at 18 months: positive association with venous umbilical DHA status and negative association with umbilical trans-fatty acids Pediatr Res, 60(3), 334-9

295 Bove, I.,Campoy, C.,Uauy, R.,Miranda, T.,Cerruti, F. (2014). Trends in early growth indices in the first 24 months of life in Uruguay over the past decade $\mathrm{J}$ Health Popul Nutr, 32(4), 600-7

296 Bradley, C. K.,Hillman, L.,Sherman, A. R.,Leedy, D.,Cordano, A. (1993). Evaluation of two iron-fortified, milk-based formulas during infancy Pediatrics, 91(5), 908-14

297 Bramhagen, A. C.,Svahn, J.,Hallstrom, I.,Axelsson, I. (2011). Factors influencing iron nutrition among one-year-old healthy children in Sweden J Clin Nurs, 20(13-14), 1887-94

298 Brams, M.,Maloney, J. (1983). "Nursing bottle caries" in breast-fed children J Pediatr, 103(3), 415-6

299 Brandenburg, A. H., Jeannet, P. Y.,Steensel-Moll, H. A.,Ott, A., Rothbarth, P. H.,Wunderli, W.,Suter, S.,Neijens, H. J.,Osterhaus, A.

Study design, Participant health D.,Siegrist, C. A. (1997). Local variability in respiratory syncytial virus disease severity Arch Dis Child, 77(5), 410-4

300 Brandstrom, A.,Brostrom, G.,Persson, L. A. (1984). The impact of feeding patterns on infant mortality in a nineteenth century Swedish parish J Trop Pediatr, 30(3), 154-9

Study design,

Intervention/exposure

301 Bray, K. K.,Branson, B. G.,Williams, K. (2003). Early childhood caries in an urban health department: an exploratory study J Dent Hyg, 77(4), 225-32

302 Brew, B. K.,Kull, I.,Garden, F.,Almqvist, C.,Bergstrom, A.,Lind, T.,Webb, K.,Wickman, M.,Marks, G. B. (2012). Breastfeeding, asthma, and allergy: a tale of two cities Pediatr Allergy Immunol, 23(1), 75-82

Study design

Study design, Redundant data with another study

303 Brew, B. K.,Marks, G. B.,Almqvist, C.,Cistulli, P. A.,Webb, K.,Marshall, N. S. (2014). Breastfeeding and snoring: a birth cohort study Outcome PLoS One, 9(1), e84956

304 Briggs, D. (1992). Baby milks and the EC. Infant nutrition Nurs Times, 88(32), 24-6

Study design

305 Brion, M. J. A.,Lawlor, D. A.,Matijasevich, A.,Horta, B.,Anselmi, L.,Araújo, C. L.,Menezes, A. M. B.,Victora, C. G.,Smith, G. D. (2011). Intervention/exposure What are the causal effects of breastfeeding on IQ, obesity and blood pressure? Evidence from comparing high-income with middleincome cohorts International Journal of Epidemiology, 40(3), 670-680

306 Broad, F. E.,Duganzich, D. M. (1983). The effects of infant feeding, birth order, occupation and socio-economic status on speech in six-year-old children N Z Med J, 96(734), 483-6

307 Brodish, M. S. (1982). Relationship of early bonding to initial infant feeding patterns in bottle-fed newborns JOGN Nurs, 11(4), 248- Intervention/exposure 52 


\begin{tabular}{|c|c|c|}
\hline 308 & Brooke OG (1983). Supplementary vitamin D in infancy and childhood Arch Dis Child, 58(\#issue\#), 573-4 & Study design \\
\hline 309 & $\begin{array}{l}\text { Brooks, J. G., Gilbert, R. E.,Flemming, P. J.,Berry, P. J.,Golding, J. (1994). Postnatal growth preceding sudden infant death } \\
\text { syndrome Pediatrics, 94(4 Pt 1), 456-61 }\end{array}$ & Outcome \\
\hline 310 & $\begin{array}{l}\text { Broor, S.,Pandey, R. M.,Ghosh, M.,Maitreyi, R. S.,Lodha, R.,Singhal, T.,Kabra, S. K. (2001). Risk factors for severe acute lower } \\
\text { respiratory tract infection in under-five children Indian Pediatr, 38(12), 1361-9 }\end{array}$ & Country \\
\hline 311 & $\begin{array}{l}\text { Brown, A.,Lee, M. (2012). Breastfeeding during the first year promotes satiety responsiveness in children aged 18-24 months } \\
\text { Pediatr Obes, } 7(5), 382-90\end{array}$ & Outcome \\
\hline 312 & $\begin{array}{l}\text { Brown, C. M.,Austin, D. W.,Busija, L. (2014). Observable essential fatty acid deficiency markers and autism spectrum disorder } \\
\text { Breastfeed Rev, 22(2), 21-6 }\end{array}$ & $\begin{array}{l}\text { Study design, Size of study } \\
\text { groups }\end{array}$ \\
\hline 313 & $\begin{array}{l}\text { Brown, J. P.,Junner, C.,Liew, V. (1985). A study of Streptococcus mutans levels in both infants with bottle caries and their mothers } \\
\text { Aust Dent J, 30(2), 96-8 }\end{array}$ & Intervention/exposure \\
\hline 314 & $\begin{array}{l}\text { Brown, K. H.,Black, R. E.,Lopez de Romana, G.,Creed de Kanashiro, H. (1989). Infant-feeding practices and their relationship with } \\
\text { diarrheal and other diseases in Huascar (Lima), Peru Pediatrics, 83(1), 31-40 }\end{array}$ & Intervention/exposure \\
\hline 315 & $\begin{array}{l}\text { Brown, K. H.,Stallings, R. Y.,de Kanashiro, H. C.,Lopez de Romana, G.,Black, R. E. (1990). Effects of common illnesses on infants' } \\
\text { energy intakes from breast milk and other foods during longitudinal community-based studies in Huascar (Lima), Peru Am J Clin } \\
\text { Nutr, 52(6), 1005-13 }\end{array}$ & Outcome \\
\hline 316 & Broxton, D. (2008). Infant feeding research summaries International Journal of Childbirth Education, 23(2), 28-31 4p & Country \\
\hline 317 & $\begin{array}{l}\text { Bruce, L.,Lieberman, L. S. (1987). Nutritional anthropometry and dietary intake of children from the Las Cuevas region of the } \\
\text { Dominican Republic Arch Latinoam Nutr, 37(2), 250-8 }\end{array}$ & Study design \\
\hline 318 & $\begin{array}{l}\text { Bruerd, B.,Kinney, M. B.,Bothwell, E. (1989). Preventing baby bottle tooth decay in American Indian and Alaska native communities: } \\
\text { a model for planning Public Health Rep, 104(6), } 631-40\end{array}$ & Intervention/exposure \\
\hline 319 & $\begin{array}{l}\text { Bruno, G.,Milita, O.,Ferrara, M.,Nisini, R.,Cantani, A.,Businco, L. (1993). Prevention of atopic diseases in high risk babies (long-term } \\
\text { follow-up) Allergy Proc, 14(3), 181-6; discussion 186-7 }\end{array}$ & Intervention/exposure \\
\hline 320 & $\begin{array}{l}\text { Brunser, O.,Espinoza, J.,Figueroa, G.,Araya, M.,Spencer, E.,Hilpert, H.,Link-Amster, H.,Brussow, H. (1992). Field trial of an infant } \\
\text { formula containing anti-rotavirus and anti-Escherichia coli milk antibodies from hyperimmunized cows J Pediatr Gastroenterol Nutr, } \\
15(1), 63-72\end{array}$ & Intervention/exposure \\
\hline 321 & Buckley, K. M. (2001). Long-term breastfeeding: nourishment or nurturance? J Hum Lact, 17(4), $304-12$ & $\begin{array}{l}\text { Study design, } \\
\text { Intervention/exposure }\end{array}$ \\
\hline 322 & $\begin{array}{l}\text { Buinauskiene, J.,Baliutaviciene, D.,Zalinkevicius, R. (2004). Glucose tolerance of 2- to 5-yr-old offspring of diabetic mothers } \\
\text { Pediatric Diabetes, 5(3), 143-146 }\end{array}$ & Intervention/exposure \\
\hline
\end{tabular}


323 Bulk-Bunschoten, A. M.,Pasker-de Jong, P. C.,van Wouwe, J. P.,de Groot, C. J. (2008). Ethnic variation in infant-feeding practices in the Netherlands and weight gain at 4 months $\mathrm{J}$ Hum Lact, 24(1), 42-9

324 Bulk-Bunschoten, A. M.,van Bodegom, S.,Reerink, J. D.,de Jong, P. C.,de Groot, C. J. (2002). Weight and weight gain at 4 months (The Netherlands 1998): influences of nutritional practices, socio-economic and ethnic factors Paediatr Perinat Epidemiol, 16(4), $361-9$

325 Bulkow, L. R.,Singleton, R. J.,DeByle, C.,Miernyk, K.,Redding, G.,Hummel, K. B.,Chikoyak, L.,Hennessy, T. W. (2012). Risk factors for hospitalization with lower respiratory tract infections in children in rural Alaska Pediatrics, 129(5), e1220-7

326 Bulkow, L. R.,Singleton, R. J.,Karron, R. A.,Harrison, L. H. (2002). Risk factors for severe respiratory syncytial virus infection among Outcome Alaska native children Pediatrics, 109(2), 210-6

327 Bunik, M.,Shobe, P.,O'Connor, M. E.,Beaty, B.,Langendoerfer, S.,Crane, L. (2007). Randomized controlled trial to evaluate a telephone support intervention for breastfeeding in low-income Latina mothers Breastfeeding medicine, 2(3), 183

328 Burd, L.,Fisher, W.,Kerbeshian, J.,Vesely, B.,Durgin, B.,Reep, P. (1988). A comparison of breastfeeding rates among children with Study design pervasive developmental disorder, and controls J Dev Behav Pediatr, 9(5), 247-51

329 Burdette, H. L.,Whitaker, R. C. (2007). Differences by race and ethnicity in the relationship between breastfeeding and obesity in preschool children Ethn Dis, 17(3), 467-70

330 Burdette, H. L.,Whitaker, R. C.,Hall, W. C.,Daniels, S. R. (2006). Breastfeeding, introduction of complementary foods, and adiposity at $5 \mathrm{y}$ of age Am J Clin Nutr, 83(3), 550-8

331 Burgess, S. W.,Dakin, C. J.,O'Callaghan, M. J. (2006). Breastfeeding does not increase the risk of asthma at 14 years Pediatrics, 117(4), e787-92

332 Burke, V.,Beilin, L. J.,Simmer, K.,Oddy, W. H.,Blake, K. V.,Doherty, D.,Kendall, G. E.,Newnham, J. P.,Landau, L. I.,Stanley, F. J. (2005). Breastfeeding and overweight: Iongitudinal analysis in an Australian birth cohort J Pediatr, 147(1), 56-61

Publication date for a non-sibling study

Publication date for a non-sibling study

Outcome

Publication date for a non-sibling study

333 Burns, E.,Schmied, V.,Sheehan, A.,Fenwick, J. (2009). Let women express themselves - breastfeeding study Australian Nursing Study design Journal, 17(2), 44-45 2p

334 Burr, M. L.,Limb, E. S.,Maguire, M. J.,Amarah, L.,Eldridge, B. A.,Layzell, J. C.,Merrett, T. G. (1993). Infant feeding, wheezing, and Outcome allergy: a prospective study Arch Dis Child, 68(6), 724-8 at high risk of allergy $\mathrm{J}$ Epidemiol Community Health, 43(2), 125-32 336 Businco, L.,Marchetti, F.,Pellegrini, G.,Cantani, A.,Perlini, R. (1983). Prevention of atopic disease in "at-risk newborns" by prolonged Intervention/exposure
breast-feeding Ann Allergy, 51(2 Pt 2), 296-9

337 Butland, B. K.,Strachan, D. P.,Lewis, S.,Bynner, J.,Butler, N.,Britton, J. (1997). Investigation into the increase in hay fever and eczema at age 16 observed between the 1958 and 1970 British birth cohorts BMJ, 315(7110), 717-21 
340 Butte, N. F.,Wong, W. W.,Ferlic, L.,Smith, E. O.,Klein, P. D., Garza, C. (1990). Energy expenditure and deposition of breast-fed and formula-fed infants during early infancy Pediatr Res, 28(6), 631-40

Study design, Size of study groups

341 Butte, N. F.,Wong, W. W.,Hopkinson, J. M.,Smith, E. O.,Ellis, K. J. (2000). Infant feeding mode affects early growth and body composition Pediatrics, 106(6), 1355-66

342 Butters, L.,McCabe, R. (1988). The influence of breast and bottle feeding on blood pressure Midwifery, 4(3), 130-2

Size of study groups

Study design, Outcome

343 Buyken, A. E.,Karaolis-Danckert, N.,Remer, T.,Bolzenius, K.,Landsberg, B.,Kroke, A. (2008). Effects of breastfeeding on trajectories Intervention/exposure of body fat and BMI throughout childhood Obesity (Silver Spring), 16(2), 389-95

344 Bystrova, K.,Matthiesen, A. S.,Widstrom, A. M.,Ransjo-Arvidson, A. B.,Welles-Nystrom, B., Vorontsov, I.,Uvnas-Moberg, K. (2007). The effect of Russian Maternity Home routines on breastfeeding and neonatal weight loss with special reference to swaddling Early Hum Dev, 83(1), 29-39

345 Cable, N.,Bartley, M.,McMunn, A.,Kelly, Y. (2010). 011 Gender differences in the effect of breast feeding on adult psychological well-being Journal of Epidemiology \& Community Health, 64(\#issue\#), A4-5 1p

346 Cable, N.,Bartley, M.,McMunn, A.,Kelly, Y. (2012). Gender differences in the effect of breastfeeding on adult psychological wellbeing Eur J Public Health, 22(5), 653-8

347 Cai, S.,Pang, W. W.,Low, Y. L.,Sim, L. W.,Sam, S. C.,Bruntraeger, M. B.,Wong, E. Q.,Fok, D.,Broekman, B. F.,Singh, L.,Richmond, J.,Agarwal, P.,Qiu, A.,Saw, S. M.,Yap, F.,Godfrey, K. M.,Gluckman, P. D.,Chong, Y. S.,Meaney, M. J.,Kramer, M. S.,Rifkin-Graboi, A. (2015). Infant feeding effects on early neurocognitive development in Asian children Am J Clin Nutr, 101(2), 326-36

\begin{tabular}{ll}
\hline 348 & Calamaro, C. J. (2000). Infant nutrition in the first year of life: tradition or science? Pediatr Nurs, 26(2), 211-5 \\
\hline 349 & Calvo, E. B.,Galindo, A. C.,Aspres, N. B. (1992). Iron status in exclusively breast-fed infants Pediatrics, 90(3 I), 375-379
\end{tabular}

350 Cama, R. I.,Parashar, U. D., Taylor, D. N.,Hickey, T.,Figueroa, D.,Ortega, Y. R.,Romero, S.,Perez, J.,Sterling, C. R., Gentsch, J. R.,Gilman, R. H.,Glass, R. I. (1999). Enteropathogens and other factors associated with severe disease in children with acute watery diarrhea in Lima, Peru Journal of Infectious Diseases, 179(5), 1139-1144

351 Camara, A. A.,Silva, J. M.,Ferriani, V. P.,Tobias, K. R.,Macedo, I. S.,Padovani, M. A.,Harsi, C. M.,Cardoso, M. R.,Chapman, M. D.,Arruda, E.,Platts-Mills, T. A.,Arruda, L. K. (2004). Risk factors for wheezing in a subtropical environment: role of respiratory viruses and allergen sensitization J Allergy Clin Immunol, 113(3), 551-7

352 Camargo-Figuera, F. A.,Barros, A. J.,Santos, I. S.,Matijasevich, A.,Barros, F. C. (2014). Early life determinants of low IQ at age 6 in Outcome children from the 2004 Pelotas Birth Cohort: a predictive approach BMC Pediatr, 14(\#issue\#), 308 
354 Cameron, S. L.,Gray, A. R.,Taylor, R. W.,Lawrence, J. A.,Galland, B. C.,Hanna, M. B.,Heath, A. L. M.,Sayers, R. M.,Taylor, B. J. (2014). Excessive growth from 6 to 24 months of age: Results from the prevention of overweight in infancy (POI) randomised controlled trial Archives of disease in childhood, 99(\#issue\#), A109

355 Campbell N (1981). The nutritional and immunological benefits of breast milk Aust Nurses J, 10(\#issue\#), 40-3, 47 Campos-Martinez, A.,Serrano- Lopez, L.,Medina- Navarro, M.,Ochoa- Herrera, J.,Pena-Caballero, M. (2012). Levels of docosahexaenoic acid in pregnant women and their children after taking a fish oil enriched diet Journal of maternal-fetal \& neonatal medicine, 25(\#issue\#), 92

357 Campus, G.,Solinas, G.,Sanna, A.,Maida, C.,Castiglia, P. (2007). Determinants of ECC in Sardinian preschool children Community Dent Health, 24(4), 253-6

358 Camurdan, M. O.,Camurdan, A. D.,Polat, S.,Beyazova, U. (2011). Growth patterns of large, small, and appropriate for gestational age infants: impacts of long-term breastfeeding: a retrospective cohort study J Pediatr Endocrinol Metab, 24(7-8), 463-8
Study design

Publication status

Intervention/exposure

Intervention/exposure

Study design

Study design, Participant health

Study design,

Intervention/exposure

Intervention/exposure

362 Capeding, R.,Gepanayao, C. P.,Calimon, N.,Lebumfacil, J.,Davis, A. M.,Stouffer, N.,Harris, B. J. (2010). Lutein-fortified infant formula fed to healthy term infants: evaluation of growth effects and safety Nutr J, 9(\#issue\#), 22

363 Caplan, L. S.,Erwin, K.,Lense, E.,Hicks, J., Jr. (2008). The potential role of breast-feeding and other factors in helping to reduce early childhood caries J Public Health Dent, 68(4), 238-41

364 Capozzi, L.,Russo, R.,Bertocco, F.,Ferrara, D.,Ferrara, M. (2010). Diet and iron deficiency in the first year of life: a retrospective study Hematology, 15(6), 410-3

365 Capozzi, L.,Russo, R.,Bertocco, F.,Ferrara, D.,Ferrara, M. (2011). Effect on haematological and anthropometric parameters of iron supplementation in the first 2 years of life. Risks and benefits Hematology, 16(5), 261-4

366 Carberry, A. E.,Colditz, P. B.,Lingwood, B. E. (2010). Body composition from birth to 4.5 months in infants born to non-obese women Pediatr Res, 68(1), 84-8

367 Carling, S. J.,Demment, M. M.,Kjolhede, C. L.,Olson, C. M. (2015). Breastfeeding duration and weight gain trajectory in infancy Pediatrics, 135(1), 111-9

368 Carlsen, K. H.,Larsen, S.,Bjerve, O.,Leegaard, J. (1987). Acute bronchiolitis: predisposing factors and characterization of infants at risk Pediatr Pulmonol, 3(3), 153-60
Study design

ntervention/exposure

Participant health

Intervention/exposure

Size of study groups

Outcome for a non-sibling study

Size of study groups 
369 Carlson, S. E.,DeVoe, P. W.,Barness, L. A. (1982). Effect of infant diets with different polyunsaturated to saturated fat ratios on circulating high-density lipoproteins J Pediatr Gastroenterol Nutr, 1(3), 303-9

370 Carlson, S. E.,Ford, A. J.,Werkman, S. H.,Peeples, J. M.,Koo, W. W. (1996). Visual acuity and fatty acid status of term infants fed human milk and formulas with and without docosahexaenoate and arachidonate from egg yolk lecithin Pediatr Res, 39(5), 882-8

371 Carpenter, R.,McGarvey, C.,Mitchell, E. A.,Tappin, D. M.,Vennemann, M. M.,Smuk, M.,Carpenter, J. R. (2013). Bed sharing when parents do not smoke: Is there a risk of SIDS? An individual level analysis of five major case-control studies BMJ Open, 3(5), \#Pages\#

372 Carr, A. (2009). Breastfeeding and the WIC program Breastfeed Med, 4 Suppl 1(\#issue\#), S57-8

Study design

373 Carrascoza, K. C.,Possobon Rde, F.,Tomita, L. M.,Moraes, A. B. (2006). Consequences of bottle-feeding to the oral facial development of initially breastfed children J Pediatr (Rio J), 82(5), 395-7

374 Carroll, T. P. (1994). Substantially increasing breastfeeding: an accomplishment of the Alabama WIC Program J Hum Lact, 10(2), $129-30$

375 Carson, C. G. (2013). Risk factors for developing atopic dermatitis Dan Med J, 60(7), B4687

Intervention/exposure

376 Carter, C. S.,Porges, E. C. (2011). Parenthood, stress, and the brain Biol Psychiatry, 70(9), 804-5

Study design

377 Carvalho, R.,Johnson, E.,Kozlosky, M.,Scheimann, A. O. (2008). Clinical profile of the overweight child in the new millennium Clin Pediatr (Phila), 47(5), 476-82

378 Casazza, Krista,Fernandez, Jose R.,Allison, David B. (2012). Modest Protective Effects of Breast-feeding on Obesity: Is the Evidence Truly Supportive? Nutrition Today, 47(1), 33-40 8p

379 Casiday, R. E.,Wright, C. M.,Panter-Brick, C.,Parkinson, K. N. (2004). Do early infant feeding patterns relate to breast-feeding continuation and weight gain? Data from a longitudinal cohort study Eur J Clin Nutr, 58(9), 1290-6

380 Caspi, A.,Williams, B.,Kim-Cohen, J.,Craig, I. W.,Milne, B. J.,Poulton, R.,Schalkwyk, L. C., Taylor, A.,Werts, H.,Moffitt, T. E. (2007) Moderation of breastfeeding effects on the IQ by genetic variation in fatty acid metabolism Proc Natl Acad Sci U S A, 104(47), 18860-5

381 Cassimos, D. C.,Tsalkidis, A.,Tripsianis, G. A.,Stogiannidou, A.,Anthracopoulos, M.,Ktenidou-Kartali, S.,Aivazis, V.,,Gardikis, S.,Chatzimichael, A. (2008). Asthma, lung function and sensitization in school children with a history of bronchiolitis Pediatr Int, $50(1), 51-6$

382 Castelo, P. M.,Gaviao, M. B.,Pereira, L. J.,Bonjardim, L. R. (2010). Maximal bite force, facial morphology and sucking habits in young children with functional posterior crossbite J Appl Oral Sci, 18(2), 143-8

Study design, Participant health

Study design

Outcome

Outcome

Study design

Castiglione, F.,Diaferia, M.,Morace, F.,Labianca, O.,Meucci, C.,Cuomo, A.,Panarese, A.,Romano, M.,Sorrentini, I.,D'Onofrio,

C.,Caporaso, N.,Rispo, A. (2012). Risk factors for inflammatory bowel diseases according to the "hygiene hypothesis": a casecontrol, multi-centre, prospective study in Southern Italy J Crohns Colitis, 6(3), 324-9 
384 Castillo, C.,Atalah, E.,Riumallo, J.,Castro, R. (1996). Breast-feeding and the nutritional status of nursing children in Chile Bull Pan Am Health Organ, 30(2), 125-33

385 Castro-Rodriguez, J. A.,Mallol, J.,Rodriguez, J.,Auger, F.,Andrade, R. (2008). Risk factors for X-ray pneumonia in the first year of life and its relation to wheezing: a longitudinal study in a socioeconomic disadvantaged population Allergol Immunopathol (Madr), $36(1), 3-8$

386 Castro-Rodriguez, J. A.,Stern, D. A.,Halonen, M.,Wright, A. L.,Holberg, C. J.,Taussig, L. M.,Martinez, F. D. (2001). Relation between infantile colic and asthma/atopy: a prospective study in an unselected population Pediatrics, 108(4), 878-82

387 Cattaneo, A. (2013). Infant and young child feeding: solid facts Breastfeed Rev, 21(2), 7-9

388 Cattaneo, A.,Ronfani, L.,Burmaz, T.,Quintero-Romero, S.,Macaluso, A.,Di Mario, S. (2006). Infant feeding and cost of health care: a Outcome cohort study Acta Paediatr, 95(5), 540-6

389 Cattaneo, A., Timmer, A.,Bomestar, T.,Bua, J.,Kumar, S.,Tamburlini, G. (2008). Child nutrition in countries of the Commonwealth of Study design Independent States: time to redirect strategies? Public Health Nutr, 11(12), 1209-19

390 Caudri, D.,Savenije, O. E.,Smit, H. A.,Postma, D. S.,Koppelman, G. H.,Wijga, A. H.,Kerkhof, M.,Gehring, U.,Hoekstra, M.

Outcome

O.,Brunekreef, B.,de Jongste, J. C. (2013). Perinatal risk factors for wheezing phenotypes in the first 8 years of life Clin Exp Allergy, 43(12), $1395-405$

391 Caulfield, L. E.,Bentley, M. E.,Ahmed, S. (1996). Is prolonged breastfeeding associated with malnutrition? Evidence from nineteen demographic and health surveys Int J Epidemiol, 25(4), 693-703

392 Caulfield, L. E.,Bose, A.,Chandyo, R. K.,Nesamvuni, C.,de Moraes, M. L.,Turab, A.,Patil, C.,Mahfuz, M.,Ambikapathi, R.,Ahmed, T. (2014). Infant feeding practices, dietary adequacy, and micronutrient status measures in the MAL-ED study Clin Infect Dis, 59 Supp 4(\#issue\#), S248-54

393 Cavalcante e Silva, A.,Correia, L. L.,Campos, J. S.,Andrade, F. M.,Silveira, D. M.,Leite, A. J.,Rocha, H. A.,Machado, M. M.,Cunha, A. J. (2015). Reducing child mortality: the contribution of Ceara state, northeast of Brazil, on achieving the Millennium Development Goal 4 in Brazil Matern Child Health J, 19(4), 700-6

394 Çelikkiran, S.,Bozkurt, H.,Coşkun, M. (2015). Denver developmental test findings and their relationship with sociodemographic variables in a large community sample of 0-4-year-old children Noropsikiyatri Arsivi, 52(2), 180-184

395 Celikkiran, S.,Bozkurt, H.,Coskun, M. (2015). Denver developmental test findings and their relationship with sociodemographic variables in a large community sample of 0-4-year-old children Noropsikiyatri Arsivi, 52(2), 180-4

396 Cerrato, P. L. (1992). Preventing food allergies Rn, 55(10), 73-5

Study design

Study design

Study design

Study design

Study design

Study design

397 Cerrato, P. L. (1993). Does milk cause juvenile diabetes? Rn, 56(1), 69-72

Study design

398 Cesar, J. A.,Victora, C. G.,Barros, F. C.,Santos, I. S.,Flores, J. A. (1999). Impact of breast feeding on admission for pneumonia during postneonatal period in Brazil: nested case-control study BMJ, 318(7194), 1316-20

Intervention/exposure 
399 Chaffee, B. W.,Feldens, C. A.,Vitolo, M. R. (2014). Association of long-duration breastfeeding and dental caries estimated with marginal structural models Ann Epidemiol, 24(6), 448-54

400 Chaimay, B.,Ruagdaraganon, N.,Thinkhamrop, B.,Thinkhamrop, J. (2015). Association between infant feeding practices and first meaningful words at first year of life: a prospective cohort study of Thai children Asia Pac J Public Health, 27(2), NP1071-84

401 Challacombe, D. N.,Mecrow, I. K.,Elliott, K.,Clarke, F. J.,Wheeler, E. E. (1997). Changing infant feeding practices and declining incidence of coeliac disease in West Somerset Arch Dis Child, 77(3), 206-9

402 Chaman, R.,Alami, A.,Emamian, M. H.,Naieni, K. H.,Mirmohammadkhani, M.,Ahmadnezhad, E.,Entezarmahdi, R.,Shati, M.,Shariati, M. (2012). Important risk factors of mortality among children aged 1-59 months in rural areas of Shahroud, Iran: A community-based nested case-control study International Journal of Preventive Medicine, 3(12), 875-879

403 Chan, G. M.,Leeper, L.,Book, L. S. (1987). Effects of soy formulas on mineral metabolism in term infants Am J Dis Child, 141(5), $527-30$

404 Chan, G. M.,Roberts, C. C.,Folland, D.,Jackson, R. (1982). Growth and bone mineralization of normal breast-fed infants and the effects of lactation on maternal bone mineral status Am J Clin Nutr, 36(3), 438-43

405 Chandra J,Jain V,Narayan S,Sharma S,Singh V,Kapoor AK,Batra S (2002). Folate and cobalamin deficiency in megaloblastic anemia in children Indian Pediatr, 39(\#issue\#), 453-7

406 Chandra, R. K. (1997). Five-year follow-up of high-risk infants with family history of allergy who were exclusively breast-fed or fed partial whey hydrolysate, soy, and conventional cow's milk formulas J Pediatr Gastroenterol Nutr, 24(4), 380-8

407 Chandra, R. K.,Hamed, A. (1991). Cumulative incidence of atopic disorders in high risk infants fed whey hydrolysate, soy, and conventional cow milk formulas Ann Allergy, 67(2 Pt 1), 129-32

408 Chandra, R. K.,Puri, S.,Cheema, P. S. (1985). Predictive value of cord blood IgE in the development of atopic disease and role of breast-feeding in its prevention Clin Allergy, 15(6), 517-22

409 Chandra, R. K.,Puri, S.,Hamed, A. (1989). Influence of maternal diet during lactation and use of formula feeds on development o atopic eczema in high risk infants BMJ 1989 Oct 7;299(6704):896 BMJ (Clinical research ed.), 299(6693), 228-30

410 Chandra, R. K.,Puri, S.,Suraiya, C.,Cheema, P. S. (1986). Influence of maternal food antigen avoidance during pregnancy and lactation on incidence of atopic eczema in infants Clin Allergy, 16(6), 563-9

Outcome

Size of study groups, Outcome

Outcome

Size of study groups

Size of study groups

Country

Retracted

Intervention/exposure

Intervention/exposure

Retracted

Reliability of the data is questionable (other articles by the author retracted)

\begin{tabular}{|c|c|c|}
\hline 411 & Chandran, L.,Gelfer, P. (2006). Breastfeeding: the essential principles Pediatr Rev, 27(11), 409-17 & Study design \\
\hline 412 & $\begin{array}{l}\text { Chang YT, Germain-Lee EL,Doran TF,Migeon CJ,Levine MA,Berkovitz GD (1992). Hypocalcemia in nonwhite breast-fed infants. } \\
\text { Vitamin D deficiency revisited Clin Pediatr (Phila), 31(\#issue\#), 695-8 }\end{array}$ & Study design \\
\hline 413 & $\begin{array}{l}\text { Chanoine, J. P.,Boulvain, M.,Bourdoux, P.,Pardou, A.,Van Thi, H. V.,Ermans, A. M.,Delange, F. (1988). Increased recall rate at } \\
\text { screening for congenital hypothyroidism in breast fed infants born to iodine overloaded mothers Arch Dis Child, 63(10), } 1207-10\end{array}$ & $\begin{array}{l}\text { Study design, } \\
\text { Intervention/exposure }\end{array}$ \\
\hline
\end{tabular}


414 Chan-Yeung, M.,Ferguson, A.,Watson, A.,Dimich, WArd H.,Dybuncio, A.,Rousseau, R.,Becker, A. (2005). Breastfeeding and risk of Publication status asthma and other allergic diseases at aged 7 years in a high-risk birth-cohort [Abstract] American Thoracic Society 2005 International Conference; May 20-25; San Diego, California, \#volume\#(\#issue\#), [C49] [Poster: A85]

415 Chan-Yeung, M.,Ferguson, A.,Watson, W.,Dimich-Ward, H.,Rousseau, R.,Lilley, M.,Dybuncio, A.,Becker, A. (2005). The Canadian Intervention/exposure Childhood Asthma Primary Prevention Study: outcomes at 7 years of age J Allergy Clin Immunol, 116(1), 49-55

416 Chan-Yeung, M.,Manfreda, J.,Dimich-Ward, H.,Ferguson, A.,Watson, W.,Becker, A. (2000). A randomized controlled study on the effectiveness of a multifaceted intervention program in the primary prevention of asthma in high-risk infants Arch Pediatr Adolesc Med, 154(7), 657-63

417 Chan-Yip, A.,Gray-Donald, K. (1987). Prevalence of iron deficiency among Chinese children aged 6 to 36 months in Montreal CMAJ, 136(4), 373-8

418 Chaparro, C. M.,Neufeld, L. M.,Tena Alavez, G.,Eguia-Liz Cedillo, R.,Dewey, K. G. (2006). Effect of timing of umbilical cord clamping on iron status in Mexican infants: a randomised controlled trial Lancet, 367(9527), 1997-2004

419 Chapman NL,Barnett DC (1982). In defense of bottle-feeding J Pract Nurs, 32(\#issue\#), 24-7, 38

Intervention/exposure

420 Chapman, D. J. (2011). Breastfeeding, brain imaging, and maternal behavior J Hum Lact, 27(3), 304-5

421 Chapman, D. J. (2012). Exclusive breastfeeding through 6 months: infant intake and growth patterns J Hum Lact, 28(2), 132-3

Intervention/exposure

422 Chapman, D. J. (2012). Longer cumulative breastfeeding duration associated with improved bone strength J Hum Lact, 28(1), 18-9

423 Chapman, D. J. (2013). Does breastfeeding result in smarter children? A closer look J Hum Lact, 29(4), 444-5

Intervention/exposure

Study design

424 Chapman, D. J.,Morel, K.,Bermudez-Millan, A.,Young, S.,Damio, G.,Perez-Escamilla, R. (2013). Breastfeeding education and support trial for overweight and obese women: a randomized trial Pediatrics, 131(1), e162-70

425 Chapman, D. J.,Nommsen-Rivers, L. (2012). Impact of maternal nutritional status on human milk quality and infant outcomes: an update on key nutrients Adv Nutr, 3(3), 351-2

426 Chatzimichael, A.,Tsalkidis, A.,Cassimos, D.,Gardikis, S.,Tripsianis, G.,Deftereos, S.,Ktenidou-Kartali, S., Tsanakas, I. (2007). The role of breastfeeding and passive smoking on the development of severe bronchiolitis in infants Minerva Pediatr, 59(3), 199-206

427 Chavalittamrong, B.,Jirapinyo, P. (1987). The weight of Thai infants exclusively breast-fed and formula-fed from birth to four months J Med Assoc Thai, 70(5), 247-51

428 Chavez-Payan, P.,Grineski, S. E.,Collins, T. W. (2015). Early Life and Environmental Risk Factors Modify the Effect of Acculturation on Hispanic Children's Asthma Hisp Health Care Int, 13(3), 119-30

429 Chellakooty, M.,Juul, A.,Boisen, K. A.,Damgaard, I. N.,Kai, C. M.,Schmidt, I. M.,Petersen, J. H.,Skakkebæk, N. E.,Main, K. M. (2006). A prospective study of serum insulin-like growth factor I (IGF-I) and IGF-binding protein-3 in 942 healthy infants:

Associations with birth weight, gender, growth velocity, and breastfeeding Journal of Clinical Endocrinology and Metabolism, 91(3), 820-826 


\begin{tabular}{|c|c|c|}
\hline 430 & Chen, A.,Rogan, W. J. (2004). Breastfeeding and the risk of postneonatal death in the United States Pediatrics, 113(5), e435-9 & Outcome \\
\hline 431 & $\begin{array}{l}\text { Chen, B. Y.,Chan, C. C.,Han, Y. Y.,Wu, H. P.,Guo, Y. L. (2012). The risk factors and quality of life in children with allergic rhinitis in } \\
\text { relation to seasonal attack patterns Paediatr Perinat Epidemiol, 26(2), 146-55 }\end{array}$ & Study design \\
\hline 432 & $\begin{array}{l}\text { Chen, C. F.,Hsu, M. C.,Shen, C. H.,Wang, C. L.,Chang, S. C.,Wu, K. G.,Wu, S. C.,Chen, S. J. (2011). Influence of breast-feeding on } \\
\text { weight loss, jaundice, and waste elimination in neonates Pediatr Neonatol, 52(2), 85-92 }\end{array}$ & Outcome \\
\hline 433 & $\begin{array}{l}\text { Chen, C. J.,Wu, F. T.,Hsiung, C. A.,Chang, W. C.,Wu, H. S.,Wu, C. Y.,Lin, J. S.,Huang, F. C.,Huang, Y. C. (2012). Risk factors for } \\
\text { salmonella gastroenteritis in children less than five years of age in Taiwan Pediatr Infect Dis J, 31(12), e239-43 }\end{array}$ & Outcome \\
\hline 434 & $\begin{array}{l}\text { Chen, K.,Chai, L.,Li, H.,Zhang, Y.,Xie, H. M.,Shang, J.,Tian, W.,Yang, P.,Jiang, A. C. (2015). Effect of bovine lactoferrin from iron- } \\
\text { fortified formulas on morbidity of diarrhea and respiratory tract infections of weaned infants in a randomized controlled trial Nutrition, } \\
\text { \#volume\#(\#issue\#), \#Pages\# }\end{array}$ & Outcome \\
\hline 435 & $\begin{array}{l}\text { Chen, M. (2005). Test a model of breast-feeding duration for Vietnamese mothers in Taiwan Communicating Nursing Research, } \\
\text { 38(\#issue\#), 461-461 1p }\end{array}$ & Study design \\
\hline 436 & $\begin{array}{l}\text { Chen, S. M.,Du, J. W.,Jin, Y. M.,Qiu, L.,Du, Z. H.,Li, D. D.,Chen, H. Y.,Watanabe, C.,Umezaki, M. (2015). Risk Factors for Severe } \\
\text { Hand-Foot-Mouth Disease in Children in Hainan, China, 2011-2012 Asia Pac J Public Health, 27(7), 715-22 }\end{array}$ & Intervention/exposure, Outcome \\
\hline 437 & $\begin{array}{l}\text { Chen, X. C.,Liu, D. S.,Fu, A. Z.,Yan, H. C.,Yin, T. A.,Jing, Y. S.,Xu, Q. M. (1989). A longitudinal study on infant growth during the } \\
\text { first sixth months of life, in relation to the nutrition of the lactating mothers and to the breastmilk output Prog Food Nutr Sci, 13(2), } \\
113-37\end{array}$ & Intervention/exposure \\
\hline 438 & $\begin{array}{l}\text { Chen, Y. (1989). Synergistic effect of passive smoking and artificial feeding on hospitalization for respiratory illness in early } \\
\text { childhood Chest, 95(5), 1004-7 }\end{array}$ & Study design \\
\hline 439 & $\begin{array}{l}\text { Chen, Y. (1994). Relationship between type of infant feeding and hospitalization for gastroenteritis in Shanghai infants J Hum Lact, } \\
\text { 10(3), 177-9 }\end{array}$ & Study design \\
\hline 440 & $\begin{array}{l}\text { Chen, Y. C.,Tsai, C. H.,Lee, Y. (2012). Gestational medication use, birth conditions, and early postnatal exposures for childhood } \\
\text { asthma Clin Dev Immunol, 2012(\#issue\#), } 913426\end{array}$ & Intervention/exposure \\
\hline 441 & Chen, Y.,Yu, S. Z.,Li, W. X. (1988). Artificial feeding and hospitalization in the first 18 months of life Pediatrics, 81(1), 58-62 & Outcome \\
\hline 442 & $\begin{array}{l}\text { Cheng, S.,Volgyi, E.,Tylavsky, F. A.,Lyytikainen, A.,Tormakangas, T.,Xu, L.,Cheng, S. M.,Kroger, H.,Alen, M.,Kujala, U. M. (2009). } \\
\text { Trait-specific tracking and determinants of body composition: a 7-year follow-up study of pubertal growth in girls BMC Med, } \\
\text { 7(\#issue\#), } 5\end{array}$ & $\begin{array}{l}\text { Publication date for a non-sibling } \\
\text { study }\end{array}$ \\
\hline 443 & Cherian, A.,Lawande, R. V. (1987). Diarrhoeal disease in bottle fed children J R Soc Health, 107(2), 62-3 & Country \\
\hline 444 & $\begin{array}{l}\text { Chertok, I. R.,Raz, I.,Shoham, I.,Haddad, H.,Wiznitzer, A. (2009). Effects of early breastfeeding on neonatal glucose levels of term } \\
\text { infants born to women with gestational diabetes J Hum Nutr Diet, 22(2), 166-9 }\end{array}$ & $\begin{array}{l}\text { Study design, } \\
\text { Intervention/exposure }\end{array}$ \\
\hline 445 & Chertok, I. R.,Shoham-Vardi, I. (2008). Infant hospitalization and breastfeeding post-caesarean section Br J Nurs, 17(12), $786-91$ & Outcome \\
\hline
\end{tabular}




\begin{tabular}{|c|c|c|}
\hline 446 & Chesney, R. W. (2003). Rickets: an old form for a new century Pediatr Int, 45(5), 509-11 & Study design \\
\hline 447 & $\begin{array}{l}\text { Chhonker, D.,Faridi, M. M.,Narang, M.,Sharma, S. B. (2015). Does type of feeding in infancy influence lipid profile in later life? } \\
\text { Indian J Pediatr, 82(4), 345-8 }\end{array}$ & Country \\
\hline 448 & $\begin{array}{l}\text { Chiasson, M. A.,Scheinmann, R.,Hartel, D.,McLeod, N.,Sekhobo, J.,Edmunds, L. S.,Findley, S. (2015). Predictors of Obesity in a } \\
\text { Cohort of Children Enrolled in WIC as Infants and Retained to } 3 \text { Years of Age J Community Health, \#volume\#(\#issue\#), \#Pages\# }\end{array}$ & Intervention/exposure \\
\hline 449 & $\begin{array}{l}\text { Chierici, R.,Sawatzki, G.,Tamisari, L.,Volpato, S.,Vigi, V. (1992). Supplementation of an adapted formula with bovine lactoferrin. } 2 . \\
\text { Effects on serum iron, ferritin and zinc levels Acta Paediatr, 81(6-7), 475-9 }\end{array}$ & $\begin{array}{l}\text { Study design, Size of study } \\
\text { groups }\end{array}$ \\
\hline 450 & $\begin{array}{l}\text { Chierici, R.,Sawatzki, G.,Thurl, S.,Tovar, K.,Vigi, V. (1997). Experimental milk formulae with reduced protein content and } \\
\text { desialylated milk proteins: influence on the faecal flora and the growth of term newborn infants Acta Paediatr, 86(6), 557-63 }\end{array}$ & Size of study groups \\
\hline 451 & Chin, K. C.,Galea, P.,Goel, K. M. (1981). Changing pattern in infant feeding practices Health Bull (Edinb), 39(1), 51-7 & Outcome \\
\hline 452 & $\begin{array}{l}\text { Chiu, W. C.,Liao, H. F.,Chang, P. J.,Chen, P. C.,Chen, Y. C. (2011). Duration of breast feeding and risk of developmental delay in } \\
\text { Taiwanese children: a nationwide birth cohort study Paediatr Perinat Epidemiol, 25(6), 519-27 }\end{array}$ & Study design \\
\hline 453 & $\begin{array}{l}\text { Chivers, P.,Hands, B.,Parker, H.,Bulsara, M.,Beilin, L. J.,Kendall, G. E.,Oddy, W. H. (2010). Body mass index, adiposity rebound } \\
\text { and early feeding in a longitudinal cohort (Raine Study) Int J Obes (Lond), 34(7), 1169-76 }\end{array}$ & Intervention/exposure \\
\hline 454 & $\begin{array}{l}\text { Chmiel, R.,Beyerlein, A.,Knopff, A.,Hummel, S.,Ziegler, A. G.,Winkler, C. (2015). Early infant feeding and risk of developing islet } \\
\text { autoimmunity and type } 1 \text { diabetes Acta Diabetol, 52(3), 621-4 }\end{array}$ & Outcome \\
\hline 455 & $\begin{array}{l}\text { Chomtho, S. (2014). Breastfeeding to prevent double burden of malnutrition Southeast Asian J Trop Med Public Health, } 45 \text { Suppl } \\
\text { 1(\#issue\#), 132-6 }\end{array}$ & Study design \\
\hline 456 & $\begin{array}{l}\text { Chong, H. L.,Soo, T. L.,Rasat, R. (2012). Childhood obesity-prevalence among } 7 \text { and } 8 \text { year old primary school students in Kota } \\
\text { Kinabalu Medical Journal of Malaysia, } 67(2), 147-150\end{array}$ & Study design \\
\hline 457 & Christopher, G. C. (2009). First food: the essential role of breastfeeding Breastfeed Med, 4 Suppl 1(\#issue\#), S9-s10 & Study design \\
\hline 458 & $\begin{array}{l}\text { Chu, L.,Retnakaran, R.,Zinman, B.,Hanley, A. J. G.,Hamilton, J. K. (2012). Impact of maternal physical activity and infant feeding } \\
\text { practices on infant weight gain and adiposity International Journal of Endocrinology, 2012(\#issue\#), \#Pages\# }\end{array}$ & Intervention/exposure \\
\hline 459 & $\begin{array}{l}\text { Chuang, C. H.,Hsieh, W. S.,Chen, Y. C.,Chang, P. J.,Hurng, B. S.,Lin, S. J.,Chen, P. C. (2011). Infant feeding practices and } \\
\text { physician diagnosed atopic dermatitis: a prospective cohort study in Taiwan Pediatr Allergy Immunol, 22(1 Pt 1), 43-9 }\end{array}$ & Outcome \\
\hline 460 & $\begin{array}{l}\text { Chuansumrit, A.,Arnutti, P.,Apivanich, S. (2002). Iron status of one-year-old infants in a well baby clinic J Med Assoc Thai, } 85 \text { Suppl } \\
4(\text { \#issue\#), S1081-8 }\end{array}$ & $\begin{array}{l}\text { Study design, Size of study } \\
\text { groups }\end{array}$ \\
\hline 461 & Chye, J. K.,Lim, C. T. (1998). Breastfeeding at 6 months and effects on infections Singapore Med J, 39(12), 551-6 & Outcome \\
\hline 462 & $\begin{array}{l}\text { Ciardelli, R.,Haumont, D.,Gnat, D.,Vertongen, F.,Delange, F. (2002). The nutritional iodine supply of Belgian neonates is still } \\
\text { insufficient Eur J Pediatr, 161(10), 519-23 }\end{array}$ & Intervention/exposure \\
\hline
\end{tabular}


464 Ciria-Martin, A.,Caravia-Bernardo, F.,Alvarez-Castello, M.,Insua-Arregui, C.,Tamargo-Barbeito, T. O.,Massip-Nicot, J. (2012). [Risk Language factors for recurrent upper airways infections in pre-school children] Rev Alerg Mex, 59(3), 113-22

465 Civelek, E.,Cakir, B.,Orhan, F.,Yuksel, H.,Boz, A. B.,Uner, A.,Sekerel, B. E. (2011). Risk factors for current wheezing and its phenotypes among elementary school children Pediatr Pulmonol, 46(2), 166-74

Study design

466 Clark MJ (1984). A case for breast feeding Ky Nurse, 32(\#issue\#), 14-5

Study design

467 Clark, K. M.,Castillo, M.,Calatroni, A.,Walter, T.,Cayazzo, M.,Pino, P.,Lozoff, B. (2006). Breast-feeding and mental and motor development at 51/2 years Ambul Pediatr, 6(2), 65-71

468 Clark-Kellerman, M. J. (1985). A case for formula feeding Ky Nurse, 33(3), 13-4

Study design

469 Clavano, N. R. (1982). Mode of feeding and its effect on infant mortality and morbidity J Trop Pediatr, 28(6), 287-93

Country

470 Closa-Monasterolo, R.,Gispert-Llaurado, M.,Luque, V.,Ferre, N.,Rubio-Torrents, C.,Zaragoza-Jordana, M.,Escribano, J. (2013). Safety and efficacy of inulin and oligofructose supplementation in infant formula: results from a randomized clinical trial Clin Nutr, $32(6), 918-27$

471 Close, C. (1987). Babies, bottles, and boobs Br Med J (Clin Res Ed), 295(6613), 1666-7

Study design

472 Cochi, S. L.,Fleming, D. W.,Hightower, A. W.,Limpakarnjanarat, K.,Facklam, R. R.,Smith, J. D.,Sikes, R. K.,Broome, C. V. (1986). Primary invasive Haemophilus influenzae type b disease: a population-based assessment of risk factors J Pediatr, 108(6), 887-96

\section{Outcome}

473 Cockburn F,Belton NR,Purvis RJ,Giles MM,Brown JK,Turner TL,Wilkinson EM,Forfar JO,Barrie WJ,McKay GS,Pocock SJ (1980).

Size of study groups, Maternal vitamin D intake and mineral metabolism in mothers and their newborn infants $\mathrm{Br}$ Med J, 281(\#issue\#), 11-4

Intervention/exposure

Study design

474 Cockburn, F. (1994). Neonatal brain and dietary lipids Arch Dis Child Fetal Neonatal Ed, 70(1), F1-2

Outcome

475 Codispoti, C. D.,Levin, L.,LeMasters, G. K.,Ryan, P.,Reponen, T., Villareal, M.,Burkle, J.,Stanforth, S.,Lockey, J. E., Khurana Hershey, G. K.,Bernstein, D. I. (2010). Breast-feeding, aeroallergen sensitization, and environmental exposures during infancy are determinants of childhood allergic rhinitis J Allergy Clin Immunol, 125(5), 1054-1060 e1

476 Cogswell, J. J.,Mitchell, E. B.,Alexander, J. (1987). Parental smoking, breast feeding, and respiratory infection in development of allergic diseases Arch Dis Child, 62(4), 338-44

477 Colchero, M. A.,Contreras-Loya, D.,Lopez-Gatell, H.,Gonzalez de Cosio, T. (2015). The costs of inadequate breastfeeding of infants Study design in Mexico Am J Clin Nutr, 101(3), 579-86

478 Collipp, P. J.,Kuo, B.,Castro-Magana, M.,Chen, S. Y.,Salvatore, S. (1983). Hair zinc levels in infants Clin Pediatr (Phila), 22(7), 512- Study design

479 Cone, T. E., Jr. (1981). The nursing bottle caries syndrome JAMA, 245(22), 2334

Study design 


\begin{tabular}{|c|c|c|}
\hline 480 & Connolly, C. (2005). Saving babies: child-saving and infant nutrition Pediatr Nurs, 31(4), 309-11 & Study design \\
\hline 481 & $\begin{array}{l}\text { Connor, S. L.,Zhu, N.,Anderson, G. J.,Hamill, D.,Jaffe, E.,Carlson, J.,Connor, W. E. (2000). Cheek cell phospholipids in human } \\
\text { infants: a marker of docosahexaenoic and arachidonic acids in the diet, plasma, and red blood cells Am J Clin Nutr, 71(1), 21-7 }\end{array}$ & Size of study groups \\
\hline 482 & Conover B (1992). Exposures during pregnancy and lactation Nebr Med J, 77(\#issue\#), 65-7 & Study design \\
\hline 483 & Coombes R (1999). Bottling out over formula feed Nurs Times, 95(\#issue\#), 12-3 & Study design \\
\hline 484 & $\begin{array}{l}\text { Coppi, S.,lacoponi, F.,Fommei, C.,Strambi, M. (2013). Growth trend during the first six months of life in male infants with different } \\
\text { type of feeding Minerva Pediatr, 65(1), 51-9 }\end{array}$ & Outcome \\
\hline 485 & $\begin{array}{l}\text { Cornish, R. P.,Tilling, K.,Boyd, A.,Davies, A.,Macleod, J. (2015). Using linked educational attainment data to reduce bias due to } \\
\text { missing outcome data in estimates of the association between the duration of breastfeeding and IQ at } 15 \text { years Int J Epidemiol, } \\
44(3), 937-45\end{array}$ & Outcome \\
\hline 486 & $\begin{array}{l}\text { Corrao, G.,Tragnone, A., Caprilli, R.,Trallori, G.,Papi, C.,Andreoli, A.,Di Paolo, M.,Riegler, G.,Rigo, G. P.,Ferrau, O.,Mansi, } \\
\text { C.,Ingrosso, M.,Valpiani, D. (1998). Risk of inflammatory bowel disease attributable to smoking, oral contraception and } \\
\text { breastfeeding in Italy: a nationwide case-control study. Cooperative Investigators of the Italian Group for the Study of the Colon and } \\
\text { the Rectum (GISC) Int J Epidemiol, } 27(3), 397-404\end{array}$ & Intervention/exposure \\
\hline 487 & $\begin{array}{l}\text { Correa-Faria, P.,Martins-Junior, P. A., Vieira-Andrade, R. G.,Marques, L. S.,Ramos-Jorge, M. L. (2013). Perinatal factors associated } \\
\text { with developmental defects of enamel in primary teeth: a case-control study Braz Oral Res, 27(4), 363-8 }\end{array}$ & Outcome \\
\hline 488 & $\begin{array}{l}\text { Corvalan, C., Kain, J.,Weisstaub, G., Uauy, R. (2009). Impact of growth patterns and early diet on obesity and cardiovascular risk } \\
\text { factors in young children from developing countries Proc Nutr Soc, } 68(3), 327-37\end{array}$ & Study design \\
\hline 489 & $\begin{array}{l}\text { Corvalan, C.,Uauy, R.,Stein, A. D.,Kain, J.,Martorell, R. (2009). Effect of growth on cardiometabolic status at } 4 \text { y of age Am J Clin } \\
\text { Nutr, 90(3), } 547-55\end{array}$ & Study design \\
\hline 490 & $\begin{array}{l}\text { Costeira, M. J.,Oliveira, P.,Ares, S.,de Escobar, G. M.,Palha, J. A. (2009). lodine status of pregnant women and their progeny in the } \\
\text { Minho Region of Portugal Thyroid, 19(2), 157-63 }\end{array}$ & $\begin{array}{l}\text { Size of study groups, } \\
\text { Intervention/exposure }\end{array}$ \\
\hline 491 & $\begin{array}{l}\text { Counsilman, J. J.,Chan, S. Y.,Haiyon, H.,Rahim, N. A.,Salim, R.,Tai, T. Y.,Tan, M. L.,Zainy, Z.,Viegas, O. (1986). Breast feeding } \\
\text { among poor Singaporeans J Trop Pediatr, 32(6), 310-2 }\end{array}$ & Outcome \\
\hline 492 & Counsilman, J. J.,Chua, S.,Viegas, O. (1986). Breast feeding among well-to-do Singaporeans J Trop Pediatr, 32(6), 313-6 & Outcome \\
\hline 493 & $\begin{array}{l}\text { Counter, S. A.,Buchanan, L. H.,Ortega, F. (2004). Current pediatric and maternal lead levels in blood and breast milk in Andean } \\
\text { inhabitants of a lead-glazing enclave J Occup Environ Med, 46(9), 967-73 }\end{array}$ & $\begin{array}{l}\text { Study design, } \\
\text { Intervention/exposure }\end{array}$ \\
\hline 494 & $\begin{array}{l}\text { Couper, J. J.,Beresford, S.,Hirte, C.,Baghurst, P. A.,Pollard, A., Tait, B. D.,Harrison, L. C.,Colman, P. G. (2009). Weight gain in early } \\
\text { life predicts risk of islet autoimmunity in children with a first-degree relative with type } 1 \text { diabetes Diabetes Care, 32(1), 94-9 }\end{array}$ & Outcome \\
\hline
\end{tabular}


of association between duration of breast-feeding or introduction of cow's milk and development of islet autoimmunity Diabetes, 48(11), 2145-9

496 Courage, M. L.,McCloy, U. R.,Herzberg, G. R.,Andrews, W. L.,Simmons, B. S.,McDonald, A. C.,Mercer, C. N.,Friel, J. K. (1998). Visual acuity development and fatty acid composition of erythrocytes in full-term infants fed breast milk, commercial formula, or evaporated milk J Dev Behav Pediatr, 19(1), 9-17

\section{Cowden, M. (1982). Infant feeding Midwives Chron, 95(1136), 319-20}

498 Crestani, A. H.,Souza, A. P.,Beltrami, L.,Moraes, A. B. (2012). Analysis of the association among types of breastfeeding, presence of child development risk, socioeconomic and obstetric variables J Soc Bras Fonoaudiol, 24(3), 205-10

499 Crewe, E.,Murphy, A. M. (1980). Further studies on neonatal rotavirus infections Med J Aust, 1(2), 61-3

500 Crocker, B.,Green, T. J.,Barr, S. I.,Beckingham, B.,Bhagat, R.,Dabrowska, B.,Douthwaite, R.,Evanson, C.,Friesen, R.,Hydamaka, K.,Li, W.,Simmons, K.,Tse, L. (2011). Very high vitamin D supplementation rates among infants aged 2 months in Vancouver and Richmond, British Columbia, Canada BMC Public Health, 11(\#issue\#), 905

501 Crossland, D. S.,Richmond, S.,Hudson, M.,Smith, K.,Abu-Harb, M. (2008). Weight change in the term baby in the first 2 weeks of life Acta Paediatr, 97(4), 425-9

502 Crouch, A. A.,Seow, W. K.,Whitman, L. M.,Thong, Y. H. (1991). Effect of human milk and infant milk formulae on adherence of Giardia intestinalis Trans R Soc Trop Med Hyg, 85(5), 617-9

503 Crouch, S.,Lightfoot, T.,Simpson, J.,Smith, A.,Ansell, P.,Roman, E. (2012). Infectious illness in children subsequently diagnosed with acute lymphoblastic leukemia: modeling the trends from birth to diagnosis Am J Epidemiol, 176(5), 402-8

504 Crow, D. R. (1992). Baby bottle tooth decay prevention--a new program for the Texas Department of Health Tex Dent J, 109(8), 141

505 Croxatto, H. B.,Diaz, S.,Peralta, O.,Juez, G.,Herreros, C.,Casado, M. E.,Salvatierra, A. M.,Miranda, P.,Duran, E. (1983). Fertility regulation in nursing women: IV. Long-term influence of a low-dose combined oral contraceptive initiated at day 30 postpartum upon lactation and infant growth Contraception, 27(1), 13-25

506 Crume, T. L.,Bahr, T. M.,Mayer-Davis, E. J.,Hamman, R. F.,Scherzinger, A. L.,Stamm, E.,Dabelea, D. (2012). Selective protection against extremes in childhood body size, abdominal fat deposition, and fat patterning in breastfed children Arch Pediatr Adolesc Med, 166(5), 437-43

507 Crume, T. L.,Ogden, L. G.,Mayer-Davis, E. J.,Hamman, R. F.,Norris, J. M.,Bischoff, K. J.,McDuffie, R.,Dabelea, D. (2012). The impact of neonatal breast-feeding on growth trajectories of youth exposed and unexposed to diabetes in utero: the EPOCH Study Int J Obes (Lond), 36(4), 529-34

508 Crume, T. L.,Ogden, L.,Maligie, M.,Sheffield, S.,Bischoff, K. J.,McDuffie, R.,Daniels, S.,Hamman, R. F.,Norris, J. M.,Dabelea, D. (2011). Long-term impact of neonatal breastfeeding on childhood adiposity and fat distribution among children exposed to diabetes in utero Diabetes Care, 34(3), 641-5
Publication date for a non-sibling study

Study design

Study design, Outcome

Study design, Outcome

Study design, Outcome

Outcome, Publication date for a non-sibling study

Non-human sample, Intervention/exposure

Intervention/exposure

Study design

Intervention/exposure

Study design

Intervention/exposure

Study design,

Intervention/exposure 
509 Cruz, M. L.,Wong, W. W.,Mimouni, F.,Hachey, D. L.,Setchell, K. D.,Klein, P. D.,Tsang, R. C. (1994). Effects of infant nutrition on cholesterol synthesis rates Pediatr Res, 35(2), 135-40

510 Cuhaci Çakir, B.,Beyazova, U.,Kemalo?lu, Y. K.,Özkan, S.,Gündüz, B.,Özdek, A. (2012). Effectiveness of pandemic influenza $\mathrm{A} / \mathrm{H} 1 \mathrm{~N} 1$ vaccine for prevention of otitis media in children European journal of pediatrics, 171(11), 1667-71

511 Cullinan, T. R.,Saunders, D. I. (1983). Prediction of infant hospital admission risk Arch Dis Child, 58(6), 423-7

tudy design,

Intervention/exposure

512 Cunningham, A. S. (1987). Breast-feeding and health J Pediatr, 110(4), 658-9

Study design

513 Curtis, J. A.,Kooh, S. W.,Fraser, D.,Greenberg, M. L. (1983). Nutritional rickets in vegetarian children Can Med Assoc J, 128(2), $150-2$

514 Cushing, A. H.,Anderson, L. (1982). Diarrhea in breast-fed and non-breast-fed infants Pediatrics, 70(6), 921-5

Study design

Size of study groups

515 Cushing, A. H.,Samet, J. M.,Lambert, W. E.,Skipper, B. J.,Hunt, W. C.,Young, S. A.,McLaren, L. C. (1998). Breastfeeding reduces Outcome risk of respiratory illness in infants Am J Epidemiol, 147(9), 863-70

516 Cusick, S. E.,Mei, Z.,Cogswell, M. E. (2007). Continuing anemia prevention strategies are needed throughout early childhood in low- Outcome income preschool children J Pediatr, 150(4), 422-8, 428 e1-2

517 Cutting, W. A. (2002). Cholera and breastfeeding Trop Doct, 32(1), 57-8

Study design

518 da Costa Lima, R.,Victora, C. G.,Menezes, A. M.,Barros, F. C. (2003). Do risk factors for childhood infections and malnutrition

protect against asthma? A study of Brazilian male adolescents Am J Public Health, 93(11), 1858-64

Outcome

519 Dada, J. H. (2010). Nutrition and type 1 diabetes: can diet reduce risk? Today's Dietitian, 12(8), 36-39 4p

Study design

520 Dadhich, J. P.,Agarwal, R. K. (2009). Mainstreaming early and exclusive breastfeeding for improving child survival Indian Pediatr, 46(1), 11-7

521 Daga, S. R. (1989). Reduction in neonatal mortality by simple interventions J Biosoc Sci Suppl, 10(\#issue\#), 127-36

Country, Study design

Country

522 Daga, S. R.,Daga, A. S. (1989). Reduction in neonatal mortality with simple interventions J Trop Pediatr, 35(4), 191-6

Country, Study design

523 Dagan, R.,Pridan, H. (1982). Relationship of breast feeding versus bottle feeding with emergency room visits and hospitalization for Outcome infectious diseases Eur J Pediatr, 139(3), 192-4

524 Dahlquist, G.,Blom, L.,Lonnberg, G. (1991). The Swedish Childhood Diabetes Study--a multivariate analysis of risk determinants for Outcome diabetes in different age groups Diabetologia, 34(10), 757-62

525 Dahlquist, G.,Mustonen, L. (2000). Analysis of 20 years of prospective registration of childhood onset diabetes time trends and birth cohort effects. Swedish Childhood Diabetes Study Group Acta Paediatr, 89(10), 1231-7 
526 Dahlquist, G.,Savilahti, E.,Landin-Olsson, M. (1992). An increased level of antibodies to $\beta$-lactoglobulin is a risk determinant for early-onset Type 1 (insulin-dependent) diabetes mellitus independent of islet cell antibodies and early introduction of cow's milk Diabetologia, 35(10), 980-984

527 Dallaire, R.,Muckle, G.,Rouget, F.,Kadhel, P.,Bataille, H.,Guldner, L.,Seurin, S.,Chajes, V.,Monfort, C.,Boucher, O., Thome, J. P.,Jacobson, S. W.,Multigner, L.,Cordier, S. (2012). Cognitive, visual, and motor development of 7-month-old Guadeloupean infants exposed to chlordecone Environ Res, 118(\#issue\#), 79-85

528 Dalmeijer, G. W.,Wijga, A. H.,Gehring, U.,Renders, C. M.,Koppelman, G. H.,Smit, H. A.,van Rossem, L. (2015). Fatty acid composition in breastfeeding and school performance in children aged 12 years Eur J Nutr, \#volume\#(\#issue\#), \#Pages\#

29 Daly, K. A.,Rich, S. S.,Levine, S.,Margolis, R. H.,Le, C. T.,Lindgren, B.,Giebink, G. S. (1996). The family study of otitis media: design and disease and risk factor profiles Genet Epidemiol, 13(5), 451-68

530 Damore, D.,Mansbach, J. M.,Clark, S.,Ramundo, M.,Camargo, C. A., Jr. (2008). Prospective multicenter bronchiolitis study: predicting intensive care unit admissions Acad Emerg Med, 15(10), 887-94

531 Daniels, L. A.,Mallan, K. M.,Nicholson, J. M.,Battistutta, D.,Magarey, A. (2013). Outcomes of an early feeding practices intervention Intervention/exposure to prevent childhood obesity Pediatrics, 132(1), e109-e118

532 Darmstadt, G. L.,Munar, W. (2013). Behavior change and community participation: Assessing causal pathways affecting neonatal Study design mortality JAMA - Journal of the American Medical Association, 310(9), 969-70

533 Darnall, B. D.,Schatman, M. E. (2015). Protecting the infant from unknown risks Pain Med, 16(4), 631-2

Study design

534 DaVanzo, J.,Habicht, J. P. (1986). Infant mortality decline in Malaysia, 1946-1975: the roles of changes in variables and changes in the structure of relationships Demography, 23(2), 143-60

535 Davanzo, R.,Cannioto, Z.,Ronfani, L.,Monasta, L.,Demarini, S. (2013). Breastfeeding and neonatal weight loss in healthy term infants J Hum Lact, 29(1), 45-53

536 David, C. B.,David, P. H.,el Lozy, M. (1983). Determinants of breastfeeding duration and nutrition in a transition society J Trop Pediatr, 29(1), 45-9

537 Davidson, R.,Roberts, S. E.,Wotton, C. J.,Goldacre, M. J. (2010). Influence of maternal and perinatal factors on subsequent hospitalisation for asthma in children: evidence from the Oxford record linkage study BMC Pulm Med, 10(\#issue\#), 14

538 Davis, D. W.,Bell, P. A. (1991). Infant feeding practices and occlusal outcomes: a longitudinal study J Can Dent Assoc, 57(7), 593-4

Study design Intervention/exposure

Intervention/exposure

Country

Intervention/exposure

Outcome

539 Davis, J. (2014). Well advised: a journey to breastfeeding success Pract Midwife, 17(8), 34, 36-8

Study design

540 Davis, J. N.,Gunderson, E. P.,Gyllenhammer, L. E.,Goran, M. I. (2013). Impact of gestational diabetes mellitus on pubertal changes in adiposity and metabolic profiles in Latino offspring J Pediatr, 162(4), 741-5

541 Davis, J. N.,Weigensberg, M. J.,Shaibi, G. Q.,Crespo, N. C.,Kelly, L. A.,Lane, C. J.,Goran, M. I. (2007). Influence of breastfeeding on obesity and type 2 diabetes risk factors in Latino youth with a family history of type 2 diabetes Diabetes Care, 30(4), 784-9

Study design, Intervention/exposure

Publication date for a non-sibling study 
542 Davis, J. N.,Whaley, S. E.,Goran, M. I. (2012). Effects of breastfeeding and low sugar-sweetened beverage intake on obesity prevalence in Hispanic toddlers Am J Clin Nutr, 95(1), 3-8

543 Davis, J. R., Jr.,Goldenring, J.,Lubin, B. H. (1981). Nutritional vitamin B12 deficiency in infants Am J Dis Child, 135(6), 566-7

545 Davis, R. E.,Icke, G. C.,Hilton, J. M.,Orr, E. (1986). Serum thiamin, pyridoxal, cobalamin and folate concentrations in young infants Acta Paediatr Scand, 75(3), 402-7

Study design,

Intervention/exposure

546 Dawodu, A.,Davidson, B.,Woo, J. G.,Peng, Y. M.,Ruiz-Palacios, G. M.,de Lourdes Guerrero, M.,Morrow, A. L. (2015). Sun exposure and vitamin $D$ supplementation in relation to vitamin $D$ status of breastfeeding mothers and infants in the global exploration of human milk study Nutrients, $7(2), 1081-93$

547 Dawodu, A.,Zalla, L.,Woo, J. G.,Herbers, P. M.,Davidson, B. S.,Heubi, J. E.,Morrow, A. L. (2014). Heightened attention to supplementation is needed to improve the vitamin $D$ status of breastfeeding mothers and infants when sunshine exposure is restricted Matern Child Nutr, 10(3), 383-97

548 de Beer, M.,Vrijkotte, T. G.,Fall, C. H.,van Eijsden, M.,Osmond, C.,Gemke, R. J. (2015). Associations of infant feeding and timing of Intervention/exposure linear growth and relative weight gain during early life with childhood body composition Int J Obes (Lond), 39(4), 586-92

549 de Boer, R. (2011). A topic in 10 questions: assessing common dietary deficiencies J Fam Health Care, 21(6), 28-9

Study design

550 de Bruin, N. C.,Degenhart, H. J.,Gal, S.,Westerterp, K. R.,Stijnen, T.,Visser, H. K. (1998). Energy utilization and growth in breast-fed Size of study groups and formula-fed infants measured prospectively during the first year of life Am J Clin Nutr, 67(5), 885-96

551 de Fátima Buco Busto Moreno, Patrícia,Trombini Schmidt, Kayna (2014). BREAST-FEEDING AND FACTORS RELATED TO EARLY WEANING Cogitare Enfermagem, 19(3), 531-537 7p

552 de Freitas, C. L.,Romani, S.,Amigo, H. (1986). Breast-feeding and malnutrition in rural areas of northeast Brazil Bull Pan Am Health Organ, 20(2), 138-46

553 de Hoog, M. L.,van Eijsden, M.,Stronks, K.,Gemke, R. J.,Vrijkotte, T. G. (2011). The role of infant feeding practices in the explanation for ethnic differences in infant growth: the Amsterdam Born Children and their Development study Br J Nutr, 106(10), $1592-601$

554 de Jong, C.,Kikkert, H. K.,Fidler, V.,Hadders-Algra, M. (2010). The Groningen LCPUFA study: no effect of postnatal long-chain polyunsaturated fatty acids in healthy term infants on neurological condition at 9 years $\mathrm{Br} \mathrm{J} \mathrm{Nutr,} \mathrm{104(4),} \mathrm{566-72}$

Intervention/exposure

Intervention/exposure

Size of study groups, Outcome

Study design, Outcome

Outcome for a non-sibling study

Outcome

de Jong, C. Kikkert, H. K. Fidler, V. Hadders-Algra, M. (2012). Effects of long-chain polyunsaturated fatty acid supplementation of

Outcome

infant formula on cognition and behaviour at 9 years of age Dev Med Child Neurol, 54(12), 1102-8

556 de Jonge, L. L.,Langhout, M. A., Taal, H. R.,Franco, O. H.,Raat, H.,Hofman, A.,van Osch-Gevers, L.,Jaddoe, V. W. (2013). Infant feeding patterns are associated with cardiovascular structures and function in childhood J Nutr, 143(12), 1959-65

Outcome 
557 de Jonge, L. L.,van Osch-Gevers, L., Geelhoed, J. J.,Hofman, A.,Steegers, E. A.,Helbing, W. A.,Jaddoe, V. W. (2010). Breastfeeding is not associated with left cardiac structures and blood pressure during the first two years of life. The Generation $R$ Study Early Hum Dev, 86(8), 463-8

Outcome

558 De Kroon, M. L.,Renders, C. M.,Buskermolen, M. P.,Van Wouwe, J. P.,van Buuren, S.,Hirasing, R. A. (2011). The Terneuzen Birth Cohort. Longer exclusive breastfeeding duration is associated with leaner body mass and a healthier diet in young adulthood BMC Pediatr, 11(\#issue\#), 33

559 de la Hunty, A. (2009). The EU Childhood Obesity Project Nutrition Bulletin, 34(4), 403-406 4p

560

de Lima, L. F.,Barbosa, F., Jr.,Navarr Trace Elem Med Biol, 27(3), 221-5

561 de Looy, A. E. (1986). Infant nutrition Nursing (Lond), 3(12), 446-9

562 De Lucia Rolfe, E.,Modi, N.,Uthaya, S.,Hughes, I. A.,Dunger, D. B.,Acerini, C.,Stolk, R. P.,Ong, K. K. (2013). Ultrasound estimates of visceral and subcutaneous-abdominal adipose tissues in infancy J Obes, 2013(\#issue\#), 951954

563 de Melo, M. C. N.,Taddei, J. A. A. C.,Diniz-Santos, D. R.,Vieira, C.,Carneiro, N. B.,Melo, R. F.,Silva, L. R. (2008). Incidence of diarrhea in children living in urban slums in Salvador, Brazil Brazilian Journal of Infectious Diseases, 12(1), 89-93

564 de Oliveira Bezerra, Joana Lidyanne,De Vasconcelos, Maria Gorete Lucena,Pereira Linhares, Francisca Márcia,Javorski, Marly,Leal, Luciana Pedrosa (2014). Maternal perception of their children's body image in exclusive breastfeeding Acta Paulista de Enfermagem, 27(4), 293-299 7p

565 de Oliveira, D. M Dahan, P Ferreira, D. F de Oliveira L. F de Paula, L I de Figueiredo, A A de Bessa, J., Jr.,Bastos Netto, J. M. (2015). Association between exclusive maternal breastfeeding during the first 4 months of life and primary enuresis $\mathrm{J}$ Pediatr Urol, \#volume\#(\#issue\#), \#Pages\#

566 de Rooy, L.,Hawdon, J. (2002). Nutritional factors that affect the postnatal metabolic adaptation of full-term small- and large-forgestational-age infants Pediatrics, 109(3), E42

567 De Souza, A. C.,Petersont, K. E.,Cufino, E.,do Amaral, M. I.,Gardner, J. (2001). Underlying and proximate determinants of diarrhoea-specific infant mortality rates among municipalities in the state of Ceara, north-east Brazil: an ecological study $\mathrm{J}$ Biosoc Sci, 33(2), 227-44

568 Deacon C (2001). Breastfeeding. Are we just bottling out? Nurs Times, 97(\#issue\#), 26-7 Decker, E.,Engelmann, G.,Findeisen, A.,Gerner, P.,Laaß, M.,Ney, D.,Posovszky, C.,Hoy, L.,Hornef, M. W. (2010). Cesarean delivery is associated with celiac disease but not inflammatory bowel disease in children Pediatrics, 125(6), e1433-e1440

570 Decsi, T.,Kelemen, B.,Minda, H.,Burus, I. (2000). Long term effect of breast feeding on essential fatty acid status in healthy, full-term infants Adv Exp Med Biol, 478(\#issue\#), 397-8
Intervention/exposure

Study design

Intervention/exposure

Study design, Size of study groups

Study design

Intervention/exposure

Intervention/exposure

Study design

Outcome

Size of study groups, Outcome

Study design

Intervention/exposure

Study design

Outcome

Study design, Size of study

groups 
571 Decsi, T.,Kelemen, B.,Minda, H.,Burus, I.,Kohn, G. (2000). Effect of type of early infant feeding on fatty acid composition of plasma lipid classes in full-term infants during the second 6 months of life J Pediatr Gastroenterol Nutr, 30(5), 547-51

572 Decsi, T.,Koletzko, B. (1995). Growth, fatty acid composition of plasma lipid classes, and plasma retinol and alpha-tocopherol concentrations in full-term infants fed formula enriched with omega- 6 and omega-3 long-chain polyunsaturated fatty acids Acta Paediatr, 84(7), 725-32

573 Decsi, T.,Thiel, I.,Koletzko, B. (1995). Essential fatty acids in full term infants fed breast milk or formula Arch Dis Child Fetal Neonatal Ed, 72(1), F23-8

574 Dedoussis, G. V.,Yannakoulia, M.,Timpson, N. J.,Manios, Y.,Kanoni, S.,Scott, R. A.,Papoutsakis, C.,Deloukas, P.,Pitsiladis, Y. P.,Davey-Smith, G.,Hirschhorn, J. N.,Lyon, H. N. (2011). Does a short breastfeeding period protect from FTO-induced adiposity in children? Int J Pediatr Obes, 6(2-2), e326-35

575 Deegan, K. L.,Jones, K. M.,Zuleta, C.,Ramirez-Zea, M.,Lildballe, D. L.,Nexo, E.,Allen, L. H. (2012). Breast milk vitamin B-12 concentrations in Guatemalan women are correlated with maternal but not infant vitamin B-12 status at 12 months postpartum $\mathrm{J}$ Nutr, 142(1), 112-6

576 Deliu, M.,Belgrave, D.,Simpson, A.,Murray, C. S.,Kerry, G.,Custovic, A. (2014). Impact of rhinitis on asthma severity in school-age children Allergy, 69(11), 1515-21

577 Dell, S.,To, T. (2001). Breastfeeding and asthma in young children: findings from a population-based study Arch Pediatr Adolesc Med, 155(11), 1261-5

578 De-Lucchi C,Pita ML,Faus MJ,Periago JL,Gil A (1988). Influences of diet and postnatal age on the lipid composition of red blood cell Study design membrane in newborn infants Ann Nutr Metab, 32(\#issue\#), 231-9

579 DeLucchi, C.,Pita, M. L.,Faus, M. J.,Molina, J. A.,Uauy, R.,Gil, A. (1987). Effects of dietary nucleotides on the fatty acid composition Size of study groups of erythrocyte membrane lipids in term infants J Pediatr Gastroenterol Nutr, 6(4), 568-74

580 Demir, A. U.,Celikel, S.,Karakaya, G.,Kalyoncu, A. F. (2010). Asthma and allergic diseases in school children from 1992 to 2007 with incidence data J Asthma, 47(10), 1128-35

581 Demment, M. M.,Haas, J. D.,Olson, C. M. (2014). Changes in family income status and the development of overweight and obesity from 2 to 15 years: a longitudinal study BMC Public Health, 14(\#issue\#), 417

582 Demmers, T. A.,Jones, P. J.,Wang, Y.,Krug, S.,Creutzinger, V.,Heubi, J. E. (2005). Effects of early cholesterol intake on cholestero biosynthesis and plasma lipids among infants until 18 months of age Pediatrics, 115(6), 1594-601

583 Dennehy, P. H.,Cortese, M. M.,Begue, R. E.,Jaeger, J. L.,Roberts, N. E.,Zhang, R.,Rhodes, P.,Gentsch, J.,Ward, R.,Bernstein, D. Intervention/exposure I.,Vitek, C.,Bresee, J. S.,Staat, M. A. (2006). A case-control study to determine risk factors for hospitalization for rotavirus gastroenteritis in U.S. children Pediatr Infect Dis J, 25(12), 1123-31

584 Der, G.,Batty, G. D.,Deary, I. J. (2006). Effect of breast feeding on intelligence in children: Prospective study, sibling pairs analysis, and meta-analysis British Medical Journal, 333(7575), 945-948

Size of study groups, Intervention/exposure

Size of study groups, Intervention/exposure

Study design

Country

Outcome

Study design

Study design

Intervention/exposure

Size of study groups

Outcome 

Dewailly, E.,Ayotte, P.,Bruneau, S.,Gingras, S.,Belles-Isles, M.,Roy, R. (2000) infants exposed to organochlorines Environ Health Perspect, 108(3), 205-11

588 Dewey, K. G. (2000). Complementary feeding and breastfeeding Pediatrics, 106(5), 1301

589 Dewey, K. G.,Hawck, M. G.,Brown, K. H.,Lartey, A.,Cohen, R. J.,Peerson, J. M. (2005). Infant weight-for-length is positively associated with subsequent linear growth across four different populations Matern Child Nutr, 1(1), 11-20

590 Dewey, K. G.,Heinig, M. J.,Nommsen, L. A.,Lonnerdal, B. (1991). Adequacy of energy intake among breast-fed infants in the DARLING study: relationships to growth velocity, morbidity, and activity levels. Davis Area Research on Lactation, Infant Nutrition and Growth J Pediatr, 119(4), 538-47

591 Dewey, K. G.,Heinig, M. J.,Nommsen, L. A.,Peerson, J. M.,Lonnerdal, B. (1992). Growth of breast-fed and formula-fed infants from Intervention/exposure 0 to 18 months: the DARLING Study Pediatrics, 89(6 Pt 1), 1035-41

592 Dewey, K. G.,Heinig, M. J.,Nommsen, L. A.,Peerson, J. M.,Lonnerdal, B. (1993). Breast-fed infants are leaner than formula-fed infants at $1 \mathrm{y}$ of age: the DARLING study Am J Clin Nutr, 57(2), 140-5 Pediatr, 126(5 Pt 1), 696-702

594 Dewey, K. G.,Nommsen-Rivers, L. A.,Heinig, M. J.,Cohen, R. J. (2003). Risk factors for suboptimal infant breastfeeding behavior, delayed onset of lactation, and excess neonatal weight loss Pediatrics, 112(3 Pt 1), 607-19

595 Dewey, K. G.,Peerson, J. M.,Brown, K. H.,Krebs, N. F.,Michaelsen, K. F.,Persson, L. A.,Salmenpera, L.,Whitehead, R. G., Yeung, D. L. (1995). Growth of breast-fed infants deviates from current reference data: a pooled analysis of US, Canadian, and European data sets. World Health Organization Working Group on Infant Growth Pediatrics, 96(3 Pt 1), 495-503

596 Dewey, K. G.,Peerson, J. M.,Heinig, M. J.,Nommsen, L. A.,Lonnerdal, B.,Lopez de Romana, G.,de Kanashiro, H. C.,Black, R. E.,Brown, K. H. (1992). Growth patterns of breast-fed infants in affluent (United States) and poor (Peru) communities: implications for timing of complementary feeding Am J Clin Nutr, 56(6), 1012-8

597 Dharmage, S. C., Rajapaksa, L. C.,Fernando, D. N. (1996). Risk factors of acute lower respiratory tract infections in children under five years of age Southeast Asian J Trop Med Public Health, 27(1), 107-10

598 Dhillon, S. K.,Davies, W. E.,Hopkins, P. C.,Rose, S. J. (1998). Effects of dietary taurine on auditory function in full-term infants Adv Exp Med Biol, 442(\#issue\#), 507-14

599 Diaz, S.,Herreros, C.,Aravena, R.,Casado, M. E.,Reyes, M. V.,Schiappacasse, V. (1995). Breast-feeding duration and growth of fully breast-fed infants in a poor urban Chilean population Am J Clin Nutr, 62(2), 371-6

Intervention/exposure

Intervention/exposure

Intervention/exposure

Study design

Study design,

Intervention/exposure

Participant health

Size of study groups

Intervention/exposure 
601 Diesel, J. C.,Eckhardt, C. L.,Day, N. L.,Brooks, M. M.,Arslanian, S. A.,Bodnar, L. M. (2015). Is gestational weight gain associated with offspring obesity at 36 months? Pediatr Obes, 10(4), 305-10

602 Dini, E. L.,Holt, R. D.,Bedi, R. (2000). Caries and its association with infant feeding and oral health-related behaviours in 3-4-yearold Brazilian children Community Dent Oral Epidemiol, 28(4), 241-8

603 Dinsmore, J.,Williams, E.,McCarthy, H.,Coghlan, D. (2011). A pilot study to explore factors affecting faltering growth in children Journal of Human Nutrition \& Dietetics, 24(3), 280-281 2p

604 Disantis, K. I.,Collins, B. N.,Fisher, J. O.,Davey, A. (2011). Do infants fed directly from the breast have improved appetite regulation and slower growth during early childhood compared with infants fed from a bottle? Int J Behav Nutr Phys Act, 8(\#issue\#), 89

605 Dixon, D. L.,Griggs, K. M.,Forsyth, K. D.,Bersten, A. D. (2010). Lower interleukin-8 levels in airway aspirates from breastfed infants with acute bronchiolitis Pediatr Allergy Immunol, 21(4 Pt 2), e691-6

606 Djalalinia, S.,Qorbani, M.,Heshmat, R.,Motlagh, M. E.,Ardalan, G.,Bazyar, N., Taheri, M.,Asayesh, H.,Kelishadi, R. (2015). Association of Breast Feeding and Birth Weight with Anthropometric Measures and Blood Pressure in Children and Adolescents: The CASPIAN-IV Study Pediatr Neonatol, 56(5), 324-33

607 Dogaru, C. M.,Strippoli, M. P.,Spycher, B. D.,Frey, U.,Beardsmore, C. S.,Silverman, M.,Kuehni, C. E. (2012). Breastfeeding and lung function at school age: does maternal asthma modify the effect? Am J Respir Crit Care Med, 185(8), 874-80

608 Dogruel, D.,Bingol, G.,Altintas, D. U.,Yilmaz, M.,Kendirli, S. G. (2015). Prevalence of and risk factors for atopic dermatitis: A birth cohort study of infants in southeast Turkey Allergol Immunopathol (Madr), \#volume\#(\#issue\#), \#Pages\#

609 Domellof, E.,Timby, N.,Domellof, M.,Lonnerdal, B.,Hernell, O. (2013). Formula feeding supplemented with milk fat globule membranes improves cognitive score in term infants at 12 months Developmental medicine and child neurology, 55(\#issue\#), 50

610 Dondi, A.,Tripodi, S.,Panetta, V.,Asero, R.,Businco, A. D.,Bianchi, A.,Carlucci, A.,Ricci, G.,Bellini, F.,Maiello, N.,del Giudice, M. M.,Frediani, T.,Sodano, S.,Dello lacono, I.,Macri, F.,Massaccesi, V.,Caffarelli, C.,Rinaldi, L.,Patria, M. F.,Varin, E.,Peroni, D.,Chinellato, I.,Chini, L.,Moschese, V.,Lucarelli, S.,Bernardini, R.,Pingitore, G.,Pelosi, U.,Tosca, M.,Paravati, F.,La Grutta, S.,Meglio, P.,Calvani, M.,Plebani, M.,Matricardi, P. M. (2013). Pollen-induced allergic rhinitis in 1360 Italian children: comorbidities and determinants of severity Pediatr Allergy Immunol, 24(8), 742-51

611 Dong, G. H.,Qian, Z. M.,Liu, M. M.,Wang, D.,Ren, W. H.,Bawa, S.,Fu, J.,Wang, J.,Lewis, R.,Zelicoff, A.,Simckes, M.,Trevathan, E. (2013). Breastfeeding as a modifier of the respiratory effects of air pollution in children Epidemiology, 24(3), 387-94

612 Dong, G. H.,Qian, Z. M.,Trevathan, E.,Zeng, X. W.,Vaughn, M. G.,Wang, J.,Zhao, Y.,Liu, Y. Q.,Ren, W. H.,Qin, X. D. (2014). Air pollution associated hypertension and increased blood pressure may be reduced by breastfeeding in Chinese children: the Seven Northeastern Cities Chinese Children's Study Int J Cardiol, 176(3), 956-61

613 Donma, M. M.,Donma, O. (1997). The influence of feeding patterns on head circumference among Turkish infants during the first 6 months of life Brain Dev, 19(6), 393-7

Study design

Size of study groups

Intervention/exposure

Size of study groups, Outcome

Study design

Outcome

Intervention/exposure, Size of study groups

Publication status

Study design 
614 Donma, M. M.,Donma, O. (1999). Infant feeding and growth: a study on Turkish infants from birth to 6 months Pediatr Int, 41(5), $542-8$

615 Donohue, L. (1994). Baby Friendly Hospitals in China Aust J Adv Nurs, 12(2), 7

Study design

616 Doran, E. (1983). Breast is best for lightweights Nurs Mirror, 156(12), 46-7

Participant health

617 Dorea, J. G. (1997). Zinc in urban infants and children from Brasilia Arch Latinoam Nutr, 47(2 Suppl 1), 39-40

Study design

618 Dorea, J. G.,Marques, R. C.,Isejima, C. (2012). Neurodevelopment of Amazonian infants: antenatal and postnatal exposure to methyl- and ethylmercury J Biomed Biotechnol, 2012(\#issue\#), 132876

Study design,

Intervention/exposure

619 Dotan, I.,Alper, A.,Rachmilewitz, D.,Israeli, E.,Odes, S.,Chermesh, I.,Naftali, T.,Fraser, G.,Shitrit, A. B.,Peles, V.,Reif, S. (2013). Maternal inflammatory bowel disease has short and long-term effects on the health of their offspring: a multicenter study in Israel $J$

Crohns Colitis, 7(7), 542-50

620 Douglas, R. M.,Woodward, A.,Miles, H.,Buetow, S.,Morris, D. (1994). A prospective study of proneness to acute respiratory illness in the first two years of life Int J Epidemiol, 23(4), 818-26

621 Doumid Borges Pretto, A.,Correa Kaufmann, C.,Ferreira Dutra, G.,Pinto Albernaz, E. (2015). Prevalence of factors related to the bone mass formation of children from a cohort in Southern Brazil Nutr Hosp, 31(3), 1122-8

622 Draaisma, E.,Garcia-Marcos, L.,Mallol, J.,Sole, D.,Perez-Fernandez, V.,Brand, P. L. (2015). A multinational study to compare prevalence of atopic dermatitis in the first year of life Pediatr Allergy Immunol, 26(4), 359-66

623 Dratva, J.,Merten, S.,Ackermann-Liebrich, U. (2006). Vitamin D supplementation in Swiss infants Swiss Med Wkly, 136(29-30), 473-81

624 Drewett, R. F.,Amatayakul, K. (1999). Energy intake, appetite and body mass in infancy Early Hum Dev, 56(1), 75-82

Intervention/exposure, Outcome

Outcome

Intervention/exposure

Study design, Outcome

Study design, Outcome

Intervention/exposure

625 Drewett, R.,Amatayakul, K.,Chiowanich, P.,Tansuhaj, A.,Ruckphaopunt, S.,Wongsawasdii, L.,Baum, D.,Imong, S.,Jackson, D.,Woolridge, M. (1991). The Chiang Mai lactation project: study design and implementation Paediatr Perinat Epidemiol, 5(3), 34760

626 Drover, J.,Hoffman, D. R.,Castaneda, Y. S.,Morale, S. E.,Birch, E. E. (2009). Three randomized controlled trials of early long-chain polyunsaturated Fatty Acid supplementation on means-end problem solving in 9-month-olds Child Dev, 80(5), 1376-84

627 Du, Y.,Ellert, U.,Lampert, T.,Mensink, G. B.,Schlaud, M. (2012). Association of breastfeeding and exposure to maternal smoking during pregnancy with children's general health status later in childhood Breastfeed Med, 7(6), 504-13

628 Dubakiene, R.,Rudzeviciene, O.,Butiene, I.,Sezaite, I.,Petronyte, M.,Vaicekauskaite, D.,Zvirbliene, A. (2012). Studies on early allergic sensitization in the Lithuanian birth cohort ScientificWorldJournal, 2012(\#issue\#), 909524

629 Dube, K.,Schwartz, J.,Mueller, M. J.,Kalhoff, H.,Kersting, M. (2010). Iron intake and iron status in breastfed infants during the first year of life Clin Nutr, 29(6), 773-8 
631 Duffy, L. C.,Byers, T. E.,Riepenhoff-Talty, M.,La Scolea, L. J.,Zielezny, M.,Ogra, P. L. (1986). The effects of infant feeding on rotavirus-induced gastroenteritis: a prospective study Am J Public Health, 76(3), 259-63

Duffy, L. C.,Faden, H. Wasielewski, R.,Wolf, J.,Krystofik, D. (1997). Exclusive breastfeeding protects against bacterial colonization and day care exposure to otitis media Pediatrics, 100(4), E7

Duffy, L. C.,Riepenhoff-Talty, M.,Byers, T. E.,La Scolea, L. J.,Zielezny, M. A.,Dryja, D. M.,Ogra, P. L. (1986). Modulation
enteritis during breast-feeding. Implications on alterations in the intestinal bacterial flora Am J Dis Child, 140(11), 1164-8

634 Dugdale, A. E. (1980). Infant feeding, growth and mortality: a 20-year study of an Australian Aboriginal community Med J Aust, 2(7) 380-5

635 Duijts, L., Jaddoe, V. W.,Hofman, A.,Moll, H. A. (2010). Prolonged and exclusive breastfeeding reduces the risk of infectious diseases in infancy Pediatrics, 126(1), e18-25

636 Dumrongwongsiri, O.,Suthutvoravut, U.,Chatvutinun, S.,Phoonlabdacha, P.,Sangcakul, A.,Siripinyanond, A.,Thiengmanee, U.,Chongviriyaphan, N. (2015). Maternal zinc status is associated with breast milk zinc concentration and zinc status in breastfed infants aged 4-6 months Asia Pac J Clin Nutr, 24(2), 273-80

637 Duncan, B.,Ey, J.,Holberg, C. J.,Wright, A. L.,Martinez, F. D.,Taussig, L. M. (1993). Exclusive breast-feeding for at least 4 months protects against otitis media Pediatrics, 91(5), 867-72

638 Dunlop, A. L.,Reichrtova, E.,Palcovicova, L.,Ciznar, P.,Adamcakova-Dodd, A.,Smith, S. J.,McNabb, S. J. (2006). Environmental and dietary risk factors for infantile atopic eczema among a Slovak birth cohort Pediatr Allergy Immunol, 17(2), 103-11

639 Dunne, A. (2012). Early infant nutrition: the importance of getting it right Br J Nurs, 21(7), 390

utcome

Publication date for a non-sibling study

Outcome

Study design

Outcome

Study design

Study design

640 Dunne, A. (2012). Nutrition in infancy: achieving nutrition needs for new mothers and children Br J Community Nurs, Suppl(\#issue\#), Study design S22

641 Dunson, D. B.,Chulada, P.,Arbes, S. J., Jr. (2003). Bayesian modeling of time-varying and waning exposure effects Biometrics $59(1), 83-91$

642 Dunstan, J. A.,Mitoulas, L. R.,Dixon, G.,Doherty, D. A.,Hartmann, P. E.,Simmer, K.,Prescott, S. L. (2007). The effects of fish oil supplementation in pregnancy on breast milk fatty acid composition over the course of lactation: a randomized controlled trial Pediat Res, 62(6), 689-94

643 Durmu, B.,Ay, L.,Duijts, L.,Moll, H. A.,Hokken-Koelega, A. C. S.,Raat, H.,Hofman, A.,Steegers, E. A. P.,Jaddoe, V. W. V. (2012) Infant diet and subcutaneous fat mass in early childhood: The Generation R Study European Journal of Clinical Nutrition, 66(2), 253-260

644 Durmus, B.,Ay, L.,Hokken-Koelega, A. C.,Raat, H.,Hofman, A.,Steegers, E. A.,Jaddoe, V. W. (2011). Maternal smoking during pregnancy and subcutaneous fat mass in early childhood. The Generation R Study Eur J Epidemiol, 26(4), 295-304 


\begin{tabular}{|c|c|c|}
\hline 645 & Dutta, P.,Lahiri, M.,Sen, D.,Pal, S. C. (1991). Prospective hospital based study on persistent diarrhoea Gut, 32(7), 787-90 & Country \\
\hline 646 & Dwyer, T.,Ponsonby, A. L. (1995). SIDS epidemiology and incidence Pediatr Ann, 24(7), 350-2, 354-6 & Study design \\
\hline 647 & $\begin{array}{l}\text { Eaton-Evans, J.,Dugdale, A. E. (1987). Effects of feeding and social factors on diarrhoea and vomiting in infants Arch Dis Child, } \\
62(5), 445-8\end{array}$ & Outcome \\
\hline 648 & $\begin{array}{l}\text { Ebina, S.,Kashiwakura, I. (2013). Relationship between feeding modes and infant weight gain in the first month of life Exp Ther Med, } \\
5(1), 28-32\end{array}$ & Outcome \\
\hline 649 & $\begin{array}{l}\text { Eckhardt, C. L.,Rivera, J.,Adair, L. S.,Martorell, R. (2001). Full breast-feeding for at least four months has differential effects on } \\
\text { growth before and after six months of age among children in a Mexican community J Nutr, 131(9), 2304-9 }\end{array}$ & Intervention/exposure \\
\hline 650 & $\begin{array}{l}\text { Ecord, J. S. (2003). Critical connections. Study finds full breastfeeding for } 6 \text { months boosts infant's resistance to respiratory illnesses } \\
\text { Advances in Neonatal Care (Elsevier Science), 3(1), 2-2 1p }\end{array}$ & Study design \\
\hline 651 & $\begin{array}{l}\text { Edwards, C. A.,Parrett, A. M.,Balmer, S. E.,Wharton, B. A. (1994). Faecal short chain fatty acids in breast-fed and formula-fed } \\
\text { babies Acta Paediatr, 83(5), 459-62 }\end{array}$ & Size of study groups, Outcome \\
\hline 652 & $\begin{array}{l}\text { Eglinton, T. W.,Roberts, R.,Pearson, J.,Barclay, M.,Merriman, T. R.,Frizelle, F. A.,Gearry, R. B. (2012). Clinical and genetic risk } \\
\text { factors for perianal Crohn's disease in a population-based cohort Am J Gastroenterol, 107(4), 589-96 }\end{array}$ & Outcome \\
\hline 653 & $\begin{array}{l}\text { Eickmann, S. H.,de Lira, P. I.,Lima Mde, C.,Coutinho, S. B., Teixeira Mde, L.,Ashworth, A. (2007). Breast feeding and mental and } \\
\text { motor development at } 12 \text { months in a low-income population in northeast Brazil Paediatr Perinat Epidemiol, 21(2), 129-37 }\end{array}$ & $\begin{array}{l}\text { Size of study groups, } \\
\text { Intervention/exposure }\end{array}$ \\
\hline 654 & Eidelman, A. I. (2013). Breastfeeding mitigates a disaster Breastfeed Med, 8(3), 344-5 & Study design \\
\hline 655 & $\begin{array}{l}\text { Eiger, M. S.,Rausen, A. R.,Silverio, J. (1984). Breast-vs. bottle-feeding. A study of morbidity in upper middle class infants Clin } \\
\text { Pediatr (Phila), 23(9), 492-5 }\end{array}$ & Size of study groups \\
\hline 656 & $\begin{array}{l}\text { Ejlerskov, K. T.,Christensen, L. B.,Ritz, C.,Jensen, S. M.,Molgaard, C.,Michaelsen, K. F. (2015). The impact of early growth patterns } \\
\text { and infant feeding on body composition at } 3 \text { years of age Br J Nutr, 114(2), 316-27 }\end{array}$ & Intervention/exposure \\
\hline 657 & $\begin{array}{l}\text { Ek, J.,Magnus, E. (1982). Plasma and red cell folate values and folate requirements in formula-fed term infants J Pediatr, 100(5), } \\
738-44\end{array}$ & Size of study groups \\
\hline 658 & $\begin{array}{l}\text { Ekstrom, A.,Abrahamsson, H.,Eriksson, R. M.,Martensson, B. L. (2014). Women's use of nipple shields-Their influence on } \\
\text { breastfeeding duration after a process-oriented education for health professionals Breastfeed Med, } 9(9), 458-66\end{array}$ & Intervention/exposure \\
\hline 659 & Elborn, G.,Kerr, M. M. (1982). Acceptability trial of "Milumil" artificial milk for infant feeding Midwives Chron, 95(1133), $210-1$ & Intervention/exposure \\
\hline 660 & $\begin{array}{l}\text { Eldeirawi, K.,McConnell, R.,Furner, S.,Freels, S.,Stayner, L.,Hernandez, E.,Amoruso, L., Torres, S.,Persky, V. W. (2009). } \\
\text { Associations of doctor-diagnosed asthma with immigration status, age at immigration, and length of residence in the United States in } \\
\text { a sample of Mexican American School Children in Chicago J Asthma, 46(8), 796-802 }\end{array}$ & Study design \\
\hline 661 & El-Gilany, A. H.,El-Wehady, A. (2007). Maternal work and infant health in Al-Hassa, Saudi Arabia Paediatrics ME, 12(4), $100-105$ & Study design \\
\hline
\end{tabular}


663 Elliott, K. G.,Kjolhede, C. L.,Gournis, E.,Rasmussen, K. M. (1997). Duration of breastfeeding associated with obesity during adolescence Obes Res, 5(6), 538-41

Publication date for a non-sibling study

664 Elliott, L.,Henderson, J.,Northstone, K.,Chiu, G. Y.,Dunson, D.,London, S. J. (2008). Prospective study of breast-feeding in relation to wheeze, atopy, and bronchial hyperresponsiveness in the Avon Longitudinal Study of Parents and Children (ALSPAC) J Allergy Clin Immunol, 122(1), 49-54, 54 e1-3

665 Elwood, P. C.,Pickering, J.,Gallacher, J. E.,Hughes, J.,Davies, D. (2005). Long term effect of breast feeding: cognitive function in the Caerphilly cohort J Epidemiol Community Health, 59(2), 130-3

666 Emamghorashi, F.,Heydari, S. T. (2007). Growth of infants in relation to type of feeding in Jahrom, Islamic Republic of Iran East Mediterr Health J, 13(4), 846-54

667 Emilsson, L.,Magnus, M. C.,Stordal, K. (2015). Perinatal risk factors for development of celiac disease in children, based on the prospective Norwegian Mother and Child Cohort Study Clin Gastroenterol Hepatol, 13(5), 921-7

668 Emmett, P. M.,Jones, L. R. (2014). Diet and growth in infancy: relationship to socioeconomic background and to health and development in the Avon Longitudinal Study of Parents and Children Nutr Rev, 72(8), 483-506

669 Emond, A.,Drewett, R.,Blair, P.,Emmett, P. (2007). Postnatal factors associated with failure to thrive in term infants in the Avon Longitudinal Study of Parents and Children Arch Dis Child, 92(2), 115-9

670 Emond, A.,Pollock, J.,Da Costa, N.,Maranhao, T.,Macedo, A. (2002). The effectiveness of community-based interventions to improve maternal and infant health in the Northeast of Brazil Rev Panam Salud Publica, 12(2), 101-10

671 Endesfelder, D.,zu Castell, W.,Ardissone, A.,Davis-Richardson, A. G.,Achenbach, P.,Hagen, M.,Pflueger, M.,Gano, K. A.,Fagen, J. R.,Drew, J. C.,Brown, C. T.,Kolaczkowski, B.,Atkinson, M.,Schatz, D.,Bonifacio, E.,,Triplett, E. W.,Ziegler, A. G. (2014) Compromised gut microbiota networks in children with anti-islet cell autoimmunity Diabetes, 63(6), 2006-14

672 Engel, J.,Anteunis, L.,Volovics, A.,Hendriks, J.,Marres, E. (1999). Risk factors of otitis media with effusion during infancy Int J Pediatr Otorhinolaryngol, 48(3), 239-49

673 Eriksen, H. L.,Kesmodel, U. S.,Underbjerg, M.,Kilburn, T. R.,Bertrand, J.,Mortensen, E. L. (2013). Predictors of intelligence at the age of 5: family, pregnancy and birth characteristics, postnatal influences, and postnatal growth PLoS One, 8(11), e79200

\section{Outcome}

\section{Outcome}

Outcome, Publication date for a non-sibling study

Intervention/exposure

Study design

Outcome, Publication date for a non-sibling study

Study design, Intervention/exposure

Intervention/exposure, Outcome Eriksson, J.,Forsen, T.,Osmond, C.,Barker, D. (2003). Obesity from cradle to grave Int J Obes Relat Metab Disord, 27(6), 722-7 Eriksson, M.,Forsgren, M.,Sjoberg, S.,von Sydow, M.,Wolontis, S. (1983). Respiratory syncytial virus infection in young hospitalized
children. Identification of risk patients and prevention of nosocomial spread by rapid diagnosis Acta Paediatr Scand, 72(1), 47-51 
677 Eronat, N.,Eden, E. (1992). A comparative study of some influencing factors of rampant or nursing caries in preschool children $J$ Clin Pediatr Dent, 16(4), 275-9

678 Escribano, J.,Luque, V.,Ferre, N.,Mendez-Riera, G.,Koletzko, B.,Grote, V.,Demmelmair, H.,Bluck, L.,Wright, A.,Closa-Monasterolo, R. (2012). Effect of protein intake and weight gain velocity on body fat mass at 6 months of age: the EU Childhood Obesity Programme Int J Obes (Lond), 36(4), 548-53

679 Esfarjani, F.,Azar, M. R.,Gafarpour, M. (2001). IDDM and early exposure of infant to cow's milk and solid food Indian J Pediatr $68(2), 107-10$

680 Eskenazi, B.,Marks, A. R.,Bradman, A.,Fenster, L.,Johnson, C.,Barr, D. B.,Jewell, N. P. (2006). In utero exposure to dichlorodiphenyltrichloroethane (DDT) and dichlorodiphenyldichloroethylene (DDE) and neurodevelopment among young Mexican American children Pediatrics, 118(1), 233-41

681 Esmail, A.,Lambert, P. C.,Jones, D. R.,Mitchell, E. A. (1995). Prevalence of risk factors for sudden infant death syndrome in south east England before the 1991 national 'Back to Sleep' health education campaign J Public Health Med, 17(3), 282-9

682 Estevez-Gonzalez, M. D.,Santana Del Pino, A.,Henriquez-Sanchez, P.,Pena-Quintana, L.,Saavedra-Santana, P. (2015). Breastfeeding during the first six months of life, adiposity rebound and overweight/obesity at eight years of age Int $\mathrm{J}$ Obes (Lond), \#volume\#(\#issue\#), \#Pages\#

683 Ethelberg, S.,Olesen, B.,Neimann, J.,Schiellerup, P.,Helms, M.,Jensen, C.,Böttiger, B.,Olsen, K. E. P.,Scheutz, F.,Gerner-Smidt, P.,Mølbak, K. (2006). Risk factors for diarrhea among children in an industrialized country Epidemiology, 17(1), 24-30

Study design

Intervention/exposure

Intervention/exposure, Size of study groups

Outcome

Study design

Study design

Confounding

Study design,

Intervention/exposure

684 Etiler, N.,Velipasaoglu, S.,Aktekin, M. (2002). Incidence of acute respiratory infections and the relationship with some factors in infancy in Antalya, Turkey Pediatr Int, 44(1), 64-9

685 Etiler, N.,Velipasaoglu, S.,Aktekin, M. (2004). Risk factors for overall and persistent diarrhoea in infancy in Antalya, Turkey: a cohort Outcome study Public Health, 118(1), 62-9

686 Etling, N.,Padovani, E., Gehin-Fouque, F., Tato, L. (1983). Iodine and thyroid hormone levels in serum and urine of full term newborn infants Helv Paediatr Acta, 38(2), 117-22

687 Evelein, A. M.,Geerts, C. C.,Visseren, F. L.,Bots, M. L.,van der Ent, C. K.,Grobbee, D. E.,Uiterwaal, C. S. (2011). The association between breastfeeding and the cardiovascular system in early childhood Am J Clin Nutr, 93(4), 712-8

688 Exl, B. M.,Deland, U.,Secretin, M. C.,Preysch, U.,Wall, M.,Shmerling, D. H. (2000). Improved general health status in an unselected infant population following an allergen-reduced dietary intervention programme: The ZUFF-STUDY-PROGRAMME - Part II: Infant growth and health status to age 6 months European Journal of Nutrition, 39(4), 145-156

689 Exl, B. M.,Deland, U.,Wall, M.,Preysch, U.,Secretin, M. C.,Shmerling, D. H. (1998). Zug-Frauenfeld nutritional survey ('Zuff Study'): Allergen-reduced nutrition in a normal infant population and its health-related effects: Results at the age of six months Nutrition research (New York, N.Y.), 18(8), 1443-62

690 Fagrell, T. G.,Ludvigsson, J.,Ullbro, C.,Lundin, S. A.,Koch, G. (2011). Aetiology of severe demarcated enamel opacities--an evaluation based on prospective medical and social data from 17,000 children Swed Dent J, 35(2), 57-67

Size of study groups, Intervention/exposure

Outcome

\section{Study design, Outcome}

Study design 
691 Fall, C. H.,Barker, D. J.,Osmond, C.,Winter, P. D.,Clark, P. M. Hales, C. N. (1992). Relation of infant feeding to adult serum cholesterol concentration and death from ischaemic heart disease BMJ, 304(6830), 801-5

Publication date for a non-sibling study

692 Fall, C. H.,Borja, J. B.,Osmond, C.,Richter, L.,Bhargava, S. K.,Martorell, R.,Stein, A. D.,Barros, F. C.,Victora, C. G. (2011). Infantfeeding patterns and cardiovascular risk factors in young adulthood: data from five cohorts in low- and middle-income countries Int $\mathrm{J}$ Epidemiol, 40(1), 47-62

693 Fallot, M. E.,Boyd, J. L., 3rd,Oski, F. A. (1980). Breast-feeding reduces incidence of hospital admissions for infection in infants Pediatrics, 65(6), 1121-4

694 Falth-Magnusson, K.,Franzen, L.,Jansson, G.,Laurin, P.,Stenhammar, L. (1996). Infant feeding history shows distinct differences between Swedish celiac and reference children Pediatr Allergy Immunol, 7(1), 1-5

695 Falth-Magnusson, K.,Kjellman, N. I. (1987). Development of atopic disease in babies whose mothers were receiving exclusion diet during pregnancy--a randomized study J Allergy Clin Immunol, 80(6), 868-75

696 Farham, B. (2006). Rethink formula feeding South African medical journal, 96(10), 1054

697 Farooqi, I. S.,Hopkin, J. M. (1998). Early childhood infection and atopic disorder Thorax, 53(11), 927-32

Intervention/exposure

698 Farris, R. P.,Frank, G. C.,Webber, L. S.,Srinivasan, S. R.,Berenson, G. S. (1982). Influence of milk source on serum lipids and lipoproteins during the first year of life, Bogalusa heart study Am J Clin Nutr, 35(1), 42-9

699 Fawcett JN (1981). Feeding from birth to 18 months Nursing (Lond), \#volume\#(\#issue\#), 956-8

Size of study groups,

ntervention/exposure

700 Fawzi, W. W.,Forman, M. R.,Levy, A., Graubard, B. I.,Naggan, L.,Berendes, H. W. (1997). Maternal anthropometry and infant feeding practices in Israel in relation to growth in infancy: the North African Infant Feeding Study Am J Clin Nutr, 65(6), 1731-7

Study design

Publication date for a non-sibling study

701 Fawzi, W. W.,Herrera, M. G.,Nestel, P.,el Amin, A.,Mohamed, K. A. (1998). A longitudinal study of prolonged breastfeeding in relation to child undernutrition Int J Epidemiol, 27(2), 255-60

702 Feig, D. S.,Lipscombe, L. L.,Tomlinson, G.,Blumer, I. (2011). Breastfeeding predicts the risk of childhood obesity in a multi-ethnic cohort of women with diabetes J Matern Fetal Neonatal Med, 24(3), 511-5

703 Feigal, R. J. (1985). Common oral diseases of children Pediatr Ann, 14(2), 133-8

704 Fein, S. B.,Grummer-Strawn, L. M.,Raju, T. N. (2008). Infant feeding and care practices in the United States: results from the Infant Study design Feeding Practices Study II Pediatrics, 122 Suppl 2(\#issue\#), S25-7

705 Feldens, C. A.,Giugliani, E. R.,Duncan, B. B.,Drachler Mde, L.,Vitolo, M. R. (2010). Long-term effectiveness of a nutritional program Outcome in reducing early childhood caries: a randomized trial Community Dent Oral Epidemiol, 38(4), $324-32$

706 Feldens, C. A., Giugliani, E. R.,Vigo, A.,Vitolo, M. R. (2010). Early feeding practices and severe early childhood caries in four-year- $\quad$ Outcome old children from southern Brazil: a birth cohort study Caries Res, 44(5), 445-52

707 Feldens, C. A.,Kramer, P. F.,Feldens, E. G.,Pacheco, L. M.,Vitolo, M. R. (2014). Socioeconomic, behavioral, and anthropometric risk factors for traumatic dental injuries in childhood: a cohort study Int J Paediatr Dent, 24(3), 234-43 
708 Feldens, C. A.,Vitolo, M. R.,Drachler Mde, L. (2007). A randomized trial of the effectiveness of home visits in preventing early childhood caries Community Dent Oral Epidemiol, 35(3), 215-23

709 Fenger-Gron J, Fenger-Gron M, Blunck CH, Schonemann-Rigel H, Wielandt HB. (2015). Low breastfeeding rates and body mass index in Danish children of women with gestational diabetes mellitus International Breastfeeding Journal, 10(1), 1-12

710 Ferguson, A. E.,Tappin, D. M.,Girdwood, R. W. A.,Kennedy, R.,Cockburn, F. (1994). Breast feeding in Scotland British Medica Journal, 308(6932), 824-825

711 Fergusson, D. M.,Beautrais, A. L.,Silva, P. A. (1982). Breast-feeding and cognitive development in the first seven years of life Soc Outcome Sci Med, 16(19), 1705-8

712 Fergusson, D. M.,Horwood, L. J. (1994). Early solid food diet and eczema in childhood: a 10-year longitudinal study Pediatr Allergy Immunol, 5(6 Suppl), 44-7 713 Fergusson, D. M.,Horwood, L. J.,Beautrais, A. L.,Shannon, F. T.,Taylor, B. (1981). Eczema and infant diet Clin Allergy, 11(4), 325- Intervention/exposure
31

714 Fergusson, D. M.,Horwood, L. J.,Shannon, F. T. (1982). Risk factors in childhood eczema J Epidemiol Community Health, 36(2), 118-22

715 Fergusson, D. M.,Horwood, L. J.,Shannon, F. T. (1983). Asthma and infant diet Arch Dis Child, 58(1), 48-51

Size of study groups, Intervention/exposure

716 Fergusson, D. M.,Horwood, L. J.,Shannon, F. T. (1987). Breastfeeding and subsequent social adjustment in six- to eight-year-old Outcome children J Child Psychol Psychiatry, 28(3), 379-86

717 Fergusson, D. M.,Horwood, L. J.,Shannon, F. T.,Taylor, B. (1981). Breast-feeding, gastrointestinal and lower respiratory illness in Outcome the first two years Aust Paediatr J, 17(3), 191-5

718 Fergusson, D. M.,McLeod, G. F.,Horwood, L. J. (2014). Breast feeding, infant growth, and body mass index at 30 and 35 years Paediatr Perinat Epidemiol, 28(6), 545-52

719 Fergusson, D. M.,Woodward, L. J. (1999). Breast feeding and later psychosocial adjustment Paediatr Perinat Epidemiol, 13(2) 144-57

720 Ferris, A. G.,Laus, M. J.,Hosmer, D. W.,Beal, V. A. (1980). The effect of diet on weight gain in infancy Am J Clin Nutr, 33(12), 263542

721 Fewtrell, M. S.,Kennedy, K.,Murgatroyd, P. R.,Williams, J. E.,Chomtho, S.,Lucas, A. (2013). Breast-feeding and formula feeding in healthy term infants and bone health at age 10 years $\mathrm{Br} \mathrm{J}$ Nutr, 110(6), 1061-7

722 Field, C. J.,Van Aerde, J. E.,Robinson, L. E.,Clandinin, M. T. (2008). Feeding a formula supplemented with long chain polyunsaturated fatty acids modifies the "ex vivo" cytokine responses to food proteins in infants at low risk for allergy Pediatr Res, 64(4), 411-7

Size of study groups, Intervention/exposure

Size of study groups

Size of study groups 
723 Field, S. S. (2014). Interaction of genes and nutritional factors in the etiology of autism and attention deficit/hyperactivity disorders: a Outcome case control study Med Hypotheses, 82(6), 654-61

724 Fildes, A.,van Jaarsveld, C. H.,Llewellyn, C.,Wardle, J.,Fisher, A. (2015). Parental control over feeding in infancy. Influence of infant Outcome weight, appetite and feeding method Appetite, 91(\#issue\#), 101-6

725 Fildes, V. (1980). Weaning: on the bottle again Nurs Mirror, 151(24), 18-21

Study design

726 Findeisen, M.,Vennemann, M.,Brinkmann, B.,Ortmann, C.,Rose, I.,Kopcke, W.,Jorch, G.,Bajanowski, T. (2004). German study on Outcome sudden infant death (GeSID): design, epidemiological and pathological profile Int J Legal Med, 118(3), 163-9

727 Firer, M. A.,Hosking, C. S.,Hill, D. J. (1981). Effect of antigen load on development of milk antibodies in infants allergic to milk Br Med J (Clin Res Ed), 283(6293), 693-6

728 Fisher C (1985). Breastfeeding. Two. Feeding the relationship Nurs Times, 81(\#issue\#), 51

Size of study groups

729 Fisher SE,Markowitz J,Lifshitz F (1984). Food intolerance in childhood Compr Ther, 10(\#issue\#), 5-11

Study design

Fisk, C. M.,Crozier, S. R.,Inskip, H. M.,Godfrey, K. M.,Cooper, C.,Roberts, G. C.,Robinson, S. M. (2011). Breastfeeding and reported morbidity during infancy: findings from the Southampton Women's Survey Matern Child Nutr, 7(1), 61-70

731 Fitzgerald, S.,Kearney, M.,Mahony, M.,O'Halloran, E. T.,Barry, R. G. (1982). Gastroenteritis 1972-1978 Ir Med J, 75(5), 155-7 Study design

Outcome

Study design

732 Flaherman, V. J.,Bokser, S.,Newman, T. B. (2010). First-day newborn weight loss predicts in-hospital weight nadir for breastfeeding Intervention/exposure infants Breastfeed Med, 5(4), 165-8

733 Flaherman, V. J.,Fuentes-Afflick, E. (2014). Social and public health perspectives of promotion of breastfeeding JAMA Pediatr 168(10), $877-8$

734 Flaherman, V. J.,Kuzniewicz, M. W.,Li, S.,Walsh, E.,McCulloch, C. E.,Newman, T. B. (2013). First-day weight loss predicts eventual weight nadir for breastfeeding newborns Arch Dis Child Fetal Neonatal Ed, 98(6), F488-92

735 Flaherman, V.,Aby, J.,Burgos, A .,Lee, K.,Cabana, M.,Newman, T. (2012). Randomized Trial of Early Limited Formula To Reduce Formula Use at 1 Week and Promote Breastfeeding at 3 Months in Infants with High Early Weight Loss Pediatric Academic Societies Annual Meeting, \#volume\#(\#issue\#), \#Pages\#

736 Fleddermann, M.,Demmelmair, H.,Grote, V.,Nikolic, T.,Koletzko, B. (2013). A protein reduced, alpha-lactalbumin and LC-PUFA containing infant formula enables an adequate growth in infants and influences the energetic efficiency for growth: A randomized controlled trial Clinical nutrition (Edinburgh, Scotland), 32(\#issue\#), S16

737 Fleming, P. J.,Blair, P. S.,Bacon, C.,Bensley, D.,Smith, I.,Taylor, E.,Berry, J.,Golding, J.,Tripp, J. (1996). Environment of infants during sleep and risk of the sudden infant death syndrome: results of 1993-5 case-control study for confidential inquiry into stillbirths and deaths in infancy. Confidential Enquiry into Stillbirths and Deaths Regional Coordinators and Researchers BMJ, 313(7051), $191-5$

Study design

Intervention/exposure, Outcome

Publication status

Publication status

Outcome 
738 Fleming, P. J.,Blair, P. S.,Ward Platt, M.,Tripp, J.,Smith, I. J. (2003). Sudden infant death syndrome and social deprivation: assessing epidemiological factors after post-matching for deprivation Paediatr Perinat Epidemiol, 17(3), 272-80

739 Fleming, T. (2008). Breast is best to avoid obesity: study Pharmacy News, \#volume\#(\#issue\#), 4-4 1p

740 Flohr, C.,Nagel, G.,Weinmayr, G.,Kleiner, A.,Strachan, D. P.,Williams, H. C. (2011). Lack of evidence for a protective effect of prolonged breastfeeding on childhood eczema: lessons from the International Study of Asthma and Allergies in Childhood (ISAAC)

Study design Phase Two Br J Dermatol, 165(6), 1280-9

741 Flohr, C.,Perkin, M.,Logan, K.,Marrs, T.,Radulovic, S.,Campbell, L. E.,Maccallum, S. F.,McLean, W. H.,Lack, G. (2014). Atopic dermatitis and disease severity are the main risk factors for food sensitization in exclusively breastfed infants $\mathrm{J}$ Invest Dermatol, $134(2), 345-50$

742 Flores, M. S.,Fairchok, M. P. (2004). The relationship of breastfeeding to antimicrobial exposure in the first year of life Clin Pediatr (Phila), 43(7), 631-6

743 Flores, M.,Pasquel, M. R.,Maulen, I.,Rivera, J. (2005). Exclusive breastfeeding in 3 rural localities in Mexico J Hum Lact, 21(3) 276-83

744 Floret, D.,Lina, B.,Pinchinat, S.,Billaud, G.,Ait-Belghiti, F.,Largeron, N.,Bellemin, B., Trang, C. N.,Fau, C.,Gaspard, C.,Mamoux, V.,Marcelon, L. (2006). Epidemiology and burden of rotavirus diarrhea in day care centers in Lyon, France Eur J Pediatr, 165(12), $905-6$

745 Florey, C. D.,Leech, A. M.,Blackhall, A. (1995). Infant feeding and mental and motor development at 18 months of age in first born singletons Int J Epidemiol, 24 Suppl 1(\#issue\#), S21-6

746 Florez, C. E.,Hogan, D. P. (1990). Women's status and infant mortality in rural Colombia Soc Biol, 37(3-4), 188-203

Intervention/exposure

Study design,

ntervention/exposure

Study design

Intervention/exposure

\section{Outcome}

\section{Study design,}

Intervention/exposure

747 Fogaca, H. R.,Marson, F. A.,Toro, A. A.,Sole, D.,Ribeiro, J. D. (2014). Epidemiological aspects of and risk factors for wheezing in the first year of life J Bras Pneumol, 40(6), 617-25

748 Fokkema MR,Smit EN,Martini IA,Woltil HA,Boersma ER,Muskiet FA (2002). Assessment of essential fatty acid and omega3-fatty acid status by measurement of erythrocyte 20:3omega9 (Mead acid), 22:5omega6/20:4omega6 and 22:5omega6/22:6omega3 Prostaglandins Leukot Essent Fatty Acids, 67(\#issue\#), 345-56

749 Foley, S., Quinn, S.,Jones, G. (2009). Tracking of bone mass from childhood to adolescence and factors that predict deviation from tracking Bone, 44(5), 752-7

750 Folic, N.,Folic, M.,Markovic, S.,Andjelkovic, M.,Jankovic, S. (2015). Risk factors for the development of metabolic syndrome in obese children and adolescents Srp Arh Celok Lek, 143(3-4), 146-52

751 Fomon, S. J. (1980). Factors influencing food consumption in the human infant Int J Obes, 4(4), 348-50

Study design

Intervention/exposure

Publication date for a non-sibling study

Study design, Size of study groups

\section{Study design}

752 Fomon, S. J. (2004). Assessment of growth of formula-fed infants: evolutionary considerations Pediatrics, 113(2), 389-93

Study design 
753 Fomon, S. J.,Rogers, R. R.,Ziegler, E. E.,Nelson, S. E.,Thomas, L. N. (1984). Indices of fatness and serum cholesterol at age eight years in relation to feeding and growth during early infancy Pediatr Res, 18(12), 1233-8

754 Fomon, S. J.,Ziegler, E. E.,Nelson, S. E. (1993). Erythrocyte incorporation of ingested 58Fe by 56-day-old breast-fed and formulafed infants Pediatr Res, 33(6), 573-6

755 Fomon, S. J.,Ziegler, E. E.,Nelson, S. E.,Rogers, R. R.,Frantz, J. A. (1999). Infant formula with protein-energy ratio of 1.7 g/100 kcal is adequate but may not be safe $\mathrm{J}$ Pediatr Gastroenterol Nutr, 28(5), 495-501

756 Fonseca, A. L.,Albernaz, E. P.,Kaufmann, C. C.,Neves, I. H.,Figueiredo, V. L. (2013). Impact of breastfeeding on the intelligence quotient of eight-year-old children J Pediatr (Rio J), 89(4), 346-53

757 Fonseca, M. J.,Moreira, A.,Moreira, P.,Delgado, L., Teixeira, V.,Padrão, P. (2010). Duration of breastfeeding and the risk of childhood asthma in children living in urban areas Journal of Investigational Allergology and Clinical Immunology, 20(4), 357-358

758 Fonseca, M. J.,Severo, M.,Barros, H.,Santos, A. C. (2014). Determinants of weight changes during the first 96 hours of life in fullterm newborns Birth, 41(2), 160-8

759 Fonseca, W.,Kirkwood, B. R.,Victora, C. G.,Fuchs, S. R.,Flores, J. A.,Misago, C. (1996). Risk factors for childhood pneumonia among the urban poor in Fortaleza, Brazil: a case--control study Bull World Health Organ, 74(2), 199-208

760 Ford, K.,Labbok, M. (1993). Breast-feeding and child health in the United States J Biosoc Sci, 25(2), 187-94

Intervention/exposure

Size of study groups

Publication date for a non-sibling study

Intervention/exposure

Study design

Study design

Intervention/exposure

Outcome

Study design

761 Ford, R. P.,Taylor, B. J.,Mitchell, E. A.,Enright, S. A.,Stewart, A. W.,Becroft, D. M.,Scragg, R.,Hassall, I. B.,Barry, D. M.,Allen, E. M.,et al., (1993). Breastfeeding and the risk of sudden infant death syndrome Int J Epidemiol, 22(5), 885-90

762 Ford-Jones, E. L.,Wang, E.,Petric, M.,Corey, P.,Moineddin, R.,Fearon, M. (2000). Hospitalization for community-acquired, rotavirusassociated diarrhea: a prospective, longitudinal, population-based study during the seasonal outbreak. The Greater Toronto Area/Peel Region PRESI Study Group. Pediatric Rotavirus Epidemiology Study for Immunization Arch Pediatr Adolesc Med, 154(6), $578-85$

763 Forman, M. R.,Graubard, B. I.,Hoffman, H. J.,Beren, R.,Harley, E. E.,Bennett, P. (1984). The Pima Infant Feeding Study: breast feeding and gastroenteritis in the first year of life Am J Epidemiol, 119(3), 335-49

764 Forman, M. R.,Graubard, B. I.,Hoffman, H. J.,Beren, R.,Harley, E. E.,Bennett, P. (1984). The Pima infant feeding study: breastfeeding and respiratory infections during the first year of life Int $\mathrm{J}$ Epidemiol, 13(4), 447-53

765 Forman, M. R., Guptill, K. S.,Chang, D. N.,Sarov, B.,Berendes, H. W.,Naggan, L.,Hundt, G. L. (1990). Undernutrition among Bedouin Arab infants: the Bedouin Infant Feeding Study Am J Clin Nutr, 51(3), 343-9

766 Forman, M. R.,Lewando-Hundt, G.,Graubard, B. I.,Chang, D.,Sarov, B.,Naggan, L.,Berendes, H. W. (1992). Factors influencing milk insufficiency and its long-term health effects: the Bedouin Infant Feeding Study Int J Epidemiol, 21(1), 53-8

767 Forns, J.,Torrent, M.,Garcia-Esteban, R.,Caceres, A.,Pilar Gomila, M.,Martinez, D.,Morales, E.,Julvez, J.,Grimalt, J. O.,Sunyer, J. (2012). Longitudinal association between early life socio-environmental factors and attention function at the age 11 years Environ Res, 117(\#issue\#), 54-9 
768 Forns, J.,Vegas, O.,Julvez, J.,Garcia-Esteban, R.,Rivera, M.,Lertxundi, N.,Guxens, M.,Fano, E.,Ferrer, M.,Grellier, J.,Ibarluzea, J.,Sunyer, J. (2014). Association between child cortisol levels in saliva and neuropsychological development during the second year of life Stress Health, 30(2), 142-8

769 Foroushani, A. R.,Mohammad, K.,Mahmoodi, M.,Siassi, F. (2010). Effect of breastfeeding on cognitive performance in a British birth Outcome cohort East Mediterr Health J, 16(2), 202-8

770 Forssell, G.,Hakansson, A.,Mansson, N. O. (2001). Risk factors for respiratory tract infections in children aged 2-5 years Scand $J$ Prim Health Care, 19(2), 122-5

771 Forster, D. A.,Johns, H.,Amir, L. H.,McLachlan, H. L.,Moorhead, A.,Ford, R.,McEgan, K. (2013). The MILC Study-Exploring the prevalence and outcomes associated with breast milk expression: A prospective cohort study Women \& Birth, 26(\#issue\#), S7-S7 preval
$1 \mathrm{p}$

772 Forsyth S,Hornstra G (2001). Essential fatty acids. Maternal and infant nutrition Pract Midwife, 4(\#issue\#), 34-7

Study design

773 Forsyth, J. S.,Willatts, P.,Agostoni, C.,Bissenden, J.,Casaer, P.,Boehm, G. (2003). Long chain polyunsaturated fatty acid supplementation in infant formula and blood pressure in later childhood: follow up of a randomised controlled trial BMJ, 326(7396), 953

774 Fort, P.,Lanes, R.,Dahlem, S.,Recker, B.,Weyman-Daum, M.,Pugliese, M.,Lifshitz, F. (1986). Breast feeding and insulin-dependent Outcome diabetes mellitus in children J Am Coll Nutr, 5(5), 439-41

775 Fosarelli, P. D.,DeAngelis, C.,Winkelstein, J.,Mellits, E. D. (1985). Infectious illnesses in the first two years of life Pediatr Infect Dis, Outcome 4(2), 153-9

776 Foulon, S.,Pingault, J. B.,Larroque, B.,Melchior, M.,Falissard, B.,Cote, S. M. (2015). Developmental predictors of inattentionhyperactivity from pregnancy to early childhood PLoS One, 10(5), e0125996

777 France, G. L.,Marmer, D. J.,Steele, R. W. (1980). Breast-feeding and Salmonella infection Am J Dis Child, 134(2), 147-52

Outcome

Study design, Size of study groups

778 Frank, A. L.,Taber, L. H.,Glezen, W. P.,Kasel, G. L.,Wells, C. R.,Paredes, A. (1982). Breast-feeding and respiratory virus infection Pediatrics, 70(2), 239-45

779 Franklin, Patricia D. (2013). Exclusive Breastfeeding Duration in Relationship to Infant Risk for Overweight and Obesity at Three Years of Age \#journal\#, Ph.D.(\#issue\#), 186 p-186 p 1p

780 Franks, A. (1989). Breastfeeding in the neonatal unit N Z Nurs J, 82(8), 23-4

781 Fransoo, R. R.,Roos, N. P.,Martens, P. J.,Heaman, M.,Levin, B.,Chateau, D. (2008). How health status affects progress and performance in school: a population-based study Can J Public Health, 99(4), 344-9

782 Frederiksen, B.,Kroehl, M.,Lamb, M. M.,Seifert, J.,Barriga, K.,Eisenbarth, G. S.,Rewers, M.,Norris, J. M. (2013). Infant exposures and development of type 1 diabetes mellitus: The Diabetes Autoimmunity Study in the Young (DAISY) JAMA Pediatr, 167(9), 80815 
Fredriksson, $\mathrm{P}$, Jaakkola, $\mathrm{N}$ BMC Pediatr, 7(\#issue\#), 39

784 Freeman, K.,Bonuck, K. A.,Trombley, M. (2008). Breastfeeding and infant illness in low-income, minority women: a prospective cohort study of the dose-response relationship J Hum Lact, 24(1), 14-22; quiz 23-6

785 Freeman, V. E.,Mulder, J.,van't Hof, M. A.,Hoey, H. M.,Gibney, M. J. (1998). A longitudinal study of iron status in children at $12,24 \quad$ Intervention/exposure and 36 months Public Health Nutr, 1(2), 93-100

786 Friel, J. K.,Andrews, W. L.,Simmons, B. S.,L'Abbe, M. R.,Mercer, C.,MacDonald, A.,McCloy, U. R. (1997). Evaluation of full-term infants fed an evaporated milk formula Acta Paediatr, 86(5), 448-53

787 Froom, J.,Culpepper, L., Green, L. A., de Melker, R. A.,Grob, P.,Heeren, T.,van Balen, F. (2001). A cross-national study of acute otitis media: risk factors, severity, and treatment at initial visit. Report from the International Primary Care Network (IPCN) and the Ambulatory Sentinel Practice Network (ASPN) J Am Board Fam Pract, 14(6), 406-17

788 Froozani, M. D.,Malekafzali, H.,Bahrini, B. (1980). Growth of a group of low income infants in the first year of life J Trop Pediatr, 26(3), 96-8

789 Froozani, M. D.,Permehzadeh, K.,Motlagh, A. R.,Golestan, B. (1999). Effect of breastfeeding education on the feeding pattern and health of infants in their first 4 months in the Islamic Republic of Iran Bull World Health Organ, 77(5), 381-5

Size of study groups

Study design

Fruhwirth, M.,Heininger, U.,Ehlken, B.,Petersen, G.,Laubereau, B.,Moll-Schuler, I.,Mutz, I.,Forster, J. (2001). International variation in disease burden of rotavirus gastroenteritis in children with community- and nosocomially acquired infection Pediatr Infect Dis $\mathrm{J}$, 20(8), 784-91

791 Frye, C.,Heinrich, J. (2003). Trends and predictors of overweight and obesity in East German children Int J Obes Relat Metab Disord, 27(8), 963-9

792 Fuchs, S. C.,Victora, C. G. (2002). Risk and prognostic factors for diarrheal disease in Brazilian infants: a special case-control design application Cad Saude Publica, 18(3), 773-82

793 Fuchs, S. C.,Victora, C. G.,Martines, J. (1996). Case-control study of risk of dehydrating diarrhoea in infants in vulnerable period after full weaning BMJ, 313(7054), 391-4

794 Fuiano, N.,Rapa, A.,Monzani, A.,Pietrobelli, A.,Diddi, G.,Limosani, A.,Bona, G. (2008). Prevalence and risk factors for overweight and obesity in a population of Italian schoolchildren: a longitudinal study J Endocrinol Invest, 31(11), 979-84

795 Fujita, H.,Okada, T.,Inami, I.,Makimoto, M.,Hosono, S.,Minato, M.,Takahashi, S.,Mugishima, H.,Yamamoto, T. (2008). Low-density lipoprotein profile changes during the neonatal period J Perinatol, 28(5), 335-40

796 Fujiwara, T.,Oguni, T.,Unishi, G.,Tanabe, T.,Ohbayashi, K.,Kaneko, K. (2014). Factors related to patterns of body mass index in early infancy: 18 month longitudinal study Pediatr Int, 56(3), 406-10

Study design

Intervention/exposure

Publication date for a non-sibling study

Participant health

Study design

Outcome

Outcome

ntervention/exposure

Size of study groups,

Intervention/exposure

Intervention/exposure 
797 Fullerton, K. E.,Ingram, L. A.,Jones, T. F.,Anderson, B. J.,McCarthy, P. V.,Hurd, S.,Shiferaw, B.,Vugia, D.,Haubert, N.,Hayes, T.,Wedel, S.,Scallan, E.,Henao, O.,Angulo, F. J. (2007). Sporadic campylobacter infection in infants: a population-based surveillance case-control study Pediatr Infect Dis J, 26(1), 19-24

798 Gabriel, C. G.,Corso, A. C.,Caldeira, G. V.,Gimeno, S. G.,Schmitz Bde, A., de Vasconcelos Fde, A. ( related factors in schoolchildren in Santa Catarina State, Brazil Arch Latinoam Nutr, 60(4), 332-9

799 Gabriele, C.,Silva, L. M.,Arends, L. R.,Raat, H.,Moll, H. A.,Hofman, A.,Jaddoe, V. W.,de Jongste, J. C. (2012). Early respiratory morbidity in a multicultural birth cohort: the Generation R Study Eur J Epidemiol, 27(6), 453-62

800 Gaffney, K. F.,Kitsantas, P.,Cheema, J. (2012). Clinical practice guidelines for feeding behaviors and weight-for-age at 12 months: a Intervention/exposure secondary analysis of the Infant Feeding Practices Study II Worldviews Evid Based Nurs, 9(4), 234-42

801 Galan-Gonzalez AF,Aznar-Martin T,Cabrera-Dominguez ME,Dominguez-Reyes A (2014). Do breastfeeding and bottle feeding influence occlusal parameters? Breastfeed Med, 9(\#issue\#), 24-8

802 Galán-Gónzalez, A. F.,Aznar-Martín, T.,Cabrera-Domínguez, M. E.,Domínguez-Reyes, A. (2014). Do breastfeeding and bottle feeding influence occlusal parameters? Breastfeeding Medicine, 9(1), 24-28

803 Gale, C. R.,Marriott, L. D.,Martyn, C. N.,Limond, J.,Inskip, H. M.,Godfrey, K. M.,Law, C. M.,Cooper, C.,West, C.,Robinson, S. M. (2010). Breastfeeding, the use of docosahexaenoic acid-fortified formulas in infancy and neuropsychological function in childhood Arch Dis Child, 95(3), 174-9

804 Gale, C. R.,Martyn, C. N. (1996). Breastfeeding, dummy use, and adult intelligence Lancet, 347(9008), 1072-5

Outcome

805 Gale, C. R.,Martyn, C. N.,Marriott, L. D.,Limond, J.,Crozier, S.,Inskip, H. M.,Godfrey, K. M.,Law, C. M.,Cooper, C.,Robinson, S. M (2009). Dietary patterns in infancy and cognitive and neuropsychological function in childhood Journal of Child Psychology and Psychiatry and Allied Disciplines, 50(7), 816-823

806 Gale, C., Thomas, E. L.,Jeffries, S.,Durighel, G.,Logan, K. M.,Parkinson, J. R.,Uthaya, S.,Santhakumaran, S.,Bell, J. D.,Modi, N (2014). Adiposity and hepatic lipid in healthy full-term, breastfed, and formula-fed human infants: a prospective short-term longitudinal cohort study Am J Clin Nutr, 99(5), 1034-40

807 Galler, J. R.,Harrison, R. H.,Ramsey, F.,Forde, V.,Butler, S. C. (2000). Maternal depressive symptoms affect infant cognitive development in Barbados J Child Psychol Psychiatry, 41(6), 747-57

808 Galler, J. R.,Ramsey, F. C.,Harrison, R. H.,Brooks, R.,Weiskopf-Bock, S. (1998). Infant feeding practices in Barbados predict later growth J Nutr, 128(8), 1328-35

809 Galler, J. R.,Ramsey, F. C.,Harrison, R. H.,Taylor, J.,Cumberbatch, G.,Forde, V. (2004). Postpartum maternal moods and infant size Outcome predict performance on a national high school entrance examination J Child Psychol Psychiatry, 45(6), 1064-75

810 Galli, E.,Picardo, M.,Chini, L.,Passi, S.,Moschese, V.,Terminali, O.,Paone, F.,Fraioli, G.,Rossi, P. (1994). Analysis of polyunsaturated fatty acids in newborn sera: a screening tool for atopic disease? $\mathrm{Br} \mathrm{J}$ Dermatol, 130(6), 752-6 
812 Garcia, M. V.,Azevedo, M. F.,Testa, J. R.,Luiz, C. B. (2012). The influence of the type of breastfeeding on middle ear conditions in infants Braz J Otorhinolaryngol, 78(1), 8-14

813 Garcia-Marcos, L.,Mallol, J.,Sole, D.,Brand, P. L. (2010). International study of wheezing in infants: risk factors in affluent and non-

Study design affluent countries during the first year of life Pediatr Allergy Immunol, 21(5), 878-88

814 Garcia-Marcos, L.,Mallol, J.,Sole, D.,Brand, P. L.,Sanchez-Bahillo, M.,Sanchez-Solis, M. (2013). Latitude modifies the effect size of Study design, Outcome factors related to recurrent wheeze in the first year of life Respir Med, 107(5), 665-72

815 Garden, F. L.,Marks, G. B.,Simpson, J. M.,Webb, K. L. (2012). Body mass index (BMI) trajectories from birth to 11.5 years: relation Confounding to early life food intake Nutrients, 4(10), 1382-98

816 Garmendia, M. L.,Corvalan, C.,Araya, M.,Casanello, P.,Kusanovic, J. P.,Uauy, R. (2015). Effectiveness of a normative nutrition intervention (diet, physical activity and breastfeeding) on maternal nutrition and offspring growth: the Chilean maternal and infant nutrition cohort study (CHiMINCs) BMC Pregnancy Childbirth, 15(\#issue\#), 175

817 Garry, P. J.,Owen, G. M.,Hooper, E. M.,Gilbert, B. A. (1981). Iron absorption from human milk and formula with and without iron supplementation Pediatr Res, 15(5), 822-8

818 Garza, C. (2014). The INTERGROWTH-21st project and the multicenter growth reference study: enhanced opportunities for monitoring growth from early pregnancy to 5 years of age Breastfeed Med, $9(7), 341-4$

819 Garza, C.,Borghi, E.,Onyango, A. W.,de Onis, M. (2013). Parental height and child growth from birth to 2 years in the WHO Multicentre Growth Reference Study Matern Child Nutr, 9 Suppl 2(\#issue\#), 58-68

820 Gathwala, G.,Narang, A. (1995). Breast is best Indian J Pediatr, 62(6), 687-90

Study design,

Intervention/exposure

Intervention/exposure

Study design

Outcome

Study design

821 Gearry, R. B.,Richardson, A. K.,Frampton, C. M.,Dodgshun, A. J.,Barclay, M. L. inflammatory bowel disease risk factors J Gastroenterol Hepatol, 25(2), 325-33

822 Geller-Bernstein, G.,Kenett, R.,Weisglass, L.,Tsur, S.,Lahav, M.,Levin, S. (1987). Atopic babies with wheezy bronchitis. Follow-up study relating prognosis to sequential IgE values, type of early infant feeding, exposure to parental smoking and incidence of lower respiratory tract infections Allergy, 42(2), 85-91

823 Gerrard, J. W. (1984). Allergies in breastfed babies to foods ingested by the mother (review) Clin Rev Allergy, 2(2), 143-9

Study design

824 Gerrard $375-9$

825 Gessner, B. D.,Plotnik, J.,Muth, P. T. (2003). 25-hydroxyvitamin D levels among healthy children in Alaska J Pediatr, 143(4), 434-7 
827 Ghosh, S.,Sengupta, P. G.,Mondal, S. K.,Banu, M. K.,Gupta, D. N.,Sircar, B. K. (1997). Risk behavioural practices of rural mothers as determinants of childhood diarrhoea J Commun Dis, 29(1), 7-14

828 Ghys, A.,Bakker, E.,Hornstra, G.,van den Hout, M. (2002). Red blood cell and plasma phospholipid arachidonic and docosahexaenoic acid levels at birth and cognitive development at 4 years of age Early Hum Dev, 69(1-2), 83-90

829 Gianino, P.,Mastretta, E.,Longo, P.,Laccisaglia, A.,Sartore, M.,Russo, R.,Mazzaccara, A. (2002). Incidence of nosocomial rotavirus infections, symptomatic and asymptomatic, in breast-fed and non-breast-fed infants Journal of Hospital Infection, 50(1), 13-17

830 Gianni, M. L.,Roggero, P.,Baudry, C.,Ligneul, A.,Morniroli, D.,Garbarino, F.,le Ruyet, P.,Mosca, F. (2012). The influence of a formula supplemented with dairy lipids and plant oils on the erythrocyte membrane omega-3 fatty acid profile in healthy full-term infants: a double-blind randomized controlled trial BMC Pediatr, 12(\#issue\#), 164

831 Gianni, M. L.,Roggero, P.,Morlacchi, L.,Garavaglia, E.,Piemontese, P.,Mosca, F. (2014). Formula-fed infants have significantly higher fat-free mass content in their bodies than breastfed babies Acta Paediatr, 103(7), e277-81

\section{Gibbs, B. G.,Forste, R. (2014). Socioeconomic status, infant feeding practices and early childhood obesity Pediatr Obes, 9(2), 135- Intervention/exposure 46}

834 Gibson RA,Makrides M,Clark KJ,Neumann MA,Lines DR (1992). Long chain omega 3 polyunsaturates in formula-fed term infants Adv Exp Med Biol, 318(\#issue\#), 341-5

835 Gibson, R. A.,Hawkes, J. S.,Makrides, M. (2005). Dietary nucleotides do not alter erythrocyte long-chain polyunsaturated fatty acids Outcome in formula-fed term infants Lipids, 40(6), 631-4

836 Gibson, R. A.,Neumann, M. A.,Makrides, M. (1997). Effect of increasing breast milk docosahexaenoic acid on plasma and erythrocyte phospholipid fatty acids and neural indices of exclusively breast fed infants Eur J Clin Nutr, 51(9), 578-84

837 Gibson-Davis, C. M.,Brooks-Gunn, J. (2006). Breastfeeding and verbal ability of 3-year-olds in a multicity sample Pediatrics, 118(5), Outcome e1444-51

838 Gigante, D. P.,Horta, B. L.,Lima, R. C.,Barros, F. C.,Victora, C. G. (2006). Early life factors are determinants of female height at age 19 years in a population-based birth cohort (Pelotas, Brazil) J Nutr, 136(2), 473-8

Outcome, Publication date for non-sibling study

839 Gil, A.,Lozano, E.,De-Lucchi, C.,Maldonado, J.,Molina, J. A.,Pita, M. (1988). Changes in the fatty acid profiles of plasma lipid fractions induced by dietary nucleotides in infants born at term Eur J Clin Nutr, 42(6), 473-81

840 Gil, A.,Pita, M.,Martinez, A.,Molina, J. A.,Sanchez Medina, F. (1986). Effect of dietary nucleotides on the plasma fatty acids in atterm neonates Hum Nutr Clin Nutr, 40(3), 185-95

841 Gilat, T.,Hacohen, D.,Lilos, P.,Langman, M. J. (1987). Childhood factors in ulcerative colitis and Crohn's disease. An international cooperative study Scand J Gastroenterol, 22(8), 1009-24 

BMJ, 310(6972), 88-90

844 Gillman, M. W.,Rifas-Shiman, S. L.,Camargo, C. A., Jr.,Berkey, C. S.,Frazier, A. L.,Rockett, H. R.,Field, A. E.,Colditz, G. A. (2001) Risk of overweight among adolescents who were breastfed as infants JAMA, 285(19), 2461-7

845 Gillman, M. W.,Rifas-Shiman, S. L.,Kleinman, K.,Oken, E.,Rich-Edwards, J. W.,Taveras, E. M. (2008). Developmental origins of childhood overweight: potential public health impact Obesity (Silver Spring), 16(7), 1651-6

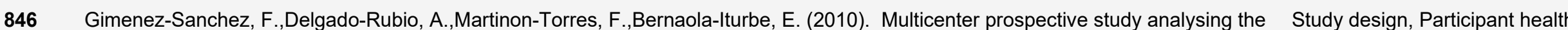
role of rotavirus on acute gastroenteritis in Spain Acta Paediatr, 99(5), 738-42

847 Gimeno, S. G.,de Souza, J. M. (1997). IDDM and milk consumption. A case-control study in Sao Paulo, Brazil Diabetes Care, 20(8), Outcome 1256-60

848 Giovannini, M.,Agostoni, C.,Fiocchi, A.,Bellu, R.,Trojan, S.,Riva, E. (1994). Antigen-reduced infant formulas versus human milk: growth and metabolic parameters in the first 6 months of life J Am Coll Nutr, 13(4), 357-63

849 Giovannini, M.,Verduci, E.,Zuccotti, G.,Biasucci, G.,Podesta, A.,Rottoli, A.,Gregori, D.,Ballali, S.,Banderali, G.,Riva, E.,Ghisleni, D.,Pogliani, L.,Cicero, C.,Tonella, M.,Frugnoli, I. (2013). Safety of a formula supplemented with galacto-oligosaccharides in term infants International journal of probiotics \& prebiotics, 8(2-3), 67-74

850 Giovannini, M.,Verduci, E.,Zuccotti, G.,Biasucci, G.,Podesta, A.,Rottoli, A.,Gregori, D.,Ballali, S.,Soldi, S.,Banderali, G.,Ghisleni, D.,Riva, E. (2013). Prebiotic effect of a formula supplemented with galacto-oligosaccharides in term infants: A randomized multicenter trial Annals of nutrition \& metabolism, 63(\#issue\#), 1667

851 Gishti, O Gaillard, R Durmus, B Hofman, A Duijs, L.Franco, O. H Jaddoe, V. W. (2014). Infant diet and metabolic outcomes in school-age children. The Generation R Study Eur J Clin Nutr, 68(9), 1008-15

852 Gishti, O.,Jaddoe, V. W.,Duijts, L.,Franco, O. H.,Hofman, A., Ikram, M. K.,Gaillard, R. (2015). Influence of breastfeeding on retina vessel calibers in school-age children. The Generation R Study Eur J Clin Nutr, \#volume\#(\#issue\#), \#Pages\#

853 Giugliano, L. G.,Meyer, C. J.,Arantes, L. C.,Ribeiro, S. T.,Giugliano, R. (1993). Mannose-resistant haemagglutination (MRHA) and haemolysin (Hly) production of strains of Escherichia coli isolated from children with diarrhoea: effect of breastfeeding $\mathrm{J}$ Trop Pediatr 39(3), 183-7

854 Giwercman, C.,Halkjaer, L. B.,Jensen, S. M.,Bonnelykke, K.,Lauritzen, L.,Bisgaard, H. (2010). Increased risk of eczema but reduced risk of early wheezy disorder from exclusive breast-feeding in high-risk infants J Allergy Clin Immunol, 125(4), 866-71

855 Glatthaar, C.,Whittall, D. E.,Welborn, T. A.,Gibson, M. J.,Brooks, B. H.,Ryan, M. M.,Byrne, G. C. (1988). Diabetes in Western Australian children: descriptive epidemiology Med J Aust, 148(3), 117-23

Size of study groups

Intervention/exposure

Study design

Outcome

Outcome

Study design, Participant health

Intervention/exposure

Intervention/exposure

Gliddon, M. L.,Sutton, G. (2001). Prediction of 8-month MEE from neonatal risk factors and test results in SCBU and full-term babies

British Journal of Audiology, 35(1), 77-85

Non-human sample, Participant

health 
857 Glueck, C. J.,Salehi, M.,Sieve, L.,Wang, P. (2006). Growth, motor, and social development in breast- and formula-fed infants of metformin-treated women with polycystic ovary syndrome J Pediatr, 148(5), 628-632

858 Gokcay, G.,Turan, J. M.,Partalci, A.,Neyzi, O. (2003). Growth of infants during the first year of life according to feeding regimen in

Intervention/exposure the first 4 months J Trop Pediatr, 49(1), 6-12

859 Goldfield, G. S.,Paluch, R.,Keniray, K.,Hadjiyannakis, S.,Lumb, A. B.,Adamo, K. (2006). Effects of breastfeeding on weight changes Participant health in family-based pediatric obesity treatment J Dev Behav Pediatr, 27(2), 93-7

860 Golding, J.,Rogers, I. S.,Emmett, P. M. (1997). Breast feeding: benefits and hazards. Methodology and summary of results Early Study design Hum Dev, 49 Suppl(\#issue\#), S1-6

861 Gomez-Sanchiz, M.,Canete, R.,Rodero, I.,Baeza, J. E.,Avila, O. (2003). Influence of breast-feeding on mental and psychomotor development Clin Pediatr (Phila), 42(1), 35-42

862 Gomez-Sanchiz, M.,Canete, R.,Rodero, I.,Baeza, J. E Gonzalez, J. A (2004). Influence of breast-feeding and parental intelligence on cognitive development in the 24-month-old child Clin Pediatr (Phila), 43(8), 753-61

863 Gong, Y. H.,Ji, C. Y.,Zheng, X. X.,Shan, J. P.,Hou, R. (2008). Correlation of 4-month infant feeding modes with their growth and iron Intervention/exposure status in Beijing Chin Med J (Engl), 121(5), 392-8

864 Gonzalez-Casanova, I.,Stein, A.,Hao, W.,Feregrino, R.,Romieu, I.,Barraza-Villarreal, A.,Rivera, J.,Martorell, R.,Ramakrishnan, U. (2014). Height and BMI at five years of age following prenatal supplementation with docosahexaenoic acid in Mexico FASEB journal, 28(1 suppl. 1), \#Pages\#

865 Gónzalez-Iglesias, H.,De La Flor St Remy, R. R.,López-Sastre, J.,Fernández-Colomer, B.,Ibáñez-Fernández, A.,Solís, G.,SanzMedel, A.,Fernández-Sánchez, M. L. (2012). Efficiency of iodine supplementation, as potassium iodide, during lactation: A study in neonates and their mothers Food Chemistry, 133(3), 859-865

866 Gopalan, S.,Puri, R. K. (1992). Breast feeding and infant growth Indian Pediatr, 29(8), 1079-86

867 Gopinath, V. K.,Muda, W. A. (2005). Assessment of growth and feeding practices in children with cleft lip and palate Southeast Asian J Trop Med Public Health, 36(1), 254-8

868 Gordon, M. (1995). Why breastfeeding is best for babies Health Visit, 68(5), 203-4

Study design

869 Gordon, R. R.,Noble, D. A.,Ward, A. M.,Allen, R. (1982). Immunoglobulin E and the eczema-asthma syndrome in early childhood Lancet, 1(8263), 72-4

870 Gore, C.,Custovic, A.,Tannock, G. W.,Munro, K.,Kerry, G.,Johnson, K.,Peterson, C.,Morris, J.,Chaloner, C.,Murray, C. S.,Woodcock A. (2012). Treatment and secondary prevention effects of the probiotics Lactobacillus paracasei or Bifidobacterium lactis on early infant eczema: randomized controlled trial with follow-up until age 3 years Clin Exp Allergy, 42(1), 112-22

871 Gore, C.,Munro, K.,Lay, C.,Bibiloni, R.,Morris, J.,Woodcock, A.,Custovic, A.,Tannock, G. W. (2008). Bifidobacterium pseudocatenulatum is associated with atopic eczema: a nested case-control study investigating the fecal microbiota of infants $\mathrm{J}$ Allergy Clin Immunol, 121(1), 135-40

Outcome

Participant health, Size of study groups 
872 Gore, N.,Emerson, E.,Brady, S. (2015). Rates of breastfeeding and exposure to socio-economic adversity amongst children with intellectual disability Res Dev Disabil, 39(\#issue\#), 12-9

873 Gormally, S. M.,Matthews, T. G. (1992). Contemporary risk factors for sudden infant death in an Irish population--a case control study Ir J Med Sci, 161(5), 131-4

874 Grabenhenrich, L. B.,Gough, H.,Reich, A.,Eckers, N.,Zepp, F.,Nitsche, O.,Forster, J.,Schuster, A.,Schramm, D.,Bauer, C.

P.,Hoffmann, U.,Beschorner, J.,Wagner, P.,Bergmann, R.,Bergmann, K.,Matricardi, P. M.,Wahn, U.,Lau, S.,Keil, T. (2014). Early-life determinants of asthma from birth to age 20 years: a German birth cohort study J Allergy Clin Immunol, 133(4), 979-88

875 Gracey, M. (1989). Maternal health, breast-feeding and infant nutrition in Australian aborigines Acta Paediatr Jpn, 31(4), 377-80

Study design

876 Grainger, M. (2006). Breastfeeding can reduce infant infections and health care costs Ala Nurse, 33(3), 23

Study design

877 Grandjean, P.,Poulsen, L. K.,Heilmann, C.,Steuerwald, U.,Weihe, P. (2010). Allergy and sensitization during childhood associated

Outcome with prenatal and lactational exposure to marine pollutants Environ Health Perspect, 118(10), 1429-33

878 Granot, E.,Golan, D.,Berry, E. M. (2000). Breast-fed and formula-fed infants do not differ in immunocompetent cell cytokine production despite differences in cell membrane fatty acid composition Am J Clin Nutr, 72(5), 1202-5

879 Graves, J.,Grandhe, S.,Weinfurtner, K.,Krupp, L.,Belman, A.,Chitnis, T.,Ness, J.,Weinstock-Guttman, B.,Gorman, M.,Patterson, M.,Rodriguez, M.,Lotze, T.,Aaen, G.,Mowry, E. M.,Rose, J. W.,Simmons, T.,Casper, T. C.,James, J.,Waubant, E. (2014). Protective environmental factors for neuromyelitis optica Neurology, 83(21), 1923-9

880 Greasley, V. (1986). Breast feeding Nursing (Lond), 3(2), 63-70

Study design

881 Greco, L.,Auricchio, S.,Mayer, M.,Grimaldi, M. (1988). Case control study on nutritional risk factors in celiac disease J Pediatr Gastroenterol Nutr, 7(3), 395-9

882 Green, Ken (2012). UC Denver Study: Breastfeeding Can Prevent Diabetes-Related Childhood Obesity Inside Childbirth Education, Study design \#volume\#(\#issue\#), 10-10 1p

883 Greene, L. C.,Lucas, A.,Livingstone, M. B.,Harland, P. S.,Baker, B. A. (1995). Relationship between early diet and subsequent cognitive performance during adolescence Biochem Soc Trans, 23(2), 376S

884 Greenop, K. R.,Bailey, H. D.,Miller, M.,Scott, R. J.,Attia, J.,Ashton, L. J.,Downie, P.,Armstrong, B. K.,Milne, E. (2015). Breastfeeding Outcome and nutrition to 2 years of age and risk of childhood acute lymphoblastic leukemia and brain tumors Nutr Cancer, 67(3), 431-41

885 Greer FR,Tsang RC (1983). Vitamin D in human milk: is there enough? J Pediatr Gastroenterol Nutr, 2 Suppl 1(\#issue\#), S277-81

Study design

886 Greer MH,Tendan SL (1998). Early childhood dental caries in Hawai'i Hawaii Dent J, 29(\#issue\#), 10, 14

Study design

887 Greer, F. R.,Marshall, S. (1989). Bone mineral content, serum vitamin D metabolite concentrations, and ultraviolet B light exposure in infants fed human milk with and without vitamin D2 supplements J Pediatr, 114(2), 204-12

Size of study groups 

25-hydroxyvitamin D concentrations in breast-fed infants with and without supplemental vitamin D: one-year follow-up $\mathrm{J}$ Pediatr, 100(6), 919-22 human milk and cobalamin-related variables in mother and child: a 9-mo longitudinal study Am J Clin Nutr, 98(2), 389-95

890 Grguric, J.,Wen, R. A.,Kylberg, E.,Ashmore, S.,Macenroe, T. (2012). International perspectives on the Baby-Friendly Initiative J Hum Lact, 28(3), 281-4

891 Grice, A. C.,McGlashan, N. D. (1981). Obstetric factors in 171 sudden infant deaths in Tasmania, 1970--1976 Med J Aust, 1(1), 26- Outcome 31

892 Griffiths, L. J.,Hawkins, S. S.,Cole, T. J.,Dezateux, C. (2010). Risk factors for rapid weight gain in preschool children: Findings from a UK-wide prospective study International Journal of Obesity, 34(4), 624-632

Publication date for a non-sibling study, Confounding

893 Griffiths, L. J.,Smeeth, L.,Hawkins, S. S.,Cole, T. J.,Dezateux, C. (2009). Effects of infant feeding practice on weight gain from birth to 3 years Arch Dis Child, 94(8), 577-82

Publication date for a non-sibling study complementary foods and the relationship to food allergy Pediatrics, 132(6), e1529-38

895 Grjibovski, A. M.,Bygren, L. O.,Yngve, A.,Sjostrom, M. (2004). Social variations in infant growth performance in Severodvinsk, Northwest Russia: community-based cohort study Croat Med J, 45(6), 757-63

896 Groen-Blokhuis, M. M.,Franic, S.,van Beijsterveldt, C. E.,de Geus, E.,Bartels, M.,Davies, G. E.,Ehli, E. A.,Xiao, X.,Scheet, P. A.,Althoff, R.,Hudziak, J. J.,Middeldorp, C. M.,Boomsma, D. I. (2013). A prospective study of the effects of breastfeeding and FADS2 polymorphisms on cognition and hyperactivity/attention problems Am J Med Genet B Neuropsychiatr Genet, 162B(5), 45765

Outcome

Publication date for a non-sibling study

Outcome

897 Groenwold, R. H.,Tilling, K.,Moons, K. G.,Hoes, A. W.,van der Ent, C. K.,Kramer, M. S.,Martin, R. M.,Sterne, J. A. (2014). Breastfeeding and health consequences in early childhood: is there an impact of time-dependent confounding? Ann Nutr Metab, 65(2-3), $139-48$

898 Grossman, X.,Chaudhuri, J. H.,Feldman-Winter, L.,Merewood, A. (2012). Neonatal weight loss at a US Baby-Friendly Hospital J Acad Nutr Diet, 112(3), 410-3

899 Grube, M. M.,von der Lippe, E.,Schlaud, M.,Brettschneider, A. K. (2015). Does breastfeeding help to reduce the risk of childhood overweight and obesity? A propensity score analysis of data from the KiGGS study PLoS One, 10(3), e0122534

900 Gruber, C.,van Stuijvenberg, M.,Mosca, F.,Moro, G.,Chirico, G.,Braegger, C. P.,Riedler, J.,Boehm, G.,Wahn, U. (2010). Reduced occurrence of early atopic dermatitis because of immunoactive prebiotics among low-atopy-risk infants $\mathrm{J}$ Allergy Clin Immunol, 126(4), 791-7

901 Gruber, M.,Marshall, J. R.,Zielezny, M.,Lance, P. (1996). A case-control study to examine the influence of maternal perinatal behaviors on the incidence of Crohn's disease Gastroenterol Nurs, 19(2), 53-9 
902 Grummer-Strawn, L. M.,Li, R.,Perrine, C. G.,Scanlon, K. S.,Fein, S. B. (2014). Infant feeding and long-term outcomes: results from the year 6 follow-up of children in the Infant Feeding Practices Study II Pediatrics, 134 Suppl 1(\#issue\#), S1-3

903 Grummer-Strawn, L. M.,Mei, Z. (2004). Does breastfeeding protect against pediatric overweight? Analysis of longitudinal data from the Centers for Disease Control and Prevention Pediatric Nutrition Surveillance System Pediatrics, 113(2), e81-6

904 Gruskay, F. L. (1982). Comparison of breast, cow, and soy feedings in the prevention of onset of allergic disease: a 15-year prospective study Clin Pediatr (Phila), 21(8), 486-91

905 Gruszfeld, D.,Weber, M.,Nowakowska-Rysz, M.,Janas, R.,Kozlik-Feldmann, R.,Xhonneux, A.,Carlier, C.,Riva, E.,Verduci, E.,ClosaMonasterolo, R.,Escribano, J.,Dobrzanska, A.,Koletzko, B. (2015). Protein intake in infancy and carotid intima media thickness at 5 years--a secondary analysis from a randomized trial Ann Nutr Metab, 66(1), 51-9

906 Gudino, S.,Rojas, N.,Castro, C.,Rodriguez, M.,Vega, M.,Lopez, L. M. (2007). Colonization of mutans streptococci in Costa Rican children from a high-risk population J Dent Child (Chic), 74(1), 36-40

Publication date for a non-sibling study

Intervention/exposure

Intervention/exposure

Study design

907 Guedes, H. T.,Souza, L. S. (2009). Exposure to maternal smoking in the first year of life interferes in breast-feeding protective effect Intervention/exposure against the onset of respiratory allergy from birth to $5 \mathrm{yr}$ Pediatr Allergy Immunol, 20(1), 30-4

908 Guerrero, M. L.,Moreno-Espinosa, S.,Tuz-Dzib, F.,Solis-Albino, J.,Ortega-Gallegos, H.,Ruiz-Palacios, G. M. (2004). Breastfeeding and natural colonization with Lactobacillus spp as protection against rotavirus-associated diarrhea Adv Exp Med Biol, 554(\#issue\#), $451-5$ Blood lipid concentrations of docosahexaenoic and arachidonic acids at birth determine their relative postnatal changes in term infants fed breast milk or formula Am J Clin Nutr, 70(2), 292-8

910 Guibas, G. V.,Xepapadaki, P.,Moschonis, G.,Douladiris, N.,Filippou, A.,Tsirigoti, L.,Manios, Y.,Papadopoulos, N. G. (2013). Breastfeeding and wheeze prevalence in pre-schoolers and pre-adolescents: the Genesis and Healthy Growth studies Pediatr Allergy Immunol, 24(8), 772-81

911 Guldan, G. S.,Fan, H. C.,Ma, X.,Ni, Z. Z.,Xiang, X.,Tang, M. Z. (2000). Culturally appropriate nutrition education improves infant feeding and growth in rural Sichuan, China J Nutr, 130(5), 1204-11

912 Gulick EE (1986). The effects of breast-feeding on toddler health Pediatr Nurs, 12(\#issue\#), 51-4 Outcome

913 Gulick, E. E. (1983). Infant health and breast-feeding Pediatr Nurs, 9(5), 359-62, 389

914 Gunderson, E. P. (2007). Breastfeeding after gestational diabetes pregnancy: subsequent obesity and type 2 diabetes in women and their offspring Diabetes Care, 30 Suppl 2(\#issue\#), S161-8

915 Gunderson, E. P.,Hurston, S. R.,Dewey, K. G.,Faith, M. S.,Charvat-Aguilar, N.,Khoury, V. C.,Nguyen, V. T.,Quesenberry, C. P., Jr. (2015). The study of women, infant feeding and type 2 diabetes after GDM pregnancy and growth of their offspring (SWIFT Offspring study): prospective design, methodology and baseline characteristics BMC Pregnancy Childbirth, 15(\#issue\#), 150 
916 Gungor, D. E.,Paul, I. M.,Birch, L. L.,Bartok, C. J. (2010). Risky vs rapid growth in infancy: refining pediatric screening for childhood overweight Arch Pediatr Adolesc Med, 164(12), 1091-7

Publication date for a non-sibling study

917 Gunnarsdottir, I.,Aspelund, T.,Birgisdottir, B. E.,Benediktsson, R.,Gudnason, V.,Thorsdottir, I. (2007). Infant feeding patterns and Intervention/exposure midlife erythrocyte sedimentation rate Acta Paediatr, 96(6), 852-6

918 Gunnarsdottir, I.,Schack-Nielsen, L.,Michaelsen, K. F.,Sorensen, T. I.,Thorsdottir, I. (2010). Infant weight gain, duration of exclusive breast-feeding and childhood BMI - two similar follow-up cohorts Public Health Nutr, 13(2), 201-7

919 Gunther, A. L.,Walz, H.,Kroke, A.,Wudy, S. A.,Riedel, C.,von Kries, R.,Joslowski, G.,Remer, T.,Cheng, G.,Buyken, A. E. (2013). Breastfeeding and its prospective association with components of the $\mathrm{GH}$-IGF-Axis, insulin resistance and body adiposity measures in young adulthood--insights from linear and quantile regression analysis PLoS One, 8(11), e79436

920 Guo, A. Y.,Stevens, B. W.,Wilson, R. G.,Russell, C. N.,Cohen, M. A.,Sturgeon, H. C., Thornton, A.,Giallourakis, C.,Khalili, H.,Nguyen, Study design, Outcome D. D.,Sauk, J.,Yajnik, V.,Xavier, R. J.,Ananthakrishnan, A. N. (2014). Early life environment and natural history of inflammatory bowel diseases BMC Gastroenterol, 14(\#issue\#), 216

921 Gurkan, F.,Davutog Lu, M.,Bilici, M.,Sincar, N.,Haspolat, K. (2002). Pulmonary functions in atopic and nonatopic asthmatic children Allergol Immunopathol (Madr), 30(2), 70-3

922 Gurkan, F.,Davutoglu, M.,Bilici, M.,Dagli, A.,Haspolat, K. (2002). Asthmatic children and risk factors at a province in the southeast of Turkey Allergol Immunopathol (Madr), 30(1), 25-9

923 Gurnida, D. A.,Rowan, A. M.,Idjradinata, P.,Muchtadi, D.,Sekarwana, N. (2012). Association of complex lipids containing gangliosides with cognitive development of 6-month-old infants Early Hum Dev, 88(8), 595-601

924 Gurwith, M.,Wenman, W.,Gurwith, D.,Brunton, J.,Feltham, S., Greenberg, H. (1983). Diarrhea among infants and young children in Canada: a longitudinal study in three northern communities J Infect Dis, 147(4), 685-92

Publication date for a non-sibling study

Intervention/exposure

Study design, Participant health

Study design

Country

925 Gurwith, M.,Wenman, W.,Hinde, D.,Feltham, S.,Greenberg, H. (1981). A prospective study of rotavirus infection in infants and young children J Infect Dis, 144(3), 218-24

926 Gustafsson, D.,Lowhagen, T.,Andersson, K. (1992). Risk of developing atopic disease after early feeding with cows' milk based formula Arch Dis Child, 67(8), 1008-10

927 Gustafsson, P. A.,Duchen, K.,Birberg, U.,Karlsson, T. (2004). Breastfeeding, very long polyunsaturated fatty acids (PUFA) and IQ at Outcome $61 / 2$ years of age Acta Paediatr, 93(10), 1280-7

928 Guxens, M.,Aguilera, I.,Ballester, F.,Estarlich, M.,Fernandez-Somoano, A.,Lertxundi, A.,Lertxundi, N.,Mendez, M. A.,Tardon, A.,Vrijheid, M.,Sunyer, J. (2012). Prenatal exposure to residential air pollution and infant mental development: modulation by antioxidants and detoxification factors Environ Health Perspect, 120(1), 144-9

929 Guxens, M.,Mendez, M. A.,Molto-Puigmarti, C.,Julvez, J.,Garcia-Esteban, R.,Forns, J.,Ferrer, M.,Vrijheid, M.,Lopez-Sabater, M. C.,Sunyer, J. (2011). Breastfeeding, long-chain polyunsaturated fatty acids in colostrum, and infant mental development Pediatrics, 128(4), e880-9

Size of study groups, Intervention/exposure

Size of study groups

Intervention/exposure

Outcome

\section{Outcome}


930 Habibzadeh, H.,Jafarizadeh, H.,Didarloo, A. (2015). Determinants of failure to thrive (FTT) among infants aged 6-24 months: a case-control study J Prev Med Hyg, 56(4), E180-6

931 Habicht, J. P.,DaVanzo, J.,Butz, W. P. (1986). Does breastfeeding really save lives, or are apparent benefits due to biases? Am J Epidemiol, 123(2), 279-90

932 Habicht, J. P.,DaVanzo, J.,Butz, W. P. (1988). Mother's milk and sewage: their interactive effects on infant mortality Pediatrics, $81(3), 456-61$

933 Hackney, A. R. (1990). Breast feeding Am J Nurs, 90(12), 70

Study design

934 Haddad, M. B.,Porucznik, C. A.,Joyce, K. E.,De, A. K.,Pavia, A. T.,Rolfs, R. T.,Byington, C. L. (2008). Risk factors for pediatric invasive pneumococcal disease in the Intermountain West, 1996-2002 Ann Epidemiol, 18(2), 139-46

935 Haider, S. J.,Chang, L. V.,Bolton, T. A.,Gold, J. G.,Olson, B. H. (2014). An evaluation of the effects of a breastfeeding support program on health outcomes Health Serv Res, 49(6), 2017-34

936 Haines, M. R.,Kintner, H. J. (2008). "Can breast feeding help you in later life? Evidence from German military heights in the early 20th century" Econ Hum Biol, 6(3), 420-30

937 Hakansson, A.,Carlsson, B. (1992). Maternal cigarette smoking, breast-feeding, and respiratory tract infections in infancy. A population-based cohort study Scand J Prim Health Care, 10(1), 60-5

938 Halchak, B. (1982). The Oxford lactation study J Nurse Midwifery, 27(5), 34-6

939 Halken, S. (2004). What causes allergy and asthma? The role of dietary factors Pediatr Pulmonol Suppl, 26(\#issue\#), 223-4

940 Halken, S.,Hansen, K. S.,Jacobsen, H. P.,Estmann, A.,Faelling, A. E.,Hansen, L. G., Kier, S. R.,Lassen, K.,Lintrup, M.,Mortensen, S.,Ibsen, K. K., Osterballe, O.,Host, A. (2000). Comparison of a partially hydrolyzed infant formula with two extensively hydrolyzed formulas for allergy prevention: a prospective, randomized study Pediatr Allergy Immunol, 11(3), 149-61

941 Halken, S.,Host, A.,Hansen, L. G.,Osterballe, O. (1992). Effect of an allergy prevention programme on incidence of atopic symptoms in infancy. A prospective study of 159 "high-risk" infants Allergy, 47(5), 545-53

942 Halken, S.,Host, A.,Hansen, L. G.,Osterballe, O. (1993). Preventive effect of feeding high-risk infants a casein hydrolysate formula or an ultrafiltrated whey hydrolysate formula. A prospective, randomized, comparative clinical study Pediatr Allergy Immunol, 4(4), $173-81$

943 Halken, S.,Host, A.,Husby, S.,Hansen, L. G.,Osterballe, O.,Nyboe, J. (1991). Recurrent wheezing in relation to environmental risk factors in infancy. A prospective study of 276 infants Allergy, 46(7), 507-14

944 Hall, K.,Frederiksen, B.,Rewers, M.,Norris, J. M. (2015). Daycare attendance, breastfeeding, and the development of type 1 diabetes: the diabetes autoimmunity study in the young Biomed Res Int, 2015(\#issue\#), 203947

945 Hallonsten, A. L.,Wendt, L. K.,Mejare, I.,Birkhed, D.,Hakansson, C.,Lindvall, A. M.,Edwardsson, S.,Koch, G. (1995). Dental caries and prolonged breast-feeding in 18-month-old Swedish children Int J Paediatr Dent, 5(3), 149-55

Study design

tudy design

Intervention/exposure

Study design, Outcome

ntervention/exposure, Outcome

Intervention/exposure

Study design

Intervention/exposure

Size of study groups,

Intervention/exposure

\section{Outcome}

Outcome

Study design 
947 Hamburger, R. N.,Heller, S.,Mellon, M. H.,O'Connor, R. D.,Zeiger, R. S. (1983). Current status of the clinical and immunologic consequences of a prototype allergic disease prevention program Ann Allergy, 51(2 Pt 2), 281-90

948 Hamilton, J. J.,Synnes, A.,Innis, S. M. (1992). Plasma cholesterol and lathosterol levels in term infants in the early neonatal period

Study design, Pediatr Res, 31(4 Pt 1), 396-400

949 Hamilton, J. R. (1985). Viral diarrhea Pediatr Ann, 14(1), 25-8

950 Han, D. H.,Ahn, J. C.,Mun, S. J.,Park, S. K.,Oh, S. Y.,Rhee, C. S. (2015). Novel risk factors for allergic rhinitis in Korean elementary Study design school children: ARCO-kids phase II in a community Allergy, Asthma and Immunology Research, 7(3), 234-240

951 Han, D. Y.,Fraser, A. G.,Dryland, P.,Ferguson, L. R. (2010). Environmental factors in the development of chronic inflammation: a case-control study on risk factors for Crohn's disease within New Zealand Mutat Res, 690(1-2), 116-22

952 Han, Y. S.,Park, H. Y.,Ahn, K. M.,Lee, J. S.,Choi, H. M.,Lee, S. I. (2003). Short-term effect of partially hydrolyzed formula on the prevention of development of atopic dermatitis in infants at high risk J Korean Med Sci, 18(4), 547-51

953 Han, Y.,Chung, S. J.,Kim, J.,Ahn, K.,Lee, S. I. (2009). High sensitization rate to food allergens in breastfed infants with atopic dermatitis Ann Allergy Asthma Immunol, 103(4), 332-6

954 Hancox, R. J.,Stewart, A. W.,Braithwaite, I.,Beasley, R.,Murphy, R.,Mitchell, E. A. (2015). Association between breastfeeding and body mass index at age 6-7 years in an international survey Pediatr Obes, 10(4), 283-7

955 Hanicar, B.,Mandic, Z.,Pavic, R. (2009). Exclusive breastfeeding and growth in Croatian infants--comparison to the WHO child growth standards and to the NCHS growth references Coll Antropol, 33(3), 735-41

\section{Intervention/exposure}

Size of study groups

\section{Study design}

Study design

Size of study groups

Study design, Outcome

Study design

Publication date for a non-sibling study

Publication date for a non-sibling study 40:60 whey: casein formula with added tryptophan Am J Clin Nutr, 56(6), 1004-11

957 Hansen, K. (2015). The Power of Nutrition and the Power of Breastfeeding Breastfeed Med, 10(8), 385-8

Study design

Outcome

958 Hansen, T. S.,Jess, T.,Vind, I.,Elkjaer, M.,Nielsen, M. F.,Gamborg, M.,Munkholm, P. (2011). Environmental factors in inflammatory bowel disease: a case-control study based on a Danish inception cohort J Crohns Colitis, 5(6), 577-84

959 Hanson, L. A.,Ashraf, R.,Zaman, S.,Karlberg, J.,Lindblad, B. S.,Jalil, F. (1994). Breast feeding is a natural contraceptive and prevents disease and death in infants, linking infant mortality and birth rates Acta Paediatr, 83(1), 3-6

960 Hanson, L. A.,Jalil, F.,Ashraf, R.,Bernini, S.,Carlsson, B.,Cruz, J. R.,Gonzalez, T.,Hahn-Zoric, M.,Mellander, L.,Minoli, Y.,et al., (1991). Characteristics of human milk antibodies and their effect in relation to the epidemiology of breastfeeding and infections in a developing country Adv Exp Med Biol, 310(\#issue\#), 1-15 
962 Haq, M. E.,Begum, K.,Muttalib, M. A.,Shahidullah, M. (1985). Prevalence of caries in urban children and its relation to feeding pattern Bangladesh Med Res Counc Bull, 11(2), 55-63

963 Hardell, L.,Dreifaldt, A. C. (2001). Breast-feeding duration and the risk of malignant diseases in childhood in Sweden Eur J Clin Nutr, Intervention/exposure $55(3), 179-85$

964 Hardy, E. E.,Vichi, A. M.,Sarmento, R. C.,Moreira, L. E.,Bosqueiro, C. M. (1982). Breastfeeding promotion: effect of an educational Outcome program in Brazil Stud Fam Plann, 13(3), 79-86

965 Harkin, T. (2011). Wellness and disease prevention begins at birth: the critically important role of breastfeeding Breastfeed Med, Study design 6(\#issue\#), 245-6

966 Harland, B. F.,Smith, S. A.,Ellis, R.,O'Brien, R.,Morris, E. R. (1992). Comparison of the nutrient intakes of blacks, Siouan Indians,

Study design, Outcome and whites in Columbus County, North Carolina Journal of the American Dietetic Association, 92(3), 348-350 Harris, J. M.,Cullinan, P.,Williams, H. C.,Mills, P.,Moffat eczema in early life $\mathrm{Br} \mathrm{J}$ Dermatol, 144(4), 795-802

968 Harris, M. C.,Kolski, G. B.,Campbell, D. E.,Deuber, C.,Marcus, M.,Douglas, S. D. (1989). Ontogeny of the antibody response to cow Size of study groups milk proteins Ann Allergy, 63(5), 439-43

969 Harrison, G. G.,Graver, E. J.,Vargas, M.,Churella, H. R.,Paule, C. L. (1987). Growth and adiposity of term infants fed wheypredominant or casein-predominant formulas or human milk J Pediatr Gastroenterol Nutr, 6(5), 739-47

970 Harrison, R.,Wong, T.,Ewan, C.,Contreras, B.,Phung, Y. (1997). Feeding practices and dental caries in an urban Canadian population of Vietnamese preschool children ASDC J Dent Child, 64(2), 112-7

971 Harsten, G.,Prellner, K.,Heldrup, J.,Kalm, O.,Kornfalt, R. (1989). Recurrent acute otitis media. A prospective study of children during Size of study groups the first three years of life Acta Otolaryngol, 107(1-2), 111-9

972 Hart, S.,Boylan, L. M.,Carroll, S.,Musick, Y. A.,Lampe, R. M. (2003). Brief report: breast-fed one-week-olds demonstrate superior neurobehavioral organization J Pediatr Psychol, 28(8), 529-34

973 Hartley, A. L.,Birch, J. M.,McKinney, P. A.,Blair, V.,Teare, M. D.,Carrette, J.,Mann, J. R.,Stiller, C. A.,Draper, G. J.,Johnston, H. E.,et al., (1988). The Inter-Regional Epidemiological Study of Childhood Cancer (IRESCC): past medical history in children with cancer J Epidemiol Community Health, 42(3), 235-42

974 Harvey, N. C.,Robinson, S. M.,Crozier, S. R.,Marriott, L. D.,Gale, C. R.,Cole, Z. A.,Inskip, H. M.,Godfrey, K. M.,Cooper, C. (2009). Breast-feeding and adherence to infant feeding guidelines do not influence bone mass at age 4 years Br J Nutr, 102(6), 915-20

Publication date for a non-sibling study

975 Haschke, F.,van't Hof, M. A. (2000). Euro-Growth references for breast-fed boys and girls: influence of breast-feeding and solids on growth until 36 months of age. Euro-Growth Study Group J Pediatr Gastroenterol Nutr, 31 Suppl 1(\#issue\#), S60-71

976 Haschke, F.,Vanura, H.,Male, C., Owen, G.,Pietschnig, B.,Schuster, E.,Krobath, E.,Huemer, C. (1993). Iron nutrition and growth of breast- and formula-fed infants during the first 9 months of life J Pediatr Gastroenterol Nutr, 16(2), 151-6 
977 Haschke, F.,Ziegler, E. E.,Grathwohl, D. (2014). Fast growth of infants of overweight mothers: Can it be slowed down? Annals of Nutrition and Metabolism, 64(\#issue\#), 19-24

978 Hashim SA (1983). Dietary fats and adipose tissue fatty acid composition Prev Med, 12(\#issue\#), 854-67

Study design

979 Hasselbalch, H.,Jeppesen, D. L.,Ersboll, A. K.,Engelmann, M. D.,Nielsen, M. B. (1997). Thymus size evaluated by sonography. A longitudinal study on infants during the first year of life Acta Radiol, 38(2), 222-7

980 Hassiotou, F.,Geddes, D. T. (2014). Programming of appetite control during breastfeeding as a preventative strategy against the obesity epidemic J Hum Lact, 30(2), 136-42

981 Hatano, S.,Aihara, K.,Nishi, Y.,Usui, T. (1985). Trace elements (copper, zinc, manganese, and selenium) in plasma and erythrocytes in relation to dietary intake during infancy J Pediatr Gastroenterol Nutr, 4(1), 87-92

982 Hathcock, A.,Krause, K.,Viera, A. J.,Fuemmeler, B. F.,Lovelady, C.,Ostbye, T. (2014). Satiety responsiveness and the relationship between breastfeeding and weight status of toddlers of overweight and obese women Matern Child Health J, 18(4), 1023-30

983 Hattab, F. N.,Al-Omari, M. A.,Angmar-Mansson, B.,Daoud, N. (1999). The prevalence of nursing caries in one-to-four-year-old children in Jordan ASDC J Dent Child, 66(1), 53-8

984 Hauck, F. R.,Herman, S. M.,Donovan, M.,Iyasu, S.,Merrick Moore, C.,Donoghue, E.,Kirschner, R. H.,Willinger, M. (2003). Sleep environment and the risk of sudden infant death syndrome in an urban population: the Chicago Infant Mortality Study Pediatrics, 111(5 Pt 2), 1207-14

985 Hawkes, J. S.,Gibson, R. A.,Roberton, D.,Makrides, M. (2006). Effect of dietary nucleotide supplementation on growth and immune function in term infants: a randomized controlled trial Eur J Clin Nutr, 60(2), 254-64

986 Hawkins, S. S.,Cole, T. J.,Law, C. (2009). An ecological systems approach to examining risk factors for early childhood overweight: findings from the UK Millennium Cohort Study J Epidemiol Community Health, 63(2), 147-55

Publication date for a non-sibling study

987 Hawley, N. L.,Johnson, W.,Nu'usolia, O.,McGarvey, S. T. (2014). The contribution of feeding mode to obesogenic growth trajectories in American Samoan infants Pediatr Obes, 9(1), e1-e13

988 Hay, A. E.,Campbell, C. M. A. (2004). Volunteer counsellors for supporting breast feeding...Graffy J, Taylor J, Williams A et al Randomised controlled trial of support from volunteer counsellors for mothers considering breast feeding. BMJ 2004;328:26. (3 January) BMJ: British Medical Journal (International Edition), 328(7435), 349-349 1p

989 Hay, D. F.,Pawlby, S.,Sharp, D.,Asten, P.,Mills, A.,Kumar, R. (2001). Intellectual problems shown by 11-year-old children whose mothers had postnatal depression J Child Psychol Psychiatry, 42(7), 871-89

990 Hay, G.,Clausen, T.,Whitelaw, A., Trygg, K.,Johnston, C.,Henriksen, T.,Refsum, H. (2010). Maternal folate and cobalamin status predicts vitamin status in newborns and 6-month-old infants J Nutr, 140(3), 557-64

991 Hay, G.,Johnston, C.,Whitelaw, A., Trygg, K.,Refsum, H. (2008). Folate and cobalamin status in relation to breastfeeding and weaning in healthy infants Am J Clin Nutr, 88(1), 105-14

Intervention/exposure

Study design

Outcome

Intervention/exposure

Study design

Intervention/exposure 
992 Hayatbakhsh, M. R.,O'Callaghan M, J.,Bor, W.,Williams, G. M.,Najman, J. M. (2012). Association of breastfeeding and adolescents' psychopathology: A large prospective study Breastfeeding Medicine, 7(6), 480-486

993 Hayes, K. C.,Pronczuk, A.,Wood, R. A.,Guy, D. G. (1992). Modulation of infant formula fat profile alters the low-density lipoprotein/high-density lipoprotein ratio and plasma fatty acid distribution relative to those with breast-feeding J Pediatr, 120(4 Pt 2), S109-16

994 Hayosh, O.,Mandel, D.,Mimouni, F. B.,Lahat, S.,Marom, R.,Lubetzky, R. (2015). Prolonged duration of breastfeeding does not affect lipid profile in adulthood Breastfeed Med, 10(4), 218-21

995 Hearst, Mary O.,Martin, Lauren, Rafdal, Brooke H.,Robinson, Ronel,McConnell, Scott R. (2013). Early childhood development and obesity risk-factors in a multi-ethnic, low-income community: Feasibility of the 'Five Hundred under Five' social determinants of health pilot study Health Education Journal, 72(2), 203-215 13p

996 Heath, A. L.,Tuttle, C. R.,Simons, M. S.,Cleghorn, C. L.,Parnell, W. R. (2002). Longitudinal study of diet and iron deficiency anaemia in infants during the first two years of life Asia Pac J Clin Nutr, 11(4), 251-7

997 Hedstrom, M. (1982). Breastfeeding and Amningshjalpen in Sweden J Trop Pediatr, 28(3), 113-5 Intervention/exposure

Study design

998 Hegde CV,Anand RK (1987). Bowel pattern and weight gain in breastfed infants Indian Pediatr, 24(\#issue\#), 859-64 Country

999 Heikkila, K.,Kelly, Y.,Renfrew, M. J.,Sacker, A.,Quigley, M. A. (2014). Breastfeeding and educational achievement at age 5 Matern Child Nutr, 10(1), 92-101

1000 Heikkilä, K.,Sacker, A.,Kelly, Y.,Renfrew, M. J.,Quigley, M. A. (2010). 012 Breast feeding and behavioural development in children: findings from the Millennium Cohort Study Journal of Epidemiology \& Community Health, 64(\#issue\#), A5-A5 1p

1001 Heikkila, K.,Sacker, A.,Kelly, Y.,Renfrew, M. J.,Quigley, M. A. (2011). Breast feeding and child behaviour in the Millennium Cohort Outcome Study Arch Dis Child, 96(7), 635-42

1002 Heine, W.,Lapsien, C. (1982). Influence of early breast milk and formula feeding on body weight in children born in Rostock since 1945 Bibl Nutr Dieta, \#volume\#(31), 17-8

\begin{tabular}{cl}
\hline 1003 & Heiner, D. C. (1984). Modern research relating to food allergy and its implications--introduction Clin Rev Allergy, 2(1), 1-5 \\
\hline 1004 & Heinig, J., Ishii, K. (2004). Exclusive breastfeeding: isn't some breastfeeding good enough? J Hum Lact, 20(4), 423-4 \\
\hline 1005 & $\begin{array}{l}\text { Heinig, M. J.,Nommsen, L. A.,Peerson, J. M.,Lonnerdal, B.,Dewey, K. G. (1993). Energy and protein intakes of breast-fed and } \\
\text { formula-fed infants during the first year of life and their association with growth velocity: the DARLING Study Am J Clin Nutr, 58(2), } \\
152-61\end{array}$
\end{tabular}

1006 Heinig, M. J.,Nommsen, L. A.,Peerson, J. M.,Lonnerdal, B.,Dewey, K. G. (1993). Intake and growth of breast-fed and formula-fed infants in relation to the timing of introduction of complementary foods: the DARLING study. Davis Area Research on Lactation, Infant Nutrition and Growth Acta Paediatr, 82(12), 999-1006

Study design,

Intervention/exposure

Study design

Study design

Intervention/exposure

Intervention/exposure 
1007 Heinonen, K.,Raikkonen, K.,Pesonen, A. K.,Andersson, S.,Kajantie, E.,Eriksson, J. G.,Wolke, D.,Lano, A. (2011). Longitudinal study of smoking cessation before pregnancy and children's cognitive abilities at 56 months of age Early Hum Dev, 87(5), 353-9

Participant health,

Hemalatha, P.,Bhaskaram, P.,Kumar, P. A.,Khan, M. M.,Islam, M. A. (1997). Zinc status of breastfed and formula-fed infants of Intervention/exposure

1008 Hemalatha, P.,Bhaskaram, P.,Kumar, P. A.,Khan, M.

1009 Henry, F. J.,Bartholomew, R. K. (1990). Epidemiology and transmission of rotavirus infections and diarrhoea in St. Lucia, West Indies West Indian Med J, 39(4), 205-12

Country, Size of study group

1010 Hepworth, S. J.,Law, G. R.,Lawlor, D. A.,McKinney, P. A. (2010). Early life patterns of common infection: a latent class analysis Eur Outcome J Epidemiol, 25(12), 875-83

1011 Herba, C. M.,Roza, S.,Govaert, P.,Hofman, A., Jaddoe, V.,Verhulst, F. C., Tiemeier, H. (2013). Breastfeeding and early brain development: the Generation R study Matern Child Nutr, 9(3), 332-49

1012 Heresi, G.,Pizarro, F.,Olivares, M.,Cayazzo, M.,Hertrampf, E.,Walter, T.,Murphy, J. R.,Stekel, A. (1995). Effect of supplementation with an iron-fortified milk on incidence of diarrhea and respiratory infection in urban-resident infants Scand J Infect Dis, 27(4), 385-9

1013 Hernell, O. (1990). The requirements and utilization of dietary fatty acids in the newborn infant Acta Paediatr Scand Suppl, 365(\#issue\#), 20-7

1014 Hernell, O.,Lonnerdal, B. (2002). Iron status of infants fed low-iron formula: no effect of added bovine lactoferrin or nucleotides Am J Clin Nutr, 76(4), 858-64

1015 Hernell, O.,Lonnerdal, B. (2003). Nutritional evaluation of protein hydrolysate formulas in healthy term infants: plasma amino acids, hematology, and trace elements Am J Clin Nutr, 78(2), 296-301

1016 Hertrampf, E.,Cayazzo, M.,Pizarro, F.,Stekel, A. (1986). Bioavailability of iron in soy-based formula and its effect on iron nutriture in Intervention/exposure infancy Pediatrics, 78(4), 640-5

1017 Hesselmar, B.,Saalman, R.,Rudin, A.,Adlerberth, I.,Wold, A. E. (2010). Early fish introduction is associated with less eczema, but not sensitization, in infants Acta Paediatrica, International Journal of Paediatrics, 99(12), 1861-1867

1018 Hetzner, N. M.,Razza, R. A.,Malone, L. M.,Brooks-Gunn, J. (2009). Associations among feeding behaviors during infancy and child Outcome illness at two years Matern Child Health J, 13(6), 795-805

1019 Hide DW,Guyer BM (1983). Cows milk intolerance in Isle of Wight infants Br J Clin Pract, 37(\#issue\#), 285-7

Outcome, Size of study groups

1020 Hide, D. W. (1980). Aspects of nutrition: Isle of Wight infant feeding survey Health Visit, 53(2), 43

1021 Hide, D. W. (1991). The clinical expression of allergy in breast-fed infants Adv Exp Med Biol, 310(\#issue\#), 475-80

Study design

Study design

1022 Hide, D. W.,Guyer, B. M. (1981). Clinical manifestations of allergy related to breast and cows' milk feeding Arch Dis Child, 56(3), $172-5$ 
1024 Hide, D. W.,Matthews, S.,Matthews, L.,Stevens, M.,Ridout, S.,Twiselton, R.,Gant, C.,Arshad, S. H. (1994). Effect of allergen avoidance in infancy on allergic manifestations at age two years J Allergy Clin Immunol, 93(5), 842-6

1025 Hide, D. W.,Matthews, S.,Tariq, S.,Arshad, S. H. (1996). Allergen avoidance in infancy and allergy at 4 years of age Allergy, 51(2), Intervention/exposure $89-93$

1026 Higashi, A.,Ikeda, T.,Uehara, I.,Matsuda, I. (1982). Effect of low-content zinc and copper formula on infant nutrition Eur J Pediatr $138(3), 237-40$

1027 Highet, A. R.,Berry, A. M.,Bettelheim, K. A.,Goldwater, P. N. (2014). Gut microbiome in sudden infant death syndrome (SIDS) differs from that in healthy comparison babies and offers an explanation for the risk factor of prone position Int J Med Microbiol, 304(5-6), $735-41$ Acta Paediatr Scand, 78(1), 23-8

1029 Hiley, C. M.,Morley, C. J. (1996). Risk factors for sudden infant death syndrome: further change in 1992-3 BMJ, 312(7043), 1397-8 Study design

1030 Hill, D. J.,Hosking, C. S. (1993). Preventing childhood allergy Med J Aust, 158(6), 367-9

Study design

1031 Hillemeier, M. M.,Landale, N. S.,Oropesa, R. S. (2015). Asthma in US Mexican-Origin Children in Early Childhood: Differences in Outcome Risk and Protective Factors by Parental Nativity Acad Pediatr, 15(4), 421-9

1032 Hillman, L. S. (1988). Bone mineral content in term infants fed human milk, cow milk-based formula, or soy-based formula J Pediatr, Size of study groups $113(1 \mathrm{Pt} 2), 208-12$

1033 Hillman, L. S.,Chow, W.,Salmons, S. S.,Weaver, E.,Erickson, M.,Hansen, J. (1988). Vitamin D metabolism, mineral homeostasis, and bone mineralization in term infants fed human milk, cow milk-based formula, or soy-based formula J Pediatr, 112(6), 864-74

1034 Hirota, T.,Nara, M. Ohguri, M. Manago, E.,Hirota, K. (1992). Effect of diet and lifestyle on bone mass in Asian young women Am J Clin Nutr, 55(6), 1168-73

1035 Hitchcock, N. E.,Coy, J. F. (1989). The growth of healthy Australian infants in relation to infant feeding and social group Med J Aust, 150(6), 306-8, 310-1

1036 Hitchcock, N. E.,Gracey, M.,Gilmour, A. I. (1985). The growth of breast fed and artificially fed infants from birth to twelve months Acta Paediatr Scand, 74(2), 240-5

1037 Hitchcock, N. E.,Gracey, M.,Owles, E. N. (1981). Growth of healthy breast-fed infants in the first six months Lancet, 2(8237), 64-5

Size of study groups

Study design

Publication date for a non-sibling study

Publication date for a non-sibling study

Study design

Intervention/exposure

1038 Hitchcock, N. E.,McGuiness, D.,Gracey, M. (1982). Growth and feeding practices of Western Australian infants Med J Aust, 1(9),

Publication date for a non-sibling study

1039 Hitchcock, N. E.,Owles, E. N.,Gracey, M. (1981). Breast feeding and growth of healthy infants Med J Aust, 2(10), 536-7

Study design 
1040 Hlavaty, T.,Toth, J.,Koller, T.,Krajcovicova, A.,Oravcova, S.,Zelinkova, Z.,Huorka, M. (2013). Smoking, breastfeeding, physical inactivity, contact with animals, and size of the family influence the risk of inflammatory bowel disease: A Slovak case-control study United European Gastroenterology Journal, 1(2), 109-119 in an American Indian community: educational strategy for obesity prevention J Okla State Med Assoc, 107(2), 55-9

1042 Hoffman, D. R.,Birch, E. E.,Birch, D. G.,Uauy, R.,Castaneda, Y. S.,Lapus, M. G.,Wheaton, D. H. (2000). Impact of early dietary intake and blood lipid composition of long-chain polyunsaturated fatty acids on later visual development J Pediatr Gastroenterol Nutr, $31(5), 540-53$

1043 Hoffman, D. R.,Birch, E. E.,Castaneda, Y. S.,Fawcett, S. L.,Birch, D. G.,Uauy, R. (2001). Dietary docosahexaenoic acid (DHA) and visual maturation in the post-weaning term infant lovs, 42(\#issue\#), ARVO Abstract 656

1044 Hoffman, D. R.,Birch, E. E.,Castaneda, Y. S.,Fawcett, S. L.,Wheaton, D. H.,Birch, D. G., Uauy, R. (2003). Visual function in breastfed term infants weaned to formula with or without long-chain polyunsaturates at 4 to 6 months: a randomized clinical trial J Pediatr, 142(6), 669-77

1045 Hoffman, D. R.,Wheaton, D. K.,James, K. J.,Tuazon, M.,Diersen-Schade, D. A.,Harris, C. L.,Stolz, S.,Berseth, C. L. (2006). Docosahexaenoic acid in red blood cells of term infants receiving two levels of long-chain polyunsaturated fatty acids $\mathrm{J}$ Pediatr Gastroenterol Nutr, 42(3), 287-92

$1046 \begin{aligned} & \text { Hoffman, D.,Birch, E.,Birch, D.,Uauy, R., Castaneda, Y.,Wheaton, D. (1996). Red blood cell (rbc) fatty acid profiles in term infants } \\ & \text { fed formulas enriched with long-chain polyunsaturates (Icp) lovs, 37(\#issue\#), ARVO Abstract } 3693\end{aligned}$

$1046 \begin{aligned} & \text { Hoffman, D.,Birch, E.,Birch, D.,Uauy, R.,Castaneda, Y.,Wheaton, D. (1996). Red blood cell (rbc) fatty } \\ & \text { fed formulas enriched with long-chain polyunsaturates (Icp) lovs, 37(\#issue\#), ARVO Abstract } 3693\end{aligned}$

Publication status

Outcome

Intervention/exposure

1047 Hoffmans, M. D.,Obermann-de Boer, G. L.,Florack, E. I.,van Kampen-Donker, M.,Kromhout, D. (1988). Determinants of growth during early infancy Hum Biol, 60(2), 237-49

1048 Hofvander Y,Hillervik C (1995). Breast-feeding in Swedish hospitals World Health Forum, 16(\#issue\#), 95-9

Study design,

Intervention/exposure

Publication date for a non-sibling study Study design, Outcome

1049 Hogendorf, A.,Stanczyk-Przyluska, A.,Sieniwicz-Luzenczyk, K.,Wiszniewska, M.,Arendarczyk, J.,Banasik, M.,Fendler, W.,Kowalski, M.,Zeman, K. (2013). Is there any association between secretory IgA and lactoferrin concentration in mature human milk and food allergy in breastfed children Med Wieku Rozwoj, 17(1), 47-52

\begin{tabular}{|c|c|c|}
\hline 1050 & $\begin{array}{l}\text { Hokama, T. (1993). A study of the hemoglobin levels in breast-fed infants in one village of Okinawa prefecture Acta Paediatr Jpn, } \\
35(2), 138-40\end{array}$ & Size of study groups \\
\hline 1051 & $\begin{array}{l}\text { Hokama, T. (1993). Levels of serum ferritin and total body iron among infants with different feeding regimens Acta Paediatr Jpn, } \\
\text { 35(4), 298-301 }\end{array}$ & $\begin{array}{l}\text { Study design, Size of study } \\
\text { groups }\end{array}$ \\
\hline 1052 & $\begin{array}{l}\text { Hokama, T.,Sakamoto, R.,Yara, A.,Asato, Y.,Takamine, F.,Itokazu, K. (1999). Incidence of Haemophilus influenzae in the throats of } \\
\text { healthy infants with different feeding methods Pediatr Int, 41(3), 277-80 }\end{array}$ & Study design \\
\hline 1053 & $\begin{array}{l}\text { Holberg, C. J.,Wright, A. L.,Martinez, F. D.,Ray, C. G., Taussig, L. M.,Lebowitz, M. D. (1991). Risk factors for respiratory syncytial } \\
\text { virus-associated lower respiratory illnesses in the first year of life Am J Epidemiol, 133(11), 1135-51 }\end{array}$ & Intervention/exposure \\
\hline
\end{tabular}



34(1-2), 78-93

1055 Holland, B. (1987). The validity of retrospective breast-feeding-duration data: an illustrative analysis of data quality in the Malaysian Study design Family Life Survey Hum Biol, 59(3), 477-87

1056 Hollen, L. I.,Din, Zu,Jones, L. R.,Emond, A. M.,Emmett, P. (2014). Are diet and feeding behaviours associated with the onset of and Intervention/exposure recovery from slow weight gain in early infancy? Br J Nutr, 111(9), 1696-704

1057 Hollis, B. W.,Wagner, C. L.,Howard, C. R.,Ebeling, M.,Shary, J. R.,Smith, P. G., Taylor, S. N.,Morella, K.,Lawrence, R. A.,Hulsey, T.

Intervention/exposure C. (2015). Maternal Versus Infant Vitamin D Supplementation During Lactation: A Randomized Controlled Trial Pediatrics, 136(4), $625-34$

1058 Holm, A. K.,Andersson, R. (1982). Enamel mineralization disturbances in 12-year-old children with known early exposure to fluorides Community Dent Oral Epidemiol, 10(6), 335-9

1059 Holman, D. J.,Yamaguchi, K. (2005). Longitudinal analysis of deciduous tooth emergence: IV. Covariate effects in Japanese children Am J Phys Anthropol, 126(3), 352-8

1060 Holme, A.,MacArthur, C.,Lancashire, R. (2010). The effects of breastfeeding on cognitive and neurological development of children at 9 years Child Care Health Dev, 36(4), 583-90

1061 Holmes, G. E.,Hassanein, K. M.,Miller, H. C. (1983). Factors associated with infections among breast-fed babies and babies fed proprietary milks Pediatrics, 72(3), 300-6

1062 Holmes, V. A.,Cardwell, C.,McKinley, M. C.,Young, I. S.,Murray, L. J.,Boreham, C. A.,Woodside, J. V. (2010). Association between breast-feeding and anthropometry and CVD risk factor status in adolescence and young adulthood: the Young Hearts Project, Northern Ireland Public Health Nutr, 13(6), 771-8

1063 Holscher, H. D.,Czerkies, L. A.,Cekola, P.,Litov, R.,Benbow, M.,Santema, S.,Alexander, D. D.,Perez, V.,Sun, S.,Saavedra, J. M.,Tappenden, K. A. (2012). Bifidobacterium lactis Bb12 enhances intestinal antibody response in formula-fed infants: a randomized, double-blind, controlled trial JPEN J Parenter Enteral Nutr, 36(1 Suppl), 106S-17S

1064 Holt, R. D.,Joels, D.,Winter, G. B. (1982). Caries in pre-school children. The Camden study Br Dent J, 153(3), 107-9

1065 Holt, R. D.,Winter, G. B.,Downer, M. C.,Bellis, W. J.,Hay, I. S. (1996). Caries in pre-school children in Camden 1993/94 Br Dent J, 181(11-12), 405-10

1066 Hon, K. L. E.,Leung, T. F.,Kam, W. Y. C.,Lam, M. C. A.,Fok, T. F.,Ng, P. C. (2006). Dietary restriction and supplementation in children with atopic eczema Clinical and Experimental Dermatology, 31(2), 187-191

1067 Hong, L.,Levy, S. M.,Warren, J. J.,Broffitt, B. (2014). Infant breast-feeding and childhood caries: a nine-year study Pediatr Dent, $36(4), 342-7$ 
1068 Hong, X.,Wang, G.,Liu, X.,Kumar, R.,Tsai, H. J.,Arguelles, L.,Hao, K.,Pearson, C.,Ortiz, K.,Bonzagni, A.,Apollon, S.,Fu, L.,Caruso, D.,Pongracic, J. A.,Schleimer, R.,Holt, P. G.,Bauchner, H.,Wang, X. (2011). Gene polymorphisms, breast-feeding, and development of food sensitization in early childhood J Allergy Clin Immunol, 128(2), 374-81 e2

1069 Hong, Z. Y.,Zhang, Y. W.,Xu, J. D.,Zhou, J. D.,Gao, X. L.,Liu, X. G.,Shi, Y. Y. (1992). Growth promoting effect of zinc supplementation in infants of high-risk pregnancies Chin Med J (Engl), 105(10), 844-8

1070 Honorio, R. F.,Costa Monteiro Hadler, M. C. (2014). Factors associated with obesity in brazilian children enrolled in the school health program: a case-control study Nutr Hosp, 30(3), 526-34

1071 Hopkins, D.,Emmett, P.,Steer, C.,Rogers, I.,Noble, S.,Emond, A. (2007). Infant feeding in the second 6 months of life related to iron Intervention/exposure status: an observational study Arch Dis Child, 92(10), 850-4

1072 Hopkins, D.,Steer, C. D.,Northstone, K.,Emmett, P. M. (2015). Effects on childhood body habitus of feeding large volumes of cow or Intervention/exposure formula milk compared with breastfeeding in the latter part of infancy Am J Clin Nutr, 102(5), 1096-103

1073 Hopkinson, J. (2003). Is it possible for a breastfed baby to be overweight? J Hum Lact, 19(2), 189-90

Study design

1074 Hoppu, U.,Isolauri, E.,Koskinen, P.,Laitinen, K. (2013). Diet and blood lipids in 1-4 year-old children Nutr Metab Cardiovasc Dis, 23(10), 980-6

1075 Hoppu, U.,Kalliomaki, M.,Isolauri, E. (2002). Cow's milk allergy--a matter of fat Allergy, 57(1), 61-2

Study design

Intervention/exposure

1076 Horby Jorgensen, M.,Holmer, G.,Lund, P.,Hernell, O.,Michaelsen, K. F. (1998). Effect of formula supplemented with

Size of study groups,

docosahexaenoic acid and gamma-linolenic acid on fatty acid status and visual acuity in term infants $\mathrm{J}$ Pediatr Gastroenterol Nutr, 26(4), 412-21

1077 Horst, C. H.,Obermann-de Boer, G. L.,Kromhout, D. (1987). Type of milk feeding and nutrient intake during infancy. The Leiden Pre- Study design, Outcome School Children Study Acta Paediatr Scand, 76(6), 865-71

1078 Horta, B. L.,Bas, A.,Bhargava, S. K.,Fall, C. H.,Feranil, A.,de Kadt, J.,Martorell, R.,Richter, L. M.,Stein, A. D.,Victora, C. G. (2013). Infant feeding and school attainment in five cohorts from low- and middle-income countries PLoS One, 8(8), e71548

1079 Horta, B. L., Victora, C. G.,Lima, R. C.,Goncalves, H.,Guimaraes, B. E.,Barros, F. C. (2006). Breastfeeding duration and blood pressure among Brazilian adolescents Acta Paediatr, 95(3), 325-31

\begin{tabular}{|c|c|c|}
\hline 1080 & Horton, C. (2012). An overview of the NUTRIMENTHE project Nutrition Bulletin, 37(2), 152-156 5p & Study design \\
\hline 1081 & Horwood, L. J.,Fergusson, D. M. (1998). Breastfeeding and later cognitive and academic outcomes Pediatrics, 101(1), E9 & Outcome \\
\hline 1082 & $\begin{array}{l}\text { Horwood, L. J.,Fergusson, D. M.,Shannon, F. T. (1985). Social and familial factors in the development of early childhood asthma } \\
\text { Pediatrics, 75(5), 859-68 }\end{array}$ & $\begin{array}{l}\text { Size of study groups, } \\
\text { Intervention/exposure }\end{array}$ \\
\hline
\end{tabular}


1083 Hosaka, M.,Asayama, K.,Staessen, J. A.,Ohkubo, T.,Hayashi, K., Tatsuta, N.,Kurokawa, N.,Satoh, M.,Hashimoto, T.,Hirose, T.,Obara, T.,Metoki, H.,Inoue, R., Kikuya, M.,Nakai, K.,Imai, Y.,Satoh, H. (2013). Breastfeeding leads to lower blood pressure in 7 year-old Japanese children: Tohoku Study of Child Development Hypertens Res, 36(2), 117-22

1084 Hosseini, S. M.,Maracy, M. R.,Sarrafzade, S.,Kelishadi, R. (2014). Child weight growth trajectory and its determinants in a sample of Intervention/exposure Iranian children from birth until 2 years of age International Journal of Preventive Medicine, 5(3), 348-355

1085 Host, A. (1991). Importance of the first meal on the development of cow's milk allergy and intolerance Allergy Proc, 12(4), 227-32 Outcome

1086 Host, A.,Husby, S.,Osterballe, O. (1988). A prospective study of cow's milk allergy in exclusively breast-fed infants. Incidence, pathogenetic role of early inadvertent exposure to cow's milk formula, and characterization of bovine milk protein in human milk Acta Paediatr Scand, 77(5), 663-70

1087 Houston M,Howie P,McNeilly A (1983). Nursing Mirror Midwifery Forum 4. Infant feeding Nurs Mirror, 156(\#issue\#), i-iv

\section{Study design,}

Intervention/exposure

Study design

1088 Hovland, V.,Riiser, A.,Mowinckel, P.,Carlsen, K. H.,Lodrup Carlsen, K. C. (2015). Early risk factors for pubertal asthma Clin Exp Allergy, 45(1), 164-76

1089 Howe, L. D.,Ellison-Loschmann, L.,Pearce, N.,Douwes, J.,Jeffreys, M.,Firestone, R. (2015). Ethnic differences in risk factors for obesity in New Zealand infants J Epidemiol Community Health, 69(6), 516-22

1090 Howie, P. W.,Forsyth, J. S.,Ogston, S. A.,Clark, A.,Du Florey, V. C. (1990). Protective effect of breat feeding against infection British Outcome Medical Journal, 300(6716), 11-16

1091 Howie, P. W.,Forsyth, J. S.,Ogston, S. A.,Clark, A.,Florey, C. (1990). Protective effect of breastfeeding against infection... this article Outcome originally appeared in the British Medical Journal, V. 300. Reproduced with permission Breastfeeding Review, 2(1), 7-15 9p

1092 Howie, P. W.,Forsyth, J. S.,Ogston, S. A.,Clark, A.,Florey, C. D. (1990). Protective effect of breast feeding against infection BMJ, Outcome $300(6716), 11-6$

1093 Hoyle, B.,Yunus, M.,Chen, L. C. (1980). Breast-feeding and food intake among children with acute diarrheal disease The American Country, Study design journal of clinical nutrition, 33(11), 2365-2371

1094 Hromadova, M. Kostalova, L Leskova, L Kapellerova, A (1997). Relationship between the duration of the breast-feeding period and the lipoprotein profile of children at the age of 13 years Physiol Res, 46(1), 21-5

1095 Huang, J.,Peters, K. E.,Vaughn, M. G.,Witko, C. (2014). Breastfeeding and trajectories of children's cognitive development Dev Sci, Outcome $17(3), 452-61$

1096 Huang, J.,Vaughn, M. G.,Kremer, K. P. (2015). Breastfeeding and child development outcomes: an investigation of the nurturing hypothesis Matern Child Nutr, \#volume\#(\#issue\#), \#Pages\#

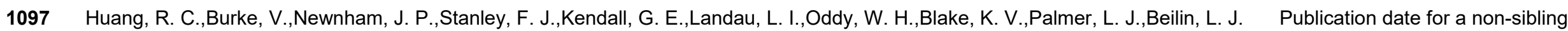
(2007). Perinatal and childhood origins of cardiovascular disease Int J Obes (Lond), 31(2), 236-44 
1098 Huang, R. C.,Mori, T. A.,Beilin, L. J. (2012). Early life programming of cardiometabolic disease in the Western Australian pregnancy cohort (Raine) study Clinical and Experimental Pharmacology and Physiology, 39(11), 973-978

1099 Huffman, S. L.,Dewey, K. G.,Schofield, D. (2010). Moving ahead with maternal, infant, and young child nutrition: need to integrate actions Food Nutr Bull, 31(2 Suppl), S99

1100 Huffman, S. L.,Lopez de Romana, G.,Madrid, S.,Brown, K. H.,Bentley, M.,Black, R. E. (1991). Do child feeding practices change due to diarrhoea in the Central Peruvian Highlands? J Diarrhoeal Dis Res, 9(4), 295-300

1101 Huh, S. Y.,Rifas-Shiman, S. L.,Taveras, E. M.,Oken, E.,Gillman, M. W. (2011). Timing of solid food introduction and risk of obesity in Intervention/exposure preschool-aged children Pediatrics, 127(3), e544-51

1102 Hummel, M.,Fuchtenbusch, M.,Schenker, M.,Ziegler, A. G. (2000). No major association of breast-feeding, vaccinations, and

Outcome childhood viral diseases with early islet autoimmunity in the German BABYDIAB Study Diabetes Care, 23(7), 969-74

1103 Hummel, S.,Pfluger, M.,Kreichauf, S.,Hummel, M.,Ziegler, A. G. (2009). Predictors of overweight during childhood in offspring of parents with type 1 diabetes Diabetes Care, 32(5), 921-5

1104 Hundt, G. A.,Forman, M. R. (1993). Interfacing anthropology and epidemiology: the Bedouin Arab Infant Feeding Study Soc Sci Med, 36(7), 957-64

1105 Hure, A. J.,Collins, C. E.,Smith, R. (2012). A longitudinal study of maternal folate and vitamin B12 status in pregnancy and postpartum, with the same infant markers at 6 months of age Matern Child Health J, 16(4), 792-801

1106 Hurtado, J. A., Iznaola, C.,Pena, M.,Ruiz, J.,Pena-Quintana, L.,Kajarabille, N.,Rodriguez-Santana, Y.,Sanjurjo, P.,AldamizEchevarria, L.,Ochoa, J.,Lara-Villoslada, F. (2015). Effects of Maternal Omega-3 Supplementation on Fatty Acids and on Visual and Cognitive Development J Pediatr Gastroenterol Nutr, 61(4), 472-80

1107 Husk, J. S.,Keim, S. A. (2015). Breastfeeding and Autism Spectrum Disorder in the National Survey of Children's Health Epidemiology, 26(4), 451-457

1108 Hutchison, B. L., Thompson, J. M.,Mitchell, E. A. (2015). Infant care practices related to sudden unexpected death in infancy: a 2013 Study design, Outcome survey N Z Med J, 128(1408), 15-22

1109 Huttunen, J. K.,Saarinen, U. M.,Kostiainen, E.,Siimes, M. A. (1983). Fat composition of the infant diet does not influence subsequent Intervention/exposure serum lipid levels in man Atherosclerosis, 46(1), 87-94

1110 Huurre, A.,Laitinen, K.,Rautava, S.,Korkeamaki, M.,Isolauri, E. (2008). Impact of maternal atopy and probiotic supplementation

Outcome during pregnancy on infant sensitization: a double-blind placebo-controlled study Clin Exp Allergy, 38(8), 1342-8

1111 Huus, K.,Ludvigsson, J. F.,Enskar, K.,Ludvigsson, J. (2008). Exclusive breastfeeding of Swedish children and its possible influence on the development of obesity: a prospective cohort study BMC Pediatr, 8(\#issue\#), 42

Publication date for a non-sibling study 
1112 Huybrechts, I.,De Vriendt, T.,Breidenassel, C.,Rogiers, J.,Vanaelst, B.,Cuenca-Garcia, M.,Moreno, L. A.,Gonzalez-Gross, M.,Roccaldo, R.,Kafatos, A.,Clays, E.,Bueno, G.,Beghin, L.,Sjostrom, M.,Manios, Y.,Molnar, D.,Pisa, P. T.,De Henauw, S. (2014). Mechanisms of stress, energy homeostasis and insulin resistance in European adolescents--the HELENA study Nutr Metab

Cardiovasc Dis, 24(10), 1082-9

1113 Hwang, J. B.,Lee, S. H.,Kang, Y. N.,Kim, S. P.,Suh, S. I.,Kam, S. (2007). Indexes of suspicion of typical cow's milk protein-induced enterocolitis J Korean Med Sci, 22(6), 993-7

1114 Hyland, F. (1988). Breastfeeding: for those who won't Community Outlook, \#volume\#(\#issue\#), 11-2

Participant health,

intervention/exposure

1115 Hysing, M.,Harvey, A. G.,Torgersen, L.,Ystrom, E.,Reichborn-Kjennerud, T.,Sivertsen, B. (2014). Trajectories and predictors of Study design nocturnal awakenings and sleep duration in infants J Dev Behav Pediatr, 35(5), 309-16

1116 lacono, G.,Merolla, R.,D'Amico, D.,Bonci, E.,Cavataio, F.,Di Prima, L.,Scalici, C.,Indinnimeo, L.,Averna, M. R.,Carroccio, A. (2005). Gastrointestinal symptoms in infancy: a population-based prospective study Dig Liver Dis, 37(6), 432-8

1117 Iannotti, L. L.,Zavaleta, N.,León, Z.,Caulfield, E. L. (2009). Growth and body composition of Peruvian infants in a peri urban setting Intervention/exposure Food and Nutrition Bulletin, 30(3), 245-253

1118 Imai, C. M.,Gunnarsdottir, I.,Thorisdottir, B.,Halldorsson, T. I.,Thorsdottir, I. (2014). Associations between infant feeding practice prior to six months and body mass index at six years of age Nutrients, 6(4), 1608-17

1119 Inamo, Y.,Hasegawa, M.,Saito, K.,Hayashi, R.,Ishikawa, T.,Yoshino, Y.,Hashimoto, K.,Fuchigami, T. (2011). Serum vitamin D concentrations and associated severity of acute lower respiratory tract infections in Japanese hospitalized children Pediatr Int, $53(2)$ 199-201

1120 Inanç, B. B.,Şahin, D. S.,Oğuzüncül, A. F.,Bindak, R.,Mungan, F. (2012). Prevalence of obesity in elementary schools in mardin, south-eastern of turkey: A preliminary study Balkan Medical Journal, 29(4), 424-430

1121 Infante-Rivard, C. (1993). Childhood asthma and indoor environmental risk factors Am J Epidemiol, 137(8), 834-44

Intervention/exposure, Size of study groups

Study design, Size of study groups

Outcome

Intervention/exposure, Outcome

Study design

Outcome

1122 Infante-Rivard, C.,Amre, D.,Gautrin, D.,Malo, J. L. (2001). Family size, day-care attendance, and breastfeeding in relation to the Outcome incidence of childhood asthma Am J Epidemiol, 153(7), 653-8

1123 Infante-Rivard, C.,Fortier, I.,Olson, E. (2000). Markers of infection, breast-feeding and childhood acute lymphoblastic leukaemia Br J Outcome Cancer, 83(11), 1559-64

1124 Innis, S. M. (1992). Human milk and formula fatty acids J Pediatr, 120(4 Pt 2), S56-61

Study design

1125 Innis, S. M.,Akrabawi, S. S.,Diersen-Schade, D. A.,Dobson, M. V.,Guy, D. G. (1997). Visual acuity and blood lipids in term infants fed human milk or formulae Lipids, 32(1), 63-72

1126 Innis, S. M.,Auestad, N.,Siegman, J. S. (1996). Blood lipid docosahexaenoic and arachidonic acid in term gestation infants fed formulas with high docosahexaenoic acid, low eicosapentaenoic acid fish oil Lipids, 31(6), 617-25 
1127 Innis, S. M.,Diersen-Schade, D. A.,Akrabawi, S. S. (1995). Prospective evaluation of preferential looking acuity in healthy term infants fed infant formula or breast fed Pediatric research, 37(4), $308 \mathrm{a}$

1128 Innis, S. M.,Friesen, R. W. (2007). Maternal DHA supplementation in pregnancy: a double blind randomized prospective trial of maternal N-3 fatty acid status, human milk fatty acids and infant development Pediatric Academic Societies Annual Meeting; 2007 May 5-8; Toronto, Canada, \#volume\#(\#issue\#), \#Pages\#

1129 Innis, S. M.,Nelson, C. M.,Lwanga, D.,Rioux, F. M.,Waslen, P. (1996). Feeding formula without arachidonic acid and docosahexaenoic acid has no effect on preferential looking acuity or recognition memory in healthy full-term infants at 9 mo of age Am J Clin Nutr, 64(1), 40-6

1130 Innis, S. M.,Nelson, C. M.,Rioux, M. F.,King, D. J. (1994). Development of visual acuity in relation to plasma and erythrocyte omega- Intervention/exposure 6 and omega-3 fatty acids in healthy term gestation infants Am J Clin Nutr, 60(3), 347-52

1131 Inostroza, J.,Haschke, F.,Steenhout, P.,Grathwohl, D.,Nelson, S. E.,Ziegler, E. E. (2014). Low-protein formula slows weight gain in Intervention/exposure infants of overweight mothers J Pediatr Gastroenterol Nutr, 59(1), 70-7

1132 Iron-Segev, S.,Peterson, K. E.,Gillman, M. W.,Williams, C. M.,Austin, S. B.,Field, A. E. (2013). Associations of breastfeeding with Outcome bulimic behaviors and eating disorders among adolescents Int J Eat Disord, 46(8), 834-40

1133 Isaacs, C. E.,Jia, J. H. (2004). The anti-infective activity of human milk is potentially greater than the sum of its microbicidal components Adv Exp Med Biol, 554(\#issue\#), 439-41

1134 Isaacs, E. B.,Fischl, B. R.,Quinn, B. T.,Chong, W. K.,Gadian, D. G.,Lucas, A. (2010). Impact of breast milk on intelligence quotient, Participant health brain size, and white matter development Pediatr Res, 67(4), 357-62

1135 Islam, M. A.,Rahman, M. M.,Mahalanabis, D. (1994). Maternal and socioeconomic factors and the risk of severe malnutrition in a Country child: a case-control study Eur J Clin Nutr, 48(6), 416-24

1136 Islam, M. A.,Rahman, M. M.,Mahalanabis, D.,Rahman, A. K. (1996). Death in a diarrhoeal cohort of infants and young children soon Country after discharge from hospital: risk factors and causes by verbal autopsy J Trop Pediatr, 42(6), 342-7

1137 Isolauri, E. (2005). Nutrition, allergy, mucosal immunology and intestinal microbiota: the effects of maternal nutrition during pregnancy and breast feeding on the risk of allergic disease ClinicalTrials.gov [http://clinicaltrials.gov], \#volume\#(\#issue\#), \#Pages\#

1138 Isomura, H., Takimoto, H.,Miura, F.,Kitazawa, S., Takeuchi, T.,Itabashi, K.,Kato, N. (2011). Type of milk feeding affects hematological Outcome parameters and serum lipid profile in Japanese infants Pediatr Int, 53(6), 807-13

1139 Ito, J.,Fujiwara, T. (2014). Breastfeeding and risk of atopic dermatitis up to the age 42 months: a birth cohort study in Japan Ann Intervention/exposure Epidemiol, 24(4), 267-72

1140 Ivakhnenko, O. S.,Nyankovskyy, S. L. (2013). Effect of the specific infant formula mixture of oligosaccharides on local immunity and development of allergic and infectious disease in young children: Randomized study Pediatria Polska, 88(5), 398-404

1141 Ivanovic, D.,Ivanovic, R.,Buitron, C. (1987). Nutritional status, birth weight and breast feeding of elementary first grade Chilean students Nutrition Reports International, 36(6), 1347-1361

Outcome

Study design 
1142 Ivarsson, A.,Hernell, O.,Stenlund, H.,Persson, L. A. (2002). Breast-feeding protects against celiac disease Am J Clin Nutr, 75(5), 914-21

1143 Ivarsson, A.,Persson, L. A.,Nystrom, L.,Ascher, H.,Cavell, B.,Danielsson, L.,Dannaeus, A.,Lindberg, T.,Lindquist, B.,Stenhammar, L.,Hernell, O. (2000). Epidemic of coeliac disease in Swedish children Acta Paediatr, 89(2), 165-71

1144 Izadi, V.,Kelishadi, R.,Qorbani, M.,Esmaeilmotlagh, M.,Taslimi, M.,Heshmat, R.,Ardalan, G.,Azadbakht, L. (2013). Duration of breast-feeding and cardiovascular risk factors among Iranian children and adolescents: the CASPIAN III study Nutrition, 29(5), 74451

1145 J. M, Hamid Jan,Mitra, Amal K.,H, Hasmiza,C. D, Pim,L. O, Ng,W. M, Wan Manan (2011). Effect of Gender and Nutritional Status on Academic Achievement and Cognitive Function among Primary School Children in a Rural District in Malaysia Malaysian Journal of Nutrition, 17(2), 189-200 12p

1146 Jaber, L. (2014). Preventive intervention for iron deficiency anaemia in a high risk population Int J Risk Saf Med, 26(3), 155-62

1147 Jackson, D. B.,Beaver, K. M. (2015). The Association Between Breastfeeding Exposure and Duration, Neuropsychological Deficits and Psychopathic Personality Traits in Offspring: The Moderating Role of 5HTTLPR Psychiatr Q, \#volume\#(\#issue\#), \#Pages\#

1148 Jackson, J. M.,Mourino, A. P. (1999). Pacifier use and otitis media in infants twelve months of age or younger Pediatr Dent, 21(4), 255-60

1149 Jacobson, J. L., Jacobson, S. W. (2002). Association of prenatal exposure to an environmental contaminant with intellectual function in childhood J Toxicol Clin Toxicol, 40(4), 467-75

1150 Jacobson, J. L.,Jacobson, S. W.,Muckle, G.,Kaplan-Estrin, M.,Ayotte, P.,Dewailly, E. (2008). Beneficial effects of a polyunsaturated Intervention/exposure fatty acid on infant development: evidence from the inuit of arctic Quebec J Pediatr, 152(3), 356-64

1151 Jacobson, S. W.,Chiodo, L. M.,Jacobson, J. L. (1999). Breastfeeding effects on intelligence quotient in 4- and 11-year-old children Outcome Pediatrics, 103(5), e71

1152 Jacoby, P.,Carville, K. S.,Hall, G.,Riley, T. V.,Bowman, J.,Leach, A. J.,Lehmann, D. (2011). Crowding and other strong predictors of upper respiratory tract carriage of otitis media-related bacteria in Australian Aboriginal and non-Aboriginal children Pediatr Infect Dis J, 30(6), 480-5

1153 Jaganath, D.,Saito, M.,Gilman, R. H.,Queiroz, D. M.,Rocha, G. A.,Cama, V.,Cabrera, L.,Kelleher, D.,Windle, H. J.,Crabtree, J. E.,Checkley, W. (2014). First detected Helicobacter pylori infection in infancy modifies the association between diarrheal disease and childhood growth in Peru Helicobacter, 19(4), 272-9

1154 Jain, L. (2014). Our babies are what we feed them Clin Perinatol, 41(2), xv-xvii

1155 Jain, M. K.,Vora, J. N.,Kale, V. V.,Iyyer, L.,Irani, S. F. (1984). A study of non-epidemic diarrhea in the newborns Indian Pediatr 21(1), 56-60

Outcome

Study design

Study design,

ntervention/exposure

Study design

Study design

Outcome

Study design

Size of study groups

Outcome

Intervention/exposure, Outcome

Country

Study design 
1157 Jakobsen, C.,Paerregaard, A.,Munkholm, P.,Wewer, V. (2013). Environmental factors and risk of developing paediatric inflammatory Outcome bowel disease -- a population based study 2007-2009 J Crohns Colitis, 7(1), 79-88

1158 Jalevik, B.,Noren, J. G.,Klingberg, G.,Barregard, L. (2001). Etiologic factors influencing the prevalence of demarcated opacities in Study design permanent first molars in a group of Swedish children Eur J Oral Sci, 109(4), 230-4

1159 James, J.,Evans, J.,Male, P.,Pallister, C.,Hendrikz, J. K.,Oakhill, A. (1988). Iron deficiency in inner city pre-school children: development of a general practice screening programme J R Coll Gen Pract, 38(311), 250-2

1160 James, M. (1986). Child's nutritional needs: nature's wonderful formula Nurs J India, 77(7), 180-1, 196

Study design

Study design

1161 Jamieson EC,Abbasi KA,Cockburn F,Farquharson J,Logan RW,Patrick WA (1994). Effect of diet on term infant cerebral cortex fatty acid composition World Rev Nutr Diet, 75(\#issue\#), 139-41

Participant health, Size of study groups

1162 Janevic, T.,Petrovic, O.,Bjelic, I.,Kubera, A. (2010). Risk factors for childhood malnutrition in Roma settlements in Serbia BMC Public Health, 10(\#issue\#), 509

1163 Janowitz, B.,Nichols, D. J. (1983). Child survivorship and pregnancy spacing in Iran J Biosoc Sci, 15(1), 35-46

Study design

Outcome

1164 Jansen, A. A. (1982). Malnutrition and child feeding practices in the Kingdom of Tonga J Trop Pediatr, 28(4), 202-8

Study design

1165 Jansen, H.,Huiting, H. G.,Scholtens, S.,Sauer, P. J.,Stolk, R. P. (2011). HbA1c in nondiabetic Dutch infants aged 8-12 months: the GECKO-Drenthe birth cohort study Diabetes Care, 34(2), 403-5

1166 Jansen, M. A.,Tromp,, II,Kiefte-de Jong, J. C.,Jaddoe, V. W.,Hofman, A.,Escher, J. C.,Hooijkaas, H.,Moll, H. A. (2014). Infant feeding and anti-tissue transglutaminase antibody concentrations in the Generation R Study Am J Clin Nutr, 100(4), 1095-101

1167 Jarvisalo, M. J.,Hutri-Kahonen, N.,Juonala, M.,Mikkila, V.,Rasanen, L.,Lehtimaki, T.,Viikari, J.,Raitakari, O. T. (2009). Breast feeding Intervention/exposure in infancy and arterial endothelial function later in life. The Cardiovascular Risk in Young Finns Study Eur J Clin Nutr, 63(5), 640-5

1168 Javed, A.,Yoo, K. H.,Agarwal, K.,Jacobson, R. M.,Li, X.,Juhn, Y. J. (2013). Characteristics of children with asthma who achieved remission of asthma J Asthma, 50(5), 472-9

1169 Jazar, A. S.,Takruri, H. R.,Khuri-Bulos, N. A. (2011). Vitamin D status in a sample of preschool children aged from 1 to 6 years visiting the pediatrics clinic at Jordan University Hospital Jordan Medical Journal, 45(4), 308-316

1170 Jedrychowski, W.,Maugeri, U.,Perera, F.,Stigter, L.,Jankowski, J.,Butscher, M.,Mroz, E.,Flak, E.,Skarupa, A.,Sowa, A. (2011). Cognitive function of 6-year old children exposed to mold-contaminated homes in early postnatal period. Prospective birth cohort study in Poland Physiol Behav, 104(5), 989-95

1171 Jedrychowski, W.,Perera, F.,Jankowski, J.,Butscher, M.,Mroz, E.,Flak, E.,Kaim, I.,Lisowska-Miszczyk, I.,Skarupa, A.,Sowa, A (2012). Effect of exclusive breastfeeding on the development of children's cognitive function in the Krakow prospective birth cohort study Eur J Pediatr, 171(1), 151-8

1172 Jeffery, A. N.,Metcalf, B. S.,Hosking, J.,Murphy, M. J.,Voss, L. D.,Wilkin, T. J. (2006). Little evidence for early programming of weight and insulin resistance for contemporary children: EarlyBird Diabetes Study report 19 Pediatrics, 118(3), 1118-23

Participant health

Study design

Intervention/exposure

Study design

Outcome

Intervention/exposure

Publication date for a non-sibling study 
1173 Jelding-Dannemand, E.,Malby Schoos, A. M.,Bisgaard, H. (2015). Breast-feeding does not protect against allergic sensitization in early childhood and allergy-associated disease at age 7 years J Allergy Clin Immunol, 136(5), 1302-1308 e13

1174 Jelliffe DB (1986). Recent developments in breastfeeding Med J Malaysia, 41(\#issue\#), 59-63

Study design

1175 Jelliffee, E. F. (1986). Breastfeeding and the prevention of malnutrition Med J Malaysia, 41(1), 88-92

Study design

1176 Jenkins, A. L.,Gyorkos, T. W.,Joseph, L.,Culman, K. N.,Ward, B. J.,Pekeles, G. S.,Mills, E. L. (2004). Risk factors for hospitalization Size of study groups and infection in Canadian Inuit infants over the first year of life--a pilot study Int J Circumpolar Health, 63(1), 61-70

1177 Jenkins, J. M.,Foster, E. M. (2014). The effects of breastfeeding exclusivity on early childhood outcomes Am J Public Health, 104 Suppl 1(\#issue\#), S128-35

1178 Jensen, B. H.,Röser, D.,Andreassen, B. U.,Olsen, K. E. P.,Nielsen, H. V.,Roldgaard, B. B.,Schjørring, S.,Mirsepasi-Lauridsen, H. C.,Jørgensen, S. L.,Mortensen, E. M.,Petersen, A. M.,Krogfelt, K. A. (2015). Childhood diarrhoea in Danish day care centres could be associated with infant colic, low birthweight and antibiotics Acta Paediatrica, International Journal of Paediatrics, \#volume\#(\#issue\#), \#Pages\#

1179 Jensen, C. L.,Chen, H.,Fraley, J. K.,Anderson, R. E.,Heird, W. C. (1996). Biochemical effects of dietary linoleic/alpha-linolenic acid Intervention/exposure ratio in term infants Lipids, 31(1), 107-13

1180 Jensen, C. L.,Prager, T. C.,Fraley, J. K.,Chen, H.,Anderson, R. E.,Heird, W. C. (1997). Effect of dietary linoleic/alpha-linolenic acid Intervention/exposure ratio on growth and visual function of term infants J Pediatr, 131(2), 200-9

1181 Jensen, C. L.,Prager, T. C.,Zou, Y.,Fraley, J. K.,Maude, M.,Anderson, R. E.,Heird, W. C. (1999). Effects of maternal docosahexaenoic acid supplementation on visual function and growth of breast-fed term infants Lipids, 34 Suppl(\#issue\#), S225

1182 Jensen, E. T.,Kappelman, M. D.,Kim, H. P.,Ringel-Kulka, T.,Dellon, E. S. (2013). Early life exposures as risk factors for pediatric eosinophilic esophagitis J Pediatr Gastroenterol Nutr, 57(1), 67-71

1183 Jensen, S. M.,Ritz, C.,Ejlerskov, K. T.,Molgaard, C.,Michaelsen, K. F. (2015). Infant BMI peak, breastfeeding, and body composition Outcome at age 3 y Am J Clin Nutr, 101(2), 319-25

1184 Jensen, T. K.,Grandjean, P.,Jorgensen, E. B.,White, R. F.,Debes, F.,Weihe, P. (2005). Effects of breast feeding on neuropsychological development in a community with methylmercury exposure from seafood J Expo Anal Environ Epidemiol, 15(5), 423-30

1185 Jeris, L. S.,Thies, P. A. (1980). Infant feeding practices and dental health. Part 1: the biological specificity of human milk Bull Mich Dent Hyg Assoc, 10(3), 9-10

1186 Jiang, M.,Foster, E. M.,Gibson-Davis, C. M. (2011). Breastfeeding and the child cognitive outcomes: a propensity score matching approach Matern Child Health J, 15(8), 1296-307

1187 Jin, C.,MacKay Rossignol, A. (1993). Effects of passive smoking on respiratory illness from birth to age eighteen months, in Shanghai, People's Republic of China Journal of Pediatrics, 123(4), 553-558 
1188 Jin, H. J.,Lee, J. H.,Kim, M. K. (2013). The prevalence of vitamin D deficiency in iron-deficient and normal children under the age of 24 months Blood Research, 48(1), 40-45

1189 Jing, H.,Gilchrist, J. M.,Badger, T. M.,Pivik, R. T. (2010). A longitudinal study of differences in electroencephalographic activity among breastfed, milk formula-fed, and soy formula-fed infants during the first year of life Early Hum Dev, 86(2), 119-25

1190 Jing, H.,Pivik, R. T.,Dykman, R. A.,Gilchrist, J. M.,Badger, T. M. (2007). Effects of breast milk and milk formula diets on synthesized Size of study groups speech sound-induced event-related potentials in 3- and 6-month-old infants Dev Neuropsychol, 31(3), 349-62

1191 Jing, H.,Xu, H.,Wan, J.,Yang, Y.,Ding, H.,Chen, M.,Li, L.,Lv, P.,Hu, J.,Yang, J. (2014). Effect of breastfeeding on childhood BMI and Study design obesity: the China Family Panel Studies Medicine (Baltimore), 93(10), e55

1192 Jochum, F.,Fuchs, A.,Cser, A.,Menzel, H.,Lombeck, I. (1995). Trace mineral status of full-term infants fed human milk, milk-based Outcome formula or partially hydrolysed whey protein formula Analyst, 120(3), 905-9

1193 Johansson, C.,Samuelsson, U.,Ludvigsson, J. (1994). A high weight gain early in life is associated with an increased risk of type 1 (insulin-dependent) diabetes mellitus Diabetologia, 37(1), 91-4

1194 Johnsen, D. C. (1982). Characteristics and backgrounds of children with "nursing caries" Pediatr Dent, 4(3), 218-24

Publication date for a non-sibling study

Study design

Intervention/exposure

1195 Johnsen, D. C.,Gerstenmaier, J. H.,DiSantis, T. A.,Berkowitz, R. J. (1986). Susceptibility of nursing-caries children to future approximal molar decay Pediatr Dent, 8(3), 168-70

1196 Johnsen, D. C.,Gerstenmaier, J. H.,Schwartz, E.,Michal, B. C.,Parrish, S. (1984). Background comparisons of pre-31/2-year-old children with nursing caries in four practice settings Pediatr Dent, 6(1), 50-4

Study design

1197 Johnson, C. A.,Lieberman, B.,Hassanein, R. E. (1985). The relationship of breast feeding to third-day bilirubin levels J Fam Pract, 20(2), 147-52

1198 Johnson, C. C.,Ownby, D. R.,Alford, S. H.,Havstad, S. L.,Williams, L. K.,Zoratti, E. M.,Peterson, E. L.,Joseph, C. L. (2005). Antibiotic Outcome exposure in early infancy and risk for childhood atopy J Allergy Clin Immunol, 115(6), 1218-24

1199 Johnson, D. L.,Swank, P. R.,Howie, V. M.,Baldwin, C. D.,Owen, M. (1996). Breast feeding and children's intelligence Psychol Rep, Outcome 79(3 Pt 2), 1179-85 1200 Johnson, L.,van Jaarsveld, C. H.,Llewellyn, C. H.,Cole, T. J.,Wardle, J. (2014). Associations between infant feeding and the size,
tempo and velocity of infant weight gain: SITAR analysis of the Gemini twin birth cohort Int J Obes (Lond), 38(7), 980-7

1201 Johnston, B. D.,Huebner, C. E.,Anderson, M. L.,Tyll, L. T.,Thompson, R. S. (2006). Healthy steps in an integrated delivery system: child and parent outcomes at 30 months Arch Pediatr Adolesc Med, 160(8), 793-800

1202 Johnston, P. K. (1984). Getting enough to grow on Am J Nurs, 84(3), 336-9 
1203 Jonas, W.,Atkinson, L.,Steiner, M.,Meaney, M. J.,Wazana, A.,Fleming, A. S. (2015). Breastfeeding and maternal sensitivity predict early infant temperament Acta Paediatr, 104(7), 678-86

1204 Jones EG,Matheny RJ (1993). Relationship between infant feeding and exclusion rate from child care because of illness $\mathrm{J}$ Am Diet Assoc, 93(\#issue\#), 809-11

1205 Jones, A. (2015). INTERGENERATIONAL EDUCATIONAL ATTAINMENT, FAMILY CHARACTERISTICS AND CHILD OBESITY J Study design Biosoc Sci, \#volume\#(\#issue\#), 1-20

1206 Jones, D. (1987). Infant feeding. Breast-feeding practices Nurs Times, 83(3), 56-7

Outcome

1207 Jones, F.,Green, M. (1996). The B.C. Baby-Friendly Initiative Nurs BC, 28(5), 7-8

Study design

1208 Jones, G.,Hynes, K. L.,Dwyer, T. (2013). The association between breastfeeding, maternal smoking in utero, and birth weight with Outcome bone mass and fractures in adolescents: a 16-year longitudinal study Osteoporos Int, 24(5), 1605-11

1209 Jones, G.,Riley, M.,Dwyer, T. (2000). Breastfeeding in early life and bone mass in prepubertal children: a longitudinal study Osteoporos Int, 11(2), 146-52

1210 Jones, I. E.,Williams, S. M.,Goulding, A. (2004). Associations of birth weight and length, childhood size, and smoking with bone fractures during growth: evidence from a birth cohort study Am J Epidemiol, 159(4), 343-50

1211 Jones, M. E.,Swerdlow, A. J.,Gill, L. E.,Goldacre, M. J. (1998). Pre-natal and early life risk factors for childhood onset diabetes mellitus: a record linkage study Int J Epidemiol, 27(3), 444-9

1212 Jones, N. A.,McFall, B. A.,Diego, M. A. (2004). Patterns of brain electrical activity in infants of depressed mothers who breastfeed and bottle feed: the mediating role of infant temperament Biol Psychol, 67(1-2), 103-24 1213 Jones, S. M.,Steele, R. W. (2012). Recurrent group B streptococcal bacteremia Clin Pediatr (Phila), 51(9), 884-7

Publication date for a non-sibling study

Outcome

Intervention/exposure

Size of study groups

Study design

1214 Jones, T. F.,Ingram, L. A.,Fullerton, K. E.,Marcus, R.,Anderson, B. J.,McCarthy, P. V.,Vugia, D.,Shiferaw, B.,Haubert, N.,Wedel, S.,Angulo, F. J. (2006). A case-control study of the epidemiology of sporadic Salmonella infection in infants Pediatrics, 118(6), 2380-7

1215 Jonsdottir, O. H.,Kleinman, R. E.,Wells, J. C.,Fewtrell, M. S.,Hibberd, P. L.,Gunnlaugsson, G.,Thorsdottir, I. (2014). Exclusive breastfeeding for 4 versus 6 months and growth in early childhood Acta Paediatr, 103(1), 105-11

1216 Jonsdottir, O. H., Thorsdottir, I.,Gunnlaugsson, G.,Fewtrell, M. S.,Hibberd, P. L., Kleinman, R. E. (2013). Exclusive breastfeeding and developmental and behavioral status in early childhood Nutrients, 5(11), 4414-28

1217 Jonsdottir, O. H., Thorsdottir, I.,Hibberd, P. L.,Fewtrell, M. S.,Wells, J. C.,Palsson, G. I.,Lucas, A., Gunnlaugsson, G.,Kleinman, R. E. Intervention/exposure (2012). Timing of the introduction of complementary foods in infancy: a randomized controlled trial Pediatrics, 130(6), 1038-45

1218 Jonville-Béra, A. P.,Autret-Leca, E.,Barbeillon, F.,Paris-Llado, J. (2001). Sudden unexpected death in infants under 3 months of age Outcome and vaccination status - A case-control study British Journal of Clinical Pharmacology, 51(3), 271-276 
1219 Jonville-Bera, A. P.,Autret-Leca, E.,Barbeillon, F Paris-Llado, J. (2001). Sudden unexpected death in infants under 3 months of age Outcome and vaccination status- -a case-control study $\mathrm{Br} \mathrm{J}$ Clin Pharmacol, 51(3), 271-6

1220 Jooste, P. L.,Rossouw, L. J.,Steenkamp, H. J.,Rossouw, J. E.,Swanepoel, A. S.,Charlton, D. O. (1991). Effect of breast feeding on Country the plasma cholesterol and growth of infants J Pediatr Gastroenterol Nutr, 13(2), 139-42

1221 Jorgensen, M. H.,Hernell, O.,Lund, P.,Holmer, G.,Michaelsen, K. F. (1996). Visual acuity and erythrocyte docosahexaenoic acid status in breast-fed and formula-fed term infants during the first four months of life Lipids, 31(1), 99-105

1222 Jørgensen, M. H.,Hølmer, G.,Lund, P.,Hernell, O.,Michaelsen, K. F. (1998). Effect of formula supplemented with docosahexaenoic acid and $y$ - linolenic acid on fatty acid status and visual acuity in term infants Journal of Pediatric Gastroenterology and Nutrition, 26(4), 412-421

1223 Jorgensen, M. H.,Nielsen, P. K.,Michaelsen, K. F.,Lund, P.,Lauritzen, L. (2006). The composition of polyunsaturated fatty acids in erythrocytes of lactating mothers and their infants Matern Child Nutr, 2(1), 29-39

1224 Jourdan-Da Silva, N.,Perel, Y.,Mechinaud, F.,Plouvier, E.,Gandemer, V.,Lutz, P.,Vannier, J. P.,Lamagnere, J. L.,Margueritte, G.,Boutard, P.,Robert, A.,Armari, C.,Munzer, M.,Millot, F.,De Lumley, L.,Berthou, C.,Rialland, X.,Pautard, B.,Hemon, D.,Clavel, J. (2004). Infectious diseases in the first year of life, perinatal characteristics and childhood acute leukaemia Br J Cancer, 90(1), 13945

1225 Jovanovic, D.,llic, N.,Miljkovic-Selimovic, B.,Djokic, D.,Relic, T.,Tambur, Z.,Doder, R.,Kostic, G. (2015). Campylobacter jejuni infection and IgE sensitization in up to 2-year-old infants Vojnosanit Pregl, 72(2), 140-7

1226 Joventino, Emanuella Silva,Gomes Coutinho, Robson, de Castro Bezerra, Karine, de Almeida, Paulo CÃ@sar,Oliveira Batista Orĩ̃ MÃ'nica,Barbosa Ximenes, Lorena (2013). Self-effectiveness in preventing diarrhea and child care: a transversal study Online Brazilian Journal of Nursing, 12(2), 1-1 1p

1227 Juambeltz, J. C.,Kula, K.,Perman, J. (1993). Nursing caries and lactose intolerance ASDC J Dent Child, 60(4), 377-84

1228 Juez, G.,Diaz, S.,Casado, M. E.,Duran, E.,Salvatierra, A. M.,Peralta, O.,Croxatto, H. B. (1983). Growth pattern of selected urban Chilean infants during exclusive breast-feeding Am J Clin Nutr, 38(3), 462-8

1229 Juliusson, P. B.,Roelants, M.,Hoppenbrouwers, K.,Hauspie, R.,Bjerknes, R. (2011). Growth of Belgian and Norwegian children compared to the WHO growth standards: prevalence below -2 and above +2 SD and the effect of breastfeeding Arch Dis Child, 96(10), 916-21

1230 Julvez, J.,Guxens, M.,Carsin, A. E.,Forns, J.,Mendez, M.,Turner, M. C.,Sunyer, J. (2014). A cohort study on full breastfeeding and child neuropsychological development: the role of maternal social, psychological, and nutritional factors Dev Med Child Neurol, 56(2), $148-56$

1231 Julvez, J.,Ribas-Fito, N.,Forns, M.,Garcia-Esteban, R.,Torrent, M.,Sunyer, J. (2007). Attention behaviour and hyperactivity at age 4 and duration of breast-feeding Acta Paediatr, 96(6), 842-7

Study design,

Intervention/exposure

Study design, Size of study groups

Study design

Intervention/exposure

Study design

Outcome

Outcome 
1232 Jung, E.,Czajka-Narins, D. (1986). Comparison of growth of black and white infants during their first two years of life J Natl Med Assoc, 78(12), 1157-60

1233 Jung, E.,Czajka-Narins, D. M. (1985). Birth weight doubling and tripling times: an updated look at the effects of birth weight, sex, race and type of feeding Am J Clin Nutr, 42(2), 182-9

1234 Just, J.,Belfar, S.,Wanin, S.,Pribil, C.,Grimfeld, A.,Duru, G. (2010). Impact of innate and environmental factors on wheezing persistence during childhood J Asthma, 47(4), 412-6

1235 Juto, P.,Moller, C.,Engberg, S.,Bjorksten, B. (1982). Influence of type of feeding on lymphocyte function and development of infantile Size of study groups allergy Clin Allergy, 12(4), 409-16

1236 Juvonen, P.,Mansson, M.,Andersson, C.,Jakobsson, I. (1996). Allergy development and macromolecular absorption in infants with different feeding regimens during the first three days of life. A three-year prospective follow-up Acta Paediatr, 85(9), 1047-52

1237 Jwa, S. C.,Fujiwara, T.,Kondo, N. (2014). Latent protective effects of breastfeeding on late childhood overweight and obesity: a nationwide prospective study Obesity (Silver Spring), 22(6), 1527-37

1238 Kaatsch, P.,Kaletsch, U.,Krummenauer, F.,Meinert, R.,Miesner, A.,Haaf, G.,Michaelis, J. (1996). Case control study on childhood leukemia in Lower Saxony, Germany. Basic considerations, methodology, and summary of results Klin Padiatr, 208(4), 179-85

Size of study groups, Intervention/exposure

Intervention/exposure

1239 Kadziela-Olech, H.,Piotrowska-Jastrzebska, J. (2005). The duration of breastfeeding and attention deficit hyperactivity disorder

Study design, Intervention/exposure Rocz Akad Med Bialymst, 50(\#issue\#), 302-6

1240 Kafouri, S.,Kramer, M.,Leonard, G.,Perron, M.,Pike, B.,Richer, L.,Toro, R.,Veillette, S.,Pausova, Z.,Paus, T. (2013). Breastfeeding Study design and brain structure in adolescence Int J Epidemiol, 42(1), 150-9

1241 Kajantie, E.,Barker, D. J.,Osmond, C.,Forsen, T.,Eriksson, J. G. (2008). Growth before 2 years of age and serum lipids 60 years later: the Helsinki Birth Cohort study Int J Epidemiol, 37(2), 280-9

1242 Kajosaari, M. (1991). Atopy prophylaxis in high-risk infants. Prospective 5-year follow-up study of children with six months exclusive Publication status breastfeeding and solid food elimination Adv Exp Med Biol, 310(\#issue\#), 453-8

1243 Kajosaari, M. (1994). Atopy prevention in childhood: the role of diet. Prospective 5-year follow-up of high-risk infants with six months Intervention/exposure exclusive breastfeeding and solid food elimination Pediatr Allergy Immunol, 5(6 Suppl), 26-8

1244 Kajosaari, M.,Saarinen, U. M. (1983). Prophylaxis of atopic disease by six months' total solid food elimination. Evaluation of 135 exclusively breast-fed infants of atopic families Acta Paediatr Scand, 72(3), 411-4

1245 Kale, A.,Deardorff, J.,Lahiff, M.,Laurent, C.,Greenspan, L. C.,Hiatt, R. A.,Windham, G.,Galvez, M. P.,Biro, F. M.,Pinney, S M.,Teitelbaum, S. L.,Wolff, M. S.,Barlow, J.,Mirabedi, A.,Lasater, M.,Kushi, L. H. (2015). Breastfeeding versus formula-feeding and girls' pubertal development Matern Child Health J, 19(3), 519-27

1246 Kalies, H.,Heinrich, J.,Borte, N.,Schaaf, B.,von Berg, A.,von Kries, R.,Wichmann, H. E.,Bolte, G. (2005). The effect of breastfeeding Intervention/exposure on weight gain in infants: results of a birth cohort study Eur J Med Res, 10(1), 36-42 
1247 Kallio, M. J.,Salmenpera, L.,Siimes, M. A.,Perheentupa, J.,Miettinen, T. A. (1992). Exclusive breast-feeding and weaning: effect on serum cholesterol and lipoprotein concentrations in infants during the first year of life Pediatrics, 89(4 Pt 1), 663-6

1248 Kallio, M. J.,Salmenpera, L.,Siimes, M. A.,Perheentupa, J.,Miettinen, T. A. (1993). Tracking of serum cholesterol and lipoprotein levels from the first year of life Pediatrics, 91(5), 949-54

1249 Kalliomaki, M.,Isolauri, E. (2000). Breastfeeding and atopic sensitisation Adv Exp Med Biol, 478(\#issue\#), 389-90

Outcome

Intervention/exposure

Study design

1250 Kalliomäki, M.,Salminen, S.,Arvilommi, H. (2001). Prenatal and postnatal administration of Lactobacillus GG reduced the occurence of atopic disease in offspring Evidence-Based Medicine, 6(6), 178

1251 Kamer, B.,Raczynska, J.,Kaczmarek, J.,Lukamowicz, J.,Pasowska, R.,Puchala, B. (1995). Genetic and environmental conditions involved in assessment of the immunological state in children with atopic dermatitis Rocz Akad Med Bialymst, 40(3), 439-47

1252 Kanazawa, S. (2015). Breastfeeding is positively associated with child intelligence even net of parental IQ Dev Psychol, 51(12), 1683-9

1253 Kaplan, B. A.,Mascie-Taylor, C. G. (1985). Biosocial factors in the epidemiology of childhood asthma in a British national sample J Intervention/exposure Epidemiol Community Health, 39(2), 152-6

1254 Karademir, F.,Suleymanoglu, S.,Ersen, A.,Aydinoz, S.,Gultepe, M.,Meral, C.,Ozkaya, H.,Gocmen, I. (2007). Vitamin B12, folate homocysteine and urinary methylmalonic acid levels in infants Journal of International Medical Research, 35(3), 384-388

1255 Karaguzel, G.,Ozer, S.,Akcurin, S.,Turkkahraman, D.,Bircan, I. (2007). Type 1 diabetes-related epidemiological, clinical and laboratory findings. An evaluation with special regard to autoimmunity in children Saudi Med J, 28(4), 584-9

1256 Karakoç, G. B.,Altintaş, D. U.,Yilmaz, M.,Kendirli, S. G. (2003). Prick Skin Test Results in Children Less Than Three Years-Old Annals of Medical Sciences, 12(3), 85-88

1257 Karaolis-Danckert, N.,Buyken, A. E.,Kulig, M.,Kroke, A.,Forster, J.,Kamin, W.,Schuster, A.,Hornberg, C.,Keil, T.,Bergmann, R. L.,Wahn, U.,Lau, S. (2008). How pre- and postnatal risk factors modify the effect of rapid weight gain in infancy and early childhood on subsequent fat mass development: results from the Multicenter Allergy Study 90 Am J Clin Nutr, 87(5), 1356-64

1258 Karaolis-Danckert, N.,Buyken, A. E.,Sonntag, A.,Kroke, A. (2009). Birth and early life influences on the timing of puberty onset: results from the DONALD (DOrtmund Nutritional and Anthropometric Longitudinally Designed) Study Am J Clin Nutr, 90(6), 1559-65

1259 Karaolis-Danckert, N.,Gunther, A. L.,Kroke, A.,Hornberg, C.,Buyken, A. E. (2007). How early dietary factors modify the effect of rapid weight gain in infancy on subsequent body-composition development in term children whose birth weight was appropriate for gestational age Am J Clin Nutr, 86(6), 1700-8

1260 Karino, S.,Okuda, T.,Uehara, Y.,Toyo-oka, T. (2008). Breastfeeding and prevalence of allergic diseases in Japanese university students Ann Allergy Asthma Immunol, 101(2), 153-9

1261 Karjalainen, S.,Ronning, O.,Lapinleimu, H.,Simell, O. (1999). Association between early weaning, non-nutritive sucking habits and occlusal anomalies in 3-year-old Finnish children Int J Paediatr Dent, 9(3), 169-73

Publication status

Study design, Participant health

Outcome

Intervention/exposure

Participant health

Participant health

Intervention/exposure

Outcome

Intervention/exposure

Study design

Outcome 
1262 Kark, J. D.,Troya, G.,Friedlander, Y.,Slater, P. E.,Stein, Y. (1984). Validity of maternal reporting of breast feeding history and the association with blood lipids in 17 year olds in Jerusalem J Epidemiol Community Health, 38(3), 218-25

1263 Karmaus, W.,Dobai, A. L.,Ogbuanu, I.,Arshard, S. H.,Matthews, S.,Ewart, S. (2008). Long-term effects of breastfeeding, maternal smoking during pregnancy, and recurrent lower respiratory tract infections on asthma in children J Asthma, 45(8), 688-95

1264 Karunasekera, K. A.,Jayasinghe, J. A.,Alwis, L. W. (2001). Risk factors of childhood asthma: a Sri Lankan study J Trop Pediatr $47(3), 142-5$

1265 Kaseb, F.,Kimiagar, M.,Ghafarpoor, M.,Valaii, N. (2002). Effect of traditional food supplementation during pregnancy on maternal weight gain and birthweight Int J Vitam Nutr Res, 72(6), 389-93

1266 Kasla, R. R.,Bavdekar, S. B.,Joshi, S. Y.,Hathi, G. S. (1995). Exclusive breastfeeding: protective efficacy Indian J Pediatr, 62(4), 449-53

1267 Kass, R. B.,Meumann, F. (1985). Hospitalisation for childhood diarrhoea in Central Australia Aust Clin Rev, 5(19), 178-83

Study design, Participant health

1268 Kaste, L. M.,Marianos, D.,Chang, R.,Phipps, K. R. (2010). The assessment of nursing caries and its relationship to high caries in the Intervention/exposure permanent dentition. $1992 \mathrm{~J}$ Indiana Dent Assoc, 89(2), 20-4

1269 Katikaneni, R.,Ponnapakkam, T.,Ponnapakkam, A.,Gensure, R. (2009). Breastfeeding does not protect against urinary tract infection in the first 3 months of life, but vitamin D supplementation increases the risk by $76 \%$ Clin Pediatr (Phila), 48(7), $750-5$

1270 Kato, T.,Yorifuji, T.,Yamakawa, M.,Inoue, S.,Saito, K.,Doi, H.,Kawachi, I. (2015). Association of breast feeding with early childhood Outcome dental caries: Japanese population-based study BMJ Open, 5(3), e006982

1271 Katoku, Y.,Yamada, M.,Yonekubo, A.,Kuwata, T.,Kobayashi, A.,Sawa, A. (1996). Effect of the cholesterol content of a formula on the lipid compositions of plasma lipoproteins and red blood cell membranes in early infancy Am J Clin Nutr, 64(6), 871-7

1272 Kaufman, H. S.,Frick, O. L. (1981). Prevention of asthma Clin Allergy, 11(6), 549-53

Size of study groups

Intervention/exposure

1273 Kaur, N.,Deol, R.,Yadav, A. (2014). Correlation of feeding practices and health profile of children Nurs J India, 105(3), 128-30

1274 Kawai, T.,Goto, A.,Watanabe, E.,Nagasawa, M.,Yasumura, S. (2011). Lower respiratory tract infections and gastrointestinal infections among mature babies in Japan Pediatr Int, 53(4), 431-45

1275 Kazemi, A.,Tabatabaie, F.,Agha-Ghazvini, M. R.,Kelishadi, R. (2006). The role of rotavirus in acute pediatric diarrhea in Isfahan, Iran Pakistan Journal of Medical Sciences, 22(3), 282-285

1276 Keim, S. A.,Daniels, J. L.,Siega-Riz, A. M.,Herring, A. H.,Dole, N.,Scheidt, P. C. (2012). Breastfeeding and long-chain polyunsaturated fatty acid intake in the first 4 post-natal months and infant cognitive development: an observational study Matern Child Nutr, 8(4), 471-82

1277 Kellberger, J.,Dressel, H.,Vogelberg, C.,Leupold, W.,Windstetter, D.,Weinmayr, G.,Genuneit, J.,Heumann, C.,Nowak, D.,von Mutius, Intervention/exposure E.,Radon, K. (2012). Prediction of the incidence and persistence of allergic rhinitis in adolescence: a prospective cohort study J Allergy Clin Immunol, 129(2), 397-402, 402 e1-3 
1278 Keller, K. M.,Burgin-Wolff, A.,Lippold, R.,Wirth, S.,Lentze, M. J. (1996). The diagnostic significance of IgG cow's milk protein antibodies re-evaluated Eur J Pediatr, 155(4), 331-7

1279 Keller, K. M.,Burgin-Wolff, A.,Menger, H.,Lippold, R.,Wirth, S.,Baumann, W. (1991). IgG, IgA, and IgE antibodies to cow milk proteins in an allergy prevention study Adv Exp Med Biol, 310(\#issue\#), 467-73

1280 Kemeny, D. M.,Price, J. F.,Richardson, V.,Richards, D.,Lessof, M. H. (1991). The IgE and IgG subclass antibody response to foods Outcome in babies during the first year of life and their relationship to feeding regimen and the development of food allergy $\mathrm{J}$ Allergy Clin Immunol, 87(5), 920-9

1281 Kennedy, K.,Fewtrell, M. S.,Morley, R.,Abbott, R.,Quinlan, P. T.,Wells, J. C.,Bindels, J. G.,Lucas, A. (1999). Double-blind, randomized trial of a synthetic triacylglycerol in formula-fed term infants: effects on stool biochemistry, stool characteristics, and bone study mineralization Am J Clin Nutr, 70(5), 920-7

1282 Kerkhof, M.,Koopman, L. P.,van Strien, R. T.,Wijga, A.,Smit, H. A.,Aalberse, R. C.,Neijens, H. J.,Brunekreef, B.,Postma, D. S.,Gerritsen, J. (2003). Risk factors for atopic dermatitis in infants at high risk of allergy: the PIAMA study Clin Exp Allergy, 33(10), 1336-41

1283 Kero, P..Piekkala, P. (1987). Factors affecting the occurrence of acute otitis media during the first year of life Acta Paediatr Scand, 76(4), 618-23

1284 Kerr, A. A. (1981). Lower respiratory tract illness in Polynesian infants N Z Med J, 93(684), 333-5

Study design, Outcome

1285 Keusch, G. T. (1980). Homing in on interventions in the malnutrition-infection complex Am J Clin Nutr, 33(4), 727-9

Study design

1286 Kew, S.,Hamilton, J. K.,Ye, C.,Hanley, A. J.,Zinman, B.,Retnakaran, R. (2013). Vitamin D status and cardiometabolic assessment in infancy Pediatr Res, 74(2), 217-22

1287 Khadivzadeh, T.,Parsai, S. (2004). Effect of exclusive breastfeeding and complementary feeding on infant growth and morbidity East Mediterr Health J, 10(3), 289-94

1288 Khalili, H.,Ananthakrishnan, A. N.,Higuchi, L. M.,Richter, J. M.,Fuchs, C. S.,Chan, A. T. (2013). Early life factors and risk of inflammatory bowel disease in adulthood Inflamm Bowel Dis, 19(3), 542-7

1289 Khan, F.,Green, F. C.,Forsyth, J. S.,Greene, S. A.,Newton, D. J.,Belch, J. J. (2009). The beneficial effects of breastfeeding on microvascular function in 11- to 14-year-old children Vasc Med, 14(2), 137-42

1290 Khanjanasthiti, P.,Nanna, P.,Sawongtrakul, S. (1986). Breast feeding in early neonatal period J Med Assoc Thai, 69 Suppl 2(\#issue\#), 100-6

1291 Khanolkar, A. R.,Sovio, U.,Bartlett, J. W.,Wallby, T.,Koupil, I. (2013). Socioeconomic and early-life factors and risk of being overweight or obese in children of Swedish- and foreign-born parents Pediatr Res, 74(3), 356-63

1292 Khedr, E. M.,Farghaly, W. M.,Amry Sel, D.,Osman, A. A. (2004). Neural maturation of breastfed and formula-fed infants Acta Paediatr, 93(6), 734-8

Study design, Size of study groups

Intervention/exposure

Intervention/exposure

Intervention/exposure

Outcome

Intervention/exposure

Country, Size of study group 
1293 Kholdi, N.,Zayeri, F.,Bagheban, A. A.,Khodakarim, S.,Ramezankhani, A. (2012). A study of growth failure and its related factors in children from 0 to 2 years in Tehran, Iran Turk J Pediatr, 54(1), 38-44

1294 Kiechl-Kohlendorfer, U.,Horak, E.,Mueller, W. Strobl, R.,Haberland, C.,Fink, F. M.,Schwaiger, M. Gutenberger, K. H.,Reich, H.,Meraner, D.,Kiechl, S. (2007). Neonatal characteristics and risk of atopic asthma in schoolchildren: results from a large prospective birth-cohort study Acta Paediatr, 96(11), 1606-10

1295 Kiechl-Kohlendorfer, U.,Peglow, U. P.,Kiechl, S.,Oberaigner, W.,Sperl, W. (2001). Epidemiology of sudden infant death syndrome (SIDS) in the Tyrol before and after an intervention campaign Wien Klin Wochenschr, 113(1-2), 27-32

1296 Kieviet, N.,Hoppenbrouwers, C.,Dolman, K. M.,Berkhof, J.,Wennink, H.,Honig, A. (2015). Risk factors for poor neonatal adaptation after exposure to antidepressants in utero Acta Paediatr, 104(4), 384-91

1297 Kim, C. S.,Jung, H. W.,Yoo, K. Y. (1993). Prevalence and risk factors of chronic otitis media in Korea: results of a nation-wide Study design survey Acta Otolaryngol, 113(3), 369-75

1298 Kim, H. S.,Kim, Y. H.,Kim, M. J.,Lee, H. S.,Han, Y. K.,Kim, K. W.,Sohn, M. H.,Kim, K. E. (2015). Effect of breastfeeding on lung function in asthmatic children Allergy Asthma Proc, 36(2), 116-22

1299 Kim, I.,Pollitt, E. (1987). Differences in the pattern of weight growth of nutritionally at-risk and well-nourished infants Am J Clin Nutr, 46(1), 31-5

1300 Kim, M. J.,Na, B.,No, S. J.,Han, H. S.,Jeong, E. H.,Lee, W.,Han, Y.,Hyeun, T. (2010). Nutritional status of vitamin D and the effect of vitamin D supplementation in Korean breast-fed infants J Korean Med Sci, 25(1), 83-9

Study design

Intervention/exposure

Outcome

Study design, Participant health

Intervention/exposure

Study design, Size of study groups

Study design, Participant health

1301 Kim, S. K.,Cheong, W. S.,Jun, Y. H.,Choi, J. W.,Son, B. K. (1996). Red blood cell indices and iron status according to feeding practices in infants and young children Acta Paediatr, 85(2), 139-44

1302 Kimpimaki, T.,Erkkola, M.,Korhonen, S.,Kupila, A.,Virtanen, S. M.,Ilonen, J.,Simell, O.,Knip, M. (2001). Short-term exclusive breastfeeding predisposes young children with increased genetic risk of Type I diabetes to progressive beta-cell autoimmunity Diabetologia, 44(1), 63-9

1303 King, D. E. (2002). Statistics. Adult intelligence and breastfeeding International Journal of Childbirth Education, 17(4), 23-23 1p

Publication status

1304 Kiris, M.,Muderris, T.,Kara, T.,Bercin, S.,Cankaya, H.,Sevil, E. (2012). Prevalence and risk factors of otitis media with effusion in school children in Eastern Anatolia International Journal of Pediatric Otorhinolaryngology, 76(7), 1030-1035

1305 Kitsantas, P.,Gaffney, K. F. (2010). Risk profiles for overweight/obesity among preschoolers Early Hum Dev, 86(9), 563-8

\section{Publication date for a non-sibling} study

1306 Kjellman, N. I. (1988). Epidemiology and prevention of allergy Allergy, 43 Suppl 8(\#issue\#), 39-40

Study design

1307 Klag, E. A.,McNamara, K.,Geraghty, S. R.,Keim, S. A. (2015). Associations Between Breast Milk Feeding, Introduction of Solid Foods, and Weight Gain in the First 12 Months of Life Clin Pediatr (Phila), 54(11), 1059-67

Study design 
1308 Klein, I.,Reif, S.,Farbstein, H.,Halak, A.,Gilat, T. (1998). Preillness non dietary factors and habits in inflammatory bowel disease Ital J Gastroenterol Hepatol, 30(3), 247-51

1309 Klein, J. O. (1997). Prevention of recurrent acute otitis media Seminars in Pediatric Infectious Diseases, 8(2), 101-104

Study design

1310 Klenovics, K. S.,Boor, P.,Somoza, V.,Celec, P.,Fogliano, V.,Sebekova, K. (2013). Advanced glycation end products in infant formulas do not contribute to insulin resistance associated with their consumption PLoS One, 8(1), e53056

Study design, Size of study groups

1311 Klinnert, M. D.,Nelson, H. S.,Price, M. R.,Adinoff, A. D.,Leung, D. Y.,Mrazek, D. A. (2001). Onset and persistence of childhood asthma: predictors from infancy Pediatrics, 108(4), E69

1312 Klonoff-Cohen, H. S.,Edelstein, S. L.,Lefkowitz, E. S.,Srinivasan, I. P.,Kaegi, D.,Chang, J. C.,Wiley, K. J. (1995). The effect of passive smoking and tobacco exposure through breast milk on sudden infant death syndrome JAMA, 273(10), 795-8

1313 Knight, S. M.,Toodayan, W.,Caique, W. C.,Kyi, W.,Barnes, A.,Desmarchelier, P. (1992). Risk factors for the transmission of diarrhoea in children: a case-control study in rural Malaysia Int J Epidemiol, 21(4), 812-8

1314 Knip, M. (2003). Cow's milk and the new trials for prevention of type 1 diabetes J Endocrinol Invest, 26(3), 265-7

Outcome

1315 Knishkowy, B.,Palti, H.,Adler, B.,Tepper, D. (1991). Effect of otitis media on development: a community-based study Early Hum Dev, 26(2), 101-11

1316 Ko, Y.,Kariyawasam, V.,Karnib, M.,Butcher, R.,Samuel, D.,Alrubaie, A.,Rahme, N.,McDonald, C.,Cowlishaw, J.,Katelaris, P.,Barr, G.,Jones, B.,Connor, S.,Paven, G.,Chapman, G.,Park, G., Gearry, R.,Leong, R. W. (2015). Inflammatory Bowel Disease Environmental Risk Factors: A Population-Based Case-Control Study of Middle Eastern Migration to Australia Clin Gastroenterol Hepatol, 13(8), 1453-63 e1

1317 Koch, A.,Molbak, K.,Homoe, P.,Sorensen, P.,Hjuler, T.,Olesen, M. E.,Pejl, J.,Pedersen, F. K.,Olsen, O. R.,Melbye, M. (2003). Risk Outcome factors for acute respiratory tract infections in young Greenlandic children Am J Epidemiol, 158(4), 374-84

1318 Kocturk, T. (1988). Infant feeding pattern in three districts of Istanbul J Trop Pediatr, 34(4), 193-7

Study design, Outcome

1319 Koehoorn, M.,Karr, C. J.,Demers, P. A.,Lencar, C.,Tamburic, L.,Brauer, M. (2008). Descriptive epidemiological features of bronchiolitis in a population-based cohort Pediatrics, 122(6), 1196-203

1320 Koenig, H. F. (2014). Breastfeeding education for healthier babies. Baby-Friendly designation improves infant, mother and community health Healthc Exec, 29(4), 46, 48-9

1321 Koh, T. H. (1981). Breast feeding among the Chinese in four countries J Trop Pediatr, 27(2), 88-91

Study design, Outcome

1322 Kohler, L.,Meeuwisse, G.,Mortensson, W. (1984). Food intake and growth of infants between six and twenty-six weeks of age on breast milk, cow's milk formula, or soy formula Acta Paediatr Scand, 73(1), 40-8 1323 Kohn, G.,Sawatzki, G.,van Biervliet, J. P. (1994). Long-chain polyunsaturated fatty acids in infant nutrition Eur J Clin Nutr, 48 Suppl
2(\#issue\#), S1-7 Outcome 
1324 Kohn, G.,Sawatzki, G.,van Biervliet, J. P.,Rosseneu, M. (1994). Diet and the essential fatty acid status of term infants Acta Paediatr Suppl, 402(\#issue\#), 69-74

Publication date for a non-sibling study

1325 Kolacek, S.,Kapetanovic, T.,Luzar, V. (1993). Early determinants of cardiovascular risk factors in adults. B. Blood pressure Acta Paediatr, 82(4), 377-82

1326 Kolacek, S.,Kapetanovic, T.,Zimolo, A.,Luzar, V. (1993). Early determinants of cardiovascular risk factors in adults. A. Plasma lipids Size of study groups Acta Paediatr, 82(8), 699-704

1327 Koletzko S (2015). 2.5 Allergy Prevention through Early Nutrition World Rev Nutr Diet, 113(\#issue\#), 113-7

Publication status

1328 Koletzko, B. (2015). 2.2 Formula feeding World Rev Nutr Diet, 113(\#issue\#), 97-103

Study design

1329 Koletzko, B.,Beyer, J.,Brands, B.,Demmelmair, H.,Grote, V.,Haile, G.,Gruszfeld, D.,Rzehak, P.,Socha, P.,Weber, M. (2013). Early Publication status influences of nutrition on postnatal growth Nestle Nutr Inst Workshop Ser, 71(\#issue\#), 11-27

1330 Koletzko, B.,Grote, V.,Schiess, S.,Verwied-Jorky, S.,Brands, B.,Demmelmair, H.,Kries, R. (2010). Prevention of pediatric obesity Language through baby nutrition. [German] Monatsschrift fur Kinderheilkunde, 158(6), 553-63

1331 Koletzko, B.,Schiess, S.,Brands, B.,Haile, G.,Demmelmair, H.,Kries, R.,Grote, V. (2010). [Infant feeding practice and later obesity risk. Indications for early metabolic programming] Bundesgesundheitsblatt, Gesundheitsforschung, Gesundheitsschutz, 53(7), 66673

Language

1332 Koletzko, B.,Toschke, A. M.,Vignerova, J.,Osancova, K., Von Kries, R. (2003). Does breast feeding protect against later overweight Publication status and obesity? Cesko-Slovenska Pediatrie, 58(1), 3-9

1333 Koletzko, B.,von Kries, R. (2002). Are there long term protective effects of breast feeding against later obesity? Pediatria Wspolczesna, 4(3), $217-223$

1334 Koletzko, B.,Von Kries, R.,Closa, R.,Escribano, J.,Scaglioni, S.,Giovannini, M.,Beyer, J.,Demmelmair, H.,Gruszfeld, D.,Dobrzanska, Intervention/exposure A.,Sengier, A.,Langhendries, J. P.,Cachera, M. F. R.,Grote, V. (2009). Lower protein in infant formula is associated with lower weight up to age 2 y: A randomized clinical trial American Journal of Clinical Nutrition, 89(6), 1836-1845

1335 Koletzko, B.,von, K. R.,Closa, R.,Escribano, J.,Scaglioni, S.,Giovannini, M.,Beyer, J.,Demmelmair, H.,Anton, B.,Gruszfeld, D.,Dobrzanska, A.,Sengier, A.,Langhendries, J. P.,Rolland Cachera, M. F.,Grote, V. (2009). Can infant feeding choices modulate later obesity risk? American journal of clinical nutrition, 89(5), 1502s-1508s

1336 Koletzko, S.,Griffiths, A.,Corey, M.,Smith, C.,Sherman, P. (1991). Infant feeding practices and ulcerative colitis in childhood BMJ 302(6792), 1580-1 $1337 \begin{aligned} & \text { Koletzko, S.,Sherman, P.,Corey, M.,Griffiths, A.,Smith, C. (1989). Role of infant feeding practices in development of Crohn's disease Outcome } \\ & \text { in childhood BMJ, 298(6688), 1617-8 }\end{aligned}$

1338 Koloski, N. A.,Jones, M.,Weltman, M.,Kalantar, J.,Bone, C.,Gowryshankar, A.,Walker, M. M.,Talley, N. J. (2015). Identification of early environmental risk factors for irritable bowel syndrome and dyspepsia Neurogastroenterol Motil, 27(9), $1317-25$ 
1340 Kosse, F. (2016). The Nutritional and Social Environment-Related Effects of Breastfeeding on Intelligence JAMA Pediatr, 170(2), Study design, Outcome $173-4$

1341 Kost, N. V.,Sokolov, O. Y.,Kurasova, O. B.,Dmitriev, A. D.,Tarakanova, J. N.,Gabaeva, M. V.,Zolotarev, Y. A.,Dadayan, A. Study design, K.,Grachev, S. A.,Korneeva, E. V.,Mikheeva, I. G.,Zozulya, A. A. (2009). Beta-casomorphins-7 in infants on different type of feeding and different levels of psychomotor development Peptides, 30(10), 1854-60

1342 Koster, E. S.,Van der Ent, C. K.,Uiterwaal, C. S.,Verheij, T. J.,Raaijmakers, J. A.,Maitland-van der Zee, A. H. (2011). Asthma medication use in infancy: determinants related to prescription of drug therapy Fam Pract, 28(4), 377-84

1343 Kostraba, J. N.,Cruickshanks, K. J.,Lawler-Heavner, J.,Jobim, L. F.,Rewers, M. J., Gay, E. C., Chase, H. P.,Klingensmith, G.,Hamman, R. F. (1993). Early exposure to cow's milk and solid foods in infancy, genetic predisposition, and risk of IDDM Diabetes, 42(2), 288-95 risk of IDDM in blacks and whites. A matched case-control study Diabetes Care, 15(5), 626-31

1345 Krabbendam, L.,Bakker, E.,Hornstra, G.,van Os, J. (2007). Relationship between DHA status at birth and child problem behaviour at Outcome 7 years of age Prostaglandins Leukot Essent Fatty Acids, 76(1), 29-34

1346 Kramer, M. S. (1981). Do breast-feeding and delayed introduction of solid foods protect against subsequent obesity? J Pediatr, 98(6), 883-7

1347 Kramer, M. S. (1988). Infant feeding, infection, and public health Pediatrics, 81(1), 164-6

Publication date for a non-sibling study

Study design

1348 Kramer, M. S. (2010). "Breast is best": The evidence Early Hum Dev, 86(11), 729-32

Publication date for a non-sibling study

1349 Kramer, M. S.,Aboud, F.,Mironova, E.,Vanilovich, I.,Platt, R. W.,Matush, L., Igumnov, S.,Fombonne, E.,Bogdanovich, N.,Ducruet, T.,Collet, J. P.,Chalmers, B.,Hodnett, E.,Davidovsky, S.,Skugarevsky, O.,Trofimovich, O.,Kozlova, L.,Shapiro, S. (2008).

Breastfeeding and child cognitive development: new evidence from a large randomized trial Arch Gen Psychiatry, 65(5), 578-84

Outcome

1350 Kramer, M. S.,Barr, R. G.,Leduc, D. G.,Boisjoly, C.,McVey-White, L.,Pless, I. B. (1985). Determinants of weight and adiposity in the first year of life J Pediatr, 106(1), 10-4

1351 Kramer, M. S.,Barr, R. G.,Pless, I. B. (1986). Determinants of weight and adiposity in early childhood Canadian Journal of Public Health, 77(SUPPL. 1), 98-103

1352 Kramer, M. S.,Chalmers, B.,Hodnett, E. D.,Sevkovskaya, Z.,Dzikovich, I.,Shapiro, S.,Collet, J. P.,Vanilovich, I.,Mezen, I.,Ducruet, T.,Shishko, G.,Zubovich, V.,Mknuik, D.,Gluchanina, E.,Dombrovskiy, V.,Ustinovitch, A., Kot, T.,Bogdanovich, N.,Ovchinikova, L.,Helsing, E. (2001). Promotion of breastfeeding intervation trial (PROBIT): A randomized trial in the Republic of Belarus Journal of the American Medical Association, 285(4), 413-420

Publication date for a non-sibling study

Publication date for a non-sibling study

Outcome 
1353 Kramer, M. S.,Fombonne, E.,Igumnov, S.,Vanilovich, I.,Matush, L.,Mironova, E.,Bogdanovich, N.,Tremblay, R. E.,Chalmers, B.,Zhang, X.,Platt, R. W. (2008). Effects of prolonged and exclusive breastfeeding on child behavior and maternal adjustment: evidence from a large, randomized trial Pediatrics, 121(3), e435-40

1354 Kramer, M. S.,Fombonne, E.,Matush, L.,Bogdanovich, N.,Dahhou, M.,Platt, R. W. (2011). Long-term behavioural consequences of infant feeding: the limits of observational studies Paediatr Perinat Epidemiol, 25(6), 500-6

1355 Kramer, M. S.,Guo, T.,Platt, R. W.,Sevkovskaya, Z.,Dzikovich, I.,Collet, J. P.,Shapiro, S.,Chalmers, B.,Hodnett, E.,Vanilovich, I.,Mezen, I.,Ducruet, T.,Shishko, G.,Bogdanovich, N. (2003). Infant growth and health outcomes associated with 3 compared with 6 mo of exclusive breastfeeding Am J Clin Nutr, 78(2), 291-5

1356 Kramer, M. S.,Guo, T.,Platt, R. W.,Shapiro, S.,Collet, J. P.,Chalmers, B.,Hodnett, E.,Sevkovskaya, Z.,Dzikovich, I., Vanilovich, I. (2002). Breastfeeding and infant growth: biology or bias? Pediatrics, $110(2 \mathrm{Pt} 1), 343-7$

Outcome

Publication date for a non-sibling study

Outcome, Publication date for non-sibling study

1357 Kramer, M. S.,Guo, T.,Platt, R. W.,Vanilovich, I.,Sevkovskaya, Z.,Dzikovich, I.,Michaelsen, K. F.,Dewey, K. (2004). Feeding effects Intervention/exposure on growth during infancy J Pediatr, 145(5), 600-5

1358 Kramer, M. S.,Martin, R. M.,Bogdanovich, N.,Vilchuk, K.,Dahhou, M.,Oken, E. (2014). Is restricted fetal growth associated with later Intervention/exposure adiposity? Observational analysis of a randomized trial Am J Clin Nutr, 100(1), 176-81

1359 Kramer, M. S.,Matush, L.,Aboud, F.,Vanilovich, I.,Bogdanovich, N.,Mironova, E. (2007). Long-term child health effects of breastfeeding in developed countries: new evidence from the PROBIT trial [abstract] Journal of human lactation, 23(1), 90

Study design

1360 Kramer, M. S.,Matush, L.,Bogdanovich, N.,Aboud, F.,Mazer, B.,Fombonne, E.,Collet, J. P.,Hodnett, E.,Mironova, E.,Igumnov, S.,Chalmers, B.,Dahhou, M.,Platt, R. W. (2009). Health and development outcomes in 6.5-y-old children breastfed exclusively for 3 or 6 mo Am J Clin Nutr, 90(4), 1070-4

1361 Kramer, M. S.,Matush, L.,Bogdanovich, N.,Dahhou, M.,Platt, R. W.,Mazer, B. (2009). The low prevalence of allergic disease in Eastern Europe: are risk factors consistent with the hygiene hypothesis? Clin Exp Allergy, 39(5), 708-16

1362 Kramer, M. S.,Matush, L.,Vanilovich, I.,Platt, R. W.,Bogdanovich, N.,Sevkovskaya, Z.,Dzikovich, I.,Shishko, G.,Collet, J. P.,Martin, R. M.,Davey Smith, G.,Gillman, M. W.,Chalmers, B.,Hodnett, E.,Shapiro, S. (2007). Effects of prolonged and exclusive breastfeeding on child height, weight, adiposity, and blood pressure at age $6.5 \mathrm{y}$ : evidence from a large randomized trial Am J Clin Nutr, 86(6), 1717-21

1363 Kramer, M. S.,Matush, L.,Vanilovich, I.,Platt, R. W.,Bogdanovich, N.,Sevkovskaya, Z.,Dzikovich, I.,Shishko, G.,Collet, J. P.,Martin, R. Study design M.,Smith, G. D.,Gillman, M. W.,Chalmers, B.,Hodnett, E.,Shapiro, S. (2009). A randomized breast-feeding promotion intervention did not reduce child obesity in Belarus J Nutr, 139(2), 417S-21S

1364 Kramer, M. S.,Matush, L.,Vanilovich, I.,Platt, R.,Bogdanovich, N.,Sevkovskaya, Z.,Dzikovich, I.,Shishko, G.,Mazer, B. (2007). Effect Outcome of prolonged and exclusive breast feeding on risk of allergy and asthma: cluster randomised trial BMJ, 335(7624), 815

1365 Kramer, M. S.,Moodie, E. E.,Dahhou, M.,Platt, R. W. (2011). Breastfeeding and infant size: evidence of reverse causality Am J

Epidemiol, 173(9), 978-83

Publication date for a non-sibling study

Intervention/exposure

Publication date for a non-sibling study

Intervention/exposure 
1366 Kramer, M. S.,Moodie, E. E.,Platt, R. W. (2012). Infant feeding and growth: can we answer the causal question? Epidemiology 23(6), $790-4$

1367 Kramer, M. S.,Moroz, B. (1981). Do breast-feeding and delayed introduction of solid foods protect against subsequent atopic eczema? J Pediatr, 98(4), 546-50

1368 Kramer, M. S.,Vanilovich, I.,Matush, L.,Bogdanovich, N.,Zhang, X.,Shishko, G.,Muller-Bolla, M.,Platt, R. W. (2007). The effect of prolonged and exclusive breast-feeding on dental caries in early school-age children. New evidence from a large randomized trial Caries Res, 41(6), 484-8

1369 Kramer, M.,Matush, L.,Vanilovich, I.,Platt, R.,Mazer, B. (2006). Does breastfeeding help prevent asthma and allergy? Evidence from Publication status a randomized trial in Belarus American journal of epidemiology, 163(Suppl 11), S85

1370 Kramer,,M, S.,Matush,,L,,Vanilovich,,I,,Platt,,R, W.,Bogdanovich,,N,,Sevkovskaya,,Z,,Dzikovich,,I,,Shishko,,G,,Collet,,J, P.,Martin,,R, Duplicate M.,Davey, Smith,G,,Gillman,,M, W.,Chalmers,,B,,Hodnett,,E,,Shapiro,,S, (2007). Effects of prolonged and exclusive breastfeeding on child height, weight, adiposity, and blood pressure at age $6.5 \mathrm{y}$ : evidence from a large randomized trial Am J Clin Nutr, 86(6), 1717-21

1371 Kraus, J. F.,Greenland, S.,Bulterys, M. (1989). Risk factors for sudden infant death syndrome in the US Collaborative Perinata Project Int J Epidemiol, 18(1), 113-20

1372 Kravetz, R. E. (2003). Infant nursing bottle Am J Gastroenterol, 98(7), 1640

1373 Krebs, N. F.,Hambidge, K. M.,Westcott, J. E.,Miller, L. V.,Sian, L.,Bell, M.,Grunwald, G. (2003). Exchangeable zinc pool size in infants is related to key variables of zinc homeostasis J Nutr, 133(5 Suppl 1), 1498S-501S milk J Pediatr, 124(1), 32-9

1375 Krebs, N. F.,Reidinger, C.,Westcott, J.,Miller, L. V.,Fennessey, P. V.,Hambidge, K. M. (1994). Whole body zinc metabolism in fullterm breasted and formula fed infants Adv Exp Med Biol, 352(\#issue\#), 223-6

Outcome

Krebs, N. F.,Westcott, J. E.,Culbertson, D. L.,Sian, L.,Miller, L. V.,Hambidge, K. M. (2012). Comparison of complementary feeding strategies to meet zinc requirements of older breastfed infants Am J Clin Nutr, 96(1), 30-5

1377 Krenz-Niedbala, M.,Puch, E. A.,Koscinski, K. (2011). Season of birth and subsequent body size: the potential role of prenatal vitamin D Am J Hum Biol, 23(2), 190-200

1378 Krishna, L. M. (1980). Breast feeding and development Public Health, 94(1), 21-4

1379 Kristiansen, A. L.,Laugsand Lillegaard, I. T.,Frost Andersen, L. (2013). Effect of changes in a food frequency questionnaire: comparing data from two national dietary survey instruments among 12-month-old infants BMC Public Health, 13(\#issue\#), 680

Study design, Outcome

Study design, Size of study groups

Intervention/exposure

Study design, Size of study groups

Intervention/exposure

Study design

Study design

Study design,

Intervention/exposure

1380 Krous, H. F.,Chadwick, A. E.,Stanley, C. (2005). Delayed infant death following catastrophic deterioration during breast-feeding J Paediatr Child Health, 41(4), 215-7 
1381 Kucukcongar A,Oguz A,Pinarli FG,Karadeniz C,Okur A,Kaya Z,Celik B (2015). Breastfeeding and Childhood Cancer: Is Breastfeeding Preventative to Childhood Cancer? Pediatr Hematol Oncol, 32(\#issue\#), 374-81

1382 Küçükçongar, A.,Ołuz, A.,Pinarli, F. G.,Karadeniz, C.,Okur, A.,Kaya, Z.,Çelik, B. (2015). Breastfeeding and Childhood Cancer: Is Breastfeeding Preventative to Childhood Cancer? Pediatric Hematology and Oncology, 32(6), 374-381

1383 Kucur, C.,Simsek, E.,Kuduban, O.,Ozbay, I. (2015). Prevalence of and risk factors for otitis media with effusion in primary school children: case control study in Erzurum, Turkey Turk J Pediatr, 57(3), 230-5

1384 Kuhn, T.,Kroke, A.,Remer, T.,Schonau, E.,Buyken, A. E. (2014). Is breastfeeding related to bone properties? A longitudinal analysis Intervention/exposure of associations between breastfeeding duration and pQCT parameters in children and adolescents Matern Child Nutr, 10(4), 642-9

1385 Kuhnisch, J.,Mach, D.,Thiering, E.,Brockow, I.,Hoffmann, U.,Neumann, C.,Heinrich-Weltzien, R.,Bauer, C. P.,Berdel, D.,von Berg, Outcome A.,Koletzko, S.,Garcia-Godoy, F.,Hickel, R.,Heinrich, J. (2014). Respiratory diseases are associated with molar-incisor hypomineralizations Swiss Dent J, 124(3), 286-93

1386 Kuiper, S.,Muris, J. W.,Dompeling, E.,Kester, A. D.,Wesseling, G.,Knottnerus, J. A.,van Schayck, C. P. (2007). Interactive effect of Outcome family history and environmental factors on respiratory tract-related morbidity in infancy J Allergy Clin Immunol, 120(2), 388-95

1387 Kukkonen, A. K.,Savilahti, E. M.,Haahtela, T.,Savilahti, E.,Kuitunen, M. (2011). Ovalbumin-specific immunoglobulins A and G levels Intervention/exposure at age 2 years are associated with the occurrence of atopic disorders Clin Exp Allergy, 41(10), 1414-21

1388 Kull, I.,Almqvist, C.,Lilja, G.,Pershagen, G.,Wickman, M. (2004). Breast-feeding reduces the risk of asthma during the first 4 years of Outcome life J Allergy Clin Immunol, 114(4), 755-60

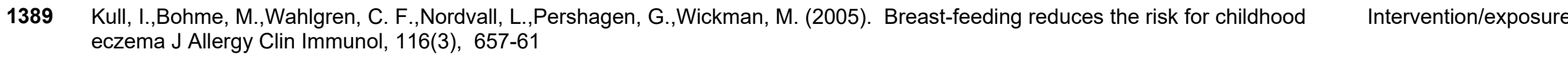

1390 Kull, I.,Melen, E.,Alm, J.,Hallberg, J.,Svartengren, M.,van Hage, M.,Pershagen, G.,Wickman, M.,Bergstrom, A. (2010). Breastfeeding in relation to asthma, lung function, and sensitization in young schoolchildren J Allergy Clin Immunol, 125(5), 1013-9

Intervention/exposure

1391 Kull, I.,Wickman, M.,Lilja, G.,Nordvall, S. L.,Pershagen, G. (2002). Breast feeding and allergic diseases in infants-a prospective birth Outcome cohort study Arch Dis Child, 87(6), 478-81

1392 Kumar, A. (1985). Breast feeding versus bottle feeding J Indian Med Assoc, 83(10), 365-6

Study design

1393 Kumar, V.,Sharma, S.,Khanna, P.,Vanaja, K. (1981). Breast vs bottle feeding-impact on growth in urban infants Indian J Pediatr 48(392), 271-5

1394 Kumari, S.,Jain, P.,Arora, U.,Pruthi, R. K. (1982). Growth of breast fed infants. A longitudinal study Indian Pediatr, 19(12), 963-8 Country

Country

1395 Kumari, S.,Pruthi, P. K.,Mehra, R.,Sehgal, H. (1985). Breast feeding: physical growth during infancy Indian J Pediatr, 52(414), 73-7

Country

1396 Kuperberg, K.,Evers, S. (2006). Feeding patterns and weight among First Nations children Can J Diet Pract Res, 67(2), 79-84 Intervention/exposure

1397 Kupers, L. K.,L'Abee, C.,Bocca, G.,Stolk, R. P.,Sauer, P. J.,Corpeleijn, E. (2015). Determinants of Weight Gain during the First Two Intervention/exposure Years of Life--The GECKO Drenthe Birth Cohort PLoS One, 10(7), e0133326 


\begin{tabular}{|c|c|c|}
\hline 1398 & Kuriakose, J. R. (2010). Nutritional status and feeding practices of infants Nurs J India, 101(8), $184-6$ & Country \\
\hline 1399 & $\begin{array}{l}\text { Kurugol, Z.,Coker, M.,Coker, C.,Egemen, A.,Ersoz, B. (1997). Comparison of growth, serum prealbumin, transferrin, IgG and amino } \\
\text { acids of term infants fed breast mild or formula Turk J Pediatr, 39(2), 195-202 }\end{array}$ & Size of study groups \\
\hline 1400 & $\begin{array}{l}\text { Kurugol, Z.,Geylani, S.,Karaca, Y.,Umay, F.,Erensoy, S.,Vardar, F.,Bak, M.,Yaprak, I.,Ozkinay, F.,Ozkinay, C. (2003). Rotavirus } \\
\text { gastroenteritis among children under five years of age in Izmir, Turkey Turk J Pediatr, 45(4), 290-4 }\end{array}$ & $\begin{array}{l}\text { Participant health, } \\
\text { Intervention/exposure }\end{array}$ \\
\hline 1401 & $\begin{array}{l}\text { Kurugöl, Z.,Geylani, S.,Karaca, Y.,Umay, F.,Erensoy, S.,Vardar, F.,Bak, M.,Yaprak, I.,Özkinay, F.,Özkinay, C. (2003). Rotavirus } \\
\text { gastroenteritis among children under five years of age in Izmir, Turkey Turkish Journal of Pediatrics, 45(4), 290-294 }\end{array}$ & $\begin{array}{l}\text { Study design, } \\
\text { Intervention/exposure }\end{array}$ \\
\hline 1402 & $\begin{array}{l}\text { Kurukulaaratchy, R. J.,Matthews, S.,Arshad, S. H. (2006). Relationship between childhood atopy and wheeze: what mediates } \\
\text { wheezing in atopic phenotypes? Ann Allergy Asthma Immunol, 97(1), 84-91 }\end{array}$ & Intervention/exposure \\
\hline 1403 & $\begin{array}{l}\text { Kurzewski, K.,Gardner, J. M. (2005). Breastfeeding patterns among six-week-old term infants at the University Hospital of the West } \\
\text { Indies West Indian Med J, 54(1), 28-33 }\end{array}$ & Study design \\
\hline 1404 & Kusel, M. M.,Holt, P. G., de Klerk, N.,Sly, P. D. (2005). Support for 2 variants of eczema J Allergy Clin Immunol, 116(5), 1067-72 & Outcome \\
\hline 1405 & $\begin{array}{l}\text { Kusunoki, T.,Morimoto, T.,Nishikomori, R.,Yasumi, T.,Heike, T.,Mukaida, K.,Fujii, T.,Nakahata, T. (2010). Breastfeeding and the } \\
\text { prevalence of allergic diseases in schoolchildren: Does reverse causation matter? Pediatric Allergy and Immunology, } 21(1 \text { PART I), } \\
60-66\end{array}$ & Study design \\
\hline 1406 & $\begin{array}{l}\text { Kuyucu, S.,Saraclar, Y.,Tuncer, A.,Sackesen, C.,Adalioglu, G.,Sumbuloglu, V.,Sekerel, B. E. (2004). Determinants of atopic } \\
\text { sensitization in Turkish school children: effects of pre- and post-natal events and maternal atopy Pediatr Allergy Immunol, 15(1), 62- } \\
71\end{array}$ & Study design \\
\hline 1407 & $\begin{array}{l}\text { Kvaavik, E.,Tell, G. S.,Klepp, K. I. (2005). Surveys of Norwegian youth indicated that breast feeding reduced subsequent risk of } \\
\text { obesity J Clin Epidemiol, 58(8), 849-55 }\end{array}$ & $\begin{array}{l}\text { Publication date for a non-sibling } \\
\text { study }\end{array}$ \\
\hline 1408 & $\begin{array}{l}\text { Kwan, M. L.,Buffler, P. A.,Wiemels, J. L., Metayer, C.,Selvin, S.,Ducore, J. M.,Block, G. (2005). Breastfeeding patterns and risk of } \\
\text { childhood acute lymphoblastic leukaemia Br J Cancer, 93(3), 379-84 }\end{array}$ & Outcome \\
\hline 1409 & $\begin{array}{l}\text { Kwok, M. K.,Leung, G. M.,Schooling, C. M. (2013). Breast feeding and early adolescent behaviour, self-esteem and depression: } \\
\text { Hong Kong's 'Children of 1997' birth cohort Arch Dis Child, 98(11), 887-94 }\end{array}$ & Outcome \\
\hline 1410 & $\begin{array}{l}\text { Kwok, M. K.,Leung, G. M.,Schooling, C. M. (2013). Breastfeeding and adolescent blood pressure: evidence from Hong Kong's } \\
\text { "Children of 1997" Birth Cohort Am J Epidemiol, 178(6), 928-36 }\end{array}$ & Outcome \\
\hline 1411 & $\begin{array}{l}\text { Kwok, M. K.,Schooling, C. M.,Lam, T. H.,Leung, G. M. (2010). Does breastfeeding protect against childhood overweight? Hong } \\
\text { Kong's 'Children of 1997' birth cohort Int J Epidemiol, 39(1), 297-305 }\end{array}$ & $\begin{array}{l}\text { Publication date for a non-sibling } \\
\text { study }\end{array}$ \\
\hline 1412 & $\begin{array}{l}\text { Kyvik, K. O.,Green, A.,Svendsen, A.,Mortensen, K. (1992). Breast feeding and the development of type } 1 \text { diabetes mellitus Diabet } \\
\text { Med, } 9(3), 233-5\end{array}$ & Outcome \\
\hline
\end{tabular}


1413 Labayen, I.,Ortega, F. B.,Ruiz, J. R.,Rodriguez, G.,Jiménez-Pavón, D.,España-Romero, V.,Widhalm, K.,Gottrand, F.,Moreno, L. A. (2015). Breastfeeding attenuates the effect of low birthweight on abdominal adiposity in adolescents: The HELENA study Maternal and Child Nutrition, 11(4), 1036-1040

1414 Labayen, I.,Ruiz, J. R.,Ortega, F. B.,Loit, H. M.,Harro, J.,Villa, I.,Veidebaum, T.,Sjostrom, M. (2012). Exclusive breastfeeding duration and cardiorespiratory fitness in children and adolescents Am J Clin Nutr, 95(2), 498-505

1415 Labbok, M. H. (1985). Consequences of breast-feeding for mother and child J Biosoc Sci Suppl, 9(\#issue\#), 43-54

Study design

Study design

1416 Ladd GA (1986). Merlin's molars Cal, 49(\#issue\#), 14-5, 31

Study design

1417 Laditan, A. A. (1983). Bilateral genu vara in childhood Cent Afr J Med, 29(11), 219-23

Country, Outcome

1418 Ladomenou, F.,Kafatos, A., Galanakis, E. (2009). Environmental tobacco smoke exposure as a risk factor for infections in infancy Acta Paediatr, 98(7), 1137-41

1419 Ladomenou, F.,Kafatos, A., Tselentis, Y.,Galanakis, E. (2010). Predisposing factors for acute otitis media in infancy J Infect, 61(1), Outcome $49-53$ infections during infancy: a prospective study Arch Dis Child, 95(12), 1004-8

1421 Lakhani SA,Chaudhri T,Jansen AA (1983). Human milk and milk formulas for infant feeding East Afr Med J, 60(\#issue\#), 181-5

1422 Lakshman, R.,Whittle, F.,Hardeman, W.,Suhrcke, M.,Wilson, E.,Griffin, S.,Ong, K. K. (2015). Effectiveness of a behavioural intervention to prevent excessive weight gain during infancy (The Baby Milk Trial): study protocol for a randomised controlled trial Trials, 16(1), 442

1423 Lamb, M. M.,Dabelea, D.,Yin, X.,Ogden, L. G.,Klingensmith, G. J.,Rewers, M.,Norris, J. M. (2010). Early-life predictors of higher body mass index in healthy children Ann Nutr Metab, 56(1), 16-22

1424 Lamb, M. M.,Simpson, M. D.,Seifert, J.,Scott, F. W.,Rewers, M.,Norris, J. M. (2013). The association between IgG4 antibodies to dietary factors, islet autoimmunity and type 1 diabetes: the Diabetes Autoimmunity Study in the Young PLoS One, 8(2), e57936

1425 Lamichhane, A. P.,Crandell, J. L.,Jaacks, L. M.,Couch, S. C.,Lawrence, J. M.,Mayer-Davis, E. J. (2015). Longitudinal associations of nutritional factors with glycated hemoglobin in youth with type 1 diabetes: the SEARCH Nutrition Ancillary Study Am J Clin Nutr, 101(6), 1278-85

1426 Lanari, M.,Adorni, F.,Silvestri, M.,Coscia, A.,Musicco, M. (2011). The multicenter Italian birth cohort study on incidence and determinants of lower respiratory tract infection hospitalization in infants at 33 weeks GA or more: preliminary results Early Hum Dev, 87 Suppl 1(\#issue\#), S43-6

1427 Lanari, M.,Prinelli, F.,Adorni, F.,Di Santo, S.,Faldella, G.,Silvestri, M.,Musicco, M. (2013). Maternal milk protects infants against bronchiolitis during the first year of life. Results from an Italian cohort of newborns Early Hum Dev, 89 Suppl 1(\#issue\#), S51-7

Outcome

Study design

Study design

Intervention/exposure

Publication date for a non-sibling study

Size of study groups, Outcome

Participant health, Outcomes

Intervention/exposure

Outcome 
1428 Lanari, M. Prinelli, F. Adorni, F. Di Santo, S. Vandini, S. Silvestri, M. Musicco, M. (2015). Risk factors for bronchiolitis hospitalization during the first year of life in a multicenter Italian birth cohort Ital J Pediatr, 41(\#issue\#), 40

1429 Lancashire, R. J.,Sorahan, T. (2003). Breastfeeding and childhood cancer risks: OSCC data Br J Cancer, 88(7), 1035-7

Outcome

Outcome

1430 Landaas, S.,Skrede, S.,Steen, J. A. (1981). The levels of serum enzymes, plasma proteins and lipids in normal infants and small children J Clin Chem Clin Biochem, 19(10), 1075-80

1431 Lande, B.,Andersen, L. F.,Henriksen, T.,Baerug, A.,Johansson, L., Trygg, K. U.,Bjorneboe, G. E.,Veierod, M. B. (2005). Relations between high ponderal index at birth, feeding practices and body mass index in infancy Eur J Clin Nutr, 59(11), 1241-9

Study design

1432 Lane, B. J.,Sellen, V. (1986). Bottle caries: a nursing responsibility Can J Public Health, 77(2), 128-30

Publication date for a non-sibling study

Study design

1433 Lane, D. M.,McConathy, W. J. (1986). Changes in the serum lipids and apolipoproteins in the first four weeks of life Pediatr Res, 20(4), 332-7

1434 Langeland, T. (1983). A clinical and immunological study of allergy to hen's egg white. I. A clinical study of egg allergy Clin Allergy, 13(4), 371-82

1435 Langman, M. J. (1986). Can epidemiology help us prevent celiac disease? Gastroenterology, 90(2), 489-91

Size of study groups

ntervention/exposure, Outcome

Study design

1436 Langnase, K.,Mast, M.,Danielzik, S.,Spethmann, C.,Muller, M. J. (2003). Socioeconomic gradients in body weight of German

Publication date for a non-sibling children reverse direction between the ages of 2 and 6 years $J$ Nutr, 133(3), 789-96 study

1437 Lanting, C. I.,Fidler, V.,Huisman, M.,Touwen, B. C.,Boersma, E. R. (1994). Neurological differences between 9-year-old children fed Intervention/exposure breast-milk or formula-milk as babies Lancet, 344(8933), 1319-22

1438 Lanting, C. I.,Patandin, S.,Weisglas-Kuperus, N.,Touwen, B. C.,Boersma, E. R. (1998). Breastfeeding and neurological outcome at Outcome 42 months Acta Paediatr, 87(12), 1224-9

1439 Laohaviranit L (1985). Milk and health J Med Assoc Thai, 68(\#issue\#), 326-9

1440 Lapillonne, A.,Brossard, N.,Claris, O.,Reygrobellet, B.,Salle, B. L. (2000). Erythrocyte fatty acid composition in term infants fed human milk or a formula enriched with a low eicosapentanoic acid fish oil for 4 months Eur J Pediatr, 159(1-2), 49-53

1441 Lapinleimu, H.,Vukari, J.,Nunikoski, H.,Tuominen, J.,Ronnemaa, T.,Valimaki, I.,Marniemi, J.,Jokinen, E.,Ehnholm, C.,Simell, O (1997). Impact of gender, apolipoprotein E phenotypes, and diet on serum lipids and lipoproteins in infancy J Pediatr, 131(6), 82532

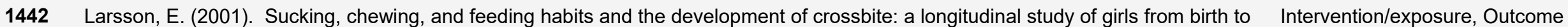
3 years of age Angle Orthod, 71(2), 116-9

1443 Larsson, J.,Aurelius, G.,Nordberg, L.,Rydelius, P.,Zetterström, R. (1999). The role of cumulative observations in identifying children Outcome in need of health promotion...including commentary by Glascoe FP Ambulatory Child Health, 5(3), 209-217 9p

1444 Larsson, M.,Hagerhed-Engman, L.,Sigsgaard, T.,Janson, S.,Sundell, J.,Bornehag, C. G. (2008). Incidence rates of asthma, rhinitis Outcome and eczema symptoms and influential factors in young children in Sweden Acta Paediatr, 97(9), 1210-5 
1445 Lasekan, J. B.,Ostrom, K. M.,Jacobs, J. R.,Blatter, M. M.,Ndife, L. I.,Gooch, lii W. M.,Cho, S. (1999). Growth of newborn, term infants fed soy formulas for 1 year Clinical Pediatrics, 38(10), 563-571

Publication date for a non-sibling study

1446 Laskey, M. A.,de Bono, S.,Smith, E. C.,Prentice, A. (2007). Influence of birth weight and early diet on peripheral bone in premenopausal Cambridge women: a pQCT study J Musculoskelet Neuronal Interact, 7(1), 83

1447 Lau, Y. L.,Karlberg, J.,Yeung, C. Y. (1995). Prevalence of and factors associated with childhood asthma in Hong Kong Acta Paediatr, 84(7), 820-2

1448 Laubereau, B.,Brockow, I.,Zirngibl, A.,Koletzko, S.,Gruebl, A.,von Berg, A.,Filipiak-Pittroff, B.,Berdel, D.,Bauer, C. P.,Reinhardt, D.,Heinrich, J.,Wichmann, H. E. (2004). Effect of breast-feeding on the development of atopic dermatitis during the first 3 years of life--results from the GINI-birth cohort study J Pediatr, 144(5), 602-7

1449 Lauer, J. A.,Betran, A. P.,Barros, A. J.,de Onis, M. (2006). Deaths and years of life lost due to suboptimal breast-feeding among children in the developing world: a global ecological risk assessment Public Health Nutr, 9(6), 673-85

1450 Lauritzen, L.,Jorgensen, M. H.,Mikkelsen, T. B.,Skovgaard I, M.,Straarup, E. M.,Olsen, S. F.,Hoy, C. E.,Michaelsen, K. F. (2004) Maternal fish oil supplementation in lactation: effect on visual acuity and n-3 fatty acid content of infant erythrocytes Lipids, 39(3), 195-206

1451 Lauver, M. A.,Hizon, L.,Bulla, A.,Connell, C.,Wagoner, B. (1981). Infant feeding practices: the effect on six month weight J Kans Med Soc, 82(9), 403-6

1452 Lauzon-Guillain, Bd,Wijndaele, K.,Clark, M.,Acerini, C. L.,Hughes, I. A.,Dunger, D. B.,Wells, J. C.,Ong, K. K. (2012). Breastfeeding Study design and infant temperament at age three months PLoS One, 7(1), e29326

1453 Lawlor, D. A.,Najman, J. M.,Batty, G. D.,O'Callaghan, M. J.,Williams, G. M.,Bor, W. (2006). Early life predictors of childhood intelligence: findings from the Mater-University study of pregnancy and its outcomes Paediatr Perinat Epidemiol, 20(2), 148-62

Outcome

1454 Lawlor, D. A.,Najman, J. M.,Sterne, J.,Williams, G. M.,Ebrahim, S.,Davey Smith, G. (2004). Associations of parental, birth, and early Intervention/exposure life characteristics with systolic blood pressure at 5 years of age: findings from the Mater-University study of pregnancy and its outcomes Circulation, 110(16), 2417-23

1455 Lawlor, D. A.,Riddoch, C. J.,Page, A. S.,Andersen, L. B.,Wedderkopp, N.,Harro, M.,Stansbie, D.,Smith, G. D. (2005). Infant feeding and components of the metabolic syndrome: findings from the European Youth Heart Study Arch Dis Child, 90(6), 582-8

Study design

Study design

Intervention/exposure

Country, Study design

Intervention/exposure

Size of study groups

1456 Lawrence, R. A. (1991). Breast-feeding trends: a cause for action Pediatrics, 88(4), 867-8 Study design

Study design,

Intervention/exposure

1457 Lawrence, R. A. (1992). Can we expect greater intelligence from human milk feedings? Birth, 19(2), 105-6

Study design

1458 Lawrence, R. A. (2001). Promotion of Breastfeeding Intervention Trial (PROBIT) a randomized trial in the Republic of Belarus J Pediatr, 139(1), 164-5

1459 Lazerov, J.,Ervin, C. (2011). Promoting breastfeeding: breastfeeding and population health Breastfeed Med, 6(\#issue\#), 305-6 


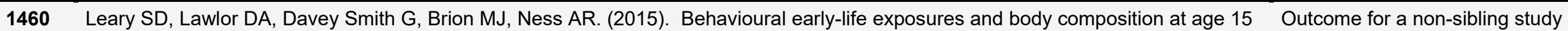
years Nutrition and Diabetes, 5(2), e150

1461 Lee, B. (1995). Breastfeeding J R Soc Med, 88(9), 537p-538p

Study design

1462 Lee, H. A.,Kim, Y. J.,Lee, H.,Gwak, H. S.,Hong, Y. S.,Kim, H. S.,Park, E. A.,Cho, S. J.,Ha, E. H.,Park, H. (2015). The preventive effect of breast-feeding for longer than 6 months on early pubertal development among children aged 7-9 years in Korea Public Health Nutr, \#volume\#(\#issue\#), 1-8

1463 Lee, L. C.,Pratt, C. A.,DeLaski-Smith, D.,Karabenick, S. A. (1999). The growth patterns of American-born Chinese infants Nutrition Research, 19(5), 697-708

1464 Leeson, C. P.,Kattenhorn, M.,Deanfield, J. E.,Lucas, A. (2001). Duration of breast feeding and arterial distensibility in early adult life: Study design population based study BMJ, 322(7287), 643-7

1465 Legovic, M.,Ostric, L. (1991). The effects of feeding methods on the growth of the jaws in infants ASDC J Dent Child, 58(3), 253-5 Study design

1466 Lemons PK,Kochanczyk M,Lemons JA (1980). Breast-feeding the newborn J Indiana State Med Assoc, 73(\#issue\#), 373-8

Study design

1467 Lenguerrand, E.,Harding, S. (2010). P46 Ethnic differences in pace of growth between birth and 5 years: results from the millennium Publication status cohort study Journal of Epidemiology \& Community Health, 64(\#issue\#), A51-A51 1p

1468 Leonard, W. R.,Dewalt, K. M.,Stansbury, J. P.,McCaston, M. K. (2000). Influence of dietary quality on the growth of highland and Outcome coastal Ecuadorian children Am J Hum Biol, 12(6), 825-837

1469 Lerman, Y.,Slepon, R.,Cohen, D. (1994). Epidemiology of acute diarrheal diseases in children in a high standard of living rura settlement in Israel Pediatr Infect Dis J, 13(2), 116-22

1470 Leung, E. Y.,Au, K. Y.,Cheng, S. S.,Kok, S. Y.,Lui, H. K.,Wong, W. C. (2006). Practice of breastfeeding and factors that affect breastfeeding in Hong Kong Hong Kong Med J, 12(6), 432-6

1471 Leung, G. M.,Lam, T. H.,Ho, L. M.,Lau, Y. L. (2005). Health consequences of breast-feeding: doctors' visits and hospitalizations Outcome during the first 18 months of life in Hong Kong Chinese infants Epidemiology, 16(3), 328-35

1472 Leung, J. Y.,Kwok, M. K.,Leung, G. M.,Schooling, C. M. (2015). Breastfeeding and childhood hospitalizations for asthma and other Outcome wheezing disorders Ann Epidemiol, \#volume\#(\#issue\#), \#Pages\#

1473 Leung, S. S. F.,Davies, D. P.,Lui, S.,Lo, L.,Yuen, P.,Swaminathan, R. (1988). Iron deficiency is uncommon in healthy Hong Kong infants at 18 months Journal of Tropical Pediatrics, 34(3), 100-103

1474 Leung, S. S.,Peng, C. X.,Xu, Y. Y.,Liu, K. M.,Quan, X. J.,Lui, S.,Davies, D. P. (1994). Comparative study of growth of Chinese infants: Hong Kong versus Guangzhou J Trop Pediatr, 40(3), 166-71

1475 Leung, S.,Davies, D. P. (1994). Infant feeding and growth of Chinese infants: birth to 2 years Paediatr Perinat Epidemiol, 8(3), 301- Intervention/exposure 13 
1476 Leventakou, V.,Roumeliotaki, T.,Koutra, K.,Vassilaki, M.,Mantzouranis, E.,Bitsios, P.,Kogevinas, M.,Chatzi, L. (2015). Breastfeeding Outcome duration and cognitive, language and motor development at 18 months of age: Rhea mother-child cohort in Crete, Greece $\mathrm{J}$ Epidemiol Community Health, 69(3), 232-9

1477 Leventhal, J. M.,Shapiro, E. D.,Aten, C. B.,Berg, A. T.,Egerter, S. A. (1986). Does breast-feeding protect against infections in infants Outcome less than 3 months of age? Pediatrics, 78(5), 896-903

1478 Lever, R. (2001). The role of food in atopic eczema J Am Acad Dermatol, 45(1 Suppl), S57-60

Study design

1479 Levine, O. S.,Farley, M.,Harrison, L. H.,Lefkowitz, L.,McGeer, A.,Schwartz, B. (1999). Risk factors for invasive pneumococcal disease in children: a population-based case-control study in North America Pediatrics, 103(3), E28

Study design,

Intervention/exposure

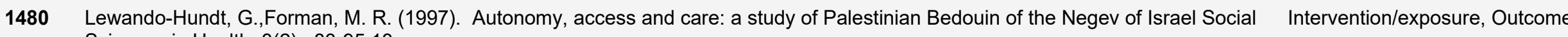
Sciences in Health, 3(2), 83-95 13p

1481 Lewis, J. K. Anderson M. Willeitner A. (2011). Powdered Versus Liquid Human Milk Fortifier: A Blinded, Randomized, Controlled Trial Pediatric Academic Societies Annual Meeting, \#volume\#(\#issue\#), \#Pages\#

1482 Lewis, S.,Butland, B.,Strachan, D.,Bynner, J.,Richards, D.,Butler, N.,Britton, J. (1996). Study of the aetiology of wheezing illness at age 16 in two national British birth cohorts Thorax, 51(7), 670-6

1483 L'Hoir, M. P.,Engelberts, A. C.,van Well, G. T.,Damste, P. H.,Idema, N. K.,Westers, P.,Mellenbergh, G. J.,Wolters, W. H.,Huber, J. $\quad$ Outcome (1999). Dummy use, thumb sucking, mouth breathing and cot death Eur J Pediatr, 158(11), 896-901

1484 I'Hoir, M. P.,Engelberts, A. C.,van Well, G. T.,Westers, P.,Mellenbergh, G. J.,Wolters, W. H.,Huber, J. (1998). Case-control study of Outcome current validity of previously described risk factors for SIDS in The Netherlands Arch Dis Child, 79(5), 386-93

1485 Li, C.,Goran, M. I.,Kaur, H.,Nollen, N.,Ahluwalia, J. S. (2007). Developmental trajectories of overweight during childhood: role of early life factors Obesity (Silver Spring), 15(3), 760-71

Publication date for a non-sibling study

1486 Li, C.,Kaur, H.,Choi, W. S.,Huang, T. T.,Lee, R. E.,Ahluwalia, J. S. (2005). Additive interactions of maternal prepregnancy BMI and breast-feeding on childhood overweight Obes Res, 13(2), 362-71

1487 Li, J.,Dykman, R. A.,Jing, H.,Gilchrist, J. M.,Badger, T. M.,Pivik, R. T. (2010). Cortical responses to speech sounds in 3- and 6month-old infants fed breast milk, milk formula, or soy formula Dev Neuropsychol, 35(6), 762-84

1488 Li, L.,Kleinman, K.,Gillman, M. W. (2014). A comparison of confounding adjustment methods with an application to early life determinants of childhood obesity J Dev Orig Health Dis, 5(6), 435-47

$1489 \mathrm{Li}$, L.,Manor, O.,Power, C. (2004). Early environment and child-to-adult growth trajectories in the 1958 British birth cohort Am J Clin Nutr, 80(1), 185-92

1490 Li, L.,Power, C. (2004). Influences on childhood height: comparing two generations in the 1958 British birth cohort Int J Epidemiol, 33(6), 1320-8

Publication date for a non-sibling study

Outcome

Study design

Intervention/exposure

Intervention/exposure

Intervention/exposure 
1491 Li, R.,Dee, D.,Li, C. M.,Hoffman, H. J.,Grummer-Strawn, L. M. (2014). Breastfeeding and risk of infections at 6 years Pediatrics, 134 Outcome Suppl 1(\#issue\#), S13-20

1492 Li, R.,Fein, S. B.,Grummer-Strawn, L. M. (2008). Association of breastfeeding intensity and bottle-emptying behaviors at early

Outcome infancy with infants' risk for excess weight at late infancy Pediatrics, 122 Suppl 2(\#issue\#), S77-84

1493 Li, R.,Fein, S. B.,Grummer-Strawn, L. M. (2010). Do infants fed from bottles lack self-regulation of milk intake compared with directly Outcome breastfed infants? Pediatrics, 125(6), e1386-93

1494 Li, R.,Magadia, J.,Fein, S. B.,Grummer-Strawn, L. M. (2012). Risk of bottle-feeding for rapid weight gain during the first year of life Outcome Arch Pediatr Adolesc Med, 166(5), 431-6

1495 Li, S. C.,Kuo, S. C.,Hsu, Y. Y.,Lin, S. J.,Chen, P. C.,Chen, Y. C. (2010). Effect of Breastfeeding Duration on Infant Growth Until 18 Months of Age: A National Birth Cohort Study Journal of Experimental and Clinical Medicine, 2(4), 165-172

1496 Li, Y.,Navia, J. M.,Caufield, P. W. (1994). Colonization by mutans streptococci in the mouths of 3-and 4-year-old Chinese children Study design with or without enamel hypoplasia Arch Oral Biol, 39(12), 1057-62

1497 Liao, S. L.,Lai, S. H.,Yeh, K. W.,Huang, Y. L.,Yao, T. C.,Tsai, M. H.,Hua, M. C.,Huang, J. L. (2014). Exclusive breastfeeding is associated with reduced cow's milk sensitization in early childhood Pediatr Allergy Immunol, 25(5), 456-61

1498 Libraty, D. H.,Capeding, R. Z.,Obcena, A.,Brion, J. D.,Tallo, V. (2013). Breastfeeding During Early Infancy is Associated with a Lower Incidence of Febrile IIInesses Open Pediatr Med Journal, 7(\#issue\#), 40-41

1499 Liebrechts-Akkerman, G.,Lao, O.,Liu, F.,Van Sleuwen, B. E.,Engelberts, A. C.,L'Hoir, M. P.,Tiemeier, H. W.,Kayser, M. (2011). Postnatal parental smoking: An important risk factor for SIDS European Journal of Pediatrics, 170(10), 1281-1291

1500 Lima, A. A.,Moore, S. R.,Barboza, M. S., Jr.,Soares, A. M.,Schleupner, M. A.,Newman, R. D.,Sears, C. L.,Nataro, J. P.,Fedorko, D. P.,Wuhib, T.,Schorling, J. B.,Guerrant, R. L. (2000). Persistent diarrhea signals a critical period of increased diarrhea burdens and nutritional shortfalls: a prospective cohort study among children in northeastern Brazil J Infect Dis, 181(5), 1643-51

1501 Lin, H.,Sun, L.,Lin, J.,He, J.,Deng, A.,Kang, M.,Zeng, H.,Ma, W.,Zhang, Y. (2014). Protective effect of exclusive breastfeeding against hand, foot and mouth disease BMC Infect Dis, 14(\#issue\#), 645

1502 Lind, J. N.,Li, R.,Perrine, C. G.,Schieve, L. A. (2014). Breastfeeding and later psychosocial development of children at 6 years of Outcome age Pediatrics, 134 Suppl 1(\#issue\#), S36-41

1503 Lindberg, S. M.,Adams, A. K.,Prince, R. J. (2012). Early predictors of obesity and cardiovascular risk among American Indian children Matern Child Health J, 16(9), 1879-86

1504 Lindenberg, C. S.,Artola, R. C.,Estrada, V. J. (1990). Determinants of early infant weaning: a multivariate approach Int J Nurs Stud, Country $27(1), 35-41$

1505 Lindfors, A. T.,Danielsson, L.,Enocksson, E.,Johansson, S. G.,Westin, S. (1992). Allergic symptoms up to 4-6 years of age in children given cow milk neonatally. A prospective study Allergy, 47(3), 207-11

Outcome

Study design, Outcome

Intervention/exposure

Intervention/exposure 
1507 Linhares Rda, S.,Gigante, D. P.,de Barros, F. C.,Horta, B. L. (2015). Carotid intima-media thickness at age 30, birth weight, accelerated growth during infancy and breastfeeding: a birth cohort study in Southern Brazil PLoS One, 10(1), e0115166

Intervention/exposure

1508 Linhares, A. C.,Gabbay, Y. B.,Freitas, R. B.,da Rosa, E. S.,Mascarenhas, J. D.,Loureiro, E. C. (1989). Longitudinal study of

Outcome rotavirus infections among children from Belem, Brazil Epidemiol Infect, 102(1), 129-45

1509 Linneberg, A.,Simonsen, J. B.,Petersen, J.,Stensballe, L. G.,Benn, C. S. (2006). Differential effects of risk factors on infant wheeze

Intervention/exposure and atopic dermatitis emphasize a different etiology J Allergy Clin Immunol, 117(1), 184-9

1510 Lionetti, E.,Castellaneta, S.,Francavilla, R.,Pulvirenti, A.,Tonutti, E.,Amarri, S.,Barbato, M.,Barbera, C.,Barera, G.,Bellantoni, A.,Castellano, E., Guariso, G.,Limongelli, M. G.,Pellegrino, S.,Polloni, C.,Ughi, C.,Zuin, G.,Fasano, A.,Catassi, C. (2014). Introduction of gluten, HLA status, and the risk of celiac disease in children N Engl J Med, 371(14), 1295-303

1511 Lionetti, E.,Castellaneta, S.,Francavilla, R.,Pulvirenti, A.,Tonutti, E.,Amarri, S.,Barbato, M.,Barbera, C.,Barera, G.,Bellantoni, A.,Castellano, E.,Limongelli, M. G.,Pellegrino, S.,Polloni, C.,Ughi, C.,Zuin, G.,Guariso, G.,Fasano, A., Catassi, C. (2014). Infant

Publication status feeding pattern, HLA status, and prevalence of celiac disease Digestive and liver disease, 46(\#issue\#), e75-e76

1512 Lionetti, E.,Castellaneta, S.,Pulvirenti, A., Tonutti, E.,Francavilla, R.,Fasano, A., Catassi, C. (2012). Prevalence and natural history of Size of study groups potential celiac disease in at-family-risk infants prospectively investigated from birth J Pediatr, 161(5), 908-14

1513 Lipsman, S.,Dewey, K. G.,Lonnerdal, B. (1985). Breast-feeding among teenage mothers: milk composition, infant growth, and maternal dietary intake J Pediatr Gastroenterol Nutr, 4(3), 426-34

1514 Litmanovitz, I.,Davidson, K.,Eliakim, A.,Regev, R. H.,Dolfin, T.,Arnon, S.,Bar-Yoseph, F.,Goren, A.,Lifshitz, Y.,Nemet, D. (2013) High Beta-palmitate formula and bone strength in term infants: a randomized, double-blind, controlled trial Calcif Tissue Int, 92(1), $35-41$

1515 Little, R. E.,Lambert, M. D., 3rd,Worthington-Roberts, B.,Ervin, C. H. (1994). Maternal smoking during lactation: relation to infant size at one year of age Am J Epidemiol, 140(6), 544-54

1516 Liu, J. (1990). Neglected problem: nursing bottle syndrome Dentistry (Loma Linda), 3(2), 57-8

Size of study groups

Study design, Size of study groups, Outcome

Publication date for a non-sibling study

Study design

1517 Liu, J.,Leung, P.,Yang, A. (2014). Breastfeeding and active bonding protects against children's internalizing behavior problems Nutrients, 6(1), 76-89

1518 Liu, Y. Q.,Qian, Z.,Wang, J.,Lu, T.,Lin, S.,Zeng, X. W.,Liu, R. Q.,Zhu, Y.,Qin, X. D.,Yuan, P.,Zhou, Y.,Li, M.,Hao, Y. T.,Dong, G. H. (2015). Breastfeeding modifies the effects of environment tobacco smoke exposure on respiratory diseases and symptoms in Chinese children: the Seven Northeast Cities Study Indoor Air, \#volume\#(\#issue\#), \#Pages\#

1519 Livingstone, V. (2006). Failure to thrive while breastfeeding Breastfeed Med, 1(2), 108-11

1520 Livny, A.,Assali, R.,Sgan-Cohen, H. D. (2007). Early Childhood Caries among a Bedouin community residing in the eastern outskirts of Jerusalem BMC Public Health, 7(\#issue\#), 167

Study design,

Intervention/exposure

Study design

Study design 
1521 Lnnerdal, B.,Timby, N.,Domellf, M.,Domellf, E.,Hernell, O. (2014). Supplementation of infant formula with milk fat globule membranes improves cognitive performance and reduces infections in formula-fed infants FASEB journal, 28(1 suppl. 1), \#Pages\#

1522 Lo, G. L. (1985). The use of comforters and dental caries in the Singaporean preschool children Singapore Dent J, 10(1), 21-4

1523 Lodge, C. J.,Zaloumis, S.,Lowe, A. J.,Gurrin, L. C.,Matheson, M. C.,Axelrad, C.,Bennett, C. M.,Hill, D. J.,Hosking, C. S.,Svanes,

Intervention/exposure

C. Abramson, M. J Allen, K. J. Dharmage S. C. (2014). Early-life risk factors for childhood wheeze phenotypes in a high-risk birth cohort J Pediatr, 164(2), 289-94 e1-2

1524 Lodinova, R.,Jouja, V.,Vinsova, N.,Vocel, J.,Melkova, J. (1980). New attempts and possibilities in prevention and treatment of intestinal coli-infections in infants Czech Med, 3(1), 47-58

1525 Lodinova-Zadnikova, R., Tlaskalova, H.,Bartakova, Z. (1991). The antibody response in infants after colonization of the intestine with E. coli O83. Artificial colonization used as a prevention against nosocomial infections Adv Exp Med Biol, 310(\#issue\#), 329-35

Size of study groups

1526 Loeb H,Mozin MJ (1983). Prevention of chronic diarrhea: nutritional implications J Pediatr Gastroenterol Nutr, 2 Suppl 1(\#issue\#), S328-34

Study design, Size of study groups

1527 Lombeck, I.,Fuchs, A. (1994). Zinc and copper in infants fed breast-milk or different formula Eur J Pediatr, 153(10), 770-6

Outcome

1528 Long, K. Z.,Wood, J. W.,Vasquez Gariby, E.,Weiss, K. M.,Mathewson, J. J.,de la Cabada, F. J.,DuPont, H. L.,Wilson, R. A. (1994).

Outcome

Proportional hazards analysis of diarrhea due to enterotoxigenic Escherichia coli and breast feeding in a cohort of urban Mexican children Am J Epidemiol, 139(2), 193-205

1529 Long, K.,Vasquez-Garibay, E.,Mathewson, J.,de la Cabada, J.,DuPont, H. (1999). The impact of infant feeding patterns on infection Intervention/exposure and diarrheal disease due to enterotoxigenic Escherichia coli Salud Publica Mex, 41(4), 263-70

1530 Long, S. A.,Bugg, K. (2015). Can't we all just get along? J Hum Lact, 31(1), 29-31

Study design

1531 Lonnerdal, B.,Chen, C. L. (1990). Effects of formula protein level and ratio on infant growth, plasma amino acids and serum trace elements. I. Cow's milk formula Acta Paediatr Scand, 79(3), 257-65

1532 Lonnerdal, B.,Havel, P. J. (2000). Serum leptin concentrations in infants: effects of diet, sex, and adiposity Am J Clin Nutr, 72(2), 484-9

1533 Lonnerdal, B.,Hernell, O. (1994). Iron, zinc, copper and selenium status of breast-fed infants and infants fed trace element fortified milk-based infant formula Acta Paediatr, 83(4), 367-73

1534 Lonnerdal, B.,Hernell, O. (1998). Effects of feeding ultrahigh-temperature (UHT)-treated infant formula with different protein concentrations or powdered formula, as compared with breast-feeding, on plasma amino acids, hematology, and trace element status Am J Clin Nutr, 68(2), 350-6

1535 Lonnerdal, B.,Kvistgaard, A. S.,Peerson, J. M.,Donovan, S. M.,Peng, Y. M. (2015). Growth, Nutrition and Cytokine Response of Breast-Fed Infants and Infants Fed Formula with Added Bovine Osteopontin J Pediatr Gastroenterol Nutr, \#volume\#(\#issue\#), \#Pages\# 
1536 Lopez Bravo, I. M.,Sepulveda, H.,Valdes, I. (1997). Acute respiratory illnesses in the first 18 months of life Rev Panam Salud Publica, 1(1), 9-17

1537 Lopez Bravo, I.,Cabiol, C.,Arcuch, S.,Rivera, E.,Vargas, S. (1984). Breast-feeding, weight gains, diarrhea, and malnutrition in the first year of life Bull Pan Am Health Organ, 18(2), 151-63

1538 Lopez Del Valle, L. M.,Singh, G. D.,Feliciano, N.,Machuca Mdel, C. (2006). Associations between a history of breast feeding, malocclusion and parafunctional habits in Puerto Rican children P R Health Sci J, 25(1), 31-4

1539 López, N.,De Barros-Mazón, S.,Dos Santos Vilela, M. M.,Silva, C. M.,Ribeiro, J. D. (1999). Genetic and environmental influences on Outcome atonic immune response in early life Journal of Investigational Allergology and Clinical Immunology, 9(6), 392-398

1540 Lopez, N.,de Barros-Mazon, S., Vilela, M. M.,Silva, C. M.,Ribeiro, J. D. (1999). Genetic and environmental influences on atopic immune response in early life J Investig Allergol Clin Immunol, 9(6), 392-8

1541 Lopez-Alarcon, M.,Garcia-Zuniga, P.,Del Prado, M.,Garza, C. (2004). Breastfeeding protects against the anorectic response to infection in infants: possible role of DHA Adv Exp Med Biol, 554(\#issue\#), 371-4

1542 Lopez-Alarcon, M.,Garza, C.,del Prado, M.,Garcia-Zuniga, P. A.,Barbosa, L. (2008). Breastfeeding's protection against illnessinduced anorexia is mediated partially by docosahexaenoic acid Eur J Clin Nutr, 62(1), 32-8

1543 Lopez-Alarcon, M.,Villalpando, S.,Fajardo, A. (1997). Breast-feeding lowers the frequency and duration of acute respiratory infection Outcome and diarrhea in infants under six months of age $\mathrm{J}$ Nutr, 127(3), 436-43

1544 Lopez-Lopez, A.,Castellote-Bargallo, A. I.,Campoy-Folgoso, C.,Rivero-Urgel, M.,Tormo-Carnice, R.,Infante-Pina, D.,Lopez-Sabater, M. C. (2001). The influence of dietary palmitic acid triacylglyceride position on the fatty acid, calcium and magnesium contents of at term newborn faeces Early Hum Dev, 65 Suppl(\#issue\#), S83-94

1545 Losonsky, G. A.,D'Alessandra de Rimer, H. (1991). Rotavirus specific breast milk antibody in two populations and possible correlates of protection Adv Exp Med Biol, 310(\#issue\#), 265-9

1546 Louzada, M. L.,Campagnolo, P. D.,Rauber, F.,Vitolo, M. R. (2012). Long-term effectiveness of maternal dietary counseling in a low- Intervention/exposure income population: a randomized field trial Pediatrics, 129(6), e1477-84

1547 Lowe, A. J.,Carlin, J. B.,Bennett, C. M.,Abramson, M. J.,Hosking, C. S.,Hill, D. J.,Dharmage, S. C. (2006). Atopic disease and breast-feeding--cause or consequence? J Allergy Clin Immunol, 117(3), 682-7

1548 Lozoff, B.,Wolf, A. W.,Jimenez, E. (1996). Iron-deficiency anemia and infant development: effects of extended oral iron therapy J Pediatr, 129(3), 382-9

1549 Lu, R.,Costello, A. (2000). Failure to exclusively breastfeed and the risk of early infant mortality due to infectious disease in poor communities in Lima, Peru J Trop Pediatr, 46(5), 309-11

1550 Lubis, I. Z.,Sinuhaji, A. B.,Sebayang, T.,Lubis, M.,Barus, N.,Sutanto, A. H. (1985). Factors influencing the duration of infantile diarrhea Paediatr Indones, 25(9-10), 175-89

Outcome, Publication date for a non-sibling study

Study design

Outcome

Size of study groups

Size of study groups

Size of study groups

Study design, Outcome

Intervention/exposure

Study design

Outcome

Country 
1551 Lucas, A.,Boyes, S.,Bloom, S. R.,Aynsley-Green, A. (1981). Metabolic and endocrine responses to a milk feed in six-day-old term infants: differences between breast and cow's milk formula feeding Acta Paediatr Scand, 70(2), 195-200

1552 Lucas, A.,Ewing, G.,Roberts, S. B.,Coward, W. A. (1987). How much energy does the breast fed infant consume and expend? Br Med J (Clin Res Ed), 295(6590), 75-7

1553 Lucas, A.,Lockton, S.,Davies, P. S. (1992). Randomised trial of a ready-to-feed compared with powdered formula Arch Dis Child, 67(7), 935-9

1554 Lucas, A.,Stafford, M.,Morley, R.,Abbott, R.,Stephenson, T.,MacFadyen, U.,Elias-Jones, A.,Clements, H. (1999). Efficacy and safety of long-chain polyunsaturated fatty acid supplementation of infant-formula milk: a randomised trial Lancet, 354(9194), 1948-54

1555 Lucas, Ruth F. (2011). Maternal Breastfeeding Experiences and Neonatal Breastfeeding Behaviors of Children Later Diagnosed with Autism \#journal\#, Ph.D.(\#issue\#), 152 p-152 p 1p

1556 Luccioli, S.,Zhang, Y.,Verrill, L.,Ramos-Valle, M.,Kwegyir-Afful, E. (2014). Infant feeding practices and reported food allergies at 6 years of age Pediatrics, 134 Suppl 1(\#issue\#), S21-8

1557 Ludvigsson, J. (2003). Cow-milk-free diet during last trimester of pregnancy does not influence diabetes-related autoantibodies in nondiabetic children Ann N Y Acad Sci, 1005(\#issue\#), 275-8

1558 Ludvigsson, J. F.,Mostrom, M.,Ludvigsson, J.,Duchen, K. (2005). Exclusive breastfeeding and risk of atopic dermatitis in some 8300 Study design infants Pediatr Allergy Immunol, 16(3), 201-8

1559 Lulic-Dukic, O.,Juric, H.,Dukic, W.,Glavina, D. (2001). Factors predisposing to early childhood caries (ECC) in children of pre-school Study design age in the city of Zagreb, Croatia Coll Antropol, 25(1), 297-302

1560 Lumia, M.,Takkinen, H. M.,Luukkainen, P.,Kaila, M.,Lehtinen-Jacks, S.,Nwaru, B. I.,Tuokkola, J.,Niemela, O.,Haapala, A. M.,Ilonen, J.,Simell, O.,Knip, M.,Veijola, R.,Virtanen, S. M. (2015). Food consumption and risk of childhood asthma Pediatr Allergy Immunol, \#volume\#(\#issue\#), \#Pages\#

1561 Lunardelli, S. E.,Peres, M. A. (2006). Breast-feeding and other mother-child factors associated with developmental enamel defects Outcome in the primary teeth of Brazilian children J Dent Child (Chic), 73(2), 70-8

1562 Lundberg, G. D. (2008). Does breast-feeding improve child cognitive development? MedGenMed Medscape General Medicine, 10(8), \#Pages\#

1563 Lund-Blix, N. A.,Stene, L. C.,Rasmussen, T.,Torjesen, P. A.,Andersen, L. F., Ronningen, K. S. (2015). Infant feeding in relation to

Outcome islet autoimmunity and type 1 diabetes in genetically susceptible children: the MIDIA Study Diabetes Care, 38(2), 257-63

1564 Lundqvist-Persson, C. (2001). Correlation between level of self-regulation in the newborn infant and developmental status at two years of age Acta Paediatrica, International Journal of Paediatrics, 90(3), 345-350

1565 Lung, F. W.,Chiang, T. L.,Lin, S. J.,Shu, B. C. (2013). Incinerator pollution and child development in the taiwan birth cohort study Int Intervention/exposure J Environ Res Public Health, 10(6), 2241-57 
1566 Luo, R.,Shi, Y.,Zhou, H.,Yue, A.,Zhang, L.,Sylvia, S.,Medina, A.,Rozelle, S. (2014). Anemia and feeding practices among infants in Study design rural Shaanxi Province in China Nutrients, 6(12), 5975-91

1567 Luo,,R,,Shi,,Y,,Zhou,,H,,Yue,,A,,Zhang,,L,,Sylvia,,S,,Medina,,A,,Rozelle,,S, (2014). Anemia and feeding practices among infants in Study design rural Shaanxi Province in China Nutrients, 6(12), 5975-91

1568 Luoma, R. (1984). Environmental allergens and morbidity in atopic and non-atopic families Acta Paediatr Scand, 73(4), 448-53

Outcome

1569 Luopajarvi, K.,Savilahti, E.,Virtanen, S. M.,Ilonen, J.,Knip, M.,Akerblom, H. K.,Vaarala, O. (2008). Enhanced levels of cow's milk antibodies in infancy in children who develop type 1 diabetes later in childhood Pediatr Diabetes, 9(5), 434-41

1570 Lutter, C. K. (2000). Breastfeeding promotion--is its effectiveness supported by scientific evidence and global changes in breastfeeding behaviors? Adv Exp Med Biol, 478(\#issue\#), 355-68

1571 Lyall, J. (1991). Growing problems Nurs Times, 87(24), 22-3

Size of study groups

Publication status

Study design

1572 Ma, D. Q.,Jones, G. (2002). Clinical risk factors but not bone density are associated with prevalent fractures in prepubertal children Study design J Paediatr Child Health, 38(5), 497-500

1573 Ma, J. Q.,Zhou, L. L.,Hu, Y. Q.,Liu, J. R.,Liu, S. S.,Zhang, J.,Sheng, X. Y. (2012). A summary index of infant and child feeding practices is associated with child growth in urban Shanghai BMC Public Health, 12(\#issue\#), 568

1574 Maas, T.,Dompeling, E.,Muris, J. W.,Wesseling, G.,Knottnerus, J. A.,van Schayck, O. C. (2011). Prevention of asthma in genetically susceptible children: a multifaceted intervention trial focussed on feasibility in general practice Pediatr Allergy Immunol, 22(8), 794802

1575 MacArthur, A. C.,McBride, M. L.,Spinelli, J. J.,Tamaro, S.,Gallagher, R. P.,Theriault, G. P. (2008). Risk of childhood leukemia associated with vaccination, infection, and medication use in childhood: the Cross-Canada Childhood Leukemia Study Am $\mathrm{J}$ Epidemiol, 167(5), 598-606

1576 MacDonald, L. D.,Gibson, R. S.,Miles, J. E. (1982). Changes in hair zinc and copper concentrations of breast fed and bottle fed infants during the first six months Acta Paediatr Scand, 71(5), 785-9

1577 Macdonald, P. D.,Ross, S. R.,Grant, L.,Young, D. (2003). Neonatal weight loss in breast and formula fed infants Arch Dis Child Fetal Neonatal Ed, 88(6), F472-6

1578 Maclntyre, E. A.,Karr, C. J.,Koehoorn, M.,Demers, P.,Tamburic, L.,Lencar, C.,Brauer, M. (2010). Otitis media incidence and risk factors in a population-based birth cohort Paediatrics and Child Health, 15(7), 437-442

1579 Macoun, E. (2005). The NSW Health Breastfeeding Project N S W Public Health Bull, 16(3-4), 62

Intervention/exposure

Outcome

\section{Outcome}

Size of study groups

Outcome, Publication date for a non-sibling study

Outcome

Study design

1580 Madar, A. A.,Stene, L. C.,Meyer, H. E. (2009). Vitamin D status among immigrant mothers from Pakistan, Turkey and Somalia and their infants attending child health clinics in Norway Br J Nutr, 101(7), 1052-8

1581 Madhavapeddi, R.,Ramachandran, P. (1990). Growth and morbidity of breastfed infants whose mothers were using combination pills Breastfeeding Review, 2(2), 66-68 3p

Study design

Country 
1582 Madhavapeddi, R.,Ramachandran, P. (1993). Growth of urban breastfed infants from low socio-economic group J Trop Pediatr, $39(6), 328-31$

1583 Madsen, A. L.,Larnkjaer, A.,Molgaard, C.,Michaelsen, K. F. (2011). IGF-I and IGFBP-3 in healthy 9 month old infants from the SKOT cohort: breastfeeding, diet, and later obesity Growth Horm IGF Res, 21(4), 199-204

1584 Magalhaes, T. C.,Vieira, S. A.,Priore, S. E.,Ribeiro, A. Q.,Lamounier, J. A.,Franceschini, S. C.,Sant'Ana, L. F. (2012). Exclusive breastfeeding and other foods in the first six months of life: effects on nutritional status and body composition of Brazilian children ScientificWorldJournal, 2012(\#issue\#), 468581

1585 Magana Cardenas, A.,Padilla Gonzalez, L. M.,Garcia de Alba, J. E.,Troyo San Roman, R.,Delgado Becerra, A. (1981). Some epidemiological aspects of maternal breast-feeding in a population entitled to social welfare services in Mexico Bull Pan Am Health Organ, 15(2), 139-47

1586 Magnus, M. C.,DeRoo, L. A.,Haberg, S. E.,Magnus, P.,Nafstad, P.,Nystad, W.,London, S. J. (2014). Prospective study of maternal alcohol intake during pregnancy or lactation and risk of childhood asthma: the Norwegian Mother and Child Cohort Study Alcohol Clin Exp Res, 38(4), 1002-11

1587 Magnusson, C. G. (1988). Cord serum IgE in relation to family history and as predictor of atopic disease in early infancy Allergy, 43(4), $241-51$

1588 Mai, X. M.,Becker, A. B.,Sellers, E. A.,Liem, J. J.,Kozyrskyj, A. L. (2007). The relationship of breast-feeding, overweight, and asthma Intervention/exposure in preadolescents J Allergy Clin Immunol, 120(3), 551-6

1589 Maisels, M. J.,Gifford, K. (1983). Breast-feeding, weight loss, and jaundice J Pediatr, 102(1), 117-8

Size of study groups

ntervention/exposure

1590 Majeed, R.,Rajar, U. D.,Shaikh, N.,Majeed, F.,Arain, A. A. (2008). Risk factors associated with childhood asthma J Coll Physicians Country Surg Pak, 18(5), 299-302

1591 Majorana, A.,Cagetti, M. G.,Bardellini, E.,Amadori, F.,Conti, G.,Strohmenger, L.,Campus, G. (2014). Feeding and smoking habits as Outcome cumulative risk factors for early childhood caries in toddlers, after adjustment for several behavioral determinants: a retrospective study BMC Pediatr, 14(\#issue\#), 45

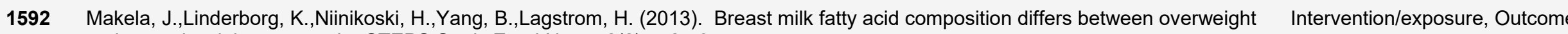
and normal weight women: the STEPS Study Eur J Nutr, 52(2), 727-35

1593 Mäkelä, J.,Vaarno, J.,Kaljonen, A.,Niinikoski, H.,Lagström, H. (2014). Maternal overweight impacts infant feeding patterns - The STEPS Study European Journal of Clinical Nutrition, 68(1), 43-49

1594 Maki, M.,Kallonen, K.,Lahdeaho, M. L.,Visakorpi, J. K. (1988). Changing pattern of childhood coeliac disease in Finland Acta Paediatr Scand, $77(3), 408-12$

1595 Makrides M,Neumann MA,Byard RW,Simmer K,Gibson RA (1994). Fatty acid composition of brain, retina, and erythrocytes in breast- and formula-fed infants Am J Clin Nutr, 60(\#issue\#), 189-94 
1596 Makrides, M. (2008). Outcomes for mothers and their babies: do n-3 long-chain polyunsaturated fatty acids and seafoods make a difference? J Am Diet Assoc, 108(10), 1622-6

1597 Makrides, M.,Gibson, R. A.,Simmer, K. (1993). The effect of dietary fat on the developing brain J Paediatr Child Health, 29(6), 409- $\quad$ Study design 10

1598 Makrides, M.,Hawkes, J. S.,Neumann, M. A.,Gibson, R. A. (2002). Nutritional effect of including egg yolk in the weaning diet of breast-fed and formula-fed infants: a randomized controlled trial Am J Clin Nutr, 75(6), 1084-92

1599 Makrides, M.,Neumann, M. A.,Jeffrey, B.,Lien, E. L.,Gibson, R. A. (2000). A randomized trial of different ratios of linoleic to alphalinolenic acid in the diet of term infants: effects on visual function and growth Am J Clin Nutr, 71(1), 120-9

1600 Makrides, M.,Neumann, M. A.,Simmer, K.,Gibson, R. A. (1995). Erythrocyte fatty acids of term infants fed either breast milk standard formula, or formula supplemented with long-chain polyunsaturates Lipids, 30(10), 941-8

1601 Makrides, M.,Neumann, M. A.,Simmer, K.,Gibson, R. A. (1999). Dietary long-chain polyunsaturated fatty acids do not influence growth of term infants: A randomized clinical trial Pediatrics, 104(3 Pt 1), 468-75

1602 Makrides, M.,Neumann, M. A.,Simmer, K.,Gibson, R. A. (2000). A critical appraisal of the role of dietary long-chain polyunsaturated Outcome fatty acids on neural indices of term infants: a randomized, controlled trial Pediatrics, 105(1 Pt 1), 32-8

1603 Makrides, M.,Neumann, M.,Gibson, R. (1997). Breast milk docosahexaenoic acid (DHA) and infant outcomes: a randomised clinical Publication status trial Journal of paediatrics and child health, 33(4), A2

1604 Makrides, M.,Neumann, M.,Simmer, K.,Pater, J.,Gibson, R. (1995). Are long-chain polyunsaturated fatty acids essential nutrients in Outcome infancy? Lancet, 345(8963), 1463-8

1605 Makrides, M.,Simmer, K.,Goggin, M.,Gibson, R. A. (1993). Erythrocyte docosahexaenoic acid correlates with the visual response of healthy, term infants Pediatr Res, 33(4 Pt 1), 425-7

1606 Malcolm, C. A.,McCulloch, D. L.,Montgomery, C.,Shepherd, A.,Weaver, L. T. (2003). Maternal docosahexaenoic acid supplementation during pregnancy and visual evoked potential development in term infants: a double blind, prospective, randomised trial Arch Dis Child Fetal Neonatal Ed, 88(5), F383-90

1607 Malcova, H.,Sumnik, Z.,Drevinek, P.,Venhacova, J.,Lebl, J.,Cinek, O. (2006). Absence of breast-feeding is associated with the risk Outcome of type 1 diabetes: a case-control study in a population with rapidly increasing incidence Eur J Pediatr, 165(2), 114-9

1608 Male, C.,Persson, L. A.,Freeman, V.,Guerra, A., van't Hof, M. A.,Haschke, F. (2001). Prevalence of iron deficiency in 12-mo-old infants from 11 European areas and influence of dietary factors on iron status (Euro-Growth study) Acta Paediatr, 90(5), 492-8

1609 Malek L,Makrides M (2015). 2.8 Nutrition in pregnancy and lactation World Rev Nutr Diet, 113(\#issue\#), 127-33

Outcome

1610 Malinowska E,Kaczmarski M,Wasilewska J (2002). Total lgE levels and skin test results in children under three years of age with food hypersensitivity Med Sci Monit, 8(\#issue\#), Cr280-7 
1611 Mallet, E.,Henocq, A. (1992). Long-term prevention of allergic diseases by using protein hydrolysate formula in at-risk infants $\mathrm{J}$ Pediatr, 121(5 Pt 2), S95-100

1612 Mallol-Mesnard, N.,Menegaux, F.,Lacour, B.,Hartmann, O.,Frappaz, D.,Doz, F.,Bertozzi, A. I.,Chastagner, P.,Hemon, D.,Clavel, J. (2008). Birth characteristics and childhood malignant central nervous sytem tumors: the ESCALE study (French Society for Childhood Cancer) Cancer Detect Prev, 32(1), 79-86

1613 Malloy, M. H.,Berendes, H. (1998). Does breast-feeding influence intelligence quotients at 9 and 10 years of age? Early Hum Dev $50(2), 209-17$

1614 Manco, M.,Alterio, A.,Bugianesi, E.,Ciampalini, P.,Mariani, P.,Finocchi, M.,Agostoni, C.,Nobili, V. (2011). Insulin dynamics of breastor formula-fed overweight and obese children Journal of the American College of Nutrition, 30(1), 29-38

1615 Mandel, E. M.,Doyle, W. J.,Winther, B.,Alper, C. M. (2008). The incidence, prevalence and burden of OM in unselected children aged 1-8 years followed by weekly otoscopy through the "common cold" season Int J Pediatr Otorhinolaryngol, 72(4), 491-9

1616 Mandhane, P. J.,Greene, J. M.,Sears, M. R. (2007). Interactions between breast-feeding, specific parental atopy, and sex on development of asthma and atopy J Allergy Clin Immunol, 119(6), 1359-66

1617 Mandic, Z.,Piricki, A. P.,Kenjeric, D.,Hanicar, B.,Tanasic, I. (2011). Breast vs. bottle: differences in the growth of Croatian infants Matern Child Nutr, 7(4), 389-96

1618 Mangskau, K. (1991). Baby bottle tooth decay: a problem affecting young children in North Dakota Northwest Dent, 70(6), 25

1619 Manjrekar, C.,Vishalakshi, M. P.,Begum, N. J.,Padma, G. N. (1985). Breast feeding ability of undernourished mothers and physical Country development of their infants during 0-1 year Indian Pediatr, 22(11), 801-9

1620 Mann, K. D.,Tennant, P. W.,Parker, L.,Unwin, N. C.,Pearce, M. S. (2011). The relatively small contribution of birth weight to blood Outcome pressure at age 49-51 years in the Newcastle Thousand Families Study J Hypertens, 29(6), 1077-84

1621 Maranhao, H. S.,Medeiros, M. C.,Scaletsky, I. C.,Fagundes-Neto, U.,Morais, M. B. (2008). The epidemiological and clinical characteristics and nutritional development of infants with acute diarrhoea, in north-eastern Brazil Ann Trop Med Parasitol, 102(4), $357-65$

1622 Marini, A.,Agosti, M.,Motta, G.,Mosca, F. (1996). Effects of a dietary and environmental prevention programme on the incidence of allergic symptoms in high atopic risk infants: three years' follow-up Acta Paediatr Suppl, 414(\#issue\#), 1-21

1623 Markestad, T. (1983). Effect of season and vitamin D supplementation on plasma concentrations of 25-hydroxyvitamin D in Norwegian infants Acta Paediatr Scand, 72(6), 817-21

1624 Markestad, T. (1983). Plasma concentrations of 1,25-dihydroxyvitamin D, 24,25-dihydroxyvitamin D, and 25,26-dihydroxyvitamin D Study design in the first year of life $\mathrm{J}$ Clin Endocrinol Metab, 57(4), 755-9

1625 Marmot, M. G.,Page, C. M.,Atkins, E.,Douglas, J. W. (1980). Effect of breast-feeding on plasma cholesterol and weight in young adults J Epidemiol Community Health, 34(3), 164-7

Intervention/exposure

Intervention/exposure

Study design,

Intervention/exposure

Intervention/exposure 
1626 Marques, R. C.,Dorea, J. G.,Bernardi, J. V.,Bastos, W. R.,Malm, O. (2008). Maternal fish consumption in the nutrition transition of the Amazon Basin: growth of exclusively breastfed infants during the first 5 years Ann Hum Biol, 35(4), 363-77

1627 Marques, R. C.,Dorea, J. G.,Bernardi, J. V.,Bastos, W. R.,Malm, O. (2009). Prenatal and postnatal mercury exposure, breastfeeding Intervention/exposure and neurodevelopment during the first 5 years Cogn Behav Neurol, 22(2), 134-41

1628 Marques, R. C.,Dorea, J. G.,Leao, R. S.,Dos Santos, V. G.,Bueno, L.,Marques, R. C.,Brandao, K. G.,Palermo, E. F.,Guimaraes, J. R. (2012). Role of methylmercury exposure (from fish consumption) on growth and neurodevelopment of children under 5 years of

Study design, age living in a transitioning (tin-mining) area of the western Amazon, Brazil Arch Environ Contam Toxicol, 62(2), 341-50

1629 Marques, R. F.,Taddei, J. A.,Lopez, F. A.,Braga, J. A. (2014). Breastfeeding exclusively and iron deficiency anemia during the first 6 Intervention/exposure months of age Rev Assoc Med Bras, 60(1), 18-22

1630 Marquis, G. S.,Habicht, J. P. (2000). Breastfeeding and stunting among toddlers in Peru Adv Exp Med Biol, 478(\#issue\#), 163-72 Publication status

1631 Marquis, G. S.,Habicht, J. P.,Lanata, C. F.,Black, R. E.,Rasmussen, K. M. (1997). Association of breastfeeding and stunting in Intervention/exposure Peruvian toddlers: an example of reverse causality Int J Epidemiol, 26(2), 349-56

1632 Marquis, G. S.,Habicht, J. P.,Lanata, C. F.,Black, R. E.,Rasmussen, K. M. (1997). Breast milk or animal-product foods improve Intervention/exposure linear growth of Peruvian toddlers consuming marginal diets Am J Clin Nutr, 66(5), 1102-9

1633 Marriage, B. J.,Buck, R. H.,Goehring, K. C.,Oliver, J. S.,Williams, J. A. (2015). Infants Fed a Lower Calorie Formula With 2'FL Show Outcome Growth and 2'FL Uptake Like Breast-Fed Infants J Pediatr Gastroenterol Nutr, 61(6), 649-58

1634 Marshall, J. (2013). Infant feeding. 6. Formula feed Pract Midwife, 16(3), 35-8

Study design

1635 Martel, M. J.,Rey, E.,Malo, J. L.,Perreault, S.,Beauchesne, M. F.,Forget, A.,Blais, L. (2009). Determinants of the incidence of childhood asthma: a two-stage case-control study Am J Epidemiol, 169(2), 195-205

1636 Martens, P. J.,Romphf, L. (2007). Factors associated with newborn in-hospital weight loss: comparisons by feeding method, demographics, and birthing procedures J Hum Lact, 23(3), 233-41, quiz 242-5

Outcome

Outcome, Publication date for a non-sibling study

1637 Martin FP,Moco S,Montoliu I,Collino S,Da Silva L,Rezzi S,Prieto R,Kussmann M,Inostroza J,Steenhout P (2014). Impact of breastfeeding and high- and low-protein formula on the metabolism and growth of infants from overweight and obese mothers Pediatr Res, 75(\#issue\#), 535-43

1638 Martin, A. J.,Landau, L. I.,Phelan, P. D. (1981). Natural history of allergy in asthmatic children followed to adult life Med J Aust, 2(9), $470-4$

1639 Martin, R. M.,Ben-Shlomo, Y.,Gunnell, D.,Elwood, P.,Yarnell, J. W.,Davey Smith, G. (2005). Breast feeding and cardiovascular disease risk factors, incidence, and mortality: the Caerphilly study J Epidemiol Community Health, 59(2), 121-9

1640 Martin, R. M.,Ebrahim, S.,Griffin, M.,Davey Smith, G.,Nicolaides, A. N.,Georgiou, N.,Watson, S.,Frankel, S.,Holly, J. M.,Gunnell, D. Intervention/exposure (2005). Breastfeeding and atherosclerosis: intima-media thickness and plaques at 65-year follow-up of the Boyd Orr cohort Arterioscler Thromb Vasc Biol, 25(7), 1482-8 
1641 Martin, R. M.,Gunnell, D.,Pemberton, J.,Frankel, S.,Smith, G. D. (2005). Cohort profile: The Boyd Orr cohort - An historical cohort study based on the 65 year follow-up of the Carnegie Survey of Diet and Health (1937-39) International Journal of Epidemiology, 34(4), 742-749

1642 Martin, R. M.,Ness, A. R.,Gunnell, D.,Emmett, P.,Davey Smith, G. (2004). Does breast-feeding in infancy lower blood pressure in childhood? The Avon Longitudinal Study of Parents and Children (ALSPAC) Circulation, 109(10), 1259-66

1643 Martin, R. M.,Patel, R.,Kramer, M. S.,Vilchuck, K.,Bogdanovich, N.,Sergeichick, N.,Gusina, N.,Foo, Y.,Palmer, T.,Thompson, J.,Gillman, M. W.,Smith, G. D.,Oken, E. (2014). Effects of promoting longer-term and exclusive breastfeeding on cardiometabolic risk factors at age 11.5 years: a cluster-randomized, controlled trial Circulation, 129(3), 321-9

1644 Martin, R. M.,Smith, G. D.,Mangtani, P.,Frankel, S.,Gunnell, D. (2002). Association between breast feeding and growth: the BoydOrr cohort study Arch Dis Child Fetal Neonatal Ed, 87(3), F193-201

Intervention/exposure

1645 Martines, F.,Bentivegna, D.,Maira, E.,Sciacca, V.,Martines, E. (2011). Risk factors for otitis media with effusion: case-control study Study design in Sicilian schoolchildren Int J Pediatr Otorhinolaryngol, 75(6), 754-9

1646 Martines, F.,Salvago, P.,Ferrara, S.,Messina, G.,Mucia, M.,Plescia, F.,Sireci, F. (2015). Factors influencing the development of otitis Outcome media among Sicilian children affected by upper respiratory tract infections Brazilian Journal of Otorhinolaryngology, \#volume\#(\#issue\#), \#Pages\#

1647 Martines, J. C.,Ashworth, A.,Kirkwood, B. (1989). Breast-feeding among the urban poor in southern Brazil: reasons for termination in Outcome the first 6 months of life Bull World Health Organ, 67(2), 151-61

1648 Martines, J. C.,Habicht, J. P.,Ashworth, A.,Kirkwood, B. R. (1994). Weaning in southern Brazil: is there a "weanling's dilemma"? J Intervention/exposure Nutr, 124(8), 1189-98

1649 Martorell, A.,Plaza, A. M.,Boné, J.,Nevot, S.,García Ara Ma, C.,Echeverria, L.,Alonso, E.,Garde, J.,Vila, B.,Alvaro, M.,Tauler, E.,Hernando, V.,Fernández, M. (2006). Cow's milk protein allergy. A multi-centre study: Clinical and epidemiological aspects Allergologia et Immunopathologia, 34(2), 46-53

1650 Martorell, R.,O'Gara, C. (1985). Breastfeeding, infant health, and socioeconomic status Med Anthropol, 9(2), 173-81

Intervention/exposure

Country

1651 Mason, J. K.,Harkness, R. A.,Elton, R. A.,Bartholomew, S. (1980). Cot deaths in Edinburgh: infant feeding and socioeconomic factors J Epidemiol Community Health, 34(1), 35-41

1652 Massoni, A. C..Chaves, A. M.,Rosenblatt, A.,Sampaio, F. C.,Oliveira, A. F. (2009). Prevalence of enamel defects related to pre-, peri- and postnatal factors in a Brazilian population Community Dent Health, 26(3), 143-9

1653 Mata, L. (1981). Epidemiologic perspective of diarrheal disease in Costa Rica and current efforts in control, prevention, and research Rev Latinoam Microbiol, 23(2), 109-19

1654 Mata, L. (1986). Cryptosporidium and other protozoa in diarrheal disease in less developed countries Pediatr Infect Dis, 5(1 Suppl), Study design S117-30 
1655 Mata, L.,Bolanos, H.,Pizarro, D.,Vives, M. (1984). Cryptosporidiosis in children from some highland Costa Rican rural and urban areas Am J Trop Med Hyg, 33(1), 24-9

Study design

Intervention/exposure

1656 Matee MI,Mikx FH,Maselle SY,Van Palenstein Helderman WH (1992). Rampant caries and linear hypoplasia (short communication) Country Caries Res, 26(\#issue\#), 205-8

1657 Matheson, M. C.,Erbas, B.,Balasuriya, A.,Jenkins, M. A.,Wharton, C. L.,Tang, M. L.,Abramson, M. J.,Walters, E. H.,Hopper, J. L.,Dharmage, S. C. (2007). Breast-feeding and atopic disease: a cohort study from childhood to middle age J Allergy Clin Immunol, 120(5), 1051-7

1658 Matsuda, I.,Higashi, A.,Ikeda, T.,Uehara, I.,Kuroki, Y. (1984). Effects of zinc and copper content of formulas on growth and on the concentration of zinc and copper in serum and hair J Pediatr Gastroenterol Nutr, 3(3), 421-5

Study design, Size of study groups

1659 Matthews, M. K.,Webber, K.,McKim, E.,Banoub-Baddour, S.,Laryea, M. (1995). Infant feeding practices in Newfoundland and

Outcome Labrador Can J Public Health, 86(5), 296-300

1660 Mattos-Graner, R. O.,Zelante, F.,Line, R. C.,Mayer, M. P. (1998). Association between caries prevalence and clinical, microbiological and dietary variables in 1.0 to 2.5-year-old Brazilian children Caries Res, 32(5), 319-23

1661 Maupome, G.,Karanja, N.,Ritenbaugh, C.,Lutz, T.,Aickin, M.,Becker, T. (2010). Dental caries in American Indian toddlers after a community-based beverage intervention Ethn Dis, 20(4), 444-50

1662 May, R.,Barber, J.,Simpson, T.,Winders, N.,Kuhler, K.,Schroeder, S. (2002). Growth pattern of overweight preschool children in the Participant health Siouxland WIC program Am J Hum Biol, 14(6), 769-76 1663 May, R.,Kim, D.,Mote-Watson, D. (2013). Change in weight-for-length status during the first three months: relationships to birth
weight and implications for metabolic risk Am J Phys Anthropol, 150(1), 5-9

1664 Mayer, E. J.,Hamman, R. F.,Gay, E. C.,Lezotte, D. C.,Savitz, D. A.,Klingensmith, G. J. (1988). Reduced risk of IDDM among breast- Outcome fed children. The Colorado IDDM Registry Diabetes, 37(12), 1625-32

1665 Mayer-Davis, E. J.,Dabelea, D.,Crandell, J. L.,Crume, T.,D'Agostino, R. B., Jr.,Dolan, L.,King, I. B.,Lawrence, J. M.,Norris, J. M.,Pihoker, C., The, N. (2013). Nutritional factors and preservation of C-peptide in youth with recently diagnosed type 1 diabetes: SEARCH Nutrition Ancillary Study Diabetes Care, 36(7), 1842-50

1666 Mayer-Davis, E. J.,Dabelea, D.,Lamichhane, A. P.,D'Agostino Jr, R. B.,Liese, A. D.,Thomas, J.,McKeown, R. E.,Hamman, R. F. (2008). Breast-feeding and type 2 diabetes in the youth of three ethnic groups: The SEARCH for diabetes in youth case-control study Diabetes Care, 31(3), 470-475

1667 Mayer-Davis, E. J.,Rifas-Shiman, S. L.,Zhou, L.,Hu, F. B.,Colditz, G. A.,Gillman, M. W. (2006). Breast-feeding and risk for childhood Study design obesity: does maternal diabetes or obesity status matter? Diabetes Care, 29(10), 2231-7

1668 McAllister, J. C.,Lane, A. T.,Buckingham, B. A. (2006). Vitamin D deficiency in the San Francisco Bay Area J Pediatr Endocrinol Study design Metab, 19(3), 205-8 
1669 McCann, M. F.,Moggia, A. V.,Higgins, J. E.,Potts, M.,Becker, C. (1989). The effects of a progestin-only oral contraceptive (levonorgestrel $0.03 \mathrm{mg}$ ) on breast-feeding Contraception, 40(6), 635-48

1670 McConnochie, K. M.,Roghmann, K. J. (1986). Breast feeding and maternal smoking as predictors of wheezing in children age 6 to Intervention/exposure 10 years Pediatr Pulmonol, 2(5), 260-8

1671 McCormick, D. P.,Grady, J. J.,Diego, A.,Matalon, R.,Revai, K.,Patel, J. A.,Han, Y.,Chonmaitree, T. (2011). Acute otitis media severity: association with cytokine gene polymorphisms and other risk factors Int J Pediatr Otorhinolaryngol, 75(5), 708-12

Outcome

\begin{tabular}{|c|c|c|}
\hline 1672 & McCrory, C.,Layte, R. (2012). Breastfeeding and risk of overweight and obesity at nine-years of age Soc Sci Med, 75(2), 323-30 & Study design \\
\hline 1673 & McCrory, C.,Murray, A. (2013). The effect of breastfeeding on neuro-development in infancy Matern Child Health J, 17(9), 1680-8 & Study design \\
\hline 1674 & $\begin{array}{l}\text { McCusker, C. (2008). Teaching tolerance: Using the neonatal immune system to prevent allergic asthma Expert Review of Clinical } \\
\text { Immunology, 4(4), 429-432 }\end{array}$ & Study design \\
\hline 1675 & $\begin{array}{l}\text { McDougall, P.,Drewett, R. F.,Hungin, A. P. S.,Wright, C. M. (2009). The detection of early weight faltering at the 6-8-week check } \\
\text { and its association with family factors, feeding and behavioural development Archives of Disease in Childhood, 94(7), 549-552 }\end{array}$ & $\begin{array}{l}\text { Outcome, Publication date for a } \\
\text { non-sibling study }\end{array}$ \\
\hline
\end{tabular}

1676 McEnery, G.,Rao, K. P. (1986). The effectiveness of antenatal education of Pakistani and Indian women living in this country Child Intervention/exposure Care Health Dev, 12(6), 385-99

1677 McGowan, E. C.,Bloomberg, G. R.,Gergen, P. J.,Visness, C. M.,Jaffee, K. F.,Sandel, M.,O'Connor, G.,Kattan, M.,Gern, J.,Wood, R. A. (2015). Influence of early-life exposures on food sensitization and food allergy in an inner-city birth cohort J Allergy Clin Immunol, $135(1), 171-8$

1678 Mclntosh, E. D.,De Silva, L. M.,Oates, R. K. (1993). Clinical severity of respiratory syncytial virus group A and B infection in Sydney, Participant health Australia Pediatr Infect Dis J, 12(10), 815-9

1679 Mclsaac, K. E.,Moineddin, R.,Matheson, F. I. (2015). Breastfeeding as a means to prevent infant morbidity and mortality in Aboriginal Canadians: A population prevented fraction analysis Can J Public Health, 106(4), e217-22

1680 McKinney, P. A.,Parslow, R., Gurney, K. A.,Law, G. R.,Bodansky, H. J.,Williams, R. (1999). Perinatal and neonatal determinants of Intervention/exposure childhood type 1 diabetes. A case-control study in Yorkshire, U.K Diabetes Care, 22(6), 928-32

1681 McMichael, A. J. (2005). Widening the horizons of 'evidence': Nutrition and disease in ecological perspective South African Journal Study design of Clinical Nutrition, 18(2), 140-148

1682 McNamara, T. M.,Melnyk, B. M. (2000). The effect of food intake on atopic disease in high-risk infants and young children Pediatric Study design nursing, 26(6), 602-604

1683 McTeer, H. (2012). Fat, young, and poor: why breastfeeding is a critical weapon in the fight against childhood obesity Breastfeed Study design Med, 7(5), 325-6 
1684 Meador, K. J.,Baker, G. A.,Browning, N.,Clayton-Smith, J.,Combs-Cantrell, D. T.,Cohen, M.,Kalayjian, L. A.,Kanner, A., Liporace, J.

D.,Pennell, P. B.,Privitera, M.,Loring, D. W. (2010). Effects of breastfeeding in children of women taking antiepileptic drugs

Neurology, 75(22), 1954-60

1685 Meador, K. J.,Baker, G. A.,Browning, N.,Cohen, M. J.,Bromley, R. L.,Clayton-Smith, J.,Kalayjian, L. A.,Kanner, A.,Liporace, J.

D.,Pennell, P. B.,Privitera, M.,Loring, D. W. (2014). Breastfeeding in children of women taking antiepileptic drugs: cognitive

Outcome

outcomes at age 6 years JAMA Pediatr, 168(8), 729-36

1686 Meah, S. (2001). A breastfeeding intervention increased breast feeding and reduced GI tract infections and atopic eczema Evidence Study design Based Nursing, \#volume\#(\#issue\#), 106-106 1p

1687 Megeid, F. Y. A.,Bakeit, Z. A. N.,Karim, B. O. I. A. A. (2011). Early introduction of cow's milk and short duration of breastfeeding is Study design associated with increasing risk of juvenile diabetes World Journal of Medical Sciences, 6(2), 54-60

1688 Megraud, F.,Boudraa, G.,Bessaoud, K.,Bensid, S.,Dabis, F.,Soltana, R.,Touhami, M. (1990). Incidence of Campylobacter infection in infants in western Algeria and the possible protective role of breast feeding Epidemiol Infect, 105(1), 73-8

Study design, Size of study groups

1689 Meinzen-Derr, J. K.,Guerrero, M. L.,Altaye, M.,Ortega-Gallegos, H.,Ruiz-Palacios, G. M.,Morrow, A. L. (2006). Risk of infant anemia Intervention/exposure is associated with exclusive breast-feeding and maternal anemia in a Mexican cohort J Nutr, 136(2), 452-8

1690 Meinzen-Derr, J. K.,Guerrero, M. L.,Altaye, M.,Ruiz-Palacios, G. M.,Morrow, A. L. (2004). Duration of exclusive breastfeeding and risk of anemia in a cohort of Mexican infants Adv Exp Med Biol, 554(\#issue\#), 395-8

1691 Mellander, M.,Noren, J. G.,Freden, H.,Kjellmer, I. (1982). Mineralization defects in deciduous teeth of low birthweight infants Acta Paediatr Scand, 71(5), 727-33

1692 Meloni, T. Marinaro, A. M Mannazzu, M. C Ogana, A.,La Vecchia, C Negri, E Colombo, C. (1997). IDDM and early infant feeding. Sardinian case-control study Diabetes Care, 20(3), 340-2 1693 Melville B (1990). The high cost of artificial feeding in Jamaica and its implications for child health West Indian Med J, 39(\#issue\#),
203-4

1694 Mendelson, M.,Cloutier, J.,Spence, L.,Sellers, E.,Taback, S.,Dean, H. (2011). Obesity and type 2 diabetes mellitus in a birth cohort of First Nation children born to mothers with pediatric-onset type 2 diabetes Pediatr Diabetes, 12(3 Pt 2), 219-28

1695 Mendez, M. A.,Torrent, M.,Julvez, J.,Ribas-Fito, N.,Kogevinas, M.,Sunyer, J. (2009). Maternal fish and other seafood intakes during Outcome pregnancy and child neurodevelopment at age 4 years Public Health Nutr, 12(10), 1702-10

1696 Menihan, C. A.,Phipps, M.,Weitzen, S. (2006). Fetal heart rate patterns and sudden infant death syndrome J Obstet Gynecol Neonatal Nurs, 35(1), 116-22

1697 Mennella, J. A., Trabulsi, J. C.,Papas, M. A. (2015). Effects of cow milk versus extensive protein hydrolysate formulas on infant cognitive development Amino Acids, \#volume\#(\#issue\#), \#Pages\#

1698 Merewood, A.,Mehta, S. D.,Grossman, X.,Chen, T. C.,Mathieu, J.,Holick, M. F.,Bauchner, H. (2012). Vitamin D status among 4 month-old infants in New England: a prospective cohort study J Hum Lact, 28(2), 159-66
Intervention/exposure,

Publication status

Participant health,

intervention/exposure

Outcome

Study design

Size of study groups, Intervention/exposure

Intervention/exposure

Intervention/exposure
Intervention/exposure 
1699 Merlob, P.,Aloni, R.,Prager, H.,Jelin, N.,Idel, M.,Kotona, J. (1994). Continued weight loss in the newborn during the third day of life as an indicator of early weaning Israel Journal of Medical Sciences, 30(8), 646-648

1700 Merlob, P.,Stahl, B.,Sulkes, J. (2004). Paroxetine during breast-feeding: infant weight gain and maternal adherence to counsel Eur J Pediatr, 163(3), 135-9

1701 Merrett, T. G.,Burr, M. L.,Butland, B. K.,Merrett, J.,Miskelly, F. G.,Vaughan Williams, E. (1988). Infant feeding and allergy: 12-month prospective study of 500 babies born into allergic families. Review 53 refs Annals of allergy, 61(6 (Pt 2)), 13-20

1702 Metcalfe, D. D. (1984). Food hypersensitivity J Allergy Clin Immunol, 73(6), 749-62

Outcome, Publication date for a non-sibling study

Redundant data with another study

Study design,

Intervention/exposure

1703 Meyers, A.,Hertzberg, J. (1988). Bottle-feeding and malocclusion: is there an association? Am J Orthod Dentofacial Orthop, 93(2), Study design 149-52

1704 Micali, N.,Simonoff, E., Treasure, J. (2009). Infant feeding and weight in the first year of life in babies of women with eating disorders J Pediatr, 154(1), 55-60 e1

1705 Michaelsen KF (2015). 1.1 Child growth World Rev Nutr Diet, 113(\#issue\#), 1-5

Publication date for a non-sibling study

Publication status

1706 Michaelsen, K. F. (1997). Nutrition and growth during infancy. The Copenhagen Cohort Study Acta Paediatr Suppl, 420(\#issue\#), 1-

Publication date for a non-sibling study

1707 Michaelsen, K. F. (2015). 2.1 Breastfeeding World Rev Nutr Diet, 113(\#issue\#), 92-6 Study design

1708 Michaelsen, K. F.,Larnkjaer, A.,Molgaard, C. (2013). Early diet, insulin-like growth factor-1, growth and later obesity \#journal\#, 106((Michaelsen K.F., kfm@life.ku.dk; Larnkjaer A.; Molgaard C.) Department of Nutrition Exercise and Sports, Faculty of Science, University of Copenhagen, DK-1958 Frederiksberg C, Denmark), 113-118

1709 Michaelsen, K. F.,Petersen, S.,Greisen, G.,Thomsen, B. L. (1994). Weight, length, head circumference, and growth velocity in a longitudinal study of Danish infants Dan Med Bull, 41(5), 577-85

Publication status

Michaelsen, K. F.,Samuelson, G.,Graham, T. W.,Lonnerdal, B. (1994). Zinc intake, zinc status and growth in a longitudinal study of healthy Danish infants Acta Paediatrica, International Journal of Paediatrics, 83(11), 1115-1121

1711 Michel, H.,Olabopo, F.,Wang, L.,Nucci, A.,Greenspan, S. L.,Rajakumar, K. (2015). Determinants of 25-hydroxyvitamin D concentrations in infants and toddlers Current Nutrition and Food Science, 11(2), 124-130

1712 Michels, K. B.,Willett, W. C.,Graubard, B. I.,Vaidya, R. L.,Cantwell, M. M.,Sansbury, L. B.,Forman, M. R. (2007). A longitudinal study Intervention/exposure of infant feeding and obesity throughout life course Int J Obes (Lond), 31(7), 1078-85

1713 Michie, C. (2016). Breast feeding could reduce the risk of childhood leukaemias Evid Based Nurs, \#volume\#(\#issue\#), \#Pages\# Study design

1714 Michie, C. A.,Gilmour, J. (2001). Breast feeding and the risks of viral transmission Arch Dis Child, 84(5), 381-2

1715 Midodzi, W. K.,Rowe, B. H.,Majaesic, C. M.,Saunders, L. D.,Senthilselvan, A. (2008). Predictors for wheezing phenotypes in the first Outcome decade of life Respirology, 13(4), 537-45 
1716 Midodzi, W. K.,Rowe, B. H.,Majaesic, C. M.,Saunders, L. D.,Senthilselvan, A. (2010). Early life factors associated with incidence of physician-diagnosed asthma in preschool children: results from the Canadian Early Childhood Development cohort study $\mathrm{J}$ Asthma, $47(1), 7-13$

1717 Midtvedt, A. C.,Midtvedt, T. (1992). Production of short chain fatty acids by the intestinal microflora during the first 2 years of human life J Pediatr Gastroenterol Nutr, 15(4), 395-403

1718 Midwinter, R. E.,Morris, A. F.,Colley, J. R. (1987). Infant feeding and atopy Arch Dis Child, 62(9), 965-7

1719 Mihrshahi, S.,Ampon, R.,Webb, K.,Almqvist, C.,Kemp, A. S.,Hector, D.,Marks, G. B. (2007). The association between infant feeding Outcome practices and subsequent atopy among children with a family history of asthma Clin Exp Allergy, 37(5), 671-9

1720 Mihrshahi, S.,Battistutta, D.,Magarey, A.,Daniels, L. A. (2011). Determinants of rapid weight gain during infancy: baseline results from the NOURISH randomised controlled trial BMC Pediatr, 11(\#issue\#), 99 1721 Mikiel-Kostyra, K.,Mazur, J. (1999). Hospital policies and their influence on newborn body weight Acta Paediatr, 88(1), 72-5

Intervention/exposure

Study design,

Intervention/exposure

1722 Milaat, W. A.,Elassouli, S. M. (1995). Epidemiology of diarrhoea in two major cities in Saudi Arabia J Commun Dis, 27(2), 84-91

Study design, Participant health

1723 Milankov, O.,Bjelica, M.,Savic, R. (2014). What kind of milk can prevent infant's sideropenic anemia--comparative study Med Pregl

Study design, Participant health $67(5-6), 167-71$

1724 Miliku, K., Voortman, T.,Bakker, H.,Hofman, A.,Franco, O. H.,Jaddoe, V. W. (2015). Infant Breastfeeding and Kidney Function in School-Aged Children Am J Kidney Dis, 66(3), 421-8

1725 Miljanovic, O.,Cikota-Aleksic, B.,Likic, D.,Vojvodic, D.,Jovicevic, O.,Magic, Z. (2016). Association of cytokine gene polymorphisms and risk factors with otitis media proneness in children Eur J Pediatr, \#volume\#(\#issue\#), \#Pages\#

\begin{tabular}{|c|c|c|}
\hline 1726 & Millard, A. V.,Graham, M. A. (1985). Abrupt weaning reconsidered: evidence from central Mexico J Trop Pediatr, 31(4), 229-34 & Study design, Outcome \\
\hline 1727 & $\begin{array}{l}\text { Miller, J. E. (2001). Predictors of asthma in young children: does reporting source affect our conclusions? Am J Epidemiol, 154(3), } \\
245-50\end{array}$ & Outcome \\
\hline 1728 & $\begin{array}{l}\text { Miller, M. R.,Seifert, J.,Szabo, N. J.,Clare-Salzler, M., Rewers, M.,Norris, J. M. (2010). Erythrocyte membrane fatty acid content in } \\
\text { infants consuming formulas supplemented with docosahexaenoic acid (DHA) and arachidonic acid (ARA): an observational study } \\
\text { Matern Child Nutr, 6(4), 338-46 }\end{array}$ & Intervention/exposure \\
\hline 1729 & Mills, A. F. (1990). Surveillance for anaemia: risk factors in patterns of milk intake Arch Dis Child, 65(4), $428-31$ & $\begin{array}{l}\text { Study design, Size of study } \\
\text { groups }\end{array}$ \\
\hline 1730 & $\begin{array}{l}\text { Mills, R. P. (1987). Persistent middle ear effusions in children with recurrent acute otitis media Clin Otolaryngol Allied Sci, 12(2), 97- } \\
101\end{array}$ & Participant health \\
\hline
\end{tabular}


1731 Milner, J. D.,Stein, D. M.,McCarter, R.,Moon, R. Y. (2004). Early infant multivitamin supplementation is associated with increased risk for food allergy and asthma Pediatrics, 114(1), 27-32

1732 Milnes, A. R.,Bowden, G. H. (1985). The microflora associated with developing lesions of nursing caries Caries Res, 19(4), 289-97

Study design, Size of study groups

1733 Mimouni-Bloch, A.,Kachevanskaya, A.,Mimouni, F. B.,Shuper, A.,Raveh, E.,Linder, N. (2013). Breastfeeding may protect from

Outcome developing attention-deficit/hyperactivity disorder Breastfeed Med, 8(4), 363-7

\begin{tabular}{|c|c|c|}
\hline 1734 & Minchin, M. (1987). Infant formula: a mass, uncontrolled trial in perinatal care Birth, 14(1), $25-35$ & Study design \\
\hline 1735 & Minchin, M. (2000). Artificial feeding and risk Pract Midwife, 3(3), 18-20 & Study design \\
\hline 1736 & $\begin{array}{l}\text { Mindru, D. E.,Moraru, E. (2012). Risk factors and their implications in the epidemiology of pediatric obesity Rev Med Chir Soc Med } \\
\text { Nat lasi, 116(3), 739-45 }\end{array}$ & Study design \\
\hline
\end{tabular}

1737 Minet, J. C.,Bisse, E.,Aebischer, C. P.,Beil, A.,Wieland, H.,Lutschg, J. (2000). Assessment of vitamin B-12, folate, and vitamin B-6 Study design status and relation to sulfur amino acid metabolism in neonates Am J Clin Nutr, 72(3), 751-7

1738 Miranda, B. H.,Milroy, C. J. (2010). A quick snip - A study of the impact of outpatient tongue tie release on neonatal growth and Intervention/exposure breastfeeding J Plast Reconstr Aesthet Surg, 63(9), e683-5

1739 Miskelly, F. G.,Burr, M. L.,Vaughan-Williams, E.,Fehily, A. M.,Butland, B. K.,Merrett, T. G. (1988). Infant feeding and allergy Arch Outcome Dis Child, 63(4), 388-93

1740 Misra, S.,Sabui, T. K.,Basu, S.,Pal, N. (2007). A prospective study of rotavirus diarrhea in children under 1 year of age Clin Pediatr Country (Phila), 46(8), 683-8

1741 Mitchell, E. A.,Blair, P. S. (2012). SIDS prevention: 3000 lives saved but we can do better N Z Med J, 125(1359), 50-7

Study design

1742 Mitchell, E. A.,Esmail, A.,Jones, D. R.,Clements, M. (1996). Do differences in the prevalence of risk factors explain the higher mortality from sudden infant death syndrome in New Zealand compared with the UK? N Z Med J, 109(1030), 352-5

Study design

1743 Mitchell, E. A.,Scragg, R.,Stewart, A. W.,Becroft, D. M.,Taylor, B. J.,Ford, R. P.,Hassall, I. B.,Barry, D. M.,Allen, E. M.,Roberts, A. P. Outcome (1991). Results from the first year of the New Zealand cot death study N Z Med J, 104(906), 71-6

1744 Mitchell, E. A.,Stewart, A. W.,Scragg, R.,Ford, R. P.,Taylor, B. J.,Becroft, D. M.,Thompson, J. M.,Hassall, I. B.,Barry, D. M.,Allen, E. M.,et al., (1993). Ethnic differences in mortality from sudden infant death syndrome in New Zealand BMJ, 306(6869), 13-6

Study design, Intervention/exposure

1745 Mitchell, E. A.,Thompson, J. M. (2001). Parental reported apnoea, admissions to hospital and sudden infant death syndrome Acta Study design, Paediatr, 90(4), 417-22

1746 Mitchell, E. A.,Tuohy, P. G.,Brunt, J. M.,Thompson, J. M.,Clements, M. S.,Stewart, A. W.,Ford, R. P.,Taylor, B. J. (1997). Risk Intervention/exposure factors for sudden infant death syndrome following the prevention campaign in New Zealand: a prospective study Pediatrics, 100(5), $835-40$

1747 Mittal, S. K. (1988). Bowel pattern and weight gain in breast fed infants Indian Pediatr, 25(2), 216-7 
1748 Mittal, S. K.,Kanwar, A.,Varghese, A.,Ramachandran, V. G. (1983). Gut flora in breast and bottle fed infants with and without diarrhea Indian Pediatr, 20(1), 21-6

1749 Miyake, Y.,Tanaka, K.,Sasaki, S.,Kiyohara, C.,Ohya, Y.,Fukushima, W.,Yokoyama, T.,Hirota, Y. (2008). Breastfeeding and the risk of wheeze and asthma in Japanese infants: the Osaka Maternal and Child Health Study Pediatr Allergy Immunol, 19(6), 490-6

1750 Miyake, Y.,Tanaka, K.,Sasaki, S.,Kiyohara, C.,Ohya, Y.,Fukushima, W.,Yokoyama, T.,Hirota, Y. (2009). Breastfeeding and atopic eczema in Japanese infants: The Osaka Maternal and Child Health Study Pediatr Allergy Immunol, 20(3), 234-41

1751 Miyamoto, S.,Murotani, K.,Yanagawa, T.,Kato, A.,Matsunaga, S. (2010). Relationship of low lean body mass with body weight increase until one year of age and current lifestyles in Japanese young women J Hum Ergol (Tokyo), 39(1), 45-51

Country

Mize, C. E.,Uauy, R.,Kramer, R.,Benser, M.,Allen, S.,Grundy, S. M. (1995). Lipoprotein-cholesterol responses in healthy infants fed defined diets from ages 1 to 12 months: comparison of diets predominant in oleic acid versus linoleic acid, with parallel observations in infants fed a human milk-based diet J Lipid Res, 36(6), 1178-87

1753 Mizuno, K.,Ueda, A., Takeuchi, T. (2002). Effects of different fluids on the relationship between swallowing and breathing during nutritive sucking in neonates Biol Neonate, 81(1), 45-50

1754 Modi, N.,Thomas, E. L.,Harrington, T. A.,Uthaya, S.,Dore, C. J.,Bell, J. D. (2006). Determinants of adiposity during preweaning postnatal growth in appropriately grown and growth-restricted term infants Pediatr Res, 60(3), 345-8

1755 Moimaz, S. A.,Garbin, A. J.,Lima, A. M.,Lolli, L. F.,Saliba, O.,Garbin, C. A. (2014). Longitudinal study of habits leading to malocclusion development in childhood BMC Oral Health, 14(\#issue\#), 96

1756 Mok, J. Y.,Simpson, H. (1982). Outcome of acute lower respiratory tract infection in infants: preliminary report of seven-year followup study Br Med J (Clin Res Ed), 285(6338), 333-7

1757 Molgaard, C.,Larnkjaer, A.,Mark, A. B.,Michaelsen, K. F. (2011). Are early growth and nutrition related to bone health in adolescence? The Copenhagen Cohort Study of infant nutrition and growth Am J Clin Nutr, 94(6 Suppl), 1865S-1869S

1758 Molla, A. M.,Badawi, M. H.,Al-Yaish, S.,Sharma, P.,El-Salam, R. S.,Molla, A. M. (2000). Risk factors for nutritional rickets among children in Kuwait Pediatrics International, 42(3), 280-284

1759 Mollborg, P.,Wennergren, G.,Almqvist, P.,Alm, B. (2015). Bed sharing is more common in sudden infant death syndrome than in explained sudden unexpected deaths in infancy Acta Paediatr, 104(8), 777-83

\begin{tabular}{|c|c|c|}
\hline 1760 & Molokhia, E. A.,Perkins, A. (2008). Preventing cancer Prim Care, 35(4), 609-23 & Study design \\
\hline 1761 & $\begin{array}{l}\text { Monobe, H.,Ishibashi, T.,Fujishiro, Y.,Shinogami, M.,Yano, J. (2003). Factors associated with poor outcome in children with acute } \\
\text { otitis media Acta Otolaryngol, 123(5), 564-8 }\end{array}$ & Study design \\
\hline 1762 & Monson, T. P. (1986). Pediatric viral gastroenteritis Am Fam Physician, 34(1), 95-9 & Study design \\
\hline 1763 & Montagu, A. (1984). The skin, touch, and human development Clin Dermatol, 2(4), 17-26 & Study design \\
\hline
\end{tabular}


1764 Monte, W. C.,Johnston, C. S.,Roll, L. E. (1994). Bovine serum albumin detected in infant formula is a possible trigger for insulindependent diabetes mellitus J Am Diet Assoc, 94(3), 314-6

1765 Montefort, S.,Muscat, H. A.,Caruana, S.,Lenicker, H. (2002). Allergic conditions in 5-8-year-old Maltese schoolchildren: prevalence, Study design severity, and associated risk factors [ISAAC] Pediatr Allergy Immunol, 13(2), 98-104

1766 Monterrosa, E. C.,Frongillo, E. A.,Vasquez-Garibay, E. M.,Romero-Velarde, E.,Casey, L. M.,Willows, N. D. (2008). Predominant Intervention/exposure breast-feeding from birth to six months is associated with fewer gastrointestinal infections and increased risk for iron deficiency among infants J Nutr, 138(8), 1499-504

1767 Montgomery, S. M.,Ehlin, A.,Sacker, A. (2006). Breast feeding and resilience against psychosocial stress Arch Dis Child, 91(12), $990-4$

1768 Moodley, A.,Spector, S. A. (2015). Single high-dose vitamin D at birth corrects vitamin D deficiency in infants in Mexico Int J Food Sci Nutr, 66(3), 336-41

1769 Moon, R. Y.,Tanabe, K. O.,Yang, D. C., Young, H. A.,Hauck, F. R. (2012). Pacifier use and SIDS: evidence for a consistently reduced risk Maternal and child health journal, 16(3), 609-614

1770 Moore, Elizabeth R. (2013). Early Skin-To-Skin Contact for Mothers and Their Healthy Newborn Infants JOGNN: Journal of Obstetric, Gynecologic \& Neonatal Nursing, 42(\#issue\#), S86-S86 1p

1771 Moore, S. R.,Lima, N. L.,Soares, A. M.,Oria, R. B.,Pinkerton, R. C.,Barrett, L. J.,Guerrant, R. L.,Lima, A. A. (2010). Prolonged episodes of acute diarrhea reduce growth and increase risk of persistent diarrhea in children Gastroenterology, 139(4), 1156-64

1772 Moore, W. J.,Midwinter, R. E.,Morris, A. F.,Colley, J. R.,Soothill, J. F. (1985). Infant feeding and subsequent risk of atopic eczema Arch Dis Child, 60(8), 722-6

1773 Mora Urda, A. I.,Pereira da Silva, R.,Bisi Molina Mdel, C.,Bresciani Salaroli, L.,Montero Lopez Mdel, P. (2015). [RELATIONSHIP BETWEEN PATTERNS OF BREASTFEEDING AND BLOOD PRESSURE IN BRAZILIAN AND SPANISH SCHOOLCHILDREN] NUT Hosp, 32(4), 1568-75

1774 Moraeus, L.,Lissner, L.,Yngve, A.,Poortvliet, E.,Al-Ansari, U.,Sjoberg, A. (2012). Multi-level influences on childhood obesity in Sweden: societal factors, parental determinants and child's lifestyle Int J Obes (Lond), 36(7), 969-76

1775 Morale, S. E.,Hoffman, D. R.,Castañeda, Y. S.,Wheaton, D. H.,Burns, R. A.,Birch, E. E. (2005). Duration of long-chain polyunsaturated fatty acids availability in the diet and visual acuity Early Human Development, 81(2), 197-203

1776 Morales, E.,Bustamante, M.,Gonzalez, J. R.,Guxens, M.,Torrent, M.,Mendez, M.,Garcia-Esteban, R.,Julvez, J.,Forns, J.,Vrijheid, M.,Molto-Puigmarti, C.,Lopez-Sabater, C.,Estivill, X.,Sunyer, J. (2011). Genetic variants of the FADS gene cluster and ELOVL gene family, colostrums LC-PUFA levels, breastfeeding, and child cognition PLoS One, 6(2), e17181

1777 Morales, E.,Garcia-Esteban, R.,Guxens, M.,Guerra, S.,Mendez, M.,Molto-Puigmarti, C.,Lopez-Sabater, M. C.,Sunyer, J. (2012). Effects of prolonged breastfeeding and colostrum fatty acids on allergic manifestations and infections in infancy Clin Exp Allergy, 42(6), 918-28 

study

1779 Moreno, M. (2014). Early infant feeding and obesity risk JAMA Pediatr, 168(11), 1084

Study design

1780 Morgan, C.,Davies, L.,Corcoran, F.,Stammers, J.,Colley, J.,Spencer, S. A.,Hull, D. (1998). Fatty acid balance studies in term infants fed formula milk containing long-chain polyunsaturated fatty acids Acta Paediatr, 87(2), 136-42

1781 Morgan, J. B.,Mumford, P. M. (1980). A follow-up study of nutrition and anthropometry in pre-school children Proc Nutr Soc, 39(1), $5 \mathrm{~A}$

1782 Morgan, J.,Taylor, A.,Fewtrell, M. (2004). Meat consumption is positively associated with psychomotor outcome in children up to 24 months of age J Pediatr Gastroenterol Nutr, 39(5), 493-8

1783 Morin, K. H. (2009). Breastfeeding immediately after birth MCN Am J Matern Child Nurs, 34(1), 63

Size of study groups

Publication status

Publication date for a non-sibling study

Study design

1784 Morley, R. (1998). Iron supplemented follow-on formula and growth and development: a randomised trial [abstract] Proc Nutr Soc

Publication status

Aust, 22(\#issue\#), 288

1785 Morley-Peet, P. (1983). Enteropathogenic Escherichia coli Nurs Times, 79(23), 24-7

Study design

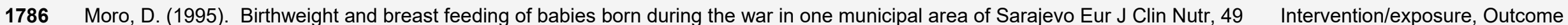
Suppl 2(\#issue\#), S37-9

1787 Morris, S. S.,Grantham-McGregor, S. M.,Lira, P. I.,Assuncao, A. M.,Ashworth, A. (1999). Effect of breastfeeding and morbidity on Intervention/exposure the development of low birthweight term babies in Brazil Acta Paediatr, 88(10), 1101-6

1788 Morrow, A. L. (2011). Infant feeding in the 21st century J Pediatr Health Care, 25(3), 195-7

Study design, Outcome

1789 Morrow, A. L., Guerrero, M. L. (2001). From bioactive substances to research on breast-feeding promotion Adv Exp Med Biol, 501(\#issue\#), 447-55

1790 Morrow, A. L.,Reves, R. R.,West, M. S.,Guerrero, M. L.,Ruiz-Palacios, G. M.,Pickering, L. K. (1992). Protection against infection with Giardia lamblia by breast-feeding in a cohort of Mexican infants J Pediatr, 121(3), 363-70

1791 Morrow-Tlucak, M.,Haude, R. H.,Ernhart, C. B. (1988). Breastfeeding and cognitive development in the first 2 years of life Soc Sci Outcome Med, 26(6), 635-9

1792 Mortensen, E. L.,Michaelsen, K. F.,Sanders, S. A.,Reinisch, J. M. (2002). The association between duration of breastfeeding and Outcome adult intelligence JAMA, 287(18), 2365-71

1793 Moschonis, G.,Grammatikaki, E.,Manios, Y. (2008). Perinatal predictors of overweight at infancy and preschool childhood: the GENESIS study Int J Obes (Lond), 32(1), 39-47

1794 Mo-Suwan, L.,Junjana, C. (1991). Breast-feeding and infant growth in the first six months J Med Assoc Thai, 74(9), 386-90

Study design

Study design,

Intervention/exposure

Intervention/exposure 
1795 Motil, K. J.,Sheng, H. P.,Montandon, C. M.,Wong, W. W. (1997). Human milk protein does not limit growth of breast-fed infants J Pediatr Gastroenterol Nutr, 24(1), 10-7

1796 Motta, M.,Tincani, A.,Faden, D.,Zinzini, E.,Lojacono, A.,Marchesi, A.,Frassi, M.,Biasini, C.,Zatti, S.,Chirico, G. (2005). Follow-up of Size of study groups infants exposed to hydroxychloroquine given to mothers during pregnancy and lactation J Perinatol, 25(2), 86-9

1797 Moxley, S.,Avni, G.,Brydon, S.,Kennedy, M. (1998). Breastfeeding and shorter hospital stays Can Nurse, 94(7), 35-9

Study design

1798 Mueller, W. H.,Pollitt, E. (1982). The Bacon Chow study: effects of nutrition supplementation on sibling-sibling anthropometric correlations Hum Biol, 54(3), 455-68

1799 Mughal, M. Z.,Salama, H.,Greenaway, T.,Laing, I.,Mawer, E. B. (1999). Lesson of the week: florid rickets associated with prolonged Study design breast feeding without vitamin D supplementation Bmj, 318(7175), 39-40

1800 Mughini-Gras, L.,Pijnacker, R.,Heusinkveld, M.,Enserink, R.,Zuidema, R.,Duizer, E.,Kortbeek, T.,van Pelt, W. (2016). Societal Burden and Correlates of Acute Gastroenteritis in Families with Preschool Children Sci Rep, 6(\#issue\#), 22144

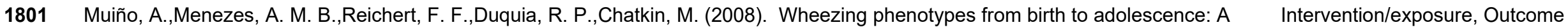
cohort study in Pelotas, Brazil, 1993-2004 Jornal Brasileiro de Pneumologia, 34(6), 347-355

1802 Muirhead, P. (1998). A randomized controlled study of the effect of organised peer support on the duration of breast feeding and the Study design consequences for infant morbidity Personal communication, \#volume\#(\#issue\#), \#Pages\#

1803 Mukherjee, D.,Stephens, D. (1997). Otitis media with effusion in intellectually disabled children Journal of Audiological Medicine, $6(1), 10-23$

1804 Mukhopadhya, J. (2001). Acute Respiratory Infection among children in an Air Force Community Medical Journal Armed Forces India, 57(4), 309-311

1805 Mukhopadhyay, S.,Lieberman, E. S.,Puopolo, K. M.,Riley, L. E.,Johnson, L. C. (2015). Effect of early-onset sepsis evaluations on in-hospital breastfeeding practices among asymptomatic term neonates Hosp Pediatr, 5(4), 203-10

1806 Mulhall AL (1984). Breast feeding: a challenge for midwives World Ir Nurs, 13(\#issue\#), 8-9

Study design

ntervention/exposure

Country

Outcome

Publication status

1807 Muller, M. (1996). Nursing-bottle syndrome: risk factors ASDC J Dent Child, 63(1), 42-50 Study design

1808 Mulrine, H. M.,Skeaff, S. A.,Ferguson, E. L.,Gray, A. R.,Valeix, P. (2010). Breast-milk iodine concentration declines over the first 6 mo postpartum in iodine-deficient women Am J Clin Nutr, 92(4), 849-56

1809 Munir M,Mustadjab I,Rampengan TH,Wulur FH (1983). Problem of infant feeding practices: implications for immediate action Paediatr Indones, 23(\#issue\#), 32-46

1810 Munir, M. (1985). Infantile diarrhoea: breast and bottle feeding compared with special reference to their clinical role Paediatr Indones, 25(5-6), 100-6 
1811 Muniz, L. C.,Menezes, A. M.,Assuncao, M. C.,Wehrmeister, F. C.,Martinez-Mesa, J.,Goncalves, H.,Domingues, M. R.,Gigante, D. P.,Horta, B. L.,Barros, F. C. (2015). Breastfeeding and bone mass at the ages of 18 and 30: prospective analysis of live births from the Pelotas (Brazil) 1982 and 1993 cohorts PLoS One, 10(4), e0122759

1812 Munns, C. F.,Simm, P. J.,Rodda, C. P.,Garnett, S. P.,Zacharin, M. R.,Ward, L. M.,Geddes, J.,Cherian, S.,Zurynski, Y.,Cowell, C. T. (2012). Incidence of vitamin D deficiency rickets among Australian children: an Australian Paediatric Surveillance Unit study Med J Aust, 196(7), 466-8

1813 Murdoch, W. (1980). Breast feeding Cent Afr J Med, 26(4), 95-7

1814 Murphy RM (1981). The hidden epidemic Can Nurse, 77(\#issue\#), 42-3

Participant health, Intervention/exposure

Study design

Study design

1815 Murrell, W. G.,Stewart, B. J.,O'Neill, C.,Siarakas, S.,Kariks, S. (1993). Enterotoxigenic bacteria in the sudden infant death syndrome Journal of Medical Microbiology, 39(2), 114-127

1816 Musaad, S. M.,Donovan, S. M.,Fiese, B. H. (2015). Parental perception of child weight in the first two years-of-life: a potential link between infant feeding and preschoolers' diet Appetite, 91(\#issue\#), 90-100

1817 Myres AW (1983). The national breast-feeding promotion program. Part 2. Public information phase--a note on its development, distribution and impact Can J Public Health, 74(\#issue\#), 404-8

1818 Myres AW,Watson J,Harrison C (1981). The national breast-feeding promotion program 1. Professional phase--a note on its development, distribution and impact Can J Public Health, 72(\#issue\#), 307-11

1819 Myres, A. W. (1988). Tradition and technology in infant feeding--achieving the best of both worlds Can J Public Health, 79(2), 78-80 Study design

1820 Nafstad, P.,Jaakkola, J. J.,Hagen, J. A.,Botten, G.,Kongerud, J. (1996). Breastfeeding, maternal smoking and lower respiratory tract Outcome infections Eur Respir J, 9(12), 2623-9

1821 Nafstad, P.,Jaakkola, J. J.,Hagen, J. A.,Pedersen, B. S.,Qvigstad, E.,Botten, G.,Kongerud, J. (1997). Weight gain during the firs year of life in relation to maternal smoking and breast feeding in Norway J Epidemiol Community Health, 51(3), 261-5

Publication date for a non-sibling study

1822 Nagahara, K.,Dobashi, K.,Itabashi, K. (2013). Feeding choice has a gender-associated effect on infant growth Pediatr Int, 55(4), $481-7$

1823 Nagendra, R.,Viswanatha, S.,Arun Kumar, S.,Krishna Murthy, B.,Venkat Rao, S. (1995). Effect of feeding milk formula containing lactulose to infants on faecal bifidobacterial flora Nutrition Research, 15(1), 15-24

1824 Naggan, L.,Forman, M. R.,Sarov, B.,Lewando-Hundt, G.,Zangwill, L.,Chang, D.,Berendes, H. W. (1991). The Bedouin Infant Feeding Study: study design and factors influencing the duration of breast feeding Paediatr Perinat Epidemiol, 5(4), 428-44

1825 Najada, A. S.,Habashneh, M. S.,Khader, M. (2004). The frequency of nutritional rickets among hospitalized infants and its relation to Study design, Participant health respiratory diseases J Trop Pediatr, 50(6), 364-8 
1826 Nakamura, Y Oki, I.,Tanihara, S.,Ojima, T.,Ito, Y Yamazaki, O.,Iwama, M., Tabata, Y.,Katsuyama, K., Sasai, Y.,Nakagawa,

M.,Matsushita, A.,Hossaka, K.,Sato, J.,Hidaka, Y.,Uda, H.,Nakamata, K.,Yanagawa, H. (2000). Relationship between breast milk feeding and atopic dermatitis in children J Epidemiol, 10(2), 74-8

1827 Nakao H (1988). Nutritional significance of human milk vitamin D in neonatal period Kobe J Med Sci, 34(\#issue\#), 121-8

1828 Nakao, R. M. (1988). Effects of an education program on the health and illness profile of rural breast-fed babies Philipp J Nurs, $58(2), 12-8$

1829 Nambiar, H. K. (1984). Acute diarrhoeal diseases: a malady in children Nurs J India, 75(8), 179

Study design

1830 Nambiar, Smita,Truby, Helen,Davies, Peter S. W. (2013). Exploring the influence of breastfeeding on abdominal adiposity in young Study design children using the waist to height ratio Nutrition \& Dietetics, 70(2), 146-152 7p

1831 Narayan, N. R.,Mendez-Lagares, G.,Ardeshir, A.,Lu, D.,Van Rompay, K. K.,Hartigan-O'Connor, D. J. (2015). Persistent effects of Non-human sample early infant diet and associated microbiota on the juvenile immune system Gut Microbes, 6(4), 284-9

1832 Narayanan, I.,Gupta, J. (1989). Human milk and neonatal infections Acta Paediatr Scand Suppl, 351(\#issue\#), 126-30

Country, Outcome

1833 Narayanan, I.,Prakash, K.,Murthy, N. S.,Gujral, V. V. (1984). Randomised controlled trial of effect of raw and holder pasteurised Country human milk and of formula supplements on incidence of neonatal infection Lancet, 2(8412), 1111-3

1834 Narayanan, I.,Singh, S.,Mathur, R.,Jain, B. K. (1989). Ear infection and infant feeding practices Indian J Pediatr, 56(3), 399-402 Country

1835 Narchi, H.,Kochiyil, J.,Zayed, R.,Abdulrazzak, W.,Agarwal, M. (2010). Maternal vitamin D status throughout and after pregnancy J Outcome Obstet Gynaecol, 30(2), 137-42

1836 Narchi, H.,Kochiyil, J.,Zayed, R.,Abdulrazzak, W.,Agarwal, M. (2011). Longitudinal study of vitamin D status in the 1st 6 months of life Ann Trop Paediatr, 31(3), 225-30 center of the femoral head in breast-fed versus formula-fed infants Nutrition, 27(11-12), 1108-11

1838 Nascimento Souza, Maria Helena,Aparecida Barbosa Nogueira, Josiê Neiber,Domingues Sodré, Vitória Regina (2015). MONITORING THE NUTRITIONAL AND HEALTH STATUS OF CHILDREN WHO ATTEND A COMMUNITY NURSERY JOURnal of Nursing UFPE / Revista de Enfermagem UFPE, 9(5), 7862-7868 7p

1839 Nassar, M. F.,Younis, N. T.,El-Arab, S. E.,Fawzi, F. A. (2011). Neuro-developmental outcome and brain-derived neurotrophic factor level in relation to feeding practice in early infancy Matern Child Nutr, 7(2), 188-97

Study design, Size of study groups

Study design

Study design,

Intervention/exposure

Study design, Size of study groups

1840 Nauta, A. (2012). Specific nutritional concepts \& clinical evidence in the management of allergy Asian Pacific Journal of Allergy and Study design Immunology, 30(4 SUPPL), S21-S24

1841 Navarro, J. I.,Sigulem, D. M.,Ferraro, A. A.,Polanco, J. J.,Barros, A. J. (2013). The double task of preventing malnutrition and overweight: a quasi-experimental community-based trial BMC Public Health, 13(\#issue\#), 212 
1842 Nelson, C. M.,Innis, S. M. (1999). Plasma lipoprotein fatty acids are altered by the positional distribution of fatty acids in infant formula triacylglycerols and human milk Am J Clin Nutr, 70(1), 62-9

1843 Nelson, C. M.,Innis, S. M.,Walsen, P.,Whitfield, M. (2002). Prospective measures of visual and cognitive development in term gestation breast-fed and formula-fed infants to 18 months of age Pediatric research, 2(\#issue\#), 315a

1844 Nelson, E. A.,Yu, L. M.,Wong, D.,Wong, H. Y.,Yim, L. (2004). Rolling over in infants: age, ethnicity, and cultural differences Dev Med Child Neurol, 46(10), 706-9

1845 Nelson, J. D. (1985). Prevention of gastrointestinal infections Pediatr Infect Dis, 4(4), 431-4

$$
431-4
$$

. Gain in weight and length during early infancy Early Hum Dev, 19(4),

Nelson, 223-39 of mutans streptococci in infants Eur J Oral Sci, 122(5), 346-52

1848 Nentwich, I.,Michkova, E.,Nevoral, J.,Urbanek, R.,Szepfalusi, Z. (2001). Cow's milk-specific cellular and humoral immune responses and atopy skin symptoms in infants from atopic families fed a partially (pHF) or extensively (eHF) hydrolyzed infant formula Allergy 56(12), 1144-56

1849 Nery Cde, G.,Buranello, F. S.,Pereira, C.,Di Francesco, R. C. (2010). Otitis media with effusion and dental occlusion: is there any relationship? Eur J Paediatr Dent, 11(3), 132-6

1850 Neutzling, M. B.,Hallal, P. R.,Araujo, C. L.,Horta, B. L.,Vieira Mde, F.,Menezes, A. M.,Victora, C. G. (2009). Infant feeding and obesity at 11 years: prospective birth cohort study Int J Pediatr Obes, 4(3), 143-9

1851 Neves, A. B. Lobo, L. A.,Pinto, K. C.,Pires, E. S.,Requejo, M.,Maia, L. C.,Antonio, A. G. (2015). Comparison between Clinical Aspects and Salivary Microbial Profile of Children with and without Early Childhood Caries: A Preliminary Study J Clin Pediatr Dent 39(3), 209-14

1852 Newburg, D. S.,Ruiz-Palacios, G. M.,Altaye, M.,Chaturvedi, P.,Guerrero, M. L.,Meinzen-Derr, J. K.,Morrow, A. L. (2004). Human milk alphal,2-linked fucosylated oligosaccharides decrease risk of diarrhea due to stable toxin of E. coli in breastfed infants Adv Exp Med Biol, 554(\#issue\#), 457-61

1853 Newman, J. (1995). How breast milk protects newborns Sci Am, 273(6), 76-9

1854 Ng, S. C.,Chong, Y. S.,Rauff, M.,Myo, Z. M.,Nurfarah, C.,Deurenberg, P. R. (2004). The influence of breast feeding compared to formula feeding on infant adiposity Ann Acad Med Singapore, 33(5 Suppl), S75

1855 Ng, S. C., Tang, W.,Leong, R. W.,Chen, M.,Ko, Y.,Studd, C.,Niewiadomski, O.,Bell, S.,Kamm, M. A., de Silva, H. J.,Kasturiratne, A.,Senanayake, Y. U.,Ooi, C. J.,Ling, K. L.,Ong, D.,Goh, K. L.,Hilmi, I.,Ouyang, Q.,Wang, Y. F.,Hu, P.,Zhu, Z.,Zeng, Z.,Wu,

K.,Wang, X.,Xia, B.,Li, J.,Pisespongsa, P.,Manatsathit, S.,Aniwan, S.,Simadibrata, M.,Abdullah, M.,Tsang, S. W.,Wong, T. C.,Hui, A.

J.,Chow, C. M.,Yu, H. H.,Li, M. F.,Ng, K. K.,Ching, J.,Wu, J. C.,Chan, F. K.,Sung, J. J. (2015). Environmental risk factors in

inflammatory bowel disease: a population-based case-control study in Asia-Pacific Gut, 64(7), 1063-71
Size of study groups

Publication status

Size of study groups

Study design,

Intervention/exposure

Outcome, Publication date for non-sibling study

Outcome

Size of study groups

Participant health, Intervention/exposure

Publication date for a non-sibling study

Study design, Size of study groups

Intervention/exposure

\section{Publication status}

Intervention/exposure 
1856 Ngale, K. M.,Santos, I. S.,Gonzalez-Chica, D. A., de Barros, A. J.,Matijasevich, A. (2013). Bed-sharing and risk of hospitalisation due Outcome to pneumonia and diarrhoea in infancy: the 2004 Pelotas Birth Cohort J Epidemiol Community Health, 67(3), 245-9

1857 Ngamphaiboon, J. (2014). Food allergy and wheezing Southeast Asian J Trop Med Public Health, 45 Suppl 1(\#issue\#), 95-9 Study design

1858 Ngamphaiboon, J.,Tansupapol, C.,Chatchatee, P. (2009). The efficacy of partially hydrolyzed formulas for allergy prevention in children under five years Asian Biomedicine, 3(3), 245-254

1859 Nguyen, N. D.,Allen, J. R.,Peat, J. K.,Beal, P.,Webster, B. H.,Gaskin, K. J. (2004). Iron status of young Vietnamese children in Australia J Paediatr Child Health, 40(8), 424-9

1860 Nguyen, N. D.,Allen, J. R.,Peat, J. K.,Schofield, W. N.,Nossar, V.,Eisenbruch, M.,Gaskin, K. J. (2004). Growth and feeding practices Intervention/exposure of Vietnamese infants in Australia Eur J Clin Nutr, 58(2), 356-62

1861 Nicolai, A.,Nenna, R.,Stefanelli, P.,Carannante, A.,Schiavariello, C.,Pierangeli, A.,Scagnolari, C.,Moretti, C.,Papoff, P.,Bonci, E.,Ferrara, M.,Papasso, S.,Midulla, F. (2013). Bordetella pertussis in infants hospitalized for acute respiratory symptoms remains a concern BMC Infect Dis, 13(\#issue\#), 526

1862 Nicoll, A.,Williams, A. (2002). Breast feeding Arch Dis Child, 87(2), 91-2

1863 Niegel, S.,Ystrom, E.,Hagtvet, K. A.,Vollrath, M. E. (2008). Difficult temperament, breastfeeding, and their mutual prospective effects: the Norwegian Mother and Child Cohort Study J Dev Behav Pediatr, 29(6), 458-62

1864 Nielsen, G. A., Thomsen, B. L.,Michaelsen, K. F. (1998). Influence of breastfeeding and complementary food on growth between 5 and 10 months Acta Paediatr, 87(9), 911-7

1865 Nielsen, S. B.,Reilly, J. J.,Fewtrell, M. S.,Eaton, S.,Grinham, J.,Wells, J. C. (2011). Adequacy of milk intake during exclusive breastfeeding: a longitudinal study Pediatrics, 128(4), e907-14

1866 Niemela, A., Jarvenpaa, A. L. (1996). Is breastfeeding beneficial and maternal smoking harmful to the cognitive development of children? Acta Paediatr, 85(10), 1202-6

1867 Niemela, M.,Uhari, M.,Mottonen, M. (1995). A pacifier increases the risk of recurrent acute otitis media in children in day care centers Pediatrics, 96(5 Pt 1), 884-8

1868 Nikpour, S.,Rahimian, Sh,Shokrabi, S.,Haghani, H. (2012). Related Factors of Acute Leukemia in Children and the Role of Breast Feeding Iranian Journal of Endocrinology \& Metabolism, 14(1), 63-97 35p

1869 Nishimura, M.,Oda, T.,Kariya, N.,Matsumura, S.,Shimono, T. (2008). Using a caries activity test to predict caries risk in early childhood J Am Dent Assoc, 139(1), 63-71

1870 Nishimura, T.,Suzue, J.,Kaji, H. (2009). Breastfeeding reduces the severity of respiratory syncytial virus infection among young infants: a multi-center prospective study Pediatr Int, 51(6), 812-6

1871 Nnanyelugo, D. O. (1982). Nutritional practices and food intake measurements and their relationship to socio-economic grouping, location and their apparent nutritional adequacy in children Appetite, 3(3), 229-41

Study design

Outcome

Study design

ntervention/exposure

Size of study groups, OUtcome

Intervention/exposure

Publication date for a non-sibling study

Outcome

Outcome

Outcome

Language

Outcome

Outcome

Country 
1872 Noda, M.,Sato, N.,Tanaka, T. (2015). Growth failure starts from early infancy in children with short stature at age 6 Clinical Pediatric Study design Endocrinology, 24(1), 1-10

1873 Nolan, L.,Goel, V. (1995). Sociodemographic factors related to breastfeeding in Ontario: results from the Ontario Health Survey Can Study design J Public Health, 86(5), 309-12

1874 Nommsen-Rivers, L. A. (2004). Does breastfeeding protect against infant mortality in the United States? J Hum Lact, 20(3), 357-8 Study design

1875 Nossar, V.,Hudson, D. (2001). Improving health outcomes for children by home visiting Medicine Today, 2(8), 135-136 Study design

1876 Nott, S. (1985). Some faults on feeding Midwife Health Visit Community Nurse, 21(6), 201-2

Study design

1877 Novotny, R.,Daida, Y. G.,Grove, J. S.,Acharya, S.,Vogt, T. M. (2003). Formula feeding in infancy is associated with adolescent body Study design fat and earlier menarche Cell Mol Biol (Noisy-le-grand), 49(8), 1289-93

1878 Novotny, R.,Mata, L. J. (1983). Breast milk consumption in rural Costa Rica Arch Latinoam Nutr, 33(2), 377-86

Size of study groups

1879 Nuesslein, T. G.,Beckers, D.,Rieger, C. H. (1999). Cotinine in meconium indicates risk for early respiratory tract infections Hum Exp Intervention/exposure Toxicol, 18(4), 283-90

1880 Nunes, A. M.,Alves, C. M.,Borba de Araujo, F.,Ortiz, T. M.,Ribeiro, M. R.,Silva, A. A.,Ribeiro, C. C. (2012). Association between prolonged breast-feeding and early childhood caries: a hierarchical approach Community Dent Oral Epidemiol, 40(6), 542-9

1881 Nwaru, B. I.,Craig, L. C.,Allan, K.,Prabhu, N.,Turner, S. W.,McNeill, G.,Erkkola, M.,Seaton, A.,Devereux, G. (2013). Breastfeeding and introduction of complementary foods during infancy in relation to the risk of asthma and atopic diseases up to 10 years Clin Exp Allergy, 43(11), 1263-73

1882 Nwaru, B. I.,Erkkola, M.,Ahonen, S.,Kaila, M.,Haapala, A. M.,Kronberg-Kippila, C.,Salmelin, R., Veijola, R., Ilonen, J.,Simell, O.,Knip, M.,Virtanen, S. M. (2010). Age at the introduction of solid foods during the first year and allergic sensitization at age 5 years Pediatrics, 125(1), 50-9

1883 Nwaru, B. I.,Takkinen, H. M.,Niemela, O.,Kaila, M.,Erkkola, M.,Ahonen, S.,Haapala, A. M.,Kenward, M. G.,Pekkanen, J.,Lahesmaa, R.,Kere, J.,Simell, O.,Veijola, R., Ilonen, J.,Hyoty, H.,Knip, M.,Virtanen, S. M. (2013). Timing of infant feeding in relation to childhood asthma and allergic diseases $\mathrm{J}$ Allergy Clin Immunol, 131(1), 78-86

1884 Nwaru, B. I.,Takkinen, H. M.,Niemela, O.,Kaila, M.,Erkkola, M.,Ahonen, S.,Tuomi, H.,Haapala, A. M.,Kenward, M. G.,Pekkanen, J.,Lahesmaa, R.,Kere, J.,Simell, O.,Veijola, R., Ilonen, J.,Hyoty, H.,Knip, M.,Virtanen, S. M. (2013). Introduction of complementary foods in infancy and atopic sensitization at the age of 5 years: timing and food diversity in a Finnish birth cohort Allergy, 68(4), 50716

1885 Nylander, G.,Lindemann, R.,Helsing, E.,Bendvold, E. (1991). Unsupplemented breastfeeding in the maternity ward. Positive longterm effects Acta Obstet Gynecol Scand, 70(3), 205-9

1886 Obel, C.,Henriksen, T. B.,Hedegaard, M.,Secher, N. J.,Ostergaard, J. (1998). Smoking during pregnancy and babbling abilities of the 8-month-old infant Paediatr Perinat Epidemiol, 12(1), 37-48

Study design

Outcome

Outcome

Outcome

Outcome

Study design,

Intervention/exposure

Intervention/exposure 
1887 Ochoa, M. C.,Moreno-Aliaga, M. J.,Martinez-Gonzalez, M. A Martinez, J. A. Marti, A. (2007). Predictor factors for childhood obesity in a Spanish case-control study Nutrition, 23(5), 379-84

1888 O'Connell, J. M.,Dibley, M. J.,Sierra, J.,Wallace, B.,Marks, J. S.,Yip, R. (1989). Growth of vegetarian children: The Farm Study Pediatrics, 84(3), 475-81

1889 O'Connor, P. A. (1980). Clouds, skin color, and rickets Pediatrics, 66(2), 332

Study design

1890 Oddy, W. H. (2000). Breastfeeding and asthma in children. A prospective cohort study Adv Exp Med Biol, 478(\#issue\#), 393-4

Publication status

1891 Oddy, W. H. (2000). Breastfeeding and asthma in children: findings from a West Australian study Breastfeed Rev, 8(1), 5-11

Redundant data with another article

1892 Oddy, W. H., de Klerk, N. H.,Sly, P. D.,Holt, P. G. (2002). The effects of respiratory infections, atopy, and breastfeeding on childhood Outcome asthma Eur Respir J, 19(5), 899-905

1893 Oddy, W. H.,Halonen, M.,Martinez, F. D.,Lohman, I. C.,Stern, D. A.,Kurzius-Spencer, M.,Guerra, S.,Wright, A. L. (2003). TGF-beta Outcome in human milk is associated with wheeze in infancy J Allergy Clin Immunol, 112(4), 723-8

1894 Oddy, W. H.,Holt, P. G.,Sly, P. D.,Read, A. W.,Landau, L. I.,Stanley, F. J.,Kendall, G. E.,Burton, P. R. (1999). Association between Outcome breast feeding and asthma in 6 year old children: findings of a prospective birth cohort study BMJ, 319(7213), 815-9

1895 Oddy, W. H.,Kendall, G. E.,Blair, E.,de Klerk, N. H.,Silburn, S.,Zubrick, S. (2004). Breastfeeding and cognitive development in children Adv Exp Med Biol, 554(\#issue\#), 365-9

1896 Oddy, W. H.,Kendall, G. E.,Blair, E.,De Klerk, N. H.,Stanley, F. J.,Landau, L. I.,Silburn, S.,Zubrick, S. (2003). Breast feeding and Outcome cognitive development in childhood: a prospective birth cohort study Paediatr Perinat Epidemiol, 17(1), 81-90

1897 Oddy, W. H.,Kendall, G. E.,Li, J.,Jacoby, P.,Robinson, M.,de Klerk, N. H.,Silburn, S. R.,Zubrick, S. R.,Landau, L. I.,Stanley, F. J. (2010). The long-term effects of breastfeeding on child and adolescent mental health: a pregnancy cohort study followed for 14 years J Pediatr, 156(4), 568-74

1898 Oddy, W. H.,Kickett-Tucker, C.,De Maio, J.,Lawrence, D.,Cox, A.,Silburn, S. R.,Stanley, F. J.,Zubrick, S. R. (2008). The association of infant feeding with parent-reported infections and hospitalisations in the West Australian Aboriginal Child Health Survey Aust $\mathrm{N} Z \mathrm{~J}$ Public Health, 32(3), 207-15

1899 Oddy, W. H.,Li, J.,Whitehouse, A. J. O.,Zubrick, S. R.,Malacova, E. (2011). Breastfeeding duration and academic achievement at 10 Outcome years Pediatrics, $127(1)$, e137-e145

1900 Oddy, W. H.,Mori, T. A.,Huang, R. C.,Marsh, J. A.,Pennell, C. E.,Chivers, P. T.,Hands, B. P.,Jacoby, P.,Rzehak, P.,Koletzko, B V.,Beilin, L. J. (2014). Early infant feeding and adiposity risk: From infancy to adulthood Annals of Nutrition and Metabolism, 64(3-4), 262-270

1901 Oddy, W. H.,Peat, J. K., de Klerk, N. H. (2002). Maternal asthma, infant feeding, and the risk of asthma in childhood J Allergy Clin Immunol, 110(1), 65-7 
1902 Oddy, W. H.,Robinson, M.,Kendall, G. E.,Li, J.,Zubrick, S. R.,Stanley, F. J. (2011). Breastfeeding and early child development: a prospective cohort study Acta Paediatr, 100(7), 992-9

1903 Oddy, W. H.,Scott, J. A.,Graham, K. I.,Binns, C. W. (2006). Breastfeeding influences on growth and health at one year of age Breastfeed Rev, 14(1), 15-23

1904 Oddy, W. H.,Sherriff, J. L., de Klerk, N. H.,Kendall, G. E.,Sly, P. D.,Beilin, L. J.,Blake, K. B.,Landau, L. I.,Stanley, F. J. (2004). The relation of breastfeeding and body mass index to asthma and atopy in children: a prospective cohort study to age 6 years Am $\mathrm{J}$ Public Health, 94(9), 1531-7

1905 Oddy, W. H.,Sly, P. D.,de Klerk, N. H.,Landau, L. I.,Kendall, G. E.,Holt, P. G.,Stanley, F. J. (2003). Breast feeding and respiratory morbidity in infancy: a birth cohort study Arch Dis Child, 88(3), 224-8

1906 Oddy, W. H.,Smith, G. J., Jacoby, P. (2014). A possible strategy for developing a model to account for attrition bias in a longitudinal cohort to investigate associations between exclusive breastfeeding and overweight and obesity at 20 years Ann Nutr Metab, 65(2-3), 234-5

1907 Odelram, H.,Vanto, T.,Jacobsen, L.,Kjellman, N. I. (1996). Whey hydrolysate compared with cow's milk-based formula for weaning at about 6 months of age in high allergy-risk infants: effects on atopic disease and sensitization Allergy, 51(3), 192-5

1908 O'Donovan, S. M.,O'B Hourihane J,Murray, D. M.,Kenny, L. C.,Khashan, A. S.,Chaoimh, C. N.,Irvine, A. D.,Kiely, M. (2015). Neonatal adiposity increases the risk of atopic dermatitis during the first year of life $\mathrm{J}$ Allergy Clin Immunol, \#volume\#(\#issue\#), \#Pages\#

1909 Ogawa, K.,Ben, R. A.,Pons, S.,de Paolo, M. I.,Bustos Fernandez, L. (1992). Volatile fatty acids, lactic acid, and pH in the stools of breast-fed and bottle-fed infants J Pediatr Gastroenterol Nutr, 15(3), 248-52

1910 Ogston, S. A.,Florey, C. D.,Walker, C. H. (1987). Association of infant alimentary and respiratory illness with parental smoking and Outcome other environmental factors J Epidemiol Community Health, 41(1), 21-5

1911 Ohlund, I.,Hornell, A.,Lind, T.,Hernell, O. (2008). Dietary fat in infancy should be more focused on quality than on quantity Eur J Clin Outcome Nutr, 62(9), 1058-64

1912 Oken, E.,Osterdal, M. L.,Gillman, M. W.,Knudsen, V. K.,Halldorsson, T. I.,Strom, M.,Bellinger, D. C.,Hadders-Algra, M.,Michaelsen, $\quad$ Outcome K. F.,Olsen, S. F. (2008). Associations of maternal fish intake during pregnancy and breastfeeding duration with attainment of developmental milestones in early childhood: a study from the Danish National Birth Cohort Am J Clin Nutr, 88(3), 789-96 red meat consumption: a randomized trial in Bogota, Colombia Am J Clin Nutr, 98(4), 983-93

1914 Oliveira, A. F.,Chaves, A. M.,Rosenblatt, A. (2006). The influence of enamel defects on the development of early childhood caries in Intervention/exposure a population with low socioeconomic status: a longitudinal study Caries Res, 40(4), 296-302

1915 Oliveira, E. A.,Bertoldi, A. D.,Domingues, M. R.,Santos, I. S.,Barros, A. J. (2012). Factors associated to medicine use among children from the 2004 Pelotas Birth Cohort (Brazil) Rev Saude Publica, 46(3), 487-96

Outcome, Publication date for

Publication date for a non-sibling study

Outcome

Study design

ntervention/exposure

Intervention/exposure

Intervention/exposure

Size of study groups, Outcome

Intervention/exposure

Outcome 
1916 Ollila, P.,Larmas, M. (2007). A seven-year survival analysis of caries onset in primary second molars and permanent first molars in different caries risk groups determined at age two years Acta Odontol Scand, 65(1), 29-35

1917 Ölmez, S.,Uzamiş, M. (2002). Risk factors of early childhood caries in Turkish children Turkish Journal of Pediatrics, 44(3), 230-236 Study design

1918 Olmez, S.,Uzamis, M.,Erdem, G. (2003). Association between early childhood caries and clinical, microbiological, oral hygiene and Study design dietary variables in rural Turkish children Turk J Pediatr, 45(3), 231-6

1919 Olson, C. M.,Baker, I. R.,Demment, M. M.,Graham, M. L.,May, J. J.,Strawderman, M. S.,Wells, N. M. (2014). The healthy start partnership: an approach to obesity prevention in young families Fam Community Health, 37(1), 74-85

1920 Ong, K. K.,Ahmed, M. L.,Sherriff, A.,Woods, K. A.,Watts, A., Golding, J.,Dunger, D. B. (1999). Cord blood leptin is associated with size at birth and predicts infancy weight gain in humans. ALSPAC Study Team. Avon Longitudinal Study of Pregnancy and Childhood J Clin Endocrinol Metab, 84(3), 1145-8

1921 Ong, K. K.,Emmett, P. M.,Noble, S.,Ness, A.,Dunger, D. B. (2006). Dietary energy intake at the age of 4 months predicts postnatal weight gain and childhood body mass index Pediatrics, 117(3), e503-8

1922 Ong, K. K.,Preece, M. A.,Emmett, P. M.,Ahmed, M. L.,Dunger, D. B. (2002). Size at birth and early childhood growth in relation to maternal smoking, parity and infant breast-feeding: longitudinal birth cohort study and analysis Pediatr Res, 52(6), 863-7

Intervention/exposure

Oppitz, I. N.,Cesar, J. A.,Neumann, N. A. (2014). Overweight among children under five years of age in municipalities of the semiarid region Rev Bras Epidemiol, 17(4), 860-72

1924 Orakzai, S. A.,Siddiqui, K. A.,Ayub, M.,Saeed, A. K. (1987). Serum proteins in infants J Pak Med Assoc, 37(10), 251-5

Intervention/exposure

Study design

1925 Orivuori, L.,Loss, G.,Roduit, C.,Dalphin, J. C.,Depner, M.,Genuneit, J.,Lauener, R.,Pekkanen, J.,Pfefferle, P.,Riedler, J.,Roponen, M.,Weber, J.,von Mutius, E.,Braun-Fahrlander, C.,Vaarala, O. (2014). Soluble immunoglobulin A in breast milk is inversely associated with atopic dermatitis at early age: the PASTURE cohort study Clin Exp Allergy, 44(1), 102-12

1926 Orozco, A. C.,Munoz, A. M.,Velasquez, C. M.,Uscategui, R. M.,Parra, M. V.,Patino, F. A.,Manjarres, L. M.,Parra, B. E.,Estrada, A.,Agudelo, G. M. (2014). Variant in CAPN10 gene and environmental factors show evidence of association with excess weight among young people in a Colombian population Biomedica, 34(4), 546-55

1927 Orr P,McDonald S,Milley D,Brown R (2001). Bronchiolitis in Inuit children from a Canadian central arctic community, 1995-1996 Int J Outcome Circumpolar Health, 60(\#issue\#), 649-58 Lopez, L. (2008). Full breastfeeding and paediatric cancer J Paediatr Child Health, 44(1-2), 10-3

1929 O'Ryan, M. L.,Lucero, Y.,Rabello, M.,Mamani, N.,Salinas, A. M.,Pena, A.,Torres-Torreti, J. P.,Mejias, A.,Ramilo, O.,Suarez, N.,Reynolds, H. E.,Orellana, A.,Lagomarcino, A. J. (2015). Persistent and transient Helicobacter pylori infections in early childhood Clin Infect Dis, 61(2), 211-8 
1930 Ostrom, K. M.,Cordle, C. T.,Schaller, J. P.,Winship, T. R., Thomas, D. J.,Jacobs, J. R.,Blatter, M. M.,Cho, S.,Gooch, W. M., 3rd,Granoff, D. M.,Faden, H.,Pickering, L. K. (2002). Immune status of infants fed soy-based formulas with or without added nucleotides for 1 year: part 1: vaccine responses, and morbidity J Pediatr Gastroenterol Nutr, 34(2), 137-44

1931 O'Sullivan, D. M.,Tinanoff, N. (1993). Social and biological factors contributing to caries of the maxillary anterior teeth Pediatr Dent, 15(1), 41-4

1932 Oti-Boateng, P.,Seshadri, R.,Petrick, S.,Gibson, R. A.,Simmer, K. (1998). Iron status and dietary iron intake of 6-24-month-old children in Adelaide J Paediatr Child Health, 34(3), 250-3

1933 Ou X,Andres A,Pivik RT,Cleves MA,Snow JH,Ding Z,Badger TM (2015). Voxel-Based Morphometry and fMRI Revealed Differences in Brain Gray Matter in Breastfed and Milk Formula-Fed Children AJNR Am J Neuroradiol, \#volume\#(\#issue\#), \#Pages\#

1934 Ou, X.,Andres, A.,Cleves, M. A.,Pivik, R. T.,Snow, J. H.,Ding, Z.,Badger, T. M. (2014). Sex-specific association between infant diet and white matter integrity in 8-y-old children Pediatr Res, 76(6), 535-43

1935 Oulis, C. J.,Berdouses, E. D.,Vadiakas, G.,Lygidakis, N. A. (1999). Feeding practices of Greek children with and without nursing caries Pediatr Dent, 21(7), 409-16

1936 Ounsted, M. K.,Moar, V. A.,Scott, A. (1983). Small-for-dates babies at the age of four years: health, handicap and developmental status Early Hum Dev, 8(3-4), 243-58

1937 Ounsted, M.,Moar, V. A.,Cockburn, J.,Redman, C. W. (1984). Factors associated with the intellectual ability of children born to women with high risk pregnancies Br Med J (Clin Res Ed), 288(6423), 1038-41

1938 Ovsenik, M. (2009). Incorrect orofacial functions until 5 years of age and their association with posterior crossbite Am J Orthod Dentofacial Orthop, 136(3), 375-81

1939 Owen, G. M.,Garry, P. J.,Hooper, E. M.,Gilbert, B. A.,Pathak, D. (1981). Iron nutriture of infants exclusively breast-fed the first five months J Pediatr, 99(2), 237-40

1940 Owen, M. J.,Baldwin, C. D.,Swank, P. R.,Pannu, A. K.,Johnson, D. L.,Howie, V. M. (1993). Relation of infant feeding practices, cigarette smoke exposure, and group child care to the onset and duration of otitis media with effusion in the first two years of life $J$ Pediatr, 123(5), 702-11

1941 Ozden, T. A.,Gokcay, G.,Cantez, M. S.,Durmaz, O.,Issever, H.,Omer, B.,Saner, G. (2015). Copper, zinc and iron levels in infants and their mothers during the first year of life: a prospective study BMC Pediatr, 15(1), 157 old Turkish children Turk J Pediatr, 51(2), 103-9

1943 Ozmert, E. N.,Yurdakok, K.,Soysal, S.,Kulak-Kayikci, M. E.,Belgin, E.,Ozmert, E.,Laleli, Y.,Saracbasi, O. (2005). Relationship between physical, environmental and sociodemographic factors and school performance in primary schoolchildren $\mathrm{J}$ Trop Pediatr, $51(1), 25-32$
Study design,

ntervention/exposure

Study design, Size of study groups

Study design, Size of study groups

Intervention/exposure

Size of study groups

Study design,

Intervention/exposure

Intervention/exposure

Outcome

Study design 
1944 Pacheco, G.,Hedges, M.,Schilling, C.,Morton, S. (2013). Pre- and postnatal drivers of childhood intelligence: evidence from Singapore J Biosoc Sci, 45(1), 41-56

1945 Paine, B. J.,Makrides, M.,Gibson, R. A. (1999). Duration of breast-feeding and Bayley's Mental Developmental Index at 1 year of age $\mathrm{J}$ Paediatr Child Health, 35(1), 82-5

1946 Paine, R.,Coble, R. J. (1982). Breast-feeding and infant health in a rural US community Am J Dis Child, 136(1), 36-8

ze of study groups,

Intervention/exposure

1947 Palloni, A.,Aguirre, G. P.,Lastiri, S. (1994). The effects of breast-feeding and the pace of childbearing on early childhood mortality in Study design,

Mexico Bull Pan Am Health Organ, 28(2), 93-111

Intervention/exposure

1948 Palloni, A.,Tienda, M. (1986). The effects of breastfeeding and pace of childbearing on mortality at early ages Demography, 23(1), Study design $31-52$

1949 Palma, G. D.,Capilla, A.,Nova, E.,Castillejo, G.,Varea, V.,Pozo, T.,Garrote, J. A.,Polanco, I.,Lopez, A.,Ribes-Koninckx, C.,Marcos, A.,Garcia-Novo, M. D.,Calvo, C.,Ortigosa, L.,Pena-Quintana, L.,Palau, F.,Sanz, Y. (2012). Influence of milk-feeding type and genetic risk of developing coeliac disease on intestinal microbiota of infants: the PROFICEL study PLoS One, 7(2), e30791

1950 Palmer, M. M.,VandenBerg, K. A. (1998). A closer look at neonatal sucking Neonatal Netw, 17(2), 77-9 Med Sci, 20(5), 395-9

1952 Palvo, F.,Toledo, E. C.,Menin, A. M.,Jorge, P. P.,Godoy, M. F.,Sole, D. (2008). Risk factors of childhood asthma in Sao Jose do Rio Study design Preto, Sao Paulo, Brazil J Trop Pediatr, 54(4), 253-7

1953 Panagiotakos, D. B.,Papadimitriou, A.,Anthracopoulos, M. B.,Konstantinidou, M.,Antonogeorgos, G.,Fretzayas, A.,Priftis, K. N (2008). Birthweight, breast-feeding, parental weight and prevalence of obesity in schoolchildren aged 10-12 years, in Greece; the Physical Activity, Nutrition and Allergies in Children Examined in Athens (PANACEA) study Pediatr Int, 50(4), 563-8

1954 Panico, L.,Stuart, B.,Bartley, M.,Kelly, Y. (2014). Asthma trajectories in early childhood: identifying modifiable factors PLoS One 9(11), e111922

1955 Papandreou, D.,Malindretos, P.,Rousso, I. (2010). Risk factors for childhood obesity in a Greek paediatric population Public Health Nutr, 13(10), 1535-9

1956 Papenburg, J.,Hamelin, M. E.,Ouhoummane, N.,Carbonneau, J.,Ouakki, M.,Raymond, F., Robitaille, L., Corbeil, J.,Caouette, G.,Frenette, L.,De Serres, G.,Boivin, G. (2012). Comparison of risk factors for human metapneumovirus and respiratory syncytial virus disease severity in young children J Infect Dis, 206(2), 178-89

1957 Papp, L. M. (2014). Longitudinal associations between breastfeeding and observed mother-child interaction qualities in early childhood Child Care Health Dev, 40(5), 740-6 Pittsburgh-area infants: prevalence and risk factors during the first two years of life Pediatrics, 99(3), 318-33

Outcome

Study design, Size of study groups

Participant health

Outcome

Outcome 
1959 Parazzini, F.,Cipriani, S.,Zinetti, C.,Chatenoud, L.,Frigerio, L.,Amuso, G.,Ciammella, M.,Di Landro, A.,Naldi, L. (2014). Perinatal factors and the risk of atopic dermatitis: a cohort study Pediatr Allergy Immunol, 25(1), 43-50

1960 Paricio Talayero JM,Lizan-Garcia M,Otero Puime A,Benlloch Muncharaz MJ,Beseler Soto B,Sanchez-Palomares M,Santos Serrano Intervention/exposure L,Rivera LL (2006). Full breastfeeding and hospitalization as a result of infections in the first year of life Pediatrics, 118(\#issue\#), e92-9

1961 Park, J.,Kim, H. S.,Chu, S. H.,Jekal, Y. S.,Lee, J. Y. (2015). The effect of predominant breast-feeding on the risk of obesity in Korean preschool children Nurs Health Sci, \#volume\#(\#issue\#), \#Pages\#

1962 Park, M. J.,Namgung, R.,Kim, D. H.,Tsang, R. C. (1998). Bone mineral content is not reduced despite low vitamin D status in breast Size of study groups milk-fed infants versus cow's milk based formula-fed infants J Pediatr, 132(4), 641-5

1963 Park, S.,Kim, B. N.,Kim, J. W.,Shin, M. S.,Yoo, H. J.,Cho, S. C. (2014). Protective effect of breastfeeding with regard to children's Study design behavioral and cognitive problems Nutr J, 13(1), 111

1964 Parra-Cabrera, S.,Moreno-Macias, H.,Mendez-Ramirez, I.,Schnaas, L.,Romieu, I. (2008). Maternal dietary omega fatty acid intake and auditory brainstem-evoked potentials in Mexican infants born at term: Cluster analysis Early Human Development, 84(1), 51-57

1965 Parsons, T. J.,Power, C.,Manor, O. (2003). Infant feeding and obesity through the lifecourse Arch Dis Child, 88(9), 793-4

Intervention/exposure

Publication date for a non-sibling study

1966 Paszkowski, J.,Lopatynski, J. (2002). Allergy to house dust mites in primary health care subjects with chronic or recurrent inflammatory states of respiratory system Ann Univ Mariae Curie Sklodowska Med, 57(1), 522-30

1967 Patel, J. A.,Alvarez-Fernandez, P.,Jennings, K.,Loeffelholz, M.,McCormick, D.,Chonmaitree, T. (2015). Factors Affecting Staphylococcus aureus Colonization of the Nasopharynx in the First 6 Months of Life Pediatr Infect Dis J, 34(8), 826-30

1968 Patel, J. A.,Nair, S.,Revai, K.,Grady, J.,Saeed, K.,Matalon, R.,Block, S.,Chonmaitree, T. (2006). Association of proinflammatory cytokine gene polymorphisms with susceptibility to otitis media Pediatrics, 118(6), 2273-9

1969 Paterson, J. E.,Gao, W.,Sundborn, G.,Cartwright, S. (2011). Maternal self-report of oral health in six-year-old Pacific children from Outcome South Auckland, New Zealand Community Dent Oral Epidemiol, 39(1), 19-28

1970 Paterson, J.,lusitini, L.,Gao, W. (2011). Child developmental assessment at two-years of age: data from the Pacific Islands Families Outcome Study Pac Health Dialog, 17(2), 51-63

1971 Patra, S.,Singh, V.,Kumar, P.,Chandra, J.,Dutta, A.,Tripathi, M. (2011). Demographic and clinical profile of children under two years of age with recurrent wheezing J Coll Physicians Surg Pak, 21(11), 715-7

1972 Patsourou, A.,Konstantinides, T.,Mantadakis, E.,Tsalkidis, A.,Zarras, C.,Balaska, A.,Simopoulos, K.,Chatzimichael, A. (2012). Growth of exclusively breastfed and self-weaned children of Greece aged 0-36 months Breastfeed Med, 7(6), 521-5

1973 Patterson, C. C.,Carson, D. J.,Hadden, D. R.,Waugh, N. R.,Cole, S. K. (1994). A case-control investigation of perinatal risk factors for childhood IDDM in Northern Ireland and Scotland Diabetes Care, 17(5), 376-81

Participant health

Outcome

Study design, Outcome

Country, Size of study group

Study design

ntervention/exposure 
1975 Paul A,Whitehead R (1986). Infant feeding: the weighting game Community Outlook, \#volume\#(\#issue\#), 11-7

Study design

1976 Paul, K.,Dittrichova, J.,Papousek, H. (1996). Infant feeding behavior: development in patterns and motivation Dev Psychobiol, 29(7), Size of study groups 563-76

1977 Pavic, I.,Jurkovic, M.,Pastar, Z. (2012). Risk factors for acute respiratory tract infections in children Coll Antropol, 36(2), 539-42

Study design

1978 Pearce, M. S.,Birrell, F. N.,Francis, R. M.,Rawlings, D. J.,Tuck, S. P.,Parker, L. (2005). Lifecourse study of bone health at age 49-51 Intervention/exposure years: the Newcastle thousand families cohort study J Epidemiol Community Health, 59(6), 475-80

1979 Pearce, M. S.,Relton, C. L.,Parker, L.,Unwin, N. C. (2009). Sex differences in the association between infant feeding and blood cholesterol in later life: the Newcastle thousand families cohort study at age 49-51 years Eur J Epidemiol, 24(7), 375-80

Publication date for a non-sibling study

1980 Pearce, M. S.,Unwin, N. C.,Parker, L.,Alberti, K. G. (2006). Life course determinants of insulin secretion and sensitivity at age 50 years: the Newcastle thousand families study Diabetes Metab Res Rev, 22(2), 118-25

1981 Pearson, Catherine (2013). Study Finds Breastfeeding May Lower Alzheimer's Risk Inside Childbirth Education, \#volume\#(\#issue\#), Study design 9-9 1p

1982 Peat, J. K. (1998). Can asthma be prevented? Evidence from epidemiological studies of children in Australia and New Zealand in the last decade Clin Exp Allergy, 28(3), 261-5

1983 Peat, J. K.,Allen, J.,Oddy, W.,Webb, K. (2003). Breastfeeding and asthma: appraising the controversy Pediatr Pulmonol, 35(5), $331-4$

1984 Pedersen, C. B.,Zachau-Christiansen, B. (1986). Otitis media in Greenland children: acute, chronic and secretory otitis media in three- to eight-year-olds J Otolaryngol, 15(6), 332-5

1985 Pehlivan, I.,Hatun, Ş,Aydoğan, M.,Babaoğlu, K.,Gökalp, S. A. (2003). Maternal vitamin D defiency and vitamin D supplementation in Intervention/exposure healthy infants Turkish Journal of Pediatrics, 45(4), 315-320

1986 Pei, Z.,Heinrich, J.,Fuertes, E.,Flexeder, C.,Hoffmann, B.,Lehmann, I.,Schaaf, B.,von Berg, A.,Koletzko, S. (2014). Cesarean delivery and risk of childhood obesity J Pediatr, 164(5), 1068-1073 e2

1987 Pelayo, L.,Nunez, F. A.,Rojas, L.,Wilke, H.,Furuseth Hansen, E.,Mulder, B.,Gjerde, B.,Robertson, L. (2008). Molecular and epidemiological investigations of cryptosporidiosis in Cuban children Ann Trop Med Parasitol, 102(8), 659-69

1988 Peltzer, K.,Mongkolchati, A.,Satchaiyan, G.,Rajchagool, S.,Pimpak, T. (2014). Sociobehavioral factors associated with caries increment: a longitudinal study from 24 to 36 months old children in Thailand Int J Environ Res Public Health, 11(10), 10838-50

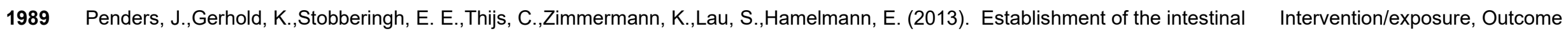
microbiota and its role for atopic dermatitis in early childhood J Allergy Clin Immunol, 132(3), 601-607 e8

1990 Peneau, S.,Hercberg, S.,Rolland-Cachera, M. F. (2014). Breastfeeding, early nutrition, and adult body fat J Pediatr, 164(6), 1363-8 Size of study groups 
1991 Penn, A. H.,Carver, L. J.,Herbert, C. A.,Lai, T. S.,McIntire, M. J.,Howard, J. T.,Taylor, S. F.,Schmid-Schonbein, G. W.,Dobkins, K. R. Outcome (2016). Breast Milk Protects Against Gastrointestinal Symptoms in Infants at High Risk for Autism During Early Development $J$ Pediatr Gastroenterol Nutr, 62(2), 317-27

\begin{tabular}{|c|c|c|}
\hline 1992 & Penwell, A. (2012). Breastfeeding and newborn survival Midwifery Today Int Midwife, \#volume\#(101), 51-3 & Study design \\
\hline 1993 & Perera, B. J. (2010). Preventive strategies for acute respiratory infections in children Ceylon Med J, 55(4), 103-5 & Study design \\
\hline 1994 & $\begin{array}{l}\text { Perera, B. J.,Ganesan, S.,Jayarasa, J.,Ranaweera, S. (1999). The impact of breastfeeding practices on respiratory and diarrhoeal } \\
\text { disease in infancy: a study from Sri Lanka J Trop Pediatr, 45(2), } 115-8\end{array}$ & Study design, Outcome \\
\hline 1995 & $\begin{array}{l}\text { Peres, K. G.,Cascaes, A. M.,Peres, M. A.,Demarco, F. F.,Santos, I. S.,Matijasevich, A.,Barros, A. J. (2015). Exclusive Breastfeeding } \\
\text { and Risk of Dental Malocclusion Pediatrics, 136(1), e60-7 }\end{array}$ & Outcome \\
\hline 1996 & $\begin{array}{l}\text { Perez-Bravo, F.,Carrasco, E.,Gutierrez-Lopez, M. D.,Martinez, M. T.,Lopez, G.,de los Rios, M. G. (1996). Genetic predisposition } \\
\text { and environmental factors leading to the development of insulin-dependent diabetes mellitus in Chilean children J Mol Med (Berl), } \\
74(2), 105-9\end{array}$ & Outcome \\
\hline 1997 & $\begin{array}{l}\text { Perez-Bravo, F., Oyarzun, A.,Carrasco, E.,Albala, C.,Dorman, J. S., Santos, J. L. (2003). Duration of breast feeding and bovine } \\
\text { serum albumin antibody levels in type } 1 \text { diabetes: a case-control study Pediatr Diabetes, 4(4), 157-61 }\end{array}$ & Outcome \\
\hline 1998 & $\begin{array}{l}\text { Peroni, D. G.,Piacentini, G. L.,Alfonsi, L.,Zerman, L.,Di Blasi, P.,Visona, G.,Nottegar, F.,Boner, A. L. (2003). Rhinitis in pre-school } \\
\text { children: prevalence, association with allergic diseases and risk factors Clin Exp Allergy, 33(10), 1349-54 }\end{array}$ & Study design \\
\hline 1999 & $\begin{array}{l}\text { Perrillat, F.,Clavel, J.,Auclerc, M. F.,Baruchel, A.,Leverger, G.,Nelken, B.,Philippe, N.,Schaison, G.,Sommelet, D.,Vilmer, E.,Hemon, } \\
\text { D. (2002). Day-care, early common infections and childhood acute leukaemia: a multicentre French case-control study Br J Cancer, } \\
\text { 86(7), 1064-9 }\end{array}$ & Outcome \\
\hline 2000 & $\begin{array}{l}\text { Perrillat, F.,Clavel, J.,Jaussent, I.,Baruchel, A.,Leverger, G.,Nelken, B.,Philippe, N.,Schaison, G.,Sommelet, D.,Vilmer, E.,Hémon, D. } \\
\text { (2002). Breast-feeding, fetal loss and childhood acute leukaemia European Journal of Pediatrics, } 161(4), 235-237\end{array}$ & Outcome \\
\hline 2001 & $\begin{array}{l}\text { Perrine, C. G.,Sharma, A. J.,Jefferds, M. E.,Serdula, M. K.,Scanlon, K. S. (2010). Adherence to vitamin D recommendations among } \\
\text { US infants Pediatrics, } 125(4), 627-32\end{array}$ & Study design \\
\hline 2002 & $\begin{array}{l}\text { Persico, M.,Podoshin, L.,Fradis, M.,Golan, D.,Wellisch, G. (1983). Recurrent middle-ear infections in infants: the protective role of } \\
\text { maternal breast feeding Ear Nose Throat J, 62(6), 297-304 }\end{array}$ & Participant health, Outcomes \\
\hline 2003 & Persson, L. A. (1985). Infant feeding and growth--a longitudinal study in three Swedish communities Ann Hum Biol, 12(1), 41-52 & $\begin{array}{l}\text { Publication date for a non-sibling } \\
\text { study }\end{array}$ \\
\hline 2004 & $\begin{array}{l}\text { Persson, L. A.,Lundstrom, M.,Lonnerdal, B.,Hernell, O. (1998). Are weaning foods causing impaired iron and zinc status in 1-year- } \\
\text { old Swedish infants? A cohort study Acta Paediatr, 87(6), 618-22 }\end{array}$ & Intervention/exposure \\
\hline 2005 & $\begin{array}{l}\text { Perumal, N.,Al Mahmud, A.,Baqui, A. H.,Roth, D. E. (2015). Prenatal vitamin D supplementation and infant vitamin D status in } \\
\text { Bangladesh Public Health Nutr, \#volume\#(\#issue\#), 1-9 }\end{array}$ & Country \\
\hline
\end{tabular}


2006 Pesonen, M.,Kallio, M. J.,Ranki, A.,Siimes, M. A. (2006). Prolonged exclusive breastfeeding is associated with increased atopic dermatitis: a prospective follow-up study of unselected healthy newborns from birth to age 20 years Clin Exp Allergy, 36(8), 1011-8

2007 Peters, D. C.,Worthington-Roberts, B. (1982). Infant feeding practices of middle-class breastfeeding and formula-feeding mothers

Outcome Birth, 9(2), 91-5

2008 Peters, K. E.,Huang, J.,Vaughn, M. G.,Witko, C. (2013). Does breastfeeding contribute to the racial gap in reading and math test scores? Ann Epidemiol, 23(10), 646-51

2009 Peters, T. J.,Golding, J. (1987). The epidemiology of childhood eczema: II. Statistical analyses to identify independent early predictors Paediatr Perinat Epidemiol, 1(1), 80-94

2010 Peters, U.,Schneeweiss, S.,Trautwein, E. A.,Erbersdobler, H. F. (2001). A case-control study of the effect of infant feeding on celiac Outcome disease Ann Nutr Metab, 45(4), 135-42

2011 Petherick, A. (2010). Development: Mother's milk: A rich opportunity Nature, 468(7327), S5-7

Study design

2012 Petridou, E.,Trichopoulos, D.,Kalapothaki, V.,Pourtsidis, A.,Kogevinas, M.,Kalmanti, M.,Koliouskas, D.,Kosmidis, H.,Panagiotou, J P.,Piperopoulou, F.,Tzortzatou, F. (1997). The risk profile of childhood leukaemia in Greece: a nationwide case-control study Br J Cancer, 76(9), $1241-7$

2013 Petti, S.,Cairella, G.,Tarsitani, G. (2000). Rampant early childhood dental decay: an example from Italy J Public Health Dent, 60(3), Study design $159-66$

2014 Pettitt, D. J.,Forman, M. R.,Hanson, R. L.,Knowler, W. C.,Bennett, P. H. (1997). Breastfeeding and incidence of non-insulindependent diabetes mellitus in Pima Indians Lancet, 350(9072), 166-8

2015 Pettitt, D. J.,Knowler, W. C. (1998). Long-term effects of the intrauterine environment, birth weight, and breast-feeding in Pima Indians Diabetes Care, 21 Suppl 2(\#issue\#), B138-41

2016 Peyre, H.,Bernard, J. Y.,Forhan, A.,Charles, M. A.,De Agostini, M.,Heude, B.,Ramus, F., Charles, M. A.,De Agostini, M.,Forhan, A.,Heude, B.,Ducimetière, P.,Kaminski, M.,Saurel-Cubizolles, M. J.,Dargent, P.,Fritel, X.,Larroque, B.,Lelong, N.,Marchand, L.,Nabet, C.,Annesi-Maesano, I.,Slama, R.,Goua, V.,Magnin, G.,Hankard, R., Thiebaugeorges, O.,Schweitzer, M.,Foliguet, B.,JobSpira, N. (2014). Predicting changes in language skills between 2 and 3 years in the EDEN mother-child cohort PeerJ, 2014(1), \#Pages\#

2017 Pfluger, M.,Winkler, C.,Hummel, S.,Ziegler, A. G. (2010). Early infant diet in children at high risk for type 1 diabetes Horm Metab Res, 42(2), 143-8

2018 Picciano, M. F.,Deering, R. H. (1980). The influence of feeding regimens on iron status during infancy Am J Clin Nutr, 33(4), 746-53

2019 Picone, T. A.,Benson, J. D.,Moro, G.,Minoli, I.,Fulconis, F.,Rassin, D. K.,Raiha, N. C. (1989). Growth, serum biochemistries, and amino acids of term infants fed formulas with amino acid and protein concentrations similar to human milk J Pediatr Gastroenterol Nutr, 9(3), 351-60

\section{Outcome}

Intervention/exposure, Outcome

Study design,

Intervention/exposure

Outcome

Intervention/exposure

Size of study groups Intervention/exposure

Intervention/exposure, Outcome 
2020 Piemontese, P.,Gianni, M. L.,Braegger, C. P.,Chirico, G.,Gruber, C.,Riedler, J.,Arslanoglu, S.,van Stuijvenberg, M.,Boehm, G., Jelinek, J.,Roggero, P. (2011). Tolerance and safety evaluation in a large cohort of healthy infants fed an innovative prebiotic formula: a randomized controlled trial PLoS One, 6(11), e28010

2021 Pinho, A. P.,Aerts, D.,Nunes, M. L. (2008). Risk factors for sudden infant death syndrome in a developing country Rev Saude Publica, 42(3), 396-401

2022 Pinzon-Rondon, A. M.,Aguilera-Otalvaro, P.,Zarate-Ardila, C.,Hoyos-Martinez, A. (2015). Acute respiratory infection in children from developing nations: a multi-level study Paediatr Int Child Health, \#volume\#(\#issue\#), $2046905515 y 0000000021$

2023 Pires, S. C.,Giugliani, E. R.,Caramez da Silva, F. (2012). Influence of the duration of breastfeeding on quality of muscle function during mastication in preschoolers: a cohort study BMC Public Health, 12(1), 934

2024 Pirila, S.,Saarinen-Pihkala, U. M.,Viljakainen, H.,Turanlahti, M.,Kajosaari, M.,Makitie, O.,Taskinen, M. (2012). Breastfeeding and determinants of adult body composition: a prospective study from birth to young adulthood Horm Res Paediatr, 77(5), 281-90

2025 Pirila, S.,Taskinen, M.,Viljakainen, H.,Kajosaari, M.,Turanlahti, M.,Saarinen-Pihkala, U. M.,Makitie, O. (2011). Infant milk feeding influences adult bone health: a prospective study from birth to 32 years PLoS One, 6(4), e19068 their later cardiovascular health: a prospective study from birth to age 32 years Br J Nutr, 111(6), 1069-76

2027 Pisacane, A.,De Vizia, B.,Valiante, A.,Vaccaro, F.,Russo, M.,Grillo, G.,Giustardi, A. (1995). Iron status in breast-fed infants J Pediatr, 127(3), 429-31

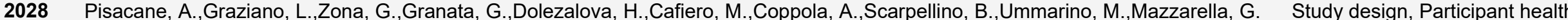
(1994). Breast feeding and acute lower respiratory infection Acta Paediatr, 83(7), 714-8

2029 Pivik, R. T.,Andres, A.,Badger, T. M. (2011). Diet and gender influences on processing and discrimination of speech sounds in 3- Outcome and 6-month-old infants: a developmental ERP study Dev Sci, 14(4), 700-12

2030 Pivik, R. T.,Andres, A.,Badger, T. M. (2012). Effects of diet on early stage cortical perception and discrimination of syllables differing Outcome in voice-onset time: a longitudinal ERP study in 3 and 6 month old infants Brain Lang, 120(1), 27-41

2031 Pivik, R. T.,Andres, A.,Tennal, K. B.,Gu, Y.,Armbya, N.,Cleves, M. A.,Badger, T. M. (2013). Infant diet, gender and the normative Outcome development of vagal tone and heart period during the first two years of life Int J Psychophysiol, 90(3), 311-20

2032 Pivik, R. T.,Andres, A.,Tennal, K. B.,Gu, Y.,Cleves, M. A.,Badger, T. M. (2015). Infant diet, gender and the development of vagal tone stability during the first two years of life Int J Psychophysiol, 96(2), 104-14

2033 Pivik, R. T.,Dykman, R. A.,Jing, H.,Gilchrist, J. M.,Badger, T. M. (2007). The influence of infant diet on early developmental changes Size of study groups in processing human voice speech stimuli: ERP variations in breast and milk formula-fed infants at 3 and 6 months after birth Dev Neuropsychol, 31(3), 279-335 resting cardiovascular activity and behavioral development during the first half-year of life Dev Neuropsychol, 34(2), 139-58 
2035 Piwoz, E. G. Creed de Kanashiro, H Lopez de Romana, G. L Black, R. E Brown, K. H (1996). Feeding practices and growth among low-income Peruvian infants: a comparison of internationally-recommended definitions Int J Epidemiol, 25(1), 103-14

2036 Pizarro, F.,Yip, R.,Dallman, P. R.,Olivares, M.,Hertrampf, E.,Walter, T. (1991). Iron status with different infant feeding regimens: relevance to screening and prevention of iron deficiency J Pediatr, 118(5), 687-92

2037 Plachta-Danielzik, S.,Kehden, B.,Landsberg, B.,Schaffrath Rosario, A.,Kurth, B. M.,Arnold, C.,Graf, C.,Hense, S.,Ahrens, W.,Muller, M. J. (2012). Attributable risks for childhood overweight: evidence for limited effectiveness of prevention Pediatrics, 130(4), e865-71

2038 Plagemann, A.,Harder, T.,Franke, K.,Kohlhoff, R. (2002). Long-term impact of neonatal breast-feeding on body weight and glucose tolerance in children of diabetic mothers Diabetes Care, 25(1), 16-22

2039 Plagemann, A.,Harder, T.,Kohlhoff, R.,Fahrenkrog, S.,Rodekamp, E.,Franke, K.,Dudenhausen, J. W. (2005). Impact of early neonatal breast-feeding on psychomotor and neuropsychological development in children of diabetic mothers Diabetes Care, 28(3), $573-8$

2040 Plagemann, A.,Harder, T.,Rodekamp, E.,Kohlhoff, R. (2012). Rapid neonatal weight gain increases risk of childhood overweight in offspring of diabetic mothers J Perinat Med, 40(5), 557-63

2041 Plancoulaine, S.,Charles, M. A.,Lafay, L.,Tauber, M.,Thibult, N.,Borys, J. M.,Eschwege, E. (2000). Infant-feeding patterns are related to blood cholesterol concentration in prepubertal children aged 5-11 y: the Fleurbaix-Laventie Ville Sante study Eur J Clin Nutr, 54(2), 114-9

2042 Plenge-Bonig, A.,Soto-Ramirez, N.,Karmaus, W.,Petersen, G.,Davis, S.,Forster, J. (2010). Breastfeeding protects against acute gastroenteritis due to rotavirus in infants Eur J Pediatr, 169(12), 1471-6

2043 Plonka, K. A.,Pukallus, M. L.,Barnett, A. G.,Walsh, L. J.,Holcombe, T. F.,Seow, W. K. (2012). A longitudinal study comparing mutans streptococci and lactobacilli colonisation in dentate children aged 6 to 24 months Caries Res, 46(4), 385-93

2044 Plonka, K. A.,Pukallus, M. L.,Barnett, A. G.,Walsh, L. J.,Holcombe, T. H.,Seow, W. K. (2012). Mutans streptococci and lactobacilli colonization in predentate children from the neonatal period to seven months of age Caries Res, 46(3), 213-20

2045 Podratz, R. O.,Broughton, D. D.,Gustafson, D. H.,Bergstralh, E. J.,Melton, L. J., 3rd (1986). Weight loss and body temperature changes in breast-fed and bottle-fed neonates Clin Pediatr (Phila), 25(2), 73-7

2046 Pohlabeln, H.,Muhlenbruch, K.,Jacobs, S.,Bohmann, H. (2010). Frequency of allergic diseases in 2-year-old children in relationship Intervention/exposure to parental history of allergy and breastfeeding J Investig Allergol Clin Immunol, 20(3), 195-200

2047 Poikonen, S.,Puumalainen, T. J.,Kautiainen, H.,Palosuo, T.,Reunala, T.,Turjanmaa, K. (2008). Sensitization to turnip rape and oilseed rape in children with atopic dermatitis: a case-control study Pediatr Allergy Immunol, 19(5), 408-11

2048 Pollock, J. I. (1994). Long-term associations with infant feeding in a clinically advantaged population of babies Dev Med Child Neurol, 36(5), 429-40

2049 Pomerance, H. H. (1987). Growth in breast-fed children Hum Biol, 59(4), 687-93
Size of study groups, Intervention/exposure

Study design

Intervention/exposure

Study design

Intervention/exposure

Intervention/exposure

Intervention/exposure

Outcome

Study design,

ntervention/exposure

Outcome

Outcome

Publication date for a non-sibling study

Intervention/exposure

Intervention/exposure

Intervention/exposure 
2050 Ponder, D. L.,Innis, S. M.,Benson, J. D.,Siegman, J. S. (1992). Docosahexaenoic acid status of term infants fed breast milk or infant Size of study groups formula containing soy oil or corn oil Pediatr Res, 32(6), 683-8

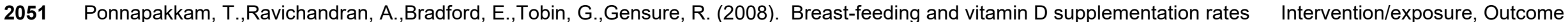
in the Ochsner health system Ochsner Journal, 8(3), 146-150

2052 Porro, E.,Indinnimeo, L.,Antognoni, G.,Midulla, F.,Criscione, S. (1993). Early wheezing and breast feeding J Asthma, 30(1), 23-8

\section{Outcome}

2053 Portela, D. S.,Vieira, T. O.,Matos, S. M.,de Oliveira, N. F.,Vieira, G. O. (2015). Maternal obesity, environmental factors, cesarean delivery and breastfeeding as determinants of overweight and obesity in children: results from a cohort BMC Pregnancy Childbirth 15(\#issue\#), 94

2054 Portoian-Shuhaiber, S.,Al-Rashied, A. A. (1986). Feeding practices and electrolyte disturbances among infants admitted with acute diarrhoea--a survey in Kuwait J Trop Pediatr, 32(4), 168-73

2055 Potera, Carol (2011). Prolonged Bottle Feeding Raises Childhood Obesity Risk: Weaning around one year is recommended American Journal of Nursing, 111(8), 17-17 1p

2056 Potter, A.,Lumley, J.,Watson, L. (1996). The 'new' risk factors for SIDS: is there an association with the ethnic and place of birth differences in incidence in Victoria, Australia? Early Hum Dev, 45(1-2), 119-31

2057 Potter, C. M.,Ulijaszek, S. J. (2013). Predicting adult obesity from measures in earlier life J Epidemiol Community Health, 67(12), 1032-7

2058

Intervention/exposure,

Confounding

Study design, Participant health

Study design

Intervention/exposure, Outcome

Study design,

Intervention/exposure

2059 Poysa, L. (1989). Atopy in children with and without a family history of atopy. II. Skin reactivity Acta Paediatr Scand, 78(6), 902-6 Study design

2060 Poysa, L.,Korppi, M.,Remes, K.,Juntunen-Backman, K. (1990). Predictive value of IgE levels in infancy Acta Paediatr Scand, 79(10), Study design, Outcome 970-2

2061 Poysa, L.,Korppi, M.,Remes, K.,Juntunen-Backman, K. (1991). Atopy in childhood and diet in infancy. A nine-year follow-up study. I. Size of study groups Clinical manifestations Allergy Proc, 12(2), 107-11

2062 Poysa, L.,Remes, K.,Korppi, M.,Juntunen-Backman, K. (1989). Atopy in children with and without a family history of atopy. I. Clinical Size of study groups manifestations, with special reference to diet in infancy Acta Paediatr Scand, 78(6), 896-901

2063 Prado-Montes de Oca, E.,Garcia-Vargas, A.,Lozano-Inocencio, R.,Gallegos-Arreola, M. P.,Sandoval-Ramirez, L.,DavalosRodriguez, N. O.,Figuera, L. E. (2007). Association of beta-defensin 1 single nucleotide polymorphisms with atopic dermatitis Int Arch Allergy Immunol, 142(3), 211-8

2064 Prado-Montes De Oca, E.,García-Vargas, A.,Lozano-Inocencio, R.,Gallegos-Arreola, M. P.,Sandoval-Ramírez, L.,DávalosRodríguez, N. O.,Figuera, L. E. (2007). Association of $\beta$-defensin 1 single nucleotide polymorphisms with atopic dermatitis International Archives of Allergy and Immunology, 142(3), 211-218 
2065 Prathanee, B.,Purdy, S. C.,Thinkhamrop, B.,Chaimay, B.,Ruangdaraganon, N.,Mo-suwan, L.,Phuphaibul, R. (2009). Early language delay and predictive factors in children aged 2 years $\mathrm{J}$ Med Assoc Thai, 92(7), 930-8

2066 Pratt, H. F. (1984). Breastfeeding and eczema Early Hum Dev, 9(3), 283-90

Outcome

Intervention/exposure

2067 Prentice, P.,Koulman, A.,Matthews, L.,Acerini, C. L.,Ong, K. K.,Dunger, D. B. (2015). Lipidomic analyses, breast- and formulafeeding, and growth in infants J Pediatr, 166(2), 276-81 e6

2068 Price, Gareth (2011). A test of temperament Midwives, 14(4), 13-13 1p

Study design

2069 Priego, T.,Sanchez, J.,Pico, C.,Ahrens, W.,Bammann, K.,De Henauw, S.,Fraterman, A.,lacoviello, L.,Lissner, L.,Molnar, D.,Moreno, L. A.,Siani, A.,Tornaritis, M.,Veidebaum, T.,Palou, A. (2014). Influence of breastfeeding on blood-cell transcript-based biomarkers of health in children Pediatr Obes, 9(6), 463-70

2070 Priya, N. Gayathri,Victoria, L. Eilean,Porkodi, A.,Eaton, Linda,Doorenbos, Ardith (2013). Effectiveness of Breastfeeding Empowerment Programme among Primigravidae Communicating Nursing Research, 46(\#issue\#), 579-579 1p

2071 Procter, S. B.,Holcomb, C. A. (2008). Breastfeeding duration and childhood overweight among low-income children in Kansas, 1998-2002 Am J Public Health, 98(1), 106-10

2072 Prodam, F.,Roccio, M.,Trovato, L.,Ricotti, R.,Moia, S.,Giglione, E.,Petri, A.,Walker, G. E.,Bellone, S.,Bona, G. (2015). Adiponectin oligomers are similarly distributed in adequate-for-gestational-age obese children irrespective of feeding in their first year Pediatr Res, 77(6), 808-13

2073 Puccio, G.,Cajozzo, C.,Meli, F.,Rochat, F.,Grathwohl, D.,Steenhout, P. (2007). Clinical evaluation of a new starter formula for infants Intervention/exposure containing live Bifidobacterium longum BL999 and prebiotics Nutrition, 23(1), 1-8

2074 Pugh, L. C.,Milligan, R. A. (1998). Nursing intervention to increase the duration of breastfeeding Appl Nurs Res, 11(4), 190-4

Study design, Outcome

2075 Pugh, L. C.,Milligan, R. A.,Frick, K. D.,Spatz, D.,Bronner, Y. (2002). Breastfeeding duration, costs, and benefits of a support program for low-income breastfeeding women Birth, 29(2), 95-100

2076 Pugo-Gunsam, P.,Guesnet, P.,Subratty, A. H.,Rajcoomar, D. A.,Maurage, C.,Couet, C. (1999). Fatty acid composition of white adipose tissue and breast milk of Mauritian and French mothers and erythrocyte phospholipids of their full-term breast-fed infants $B$ J Nutr, 82(4), 263-71

2077 Puig, C.,Sunyer, J.,Garcia-Algar, O.,Munoz, L.,Pacifici, R.,Pichini, S.,Vall, O. (2008). Incidence and risk factors of lower respiratory tract illnesses during infancy in a Mediterranean birth cohort Acta Paediatr, 97(10), 1406-11

2078 Pukander J,Luotonen J,Timonen M,Karma P (1985). Risk factors affecting the occurrence of acute otitis media among 2-3-year-old Outcome urban children Acta Otolaryngol, 100(\#issue\#), 260-5

2079 Pukander, J. (1982). Acute otitis media among rural children in Finland Int J Pediatr Otorhinolaryngol, 4(4), 325-32 virus infection $\mathrm{Br}$ Med J, 281(6247), 1034-6 
2081 Purssell, E. (2012). A topic in 10 questions: Gastrointestinal infections from a nutritional perspective J Fam Health Care, 22(1), 28-9 Study design

2082 Purvis, D. J.,Thompson, J. M.,Clark, P. M.,Robinson, E.,Black, P. N.,Wild, C. J.,Mitchell, E. A. (2005). Risk factors for atopic dermatitis in New Zealand children at 3.5 years of age Br J Dermatol, 152(4), 742-9

2083 Putet, G.,Labaune, J. M.,Mace, K.,Steenhout, P.,Grathwohl, D.,Raverot, V.,Morel, Y.,Picaud, J. C. (2015). Effect of dietary protein on plasma insulin-like growth factor-1, growth, and body composition in healthy term infants: a randomised, double-blind, controlled trial (Early Protein and Obesity in Childhood (EPOCH) study) Br J Nutr, \#volume\#(\#issue\#), 1-14

2084 Putnam, J. C.,Carlson, S. E.,DeVoe, P. W.,Barness, L. A. (1982). The effect of variations in dietary fatty acids on the fatty acid composition of erythrocyte phosphatidylcholine and phosphatidylethanolamine in human infants Am J Clin Nutr, 36(1), 106-14

2085 Putra, S. T.,Mansyur, M.,Sastroasmoro, S. (2015). Effects of duration of breastfeeding during infancy on vascular dysfunction in adolescents Acta Med Indones, 47(1), 24-30

2086 Qudsia, F.,Saboor, M.,Khosa, S. M.,Ayub, Q.,Moinuddin, (2015). Comparative analysis of serum iron, serum ferritin and red cell folate levels among breast fed, fortified milk and cow's milk fed infants Pakistan Journal of Medical Sciences, 31(3), 706-709

2087 Queiroz, V. A.,Assis, A. M.,Pinheiro, S. M.,Ribeiro, H. C., Jr. (2012). Predictors of linear growth in the first year of life of a prospective cohort of full term children with normal birth weight J Pediatr (Rio J), 88(1), 79-86

2088 Quialey, M. A.,Cumberland, P.,Cowden, J. M.,Rodrigues, L. C. (2006). How protective is breast feeding against diarrhoeal disease in infants in 1990s England? A case-control study Archives of Disease in Childhood, 91(3), 245-250

2089 Quigley, M. A.,Hockley, C.,Carson, C.,Kelly, Y.,Renfrew, M. J.,Sacker, A. (2012). Breastfeeding is associated with improved child Outcome cognitive development: a population-based cohort study J Pediatr, 160(1), 25-32

2090 Quigley, M. A.,Kelly, Y. J.,Sacker, A. (2007). Breastfeeding and hospitalization for diarrheal and respiratory infection in the United Study design Kingdom Millennium Cohort Study Pediatrics, 119(4), e837-42

2091 Quigley, M. A.,Kelly, Y. J.,Sacker, A. (2009). Infant feeding, solid foods and hospitalisation in the first 8 months after birth Arch Dis Intervention/exposure Child, 94(2), 148-50

2092 Quinn, P. J.,O'Callaghan, M.,Williams, G. M.,Najman, J. M.,Andersen, M. J.,Bor, W. (2001). The effect of breastfeeding on child development at 5 years: a cohort study J Paediatr Child Health, 37(5), 465-9

2093 Quinonez, R.,Santos, R. G.,Wilson, S.,Cross, H. (2001). The relationship between child temperament and early childhood caries Pediatr Dent, 23(1), 5-10

2094 Quiroga, M.,Oviedo, P.,Chinen, I.,Pegels, E.,Husulak, E.,Binztein, N.,Rivas, M.,Schiavoni, L.,Vergara, M. (2000). Asymptomatic infections by diarrheagenic Escherichia coli in children from Misiones, Argentina, during the first twenty months of their lives Rev Inst Med Trop Sao Paulo, 42(1), 9-15

2095 Qureshi, B.,Morgan, J. B.,Kimer, A. C.,Donaldson, D.,Dickerson, J. W. (1988). Feeding practices and birth weights of infants in Southall, Middlesex J R Soc Health, 108(3), 77-80

Outcome 
2096 Rabiei, S. (2011). The Association of Nutrition Style through the First 2 Years of Life with Type 1 Diabetes Mellitus and Some of the Other Effective Factors in 2-15 Years Old Children Iranian Journal of Endocrinology \& Metabolism, 13(1), 9-113 105p

2097 Radlovic, N. P.,Mladenovic, M. M.,Lekovic, Z. M.,Stojsic, Z. M.,Radlovic, V. N. (2010). Influence of early feeding practices on celiac disease in infants Croat Med J, 51(5), 417-22

2098 Rady, H. I.,Samir, H.,Tomerak, R.,Gaafar, M. (2014). Occult blood in stool in exclusively formula fed infants versus exclusively breast fed infants in the first six months of life Egyptian Pediatric Association Gazette, 62(1), 8-13

2099 Raftowicz-Wójcik, K.,Matthews-Brzozowska, T.,Kawala, B.,Antoszewska, J. (2011). The effects of breast feeding on occlusion in primary dentition Advances in Clinical and Experimental Medicine, 20(3), 371-375

2100 Rahman, M.,Roy, S. K.,Ali, M.,Mitra, A. K.,Alam, A. N.,Akbar, M. S. (1993). Maternal nutritional status as a determinant of child health J Trop Pediatr, 39(2), 86-8

2101 Raiha, N. C.,Fazzolari-Nesci, A.,Boehm, G. (1996). Taurine supplementation prevents hyperaminoacidemia in growing term infants fed high-protein cow's milk formula Acta Paediatr, 85(12), 1403-7

2102 Raiha, N. C.,Fazzolari-Nesci, A.,Cajozzo, C.,Puccio, G.,Monestier, A.,Moro, G.,Minoli, I.,Haschke-Becher, E.,Bachmann, C.,Van't Hof, M.,Carrie Fassler, A. L.,Haschke, F. (2002). Whey predominant, whey modified infant formula with protein/energy ratio of 1.8 $\mathrm{g} / 100 \mathrm{kcal}$ : adequate and safe for term infants from birth to four months J Pediatr Gastroenterol Nutr, 35(3), 275-81

2103 Räihä, N.,Fazzolari, A.,Cayozzo, C.,Puccio, G.,Minoli, I.,Moro, G.,Monestier, A.,Haschke-Becher, E.,Carrié, A. L.,Haschke, F. (2002). Size of study groups Infant formula with $1.8 \mathrm{~g}$ Protein/100 Kcal is adequate and safe from birth to 4 months Revue Medicale Libanaise, 14(1), 29-31

2104 Raiha, N.,Minoli, I.,Moro, G. (1986). Milk protein intake in the term infant. I. Metabolic responses and effects on growth Acta Paediatr Scand, 75(6), 881-6

2105 Raisler, J.,Alexander, C.,O'Campo, P. (1999). Breast-feeding and infant illness: a dose-response relationship? Am J Public Health $89(1), 25-30$

2106 Ramezani, G. H.,Norozi, A.,Valael, N. (2003). The prevalence of nursing caries in 18 to 60 months old children in Qazvin J Indian Soc Pedod Prev Dent, 21(1), 19-26

2107 Rami, B.,Schneider, U.,Imhof, A.,Waldhor, T.,Schober, E. (1999). Risk factors for type I diabetes mellitus in children in Austria Eur J Outcome Pediatr, 158(5), 362-6

2108 Ramirez, G. B.,Pagulayan, O.,Akagi, H.,Francisco Rivera, A.,Lee, L. V.,Berroya, A.,Vince Cruz, M. C.,Casintahan, D. (2003). Tagum Country study II: follow-up study at two years of age after prenatal exposure to mercury Pediatrics, 111(3), e289-95

2109 Ramirez-Silva, I.,Rivera, J. A.,Trejo-Valdivia, B.,Martorell, R.,Stein, A. D.,Romieu, I.,Barraza-Villarreal, A.,Ramakrishnan, U. (2015) Breastfeeding status at age 3 months is associated with adiposity and cardiometabolic markers at age 4 years in Mexican children $\mathrm{J}$ Nutr, 145(6), 1295-302

2110 Ramirez-Silva, I.,Rivera, J.,Martorell, R.,Stein, A.,Ramakrishnan, U. (2013). Breastfeeding at 3 months is associated with lower risk of adiposity and lipid metabolism alterations at 4 y of age Annals of nutrition \& metabolism, 63(\#issue\#), 774-5 
2112 Ramos-Gomez, F. J.,Tomar, S. L.,Ellison, J.,Artiga, N.,Sintes, J.,Vicuna, G. (1999). Assessment of early childhood caries and dietary habits in a population of migrant Hispanic children in Stockton, California ASDC J Dent Child, 66(6), 395-403, 366

2113 Rannan-Eliya, R. P.,Hossain, S. M.,Anuranga, C.,Wickramasinghe, R., Jayatissa, R.,Abeykoon, A. T. (2013). Trends and determinants of childhood stunting and underweight in Sri Lanka Ceylon Med J, 58(1), 10-8

2114 Ransome, O. J.,Chalmers, B.,Herman, A. A.,Reinach, S. G. (1988). Infant feeding in an urban community S Afr Med J, 74(8), 393-5

2115 Rao, M. R.,Hediger, M. L.,Levine, R. J.,Naficy, A. B.,Vik, T. (2002). Effect of breastfeeding on cognitive development of infants born small for gestational age Acta Paediatr, 91(3), 267-74

2116 Rao, S.,Kanade, A. N. (1992). Prolonged breast-feeding and malnutrition among rural Indian children below 3 years of age Eur J Country Clin Nutr, 46(3), 187-95

2117 Rao, S.,Rajpathak, V. (1992). Breastfeeding and weaning practices in relation to nutritional status of infants Indian pediatrics, 29(12), 1533-1539

2118 Rasmussen, K. M.,Kjolhede, C. L. (2008). Maternal obesity: a problem for both mother and child Obesity (Silver Spring), 16(5), 929- Study design 31

2119 Rassin, D. K.,Raiha, N. C.,Minoli, I.,Moro, G. (1990). Taurine and cholesterol supplementation in the term infant: responses of growth and metabolism JPEN J Parenter Enteral Nutr, 14(4), 392-7

2120 Ratageri, V. H.,Kabra, S. K.,Dwivedi, S. N.,Seth, V. (2000). Factors associated with severe asthma Indian Pediatr, 37(10), 1072-82

2121 Rathnayake, K. M.,Satchithananthan, A.,Mahamithawa, S.,Jayawardena, R. (2013). Early life predictors of preschool overweight and obesity: a case-control study in Sri Lanka BMC Public Health, 13(\#issue\#), 994

Study design

Study design

Country, Study design

Participant health, Intervention/exposure

Country

Size of study groups

Country

Study design,

Intervention/exposure

2122 Ravelli, A. C.,van der Meulen, J. H.,Osmond, C.,Barker, D. J.,Bleker, O. P. (2000). Infant feeding and adult glucose tolerance, lipid Intervention/exposure profile, blood pressure, and obesity Arch Dis Child, 82(3), 248-52

2123 Rawashdeh, M. O.,Khalil, B.,Raweily, E. (1996). Celiac disease in Arabs J Pediatr Gastroenterol Nutr, 23(4), 415-8

Study design,

Intervention/exposure,

Participant health

2124 Ray G (1985). Infant feeding. Psychology of choice Nurs Mirror, 160(\#issue\#), 25-8

Study design

2125 Reading, R. (2008). Effects of prolonged and exclusive breastfeeding on child behavior and maternal adjustment: evidence from a large, randomized trial...Kramer MS, Fombonne E, Igumnov S, Vanilovich L, Matush L, Mironova E, Bogdanovich N, Tremblay RE, Chalmers B, Zhang X \& Platt RW for the PROBIT study group (2008) Pediatrics, 121, E435-40 Child: Care, Health \& Development $34(4), 547-5471 \mathrm{p}$

2126 Rebhan, B.,Kohlhuber, M.,Schwegler, U.,Fromme, H.,Abou-Dakn, M.,Koletzko, B. V. (2009). Breastfeeding duration and exclusivity associated with infants' health and growth: data from a prospective cohort study in Bavaria, Germany Acta Paediatr, 98(6), 974-80 
2127 Regnault, N.,Botton, J.,Blanc, L.,Hankard, R.,Forhan, A.,Goua, V.,Thiebaugeorges, O.,Kaminski, M.,Heude, B.,Charles, M. A. (2011). Determinants of neonatal weight loss in term-infants: specific association with pre-pregnancy maternal body mass index and infant feeding mode Arch Dis Child Fetal Neonatal Ed, 96(3), F217-22

2128 Regnault, N.,Botton, J.,Forhan, A.,Hankard, R.,Thiebaugeorges, O.,Hillier, T. A.,Kaminski, M.,Heude, B.,Charles, M. A. (2010) Determinants of early ponderal and statural growth in full-term infants in the EDEN mother-child cohort study Am J Clin Nutr, 92(3), $594-602$

2129 Reid, A. (2002). Infant feeding and post-neonatal mortality in Derbyshire, England, in the early twentieth century Popul Stud (Camb), Intervention/exposure, Outcome $56(2), 151-66$

2130 Renn, M. (1987). Baby milk: is breast second best? Nurs Times, 83(6), 19-20

Study design

2131 Rennie, A. M.,Rowand, J. (2012). The beautiful game and breastfeeding Pract Midwife, 15(9), 46

Study design

2132 Renz, H.,Brehler, C.,Petzoldt, S.,Prinz, H.,Rieger, C. H. (1991). Breast feeding modifies production of SlgA cow's milk-antibodies in Outcome infants Acta Paediatr Scand, 80(2), 149-54

2133 Reyes Romagosa, D. E.,Paneque Gamboa, M. R.,Almeida Muniz, Y.,Quesada Oliva, L. M.,Escalona Oliva, D.,Torres Naranjo, S. (2014). Risk factors associated with deforming oral habits in children aged 5 to 11: a case-control study Medwave, 14(2), e5927

2134 Reyes, H.,Perez-Cuevas, R.,Salmeron, J.,Tome, P.,Guiscafre, H.,Gutierrez, G. (1997). Infant mortality due to acute respiratory infections: the influence of primary care processes Health Policy Plan, 12(3), 214-23

2135 Reyes, H.,Perez-Cuevas, R., Sandoval, A.,Castillo, R.,Santos, J. I.,Doubova, S. V.,Gutierrez, G. (2004). The family as a determinant Study design of stunting in children living in conditions of extreme poverty: a case-control study BMC Public Health, 4(\#issue\#), 57

2136 Reyes, M.,Hoyos, V.,Martinez, S. M.,Lozoff, B.,Castillo, M.,Burrows, R.,Blanco, E.,Gahagan, S. (2014). Satiety responsiveness and Intervention/exposure eating behavior among Chilean adolescents and the role of breastfeeding Int J Obes (Lond), 38(4), 552-7

2137 Reynolds, D.,Hennessy, E.,Polek, E. (2014). Is breastfeeding in infancy predictive of child mental well-being and protective against Study design obesity at 9 years of age? Child Care Health Dev, 40(6), 882-90

2138 Rhodes C (1982). The benefits of breast-feeding J Pract Nurs, 32(\#issue\#), 19-21, 54-5

Study design

2139 Ribadeau-Dumas, B. (1983). Human milk Endeavour, 7(2), 80-7

Study design

2140 Ribas-Fito, N.,Cardo, E.,Sala, M.,Eulalia de Muga, M.,Mazon, C.,Verdu, A.,Kogevinas, M.,Grimalt, J. O.,Sunyer, J. (2003) Breastfeeding, exposure to organochlorine compounds, and neurodevelopment in infants Pediatrics, 111(5 Pt 1), e580-5

2141 Ribas-Fito, N.,Julvez, J.,Torrent, M.,Grimalt, J. O.,Sunyer, J. (2007). Beneficial effects of breastfeeding on cognition regardless of DDT concentrations at birth Am J Epidemiol, 166(10), 1198-202

2142 Ricco, R. G.,Nogueira-de-Almeida, C. A.,Del Ciampo, L. A.,Daneluzzi, J. C.,Ferlin, M. L.,Muccillo, G. (2001). Growth of exclusively breast-fed infants from a poor urban population Arch Latinoam Nutr, 51(2), 122-6 
2143 Richards, M.,Hardy, R.,Wadsworth, M. E. (2002). Long-term effects of breast-feeding in a national birth cohort: educational attainment and midlife cognitive function Public Health Nutr, 5(5), 631-5

Publication date for a non-sibling study

2144 Richards, M.,Wadsworth, M.,Rahimi-Foroushani, A.,Hardy, R.,Kuh, D.,Paul, A. (1998). Infant nutrition and cognitive development in Intervention/exposure the first offspring of a national UK birth cohort Dev Med Child Neurol, 40(3), 163-7

2145 Richardson, B. D.,Cleaton-Jones, P. E.,Mclnnes, P. M.,Rantsho, J. M. (1981). Infant feeding practices and nursing bottle caries Study design, Outcome ASDC J Dent Child, 48(6), 423-9

2146 Rich-Edwards, J. W.,Stampfer, M. J.,Manson, J. E.,Rosner, B.,Hu, F. B.,Michels, K. B.,Willett, W. C. (2004). Breastfeeding during Intervention/exposure infancy and the risk of cardiovascular disease in adulthood Epidemiology, 15(5), 550-6

2147 Richman, D.,Dixon, S. (1985). Comparative study of Cambodian, Hmong, and Caucasian infant and maternal perinatal profiles J Intervention/exposure Nurse Midwifery, 30(6), 313-9 maternal smoking in the etiology of Crohn's disease and ulcerative colitis in childhood Annals of Epidemiology, 3(4), 387-392

2149 Rigby, A. S.,Sanderson, C.,Desforges, M. F.,Lindsay, G.,Hall, D. M. (1999). The infant index: a new outcome measure for preschool children's services J Public Health Med, 21(2), 172-8

2150 Rigo, J.,Salle, B. L.,Cavero, E.,Richard, P.,Putet, G.,Senterre, J. (1994). Plasma amino acid and protein concentrations in infants fed human milk or a whey protein hydrolysate formula during the first month of life Acta Paediatr, 83(2), 127-31

2151 Rigo, J.,Salle, B. L.,Picaud, J. C.,Putet, G.,Senterre, J. (1995). Nutritional evaluation of protein hydrolysate formulas Eur J Clin Nutr, Size of study groups 49 Suppl 1(\#issue\#), S26-38

2152 Riordan, J.,Countryman, B. A. (1980). Basics of breastfeeding. Part IV: Preparation for breastfeeding and early optimal functioning Study design, Outcome JOGN Nurs, 9(5), 277-83 associated with overweight in 7-year-old low-income Chilean children Matern Child Nutr, 11(4), 595-605

2154 Riva, V.,Battaglia, M.,Nobile, M.,Cattaneo, F.,Lazazzera, C.,Mascheretti, S.,Giorda, R.,Merette, C.,Emond, C.,Maziade, M.,Marino, Study design C. (2015). GRIN2B predicts attention problems among disadvantaged children Eur Child Adolesc Psychiatry, 24(7), 827-36

2155 Roberts AK (1987). Prospects for further approximation of infant formulae to human milk Midwife Health Visit Community Nurse 23(\#issue\#), 140-6

2156 Roberts, C. C.,Chan, G. M.,Folland, D.,Rayburn, C., Jackson, R. (1981). Adequate bone mineralization in breast-fed infants J Pediatr, 99(2), 192-6

2157 Roberts, D. W. (1980). Growth of breast fed and bottle fed infants N Z Med J, 92(664), 45-6

2158 Roberts, G. J. (1982). Is breast feeding a possible cause of dental caries? J Dent, 10(4), 346-52 
2159 Roberts, S. E.,Wotton, C. J.,Williams, J. G.,Griffith, M.,Goldacre, M. J. (2011). Perinatal and early life risk factors for inflammatory bowel disease World J Gastroenterol, 17(6), 743-9

2160 Robertson, L.,Harrild, K. (2010). Maternal and neonatal risk factors for childhood type 1 diabetes: a matched case-control study BMC Public Health, 10(\#issue\#), 281

2161 Robinson, M.,Oddy, W. H.,Li, J.,Kendall, G. E.,de Klerk, N. H.,Silburn, S. R.,Zubrick, S. R.,Newnham, J. P.,Stanley, F. J.,Mattes, E. (2008). Pre- and postnatal influences on preschool mental health: a large-scale cohort study J Child Psychol Psychiatry, 49(10), $1118-28$

2162 Robinson, S. M.,Crozier, S. R.,Harvey, N. C.,Barton, B. D.,Law, C. M.,Godfrey, K. M.,Cooper, C.,Inskip, H. M. (2015). Modifiable early-life risk factors for childhood adiposity and overweight: an analysis of their combined impact and potential for prevention Am $\mathrm{J}$ Clin Nutr, 101(2), 368-75

2163 Robinson, S. M.,Marriott, L. D.,Crozier, S. R.,Harvey, N. C.,Gale, C. R.,Inskip, H. M.,Baird, J.,Law, C. M.,Godfrey, K. M.,Cooper, C. (2009). Variations in infant feeding practice are associated with body composition in childhood: a prospective cohort study $\mathrm{J}$ Clin Endocrinol Metab, 94(8), 2799-805

2164 Rochat, F.,Cherbut, C.,Barclay, D.,Puccio, G.,Fazzolari-Nesci, A.,Grathwohl, D.,Haschke, F. (2007). A whey-predominant formula induces fecal microbiota similar to that found in breast-fed infants Nutrition Research, 27(12), 735-740

2165 Roche, A. F.,Guo, S.,Siervogel, R. M.,Khamis, H. J.,Chandra, R. K. (1993). Growth comparison of breast-fed and formula-fed infants Can J Public Health, 84(2), 132-5

2166 Rodekamp, E.,Harder, T.,Kohlhoff, R.,Dudenhausen, J. W.,Plagemann, A. (2006). Impact of breast-feeding on psychomotor and neuropsychological development in children of diabetic mothers: role of the late neonatal period J Perinat Med, 34(6), 490-6

2167 Rodekamp, E.,Harder, T.,Kohlhoff, R.,Franke, K.,Dudenhausen, J. W.,Plagemann, A. (2005). Long-term impact of breast-feeding on body weight and glucose tolerance in children of diabetic mothers: role of the late neonatal period and early infancy Diabetes Care, 28(6), 1457-62 of Bogota, Colombia J Asthma, 45(2), 141-7

2169 Rodriguez-Lopez, M.,Osorio, L.,Acosta-Rojas, R.,Figueras, J.,Cruz-Lemini, M.,Figueras, F.,Bijnens, B.,Gratacos, E.,Crispi, F. (2015). Participant health, Influence of breastfeeding and postnatal nutrition on cardiovascular remodeling induced by fetal growth restriction Pediatr Res, \#volume\#(\#issue\#), \#Pages\#

2170 Roelants, M.,Hauspie, R.,Hoppenbrouwers, K. (2010). Breastfeeding, growth and growth standards: Performance of the WHO growth standards for monitoring growth of Belgian children Ann Hum Biol, 37(1), 2-9

2171 Rogan, W. J.,Gladen, B. C. (1993). Breast-feeding and cognitive development Early Hum Dev, 31(3), 181-93

2172 Rolland-Cachera, M. F.,Peneau, S. (2011). Assessment of growth: variations according to references and growth parameters used Am J Clin Nutr, 94(6 Suppl), 1794S-1798S

Intervention/exposure

Size of study groups, Outcome

Publication date for a non-sibling study

Intervention/exposure

Publication date for a non-sibling study

Study design

intervention/exposure

Intervention/exposure

Study design 
2173 Romano, A. M. (2006). Longer duration of breastfeeding is associated with lower risk of type-2 diabetes (abst; commentary) Journal of Perinatal Education, 15(2), 54-55 2p

2174 Romero, C. C.,Scavone Jr, H.,Garib, D. G.,Cotrim-Ferreira, F. A.,Ferreira, I. R. (2011). Breastfeeding and non-nutritive sucking patterns related to the prevalence of anterior open bite in primary dentition Journal of Applied Oral Science, 19(2), 161-168

2175 Romieu, I.,Werneck, G.,Ruiz Velasco, S.,White, M.,Hernandez, M. (2000). Breastfeeding and asthma among Brazilian children J Study design Asthma, 37(7), 575-83

2176 Rona, R. J.,Smeeton, N. C.,Bustos, P.,Amiga, H.,Diaz, P. V. (2005). The early origins hypothesis with an emphasis on growth rate Outcome in the first year of life and asthma: A prospective study in Chile Thorax, 60(7), 549-554

2177 Rosas-Salazar, C.,Forno, E.,Brehm, J. M.,Han, Y. Y.,Acosta-Perez, E.,Cloutier, M. M.,Wakefield, D. B.,Alvarez, M.,Colon-Semidey, A.,Canino, G.,Celedon, J. C. (2015). Breastfeeding duration and asthma in Puerto Rican children Pediatr Pulmonol, 50(6), 527-34

2178 Rose, C. M.,Savage, J. S.,Birch, L. L. (2016). Patterns of early dietary exposures have implications for maternal and child weight outcomes Obesity (Silver Spring), 24(2), 430-8

2179 Rosenbauer, J.,Herzig, P.,Giani, G. (2008). Early infant feeding and risk of type 1 diabetes mellitus-a nationwide population-based Outcome case-control study in pre-school children Diabetes Metab Res Rev, 24(3), 211-22

2180 Rosenbauer, J.,Herzig, P.,Kaiser, P.,Giani, G. (2007). Early nutrition and risk of Type 1 diabetes mellitus--a nationwide case-control Duplicate study in preschool children Exp Clin Endocrinol Diabetes, 115(8), 502-8

2181 Rosenberg, M. (1989). Breast-feeding and infant mortality in Norway 1860-1930 J Biosoc Sci, 21(3), 335-48

Intervention/exposure

2182 Rosenblatt, A.,Zarzar, P. (2002). The prevalence of early childhood caries in 12- to 36-month-old children in Recife, Brazil ASDC J Study design Dent Child, 69(3), 319-24, 236

2183 Rosenblatt, W. H.,Brown, E. G. (1988). The nutritional status of breast-fed infants in a rural Peruvian community J Trop Pediatr $34(6), 319-22$

2184 Rossiter, J. C. (1993). Breast-feeding, the better option: getting the message across World Health Forum, 14(3), 316-8

Study design

Study design

2185 Rossiter, M. D.,Colapinto, C. K.,Khan, M. K.,Mclsaac, J. L.,Williams, P. L.,Kirk, S. F., Veugelers, P. J. (2015). Breast, Formula and Combination Feeding in Relation to Childhood Obesity in Nova Scotia, Canada Matern Child Health J, 19(9), 2048-56

2186 Rossiter, M. D.,Evers, S. E. (2013). Infant feeding practices and children's weight status Can J Diet Pract Res, 74(3), 107-13

2187 Roszkowska, R.,Taranta-Janusz, K.,Tenderenda-Banasiuk, E.,Wasilewska, A. (2014). Increased circulating inflammatory markers may indicate that formula-fed children are at risk of atherosclerosis Acta Paediatr, 103(8), e354-8

2188 Roszkowska, R.,Taranta-Janusz, K., Tenderenda-Banasiuk, E.,Wasilewska, A. (2015). The effects of breastfeeding on serum asymmetric dimethylarginine levels and body composition in children Breastfeed Med, 10(\#issue\#), 38-44 
2189 Roth DE (2016). Maternal postpartum high-dose vitamin D3 supplementation (6400 IU/day) or conventional infant vitamin D3 supplementation (400 IU/day) lead to similar vitamin D status of healthy exclusively/fully breastfeeding infants by 7 months of age

Study design

Evid Based Med, 21(\#issue\#), 75

2190 Rothenbacher, D.,Weyermann, M.,Beermann, C. Brenner, H. (2005) Breastfeeding soluble CD14 concentration in breast milk and risk of atopic dermatitis and asthma in early childhood: birth cohort study Clin Exp Allergy, 35(8), 1014-21

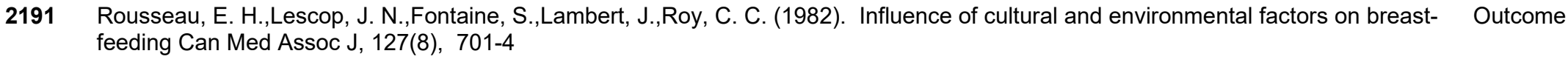

2192 Routi, T.,Ronnemaa, T.,Viikari, J. S.,Leino, A.,Valimaki, I. A.,Simell, O. G. (1997). Tracking of serum lipoprotein (a) concentration and its contribution to serum cholesterol values in children from 7 to 36 months of age in the STRIP Baby Study. Special Turku Coronary Risk Factor Intervention Project for Babies Ann Med, 29(6), 541-7 2193 Rowland, M. G. (1985). The "why" and "when" of introducing food to infants: growth in young breast-fed infants and some nutritional Study design
implications Am J Clin Nutr, 41(2 Suppl), 459-63

2194 Rowntree, S.,Cogswell, J. J.,Platts-Mills, T. A.,Mitchell, E. B. (1985). Development of IgE and IgG antibodies to food and inhalant allergens in children at risk of allergic disease Arch Dis Child, 60(8), 727-35

2195 Rubin, D. H.,Leventhal, J. M.,Krasilnikoff, P. A.,Kuo, H. S.,Jekel, J. F.,Weile, B.,Levee, A.,Kurzon, M.,Berget, A. (1990). Relationship Intervention/exposure between infant feeding and infectious illness: a prospective study of infants during the first year of life Pediatrics, 85(4), 464-71

2196 Rudant, J.,Lightfoot, T.,Urayama, K. Y.,Petridou, E.,Dockerty, J. D.,Magnani, C.,Milne, E.,Spector, L. G.,Ashton, L. J.,Dessypris, N.,Kang, A. Y.,Miller, M.,Rondelli, R.,Simpson, J.,Stiakaki, E.,Orsi, L.,Roman, E.,Metayer, C.,Infante-Rivard, C.,Clavel, J. (2015) Childhood acute lymphoblastic leukemia and indicators of early immune stimulation: A childhood leukemia international consortium study American Journal of Epidemiology, 181(8), 549-562

2197 Rudant, J.,Orsi, L.,Bonaventure, A., Goujon-Bellec, S.,Baruchel, A.,Petit, A.,Bertrand, Y.,Nelken, B.,Pasquet, M.,Michel, G.,Saumet L.,Chastagner, P.,Ducassou, S.,Reguerre, Y.,Hemon, D.,Clavel, J. (2015). ARID5B, IKZF1 and non-genetic factors in the etiology of childhood acute lymphoblastic leukemia: the ESCALE study PLoS One, 10(3), e0121348

2198 Rudant, J.,Orsi, L.,Menegaux, F.,Petit, A.,Baruchel, A.,Bertrand, Y.,Lambilliotte, A.,Robert, A.,Michel, G.,Margueritte, G.,Tandonnet, J.,Mechinaud, F.,Bordigoni, P.,Hemon, D.,Clavel, J. (2010). Childhood acute leukemia, early common infections, and allergy: The ESCALE Study Am J Epidemiol, 172(9), 1015-27

2199 Rudnicka, A. R.,Owen, C. G.,Richards, M.,Wadsworth, M. E.,Strachan, D. P. (2008). Effect of breastfeeding and sociodemographic Outcome factors on visual outcome in childhood and adolescence Am J Clin Nutr, 87(5), 1392-9

2200 Rudnicka, A. R.,Owen, C. G.,Strachan, D. P. (2007). The effect of breastfeeding on cardiorespiratory risk factors in adult life Pediatrics, 119(5), e1107-15 
2202 Ruiz-Charles, M. G.,Castillo-Rendón, R.,Bermúdez-Felizardo, F. (2002). Risk factors associated to bronchiolitis in infants less than two years of age Revista de Investigacion Clinica, 54(2), 125-132

2203 Ruiz-Palacios, G. M.,Calva, J. J.,Pickering, L. K.,Lopez-Vidal, Y.,Volkow, P.,Pezzarossi, H.,West, M. S. (1990). Protection of breast- $\quad$ Size of study groups fed infants against Campylobacter diarrhea by antibodies in human milk J Pediatr, 116(5), 707-13

2204 Rullo, V. E.,Arruda, L. K.,Cardoso, M. R.,Valente, V.,Zampolo, A. S.,Nobrega, F.,Naspitz, C. K.,Sole, D. (2009). Respiratory infection, exposure to mouse allergen and breastfeeding: role in recurrent wheezing in early life Int Arch Allergy Immunol, 150(2), $172-8$

2205 Rusconi, F.,Galassi, C.,Corbo, G. M.,Forastiere, F.,Biggeri, A.,Ciccone, G.,Renzoni, E.,Camerlengo, A.,Bugiani, M.,Dalmasso, P.,Faggiano, F.,Volante, T. F.,Magnani, C.,Natale, P.,Piccioni, P.,Bisanti, L.,Gianelle, V.,Sideri, S.,Piffer, S.,Filippetti, F.,Nava,

E.,Biocca, M.,Canossa, E.,Cavalchi, B.,Cervino, D.,Cattani, S.,De'Munari, E.,Deserti, M.,Ferro, S.,Fortezza, F.,Frigo, F.,Martini,

M.,Mazzali, P.,Paterlini, L.,Sogni, R.,Zanini, M.,Romagna, E.,Chellini, E.,Agati, L.,Barletta, E.,Bini, G.,Bini, M.,Chetoni, L.,,Grechi,

D.,Costantini, A. S.,Sestini, P.,Viegi, G.,Agabiti, N.,Dell'Orco, V.,Mallone, S.,Micera, C.,Palermo, P.,Pallotti, G.,Piras, C.,Pistelli,

R.,Salera, E.,Argentini, D.,Chiarucci, G. (1999). Risk factors for early, persistent, and late-onset wheezing in young children

American Journal of Respiratory and Critical Care Medicine, 160(5 I), 1617-1622

2206 Rush, E. C.,Paterson, J.,Obolonkin, V. V.,Puniani, K. (2008). Application of the 2006 WHO growth standard from birth to 4 years to Pacific Island children Int J Obes (Lond), 32(3), 567-72

2207 Rush, E.,Gao, W.,Funaki-Tahifote, M.,Ngamata, R.,Matenga-Smith, T.,Cassidy, M.,Paterson, J. (2010). Birth weight and growth trajectory to six years in Pacific children Int J Pediatr Obes, 5(2), 192-9

2208 Russo, R. M.,Patel, R.,Laude, T. A.,Rajkumar, S. V.,Gururaj, V. J. (1981). Infant feeding practices by ethno-cultural grouping J Med Study design, Outcome Soc N J, 78(11), 737-40

2209 Rutishauser, I. H.,McKay, H. M.,Wahlqvist, M. L. (1982). Does breast feeding have nutritional advantages over bottle feeding? Aust Study design Fam Physician, 11(4), 249-50, 252-3, 255-6

2210 Ruuska, T. (1992). Occurrence of acute diarrhea in atopic and nonatopic infants: the role of prolonged breast-feeding J Pediatr Gastroenterol Nutr, 14(1), 27-33

2211 Ruuska, T.,Vesikari, T. (1991). A prospective study of acute diarrhoea in Finnish children from birth to $21 / 2$ years of age Acta Paediatr Scand, 80(5), 500-7

2212 Ruys, J. H.,de Jonge, G. A.,Brand, R.,Engelberts, A. C.,Semmekrot, B. A. (2007). Bed-sharing in the first four months of life: a risk factor for sudden infant death Acta Paediatr, 96(10), 1399-403

2213 Rylander, E.,Pershagen, G.,Eriksson, M.,Nordvall, L. (1993). Parental smoking and other risk factors for wheezing bronchitis in children Eur J Epidemiol, 9(5), 517-26

2214 Rzehak, P.,Sausenthaler, S.,Koletzko, S.,Bauer, C. P.,Schaaf, B.,von Berg, A.,Berdel, D.,Borte, M.,Herbarth, O.,Kramer, U.,Fenske, N.,Wichmann, H. E.,Heinrich, J. (2009). Period-specific growth, overweight and modification by breastfeeding in the GINI and LISA birth cohorts up to age 6 years Eur J Epidemiol, 24(8), 449-67 
2215 Rzehak, P., Sausenthaler, S.,Koletzko, S. Reinhardt, D.,von Berg, A.,Kramer, U.,Berdel, D.,Bollrath, C., Grubl, A.,Bauer, C. P.,Wichmann, H. E.,Heinrich, J. (2009). Short- and long-term effects of feeding hydrolyzed protein infant formulas on growth at $<$ or = 6 y of age: results from the German Infant Nutritional Intervention Study Am J Clin Nutr, 89(6), 1846-56

2216 Saarinen, K. M.,Juntunen-Backman, K.,Jarvenpaa, A. L.,Klemetti, P.,Kuitunen, P.,Lope, L.,Renlund, M.,Siivola, M.,Vaarala, O.,Savilahti, E. (2000). Breast-feeding and the development of cows' milk protein allergy Adv Exp Med Biol, 478(\#issue\#), 121-30

Saarinen, K. M.,Juntunen-Backman, K.,Jarvenpaa, A. L.,Kuitunen, P.,Lope, L.,Renlund, M.,Siivola, M.,Savilahti, E. (1999). Supplementary feeding in maternity hospitals and the risk of cow's milk allergy: A prospective study of 6209 infants J Allergy Clin Immunol, 104(2 Pt 1), 457-61

2218 Saarinen, K. M.,Savilahti, E. (2000). Infant feeding patterns affect the subsequent immunological features in cow's milk allergy Clin Exp Allergy, 30(3), 400-6

2219 Saarinen, U. M. (1982). Prolonged breast feeding as prophylaxis for recurrent otitis media Acta Paediatr Scand, 71(4), 567-71

2220 Saarinen, U. M.,Kajosaari, M. (1995). Breastfeeding as prophylaxis against atopic disease: prospective follow-up study until 17 years old Lancet, 346(8982), 1065-9 of life Allergy, 37(5), 345-50

2222 Sabanayagam, C.,Shankar, A.,Chong, Y. S.,Wong, T. Y.,Saw, S. M. (2009). Breast-feeding and overweight in Singapore schoo children Pediatr Int, 51(5), 650-6

2223 Sabuncuoglu, O.,Orengul, C.,Bikmazer, A.,Kaynar, S. Y. (2014). Breastfeeding and parafunctional oral habits in children with and without attention-deficit/hyperactivity disorder Breastfeed Med, 9(5), 244-50

2224 Sacker, A.,Kelly, Y.,lacovou, M.,Cable, N.,Bartley, M. (2013). Breast feeding and intergenerational social mobility: what are the mechanisms? Arch Dis Child, 98(9), 666-71

2225 Sacker, A.,Quigley, M. A.,Kelly, Y. J. (2006). Breastfeeding and developmental delay: findings from the millennium cohort stud Pediatrics, 118(3), e682-9

2226 Sadauskaite-Kuehne, V.,Ludvigsson, J.,Padaiga, Z.,Jasinskiene, E.,Samuelsson, U. (2004). Longer breastfeeding is an independent protective factor against development of type 1 diabetes mellitus in childhood Diabetes Metab Res Rev, 20(2), 150-7 in breast milk protect the child from enterovirus infections Pediatrics, 119(5), 941-6

2228 Saeed, M.,Waseem, Q.,Ali Shair, Q.,Omonogun, B. A.,Al Husein, A. (2008). Vitamin D deficiency rickets in Maternity and Children's Hospital, Najran, Saudi Arabia Pakistan Paediatric Journal, 32(3), 145-148

2229 Sahakyan, A.,Armenian, H. K.,Breitscheidel, L.,Thompson, M. E.,Enokyan, G. (2006). Feeding practices of babies and the development of atopic dermatitis in children after 12 months of age in Armenia: Is there a signal? European Journal of Epidemiology, 21(9), 723-725
Intervention/exposure,

Publication status

Study design,

Intervention/exposure

Participant health, Outcomes

Intervention/exposure

Intervention/exposure

Outcome

Study design

Outcome

Intervention/exposure, Outcome

Study design

Outcome

Outcome

Publication status

Intervention/exposure 


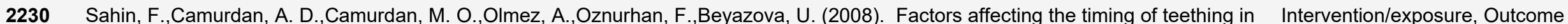
healthy Turkish infants: a prospective cohort study Int J Paediatr Dent, 18(4), 262-6

2231 Saijad, A.,Tharner, A.,Kiefte-de Jong, J. C.,Jaddoe, V. V.,Hofman, A.,Verhulst, F. C.,Franco, O. H.,Tiemeier, H.,Roza, S. J. (2015). Outcome Breastfeeding duration and non-verbal IQ in children J Epidemiol Community Health, 69(8), 775-81

2232 Saki Malehi, A.,Hajizadeh, E.,Ahmadi, K.,Kholdi, N. (2014). Modeling the recurrent failure to thrive in less than two-year children: recurrent events survival analysis J Res Health Sci, 14(1), 96-9

2233 Salah, M.,Abdel-Aziz, M.,Al-Farok, A.,Jebrini, A. (2013). Recurrent acute otitis media in infants: analysis of risk factors Int J Pediatr Otorhinolaryngol, 77(10), 1665-9

2234 Salariya, E. M. (1993). Breast versus bottle feeding Nutr Health, 9(1), 33-6 health

2235 Salariya, E. M.,Easton, P. M.,Cater, J. I. (1979). Early and often for best results. RCT on breast feeding Nursing mirror, 148(\#issue\#), 15-7

2236 Salariya, E. M.,Robertson, C. M. (1993). Relationships between baby feeding types and patterns, gut transit time of meconium and the incidence of neonatal jaundice Midwifery, $9(4), 235-42$

2237 Sala-Vila, A.,Campoy, C.,Castellote, A. I.,Garrido, F. J.,Rivero, M.,Rodríguez-Palmero, M.,López-Sabater, M. C. (2006). Influence of dietary source of docosahexaenoic and arachidonic acids on their incorporation into membrane phospholipids of red blood cells in term infants Prostaglandins Leukotrienes and Essential Fatty Acids, 74(2), 143-148

2238 Sala-Vila, A.,Castellote, A. I.,Campoy, C.,Rivero, M.,Rodriguez-Palmero, M.,Lopez-Sabater, M. C. (2004). The source of long-chain PUFA in formula supplements does not affect the fatty acid composition of plasma lipids in full-term infants $\mathrm{J} \mathrm{Nutr,} \mathrm{134(4),868-73}$

2239 Salazar, J. C.,Daly, K. A.,Giebink, G. S.,Lindgren, B. R.,Liebeler, C. L.,Meland, M.,Le, C. T. (1997). Low cord blood pneumococcal immunoglobulin $\mathrm{G}(\mathrm{IgG})$ antibodies predict early onset acute otitis media in infancy Am J Epidemiol, 145(11), 1048-56

2240 Salim, S.,Farquharson, J.,Arneil, G. C.,Cockburn, F.,Forbes, G. I.,Logan, R. W.,Sherlock, J. C., Wilson, T. S. (1986). Dietary copper Outcome intake in artificially fed infants Arch Dis Child, 61(11), 1068-75

2241 Salmenpera, L.,Perheentupa, J.,Siimes, M. A. (1985). Exclusively breast-fed healthy infants grow slower than reference infants Pediatr Res, 19(3), 307-12

2242 Salmenpera, L.,Perheentupa, J.,Siimes, M. A.,Adrian, T. E.,Bloom, S. R.,Aynsley-Green, A. (1988). Effects of feeding regimen on blood glucose levels and plasma concentrations of pancreatic hormones and gut regulatory peptides at 9 months of age: comparison between infants fed with milk formula and infants exclusively breast-fed from birth J Pediatr Gastroenterol Nutr, 7(5), 651-6

2243 Salmon, T. G., Jr. (1997). Early childhood caries: a private practitioner's perspective Pediatr Dent, 19(1), 63-4

2244 Salo, P.,Viikari, J.,Hamalainen, M.,Lapinleimu, H.,Routi, T.,Ronnemaa, T.,Seppanen, R.,Jokinen, E.,Valimaki, I.,Simell, O. (1999). Serum cholesterol ester fatty acids in 7- and 13-month-old children in a prospective randomized trial of a low-saturated fat, lowcholesterol diet: the STRIP baby project. Special Turku coronary Risk factor Intervention Project for children Acta Paediatr, 88(5), 505-12

\section{Size of study groups}

Size of study groups

Intervention/exposure

Intervention/exposure

Size of study groups 
2245 Salo, P.,Viikari, J.,Ronnemaa, T.,Hamalainen, M.,Jokinen, E.,Valimaki, I.,Simell, O. (1997). Milk type during mixed feeding: contribution to serum cholesterol ester fatty acids in late infancy J Pediatr, 130(1), 110-6

2246 Salsberry, P. J.,Reagan, P. B. (2005). Dynamics of early childhood overweight Pediatrics, 116(6), 1329-38

2247 Salsberry, P. J.,Reagan, P. B. (2007). Taking the long view: the prenatal environment and early adolescent overweight Res Nurs Health, 30(3), 297-307

Publication date for a non-sibling study

2248 Salvioli, G. P.,Faldella, G.,Alessandroni, R.,Lanari, M.,Di Turi, R. P. (1995). Iron nutrition and iron status changes in Italian infants in Study design the last decade Ann Ist Super Sanita, 31(4), 455-9

2249 Samarakkody, D.,Fernando, D.,McClure, R.,Perera, H.,De Silva, H. (2012). Prevalence of externalizing behavior problems in Sri Lankan preschool children: birth, childhood, and sociodemographic risk factors Soc Psychiatry Psychiatr Epidemiol, 47(5), 757-62

2250 Samuelsson, U.,Johansson, C.,Ludvigsson, J. (1993). Breast-feeding seems to play a marginal role in the prevention of insulindependent diabetes mellitus Diabetes Res Clin Pract, 19(3), 203-10

2251 Samuelsson, U.,Ludvigsson, J. (2001). Seasonal variation of birth month and breastfeeding in children with diabetes mellitus J Pediatr Endocrinol Metab, 14(1), 43-6

2252 Sanchez-Molins, M.,Grau Carbo, J.,Lischeid Gaig, C.,Ustrell Torrent, J. M. (2010). Comparative study of the craniofacial growth depending on the type of lactation received Eur J Paediatr Dent, 11(2), 87-92

2253 Sánchez-Uribe, E.,Esparza-Aguilar, M.,Gastañaduy, P. A.,Desai, R.,Patel, M.,Richardson, V. (2013). Risk factors associated with rotavirus gastroenteritis during a community outbreak in Chiapas, Mexico during the postvaccination Era Journal of the Pediatric Infectious Diseases Society, 2(1), 15-20

2254 Sanchez-Valverde, F.,Gil, F.,Martinez, D.,Fernandez, B.,Aznal, E.,Oscoz, M.,Olivera, J. E. (2009). The impact of caesarean delivery and type of feeding on cow's milk allergy in infants and subsequent development of allergic march in childhood Allergy, 64(6), 884-9

2255 Sanders TA,Reddy S (1992). The influence of a vegetarian diet on the fatty acid composition of human milk and the essential fatty acid status of the infant $\mathrm{J}$ Pediatr, 120(\#issue\#), S71-7

Sandini, U.,Kukkonen, A. K.,Poussa, T.,Sandini, L.,Savilahti, E.,Kuitunen, M. (2011). Protective an in high-risk children at the ages of two and five years Int Arch Allergy Immunol, 156(3), 339-48

2257 Sandstrom, O.,Lonnerdal, B.,Graverholt, G.,Hernell, O. (2008). Effects of alpha-lactalbumin-enriched formula containing different concentrations of glycomacropeptide on infant nutrition Am J Clin Nutr, 87(4), 921-8

2258 Sanger, R. G.,Bystrom, E. B. (1982). Breast feeding: does it affect oral facial growth? Dent Hyg (Chic), 56(6), 44-7

Study design

Outcome

Outcome

Intervention/exposure

Intervention/exposure

Participant health

Size of study groups

Outcome

Size of study groups

Study design

2259 Sangun, O.,Dundar, B.,Kosker, M.,Pirgon, O.,Dundar, N. (2011). Prevalence of metabolic syndrome in obese children and adolescents using three different criteria and evaluation of risk factors $\mathrm{J}$ Clin Res Pediatr Endocrinol, 3(2), 70-6

Outcome 
2260 Sanin, L. H.,Gonzalez-Cossio, T.,Romieu, I.,Peterson, K. E.,Ruiz, S.,Palazuelos, E.,Hernandez-Avila, M.,Hu, H. (2001). Effect of maternal lead burden on infant weight and weight gain at one month of age among breastfed infants Pediatrics, 107(5), 1016-23

2261 Sanjurjo, P.,Rodriguez-Alarcon, J.,Rodriguez-Soriano, J. (1988). Plasma fatty acid composition during the first week of life following feeding with human milk or formula Acta Paediatr Scand, 77(2), 202-6

2262 Santorelli, G.,Fairley, L.,Petherick, E. S.,Cabieses, B.,Sahota, P. (2014). Ethnic differences in infant feeding practices and their relationship with BMI at 3 years of age - results from the Born in Bradford birth cohort study Br J Nutr, 111(10), 1891-7

2263 Santos, C. A.,Strina, A.,Amorim, L. D.,Genser, B.,Assis, A. M.,Prado, M. S.,Barreto, M. L. (2012). Individual and contextual determinants of the duration of diarrhoeal episodes in preschool children: a longitudinal study in an urban setting Epidemiol Infect, $140(4), 689-96$

2264 Santos, I. S.,Matijasevich, A.,Assuncao, M. C., Valle, N. C.,Horta, B. L.,Goncalves, H. D.,Gigante, D. P.,Martines, J. C.,Pelto, G.,Victora, C. G. (2015). Promotion of Weight Gain in Early Childhood Does Not Increase Metabolic Risk in Adolescents: A 15-Year Follow-Up of a Cluster-Randomized Controlled Trial J Nutr, 145(12), 2749-55

2265 Santos, I. S.,Matijasevich, A.,Barros, A. J.,Albernaz, E. P.,Domingues, M. R.,Valle, N. C.,Malta, D. C.,Gorgot, L. R.,Barros, F. C. (2011). Avoidable deaths in the first four years of life among children in the 2004 Pelotas (Brazil) birth cohort study Cad Saude Publica, 27 Suppl 2(\#issue\#), S185-97

2266 Santos, I.,Victora, C. G.,Martines, J.,Goncalves, H.,Gigante, D. P.,Valle, N. J.,Pelto, G. (2001). Nutrition counseling increases weight gain among Brazilian children J Nutr, 131(11), 2866-73

2267 Sarasa Munoz, N. L. (2013). Mother's milk still best--and we must do better MEDICC Rev, 15(1), 48

Study design

2268 Sariachvili, M.,Droste, J.,Dom, S.,Wieringa, M.,Vellinga, A.,Hagendorens, M.,Bridts, C.,Stevens, W.,Sprundel, M. V.,Desager, K.,Weyler, J. (2007). Is breast feeding a risk factor for eczema during the first year of life? Pediatr Allergy Immunol, 18(5), 410-7

2269 Sartorius, N. (2007). Learning how to speak Croat Med J, 48(2), 259-60

2270 Sasai, K.,Furukawa, S.,Kaneko, K.,Yabuta, K.,Baba, M. (1994). Fecal lgE levels in infants at 1 month of age as indicator of atopic disease Allergy, 49(9), 791-4

2271 Sassen, M. L.,Brand, R.,Grote, J. J. (1994). Breast-feeding and acute otitis media Am J Otolaryngol, 15(5), 351-7

Size of study groups

Outcome for a non-sibling study

Participant health

Intervention/exposure

Outcome

Intervention/exposure

Outcome

\section{Study design}

Study design, Size of study groups

Outcome

2272 Sastry, N.,Burgard, S. (2011). Changes in Diarrheal Disease and Treatment Among Brazilian Children from 1986 to 1996 Popul Res Policy Rev, 30(1), 81-100

2273 Saukkonen, T.,Virtanen, S. M.,Karppinen, M.,Reijonen, H.,Ilonen, J.,Räsänen, L., (1998). Significance of cow's milk protein antibodies as risk factor for childhood IDDM: Interactions with dietary cow's milk intake and HLA-DQB1 genotype Diabetologia, 41(1), study $72-78$ 
2275 Savilahti, E.,Salmenpera, L.,Tainio, V. M.,Halme, H.,Perheentupa, J.,Siimes, M. A. (1987). Prolonged exclusive breast-feeding results in low serum concentrations of immunoglobulin G, A and M Acta Paediatr Scand, 76(1), 1-6

2276 Savilahti, E.,Siltanen, M.,Kajosaari, M.,Vaarala, O.,Saarinen, K. M. (2005). IgA antibodies, TGF-beta1 and -beta2, and soluble CD14 Outcome in the colostrum and development of atopy by age 4 Pediatr Res, 58(6), 1300-5

2277 Savilahti, E.,Tainio, V. M.,Salmenpera, L.,Arjomaa, P.,Kallio, M.,Perheentupa, J.,Siimes, M. A. (1991). Levels of IgA and cow milk antibodies in breast milk vs. the development of atopy in children. Low colostral IgA associated with cow milk allergy Adv Exp Med Biol, 310(\#issue\#), 417-25

2278 Savilahti, E.,Tainio, V. M.,Salmenpera, L.,Siimes, M. A.,Perheentupa, J. (1987). Prolonged exclusive breast feeding and heredity as Outcome determinants in infantile atopy Arch Dis Child, 62(3), 269-73

2279 Savino, F.,Liguori, S. A.,Benetti, S.,Sorrenti, M.,Fissore, M. F.,Cordero di Montezemolo, L. (2013). High serum leptin levels in infancy can potentially predict obesity in childhood, especially in formula-fed infants Acta Paediatr, 102(10), e455-9

2280 Savino, F.,Maccario, S.,Cresi, F.,Grasso, G.,Oggero, R.,Silvestro, L.,Mussa, G. C. (2004). Bioimpedance vector analysis in breastfed and formula-fed infants in the first six months of life Adv Exp Med Biol, 554(\#issue\#), 501-4

2281 Savino, F.,Oggero, R.,Prino, A.,Mostert, M. (1997). Hypoantigenic (HA) milk formula and blood cholesterol level in infants at 3 months of age Acta Paediatr, 86(9), 1003-5

2282 Savino, F.,Serraino, P.,Prino, A.,Oggero, R.,Bretto, R.,Mostert, M. (2000). Arachidonic (AA) and docosahexaenoic (DHA) acid content in healthy infants fed with an HA milk formula supplemented with LCPUFA and in breast fed infants Adv Exp Med Biol 478(\#issue\#), 411-2

2283 Savion I,Savion I (2007). Nursing of malnourished children with emphasis on polyunsaturated fatty acids Appl Nurs Res 20(\#issue\#), 140-5

2284 Sawchuk, L. A.,Burke, S. D. (2000). Mortality in an early Ontario community: Belleville 1876-1885 Urban Hist Rev, 29(1), 33-47

2285 Sawley, L. (1985). Bottle feeding Nurs Mirror, 160(3), 31-3

2286 Sawley, L. (1985). Breast is best Nurs Mirror, 160(2), 15-9

\section{Intervention/exposure}

Intervention/exposure

Size of study groups

Outcome

Study design, Size of study groups

Intervention/exposure, Study design

Study design

Study design

Study design

2287 Sawley, L. (1989). Infant feeding Nursing (Lond), 3(39), 18-23

Study design

2288 Say, G. N.,Karabekiroglu, K.,Babadagi, Z.,Yuce, M. (2015). Maternal stress and perinatal features in autism \& attention deficit/ hyperactivity disorder Pediatr Int, \#volume\#(\#issue\#), \#Pages\#

2289 Sayegh, A.,Dini, E. L.,Holt, R. D.,Bedi, R. (2002). Caries prevalence and patterns and their relationship to social class, infant feeding Study design and oral hygiene in 4-5-year-old children in Amman, Jordan Community Dent Health, 19(3), 144-51

2290 Sayegh, A.,Dini, E. L.,Holt, R. D.,Bedi, R. (2005). Oral health, sociodemographic factors, dietary and oral hygiene practices in Jordanian children J Dent, 33(5), 379-88 
2291 Sayyed, T.,Kandil, M.,Bashir, O.,Alnaser, H. (2014). The relationship between term pre-eclampsia and the risk of early childhood caries J Matern Fetal Neonatal Med, 27(1), 62-5

2292 Scaglioni, S.,Agostoni, C.,Notaris, R. D.,Radaelli, G.,Radice, N.,Valenti, M.,Giovannini, M.,Riva, E. (2000). Early macronutrient intake and overweight at five years of age Int J Obes Relat Metab Disord, 24(6), 777-81

2293 Scalabrin, D.,Mitmesser, S.,Birch, E.,Khoury, J.,Bean, J.,Harris, C.,Berseth, C. (2011). Lower incidence and less recurrence of allergic manifestations is observed in children who received docosahexaenoic acid/arachidonic acid in infancy via breast milk or supplemented formula Allergy: European Journal of Allergy and Clinical Immunology. Conference: 30th Congress of the European Academy of Allergy and Clinical Immunology Istanbul Turkey. Conference Start: 20110611 Conference End: 20110615. Conference Publication: (var.pagings), 66(94), 711

2294 Scariati, P. D.,Grummer-Strawn, L. M.,Fein, S. B. (1997). A longitudinal analysis of infant morbidity and the extent of breastfeeding in the United States Pediatrics, 99(6), E5

2295 Scariati, P. D.,Grummer-Strawn, L. M.,Fein, S. B.,Yip, R. (1997). Risk of diarrhea related to iron content of infant formula: lack of evidence to support the use of low-iron formula as a supplement for breastfed infants Pediatrics, 99(3), E2

2296 Scarlett D,Cargill M,Lyn-Sue J,Richardson S,McCaw-Binns A (1996). Breastfeeding prevalence among six-week-old infants at University Hospital of the West Indies West Indian Med J, 45(\#issue\#), 14-7

2297 Scerri, C.,Savona-Ventura, C. (2010). Early metabolic imprinting as a determinant of childhood obesity International Journal of Diabetes Mellitus, 2(3), 175-178

2298 Schach, B.,Haight, M. (2002). Colic and food allergy in the breastfed infant: is it possible for an exclusively breastfed infant to suffer Study design from food allergy? J Hum Lact, 18(1), 50-2

2299 Schack-Nielsen, L.,Michaelsen, K. F.,Mortensen, E. L.,Sorensen, T. I.,Reinisch, J. M. (2004). Is duration of breastfeeding influencing the risk of obesity in adult males? Adv Exp Med Biol, 554(\#issue\#), 383-5

2300 Schack-Nielsen, L.,Molgaard, C.,Larsen, D.,Martyn, C.,Michaelsen, K. F. (2004). Arterial compliance in 10-year-old children in relation to breastfeeding Adv Exp Med Biol, 554(\#issue\#), 391-3

2301 Schack-Nielsen, L.,Molgaard, C.,Larsen, D.,Martyn, C.,Michaelsen, K. F. (2005). Arterial stiffness in 10-year-old children: current and early determinants $\mathrm{Br} \mathrm{J}$ Nutr, 94(6), 1004-11

2302 Schack-Nielsen, L.,Sorensen, Tla,Mortensen, E. L.,Michaelsen, K. F. (2010). Late introduction of complementary feeding, rather than duration of breastfeeding, may protect against adult overweight Am J Clin Nutr, 91(3), 619-27

Outcome study

Publication status

Intervention/exposure

Study design

Study design

Study design

Duplicate

Outcome

Publication date for a non-sibling study

2303 Schaefer-Graf, U. M.,Hartmann, R.,Pawliczak, J.,Passow, D.,Abou-Dakn, M.,Vetter, K.,Kordonouri, O. (2006). Association of breast- $\quad$ Study design feeding and early childhood overweight in children from mothers with gestational diabetes mellitus Diabetes Care, 29(5), 1105-7

2304 Scheer, B. (1985). Caries in children--the dietary factor Middle East Dent Oral Health, \#volume\#(3), 20-2

Study design

2305 Scheiwe, A.,Hardy, R.,Watt, R. G. (2010). Four-year follow-up of a randomized controlled trial of a social support intervention on infant feeding practices Matern Child Nutr, 6(4), 328-37 
2306 Schellscheidt, J.,Ott, A.,Jorch, G. (1997). Epidemiological features of sudden infant death after a German intervention campaign in 1992 Eur J Pediatr, 156(8), 655-60

2307 Scherdel, P.,Botton, J.,Rolland-Cachera, M. F.,Leger, J.,Pele, F.,Ancel, P. Y.,Simon, C.,Castetbon, K.,Salanave, B., Thibault, H.,Lioret, S.,Peneau, S.,Gusto, G.,Charles, M. A.,Heude, B. (2015). Should the WHO growth charts be used in France? PLoS One, 10(3), e0120806

2308 Schilithz, A. O., Kale, P. L., Gama, S. G.,Nobre, F. F. (2014). Risk groups in children under six months of age using self-organizing maps Comput Methods Programs Biomed, 115(1), 1-10

2309 Schluter, P. J.,Durward, C.,Cartwright, S.,Paterson, J. (2007). Maternal self-report of oral health in 4-year-old Pacific children from South Auckland, New Zealand: findings from the Pacific Islands Families Study J Public Health Dent, 67(2), 69-77

2310 Schluter, P. J.,Ford, R. P.,Mitchell, E. A., Taylor, B. J. (1998). Residential mobility and sudden infant death syndrome J Paediat Child Health, 34(5), 432-7

2311 Schluter, P. J.,Paterson, J.,Percival, T. (2007). Infant care practices associated with sudden infant death syndrome: findings from Study design the Pacific Islands Families study J Paediatr Child Health, 43(5), 388-93

2312 Schmidt BJ (1983). Breast-feeding and infant morbidity and mortality in developing countries J Pediatr Gastroenterol Nutr, 2 Suppl Study design 1(\#issue\#), S127-30

2313 Schmidt, M. E.,Rich, M.,Rifas-Shiman, S. L.,Oken, E.,Taveras, E. M. (2009). Television viewing in infancy and child cognition at $3 \quad$ Intervention/exposure years of age in a US cohort Pediatrics, 123(3), e370-5

2314 Schmidt, R. J.,Tancredi, D. J.,Krakowiak, P.,Hansen, R. L.,Ozonoff, S. (2014). Maternal intake of supplemental iron and risk of autism spectrum disorder Am J Epidemiol, 180(9), 890-900

2315 Schmitt, J.,Romanos, M. (2012). Prenatal and perinatal risk factors for attention-deficit/hyperactivity disorder Arch Pediatr Adolesc Med, 166(11), 1074-5

2316 Schnitzer, M. E.,Moodie, E. E.,Platt, R. W. (2013). Targeted maximum likelihood estimation for marginal time-dependent treatment effects under density misspecification Biostatistics, 14(1), 1-14

2317 Schnitzer, M. E.,van der Laan, M. J.,Moodie, E. E.,Platt, R. W. (2014). EFFECT OF BREASTFEEDING ON GASTROINTESTINAL INFECTION IN INFANTS: A TARGETED MAXIMUM LIKELIHOOD APPROACH FOR CLUSTERED LONGITUDINAL DATA Ann Appl Stat, 8(2), 703-725

2318 Schoen, S.,Sichert-Hellert, W.,Kersting, M. (2009). Validation of energy requirement equations for estimation of breast milk consumption in infants Public Health Nutr, 12(12), 2309-16

2319 Schoetzau, A.,Filipiak-Pittroff, B.,Franke, K.,Koletzko, S.,Von Berg, A.,Gruebl, A.,Bauer, C. P.,Berdel, D.,Reinhardt, D.,Wichmann, H. Intervention/exposure E. (2002). Effect of exclusive breast-feeding and early solid food avoidance on the incidence of atopic dermatitis in high-risk infants at 1 year of age Pediatr Allergy Immunol, 13(4), 234-42 
2320 Scholtens, S.,Brunekreef, B.,Smit, H. A.,Gast, G. C.,Hoekstra, M. O., de Jongste, J. C.,Postma, D. S.,Gerritsen, J.,Seidell, J. C.,Wijga, A. H. (2008). Do differences in childhood diet explain the reduced overweight risk in breastfed children? Obesity (Silver Spring), 16(11), 2498-503

2321 Scholtens, S.,Gehring, U.,Brunekreef, B.,Smit, H. A.,de Jongste, J. C.,Kerkhof, M.,Gerritsen, J.,Wijga, A. H. (2007). Breastfeeding, weight gain in infancy, and overweight at seven years of age: the prevention and incidence of asthma and mite allergy birth cohort study Am J Epidemiol, 165(8), 919-26

2322 Scholtens, S.,Wijga, A. H.,Brunekreef, B.,Kerkhof, M.,Hoekstra, M. O.,Gerritsen, J.,Aalberse, R.,de Jongste, J. C.,Smit, H. A. (2009). Breast feeding, parental allergy and asthma in children followed for 8 years. The PIAMA birth cohort study Thorax, 64(7), 604-9

2323 Schraw, J. M.,Dong, Y. Q.,Okcu, M. F.,Scheurer, M. E.,Forman, M. R. (2014). Do longer formula feeding and later introduction of solids increase risk for pediatric acute lymphoblastic leukemia? Cancer Causes and Control, 25(1), 73-80

2324 Schroeder, N.,Rushovich, B.,Bartlett, E.,Sharma, S.,Gittelsohn, J.,Caballero, B. (2015). Early Obesity Prevention: A Randomized Trial of a Practice-Based Intervention in 0-24-Month Infants J Obes, 2015(\#issue\#), 795859

2325 Schuz, J.,Kaletsch, U.,Meinert, R.,Kaatsch, P.,Michaelis, J. (1999). Association of childhood leukaemia with factors related to the immune system $\mathrm{Br} \mathrm{J}$ Cancer, 80(3-4), 585-90

2326 Schwartz, J.,Drossard, C.,Dube, K.,Kannenberg, F.,Kunz, C.,Kalhoff, H.,Kersting, M. (2010). Dietary intake and plasma concentrations of PUFA and LC-PUFA in breastfed and formula fed infants under real-life conditions Eur J Nutr, 49(3), 189-95

Publication date for a non-sibling study

Publication date for a non-sibling study

Outcome

Outcome

Intervention/exposure

Outcome

Size of study groups

2327 Schwartz, R.,Vigo, A., de Oliveira, L. D.,Justo Giugliani, E. R. (2015). The Effect of a Pro-Breastfeeding and Healthy Complementary Intervention/exposure Feeding Intervention Targeting Adolescent Mothers and Grandmothers on Growth and Prevalence of Overweight of Preschool Children PLoS One, 10(7), e0131884

2328 Schwartzbaum, J. A., George, S. L.,Pratt, C. B.,Davis, B. (1991). An exploratory study of environmental and medical factors potentially related to childhood cancer Med Pediatr Oncol, 19(2), 115-21

2329 Schwarz, T. (1990). Bottle or breast. The first big decision Nurs Times, 86(35), 63-5

2330 Schwarze, C. E.,Hellhammer, D. H.,Stroehle, V.,Lieb, K.,Mobascher, A. (2015). Lack of Breastfeeding: A Potential Risk Factor in the Multifactorial Genesis of Borderline Personality Disorder and Impaired Maternal Bonding J Pers Disord, 29(5), 610-26

2331 Schweitzer, F. C.,Prager, T. C.,Zou, Y.,Ruiz, R. S.,Chen, H.,Anderson, R. E.,Jensen, C. L.,Heird, W. C. (1995). Effect of 18:3ù3 Publication status intake on pattern visual evoked potentials in term infants lovs, 36(\#issue\#), ARVO Abstract 235

2332 Sclavos S,Porter S,Kim Seow W (1988). Future caries development in children with nursing bottle caries J Pedod, 13(\#issue\#), 1- Intervention/exposure 10

2333 Scott, D. T.,Janowsky, J. S.,Carroll, R. E.,Taylor, J. A.,Auestad, N.,Montalto, M. B. (1998). Formula supplementation with long-chain Outcome polyunsaturated fatty acids: are there developmental benefits? Pediatrics, 102(5), E59

2334 Scott, F. W.,Kolb, H. (1998). Dietary intervention for diabetes prevention in the neonate Diabetes Metab Rev, 14(1), 106

Study design 
2335 Scott, J. A.,Ng, S. Y.,Cobiac, L. (2012). The relationship between breastfeeding and weight status in a national sample of Australian children and adolescents BMC Public Health, 12(\#issue\#), 107

2336 Scott, M.,Roberts, G.,Kurukulaaratchy, R. J.,Matthews, S.,Nove, A.,Arshad, S. H. (2012). Multifaceted allergen avoidance during infancy reduces asthma during childhood with the effect persisting until age 18 years Thorax, 67(12), 1046-51

2337 Seach, K. A.,Dharmage, S. C.,Lowe, A. J.,Dixon, J. B. (2010). Delayed introduction of solid feeding reduces child overweight and obesity at 10 years Int J Obes (Lond), 34(10), 1475-9

2338 Seal, N.,Broome, M. E. (2013). Prepregnancy Body Mass Index and Feeding Practices in Relation to Infants' Growth J Nurse Pract, 9(5), \#Pages\#

2339 Sears, M. R.,Greene, J. M.,Willan, A. R.,Taylor, D. R.,Flannery, E. M.,Cowan, J. O.,Herbison, G. P.,Poulton, R. (2002). Long-term relation between breastfeeding and development of atopy and asthma in children and young adults: a longitudinal study Lancet, 360(9337), 901-7

2340 Seethalakshmi,,Rao, K. M. (1985). No substitute to mother's milk Nurs J India, 76(2), 48-9

Study design

2341 Seipel, M. M.,Shafer, K. (2013). The effect of prenatal and postnatal care on childhood obesity Soc Work, 58(3), 241-52

Intervention/exposure

2342 Selvakumar, B.,Vishnu Bhat, B. (2007). Infant feeding practice and its effect on the growth and development of babies Current Pediatric Research, 11(1-2), 13-16

2343 Serino, R. J.,Gold, S. B. (1997). Infant and early childhood oral health care N Y State Dent J, 63(2), 34-5

Study design

2344 Serva, V.,Karim, H.,Ebrahim, G. J. (1986). Breast-feeding and the urban poor in developing countries J Trop Pediatr, 32(3), 127-9 Pediatr, 5(9), 501-4 status of exclusively breast fed infants and their mothers J Pediatr Endocrinol Metab, 22(\#issue\#), 241-6

2347 Sethi, D.,Cumberland, P.,Hudson, M. J.,Rodrigues, L. C.,Wheeler, J. G.,Roberts, J. A., Tompkins, D. S.,Cowden, J. M.,Roderick, P. J. (2001). A study of infectious intestinal disease in England: risk factors associated with group A rotavirus in children Epidemiol Infect, 126(1), 63-70

2348 Sethi, V.,Kashyap, S.,Seth, V. (2003). Effect of nutrition education of mothers on infant feeding practices Indian J Pediatr, 70(6) 463-6

2349 Sezer, R. G.,Aydemir, G.,Akcan, A. B.,Bayoglu, D. S.,Guran, T.,Bozaykut, A. (2013). Effect of breastfeeding on serum zinc levels and growth in healthy infants Breastfeed Med, 8(\#issue\#), 159-63

2350 Shaaban, K. M.,Hamadnalla, I. (1993). The effect of duration of breast feeding on the occurrence of acute otitis media in children under three years East Afr Med J, 70(10), 632-4

Outcome

Study design

Country, Intervention/exposure

Intervention/exposure

Country

Study design

Country 
2351 Shalofsky, Teresa (2015). Telephone peer counselling of breastfeeding among WIC participants: a randomized controlled trial MIDIRS Midwifery Digest, 25(1), 97-98 2p

2352 Shamberger R (2012). Attention-deficit disorder associated with breast-feeding: a brief report J Am Coll Nutr, 31(\#issue\#), 239-42

Study design

2353 Shamir, R.,Nganga, A.,Berkowitz, D.,Diamond, E.,Lischinsky, S.,Lombardo, D.,Shehadeh, N. (2003). Serum levels of bile saltstimulated lipase and breast feeding J Pediatr Endocrinol Metab, 16(9), 1289-94

2354 Shand, N. (1981). The reciprocal impact of breast-feeding and culture form on maternal behaviour and infant development $\mathrm{J}$ Biosoc Sci, 13(1), 1-17

2355 Shariff, A. H.,Sazlina, S. G.,Shamsul, A. S. (2007). Obesity among urban primary schoolchildren Journal of Health and Translational Study design Medicine, 10(1), 17-20

2356 Sharifzadeh, G. R.,Namakin, K.,Mehrjoofard, H. (2008). An epidemiological study on infant mortatity and factors affecting it in rural areas of Birjand, Iran Iranian Journal of Pediatrics, 18(4), 335-342

2357 Sharma, S.,Sood, M.,Sood, A. (2011). Environmental risk factors in relation to childhood asthma in rural area Current Pediatric Country Research, 15(1), 29-32

2358 Shaternikov, V. A.,Fateeva, E. M.,Chernikov, M. N. (1982). Protein nutrition in early infancy and subsequent periods: its effect on Study design further development Bibl Nutr Dieta, \#volume\#(31), 95-111

2359 Shearrer, G. E.,Whaley, S. E.,Miller, S. J.,House, B. T.,Held, T.,Davis, J. N. (2015). Association of gestational diabetes and breastfeeding on obesity prevalence in predominately Hispanic low-income youth Pediatr Obes, 10(3), 165-71

2360 Shehadeh, N.,Weitzer-Kish, H.,Shamir, R.,Shihab, S.,Weiss, R. (2008). Impact of early postnatal weight gain and feeding patterns on body mass index in adolescence J Pediatr Endocrinol Metab, 21(1), 9-15

2361 Shelton, K. H.,Collishaw, S.,Rice, F. J.,Harold, G. T.,Thapar, A. (2011). Using a genetically informative design to examine the relationship between breastfeeding and childhood conduct problems Eur Child Adolesc Psychiatry, 20(11-12), 571-9

2362 Shepherd, J. (2002). Thrush and breastfeeding Pract Midwife, 5(11), 24-7

Study design

Intervention/exposure

Study design

Study design

2363 Shepherd, R. W.,Oxborough, D. B.,Holt, T. L., Thomas, B. J., Thong, Y. H. (1988). Longitudinal study of the body composition of weight gain in exclusively breast-fed and intake-measured whey-based formula-fed infants to age 3 months $\mathrm{J}$ Pediatr Gastroentero Nutr, 7(5), 732-9

2364 Sherlock, R. L.,Synnes, A. R.,Koehoorn, M. (2008). Working mothers and early childhood outcomes: lessons from the Canadian National Longitudinal Study on Children and Youth Early Hum Dev, 84(4), 237-42

2365 Shi, Y.,De Groh, M.,Morrison, H. (2013). Perinatal and early childhood factors for overweight and obesity in young Canadian children Can J Public Health, 104(1), e69-74

2366 Shields, B. M.,Knight, B.,Shakespeare, L.,Babrah, J.,Powell, R. J.,Clark, P. M.,Hattersley, A. T. (2006). Determinants of insulin concentrations in healthy 1-week-old babies in the community: applications of a bloodspot assay Early Hum Dev, 82(2), 143-8

Publication date for a non-sibling study

\section{Outcome}

Study design

Study design, Outcome 
2367 Shields, L.,Mamun, A. A.,O'Callaghan, M.,Williams, G. M.,Najman, J. M. (2010). Breastfeeding and obesity at 21 years: a cohort study J Clin Nurs, 19(11-12), 1612-7

Publication date for a non-sibling study

2368 Shields, L.,O'Callaghan, M.,Williams, G. M.,Najman, J. M.,Bor, W. (2006). Breastfeeding and obesity at 14 years: a cohort study J Paediatr Child Health, 42(5), 289-96

Publication date for a non-sibling study

2369 Shohet, L.,Shahar, E.,Davidson, S. (1985). Breast feeding as prophylaxis for atopic eczema: a controlled study of 368 cases Acta Intervention/exposure Paediatr Hung, 26(1), 35-9

2370 Shortridge, K. F.,Lawton, J. W.,Choi, E. K. (1990). Protective potential of colostrum and early milk against prospective influenza viruses J Trop Pediatr, 36(2), 94-5

2371 Shu, X. O.,Clemens, J.,Zheng, W.,Ying, D. M.,Ji, B. T.,Jin, F. (1995). Infant breastfeeding and the risk of childhood lymphoma and Outcome leukaemia Int J Epidemiol, 24(1), 27-32

2372 Shu, X. O.,Linet, M. S.,Steinbuch, M.,Wen, W. Q.,Buckley, J. D.,Neglia, J. P.,Potter, J. D.,Reaman, G. H.,Robison, L. L. (1999). Breast-feeding and risk of childhood acute leukemia J Natl Cancer Inst, 91(20), 1765-72

2373 Shultis, W. A.,Leary, S. D.,Ness, A. R.,Scott, J.,Martin, R. M.,Whincup, P. H.,Smith, G. D. (2006). Haemoglobin A1c is not a surrogate for glucose and insulin measures for investigating the early life and childhood determinants of insulin resistance and Type 2 diabetes in healthy children. An analysis from the Avon Longitudinal Study of Parents and Children (ALSPAC) Diabet Med, 23(12) $1357-63$

2374 Sickles, V. S.,Tuley, R. J.,Bader, P.,Carnaggio, V. A.,Exon, W. J.,Hargett, I. R.,Keathley, S. E.,Wolf, R.,Cordano, A. (1984). Growth

Intervention/exposure and tolerance studies of a new infant formula Clin Pediatr (Phila), 23(11), 617-22

2375 Sidhu, L. S.,Grewal, R.,Bhatnagar, D. P. (1981). A study of physical growth in breast-fed and bottle-fed male infants Indian journal of Country pediatrics, 48(390), 75-79

2376 Siemiatycki, J.,Colle, E.,Campbell, S.,Dewar, R. A.,Belmonte, M. M. (1989). Case-control study of IDDM Diabetes Care, 12(3), 209- Outcome 16

2377 Sievers, E.,Clausen, U.,Oldigs, H. D.,Schaub, J. (2002). Supplemental feeding in the first days of life - Effects on the recipient infant Intervention/exposure Annals of Nutrition and Metabolism, 46(2), 62-67

2378 Sievers, E.,Oldigs, H. D.,Dorner, K.,Schaub, J. (1992). Longitudinal zinc balances in breast-fed and formula-fed infants Acta Paediatr, 81(1), 1-6

2379 Sievers, E.,Schleyerbach, U.,Garbe-Schonberg, D.,Arpe, T.,Schaub, J. (2000). Zinc intakes and plasma concentrations in infancy Adv Exp Med Biol, 478(\#issue\#), 383-4

2380 Sigurs, N.,Bjarnason, R.,Sigurbergsson, F.,Kjellman, B.,Bjorksten, B. (1995). Asthma and immunoglobulin E antibodies after respiratory syncytial virus bronchiolitis: a prospective cohort study with matched controls Pediatrics, 95(4), 500-5

2381 Siigur, U.,Ormisson, A.,Tamm, A. (1993). Faecal short-chain fatty acids in breast-fed and bottle-fed infants Acta Paediatrica International Journal of Paediatrics, 82(6-7), 536-538

Study design, Size of study groups

Study design

Outcome

Size of study groups, Outcome 
2382 Siimes, M. A.,Salmenpera, L.,Perheentupa, J. (1984). Exclusive breast-feeding for 9 months: Risk of iron deficiency Journal of Pediatrics, 104(2), 196-199

2383 Silberman, S. L.,Trubman, A.,Duncan, W. K.,Meydrech, E. F. (1991). Prevalence of primary canine hypoplasia of the mandibular teeth Pediatr Dent, 13(6), 356-60

2384 Siltanen, M.,Kajosaari, M.,Poussa, T.,Saarinen, K. M.,Savilahti, E. (2003). A dual long-term effect of breastfeeding on atopy in relation to heredity in children at 4 years of age Allergy, 58(6), 524-30

2385 Silva, A. A.,Mehta, Z.,O'Callaghan, F. J. (2006). Duration of breast feeding and cognitive function: Population based cohort study Eur J Epidemiol, 21(6), 435-41

2386 Silver, D. H. (1982). Improvements in the dental health of 3-year-old Hertfordshire children after 8 years. The relationship to social class Br Dent J, 153(5), 179-83

2387 Silvers, K. M.,Frampton, C. M.,Wickens, K.,Epton, M. J.,Pattemore, P. K.,Ingham, T.,Fishwick, D., Crane, J.,Town, G. I. (2009) Breastfeeding protects against adverse respiratory outcomes at 15 months of age Matern Child Nutr, 5(3), 243-50

2388 Silvers, K. M.,Frampton, C. M.,Wickens, K.,Pattemore, P. K.,Ingham, T.,Fishwick, D.,Crane, J.,Town, G. I.,Epton, M. J. (2012). Breastfeeding protects against current asthma up to 6 years of age J Pediatr, 160(6), 991-6 e1

2389 Simhon, A.,Mata, L. (1985). Fecal rotaviruses, adenoviruses, coronavirus-like particles, and small round viruses in a cohort of rural Intervention/exposure Costa Rican children Am J Trop Med Hyg, 34(5), 931-6

2390 Simhon, A.,Mata, L.,Vives, M.,Rivera, L.,Vargas, S.,Ramirez, G.,Lizano, L.,Catarinella, G.,Azofeifa, J. (1985). Low endemicity and Study design, low pathogenicity of rotaviruses among rural children in Costa Rica J Infect Dis, 152(6), 1134-42

Intervention/exposure

2391 Simon, M. R.,Havstad, S. L.,Wegienka, G. R.,Ownby, D. R.,Johnson, C. C. (2008). Risk factors associated with transient wheezing Outcome in young children Allergy Asthma Proc, 29(2), 161-5

2392 Sims, D. G.,Gardner, P. S.,Weightman, D.,Turner, M. W.,Soothill, J. F. (1981). Atopy does not predispose to RSV bronchiolitis or postbronchiolitic wheezing Br Med J (Clin Res Ed), 282(6282), 2086-8

2393 Singhal, A. (2002). Early nutrition and later blood pressure: an experimental approach Journal of Nutritional \& Environmenta Medicine, 12(3), 251-252 2p

2394 Singhal, A.,Kennedy, K.,Lanigan, J.,Clough, H.,Jenkins, W.,Elias-Jones, A.,Stephenson, T.,Dudek, P.,Lucas, A. (2010). Dietary nucleotides and early growth in formula-fed infants: a randomized controlled trial Pediatrics, 126(4), e946-53

Size of study groups

Study design

Publication date for a non-sibling study

2395 Singhal, A.,Lucas, A. (2004). Early origins of cardiovascular disease: is there a unifying hypothesis? Lancet, 363(9421), 1642-5

Study design

2396 Singhal, A.,Morley, R.,Cole, T. J.,Kennedy, K.,Sonksen, P.,Isaacs, E.,Fewtrell, M.,Elias-Jones, A.,Stephenson, T.,Lucas, A. (2007). Outcome Infant nutrition and stereoacuity at age 4-6 y Am J Clin Nutr, 85(1), 152-9

2397 Singhi, P.,Singhi, S.,Bhalla, A. K. (1985). Growth of term infants in early neonatal period Indian Pediatr, 22(7), 485-91

Country

2398 Singhi, S.,Singhi, P. (1987). Prevention of acute respiratory infections Indian J Pediatr, 54(2), 161-70 
2399 Singleton, R.,Lescher, R.,Gessner, B. D.,Benson, M.,Bulkow, L.,Rosenfeld, J.,Thomas, T.,Holman, R. C.,Haberling, D.,Bruce, M.,Bartholomew, M.,Tiesinga, J. (2015). Rickets and Vitamin D deficiency in Alaska native children Journal of Pediatric

Endocrinology and Metabolism, 28(7-8), 815-823

2400 Sinha, A.,Madden, J.,Ross-Degnan, D.,Soumerai, S.,Platt, R. (2003). Reduced risk of neonatal respiratory infections among breastfed girls but not boys Pediatrics, 112(4), e303

2401 Sipetic, S., Vlajinac, H.,Kocev, N.,Bjekic, M.,Sajic, S. (2005). Early infant diet and risk of type 1 diabetes mellitus in Belgrade children Outcome Nutrition, 21(4), 474-9

2402 Sipila, M.,Karma, P.,Pukander, J.,Timonen, M.,Kataja, M. (1988). The Bayesian approach to the evaluation of risk factors in acute and recurrent acute otitis media Acta Otolaryngol, 106(1-2), 94-101

2403 Siriaksorn, S.,Suchaitanawanit, S.,Trakultivakorn, M. (2011). Allergic rhinitis and immunoglobulin deficiency in preschool children with frequent upper respiratory illness Asian Pac J Allergy Immunol, 29(1), 73-7

2404 Sjolin, S.,Hofvander, Y.,Hillervik, C. (1979). A prospective study of individual courses of breast feeding Acta paediatrica Scandinavica, 68(\#issue\#), 521-9

2405 Skilton, M. R.,Marks, G. B.,Ayer, J. G.,Garden, F. L.,Garnett, S. P.,Harmer, J. A.,Leeder, S. R., Toelle, B. G.,Webb, K.,Baur, L. A.,Celermajer, D. S. (2013). Weight gain in infancy and vascular risk factors in later childhood Pediatrics, 131(6), e1821-8

Skrodeniene, E.,Marciulionyte, D.,Padaiga, Z.,Jasinskiene, E.,Sadauskaite-Kuehne, V.,Ludvigsson, J. (2008). Environmental risk factors in prediction of childhood prediabetes Medicina (Kaunas), 44(1), 56-63

2407 Skrodeniene, E.,Marčiulionyte, D.,Padaiga, Z.,Jašinskiene, E.,Sadauskaite-Kuehne, V.,Sanjeevi, C. B., Vitkauskiene, A.,Ludvigsson, J. (2010). Associations between HLA class II haplotypes, environmental factors and type 1 diabetes mellitus in Lithuanian children with type 1 diabetes and controls Polish Annals of Medicine, 17(1), 7-15

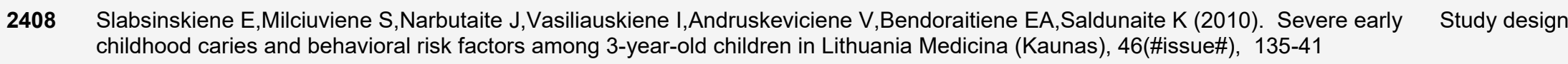

2409 Slae, M.,Persad, R.,Leung, A. J. T.,Gabr, R.,Brocks, D.,Huynh, H. Q. (2015). Role of Environmental Factors in the Development of Pediatric Eosinophilic Esophagitis Digestive Diseases and Sciences, 60(11), 3364-3372

2410 Slavkin, H. C. (1999). Streptococcus mutans, early childhood caries and new opportunities J Am Dent Assoc, 130(12), 1787-92

2411 Slykerman, R. F.,Thompson, J. M.,Becroft, D. M.,Robinson, E.,Pryor, J. E.,Clark, P. M.,Wild, C. J.,Mitchell, E. A. (2005). Breastfeeding and intelligence of preschool children Acta Paediatr, 94(7), 832-7

2412 Smith, D. P. (1985). Breastfeeding in the United States Soc Biol, 32(1-2), 53-60

Outcome

Study design

Outcome

Outcome

Outcome

Outcome

Study design

Outcome

Study design, Outcome

2413 Smith, R. M.,Smith, P. A.,McKinnon, M.,Gracey, M. (2000). Birthweights and growth of infants in five Aboriginal communities Aust N Study design Z J Public Health, 24(2), 124-35 
2414 Smithers, L. G., Golley, R. K.,Brazionis, L.,Emmett, P.,Northstone, K.,Lynch, J. W. (2012). Dietary patterns of infants and toddlers are associated with nutrient intakes Nutrients, 4(8), 935-48

2415 Smithers, L. G.,Golley, R. K.,Mittinty, M. N.,Brazionis, L.,Northstone, K.,Emmett, P.,Lynch, J. W. (2012). Dietary patterns at 6, 15 and 24 months of age are associated with IQ at 8 years of age Eur J Epidemiol, 27(7), 525-35

2416 Smithers, L. G.,Golley, R. K.,Mittinty, M. N.,Brazionis, L.,Northstone, K.,Emmett, P.,Lynch, J. W. (2013). Do dietary trajectories between infancy and toddlerhood influence IQ in childhood and adolescence? Results from a prospective birth cohort study PLoS One, 8(3), e58904

2417 Smulevich, V. B.,Solionova, L. G.,Belyakova, S. V. (1999). Parental occupation and other factors and cancer risk in children: I. Study methodology and non-occupational factors Int J Cancer, 83(6), 712-7

2418 Smyth, P. P. (1999). Variation in iodine handling during normal pregnancy Thyroid, 9(7), 637-42

2419 Smyth, P. P.,Hetherton, A. M.,Smith, D. F.,Radcliff, M.,O'Herlihy, C. (1997). Maternal iodine status and thyroid volume during pregnancy: correlation with neonatal iodine intake J Clin Endocrinol Metab, 82(9), 2840-3

Study design,

Intervention/exposure

2420 Smyth, P. P.,Smith, D. F.,Sheehan, S.,Higgins, M.,Burns, R.,O'Herlihy, C. (2007). Short-term changes in maternal and neonatal Size of study groups urinary iodine excretion Thyroid, 17(3), 219-22

2421 Snijders, B. E., Thijs, C.,Dagnelie, P. C.,Stelma, F. F.,Mommers, M.,Kummeling, I.,Penders, J.,van Ree, R.,van den Brandt, P. A. (2007). Breast-feeding duration and infant atopic manifestations, by maternal allergic status, in the first 2 years of life (KOALA study) J Pediatr, 151(4), 347-51, 351 e1-2

2423 Socha, P.,Grote, V.,Gruszfeld, D.,Janas, R.,Demmelmair, H.,Closa-Monasterolo, R.,Subias, J. E.,Scaglioni, S., Verduci, E.,Dain, E.,Langhendries, J. P.,Perrin, E.,Koletzko, B. (2011). Milk protein intake, the metabolic-endocrine response, and growth in infancy: data from a randomized clinical trial Am J Clin Nutr, 94(6 Suppl), 1776S-1784S

2424 Socha, P.,Janas, R.,Dobrzanska, A.,Koletzko, B.,Broekaert, I.,Brasseur, D.,Sengier, A., Giovannini, M.,Agostoni, C.,Monasterolo, R. C.,Mendezs, G. (2005). Insulin like growth factor regulation of body mass in breastfed and milk formula fed infants. Data from the E.U. Childhood Obesity Programme Adv Exp Med Biol, 569(\#issue\#), 159-63

2425 Soltesz, G.,Jeges, S.,Dahlquist, G. (1994). Non-genetic risk determinants for type 1 (insulin-dependent) diabetes mellitus in childhood. Hungarian Childhood Diabetes Epidemiology Study Group Acta Paediatr, 83(7), 730-5

2426 Somech, R., Tal, G., Gilad, E.,Mandelberg, A.,Tal, A.,Dalal, I. (2006). Epidemiologic, socioeconomic, and clinical factors associated with severity of respiratory syncytial virus infection in previously healthy infants Clin Pediatr (Phila), 45(7), 621-7

2427 Sommerfelt, K.,Ellertsen, B.,Markestad, T. (1996). Low birthweight and neuromotor development: a population based, controlled study Acta Paediatr, 85(5), 604-10 
2428 Sommerfield, T.,Chalmers, J.,Youngson, G.,Heeley, C.,Fleming, M.,Thomson, G. (2008). The changing epidemiology of infantile hypertrophic pyloric stenosis in Scotland Arch Dis Child, 93(12), 1007-11

Study design

Intervention/exposure,

Participant health

2429 Song, N.,Shamssain, M.,Zhang, J.,Wu, J.,Fu, C.,Hao, S.,Guan, J.,Yan, X. (2014). Prevalence, severity and risk factors of asthma, Study design rhinitis and eczema in a large group of Chinese schoolchildren J Asthma, 51(3), 232-42

2430 Sonnenschein-van der Voort, A. M.,Jaddoe, V. W.,van der Valk, R. J.,Willemsen, S. P.,Hofman, A.,Moll, H. A.,de Jongste, J. C.,Duijts, L. (2012). Duration and exclusiveness of breastfeeding and childhood asthma-related symptoms Eur Respir J, 39(1), 81-9

2431 Soto-Ramirez, N.,Karmaus, W.,Zhang, H.,Davis, S.,Agarwal, S.,Albergottie, A. (2013). Modes of infant feeding and the occurrence of coughing/wheezing in the first year of life J Hum Lact, 29(1), 71-80

2432 Soylu, H.,Özgen, Ü,Babalioğlu, M.,Aras, Ş,Sazak, S. (2001). Iron deficiency and iron deficiency anemia in infants and young children at different socioeconomic groups in Istanbul Turkish Journal of Haematology, 18(1), 19-25

\section{Outcome}

\section{Outcome}

Study design, Size of study groups

2433 Specker, B. L.,Beck, A.,Kalkwarf, H.,Ho, M. (1997). Randomized trial of varying mineral intake on total body bone mineral accretion Intervention/exposure during the first year of life Pediatrics, 99(6), E12

2434 Specker, B. L.,Black, A.,Allen, L.,Morrow, F. (1990). Vitamin B-12: low milk concentrations are related to low serum concentrations in vegetarian women and to methylmalonic aciduria in their infants Am J Clin Nutr, 52(6), 1073-6

Study design, Size of study groups

Study design, Intervention/exposure milk Am J Clin Nutr, 51(2), 209-11

2436 Specker, B. L.,Miller, D.,Norman, E. J.,Greene, H.,Hayes, K. C. (1988). Increased urinary methylmalonic acid excretion in breast-fed infants of vegetarian mothers and identification of an acceptable dietary source of vitamin B-12 Am J Clin Nutr, 47(1), 89-92

Study design, Size of study groups

2437 Spyrides, M. H.,Struchiner, C. J.,Barbosa, M. T.,Kac, G. (2008). Effect of predominant breastfeeding duration on infant growth: a Language prospective study using nonlinear mixed effect models J Pediatr (Rio J), 84(3), 237-43

2438 Srivastava, S. P.,Sharma, V. K.,Jha, S. P. (1994). Mortality patterns in breast versus artificially fed term babies in early infancy: a Country longitudinal study Indian Pediatr, 31(11), 1393-6

2439 Stadler, D. D.,Musser, E. D.,Holton, K. F.,Shannon, J.,Nigg, J. T. (2015). Recalled Initiation and Duration of Maternal Breastfeeding Among Children with and Without ADHD in a Well Characterized Case-Control Sample J Abnorm Child Psychol, \#volume\#(\#issue\#), \#Pages\#

2440 Stahl, M. D.,Guida, D. A. (1984). Slow weight gain in the breast-fed infant: management options Pediatr Nurs, 10(2), 117-20, 164

Study design

2441 Stahlberg, M. R. (1985). Breast feeding, cow milk feeding, and allergy Allergy, 40(8), 612-5

Outcome

2442 Stahlberg, M. R.,Ruuskanen, O.,Virolainen, E. (1986). Risk factors for recurrent otitis media Pediatr Infect Dis, 5(1), 30-2

Outcome 
2443 Standl, M.,Sausenthaler, S.,Lattka, E.,Koletzko, S.,Bauer, C. P.,Wichmann, H. E.,von Berg, A.,Berdel, D.,Kramer, U.,Schaaf

B.,Lehmann, I.,Herbarth, O.,Klopp, N.,Koletzko, B.,Heinrich, J. (2012). FADS gene cluster modulates the effect of breastfeeding on asthma. Results from the GINIplus and LISAplus studies Allergy, 67(1), 83-90

2444 Stanfield JP (1982). The influence of malnutrition on development Practitioner, 226(\#issue\#), 1929-40

Study design

2445 Stanley, E. O.,Lundeen, D. J. (1980). Tongue thrust in breast fed and bottle-fed school children: a cross-cultural investigation Int J Oral Myol, 6(1), 6-17

2446 Stanner, S. (2001). Is breast best for the heart? Nutrition Bulletin, 26(3), 199-200

Study design

2447 Steady, F. C. (1981). Infant feeding in developing countries: combating the multinationals imperative J Trop Pediatr, 27(4), 215-20

2448 Stecksen-Blicks, C.,Granstrom, E.,Silfverdal, S. A.,West, C. E. (2015). Prevalence of oral Candida in the first year of life Mycoses, 58(9), 550-6

2449 Steer, C. D.,Davey Smith, G.,Emmett, P. M.,Hibbeln, J. R.,Golding, J. (2010). FADS2 polymorphisms modify the effect of breastfeeding on child IQ PLoS One, 5(7), e11570

2450 Steichen, J. J.,Tsang, R. C. (1987). Bone mineralization and growth in term infants fed soy-based or cow milk-based formula J Pediatr, 110(5), 687-92

2451 Stein, A. D.,Melgar, P.,Hoddinott, J.,Martorell, R. (2008). Cohort profile: The institute of nutrition of central America and Panama (INCAP) nutrition trial cohort study International Journal of Epidemiology, 37(4), 716-720

2452 Stelmach, I.,Bobrowska-Korzeniowska, M.,Smejda, K.,Majak, P.,Jerzynska, J.,Stelmach, W.,Polanska, K.,Sobala, W.,Krysicka, J.,Hanke, W. (2014). Risk factors for the development of atopic dermatitis and early wheeze Allergy Asthma Proc, 35(5), 382-9

2453 Stene, L. C.,Joner, G. (2004). Atopic disorders and risk of childhood-onset type 1 diabetes in individuals Clin Exp Allergy, 34(2), 201-6

2454 Stenstrom, C.,Ingvarsson, L. (1997). Otitis-prone children and controls: a study of possible predisposing factors. 1. Heredity, family background and perinatal period Acta Otolaryngol, 117(1), 87-93

2455 Stepans, M. F. (1998). Birthing briefs Journal of Perinatal Education, 7(1), 39-40 2p

2456 Stevens, F. M.,Egan-Mitchell, B.,Cryan, E.,McCarthy, C. F.,McNicholl, B. (1987). Decreasing incidence of coeliac disease Arch Dis Child, 62(5), 465-8

2457 Stevens, T. (1996). Infant nutrition perspectives Midwives (1995), 109(1300), 120

Study design, Size of study

2458 Stewart, A. J.,Williams, S. M.,Mitchell, E. A.,Taylor, B. J.,Ford, R. P.,Allen, E. M. (1995). Antenatal and intrapartum factors associated with sudden infant death syndrome in the New Zealand Cot Death Study J Paediatr Child Health, 31(5), 473-8

2459 Stoeckel, J. (1992). The intervention research approach to child survival Asia Pac J Public Health, 6(1), 40-5 groups

Study design

Study design

Size of study groups

Outcome

Size of study groups Intervention/exposure

Study design

Study design

Study design

ntervention/exposure

Outcome

Study design

Intervention/exposure

Study design 
2460 Stoll, B. J.,Glass, R. I.,Banu, H.,Huq, M. I.,Khan, M. U.,Ahmed, M. (1983). Value of stool examination in patients with diarrhoea Br Med J (Clin Res Ed), 286(6383), 2037-40

2461 Stordal, K.,White, R. A.,Eggesbo, M. (2013). Early feeding and risk of celiac disease in a prospective birth cohort Pediatrics, 132(5), Outcome e1202-9

2462 Strabelli, T. M. B.,Botura, C. A.,Maciel, M. A.,Mazzutti, C.,Bridi, A.,Freitas, L. P. (2013). Socioeconomic profile of children hospitalized by community acquired pneumonia Acta Scientiarum - Health Sciences, 35(2), 175-179

2463 Strachan, D. P.,Harkins, L. S.,Johnston, I. D.,Anderson, H. R. (1997). Childhood antecedents of allergic sensitization in young British adults J Allergy Clin Immunol, 99(1 Pt 1), 6-12 Child, 74(5), 422-6

2465 Strand, T. A.,Sharma, P. R.,Gjessing, H. K.,Ulak, M.,Chandyo, R. K.,Adhikari, R. K., Sommerfelt, H. (2012). Risk factors for extended Country duration of acute diarrhea in young children PLoS One, 7(5), e36436

2466 Strand, T. A.,Taneja, S.,Bhandari, N.,Refsum, H.,Ueland, P. M.,Gjessing, H. K.,Bahl, R.,Schneede, J.,Bhan, M. K.,Sommerfelt, H. (2007). Folate, but not vitamin B-12 status, predicts respiratory morbidity in north Indian children Am J Clin Nutr, 86(1), 139-44

2467 Strandvik B,Chen Y,Dangardt F,Eriksson S,Friberg P,Garemo M,Pickova J (2011). From the Swedish to the Mediterranean diet and Study design the omega-6/omega-3 balance World Rev Nutr Diet, 102(\#issue\#), 73-80

2468 Strassburger, S. Z.,Vitolo, M. R.,Bortolini, G. A.,Pitrez, P. M.,Jones, M. H.,Stein, R. T. (2010). Nutritional errors in the first months of Outcome life and their association with asthma and atopy in preschool children J Pediatr (Rio J), 86(5), 391-9

2469 Strbak, V.,Hromadova, M.,Kostalova, L.,Kapellerova, A. (1993). Search for optimal age for weaning. Ten-year prospective study Size of study groups Endocr Regul, 27(4), 215-21

2470 Strbak, V.,Skultetyova, M.,Hromadova, M.,Randuskova, A.,Macho, L. (1991). Late effects of breast-feeding and early weaning: seven-year prospective study in children Endocr Regul, 25(1-2), 53-7

2471 Stremler, R.,Hodnett, E.,Kenton, L.,Lee, K.,Weiss, S.,Weston, J.,Willan, A. (2013). Effect of behavioural-educational intervention on sleep for primiparous women and their infants in early postpartum: multisite randomised controlled trial BMJ, 346(\#issue\#), f1164

2472 Strimas, J. H.,Chi, D. S. (1988). Significance of IgE level in amniotic fluid and cord blood for the prediction of allergy Ann Allergy, $61(2), 133-6$

2473 Strina, A.,Rodrigues, L. C.,Cairncross, S.,Ferrer, S. R.,Fialho, A. M.,Leite, J. P.,Ribeiro, H. C., Jr.,Barreto, M. L. (2012). Factors associated with rotavirus diarrhoea in children living in a socially diverse urban centre in Brazil Trans R Soc Trop Med Hyg, 106(7), 445-51

2474 Strobl, W.,Widhalm, K. (1985). The natural history of serum lipids and lipoproteins during childhood Prog Clin Biol Res, 188(\#issue\#), 101-21

Publication date for a non-sibling study

Intervention/exposure

Size of study groups, Intervention/exposure

Study design,

Intervention/exposure

Study design 
2475 Su, D.,Zhao, Y.,Binns, C.,Scott, J.,Oddy, W. (2007). Breast-feeding mothers can exercise: results of a cohort study Public Health Nutr, 10(10), 1089-93

2476 Suaini, N. H.,Koplin, J. J.,Ellis, J. A.,Peters, R. L.,Ponsonby, A. L.,Dharmage, S. C.,Matheson, M. C.,Wake, M.,Panjari, M.,Tan, H. T.,Martin, P. E.,Pezic, A.,Lowe, A. J.,Martino, D.,Gurrin, L. C., Vuillermin, P. J.,Tang, M. L.,Allen, K. J. (2014). Environmental and genetic determinants of vitamin D insufficiency in 12-month-old infants J Steroid Biochem Mol Biol, 144 Pt B(\#issue\#), 445-54

Subbarao, P.,Anand, S. S.,Becker, A. B.,Befus, A. D.,Brauer, M.,Brook, J. R.,Denburg, J. A.,Hayglass, K. T.,Kobor, M. S.,Kollmann,

T. R.,Kozyrskyj, A. L.,Lou, W. Y. W.,Mandhane, P. J.,Miller, G. E.,Moraes, T. J.,Pare, P. D.,Scott, J. A.,Takaro, T. K.,Turvey, S.

E.,Duncan, J. M.,Lefebvre, D. L.,Sears, M. R. (2015). The Canadian Healthy Infant Longitudinal Development (CHILD) study:

Examining developmental origins of allergy and asthma Thorax, 70(10), 998-1000

2478 Suganuma, E. K.,Alexander, G. R.,Baruffi, G.,Gilden, S. R. (1988). Infant feeding practices in Hawaii Hawaii Med J, 47(3), 112, $117-9$

2479 Sun, G.,Jia, G.,Peng, H.,Dickerman, B.,Compher, C.,Liu, J. (2015). Trends of childhood obesity in China and associated factors Clin Nurs Res, 24(2), 156-71

2480 Sun, J.,Huo, J.,Zhao, L.,Fu, P.,Wang, J.,Huang, J.,Wang, L.,Song, P.,Fang, Z.,Chang, S.,Yin, S.,Zhang, J.,Ma, G. (2013). The nutritional status of young children and feeding practices two years after the Wenchuan Earthquake in the worst-affected areas in China Asia Pac J Clin Nutr, 22(1), 100-8

2481 Sunoto, (1982). Diarrhoeal problems in Southeast Asia Southeast Asian J Trop Med Public Health, 13(3), 306-18

Intervention/exposure

Study design

2482 Sunyer, J.,Torrent, M.,Garcia-Esteban, R.,Ribas-Fito, N.,Carrizo, D.,Romieu, I.,Anto, J. M.,Grimalt, J. O. (2006). Early exposure to dichlorodiphenyldichloroethylene, breastfeeding and asthma at age six Clin Exp Allergy, 36(10), 1236-41

2483 Suoglu, O. D.,Gokce, S.,Saglam, A. T.,Sokucu, S.,Saner, G. (2007). Association of Helicobacter pylori infection with gastroduodenal Intervention/exposure disease, epidemiologic factors and iron-deficiency anemia in Turkish children undergoing endoscopy, and impact on growth Pediatr Int, 49(6), 858-63

2484 Surdu, S.,Montoya, L. D.,Tarbell, A.,Carpenter, D. O. (2006). Childhood asthma and indoor allergens in Native Americans in New York Environ Health, 5(\#issue\#), 22

2485 Sussmann, J. E.,Mclntosh, A. M.,Lawrie, S. M.,Johnstone, E. C. (2009). Obstetric complications and mild to moderate intellectual disability Br J Psychiatry, 194(3), 224-8

2486 Sutmoller, F.,Maia, P. R. (1995). Acute respiratory infections in children living in two low income communities of Rio de Janeiro, Brazil Mem Inst Oswaldo Cruz, 90(6), 665-74 2487 Syafruddin, M.,Djauhariah, A. M.,Dasril, D. (1988). A study comparing rooming-in with separate nursing Paediatr Indones, 28(5-6),
116-23 Country

2488 Tada, A.,Ando, Y.,Hanada, N. (1999). Caries risk factors among three-year old children in Chiba, Japan Asia Pac J Public Health, 11(2), 109-12

Study design

Study design, Size of study groups

Study design,

Intervention/exposure

Study design

Outcome

Size of study groups

Size of study groups,

Intervention/exposure

Outcome

Outcome 
2489 Tai, T. Y.,Wang, C. Y.,Lin, L. L.,Lee, L. T., Tsai, S. T.,Chen, C. J. (1998). A case-control study on risk factors for Type 1 diabetes in Taipei City Diabetes Res Clin Pract, 42(3), 197-203

2490 Tainio, V. M. (1985). Lymphocyte subsets in infants: relationships to feeding, atopy, atopic heredity and infections Int Arch Allergy Appl Immunol, 78(3), 305-10

2491 Tainio, V. M.,Savilahti, E.,Salmenpera, L.,Arjomaa, P.,Siimes, M. A.,Perheentupa, J. (1988). Risk factors for infantile recurrent otitis Outcome media: atopy but not type of feeding Pediatr Res, 23(5), 509-12

2492 Taittonen, L.,Nuutinen, M.,Turtinen, J.,Uhari, M. (1996). Prenatal and postnatal factors in predicting later blood pressure among children: cardiovascular risk in young Finns Pediatr Res, 40(4), 627-32 Biol, 53(3), 313-20

2494 Takala, A. K.,Eskola, J.,Palmgren, J.,Ronnberg, P. R.,Kela, E.,Rekola, P.,Makela, P. H. (1989). Risk factors of invasive Haemophilus influenzae type b disease among children in Finland J Pediatr, 115(5 Pt 1), 694-701

2495 Takemura, Y.,Sakurai, Y.,Honjo, S.,Kusakari, A.,Hara, T.,Gibo, M.,Tokimatsu, A.,Kugai, N. (2001). Relation between breastfeeding Study design and the prevalence of asthma : the Tokorozawa Childhood Asthma and Pollinosis Study Am J Epidemiol, 154(2), 115-9

2496 Taki, M.,Mizuno, K.,Murase, M.,Nishida, Y.,Itabashi, K.,Mukai, Y. (2010). Maturational changes in the feeding behaviour of infants a comparison between breast-feeding and bottle-feeding Acta Paediatr, 99(1), 61-7

2497 Talayero, J. M. P.,Lizán-García, M.,Puime Á, O.,Muncharaz, M. J. B.,Soto, B. B.,Sánchez-Palomares, M.,Serrano, L. S.,Rivera, L. L. Outcome (2006). Full breastfeeding and hospitalization as a result of infections in the first year of life Pediatrics, 118(1), e92-e99

2498 Tanaka, H.,Ishii, H.,Yamada, T.,Akazawa, K.,Nagata, S.,Yamashiro, Y. (2013). Growth of Japanese breastfed infants compared to Intervention/exposure national references and World Health Organization growth standards Acta Paediatr, 102(7), 739-43

2499 Tanaka, K.,Miyake, Y.,Sasaki, S. (2010). Association between breastfeeding and allergic disorders in Japanese children Int $J$ Tuberc Lung Dis, 14(4), 513-8 2500 Tanaka, K.,Miyake, Y.,Sasaki, S.,Hirota, Y. (2013). Infant feeding practices and risk of dental caries in Japan: the Osaka Maternal
And Child Health Study Pediatr Dent, 35(3), 267-71

2501 Tanaka, T.,Kato, N. (2001). Evaluation of child care practice factors that affect the occurrence of sudden infant death syndrome: Interview conducted by public health nurses Environmental Health and Preventive Medicine, 6(2), 117-120

2502 Taneja, S.,Bhandari, N.,Bahl, R.,Bhan, M. K. (2005). Impact of zinc supplementation on mental and psychomotor scores of children Country aged 12 to 18 months: a randomized, double-blind trial J Pediatr, 146(4), 506-11

2503 Taneja, S.,Bhandari, N.,Strand, T. A.,Sommerfelt, H.,Refsum, H.,Ueland, P. M.,Schneede, J.,Bahl, R.,Bhan, M. K. (2007). Cobalamin and folate status in infants and young children in a low-to-middle income community in India Am J Clin Nutr, 86(5), 1302 9 
2505 Tantracheewathorn, S. (2005). Growth of breast-fed and formula-fed infants compared with national growth references of Thai Intervention/exposure children J Med Assoc Thai, 88(2), 168-75

2506 Tantracheewathorn, S.,Lohajaroensub, S. (2005). Incidence and risk factors of iron deficiency anemia in term infants J Med Assoc Intervention/exposure Thai, 88(1), 45-51

2507 Tanzer, F.,Gumuser, C. (1989). A study of the growth of 200 newborn babies for a period of 6 months according to the type of nutrition Ann Trop Paediatr, 9(1), 54-8

2508 Targino, A. G.,Rosenblatt, A.,Oliveira, A. F.,Chaves, A. M.,Santos, V. E. (2011). The relationship of enamel defects and caries: a cohort study Oral Dis, 17(4), 420-6

2509 Tariq, S.,Memon, I. A. (1999). Acute otitis media in children Journal of the College of Physicians and Surgeons Pakistan, 9(12), 507-510

2510 Tarrant, M.,Fong, D. Y.,Heys, M.,Lee, I. L.,Sham, A.,Hui Choi, E. W. (2014). Professional breastfeeding support to increase the exclusivity and duration of breastfeeding: a randomised controlled trial Hong Kong Med J, 20 Suppl 7(\#issue\#), 34-5

2511 Tarrant, M.,Kwok, M. K.,Lam, T. H.,Leung, G. M.,Schooling, C. M. (2010). Breast-feeding and childhood hospitalizations for infections Epidemiology, 21(6), 847-54

2512 Tarrant, M.,Schooling, C. M.,Leung, S. L.,Mak, K. H.,Ho, L. M.,Leung, G. M. (2014). Impact of breastfeeding on infectious disease hospitalisation: the children of 1997 cohort Hong Kong Med J, 20 Suppl 4(\#issue\#), 5-6

2513 Tarrant, R. C.,Sheridan-Pereira, M.,Younger, K. M.,Kearney, J. M. (2012). The positive role of breastfeeding on infant health during Study design the first 6 weeks: findings from a prospective observational study based on maternal reports Ir Med J, 105(3), 75-8

2514 Taveras, E. M.,Gillman, M. W.,Kleinman, K. P.,Rich-Edwards, J. W.,Rifas-Shiman, S. L. (2013). Reducing racial/ethnic disparities in Intervention/exposure childhood obesity: the role of early life risk factors JAMA Pediatr, 167(8), 731-8

2515 Taveras, E. M.,Gillman, M. W.,Kleinman, K.,Rich-Edwards, J. W.,Rifas-Shiman, S. L. (2010). Racial/ethnic differences in early-life risk factors for childhood obesity Pediatrics, 125(4), 686-95

2516 Taveras, E. M.,Rifas-Shiman, S. L.,Scanlon, K. S.,Grummer-Strawn, L. M.,Sherry, B.,Gillman, M. W. (2006). To what extent is the protective effect of breastfeeding on future overweight explained by decreased maternal feeding restriction? Pediatrics, 118(6) 2341-8

2517 Tawia S (2013). Childhood obesity and being breastfed Breastfeed Rev, 21(\#issue\#), 42-8

Publication date for a non-sibling study

Publication date for a non-sibling study

Study design

2518 Taylor, B. (1984). Infant feeding and allergy: fact and fiction Midwife Health Visit Community Nurse, 20(10), 354-60

Study design

2519 Taylor, B.,Wadsworth, J. (1984). Breast feeding and child development at five years Dev Med Child Neurol, 26(1), 73-80

Study design 
2520 Taylor, B.,Wadsworth, J.,Golding, J.,Butler, N. (1982). Breast-feeding, bronchitis, and admissions for lower-respiratory illness and gastroenteritis during the first five years Lancet, 1(8283), 1227-9

Study design

Intervention/exposure

2521 Taylor, B.,Wadsworth, J.,Golding, J.,Butler, N. (1983). Breast feeding, eczema, asthma, and hayfever J Epidemiol Community Intervention/exposure Health, 37(2), 95-9

2522 Taylor, R. (2014). Providing additional guidance and support to parents about sleep, diet and physical activity from birth to 2 years of Publication status age: The Prevention of Overweight in Infancy study Obesity research \& clinical practice, 8(\#issue\#), 102-3

2523 Taylor-Robinson, D. C.,Williams, H.,Pearce, A.,Law, C.,Hope, S. (2015). Do early life exposures explain why more advantaged children get eczema? Findings from the UK Millennium Cohort Study Br J Dermatol, \#volume\#(\#issue\#), \#Pages\#

2524 Tee, J. H. (1987). Some characteristics of 5-year-old children with a dmf of six or more in Gloucestershire, England Community Dent Study design Health, 4(2), 121-8

2525 Teele, D. W.,Klein, J. O.,Rosner, B. (1989). Epidemiology of otitis media during the first seven years of life in children in greater Boston: a prospective, cohort study J Infect Dis, 160(1), 83-94

2526 Teixeira Mde, L.,Lira, P. I.,Coutinho, S. B.,Eickmann, S. H.,Lima, M. C. (2010). Influence of breastfeeding type and maternal anemia Study design on hemoglobin concentration in 6-month-old infants J Pediatr (Rio J), 86(1), 65-72

2527 Teixeira, Ana Karine Macedo,Menezes, LÃ@a Maria Bezerra de,Dias, Aldo Angelim,Alencar, Carlos Henrique Morais de,Almeida, Maria Eneide LeitÃ£o de (2010). Analysis of protection or risk factors for dental fluorosis in 6 to 8 year-old children in Fortaleza, Brazil Revista Panamericana de Salud Publica, 28(6), 421-428 8p

2528 Teka, T.,Faruque, A. S.,Fuchs, G. J. (1996). Risk factors for deaths in under-age-five children attending a diarrhoea treatment centre Acta Paediatr, 85(9), $1070-5$

2529 Telahun, M.,Abdulkadir, J.,Kebede, E. (1994). The relation of early nutrition, infections and socio-economic factors to the development of childhood diabetes Ethiop Med J, 32(4), 239-44

2530 Temboury, M. C.,Otero, A.,Polanco, I.,Arribas, E. (1994). Influence of breast-feeding on the infant's intellectual development $J$ Pediatr Gastroenterol Nutr, 18(1), 32-6

2531 Tenebaum, D.,Gambert, P.,Meunier, S.,d'Athis, P.,Nivelon, J. L.,Lallemand, C. (1988). Serum lipoproteins in venous blood serum from birth to the end of the first week: feeding influences Biol Neonate, 53(3), 126-31

2532 Thacher, T. D.,Fischer, P. R.,Tebben, P. J.,Singh, R. J.,Cha, S. S.,Maxson, J. A.,Yawn, B. P. (2013). Increasing incidence of nutritional rickets: a population-based study in Olmsted County, Minnesota Mayo Clin Proc, 88(2), 176-83

Outcome

Language

Thakur, R.,Singh, M. G.,Chaudhary, S.,Manuja, N. (2012). Effect of mode of delivery and feeding practices on acquisition of oral Streptococcus mutans in infants Int J Paediatr Dent, 22(3), 197-202

2534 Thapa, S.,Short, R. V.,Potts, M. (1988). Breast feeding, birth spacing and their effects on child survival Nature, 335(6192), 679-82 
2535 Thaver, I. H. (1990). "Risk approach" for reducing malnutrition in children from a privileged community J Pak Med Assoc, 40(3), 59- Country 61

2536 Thiering, E.,Bruske, I.,Kratzsch, J.,Thiery, J.,Sausenthaler, S.,Meisinger, C.,Koletzko, S.,Bauer, C. P.,Schaaf, B.,von Berg,

Intervention/exposure A.,Berdel, D.,Lehmann, I.,Herbarth, O.,Kramer, U.,Wichmann, H. E.,Heinrich, J. (2011). Prenatal and postnatal tobacco smoke exposure and development of insulin resistance in 10 year old children Int J Hyg Environ Health, 214(5), 361-8

2537 Thies, P. A.,Jeris, L. S. (1981). Infant feeding practices and dental health. Part 2: breastfeeding and dental caries Bull Mich Dent Hyg Assoc, 11(1), 6-7, 20

2538 Thitasomakul, S.,Piwat, S.,Thearmontree, A.,Chankanka, O.,Pithpornchaiyakul, W.,Madyusoh, S. (2009). Risks for early childhood caries analyzed by negative binomial models J Dent Res, 88(2), 137-41

2539 Thomas, G. P.,Soni, N. N. (1987). Clinical manifestations and management of nursing bottle syndrome J Md State Dent Assoc, $30(2), 62-4$

2540 Thomaz, E. B.,Cangussu, M. C.,Assis, A. M. (2012). Maternal breastfeeding, parafunctional oral habits and malocclusion in adolescents: a multivariate analysis Int J Pediatr Otorhinolaryngol, 76(4), 500-6

2541 Thompson, A. L.,Adair, L. S.,Bentley, M. E. (2013). Pressuring and restrictive feeding styles influence infant feeding and size among Intervention/exposure a low-income African-American sample Obesity (Silver Spring), 21(3), 562-71

2542 Thompson, A. L.,Lampl, M. (2013). Prenatal and postnatal energetic conditions and sex steroids levels across the first year of life Am J Hum Biol, 25(5), 643-54

2543 Thompson, M. (1987). Think zinc Neonatal Netw, 6(1), 44-5

2544 Thompson, N. P.,Montgomery, S. M.,Wadsworth, M. E.,Pounder, R. E.,Wakefield, A. J. (2000). Early determinants of inflammatory bowel disease: use of two national longitudinal birth cohorts Eur J Gastroenterol Hepatol, 12(1), 25-30

2545 Thomsen, S. F.,Ulrik, C. S.,Porsbjerg, C.,Backer, V. (2006). Early life exposures and risk of atopy among Danish children Allergy Asthma Proc, 27(2), 110-4

2546 Thomson, J. L., Tussing-Humphreys, L. M.,Goodman, M. H. (2014). Delta Healthy Sprouts: a randomized comparative effectiveness trial to promote maternal weight control and reduce childhood obesity in the Mississippi Delta Contemp Clin Trials, 38(1), 82-91

2547 Thomson, K.,Morley, R.,Grover, S. R.,Zacharin, M. R. (2004). Postnatal evaluation of vitamin D and bone health in women who were vitamin D-deficient in pregnancy, and in their infants Med J Aust, 181(9), 486-8

2548 Thomson, M. (1994). Otitis media. How are First Nations children affected? Can Fam Physician, 40(\#issue\#), 1943-50

Outcome, Size of study groups

Study design

Study design, of study groups

Study design

Study design

Size of study groups

Study design, Outcome

Study design, Outcome

Size of study groups

Study design,

Intervention/exposure

Intervention/exposure

2549 Thorisdottir, A. V.,Ramel, A.,Palsson, G. I.,Tomassson, H.,Thorsdottir, I. (2013). Iron status of one-year-olds and association with breast milk, cow's milk or formula in late infancy Eur J Nutr, 52(6), 1661-8 
2550 Thorisdottir, B.,Gunnarsdottir, I.,Steingrimsdottir, L.,Palsson, G. I.,Thorsdottir, I. (2014). Vitamin D intake and status in 12-month-old infants at 63-66 degrees N Nutrients, 6(3), 1182-93

2551 Thorpe, K.,Rutter, M.,Greenwood, R. (2003). Twins as a natural experiment to study the causes of mild language delay: II: Family interaction risk factors J Child Psychol Psychiatry, 44(3), 342-55

2552 Thorsdottir, I.,Birgisdottir, B. E.,Johannsdottir, I. M.,Harris, D. P.,Hill, J.,Steingrimsdottir, L.,Thorsson, A. V. (2000). Different betacasein fractions in Icelandic versus Scandinavian cow's milk may influence diabetogenicity of cow's milk in infancy and explain low incidence of insulin-dependent diabetes mellitus in Iceland Pediatrics, 106(4), 719-24

2553 Thorsdottir, I.,Gunnarsdottir, I.,Kvaran, M. A.,Gretarsson, S. J. (2005). Maternal body mass index, duration of exclusive breastfeeding and children's development status at the age of 6 years European Journal of Clinical Nutrition, 59(3), 426-431

2554 Thorsdottir, I.,Gunnarsdottir, I.,Kvaran, M. A.,Gretarsson, S. J. (2005). Maternal body mass index, duration of exclusive

breastfeeding and children's developmental status at the age of 6 years Eur J Clin Nutr, 59(3), 426-31

2555 Thorsdottir, I.,Gunnarsdottir, I.,Palsson, G. I. (2003). Association of birth weight and breast-feeding with coronary heart disease risk factors at the age of 6 years Nutr Metab Cardiovasc Dis, 13(5), 267-72

Intervention/exposure

Publication date for a non-sibling

study

2556 Thorsdottir, I.,Gunnarsdottir, I.,Palsson, G. I. (2003). Birth weight, growth and feeding in infancy: relation to serum lipid concentration Outcome in 12-month-old infants Eur J Clin Nutr, 57(11), 1479-85

2557 Thorsdottir, I.,Gunnarsson, B. S. (2006). Dietary quality and adequacy of micronutrient intakes in children Proc Nutr Soc, 65(4), 366-75

2558 Thorsdottir, I.,Gunnarsson, B. S.,Atladottir, H.,Michaelsen, K. F.,Palsson, G. (2003). Iron status at 12 months of age -- effects of body size, growth and diet in a population with high birth weight Eur J Clin Nutr, 57(4), 505-13

2559 Thurtle, V. (1985). Infant feeding Nurs Mirror, 160(19), 44-5

Outcome

Study design, Outcome

2560 Timby, N.,Domellof, E.,Hernell, O.,Lonnerdal, B.,Domellof, M. (2014). Neurodevelopment, nutrition, and growth until 12 mo of age in Intervention/exposure infants fed a low-energy, low-protein formula supplemented with bovine milk fat globule membranes: a randomized controlled trial Am J Clin Nutr, 99(4), 860-8

2561 Timby, N.,Hernell, O.,Lonnerdal, B.,Domellof, M. (2014). Parental feeding control in relation to feeding mode and growth pattern during early infancy Acta Paediatr, 103(10), 1072-7

2562 Timby, N.,Hernell, O.,Vaarala, O.,Melin, M.,Lonnerdal, B.,Domellof, M. (2015). Infections in infants fed formula supplemented with bovine milk fat globule membranes J Pediatr Gastroenterol Nutr, 60(3), 384-9

2563 Timby, N.,Lonnerdal, B.,Hernell, O.,Domellof, M. (2014). Cardiovascular risk markers until 12 mo of age in infants fed a formula supplemented with bovine milk fat globule membranes Pediatr Res, 76(4), 394-400

2564 Timmermans, F. J.,Gerson, S. (1980). Chronic granulomatous otitis media in bottle-fed Inuit children Can Med Assoc J, 122(5), $545-7$

Intervention/exposure

Intervention/exposure

Intervention/exposure

Study design,

Intervention/exposure 
2565 Timmermans, M. J.,Dagnelie, P. C., Theunisz, E. H.,Ewalds, D.,Thijs, C.,Mommers, M.,Arts, I. C. (2015). Dietary nucleotide and nucleoside exposure in infancy and atopic dermatitis, recurrent wheeze, and allergic sensitization J Pediatr Gastroenterol Nutr, $60(5), 691-3$

2566 Tiwari, S. (2015). Age of Introduction of Complementary Feeding and Iron Deficiency Anemia in Breastfed Infants,Child Health Viewpoint Indian Pediatr, 52(11), 977-8 $168(1), 24-6$

2568 Togo, A.,Espadas Macia, D.,Blanes Segura, S.,Sivo Diaz, N.,Villalba Martinez, C. (2015). [Is there vitamin D deficiency in children in a sunny Mediterranean city?] An Pediatr (Barc), \#volume\#(\#issue\#), \#Pages\#

2569 Tom, W. L. (2012). Atopic dermatitis: Recent findings and insights Pediatric Annals, 41(1), 1-5

Intervention/exposure

Study design

2570 Tomblin, J. B.,Smith, E.,Zhang, X. (1997). Epidemiology of specific language impairment: prenatal and perinatal risk factors J Commun Disord, 30(4), 325-43; quiz 343-4

2571 Toms, G. L.,Scott, R. (1987). Respiratory syncytial virus and the infant immune response Arch Dis Child, 62(6), 544-6

Outcome

Study design,

Intervention/exposure

2572 Toro Monjaraz, E. M. Ramirez Mayans, J. A. Cervantes Bustamante, R. Gomez Morales, E.,Molina Rosales, A.,Montijo Barrios, E.,Zarate Mondragon, F.,Cadena Leon, J.,Cazares Mendez, M.,Lopez-Ugalde, M. (2015). Perinatal factors associated with the development of cow's milk protein allergy Rev Gastroenterol Mex, 80(1), 27-31

2573 Toro, K.,Sotonyi, P. (2001). Distribution of prenatal and postnatal risk factors for sudden infant death in Budapest Scand J Prim Health Care, 19(3), 178-80

2574 Torowicz, Deborah L.,Spatz, Diane L.,Seelhorst, Amanda (2013). Human Milk and Breastfeeding in the Cardiac Center: A

Prospective, Descriptive Study Journal of Pediatric Healthcare, 27(5), 325-325 $1 \mathrm{p}$

Language

2575 Torsvik, I. K.,Markestad, T.,Ueland, P. M.,Nilsen, R. M.,Midttun, O.,Bjorke Monsen, A. L. (2013). Evaluating iron status and the risk of anemia in young infants using erythrocyte parameters Pediatr Res, 73(2), 214-20

2576 Toschke, A. M.,Beyerlein, A.,von Kries, R. (2005). Children at high risk for overweight: a classification and regression trees analysis Study design approach Obes Res, 13(7), 1270-4

2577 Toschke, A. M.,Martin, R. M.,von Kries, R.,Wells, J.,Smith, G. D.,Ness, A. R. (2007). Infant feeding method and obesity: body mass index and dual-energy X-ray absorptiometry measurements at 9-10 y of age from the Avon Longitudinal Study of Parents and Children (ALSPAC) Am J Clin Nutr, 85(6), 1578-85

2578 Toselli, S.,Zaccagni, L.,Celenza, F.,Albertini, A.,Gualdi-Russo, E. (2015). Risk factors of overweight and obesity among preschool children with different ethnic background Endocrine, 49(3), 717-25

Publication date for a non-sibling study

Study design 
2579 Tozzi, A. E.,Bisiacchi, P.,Tarantino, V.,Chiarotti, F.,D'Elia, L.,De Mei, B.,Romano, M.,Gesualdo, F.,Salmaso, S. (2012). Effect of duration of breastfeeding on neuropsychological development at 10 to 12 years of age in a cohort of healthy children Dev Med Child Neurol, 54(9), 843-8

2580 Trabulsi, J.,Capeding, R.,Lebumfacil, J.,Ramanujam, K.,Feng, P.,McSweeney, S.,Harris, B.,DeRusso, P. (2011). Effect of an alphalactalbumin-enriched infant formula with lower protein on growth Eur J Clin Nutr, 65(2), 167-74

Tran, T. D.,Biggs, B. A.,Tran, T.,Simpson, J. A.,Hanieh, S.,Dwyer, T.,Fisher, J. (2013). Impact on infants' cognitive development of antenatal exposure to iron deficiency disorder and common mental disorders PLoS One, 8(9), e74876

2582 Trapp, P. G.,Mielke, J. H.,Jorde, L. B.,Eriksson, A. W. (1983). Infant mortality patterns in Aland, Finland Hum Biol, 55(1), 131-49

Study design

Intervention/exposure

2583 Trevino-Garza, C.,Mancillas-Adame, L.,Villarreal-Perez, J. Z.,De la, O. Cavazos M. E.,Estrada-Zuniga, C. M.,Bosques-Padilla, F.

Outcome

J.,Argente, J. (2012). Association between umbilical cord leptin and weight gain according to feeding type in the early postnatal period, a brief report Rev Invest Clin, 64(6 Pt 2), 615-9

2584 Truswell, A. S. (1985). ABC of nutrition. Infant feeding Br Med J (Clin Res Ed), 291(6491), 333-7

Study design

2585 Tsai, A. I.,Johnsen, D. C.,Lin, Y. H.,Hsu, K. H. (2001). A study of risk factors associated with nursing caries in Taiwanese children aged 24-48 months Int J Paediatr Dent, 11(2), 147-9

2586 Tsai, S. F., Chen, S. J.,Yen, H. J.,Hung, G. Y.,Tsao, P. C.,Jeng, M. J.,Lee, Y. S.,Soong, W. J.,Tang, R. B. (2014). Iron deficiency anemia in predominantly breastfed young children Pediatr Neonatol, 55(6), 466-9

2587 Tsang RC (1983). The quandary of vitamin D in the newborn infant Lancet, 1(\#issue\#), 1370-2

Study design

2588 Tsao, P. C., Chang, F. Y.,Chen, S. J.,Soong, W. J.,Jeng, M. J.,Lee, Y. S., Yen, H. J., Yang, C. F., Tang, R. B. (2012). Sudden and unexpected and near death during the early neonatal period: a multicenter study J Chin Med Assoc, 75(2), 65-9

Study design, Size of study groups

2589 Tse, S. M.,Coull, B. A.,Sordillo, J. E.,Datta, S.,Gold, D. R. (2015). Gender- and age-specific risk factors for wheeze from birth through adolescence Pediatric Pulmonology, 50(10), 955-962 2590 Tseng, E.,Potter, S. M.,Picciano, M. F. (1990). Dietary protein source and plasma lipid profiles of infants Pediatrics, 85(4), 548-52

Outcome

Size of study groups

2591 Tsubouchi, J.,Higashi, T.,Shimono, T.,Domoto, P. K.,Weinstein, P. (1994). A study of baby bottle tooth decay and risk factors for 18- Study design month old infants in rural Japan ASDC J Dent Child, 61(4), 293-8

2592 Tsubouchi, J.,Tsubouchi, M.,Maynard, R. J.,Domoto, P. K.,Weinstein, P. (1995). A study of dental caries and risk factors among Native American infants ASDC J Dent Child, 62(4), 283-7

2593 Tsutie S,Kurihara N,Sasaki A,Takagi A,Seguti H,Inatome T (2010). Formulas providing adequate pantothenic acid, vitamin D, Intakes prepared by the Ministry of Health, Labour and Welfare (2005 edition) Matern Child Nutr, 6(\#issue\#), 147-58 
2595 Tulldahl, J.,Pettersson, K.,Andersson, S. W.,Hulthen, L. (1999). Mode of infant feeding and achieved growth in adolescence: early feeding patterns in relation to growth and body composition in adolescence Obes Res, 7(5), 431-7

2596 Tuncbilek, E.,Uner, S.,Ulusoy, M. (1983). Breastfeeding in Turkey: the demographic and socio-economic aspects and relationship with infant/child mortality Turk J Pediatr, 25(1), 3-23

2597 Turati, F.,Bertuccio, P.,Galeone, C.,Pelucchi, C.,Naldi, L.,Bach, J. F.,La Vecchia, C.,Chatenoud, L. (2016). Early weaning is beneficial to prevent atopic dermatitis occurrence in young children Allergy, \#volume\#(\#issue\#), \#Pages\#

2598 Turck, D.,Grillon, C.,Lachambre, E.,Robiliard, P.,Beck, L.,Maurin, J. L.,Kempf, C.,Bernet, J. P.,Marx, J.,Lebrun, F.,Van Egroo, L. D. (2006). Adequacy and safety of an infant formula with a protein/energy ratio of $1.8 \mathrm{~g} / 100 \mathrm{kcal}$ and enhanced protein efficiency for term infants during the first 4 months of life J Pediatr Gastroenterol Nutr, 43(3), 364-71

2599 Turkoglu, S.,Bilgic, A.,Akca, O. F. (2015). ADHD symptoms, breast-feeding and obesity in children and adolescents Pediatr Int, $57(4), 546-51$

2600 Turner, S.,Zhang, G.,Young, S.,Cox, M., Goldblatt, J.,Landau, L.,Le Souef, P. (2008). Associations between postnatal weight gain, change in postnatal pulmonary function, formula feeding and early asthma Thorax, 63(3), 234-9

2601 Tuthill, D. P.,Cosgrove, M.,Dunstan, F.,Stuart, M. L.,Wells, J. C.,Davies, D. P. (2002). Randomized double-blind controlled trial on the effects on iron status in the first year between a no added iron and standard infant formula received for three months Acta Paediatr, 91(2), 119-24

2602 Tyler, M.,Hellings, P. (2005). Feeding method and rehospitalization in newborns less than 1 month of age J Obstet Gynecol Neonatal Nurs, 34(1), $70-9$

2603 Tyson, J.,Burchfield, J.,Sentance, F.,Mize, C.,Uauy, R.,Eastburn, J. (1992). Adaptation of feeding to a low fat yield in breast milk Pediatrics, 89(2), 215-20

2604 Uauy, R.,Mize, C. E.,Castillo-Duran, C. (2000). Fat intake during childhood: metabolic responses and effects on growth Am J Clin Nutr, $72(5$ Suppl), 1354S-1360S

2605 Ugur, S.,Haktan, M.,Cakir, E.,Senocak, M.,Telci, A. (1988). Serum insulin and blood glucose levels in breast-fed and formula-fed infants in the first week of life Clin Ther, 10(6), 678-87

2606 Uhl, O.,Hellmuth, C.,Demmelmair, H.,Zhou, S. J.,Makrides, M.,Prosser, C.,Lowry, D.,Gibson, R. A.,Koletzko, B. (2015). Dietary Effects on Plasma Glycerophospholipids J Pediatr Gastroenterol Nutr, 61(3), 367-72

2607 Uijterschout, L., Vloemans, J.,Vos, R.,Teunisse, P. P.,Hudig, C.,Bubbers, S.,Verbruggen, S., Veldhorst, M.,De Leeuw, T.,Van Goudoever, J. B.,Brus, F. (2014). Prevalence and risk factors of iron deficiency in healthy young children in the southwestern netherlands Journal of Pediatric Gastroenterology and Nutrition, 58(2), 193-198

2608 Ulak, M.,Chandyo, R. K.,Adhikari, R. K.,Sharma, P. R.,Sommerfelt, H.,Refsum, H.,Strand, T. A. (2014). Cobalamin and folate status in 6 to 35 months old children presenting with acute diarrhea in Bhaktapur, Nepal PLoS One, 9(3), e90079 
2609 Ulbak, J.,Lauritzen, L.,Hansen, H. S.,Michaelsen, K. F. (2004). Diet and blood pressure in 2.5-y-old Danish children Am J Clin Nutr, $79(6), 1095-102$

2610 Umer, A.,Hamilton, C.,Britton, C. M.,Mullett, M. D.,John, C.,Neal, W.,Lilly, C. L. (2015). Association between Breastfeeding and Childhood Obesity: Analysis of a Linked Longitudinal Study of Rural Appalachian Fifth-Grade Children Child Obes, 11(4), 449-55

2611 Unay, B.,Sarici, S. U.,Ulas, U. H.,Akin, R.,Alpay, F.,Gokcay, E. (2004). Nutritional effects on auditory brainstem maturation in healthy term infants Arch Dis Child Fetal Neonatal Ed, 89(2), F177-9 P.,Dahl, G. V.,Moonsamy, P.,Erlich, H. A.,Trachtenberg, E.,Buffler, P. A. (2012). HLA-DP genetic variation, proxies for early life immune modulation and childhood acute lymphoblastic leukemia risk Blood, 120(15), 3039-47

2613 Vaarala, O.,Ilonen, J.,Ruohtula, T.,Pesola, J.,Virtanen, S. M.,Harkonen, T.,Koski, M.,Kallioinen, H.,Tossavainen, O.,Poussa, T.,Jarvenpaa, A. L.,Komulainen, J.,Lounamaa, R.,Akerblom, H. K.,Knip, M. (2012). Removal of bovine insulin from cow's milk formula and early initiation of beta-cell autoimmunity in the FINDIA pilot study Archives of pediatrics \& adolescent medicine, 166(7), 608-14

2614 Vaarala, O.,Knip, M.,Paronen, J.,Hamalainen, A. M.,Muona, P.,Vaatainen, M.,llonen, J.,Simell, O.,Akerblom, H. K. (1999). Cow's milk formula feeding induces primary immunization to insulin in infants at genetic risk for type 1 diabetes Diabetes, 48(7), 1389-94

2615 Vafa, M.,Heshmati, J.,Sadeghi, H.,Shidfar, F.,Namazi, N.,Baradaran, H.,Heydarpour, B.,Jalili, Z. (2015). Is exclusive breastfeeding and its duration related to cardio respiratory fitness in childhood? J Matern Fetal Neonatal Med, \#volume\#(\#issue\#), 1-6

2616 Vaidergorn, B. (1991). Oral habits and atypical deglutition in certain Sao Paulo children Int J Orofacial Myology, 17(3), 11-5

Study design,

Intervention/exposure

2617 Valaitis, R. K.,Ciliska, D. K.,Sheeshka, J. D.,Sword, W. A. (1996). Surveying infant feeding practices Can Nurse, 92(4), 21

Study design

2618 Valentin-Blasini, L.,Blount, B. C.,Otero-Santos, S.,Cao, Y.,Bernbaum, J. C.,Rogan, W. J. (2011). Perchlorate exposure and dose estimates in infants Environ Sci Technol, 45(9), 4127-32

2619 Valman, H. B. (1980). The first year of life: feeding and feeding problems Br Med J, 280(6212), 457-60

Study design

Study design

2620 Valvi, D.,Mendez, M. A.,Garcia-Esteban, R.,Ballester, F.,Ibarluzea, J.,Goni, F.,Grimalt, J. O.,Llop, S.,Marina, L. S.,Vizcaino,

Intervention/exposure

E.,Sunyer, J.,Vrijheid, M. (2014). Prenatal exposure to persistent organic pollutants and rapid weight gain and overweight in infancy Obesity (Silver Spring), 22(2), 488-96

2621 Van Asperen, P. P.,Kemp, A. S.,Mellis, C. M. (1984). Relationship of diet in the development of atopy in infancy Clin Allergy, 14(6), 525-32

Intervention/exposure, Size of

study groups

2622 van Beijsterveldt, T. C.,Boomsma, D. I. (2008). An exploration of gene-environment interaction and asthma in a large sample of 5-

Outcome year-old Dutch twins Twin Res Hum Genet, 11(2), 143-9

2623 Van Biervliet, J. P.,Rosseneu, M.,Caster, H. (1986). Influence of dietary factors on the plasma lipoprotein composition and content in Size of study groups neonates Eur J Pediatr, 144(5), 489-93 
2624 Van Biervliet, J. P.,Vinaimont, N.,Caster, H.,Vercaemst, R.,Rosseneu, M. (1981). Lipoprotein patterns in newborns. Influence of nutritional factors Acta Cardiol Suppl, 27(\#issue\#), 69-81

2625 van Biervliet, J. P.,Vinaimont, N.,Caster, H.,Vercaemst, R.,Rosseneu, M. (1981). Plasma apoprotein and lipid patterns in newborns: Size of study groups influence of nutritional factors Acta Paediatr Scand, 70(6), 851-6

2626 Van Biervliet, J. P.,Vinaimont, N.,Vercaemst, R.,Rosseneu, M. (1992). Serum cholesterol, cholesteryl ester, and high-density lipoprotein development in newborn infants: response to formulas supplemented with cholesterol and gamma-linolenic acid $\mathrm{J}$ Pediatr 120(4 Pt 2), S101-8

2627 Van Biervliet, S.,Van Biervliet, J. P.,Bernard, D.,Vercaemst, R.,Blaton, V. (2003). Serum zinc in healthy Belgian children Biological Trace Element Research, 94(1), 33-40

2628 van Buuren, S. (2010). Effects of selective dropout on infant growth standards Nestle Nutr Workshop Ser Pediatr Program, 65(\#issue\#), 167-75; discussion 175-9

2629 van den Berg, G.,van Eijsden, M.,Galindo-Garre, F.,Vrijkotte, T. G.,Gemke, R. J. (2013). Explaining socioeconomic inequalities in childhood blood pressure and prehypertension: the ABCD study Hypertension, 61(1), 35-41

2630 Van Den Berg, G.,Van Eijsden, M.,Galindo-Garre, F.,Vrijkotte, T.,Gemke, R. (2013). Low maternal education is associated with increased growth velocity in the first year of life and in early childhood: the ABCD study Eur J Pediatr, 172(11), 1451-7

2631 van den Bogaard, C.,van den Hoogen, H. J.,Huygen, F. J.,van Weel, C. (1991). The relationship between breast-feeding and early Study design childhood morbidity in a general population Fam Med, 23(7), 510-5

2632 van den Bogaard, C.,van den Hoogen, H. J.,Huygen, F. J.,van Weel, C. (1993). Is the breast best for children with a family history of Intervention/exposure atopy? The relation between way of feeding and early childhood morbidity Fam Med, 25(7), 471-5

2633 Van der Elst, C. W.,Dempster, W. S.,Woods, D. L.,Heese, H. D. (1986). Serum zinc and copper in thin mothers, their breast milk and their infants J Trop Pediatr, 32(3), 111-4

2634 van der Willik, E. M.,Vrijkotte, T. G.,Altenburg, T. M.,Gademan, M. G.,Kist-van Holthe, J. (2015). Exclusively breastfed overweight infants are at the same risk of childhood overweight as formula fed overweight infants Arch Dis Child, 100(10), 932-7

2635 van Dijk, C. E.,Innis, S. M. (2009). Growth-curve standards and the assessment of early excess weight gain in infancy Pediatrics 123(1), 102-8

2636 van Elten, T. M.,van Rossem, L.,Wijga, A. H.,Brunekreef, B.,de Jongste, J. C.,Koppelman, G. H.,Smit, H. A. (2015). Breast milk fatty acid composition has a long-term effect on the risk of asthma, eczema, and sensitization Allergy, 70(11), 1468-76

2637 Van Howe, R. S.,Storms, M. R. (2008). Blood glucose determinations in large for gestational age infants Am J Perinatol, 25(5), 2839

2638 van Merode, T.,Maas, T.,Twellaar, M.,Kester, A.,van Schayck, C. P. (2007). Gender-specific differences in the prevention of asthma-like symptoms in high-risk infants Pediatr Allergy Immunol, 18(3), 196-200 
2639 van Odijk, J.,Hulthen, L.,Ahlstedt, S.,Borres, M. P. (2004). Introduction of food during the infant's first year: a study with emphasis on introduction of gluten and of egg, fish and peanut in allergy-risk families Acta Paediatr, 93(4), 464-70

Study design

Intervention/exposure

2640 van Palenstein Helderman, W. H.,Soe, W.,van 't Hof, M. A. (2006). Risk factors of early childhood caries in a Southeast Asian population J Dent Res, 85(1), 85-8

2641 van Rossem, L.,Wijga, A. H.,Brunekreef, B.,de Jongste, J. C.,Kerkhof, M.,Postma, D. S.,Gehring, U.,Smit, H. A. (2014). Overweight in infancy: which pre- and perinatal factors determine overweight persistence or reduction? A birth cohort followed for 11 years Ann Nutr Metab, 65(2-3), 211-9

2642 van Rossem, L.,Wijga, A. H.,de Jongste, J. C.,Koppelman, G. H.,Oldenwening, M.,Postma, D. S.,Abrahamse-Berkeveld, M.,van de Heijning, B.,Brunekreef, B.,Smit, H. A. (2012). Blood pressure in 12-year-old children is associated with fatty acid composition of human milk: the prevention and incidence of asthma and mite allergy birth cohort Hypertension, 60(4), 1055-60

2643 van Stuijvenberg, M.,Eisses, A. M.,Gruber, C.,Mosca, F.,Arslanoglu, S.,Chirico, G.,Braegger, C. P.,Riedler, J.,Boehm, G.,Sauer, P. J. (2011). Do prebiotics reduce the number of fever episodes in healthy children in their first year of life: a randomised controlled tria Br J Nutr, 106(11), 1740-8

2644 van Stuijvenberg, M.,Stam, J.,Gruber, C.,Mosca, F.,Arslanoglu, S.,Chirico, G.,Braegger, C. P.,Riedler, J.,Boehm, G.,Sauer, P. J. (2015). Similar Occurrence of Febrile Episodes Reported in Non-Atopic Children at Three to Five Years of Age after Prebiotics Supplemented Infant Formula PLoS One, 10(6), e0129927

2645 van t Hof Msc, M. A. (2000). The influence of breastfeeding and complementary foods on growth until three years of age in the Euro-Growth Study Pediatrics, 106(5), 1281a-1281

2646 van Wouwe, J. P.,van den Hamer, C. J.,van Tricht, J. B. (1986). Serum zinc concentrations in exclusively breast-fed infants and in infants fed an adapted formula Eur J Pediatr, 144(6), 598-600

2647 Vandenplas, Y.,Deneyer, M.,Sacre, L.,Loeb, H. (1988). Preliminary data on a field study with a new hypo-allergic formula European Journal of Pediatrics, 148(3), 274-277

2648 Vandenplas, Y.,Sacre, L. (1986). Influences of neonatal serum IgE concentration, family history and diet on the incidence of cow's milk allergy Eur J Pediatr, 145(6), 493-5

2649 Vanderhoof, J. A.,Murray, N. D.,Antonson, D. L.,Kaufman, S. S. (1986). Familial occurrence of protracted diarrhea of infancy J Pediatr, 109(5), 845-7

2650 Vanella, L.,de Gonzalez Lascano, A. M. (1988). CD4+, CD8+ cells, IgE and prick test in infants allergic to cow's milk Allergol Immunopathol (Madr), 16(5), 327-31

2651 Vanessa Nazareth, Isis,Maria Meneses dos Santos, InÃas,Paula Oliveira GonÃßalves, Ana,Sena Souza, Ester (2013). RISK FOR CHILD DEVELOPMENT ACCORDING TO THE INTEGRATED ATTENTION STRATEGY TO THE PREVALENT ILLNESSES IN CHILDHOOD Journal of Nursing UFPE / Revista de Enfermagem UFPE, 7(2), 328-336 9p 
2653 Vasallo, M. I.,Martinez, R.,Ballesta, M. J.,Vives, I.,Sanchez-Solis, M.,Peso, P.,Martinez, C. (2011). Effect of an infant formula containing milk fat, Alpha-lactalbumin, Nucleotides and Icpufa on stool patterns in infants Journal of pediatric gastroenterology and nutrition, 52(\#issue\#), E166

2654 Vazquez, E. (2007). 14th annual retrovirus conference (CROI). Astounding choice in breastfeeding: infection or death Posit Aware, $18(3), 29-30$

2655 Veereman-Wauters, G.,Staelens, S.,Van de Broek, H.,Plaskie, K.,Wesling, F.,Roger, L. C.,McCartney, A. L.,Assam, P. (2011). Physiological and bifidogenic effects of prebiotic supplements in infant formulae J Pediatr Gastroenterol Nutr, 52(6), 763-71

2656 Vehapoglu, A.,Yazici, M.,Demir, A. D.,Turkmen, S.,Nursoy, M.,Ozkaya, E. (2014). Early infant feeding practice and childhood obesity: the relation of breast-feeding and timing of solid food introduction with childhood obesity J Pediatr Endocrinol Metab, 27(1112), 1181-7

2657 Venkataraman, P. S.,Luhar, H.,Neylan, M. J. (1992). Bone mineral metabolism in full-term infants fed human milk, cow milk-based and soy-based formulas Am J Dis Child, 146(11), 1302-5

2658 Vennemann, M.,Bajanowski, T.,Butterfass-Bahloul, T.,Sauerland, C.,Jorch, G.,Brinkmann, B.,Mitchell, E. A. (2007). Do risk factors differ between explained sudden unexpected death in infancy and sudden infant death syndrome? Arch Dis Child, 92(2), 133-6

2659 Venter, C.,Pereira, B.,Voigt, K.,Grundy, J.,Clayton, C. B.,Higgins, B.,Arshad, S. H.,Dean, T. (2009). Factors associated with maternal dietary intake, feeding and weaning practices, and the development of food hypersensitivity in the infant Pediatr Allergy Immunol, 20(4), 320-7

2660 Ventura, A. K.,Loken, E.,Birch, L. L. (2009). Developmental trajectories of girls' BMl across childhood and adolescence Obesity (Silver Spring), 17(11), 2067-74

2661 Ventura, A.,Longo, G.,Longo, F.,Florean, P.,Scornavacca, G. (1989). Diet and atopic eczema in children Allergy, 44 Suppl 9(\#issue\#), 159-64

2662 Verga, M. E.,Widmeier-Pasche, V.,Beck-Popovic, M.,Pauchard, J. Y.,Gehri, M. (2014). Iron deficiency in infancy: is an immigrant more at risk? Swiss Med Wkly, 144(\#issue\#), w14065

2663 Verge, C. F.,Howard, N. J.,Irwig, L.,Simpson, J. M.,Mackerras, D.,Silink, M. (1994). Environmental factors in childhood IDDM. A population-based, case-control study Diabetes Care, 17(12), 1381-9

2664 Verkasalo, M.,Kuitunen, P.,Savilahti, E.,Tiilikainen, A. (1981). Changing pattern of cow's milk intolerance. An analysis of the occurrence and clinical course in the 60s and mid-70s Acta Paediatr Scand, 70(3), 289-95

Outcome Vernacchio, L.,Lesko, S. M.,Vezina, R. M., Corwin, M. J.,Hunt, C. E Hoffman, H. J., Mitchell,

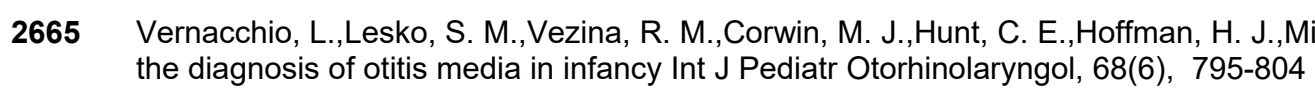

2666 Verstraete, S. G.,Heyman, M. B.,Wojcicki, J. M. (2014). Breastfeeding offers protection against obesity in children of recently immigrated Latina women J Community Health, 39(3), 480-6

Participant health, Intervention/exposure

Study design

Intervention/exposure 
2667 Vesel, L.,Bahl, R.,Martines, J.,Penny, M.,Bhandari, N.,Kirkwood, B. R. (2010). Use of new World Health Organization child growth standards to assess how infant malnutrition relates to breastfeeding and mortality Bull World Health Organ, 88(1), 39-48

2668 Vesikari, T.,Prymula, R.,Schuster, V.,Tejedor, J. C.,Cohen, R.,Bouckenooghe, A.,Damaso, S.,Han, H. H. (2012). Efficacy and immunogenicity of live-attenuated human rotavirus vaccine in breast-fed and formula-fed European infants Pediatr Infect Dis $\mathrm{J}$ $31(5), 509-13$

2669 Vestergaard, M.,Obel, C.,Henriksen, T. B.,Sorensen, H. T.,Skajaa, E.,Ostergaard, J. (1999). Duration of breastfeeding and developmental milestones during the latter half of infancy Acta Paediatr, 88(12), 1327-32

2670 Vestman, N. R.,Timby, N.,Holgerson, P. L.,Kressirer, C. A.,Claesson, R.,Domellof, M.,Ohman, C.,Tanner, A. C.,Hernell, O.,Johansson, I. (2013). Characterization and in vitro properties of oral lactobacilli in breastfed infants BMC Microbiol, 13(\#issue\#), 193

2671 Vichyanond, P. (1990). IgE regulation and the control of allergic diseases Asian Pac J Allergy Immunol, 8(1), 1-4

Study design

2672 Victora, C. G.,Barros, F. C.,Horta, B. L.,Lima, R. C. (2005). Breastfeeding and school achievement in Brazilian adolescents Acta Paediatr, 94(11), 1656-60

2673 Victora, C. G.,Barros, F.,Lima, R. C.,Horta, B. L.,Wells, J. (2003). Anthropometry and body composition of 18 year old men according to duration of breast feeding: birth cohort study from Brazil BMJ, 327(7420), 901

Victora, C. G.,Fuchs, S. C.,Flores, J. A.,Fonseca, W.,Kirkwood, B. (1994). Risk factors for pneumonia among children in a Brazilian metropolitan area Pediatrics, 93(6 Pt 1), 977-85

2675 Victora, C. G.,Hallal, P. C.,Araújo, C. L. P.,Menezes, A. M. B.,Wells, J. C. K.,Barros, F. C. (2008). Cohort profile: The 1993 pelotas (Brazil) birth cohort study International Journal of Epidemiology, 37(4), 704-709

2676 Victora, C. G.,Horta, B. L.,Loret de Mola, C.,Quevedo, L.,Pinheiro, R. T.,Gigante, D. P.,Goncalves, H.,Barros, F. C. (2015). Association between breastfeeding and intelligence, educational attainment, and income at 30 years of age: a prospective birth cohort study from Brazil Lancet Glob Health, 3(4), e199-205

2677 Victora, C. G.,Horta, B. L.,Post, P.,Lima, R. C.,De Leon Elizalde, J. W.,Gerson, B. M.,Barros, F. C. (2006). Breast feeding and blood Outcome lipid concentrations in male Brazilian adolescents J Epidemiol Community Health, 60(7), 621-5

2678 Victora, C. G.,Huttly, S. R.,Barros, F. C.,Martines, J. C.,Vaughan, J. P. (1991). Prolonged breastfeeding and malnutrition: confounding and effect modification in a Brazilian cohort study Epidemiology, 2(3), 175-81

Publication date for a non-sibling study

2679 Victora, C. G.,Huttly, S. R.,Fuchs, S. C.,Nobre, L. C.,Barros, F. C. (1992). Deaths due to dysentery, acute and persistent diarrhoea among Brazilian infants Acta Paediatr Suppl, 381(\#issue\#), 7-11

2680 Victora, C. G.,Morris, S. S.,Barros, F. C.,de Onis, M.,Yip, R. (1998). The NCHS reference and the growth of breast- and bottle-fed Intervention/exposure infants J Nutr, 128(7), 1134-8

2681 Victora, C. G.,Morris, S. S.,Barros, F. C.,Horta, B. L.,Weiderpass, E., Tomasi, E. (1998). Breast-feeding and growth in Brazilian infants Am J Clin Nutr, 67(3), 452-8 
2682 Victora, C. G.,Rivera, J. A. (2014). Optimal child growth and the double burden of malnutrition: Research and programmatic implications American Journal of Clinical Nutrition, 100(6), 1611S-1612S

2683 Victora, C. G.,Smith, P. G.,Barros, F. C.,Vaughan, J. P.,Fuchs, S. C. (1989). Risk factors for deaths due to respiratory infections among Brazilian infants Int J Epidemiol, 18(4), 918-25

2684 Victora, C. G.,Smith, P. G.,Vaughan, J. P.,Nobre, L. C.,Lombardi, C., Teixeira, A. M.,Fuchs, S. C.,Moreira, L. B.,Gigante, L. P.,Barros, F. C. (1989). Infant feeding and deaths due to diarrhea. A case-control study Am J Epidemiol, 129(5), 1032-41

2685 Victora, C. G.,Smith, P. G.,Vaughan, J. P.,Nobre, L. C.,Lombardi, C., Teixeira, A. M.,Fuchs, S. M.,Moreira, L. B., Gigante, L. P.,Barros, F. C. (1987). Evidence for protection by breast-feeding against infant deaths from infectious diseases in Brazil Lancet, 2(8554), 319-22

2686 Victora, C. G.,Vaughan, J. P.,Martines, J. C.,Barcelos, L. B. (1984). Is prolonged breast-feeding associated with malnutrition? Am J Study design Clin Nutr, 39(2), 307-14

2687 Viggiano, D.,Fasano, D.,Monaco, G.,Strohmenger, L. (2004). Breast feeding, bottle feeding, and non-nutritive sucking; effects on Study design occlusion in deciduous dentition Arch Dis Child, 89(12), 1121-3 fed an adapted formula Eur J Pediatr, 142(4), 245-7

2689 Vignerova, J.,Shriver, L.,Paulova, M.,Brabec, M.,Schneidrova, D.,Ruzkova, R.,Prochazka, B.,Riedlovia, J. (2015). Growth of Czech breastfed infants in comparison with the World Health Organization standards Cent Eur J Public Health, 23(1), 32-8

2690 Villalpando, S. (2000). Feeding mode, infections, and anthropometric status in early childhood Pediatrics, 106(5), 1282-3

Size of study groups

Intervention/exposure

Study design

2691 Villalpando, S.,Lopez-Alarcon, M. (2000). Growth faltering is prevented by breast-feeding in underprivileged infants from Mexico City J Nutr, 130(3), 546-52

2692 Viner, R. M.,Hindmarsh, P. C.,Taylor, B.,Cole, T. J. (2008). Childhood body mass index (BMI), breastfeeding and risk of Type 1 diabetes: findings from a longitudinal national birth cohort Diabet Med, 25(9), 1056-61

2693 Violato, M.,Petrou, S.,Gray, R.,Redshaw, M. (2011). Family income and child cognitive and behavioural development in the United Kingdom: does money matter? Health Econ, 20(10), 1201-25

2694 Virtanen, S. M.,Kenward, M. G.,Erkkola, M.,Kautiainen, S.,Kronberg-Kippila, C.,Hakulinen, T.,Ahonen, S.,Uusitalo, L.,Niinisto, S.,Veijola, R.,Simell, O.,llonen, J.,Knip, M. (2006). Age at introduction of new foods and advanced beta cell autoimmunity in young children with HLA-conferred susceptibility to type 1 diabetes Diabetologia, 49(7), 1512-21

2695 Virtanen, S. M.,Rasanen, L.,Aro, A.,Lindstrom, J.,Sippola, H.,Lounamaa, R., Toivanen, L., Tuomilehto, J.,Akerblom, H. K. (1991). Infant feeding in Finnish children less than $7 \mathrm{yr}$ of age with newly diagnosed IDDM. Childhood Diabetes in Finland Study Group Diabetes Care, 14(5), 415-7

2696 Virtanen, S. M.Rasanen, L.Aro, A Ylonen, K. Lounamaa, R.,Tuomilehto, J.Akerblom, H. K. (1992). Feeding in infancy and the risk of type 1 diabetes mellitus in Finnish children. The 'Childhood Diabetes in Finland' Study Group Diabet Med, 9(9), 815-9

Outcome, Publication date for a non-sibling study

Intervention/exposure

Study design,

Intervention/exposure

Intervention/exposure, Outcome 
2697 Virtanen, S. M.,Rasanen, L.,Ylonen, K.,Aro, A.,Clayton, D.,Langholz, B.,Pitkaniemi, J.,Savilahti, E.,Lounamaa, R.,Tuomilehto, J.,et al., (1993). Early introduction of dairy products associated with increased risk of IDDM in Finnish children. The Childhood in Diabetes in Finland Study Group Diabetes, 42(12), 1786-90

2698 Virtanen, S. M.,Saukkonen, T.,Savilahti, E.,Ylonen, K.,Rasanen, L.,Aro, A.,Knip, M.,Tuomilehto, J.,Akerblom, H. K. (1994). Diet, cow's milk protein antibodies and the risk of IDDM in Finnish children. Childhood Diabetes in Finland Study Group Diabetologia, $37(4), 381-7$

2699 Visalli, N.,Sebastiani, L.,Adorisio, E.,Conte, A.,De Cicco, A. L.,D'Elia, R.,Manfrini, S.,Pozzilli, P. (2003). Environmental risk factors for type 1 diabetes in Rome and province Arch Dis Child, 88(8), 695-8 2700 Vithayasai, N.,Jennuvat, S. (2014). Persistent diarrhea: 15 years experience at a tertiary care hospital J Med Assoc Thai, 97 Suppl Participant health
6(\#issue\#), S95-100

2701 Vitolo, M. R.,Bortolini, G. A.,Dal Bo Campagnolo, P.,Feldens, C. A. (2008). Effectiveness of a nutrition program in reducing symptoms of respiratory morbidity in children: a randomized field trial Prev Med, 47(4), 384-8

2702 Vitolo, M. R.,Bortolini, G. A.,Feldens, C. A.,Drachler Mde, L. (2005). [Impacts of the 10 Steps to Healthy Feeding in Infants: a randomized field trial] Cadernos de saúde pública, 21(5), 1448-57

2703 Vitolo, M. R., da Costa Louzada, M. L.,Rauber, F.,Campagnolo, P. D. (2013). Risk factors for high blood pressure in low income children aged 3-4 years Eur J Pediatr, 172(8), 1097-103

2704 Vivatvakin, B.,Mahayosnond, A., Theamboonlers, A.,Steenhout, P. G.,Conus, N. J. (2010). Effect of a whey-predominant starter formula containing LCPUFAs and oligosaccharides (FOS/GOS) on gastrointestinal comfort in infants Asia Pac J Clin Nutr, 19(4), $473-80$ article

Outcome

Outcome

Outcome

Language

Outcome

Outcome

Outcome

2705 Vobecky, J. S.,Vobecky, J.,Shapcott, D.,Demers, P. P. (1983). Nutrient intake patterns and nutritional status with regard to relative weight in early infancy Am J Clin Nutr, 38(5), 730-8

2706 Vogazianos, E.,Vogazianos, P.,Fiala, J.,Janecek, D.,Slapak, I. (2007). The effect of breastfeeding and its duration on acute otitis media in children in Brno, Czech Republic Cent Eur J Public Health, 15(4), 143-6

2707 Volz, V. R.,Book, L. S.,Churella, H. R. (1983). Growth and plasma amino acid concentrations in term infants fed either wheypredominant formula or human milk J Pediatr, 102(1), 27-31

2708 von Berg, A.,Koletzko, S.,Filipiak-Pittroff, B.,Laubereau, B.,Grubl, A.,Wichmann, H. E.,Bauer, C. P.,Reinhardt, D.,Berdel, D. (2007) Certain hydrolyzed formulas reduce the incidence of atopic dermatitis but not that of asthma: three-year results of the German Infant Nutritional Intervention Study J Allergy Clin Immunol, 119(3), 718-25

2709 von Berg, A.,Koletzko, S.,Grubl, A.,Filipiak-Pittroff, B.,Wichmann, H. E.,Bauer, C. P.,Reinhardt, D.,Berdel, D. (2003). The effect of hydrolyzed cow's milk formula for allergy prevention in the first year of life: the German Infant Nutritional Intervention Study, a randomized double-blind trial J Allergy Clin Immunol, 111(3), 533-40 is strongly associated with the development of asthma and rhinitis in a prospective cohort BMC Dermatol, 12(\#issue\#), 11

Publication date for a non-sibling study

Study design

Size of study groups

Intervention/exposure

Intervention/exposure

Outcome 
2711 von Linstow, M. L.,Hogh, M.,Nordbo, S. A.,Eugen-Olsen, J.,Koch, A.,Hogh, B. (2008). A community study of clinical traits and risk factors for human metapneumovirus and respiratory syncytial virus infection during the first year of life Eur J Pediatr, 167(10), 112533

2712 von Mutius, E.,Hartert, T. (2013). Update in asthma 2012 Am J Respir Crit Care Med, 188(2), 150-6

2713 von Stumm, S.,Plomin, R. (2015). Breastfeeding and IQ Growth from Toddlerhood through Adolescence PLoS One, 10(9), e0138676

2714 Vriezinga, S. L.,Auricchio, R.,Bravi, E.,Castillejo, G.,Chmielewska, A.,Crespo Escobar, P.,Kolacek, S.,Koletzko, S.,Korponay-Szabo, I. R.,Mummert, E.,Polanco, I.,Putter, H.,Ribes-Koninckx, C.,Shamir, R.,Szajewska, H.,Werkstetter, K., Greco, L., Gyimesi, J.,Hartman, C.,Hogen Esch, C.,Hopman, E.,Ivarsson, A.,Koltai, T.,Koning, F.,Martinez-Ojinaga, E.,te Marvelde, C.,Pavic, A.,Romanos, J.,Stoopman, E.,Villanacci, V.,Wijmenga, C.,Troncone, R.,Mearin, M. L. (2014). Randomized feeding intervention in infants at high risk for celiac disease N Engl J Med, 371(14), 1304-15

2715 Wachs, T. D.,Kanashiro, H. C.,Gurkas, P. (2008). Intra-individual variability in infancy: structure, stability, and nutritional correlates Dev Psychobiol, 50(3), 217-31

2716 Wadsworth, E. J.,Shield, J. P.,Hunt, L. P.,Baum, J. D. (1997). A case-control study of environmental factors associated with diabetes in the under 5s Diabet Med, 14(5), 390-6

2717 Wadsworth, M. E.,Hardy, R. J.,Paul, A. A.,Marshall, S. F.,Cole, T. J. (2002). Leg and trunk length at 43 years in relation to childhood Intervention/exposure health, diet and family circumstances; evidence from the 1946 national birth cohort Int J Epidemiol, 31(2), 383-90

2718 Wagner, C. L.,Hulsey, T. C.,Fanning, D.,Ebeling, M.,Hollis, B. W. (2006). High-dose vitamin D3 supplementation in a cohort of breastfeeding mothers and their infants: a 6-month follow-up pilot study Breastfeed Med, 1(2), 59-70

2719 Wagner, V.,von Stockhausen, H. B. (1988). The effect of feeding human milk and adapted milk formulae on serum lipid and lipoprotein levels in young infants Eur J Pediatr, 147(3), 292-5 21/2 year-old Swedish children Br J Nutr, 95(3), 603-8

2721 Walker, W. A. (1994). Nucleotides and nutrition: role as dietary supplement J Nutr, 124(1 Suppl), 121s-123s

Study design

Outcome

Intervention/exposure

Intervention/exposure, Outcome

Outcome

Intervention/exposure

Study design

Outcome

Study design,

Intervention/exposure, Outcome

Study design

2722 Wallis, J. (2012). Positive role of breastfeeding during the first six weeks Midwives, 15(3), 31

Intervention/exposure

Intervention/exposure Child, 93(4), 292-6

2724 Walter, T.,Pino, P.,Pizarro, F.,Lozoff, B. (1998). Prevention of iron-deficiency anemia: comparison of high- and low-iron formulas in term healthy infants after six months of life J Pediatr, 132(4), 635-40 
2726 Waly, M. I.,Ali, A.,Al-Saadoon, M.,Al-Mukhaini, Y. K.,Wali, Y. A. (2011). Breastfeeding is not associated with risk of developing childhood leukemia in the Sultanate of Oman Asian Pac J Cancer Prev, 12(8), 2087-91

2727 Wan, A. K.,Seow, W. K.,Purdie, D. M.,Bird, P. S.,Walsh, L. J.,Tudehope, D. I. (2001). Oral colonization of Streptococcus mutans in Study design six-month-old predentate infants J Dent Res, 80(12), 2060-5

2728 Wandera, A. (1998). Anticipatory guidance in infant oral health J Mich Dent Assoc, 80(9), 28, 55-9

Study design

2729 Wang, H.,Wang, A.,Wang, D.,Bright, A.,Sency, V.,Zhou, A.,Xin, B. (2015). Early growth and development impairment in patients with ganglioside GM3 synthase deficiency Clin Genet, \#volume\#(\#issue\#), \#Pages\#

2730 Wang, I. J.,Guo, Y. L.,Hwang, K. C.,Hsieh, W. S.,Chuang, Y. L.,Lin, S. J.,Chen, P. C. (2006). Genetic and environmental predictors Study design for pediatric atopic dermatitis Acta Paediatrica Taiwanica, 47(5), 238-242

2731 Wang, L.,Mamudu, H. M.,Alamian, A.,Anderson, J. L.,Brooks, B. (2014). Independent and joint effects of prenatal maternal smoking Intervention/exposure and maternal exposure to second-hand smoke on the development of adolescent obesity: a longitudinal study J Paediatr Child Health, 50(11), 908-15

2732 Wang, X.,Xing, K. H.,Qi, J.,Guan, Y.,Zhang, J. (2013). Analysis of the relationship of insulin-like growth factor-1 to the growth velocity and feeding of healthy infants Growth Horm IGF Res, 23(6), 215-9

2733 Wang, Y. F.,Ou-Yang, Q.,Xia, B.,Liu, L. N.,Gu, F.,Zhou, K. F.,Mei, Q.,Shi, R. H.,Ran, Z. H.,Wang, X. D.,Hu, P. J.,Wu, K. C.,Liu, X. G.,Miao, Y. L.,Han, Y.,Wu, X. P.,He, G. B.,Zhong, J.,Liu, G. J. (2013). Multicenter case-control study of the risk factors for ulcerative colitis in China World J Gastroenterol, 19(11), 1827-33

2734 Wang, Y. S.,Shen, Y. H.,Wang, J. J.,Yang, M. J.,Ding, S. W.,Shi, Y. Y. (1994). Preliminary study on the blood glucose level in the exclusively breastfed newborn J Trop Pediatr, 40(3), 187-8

2735 Wang, Y. S.,Wu, S. Y. (1996). The effect of exclusive breastfeeding on development and incidence of infection in infants J Hum Lact, 12(1), 27-30

2736 Wang, Y.,Wang, A.,Donovan, S. M.,Teran-Garcia, M. (2013). Individual genetic variations related to satiety and appetite control increase risk of obesity in preschool-age children in the STRONG kids program Hum Hered, 75(2-4), 152-9

2737 Wang, Y.,Zhang, Z.,Ge, P.,Wang, Y.,Wang, S. (2009). lodine status and thyroid function of pregnant, lactating women and infants (0-1 yr) residing in areas with an effective Universal Salt lodization program Asia Pac J Clin Nutr, 18(1), 34-40

2738 Warner, J. O. (1980). Food allergy in fully breast-fed infants Clin Allergy, 10(2), 133-6

2739 Warren, J. J.,Bishara, S. E. (2002). Duration of nutritive and nonnutritive sucking behaviors and their effects on the dental arches in the primary dentition Am J Orthod Dentofacial Orthop, 121(4), 347-56

2740 Warrington, S.,Storey, D. M. (1988). Comparative studies on Asian and Caucasian children. 2: Nutrition, feeding practices and health Eur J Clin Nutr, 42(1), 69-79

Outcome

Outcome

Intervention/exposure

Intervention/exposure

Study design,

Intervention/exposure

Study design,

Intervention/exposure

Study design

Size of study groups

Study design,

Intervention/exposure

274

Watase, S.,Mourino, A. P.,Tipton, G. A. (1998). An analysis of malocclusion in children with otitis media Pediatr Dent, 20(5), 327-30 Study design 
2742 Watkinson, M. (1981). Delayed onset of weanling diarrhoea associated with high breast milk intake Trans R Soc Trop Med Hyg 75(3), 432-5

2743 Watson, E.,Gardner, A.,Carpenter, R. G. (1981). An epidemiological and sociological study of unexpected death in infancy in nine areas of southern England. I: Epidemiology Med Sci Law, 21(2), 78-88

2744 Watson, P. E.,McDonald, B. W. (2013). Subcutaneous body fat in pregnant New Zealand women: association with wheeze in their Study design infants at 18 months Matern Child Health J, 17(5), 959-67

2745 Waylen, A.,Ford, T.,Goodman, R.,Samara, M.,Wolke, D. (2009). Can early intake of dietary omega-3 predict childhood externalizing Outcome behaviour? Acta Paediatr, 98(11), 1805-8

2746 Weber, F.,Woolridge, M. W.,Baum, J. D. (1986). An ultrasonographic study of the organisation of sucking and swallowing by newborn infants Dev Med Child Neurol, 28(1), 19-24

2747 Weber, M.,Grote, V.,Closa-Monasterolo, R.,Escribano, J.,Langhendries, J. P.,Dain, E.,Giovannini, M.,Verduci, E.,Gruszfeld, D.,Socha, P.,Koletzko, B. (2014). Lower protein content in infant formula reduces BMl and obesity risk at school age: follow-up of a randomized trial Am J Clin Nutr, 99(5), 1041-51

2748 Weden, M. M.,Brownell, P.,Rendall, M. S. (2012). Prenatal, perinatal, early life, and sociodemographic factors underlying racial differences in the likelihood of high body mass index in early childhood Am J Public Health, 102(11), 2057-67

2749 Weerheijm, K. L.,Uyttendaele-Speybrouck, B. F.,Euwe, H. C., Groen, H. J. (1998). Prolonged demand breast-feeding and nursing caries Caries Res, 32(1), 46-50

2750 Weggemann, T.,Brown, J. K.,Fulford, G. E.,Minns, R. A. (1987). A study of normal baby movements Child Care Health Dev, 13(1), $41-58$

2751 Wegienka, G.,Ownby, D. R.,Havstad, S.,Williams, L. K.,Johnson, C. C. (2006). Breastfeeding history and childhood allergic status in Outcome a prospective birth cohort Ann Allergy Asthma Immunol, 97(1), 78-83

2752 Wehby, G. L. (2014). Breastfeeding and child disability: a comparison of siblings from the United States Econ Hum Biol, 15(\#issue\#), 13-22

2753 Weijs, P. J.,Kool, L. M.,van Baar, N. M.,van der Zee, S. C. (2011). High beverage sugar as well as high animal protein intake at infancy may increase overweight risk at 8 years: a prospective longitudinal pilot study Nutr J, 10(\#issue\#), 95

2754 Weile, B.,Cavell, B.,Nivenius, K.,Krasilnikoff, P. A. (1995). Striking differences in the incidence of childhood celiac disease between Denmark and Sweden: a plausible explanation J Pediatr Gastroenterol Nutr, 21(1), 64-8

Outcome, Size of study groups

Intervention/exposure

Intervention/exposure

Study design

Size of study groups

Outcome

Study design

Study design

Intervention/exposure,

Participant health

2755 Weinstein, P.,Domoto, P.,Wohlers, K.,Koday, M. (1992). Mexican-American parents with children at risk for baby bottle tooth decay: $\quad$ Study design pilot study at a migrant farmworkers clinic ASDC J Dent Child, 59(5), 376-83 caloric intake and length of hospital stay for infants with bronchiolitis Hosp Pediatr, 3(1), 24-30

Participant health 
2757 Welander, A.,Montgomery, S. M.,Ludvigsson, J.,Ludvigsson, J. F. (2014). Infectious disease at gluten introduction and risk of childhood diabetes mellitus J Pediatr, 165(2), 326-331 e1

2758 Welander, A.,Tjernberg, A. R.,Montgomery, S. M.,Ludvigsson, J.,Ludvigsson, J. F. (2010). Infectious disease and risk of later celiac Outcome disease in childhood Pediatrics, 125(3), e530-6

2759 Welch, K. R.,Ariza, A. J.,Wieczorek, J. L.,Binns, H. J. (2008). Characteristics of obese children aged 1-4 years at a referral clinic J Study design Natl Med Assoc, 100(8), 884-91

2760 Welford H (1995). Breastfeeding: promoting good practice Mod Midwife, 5(\#issue\#), 29-30

Study design

2761 Weller, B. F. (1988). When is breast best? Nurs Stand, 3(11), 34-5

Study design

2762 Welliver, R. C.,Wong, D. T.,Sun, M.,McCarthy, N. (1986). Parainfluenza virus bronchiolitis. Epidemiology and pathogenesis Am J Outcome Dis Child, 140(1), 34-40

2763 Wells, J. C.,Jonsdottir, O. H.,Hibberd, P. L.,Fewtrell, M. S., Thorsdottir, I.,Eaton, S., Lucas, A.,Gunnlaugsson, G.,Kleinman, R. E. (2012). Randomized controlled trial of 4 compared with 6 mo of exclusive breastfeeding in Iceland: differences in breast-milk intake by stable-isotope probe Am J Clin Nutr, 96(1), 73-9

2764 Wells, J. C.,Stanley, M.,Laidlaw, A. S.,Day, J. M.,Davies, P. S. (1998). Energy intake in early infancy and childhood fatness Int J Size of study groups Obes Relat Metab Disord, 22(5), 387-92

2765 Wen, L. M.,Baur, L. A.,Rissel, C.,Simpson, J. M. (2011). A randomized controlled trial of an early intervention on childhood obesity:

Publication status Results from the first 12 months Obesity (Silver Spring, Md.), 19(\#issue\#), S67

2766 Wen, L. M.,Baur, L. A.,Rissel, C.,Xu, H.,Simpson, J. M. (2014). Correlates of body mass index and overweight and obesity of children aged 2 years: findings from the healthy beginnings trial Obesity (Silver Spring), 22(7), 1723-30

2767 Wen, L. M.,Baur, L. A.,Simpson, J. M.,Rissel, C.,Wardle, K.,Flood, V. M. (2013). Healthy beginnings trial: The journey from the beginning Obesity research \& clinical practice, 7(\#issue\#), e2

2768 Wen, X.,Kong, K. L.,Eiden, R. D.,Sharma, N. N.,Xie, C. (2014). Sociodemographic differences and infant dietary patterns Pediatrics, Intervention/exposure 134(5), e1387-98

2769 Wen, X.,Shenassa, E. D.,Paradis, A. D. (2013). Maternal smoking, breastfeeding, and risk of childhood overweight: findings from a Intervention/exposure national cohort Matern Child Health J, 17(4), 746-55

2770 Werneck, R. I.,Lawrence, H. P.,Kulkarni, G. V.,Locker, D. (2008). Early childhood caries and access to dental care among children Study design of Portuguese-speaking immigrants in the city of Toronto J Can Dent Assoc, 74(9), 805

2771 Weston, J. (1986). Bottle feeding Nursing (Lond), 3(2), 61-2

Study design

2772 Wetzig, H.,Schulz, R.,Diez, U.,Herbarth, O.,Viehweg, B.,Borte, M. (2000). Associations between duration of breast-feeding, sensitization to hens' eggs and eczema infantum in one and two year old children at high risk of atopy Int $\mathrm{J}$ Hyg Environ Health, 203(1), 17-21 
2773 Weyermann, M.,Brenner, H.,Rothenbacher, D. (2007). Adipokines in human milk and risk of overweight in early childhood: a prospective cohort study Epidemiology, 18(6), 722-9

Publication date for a non-sibling study

2774 Weyermann, M.,Rothenbacher, D.,Brenner, H. (2006). Duration of breastfeeding and risk of overweight in childhood: a prospective birth cohort study from Germany Int J Obes (Lond), 30(8), 1281-7

Publication date for a non-sibling study

2775 Wheeler, B. J.,Dickson, N. P.,Houghton, L. A.,Ward, L. M.,Taylor, B. J. (2015). Incidence and characteristics of vitamin D deficiency rickets in New Zealand children: a New Zealand Paediatric Surveillance Unit study Aust N Z J Public Health, 39(4), 380-3

Study design

Intervention/exposure

2776 While A (1985). Infant feeding. Breast versus bottle Nurs Mirror, 160(\#issue\#), 30-4

Study design

2777 White, C. (2000). Breast milk is still a winning formula, says study Nursing Times, 96(11), 12-12 1p

Study design

2778 White, V. (2008). Breastfeeding and the risk of early childhood caries Evid Based Dent, 9(3), 86-8

Study design

2779 Whitehead, R. G. (1983). Nutritional aspects of human lactation Lancet, 1(8317), 167-9

Study design

2780 Whitehead, R. G. (1985). Infant physiology, nutritional requirements, and lactational adequacy Am J Clin Nutr, 41(2 Suppl), 447-58

Study design,

Intervention/exposure

2781 Whitehead, R. G.,Paul, A. A. (1981). Infant growth and human milk requirements. A fresh approach Lancet, 2(8239), 161-3

Size of study groups,

Intervention/exposure

2782 Whitehead, R. G.,Paul, A. A.,Ahmed, E. A. (1986). Weaning practices in the United Kingdom and variations in anthropometric development Acta Paediatr Scand Suppl, 323(\#issue\#), 14-23

2783 Whitehouse, A. J.,Robinson, M.,Li, J.,Oddy, W. H. (2011). Duration of breast feeding and language ability in middle childhood Paediatr Perinat Epidemiol, 25(1), 44-52

Size of study groups

Outcome

Whitley, E.,Gunnell, D.,Davey Smith, G.,Holly, J. M.,Martin, R. M. (2008). Childhood circumstances and anthropometry: the Boyd Orr cohort Ann Hum Biol, 35(5), 518-34

2785 Whitley, E.,Martin, R. M.,Davey Smith, G.,Holly, J. M.,Gunnell, D. (2012). The association of childhood height, leg length and other measures of skeletal growth with adult cardiovascular disease: the Boyd-Orr cohort J Epidemiol Community Health, 66(1), 18-23

2786 Whu, R.,Cirilo, G.,Wong, J.,Finkel, M. L.,Mendez, H. A.,Leggiadro, R. J. (2007). Risk factors for pediatric asthma in the South Bronx J Asthma, 44(10), 855-9

2787 Wi, C. I.,Park, M. A.,Juhn, Y. J. (2015). Development and initial testing of Asthma Predictive Index for a retrospective study: an exploratory study J Asthma, 52(2), 183-90

2788 Wiberger, M.,Eiben, G.,Lissner, L.,Mehlig, K.,Papoutsou, S.,Hunsberger, M. (2014). Children consuming milk cereal drink are at increased risk for overweight: The IDEFICS Sweden study, on behalf of the IDEFICS Consortium Scand J Public Health, 42(6), 51824

2789 Wickens, K.,Black, P.,Stanley, T. V.,Mitchell, E.,Barthow, C.,Fitzharris, P. (2012). A protective effect of Lactobacillus rhamnosus HN001 against eczema in the first 2 years of life persists to age 4 years Clinical and Experimental Allergy, 42(7), 1071-9

Study design

Intervention/exposure

Size of study groups Intervention/exposure

Study design, Size of study groups

Intervention/exposure 
2790 Wickman, M.,Melen, E.,Berglind, N.,Lennart Nordvall, S.,Almqvist, C.,Kull, I.,Svartengren, M.,Pershagen, G. (2003). Strategies for preventing wheezing and asthma in small children Allergy, 58(8), 742-7

2791 Wigg, N. R.,Tong, S.,McMichael, A. J.,Baghurst, P. A.,Vimpani, G.,Roberts, R. (1998). Does breastfeeding at six months predict cognitive development? Aust N Z J Public Health, 22(2), 232-6

2792 Wijga, A. H.,Scholtens, S.,Bemelmans, W. J. E.,Kerkhof, M.,Koppelman, G. H.,Brunekreef, B.,Smit, H. A. (2010). Diet, screen time, physical activity, and childhood overweight in the general population and in high risk subgroups: prospective analyses in the PIAMA birth cohort Journal of Obesity, \#volume\#(\#issue\#), 9p-9p $1 p$

2793 Willatts, P.,Forsyth, S.,Agostoni, C.,Casaer, P.,Riva, E.,Boehm, G. (2013). Effects of long-chain PUFA supplementation in infant formula on cognitive function in later childhood Am J Clin Nutr, 98(2), 536S-42S

2794 Williams, C.,Birch, E. E.,Emmett, P. M.,Northstone, K. (2001). Stereoacuity at age 3.5 y in children born full-term is associated with Outcome prenatal and postnatal dietary factors: a report from a population-based cohort study Am J Clin Nutr, 73(2), 316-22

2795 Williams, D. M.,Martin, R. M.,Davey Smith, G.,Alberti, K. G.,Ben-Shlomo, Y.,McCarthy, A. (2012). Associations of infant nutrition with insulin resistance measures in early adulthood: evidence from the Barry-Caerphilly Growth (BCG) study PLoS One, 7(3), e34161

2796 Williams, S. A.,Hargreaves, J. A. (1990). An inquiry into the effects of health related behaviour on dental health among young Asian Study design children resident in a fluoridated city in Canada Community Dent Health, 7(4), 413-20

2797 Williams, S. M.,Taylor, B. J.,Ford, R. P.,Nelson, E. A. (1990). Growth velocity before sudden infant death Arch Dis Child, 65(12), Intervention/exposure $1315-8$

2798 Williams, S. M.,Taylor, B. J.,Mitchell, E. A.,Scragg, R.,Ford, R. P.,Stewart, A. W. (1995). Sudden infant death syndrome in New Zealand: are risk scores useful? New Zealand National Cot Death Study Group J Epidemiol Community Health, 49(1), 94-101

2799 Williams, S. M.,Taylor, R. W.,Taylor, B. J. (2013). Secular changes in BMI and the associations between risk factors and BMI in children born 29 years apart Pediatr Obes, 8(1), 21-30

2800 Williamson, E.,Morley, R.,Lucas, A.,Carpenter, J. (2012). Propensity scores: from naive enthusiasm to intuitive understanding Stat Methods Med Res, 21(3), 273-93

2801 Williamson, I. G.,Dunleavey, J.,Robinson, D. (1994). Risk factors in otitis media with effusion. A 1 year case control study in 5-7 year Study design old children Fam Pract, 11(3), 271-4

2802 Willows, N. D.,Dewailly, E.,Gray-Donald, K. (2000). Anemia and iron status in Inuit infants from northern Quebec Can J Public Health, 91(6), 407-10

2803 Wilson, A. C.,Forsyth, J. S.,Greene, S. A., Irvine, L.,Hau, C.,Howie, P. W. (1998). Relation of infant diet to childhood health: seven year follow up of cohort of children in Dundee infant feeding study BMJ, 316(7124), 21-5

Outcome

Intervention/exposure

Study design, Participant health

Study design

Intervention/exposure

Publication date for a non-sibling study

2804 Wilson, C. E. (2000). Cree infant care practices and sudden infant death syndrome Can J Public Health, 91(2), 133-6

Study design, Outcome 
2805 Wingard, D. L.,Criqui, M. H.,Edelstein, S. L.,Tucker, J.,Tomlinson-Keasey, C.,Schwartz, J. E.,Friedman, H. S. (1994). Is breastfeeding in infancy associated with adult longevity? Am J Public Health, 84(9), 1458-62

2806 Winkler, C.,Hummel, S.,Pfluger, M.,Ziegler, A. G.,Geppert, J.,Demmelmair, H.,Koletzko, B. (2008). The effect of maternal T1DM on the fatty acid composition of erythrocyte phosphatidylcholine and phosphatidylethanolamine in infants during early life Eur $\mathrm{J}$ Nutr, $47(3), 145-52$

2807 Wolman, P. G. (1984). Feeding practices in infancy and prevalence of obesity in preschool children J Am Diet Assoc, 84(4), 436-8

Publication date for a non-sibling study

2808 Wong, H. B. (1982). Child health in Singapore--past, present and future Ann Acad Med Singapore, 11(3), $322-35$

Study design

2809 Wong, W. W.,Hachey, D. L.,Insull, W.,Opekun, A. R., Klein, P. D. (1993). Effect of dietary cholesterol on cholesterol synthesis in breast-fed and formula-fed infants J Lipid Res, 34(8), 1403-11

2810 Woo, J. G.,Guerrero, M. L.,Ruiz-Palacios, G. M.,Peng, Y. M.,Herbers, P. M.,Yao, W.,Ortega, H.,Davidson, B. S.,McMahon, R. J.,Morrow, A. L. (2013). Specific infant feeding practices do not consistently explain variation in anthropometry at age 1 year in urban United States, Mexico, and China cohorts J Nutr, 143(2), 166-74

2811 Wood, C. S.,Isaacs, P. C.,Jensen, M.,Hilton, H. G. (1988). Exclusively breast-fed infants: growth and caloric intake Pediatr Nurs $14(2), 117-24$

2812 Wood, R.,Stockton, D.,Brown, H. (2013). Moving from a universal to targeted child health programme: which children receive enhanced care? A population-based study using routinely available data Child Care Health Dev, 39(6), 772-81 2813 Woodward, A.,Douglas, R. M.,Graham, N. M.,Miles, H. (1990). Acute respiratory illness in Adelaide children: breast feeding modifies Outcome
the effect of passive smoking J Epidemiol Community Health, 44(3), 224-30

2814 Worobey, J. (1993). Effects of feeding method on infant temperament Adv Child Dev Behav, 24(\#issue\#), 37-61

Study design

2815 Wray, J. (2008). Breastfeeding and primitive neonatal reflexes Pract Midwife, 11(5), 53-6

2816 Wright Mda, G.,Dutra de Oliveira, J. E. (1986). Is breast feeding the solution to the infant nutrition problem in underdeveloped countries? Child Care Health Dev, 12(6), 359-68

2817 Wright, A. L.,Bauer, M.,Naylor, A.,Sutcliffe, E.,Clark, L. (1998). Increasing breastfeeding rates to reduce infant illness at the community level Pediatrics, 101(5), 837-44

2818 Wright, A. L.,Holberg, C. J.,Martinez, F. D.,Morgan, W. J.,Taussig, L. M. (1989). Breast feeding and lower respiratory tract illness in Outcome the first year of life. Group Health Medical Associates BMJ, 299(6705), 946-9

2819 Wright, A. L.,Holberg, C. J.,Taussig, L. M.,Martinez, F. (2000). Maternal asthma status alters relation of infant feeding to asthma in Intervention/exposure childhood Adv Exp Med Biol, 478(\#issue\#), 131-7

2820 Wright, A. L.,Holberg, C. J.,Taussig, L. M.,Martinez, F. D. (1995). Relationship of infant feeding to recurrent wheezing at age 6 years Outcome Arch Pediatr Adolesc Med, 149(7), 758-63 
2821 Wright, A. L.,Holberg, C. J.,Taussig, L. M.,Martinez, F. D. (2001). Factors influencing the relation of infant feeding to asthma and recurrent wheeze in childhood Thorax, 56(3), 192-7

2822 Wright, A. L.,Stern, D. A.,Halonen, M. (2001). The association of allergic sensitization in mother and child in breast-fed and formula- $\quad$ Outcome fed infants Adv Exp Med Biol, 501(\#issue\#), 249-55

2823 Wright, C. J.,Atkinson, F. S.,Ramalingam, N.,Buyken, A. E.,Brand-Miller, J. C. (2015). Effects of human milk and formula on postprandial glycaemia and insulinaemia Eur J Clin Nutr, 69(8), 939-43

2824 Wright, C. M.,Parkinson, K.,Scott, J. (2006). Breast-feeding in a UK urban context: who breast-feeds, for how long and does it matter? Public Health Nutr, 9(6), 686-91

articipant age

Publication date for a non-sibling study

2825 Wright, C. M.,Stone, D. H.,Parkinson, K. N. (2010). Undernutrition in British Haredi infants within the Gateshead Millennium cohort study Arch Dis Child, 95(8), 630-3

Publication date for a non-sibling study

2826 Wright, C.,Lakshman, R.,Emmett, P.,Ong, K. K. (2008). Implications of adopting the WHO 2006 Child Growth Standard in the UK: two prospective cohort studies Arch Dis Child, 93(7), 566-9

2827 Wright, P. (1981). Development of feeding behaviour in early infancy: implications for obesity Health Bull (Edinb), 39(3), 197-205

Intervention/exposure

Study design,

Intervention/exposure

2828 Wu, T. C.,Huang, I. F.,Chen, Y. C.,Chen, P. H.,Yang, L. Y. (2011). Differences in serum biochemistry between breast-fed and formula-fed infants J Chin Med Assoc, 74(11), 511-5

Outcome

2829 Wu, T. C.,Hwang, B. (1997). Blood nutrient indices in breast and formula fed infants: amino acids metabolic responses Zhonghua Min Guo Xiao Er Ke Yi Xue Hui Za Zhi, 38(5), 345-51

2830 Wyne, A. H.,Adenubi, J. O.,Shalan, T.,Khan, N. (1995). Feeding and socioeconomic characteristics of nursing caries children in a Study design Saudi population Pediatr Dent, 17(7), 451-4

2831 Xenellis, J.,Paschalidis, J.,Georgalas, C.,Davilis, D.,Tzagaroulakis, A.,Ferekidis, E. (2005). Factors influencing the presence of otitis Participant health media with effusion 16 months after initial diagnosis in a cohort of school-age children in rural Greece: a prospective study Int $J$ Pediatr Otorhinolaryngol, 69(12), 1641-7

2832 Xie, L. L.,Jiang, L. (2014). Arterial ischemic stroke and hemorrhagic stroke in Chinese children: a retrospective analysis Brain Dev $36(2), 153-8$

2833 Yadav, M.,Akobeng, A. K.,Thomas, A. G. (2000). Breast-feeding and childhood obesity J Pediatr Gastroenterol Nutr, 30(3), 345-6

Participant health, Outcomes Yakubov, R.,Nadir, E.,Stein, R.,Klein-Kremer, A. (2015). The Duration of Breastfeeding and Its Association with Metabolic

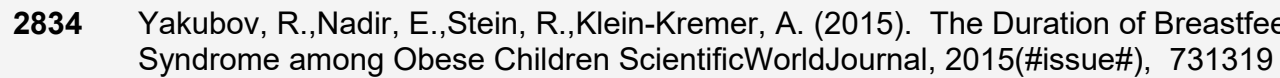

2835 Yalcin, S. S.,Hizli, S.,Yurdakok, K.,Ozmert, E. (2005). Risk factors for hospitalization in children with acute diarrhea: a case control study Turk J Pediatr, 47(4), 339-42 
2836 Yalcin, S. S.,Turul, B.,Cetinkaya, S.,Cakir, B.,Yilmaz, A. (2004). Effect of total attending period on infection episode rate in a childcare center Pediatr Int, 46(5), 555-60

2837 Yamakawa, M.,Yorifuji, T.,Inoue, S.,Kato, T.,Doi, H. (2013). Breastfeeding and obesity among schoolchildren: a nationwide longitudinal survey in Japan JAMA Pediatr, 167(10), 919-25

2838 Yamakawa, M.,Yorifuji, T.,Kato, T.,Inoue, S.,Tokinobu, A.,Tsuda, T.,Doi, H. (2015). Long-Term Effects of Breastfeeding on Children's Hospitalization for Respiratory Tract Infections and Diarrhea in Early Childhood in Japan Matern Child Health J, 19(9), 1956-65

2839 Yamakawa, M.,Yorifuji, T.,Kato, T.,Yamauchi, Y.,Doi, H. (2015). Breast-feeding and hospitalization for asthma in early childhood: a Intervention/exposure nationwide longitudinal survey in Japan Public Health Nutr, 18(10), 1756-61

2840 Yamauchi, Y.,Yamanouchi, I. (1990). The relationship between rooming-in/not rooming-in and breast-feeding variables Acta Paediatr Scand, 79(11), 1017-22

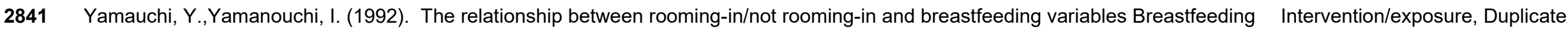
Review, 2(5), 238-241 4p

2842 Yamborisut, U.,Kosulwat, V.,Chittchang, U.,Wimonpeerapattana, W.,Suthutvoravut, U. (2006). Factors associated with dual form of malnutrition in school children in Nakhon Pathom and Bangkok J Med Assoc Thai, 89(7), 1012-23

2843 Yang, S.,Fombonne, E.,Kramer, M. S. (2011). Duration of gestation, size at birth and later childhood behaviour Paediatr Perinat Epidemiol, 25(4), 377-87

2844 Yang, S.,Platt, R. W.,Dahhou, M.,Kramer, M. S. (2014). Do population-based interventions widen or narrow socioeconomic inequalities? The case of breastfeeding promotion Int J Epidemiol, 43(4), 1284-92

2845 Ye, M.,Mandhane, P. J.,Senthilselvan, A. (2012). Association of breastfeeding with asthma in young Aboriginal children in Canada Can Respir J, 19(6), 361-6

2846 Ye, W.,Feng, X. P.,Liu, Y. L. (1999). Epidemiological study of the risk factors of rampant caries in Shanghai children Chin J Dent Res, 2(2), 58-62

2847 Yeung, D. L.,Pennell, M. D.,Leung, M.,Hall, J. (1981). Infant fatness and feeding practices: a longitudinal assessment J Am Diet Assoc, 79(5), 531-5

2848 Yeung, K. A., Taylor, T.,Scheimann, A.,Carvalho, R.,Reinhardt, E.,Girolami, P.,Wood, R. (2015). The Prevalence of Food Allergies in Children Referred to a Multidisciplinary Feeding Program Clin Pediatr (Phila), 54(11), 1081-6

2849 Yi, M. J.,Sun, D. F.,Zhou, X. B. (2003). Relationship between infant breast feeding and simple obesity in preschool children: A case- Study design control study Chinese Journal of Clinical Rehabilitation, 7(30), 4088-4089

2850 Yi, M. J.,Sun, M. H.,Liu, F.,Liu, Y. (2007). Association between infant breastfeeding and temperamental characteristics development Study design in children aged 4-5 years Journal of Clinical Rehabilitative Tissue Engineering Research, 11(30), 6100-6102

Publication date for a non-sibling study

Participant health

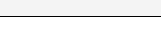


2851 Yildirim, S,,Binnetoğlu, F. K.,Aylanç, H.,Battal, F.,Tekin, M.,Kaymaz, N.,Topaloğlu, N.,A§lk, Z. (2015). Effect of infant feeding on epicardial fat thickness in normal weighted children Anatolian Journal of Clinical Investigation, 9(3), 92-97

2852 Yimyaem, P.,Chongsrisawat, V.,Vivatvakin, B.,Wisedopas, N. (2003). Gastrointestinal manifestations of cow's milk protein allergy during the first year of life J Med Assoc Thai, 86(2), 116-23

2853 Yin, J.,Quinn, S.,Dwyer, T.,Ponsonby, A. L.,Jones, G. (2012). Maternal diet, breastfeeding and adolescent body composition: a 16year prospective study Eur J Clin Nutr, 66(12), 1329-34

2854 Yip R,Parvanta I,Scanlon K,Borland EW,Russell CM,Trowbridge FL (1992). Pediatric nutrition surveillance system--United States, 1980-1991 MMWR CDC Surveill Summ, 41(\#issue\#), 1-24

2855 Yiş, U.,Öztürk, Y.,Şişman, A. R.,Uysal, S.,Soylu Ö, B.,Büyükgebiz, B. (2010). The relation of serum ghrelin, leptin and insulin levels to the growth patterns and feeding characteristics in breast-fed versus formula-fed infants Turkish Journal of Pediatrics, 52(1), 35-41

2856 Yoneyama, K.,Nagata, H.,Asano, H. (1994). Growth of Japanese breast-fed and bottle-fed infants from birth to 20 months Ann Hum Biol, 21(6), 597-608

2857 Yonezu, T.,Ushida, N.,Yakushiji, M. (2006). Longitudinal study of prolonged breast- or bottle-feeding on dental caries in Japanese children Bull Tokyo Dent Coll, 47(4), 157-60

2858 Yonezu, T.,Yotsuya, K.,Yakushiji, M. (2006). Characteristics of breast-fed children with nursing caries Bull Tokyo Dent Coll, 47(4), $161-5$

2859 Yoon, H. S., Shin, Y. J., Ki, M. (2008). Risk factors for neonatal infections in full-term babies in South Korea Yonsei Medical 49(4), 530-536

2860 Yorifuji, J.,Yorifuji, T., Tachibana, K.,Nagai, S.,Kawai, M.,Momoi, T.,Nagasaka, H.,Hatayama, H.,Nakahata, T. (2008). Craniotabes in Intervention/exposure normal newborns: the earliest sign of subclinical vitamin D deficiency J Clin Endocrinol Metab, 93(5), 1784-8 nationwide longitudinal survey in Japan J Pediatr, 164(5), 1019-1025 e3

2862 Yorifuji, T.,Murata, K.,Bjerve, K. S.,Choi, A. L.,Weihe, P.,Grandjean, P. (2013). Visual evoked potentials in children prenatally exposed to methylmercury Neurotoxicology, 37(\#issue\#), 15-8

2863 Young, H. B.,Buckley, A. E.,Hamza, B.,Mandarano, C. (1982). Milk and lactation: some social and developmental correlates among 1,000 infants Pediatrics, 69(2), 169-75

2864 Young, R. J.,Antonson, D. L.,Ferguson, P. W.,Murray, N. D.,Merkel, K.,Moore, T. E. (2005). Neonatal and infant feeding: effect on bone density at 4 years $\mathrm{J}$ Pediatr Gastroenterol Nutr, 41(1), 88-93

2865 Young, S.,O'Keeffe, P. T.,Arnott, J.,Landau, L. I. (1995). Lung function, airway responsiveness, and respiratory symptoms before and after bronchiolitis Arch Dis Child, 72(1), 16-24

Intervention/exposure, Outcome

Publication status

Size of study groups

Intervention/exposure

Outcome

Study design

intervention/exposure

Outcome

Outcome

Intervention/exposure

Size of study groups Intervention/exposure

Publication date for a non-sibling study

Study design,

Intervention/exposure, Size of study groups 
2866 Young, T. K.,Martens, P. J.,Taback, S. P.,Sellers, E. A.,Dean, H. J.,Cheang, M.,Flett, B. (2002). Type 2 diabetes mellitus in children: Outcome prenatal and early infancy risk factors among native canadians Arch Pediatr Adolesc Med, 156(7), 651-5

2867 Yu, C.,Binns, C. W.,Lee, A. H. (2015). Comparison of breastfeeding rates and health outcomes for infants receiving care from hospital outpatient clinic and community health centres in China J Child Health Care, \#volume\#(\#issue\#), \#Pages\#

2868 Yu, L. X.,Tao, Y.,Qiu, R. M.,Zhou, Y.,Zhi, Q. H.,Lin, H. C. (2015). Genetic polymorphisms of the sortase A gene and socialbehavioural factors associated with caries in children: a case-control study BMC Oral Health, 15(\#issue\#), 54

2869 Yuksel, H.,Sakar, A.,Dinc, G.,Yilmaz, O.,Gozmen, S.,Yorgancioglu, A.,Ozcan, C. (2007). The frequency of wheezing phenotypes Study design and risk factors for persistence in aegean region of Turkey J Asthma, 44(2), 89-93

2870 Yung, J.,Yuen, J. W. M.,Ou, Y.,Loke, A. Y. (2015). Factors associated with atopy in toddlers: A case-control study Internationa Journal of Environmental Research and Public Health, 12(3), 2501-2520

2871 Yurdakok, K.,Ozmert, E.,Yalcin, S. S. (1997). Physical examination of breast-fed infants Arch Pediatr Adolesc Med, 151(4), 429-30

2872 Zadik, Z.,Borondukov, E.,Zung, A.,Reifen, R. (2003). Adult height and weight of breast-fed and bottle-fed Israeli infants J Pediatr Gastroenterol Nutr, 37(4), 462-7

2873 Zadzinska E,Sitek A,Rosset I (2016). Relationship between pre-natal factors, the perinatal environment, motor development in the Study design first year of life and the timing of first deciduous tooth emergence Ann Hum Biol, 43(\#issue\#), 25-33

2874 Zaini, M. Z.,Lim, C. T.,Low, W. Y.,Harun, F. (2005). Factors affecting nutritional status of Malaysian primary school children Asia Pac Study design J Public Health, 17(2), 71-80

2875 Zamboni, G.,Piemonte, G.,Bolner, A.,Antoniazzi, F.,Dall'Agnola, A.,Messner, H.,Gambaro, G., Tato, L. (1993). Influence of dietary taurine on vitamin D absorption Acta Paediatrica, International Journal of Paediatrics, 82(10), 811-815

2876 Zamora, G.,Lutter, C. K.,Pena-Rosas, J. P. (2015). Using an equity lens in the implementation of interventions to protect, promote, and support optimal breastfeeding practices J Hum Lact, 31(1), 21-5

2877 Zarnani, A. H.,Modarres, Sh,Jadali, F.,Sabahi, F.,Moazzeni, S. M.,Vazirian, F. (2004). Role of rotaviruses in children with acute diarrhea in Tehran, Iran Journal of Clinical Virology, 29(3), 189-193

2878 Zedan, M.,Nasef, N.,El-Bayoumy, M.,El-Assmy, M.,Attia, G.,Zedan, M.,AlWakeel, A.,Kandil, S.,Laimon, W.,Fouda, A. (2012). Does decline of lung function in wheezy infants justify the early start of controller medications? Indian J Pediatr, 79(9), 1176-80

2879 Zell, B. L. (2011). Breastfeeding as a community health imperative Breastfeed Med, 6(\#issue\#), 303-4

2880 Zetterstrom, R. (1998). Human milk and infant development. Foreword Biol Neonate, 74(2), 80-3

2881 Zhang, J.,Himes, J. H.,Guo, Y.,Jiang, J.,Yang, L.,Lu, Q.,Ruan, H.,Shi, S. (2013). Birth weight, growth and feeding pattern in early infancy predict overweight/obesity status at two years of age: a birth cohort study of Chinese infants PLoS One, 8(6), e64542

2882 Zhang, J.,Jiang, J.,Himes, J. H.,Zhang, J.,Liu, G.,Huang, X., Guo, Y.,Shi, J.,Shi, S. (2012). Determinants of high weight gain and high BMI status in the first three months in urban Chinese infants Am J Hum Biol, 24(5), 633-9

Study design

Size of study groups

Study design, Outcome

Study design, Participant health

Country

Intervention/exposure

Outcome for a non-sibling study 
2883 Zhang, S.,Liu, J.,Lo, E. C.,Chu, C. H. (2013). Dental caries status of Dai preschool children in Yunnan Province, China BMC Oral Health, 13(\#issue\#), 68

2884 Zheng, J. S.,Liu, H.,Li, J.,Chen, Y.,Wei, C.,Shen, G.,Zhu, S.,Chen, H.,Zhao, Y. M.,Huang, T.,Li, D. (2014). Exclusive breastfeeding Intervention/exposure is inversely associated with risk of childhood overweight in a large Chinese cohort J Nutr, 144(9), 1454-9

2885 Zheng, W.,Suzuki, K.,Shinohara, R.,Sato, M.,Yokomichi, H.,Yamagata, Z. (2015). Maternal smoking during pregnancy and growth in Intervention/exposure infancy: a covariance structure analysis J Epidemiol, 25(1), 44-9

2886 Zhong, B. L.,Ding, J.,Chen, H. H.,Li, Y.,Xu, H. M.,Tong, J.,Wang, A. Q.,Tang, G. Z.,Zhu, J. S., Yang, D. Q.,Liu, B.,Wang, Q.,Cheng, W. F.,Yin, E.,Xu, M. J.,Zhang, T.,Hu, T. M.,Feng, X. W.,Li, H.,Dan, T. Q.,Cheng, G. M.,Zhang, J. F.,Li, H. J.,Zhu, J. H. (2013). Depressive disorders among children in the transforming China: an epidemiological survey of prevalence, correlates, and service use Depress Anxiety, 30(9), 881-92

2887 Zhou, S. J.,Baghurst, P.,Gibson, R. A.,Makrides, M. (2007). Home environment, not duration of breast-feeding, predicts intelligence Outcome quotient of children at four years Nutrition, 23(3), 236-41

2888 Zhou, S. J.,Sullivan, T.,Gibson, R. A.,Lonnerdal, B.,Prosser, C. G.,Lowry, D. J.,Makrides, M. (2014). Nutritional adequacy of goat milk infant formulas for term infants: a double-blind randomised controlled trial Br J Nutr, 111(9), 1641-51

2889 Zhou, S. J.,Sullivan, T.,Gibson, R. A.,Makrides, M. (2011). How does goat milk infant formula compare to cow milk formula? A randomised controlled trial [conference abstract] Journal of pediatric gastroenterology and nutrition, 52(\#issue\#), E208-e209

2890 Ziajka, S.,Zbikowski, Z. (1986). Characterization and properties of infant milk formulae with addition of enzymatically digested casein Nahrung, 30(3-4), 413-4

2891 Ziegler, A. G.,Schmid, S.,Huber, D.,Hummel, M.,Bonifacio, E. (2003). Early infant feeding and risk of developing type 1 diabetesassociated autoantibodies JAMA, 290(13), 1721-8

2892 Ziegler, E. E.,Fields, D. A.,Chernausek, S. D.,Steenhout, P.,Grathwohl, D.,Jeter, J. M.,Nelson, S. E.,Haschke, F. (2015). Adequacy of Infant Formula With Protein Content of $1.6 \mathrm{~g} / 100 \mathrm{kcal}$ for Infants Between 3 and 12 Months J Pediatr Gastroenterol Nutr, 61(5), 596-603

2893 Ziegler, E. E.,Hollis, B. W.,Nelson, S. E.,Jeter, J. M. (2006). Vitamin D deficiency in breastfed infants in lowa Pediatrics, 118(2), 603-10

2894 Ziegler, E. E.,Jiang, T.,Romero, E.,Vinco, A.,Frantz, J. A.,Nelson, S. E. (1999). Cow's milk and intestinal blood loss in late infancy J Pediatr, 135(6), 720-6

2895 Ziegler, E. E.,Nelson, S. E.,Jeter, J. M. (2014). Iron stores of breastfed infants during the first year of life Nutrients, 6(5), 2023-34

Intervention/exposure

2896 Ziegler, E.,Vanderhoof, J. A.,Petschow, B.,Mitmesser, S. H.,Stolz, S. I.,Harris, C. L.,Berseth, C. L. (2007). Term infants fed formula supplemented with selected blends of prebiotics grow normally and have soft stools similar to those reported for breast-fed infants $\mathrm{J}$ Pediatr Gastroenterol Nutr, 44(3), 359-64 
2897 Zielhuis, G. A.,Heuvelmans-Heinen, E. W.,Rach, G. H.,van den Broek, P. (1989). Environmental risk factors for otitis media with effusion in preschool children Scand J Prim Health Care, 7(1), 33-8

2898 Zive, M. M.,McKay, H.,Frank-Spohrer, G. C.,Broyles, S. L.,Nelson, J. A.,Nader, P. R. (1992). Infant-feeding practices and adiposity Study design in 4-y-old Anglo- and Mexican-Americans Am J Clin Nutr, 55(6), 1104-8

2899 Zollner, M. S.,Jorge, A. O. (2003). Candida spp. occurrence in oral cavities of breastfeeding infants and in their mothers' mouths and Study design breasts Pesqui Odontol Bras, 17(2), 151-5

2900 Zoppi, G.,Ferrarini, G.,Rigolin, F.,Bogaerts, H.,Andre, F. E. (1986). Response to RIT 4237 oral rotavirus vaccine in breast-fed and Size of study groups formula-fed infants Helv Paediatr Acta, 41(3), 203-8

2901 Zoppi, G.,Mantovanelli, F.,Gobio Casali, L.,Astolfi, R.,Cecchettin, M. (1986). Effects of the composition and caloric value of infant Size of study groups formulas on intake and hormone levels J Pediatr Gastroenterol Nutr, 5(5), 756-61

2902 Zuccotti, G.,Vigano, A.,Cafarelli, L.,Pivetti, V.,Pogliani, L.,Puzzovio, M.,Mora, S. (2011). Longitudinal changes of bone ultrasound measurements in healthy infants during the first year of life: influence of gender and type of feeding Calcif Tissue Int, 89(4), 312-7

2903 (1980). Nutritional adequacy of breast feeding Nutr Rev, 38(\#issue\#), 145-7

Size of study groups, Outcome

Publication status

2904 (1983). Breast-feeding and human milk Eur J Obstet Gynecol Reprod Biol, 15(4-6), 385-94

Publication status

2905 (1984). Bioavailability of milk zinc in infants Nutr Rev, 42(\#issue\#), 220-2

2906 (1984). Project report. Results and policy implications of the cross-national investigation: Rethinking Infant Nutrition Policies under

Publication status changing Socio-Economic Conditions Acta Paediatr Scand Suppl, 314(\#issue\#), 1-61

2907 (1985). Breast feeding and child development at five years Nutr Rev, 43(\#issue\#), 173-4

Publication status

2908 (1985). Current issues in feeding the normal infant Pediatrics, 75(1 Pt 2), 135-215

Publication status

2909 (1986). Allergy in your baby Aust Fam Physician, 15(2), 176, 178

Publication status

2910 (1986). Catch-up growth following severe malnutrition Nutr Rev, 44(5), 173-5

Publication status

2911 (1986). Significance of food hypersensitivity in children with atopic dermatitis Pediatr Dermatol, 3(2), 161-74

Publication status

2912 (1988). Breast versus bottle: an in-house debate Midwife Health Visit Community Nurse, 24(7), 254-5

Publication status

2913 (1988). Cow's milk allergy in the first year of life. An Italian Collaborative Study Acta Paediatr Scand Suppl, 348(\#issue\#), 1-14

Publication status

2914 (1988). Progress toward the 1990 objectives for improved nutrition MMWR Morb Mortal Wkly Rep, 37(\#issue\#), 475-9

Publication status

2915 (1989). American Academy of Pediatrics Committee on Nutrition: Follow-up or weaning formulas Pediatrics, 83(6), 1067

Publication status 
2918 (1993). Diarrhoeal disease control (CDD) and acute respiratory infections (ARI). Combined CDD/ARI/breast-feeding survey, 1992

Publication status Wkly Epidemiol Rec, 68(17), 120-2

2919 (1993). Diarrhoeal Disease Control (CDD) Programme Wkly Epidemiol Rec, 68(\#issue\#), 345-9

Publication status

2920 (1994). Dietary and other risk factors of ulcerative colitis. A case-control study in Japan. Epidemiology Group of the Research Committee of Inflammatory Bowel Disease in Japan J Clin Gastroenterol, 19(2), 166-71

2921 (1994). Infant feeding practices and their possible relationship to the etiology of diabetes mellitus. American Academy of Pediatrics Publication status Work Group on Cow's Milk Protein and Diabetes Mellitus Pediatrics, 94(5), 752-4

2922 (1997). Breast feeding: benefits and hazards Early Hum Dev, 49 Suppl(\#issue\#), S1-203

Publication status

2923 (1998). The Baby-Friendly Hospital Initiative Birth Gaz, 14(\#issue\#), 30

Publication status

2924 (1999). Breast feeding seems to reduce the risk of obesity in children Bmj, 319(7203), B

Publication status

2925 (1999). Exclusive breast feeding is protective against asthma and atopy in children Bmj, 319(7213), D

Publication status

2926 (1999). Protective effect of breast milk against pneumonia is greatest for young infants Bmj, 318(7194), C

Publication status

2927 (1999). Sudden infant death syndrome (SIDS). Canadian Foundation for the Study of Infant Deaths. Canadian Institute of Child Health. Canadian Paediatric Society Can Fam Physician, 45(\#issue\#), 702, 709-10

2928 (1999). Vitamin D supplement in early childhood and risk for Type I (insulin-dependent) diabetes mellitus. The EURODIAB Substudy 2 Study Group Diabetologia, 42(1), 51-4

2929 (2000). Growth patterns of breastfed infants in seven countries Acta Paediatr, 89(2), 215-22

\section{Publication status}

2930 (2001). Breastfeeding and childhood cancer Br J Cancer, 85(11), 1685-94

Outcome

2931 (2001). Controversial breastfeeding study Practising Midwife, 4(5), 6-6 1p

Publication status

2932 (2001). RC currents. Children breast-fed by asthmatic mothers at risk, says study AARC Times, 25(4), 70-70 1p

Publication status

2933 (2002). Rapid early growth is associated with increased risk of childhood type 1 diabetes in various European populations Diabete Care, 25(10), 1755-60

2934 (2004). Does breastfeeding prevent obesity?...and what about dairy foods? Child Health Alert, 22(\#issue\#), 3-4

Publication status

2935 (2004). Further evidence that breast is best RCM Midwives, \#volume\#(\#issue\#), 2-2 1p

Publication status

2936 (2006). Study hints at link between breastfeeding and intelligence AHRQ Research Activities, \#volume\#(308), 10-10 1p

Publication status

2937

(2008). Effects of breast-feeding: new results from a large randomised trial Journal of Family Health Care, 18(1), $34-34$ 1p

Publication status 
2938 (2008). POEMs. Breastfeeding does not decrease risk of asthma and allergy JAAPA: Journal of the American Academy of Physician Assistants (Haymarket Media, Inc.), 21(1), 66-66 1p

2939 (2009). Prolonged breast feeding reduces later cardiovascular risk Arch Dis Child, 94(11), 882

Publication status

2940 (2009). Promoting breast-feeding: fewer infections than in bottle-fed babies. Very few contraindications to breast-feeding Prescrire

Publication status international, 18(102), 178

\begin{tabular}{|c|c|c|}
\hline 2941 & (2011). ABM Clinical Protocol \#24: Allergic Proctocolitis in the Exclusively Breastfed Infant Breastfeed Med, 6(6), 435-40 & Publication status \\
\hline 2942 & (2011). Breastfeeding for the health of baby and mother Nurs J India, 102(8), 179 & Publication status \\
\hline 2943 & (2012). Breastfeeding study looks at behaviour Midwives, 15(1), 9-9 1p & Publication status \\
\hline 2944 & $\begin{array}{l}\text { (2012). UP11 The Feeding Young Children Study: Preliminary Results from a WIC-based Bottle Weaning Intervention Journal of } \\
\text { Nutrition Education \& Behavior, 44(4S1), S83-S83 1p }\end{array}$ & Publication status \\
\hline 2945 & (2013). Does breastfeeding increase risk of early childhood caries? J Can Dent Assoc, 79(\#issue\#), d123 & Publication status \\
\hline 2946 & (2013). Start smart: healthy weight in early childhood Issue Brief (Grantmakers Health), \#volume\#(\#issue\#), 1-14 & Publication status \\
\hline 2947 & (2015). Breastfeeding could be linked to higher IQ Perspect Public Health, 135(3), 114 & Publication status \\
\hline 2948 & (2015). Breastfeeding Nurs Womens Health, 19(1), 83-8 & Publication status \\
\hline 2949 & (2015). Breastfeeding: sensitive mothers and intelligent offspring Arch Dis Child, 100(6), 601 & Publication status \\
\hline 2950 & $\begin{array}{l}\text { (2015). Immediate Post-Partum Initiation of Etonogestrel-Releasing Implant: A Randomized Controlled Trial on Breastfeeding } \\
\text { Impact \#journal\#, 70(\#issue\#), 702-704 3p }\end{array}$ & Publication status \\
\hline 2951 & (2015). Study Looks at Breastfeeding Impact on Leukemia Neonatal Intensive Care, 28(4), 12-14 3p & Publication status \\
\hline 2952 & $\begin{array}{l}\text { (2015). The Optimal Duration of Exclusive Breastfeeding for Physical Growth Nutritional Perspectives: Journal of the Council on } \\
\text { Nutrition, 38(4), } 21-3311 \mathrm{p}\end{array}$ & Publication status \\
\hline
\end{tabular}


Table 13. Full-text exclusions from the update to the Pregnancy and Birth to 24 Months Project literature search

The table below lists the articles excluded after full-text screening of the update to the Pregnancy and Birth to 24 Months Project literature search. At least one reason for exclusion is provided for each article, though this may not reflect all possible reasons for exclusion. Information about articles excluded after title and abstract screening is available upon request.

Full-text article screened

Reason for exclusion

1 Abrahamse-Berkeveld, M.,Alles, M.,Franke-Beckmann, E.,Helm, K.,Knecht, R.,Kollges, R.,Sandner, B.,Knol, J.,Ben Amor, K.,Bufe, A. Intervention/exposure (2016). Infant formula containing galacto-and fructo-oligosaccharides and Bifidobacterium breve M-16V supports adequate growth and tolerance in healthy infants in a randomised, controlled, double-blind, prospective, multicentre study J Nutr Sci, 5(\#issue\#), e42

2 Abrams, E. M.,Greenhawt, M.,Fleischer, D. M.,Chan, E. S. (2017). Early Solid Food Introduction: Role in Food Allergy Prevention and Implications for Breastfeeding J Pediatr, 184(\#issue\#), 13-18

3 Adeyeye, T. E.,Yeung, E. H.,McLain, A. C.,Lin, S.,Lawrence, D. A.,Bell, E. M. (2019). Wheeze and Food Allergies in Children Born via Cesarean Delivery American Journal of Epidemiology, 188(2), 355-362

4 Adeyeye, T. E.,Yeung, E. H.,McLain, A. C.,Lin, S.,Lawrence, D. A.,Bell, E. M. (2019). Wheeze and Food Allergies in Children Born via Cesarean Delivery: The Upstate KIDS Study Am J Epidemiol, 188(2), 355-362

Publication status, Outcome

$5 \quad$ Aghajafari, F.,Field, C. J.,Weinberg, A. R.,Letourneau, N. (2018). Both Mother and Infant Require a Vitamin D Supplement to Ensure That Infants' Vitamin D Status Meets Current Guidelines Nutrients, 10(4), \#Pages\#

6 Ahrens, B.,Hellmuth, C.,Haiden, N.,Olbertz, D.,Hamelmann, E., Vusurovic, M.,Fleddermann, M.,Roehle, R.,Knoll, A.,Koletzko, B.,Wahn, U.,Beyer, K. (2018). Hydrolyzed Formula With Reduced Protein Content Supports Adequate Growth: A Randomized Controlled Noninferiority Trial J Pediatr Gastroenterol Nutr, 66(5), 822-830

7 Akkermans, M. D.,Eussen, S. R.,van der Horst-Graat, J. M.,van Elburg, R. M.,van Goudoever, J. B.,Brus, F. (2017). A micronutrientfortified young-child formula improves the iron and vitamin $D$ status of healthy young European children: a randomized, double-blind controlled trial Am J Clin Nutr, 105(2), 391-399

8 Alamian, A.,Wang, L.,Hall, A. M.,Pitts, M.,Ikekwere, J. (2016). Infant sleep problems and childhood overweight: Effects of three definitions of sleep problems Prev Med Rep, 4(\#issue\#), 463-8

9 Albaum, J. M.,Carsley, S.,Chen, Y.,Dai, D. W. H.,Lebovic, G.,McCrindle, B. W.,Maguire, J. L.,Parkin, P. C.,Birken, C. S. (2017). Persistent High Non-High-Density Lipoprotein Cholesterol in Early Childhood: A Latent Class Growth Model Analysis J Pediatr, 191(\#issue\#), 152-157

10 Alexander, D. D.,Yan, J.,Bylsma, L. C.,Northington, R. S.,Grathwohl, D.,Steenhout, P.,Erdmann, P.,Spivey-Krobath, E.,Haschke, F. (2016). Growth of infants consuming whey-predominant term infant formulas with a protein content of $1.8 \mathrm{~g} / 100 \mathrm{kcal}$ : a multicenter pooled analysis of individual participant data Am J Clin Nutr, 104(4), 1083-1092

11 Al-Mesad, Y.,Davidsson, L. (2018). Assessment of body composition of kuwaiti infants by using air displacement plethysmography (PEA POD®) Irish journal of medical science, 187(\#issue\#), S341-

Outcome

Intervention/exposure,

Outcome

Intervention/exposure

Intervention/exposure

Intervention/exposure

Intervention/exposure

Outcome

Study design,

Intervention/exposure

Publication status 
12 Altobelli, E.,Petrocelli, R.,Verrotti, A.,Chiarelli, F.,Marziliano, C. (2016). Genetic and environmental factors affect the onset of type 1 diabetes mellitus Pediatr Diabetes, 17(8), 559-566

13 Amano, I.,Murakami, A. (2019). Prevalence of infant and maternal anemia during the lactation period in Japan Pediatr Int, 61(5),

Outcome 495-503

14 Amaro-Rivera, K.,Molina, J.,Perez, C. M.,Palacios, C. (2019). Longitudinal Associations between Dietary Patterns and Weight Status Intervention/exposure in Puerto Rican Infants and Toddlers' Participants of the WIC Program P R Health Sci J, 38(2), 75-80

15 Ames, J.,Warner, M.,Siracusa, C.,Signorini, S.,Brambilla, P.,Mocarelli, P.,Eskenazi, B. (2019). Prenatal dioxin exposure and neuropsychological functioning in the Seveso Second Generation Health Study Int J Hyg Environ Health, 222(3), 425-433

Study design

16 Amoros, R.,Murcia, M.,Gonzalez, L.,Rebagliato, M.,Iniguez, C.,Lopez-Espinosa, M. J., Vioque, J.,Broberg, K.,Ballester, F.,Llop, S. (2018). Maternal selenium status and neuropsychological development in Spanish preschool children Environ Res, 166(\#issue\#),

Intervention/exposure 215-222

17 Andersen, A. T. N.,Husby, S.,Sander, S. D.,Kyhl, H. B.,Sandberg, M. B.,Molgaard, C. (2016). Iron deficiency in healthy 18-month-old danish children: prevalence and associated factors: a subproject in the odense child cohort Journal of pediatric gastroenterology and nutrition, 63(\#issue\#), S258-S259

18 Andersen, K. R.,Harslof, L. B.,Schnurr, T. M.,Hansen, T.,Hellgren, L. I.,Michaelsen, K. F.,Lauritzen, L. (2017). A study of associations between early DHA status and fatty acid desaturase (FADS) SNP and developmental outcomes in children of obese mothers Br $\mathrm{J}$ Nutr, 117(2), 278-286

Study design

20 Andres, Aline (2017). CHILDREN'S NUTRITION CENTER FOCUSED ON SOY FORMULA Soy Connection, 25(3), 6-7

Publication status

21 Anusha, K.,Hettiaratchi, U.,Gunasekera, D.,Prathapan, S.,Liyanage, G. (2019). Maternal Vitamin D Status and Its Effect on Vitamin D Intervention/exposure Levels in Early Infancy in a Tertiary Care Centre in Sri Lanka Int J Endocrinol, 2019(\#issue\#), 9017951

22 Ardıç, C.,Omar, E. (2019). Obesity frequency and related risk factors in primary school children European Research Journal, 5(3), 467-472

23 Ardic, C.,Usta, O.,Omar, E.,Yildiz, C.,Memis, E. (2019). Effects of infant feeding practices and maternal characteristics on early childhood obesity Arch Argent Pediatr, 117(1), 26-33

24 Aris, I. M.,Bernard, J. Y.,Chen, L. W.,Tint, M. T.,Pang, W. W.,Lim, W. Y.,Soh, S. E.,Saw, S. M.,Godfrey, K. M.,Gluckman, P D.,Chong, Y. S.,Yap, F.,Kramer, M. S.,Lee, Y. S. (2017). Infant body mass index peak and early childhood cardio-metabolic risk markers in a multi-ethnic Asian birth cohort Int J Epidemiol, 46(2), 513-525 
27 Aris, I. M.,Soh, S. E.,Tint, M. T.,Saw, S. M.,Rajadurai, V. S.,Godfrey, K. M.,Gluckman, P. D.,Yap, F.,Chong, Y. S.,Lee, Y. S. (2017). Associations of infant milk feed type on early postnatal growth of offspring exposed and unexposed to gestational diabetes in utero Eur J Nutr, 56(1), 55-64

28 Ayonrinde, O. T.,Oddy, W. H.,Adams, L. A.,Mori, T. A.,Beilin, L. J.,de Klerk, N.,Olynyk, J. K. (2017). Infant nutrition and maternal

obesity influence the risk of non-alcoholic fatty liver disease in adolescents $\mathrm{J}$ Hepatol, 67(3), 568-576

Duplicate from 1980 to 2016 search

29 Azad, M. B.,Vehling, L.,Chan, D.,Klopp, A.,Nickel, N. C.,McGavock, J. M.,Becker, A. B.,Mandhane, P. J.,Turvey, S. E.,Moraes, T. J.,Taylor, M. S.,Lefebvre, D. L.,Sears, M. R.,Subbarao, P. (2018). Infant Feeding and Weight Gain: Separating Breast Milk From Breastfeeding and Formula From Food Pediatrics, 142(4), \#Pages\#

30 Baïz, N.,Macchiaverni, P.,Tulic, M. K.,Rekima, A.,Annesi-Maesano, I.,Verhasselt, V.,Bernard, J. Y.,Botton, J.,Charles, M. A.,DargentMolina, P., de Lauzon-Guillain, B.,Ducimetière, P., de Agostini, M.,Foliguet, B.,Forhan, A.,Fritel, X., Germa, A., Goua, V.,Hankard, R.,Heude, B.,Kaminski, M.,Larroque, B.,Lelong, N.,Lepeule, J.,Magnin, G.,Pierre, F.,Marchand, L.,Nabet, C.,Slama, R.,SaurelCubizolles, M. J.,Schweitzer, M.,Thiebaugeorges, O. (2017). Early oral exposure to house dust mite allergen through breast milk: A potential risk factor for allergic sensitization and respiratory allergies in children Journal of Allergy and Clinical Immunology, 139(1), 369-372.e10

31 Baran, J.,Weres, A.,Czenczek-Lewandowska, E.,Luszczki, E.,Sobek, G.,Pitucha, G.,Leszczak, J.,Mazur, A. (2019). Early Eating Patterns and Overweight and Obesity in a Sample of Preschool Children in South-East Poland Int J Environ Res Public Health, 16(17), \#Pages\#

32 Barrera, C. M.,Perrine, C. G.,Li, R.,Scanlon, K. S. (2016). Age at Introduction to Solid Foods and Child Obesity at 6 Years Child Obes, 12(3), 188-92

33 Barros, V. O.,Amorim, M. R.,Melo, A. O.,Tavares, J. S.,Silva, A. C.,Alves, J. G. (2016). Abdominal Fat Distribution Among Breastfed and Formula-Fed Infants Breastfeed Med, 11(\#issue\#), 231-4

34 Béghin, L.,Marchandise, X.,Lien, E.,Bricout, M.,Bernet, J. P.,Lienhardt, J. F.,Jeannerot, F.,Menet, V.,Requillart, J. C.,Marx, J.,De Groot, N.,Jaeger, J.,Steenhout, P.,Turck, D. (2019). Growth, stool consistency and bone mineral content in healthy term infants fed sn-2-palmitate-enriched starter infant formula: A randomized, double-blind, multicentre clinical trial Clinical Nutrition, 38(3), 10231030

35 Bekhet, O. H.,Vekic, J.,Zeljkovic, A.,Paripovic, D.,Gojkovic, T.,Janac, J.,Spasojevic-Kalimanovska, V.,Peco-Antic, A.,MilosevskiLomic, G.,Jelic-Ivanovic, Z., Stefanovic, A. (2017). Associations of Apgar score and size at birth with lipoprotein subclasses in juvenile obesity Turk J Med Sci, 47(6), 1804-1812

36 Belfort, M. B.,Rifas-Shiman, S. L.,Kleinman, K. P.,Bellinger, D. C.,Harris, M. H., Taveras, E. M.,Gillman, M. W.,Oken, E. (2016). Infant Breastfeeding Duration and Mid-Childhood Executive Function, Behavior, and Social-Emotional Development J Dev Behav Pediatr, $37(1), 43-52$ 

the Risk of Overweight and Obesity in Children Aged 24 to 36 Months: Findings from an Australian Cohort Study Int J Environ Res Public Health, 15(4), \#Pages\# Berger, P. K.,Lavner, J. A.,Smith, J. J.,Birch, L. L. (2017). Differences in early risk factors for obesity between African American formula-fed infants and White breastfed controls Pilot Feasibility Stud, 3(\#issue\#), 58 Berghuis, S. A.,Van Braeckel, Knja,Sauer, P. J. J.,Bos, A. F. (2018). Prenatal exposure to persistent organic pollutants and cognition and motor performance in adolescence Environ Int, 121(Pt 1), 13-22 Bernard, J. Y.,Armand, M.,Peyre, H.,Garcia, C.,Forhan, A.,De Agostini, M.,Charles, M. A.,Heude, B. (2017). Breastfeeding, Polyunsaturated Fatty Acid Levels in Colostrum and Child Intelligence Quotient at Age 5-6 Years J Pediatr, 183(\#issue\#), 43-50.e3

42 Besharat Pour, M.,Bergstrom, A.,Bottai, M.,Magnusson, J.,Kull, I.,Moradi, T. (2017). Age at adiposity rebound and body mass index trajectory from early childhood to adolescence; differences by breastfeeding and maternal immigration background Pediatr Obes, 12(1), $75-84$

43 Betoko, A.,Lioret, S.,Heude, B.,Hankard, R.,Carles, S.,Forhan, A.,Regnault, N.,Botton, J.,Charles, M. A.,de Lauzon-Guillain, B. (2017). Influence of infant feeding patterns over the first year of life on growth from birth to 5 years Pediatr Obes, 12 Suppl 1(\#issue\#), 94-101

44 Bider-Canfield, Z.,Martinez, M. P.,Wang, X.,Yu, W.,Bautista, M. P.,Brookey, J.,Page, K. A.,Buchanan, T. A.,Xiang, A. H. (2017). Maternal obesity, gestational diabetes, breastfeeding and childhood overweight at age 2 years Pediatr Obes, 12(2), 171-178

45 Bion, V.,Lockett, G. A.,Soto-Ramirez, N.,Zhang, H.,Venter, C.,Karmaus, W.,Holloway, J. W.,Arshad, S. H. (2016). Evaluating the efficacy of breastfeeding guidelines on long-term outcomes for allergic disease Allergy, 71(5), 661-70

46 Bjarnadottir, E.,Stokholm, J.,Chawes, B., Thorsen, J.,Mora-Jensen, A. C.,Deleuran, M.,Bonnelykke, K.,Lauritzen, L.,Bisgaard, H. (2019). Determinants of neurodevelopment in early childhood - results from the Copenhagen prospective studies on asthma in childhood (COPSAC2010 ) mother-child cohort Acta Paediatr, 108(9), 1632-1641

47 Bjarnadóttir, E.,Stokholm, J.,Chawes, B., Thorsen, J.,Mora-Jensen, A. R. C.,Deleuran, M.,Bønnelykke, K.,Lauritzen, L.,Bisgaard, H. (2019). Determinants of neurodevelopment in early childhood - results from the Copenhagen prospective studies on asthma in childhood (COPSAC2010) mother-child cohort Acta Paediatrica, International Journal of Paediatrics, 108(9), 1632-1641

48 Bjerregaard, L. G.,Pedersen, D. C.,Mortensen, E. L.,Sorensen, T. I. A.,Baker, J. L. (2019). Breastfeeding duration in infancy and adult risks of type 2 diabetes in a high-income country Matern Child Nutr, \#volume\#(\#issue\#), e12869

49 Bonato, M.,Bazzan, E.,Snijders, D.,Tine, M.,Biondini, D.,Turato, G.,Balestro, E.,Papi, A.,Cosio, M. G.,Barbato, A.,Baraldo, S.,Saetta M. (2018). Clinical and Pathologic Factors Predicting Future Asthma in Wheezing Children. A Longitudinal Study Am J Respir Cell Mol Biol, 59(4), 458-466

50 Bornhorst, C.,Siani, A.,Russo, P.,Kourides, Y.,Sion, I.,Molnar, D.,Moreno, L. A.,Rodriguez, G.,Ben-Shlomo, Y.,Howe, L.,Lissner, L.,Mehlig, K.,Regber, S.,Bammann, K.,Foraita, R.,Ahrens, W.,Tilling, K. (2016). Early Life Factors and Inter-Country Heterogeneity in BMI Growth Trajectories of European Children: The IDEFICS Study PLoS One, 11(2), e0149268
Intervention/exposure

Intervention/exposure

Intervention/exposure

Outcome

Intervention/exposure

Outcome

Intervention/exposure

Outcome

Intervention/exposure

Duplicate within 2016 to 2019

search

Intervention/exposure

Outcome

Outcome 

168 development, autistic traits and ADHD symptoms: a multicenter study in Spain Pediatr Res, 81(3), 434-442

53 Boucher, Olivier,Julvez, Jordi,Guxens, Mònica,Arranz, Enrique,Ibarluzea, Jesús,Sánchez de Miguel, Manuel,Fernández-Somoano, Ana,Tardon, Adonina, Rebagliato, Marisa,Garcia-Esteban, Raquel,O'Connor, Giselle,Ballester, Ferran,Sunyer, Jordi (2016).

\section{Outcome}

55 Bove, M. I.,Zelmonovich, C.,Bia, D.,Iturralde, A., Ghiachetto, G.,Klaps, L.,Guillermo, V. (2017). Modifiable risk factors present from conception to age 2 years and their association with obesity at 5 years old Annals of nutrition \& metabolism, 71(\#issue\#), 622-623 millennium baby" study Nutr Metab Cardiovasc Dis, 26(8), 706-12

58 Breij, L. M.,Abrahamse-Berkeveld, M.,Acton, D.,De Lucia Rolfe, E.,Ong, K. K.,Hokken-Koelega, A. C. S. (2017). Impact of Early Infant Growth, Duration of Breastfeeding and Maternal Factors on Total Body Fat Mass and Visceral Fat at 3 and 6 Months of Age Ann Nutr Metab, 71(3-4), 203-210

59 Breij, L. M.,Mulder, M. T.,van Vark-van der Zee, L. C.,Hokken-Koelega, A. C. S. (2017). Appetite-regulating hormones in early life and relationships with type of feeding and body composition in healthy term infants Eur J Nutr, 56(4), 1725-1732

Duplicate within 2016 to 2019 search

Publication status

Outcome

60 Bridgman, S. L.,Azad, M. B.,Persaud, R. R.,Chari, R. S.,Becker, A. B.,Sears, M. R.,Mandhane, P. J.,Turvey, S. E.,Subbarao P.,Haqq, A. M.,Kozyrskyj, A. L. (2018). Impact of maternal pre-pregnancy overweight on infant overweight at 1 year of age: associations and sex-specific differences Pediatr Obes, 13(10), 579-589

61 Brouwer-Brolsma, E. M.,van de Rest, O.,Godschalk, R.,Zeegers, M. P. A.,Gielen, M.,de Groot, R. H. M. (2017). Associations between maternal long-chain polyunsaturated fatty acid concentrations and child cognition at 7 years of age: The MEFAB birth cohort Prostaglandins Leukot Essent Fatty Acids, 126(\#issue\#), 92-97

62 Buccigrossi, V.,Ranucci, G.,Felisi, M. G.,Cantarutti, L.,Visentin, F.,Piacentini, D.,Spagnuolo, M. I.,Giaquinto, C.,Guarino, A. (2017). Early administration of prebiotics protects from respiratory infections and atopy by modifying intestinal microbial structure Journal of pediatric gastroenterology and nutrition, 64(\#issue\#), 973-

Intervention/exposure,

Outcome

Intervention/exposure

No key confounders accounted for, Study design

Intervention/exposure

Outcome

Publication status 

24(3), $12-12$

Buckley, J. P.,Engel, S. M.,Mendez, M. A.,Richardson, D. B.,Daniels, J. L.,Calafat, A. M.,Wolff, M. S.,Herring, A. H. (2016). Prenatal Phthalate Exposures and Childhood Fat Mass in a New York City Cohort Environ Health Perspect, 124(4), 507-13

65 Byrne, M. L.,Schwartz, O. S.,Simmons, J. G.,Sheeber, L.,Whittle, S.,Allen, N. B. (2018). Duration of Breastfeeding and Subsequent Study design Adolescent Obesity: Effects of Maternal Behavior and Socioeconomic Status J Adolesc Health, 62(4), 471-479

66 Cabana, M. D. (2018). Does longer breastfeeding duration decrease the risk of asthma? Journal of Pediatrics, 195(\#issue\#), 1-2 Publication status

67 Cai, X.,Lian, F.,Kong, Y.,Huang, L.,Xu, L.,Wu, Y.,Ma, H.,Yang, L. (2019). Carotenoid metabolic (BCO1) polymorphisms and personal Intervention/exposure behaviors modify the risk of coronary atherosclerosis: a nested case-control study in Han Chinese with dyslipidaemia (2013-2016) Asia Pac J Clin Nutr, 28(1), 192-202

Campoy, C.,Nieto-Ruiz, A.,Arias, M.,Dieguez, E.,Herrmann, F.,Miranda, M. T.,De Castellar, R. (2018). Long-term influence of a milk fat globule membrane (MFGM)-enriched formula on language development in healthy children at 4 years old Journal of pediatric gastroenterology and nutrition, 66(\#issue\#), 92945

Campoy, C.,Ruiz, A. N. (2016). Nutritional intervention in early life influences the head circumference in healthy male children at 2.5 years Journal of pediatric gastroenterology and nutrition. Conference: 49th annual meeting of the european society for paediatric gastroenterology, hepatology and nutrition, ESPGHAN 2016. Athens greece. Conference start: 20160525. Conference end: 20160528. Conference publication: (var.pagings), 62(\#issue\#), 883

71 Canani, R. B.,Nocerino, R.,Frediani, T.,Lucarelli, S.,Di Scala, C.,Varin, E.,Leone, L.,Muraro, A.,Agostoni, C. (2017). Amino Acidbased Formula in Cow's Milk Allergy: Long-term Effects on Body Growth and Protein Metabolism J Pediatr Gastroenterol Nutr, 64(4), $632-638$

72 Candy, D. C. A.,Van Ampting, M. T. J.,Oude Nijhuis, M. M.,Wopereis, H.,Butt, A. M.,Peroni, D. G.,West, C. E.,Vandenplas, Y.,Fox, A. T.,Harthoorn, L. F., et al., (2016). Dietary management of non-ige mediated cow's milk allergic infants with a synbiotics-supplemented amino acid-based formula: effects on faecal microbiota and clinical symptoms Journal of pediatric gastroenterology and nutrition, 63(\#issue\#), S402-

73 Cebolla-Boado, H.,Jimenez-Buedo, M.,Salazar, L. (2017). Avoiding selection bias without random assignment? The effect of breastfeeding on cognitive outcomes in China Soc Sci Med, 194(\#issue\#), 151-159

74 Cetinkaya, M.,Semerci, S. Y.,Ugurel, O.,Balik, D. T. (2017). Evaluation of the effect of palm olein free formula on intestinal flora and gastrointestinal tolerance in infants Journal of pediatric gastroenterology and nutrition, 65(\#issue\#), S320-S321

Publication status

Publication status

Publication status

Cethakrkul, N. Topothai, C. Suphanchaimat, R., Tisayaticom, K. Limwattananon, S. Tangcharoensathien, V. (201

Cetthakrikul, N.,Topothai, C.,Suphanchaimat, R.,Tisayaticom, K.,Limwattananon, S.,Tangcharoensathien, V. (2018). Childhood stunting in Thailand: when prolonged breastfeeding interacts with household poverty BMC Pediatr, 18(1), 395 

first year of life Int $\mathrm{J}$ Obes (Lond), 42(1), 36-43

77 Chan, K. C.,Tam, W. H.,Chan, M. H.,Chan, R. S.,Li, A. M. (2018). Vitamin D deficiency among healthy infants in Hong Kong: a pilot study Hong Kong Med J, 24 Suppl 3(3), 32-35

78 Chen, F.,Lin, Z.,Chen, R.,Norback, D.,Liu, C.,Kan, H.,Deng, Q.,Huang, C.,Hu, Y.,Zou, Z.,Liu, W.,Wang, J.,Lu, C., Qian, H.,Yang X.,Zhang, X.,Qu, F.,Sundell, J.,Zhang, Y.,Li, B.,Sun, Y.,Zhao, Z. (2018). The effects of PM2.5 on asthmatic and allergic diseases or symptoms in preschool children of six Chinese cities, based on China, Children, Homes and Health (CCHH) project Environ Pollut, 232(\#issue\#), 329-337

79 Cheng, T. S.,Kwok, M. K.,Leung, G. M.,Schooling, C. M. (2018). The Associations of Breast Feeding with Infant Growth and Body Mass Index to 16 years: 'Children of 1997' Paediatr Perinat Epidemiol, 32(2), 200-209

80 Cheng, T. S.,Loy, S. L.,Cheung, Y. B.,Chan, J. K.,Pang, W. W.,Godfrey, K. M.,Gluckman, P. D.,Kwek, K.,Saw, S. M.,Chong, Y. S.,Lee, Y. S.,Lek, N.,Yap, F. (2016). Sexually dimorphic response to feeding mode in the growth of infants Am J Clin Nutr, 103(2), $398-405$

81 Chiu, C. Y.,Liao, S. L.,Su, K. W.,Tsai, M. H.,Hua, M. C.,Lai, S. H.,Chen, L. C.,Yao, T. C., Yeh, K. W.,Huang, J. L. (2016). Exclusive or Partial Breastfeeding for 6 Months Is Associated with Reduced Milk Sensitization and Risk of Eczema in Early Childhood Medicine (United States), 95(15), \#Pages\# PATCH Birth Cohort Study Medicine (Baltimore), 95(15), e3391

83 Choi, H. J.,Kang, S. K.,Chung, M. R. (2018). The relationship between exclusive breastfeeding and infant development: A 6- and 12- Outcome month follow-up study Early Hum Dev, 127(\#issue\#), 42-47

84 Choi, J.,Chang, J. Y.,Hong, J.,Shin, S.,Park, J. S.,Oh, S. (2017). Low-Level Toxic Metal Exposure in Healthy Weaning-Age Infants: Association with Growth, Dietary Intake, and Iron Deficiency Int J Environ Res Public Health, 14(4), \#Pages\#

85 Chowning, R.,Radmacher, P.,Lewis, S.,Serke, L.,Pettit, N.,Adamkin, D. H. (2016). A retrospective analysis of the effect of human milk on prevention of necrotizing enterocolitis and postnatal growth Journal of Perinatology, 36(3), 221-224

86 Christensen, L. H.,Hoyer, B. B.,Pedersen, H. S.,Zinchuk, A.,Jonsson, B. A. G.,Lindh, C.,Durr, D. W.,Bonde, J. P.,Toft, G. (2016) Prenatal smoking exposure, measured as maternal serum cotinine, and children's motor developmental milestones and motor function: A follow-up study Neurotoxicology, 53(\#issue\#), 236-245

87 Chu, S.,Zhang, Y.,Jiang, Y.,Sun, W.,Zhu, Q.,Wang, B.,Jiang, F.,Zhang, J. (2017). Cesarean section without medical indication and risks of childhood allergic disorder, attenuated by breastfeeding Sci Rep, 7(1), 9762

Study design,

Intervention/exposure

\section{Outcome}

\section{Outcome}

\section{Outcome}

Outcome

\section{Outcome}

Study design

Participant health

Civardi, E.,Garofoli, F.,Longo, S.,Mongini, M. E.,Grenci, B.,Mazzucchelli, I.,Angelini, M.,Castellazzi, A.,Fasano, F.,Grinzato, A.,Fanos, V.,Budelli, A.,Stronati, M. (2017). Safety, growth, and support to healthy gut microbiota by an infant formula enriched with functional compounds Clin Nutr, 36(1), 238-245 

Restriction Program for Obese Pregnant Women: Children's Weight Development during the First Five Years of Life Childhood Obesity, 12(3), 162-170

Clark, K. M.,Li, M.,Zhu, B.,Liang, F.,Shao, J.,Zhang, Y.,Ji, C.,Zhao, Z.,Kaciroti, N.,Lozoff, B. (2017). Breastfeeding, Mixed, or Formula Feeding at 9 Months of Age and the Prevalence of Iron Deficiency and Iron Deficiency Anemia in Two Cohorts of Infants in China J Pediatr, 181(\#issue\#), 56-61

91 Cloutier, M. M.,Wiley, J. F.,Kuo, C. L.,Cornelius, T.,Wang, Z.,Gorin, A. A. (2018). Outcomes of program in a low-income community: a pilot, randomized trial Pediatr Obes, 13(11), 677-685

Collell, R.,Closa-Monasterolo, R.,Ferre, N.,Luque, V.,Koletzko, B.,Grote, V.,Janas, R.,Verduci, E.,Escribano, J. (2016). Higher protein intake increases cardiac function parameters in healthy children: metabolic programming by infant nutrition-secondary analysis from a clinical trial Pediatr Res, 79(6), 880-8

Colombo, J., Jill Shaddy, D.,Kerling, E. H.,Gustafson, K. M.,Carlson, S. E. (2017). Docosahexaenoic acid (DHA) and arachidonic acid (ARA) balance in developmental outcomes Prostaglandins Leukot Essent Fatty Acids, 121(\#issue\#), 52-56

94 Comba, A.,Demir, E.,Baris Eren, N. (2019). Nutritional status and related factors of schoolchildren in Corum, Turkey Public Health Nutr, 22(1), 122-131

95 Contarato, A. A.,Rocha, E. D.,Czarnobay, S. A.,Mastroeni, S. S.,Veugelers, P. J.,Mastroeni, M. F. (2016). Independent effect of type of breastfeeding on overweight and obesity in children aged 12-24 months Cad Saude Publica, 32(12), e00119015

96 Coo, H.,Fabrigar, L.,Davies, G.,Fitzpatrick, R.,Flavin, M. (2019). Are observed associations between a high maternal prepregnancy body mass index and offspring IQ likely to be causal? J Epidemiol Community Health, \#volume\#(\#issue\#), \#Pages\#

97 Corkins, M.,Czerkies, L. A.,Storm, H. M.,Sun, S.,Saavedra, J. M. (2016). Assessment of Growth of Infants Fed an Amino Acid-Based Intervention/exposure Formula Clin Med Insights Pediatr, 10(\#issue\#), 3-9

98 Costa, C. S.,Campagnolo, P. D.,Lumey, L. H., Vitolo, M. R. (2017). Effect of maternal dietary counselling during the 1st year of life on glucose profile and insulin resistance at the age of 8 years: a randomised field trial $\mathrm{Br} \mathrm{J}$ Nutr, 117(1), 134-141

99 Cronin, F. M.,Segurado, R.,McAuliffe, F. M.,Kelleher, C. C.,Tremblay, R. E. (2017). Gestational age and chronic 'body-mind' health problems in childhood: dose-response association and risk factors European Child and Adolescent Psychiatry, 26(1), 57-65

Duplicate within 2016 to 2019

100 Cronin, Frances,Segurado, Ricardo,McAuliffe, Fionnuala,Kelleher, Cecily,Tremblay, Richard (2017). Gestational age and chronic 'body-mind' health problems in childhood: dose-response association and risk factors European Child \& Adolescent Psychiatry, 26(1) $57-65$

101 Cunha, M. P. L.,Marques, R. C.,Dorea, J. G. (2018). Influence of Maternal Fish Intake on the Anthropometric Indices of Children in the Western Amazon Nutrients, 10(9), \#Pages\#

102 Cuppari, C.,Manti, S.,Salpietro, A.,Alterio, T.,Arrigo, T.,Leonardi, S.,Salpietro, C. (2016). Mode of delivery and atopic phenotypes: Old questions new insights? A retrospective study Immunobiology, 221(12), 1418-1423 search

Study design

Intervention/exposure

Intervention/exposure,

Outcome

Intervention/exposure

Study design

Intervention/exposure

Intervention/exposure

Itervention/exposure

Intervention/exposure,

Outcome

Outcome

\section{Outcome}



composition in breastfeeding and school performance in children aged 12 years Eur J Nutr, 55(7), 2199-207

104 Daniels, S. R. (2018). BMI in early childhood Journal of Pediatrics, 202(\#issue\#), 2

105 Davisse-Paturet, C.,Raherison, C.,Adel-Patient, K.,Divaret-Chauveau, A.,Bois, C.,Dufourg, M. N.,Lioret, S.,Charles, M. A., de LauzonGuillain, B. (2019). Use of partially hydrolysed formula in infancy and incidence of eczema, respiratory symptoms or food allergies in toddlers from the ELFE cohort Pediatr Allergy Immunol, 30(6), 614-623

106 de Beer, M.,Vrijkotte, T. G.,Fall, C. H.,van Eijsden, M.,Osmond, C.,Gemke, R. J. (2016). Associations of Infant Feeding and Timing of Intervention/exposure Weight Gain and Linear Growth during Early Life with Childhood Blood Pressure: Findings from a Prospective Population Based Cohort Study PLoS One, 11(11), e0166281

107 De Regnier, R. A. (2017). Nutrition and brain development: it's complicated Journal of Pediatrics, 183(\#issue\#), 1-2

Publication status

108 Delgado, C. A.,Munhoz, T. N.,Santos, I. S.,Barros, F. C.,Matijasevich, A. (2017). Prolonged breastfeeding for 24 months or more and mental health at 6 years of age: evidence from the 2004 Pelotas Birth Cohort Study, Brazil Child and Adolescent Mental Health, 22(4), 209-215

109 den Dekker, H. T.,Sonnenschein-van der Voort, A. M.,Jaddoe, V. W.,Reiss, I. K., de Jongste, J. C.,Duijts, L. (2016). Breastfeeding and asthma outcomes at the age of 6 years: The Generation R Study Pediatr Allergy Immunol, 27(5), 486-92

110 Deoni, S.,Dean, D., 3rd,Joelson, S.,O'Regan, J.,Schneider, N. (2018). Early nutrition influences developmental myelination and cognition in infants and young children Neuroimage, 178(\#issue\#), 649-659

111 Dhudasia, Miren B.,Flannery, Dustin D.,Mukhopadhyay, Sagori (2019). Early limited formula for breastfeeding infants: too much or just enough? Journal of Perinatology, 39(8), 1149-1152

112 Diepeveen, F. B.,van Dommelen, P.,Oudesluys-Murphy, A. M.,Verkerk, P. H. (2017). Specific language impairment is associated with maternal and family factors Child Care Health Dev, 43(3), 401-405

Outcome

Outcome

\section{Outcome}

\section{Publication status, Outcome}

113 Ditomasso, Diane,Paiva, Andrea L. (2018). Neonatal Weight Matters: An Examination of Weight Changes in Full-Term Breastfeeding Newborns During the First 2 Weeks of Life Journal of Human Lactation, 34(1), 86-92

Study design

Intervention/exposure

Outcome

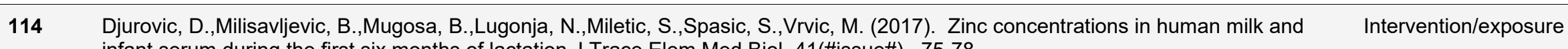
infant serum during the first six months of lactation J Trace Elem Med Biol, 41(\#issue\#), 75-78

115 Dogan, E.,Yilmaz, G.,Caylan, N.,Turgut, M.,Gokcay, G.,Oguz, M. M. (2018). Baby-led complementary feeding: Randomized controlled study Pediatr Int, 60(12), 1073-1080

116 Dogruel, D.,Bingol, G.,Altintas, D. U.,Yilmaz, M.,Kendirli, S. G. (2016). Prevalence of and risk factors for atopic dermatitis: A birth cohort study of infants in southeast Turkey Allergol Immunopathol (Madr), 44(3), 214-20 117 Doğruel, D.,Bingöl, G.,AltIntaş, D. U.,Yllmaz, M.,Kendirli, S. G. (2016). Clinical features of food allergy during the 1st year of life: The
ADAPAR birth cohort study International Archives of Allergy and Immunology, 169(3), 171-180 
118 Williams, A. J.Frank, J. (2016). How has child growth around adiposity rebound altered in Scotland since 1990 and what are the risk factors for weight gain using the Growing Up in Scotland birth cohort 1? BMC Public Health, 16(1), 1081

119 Donkor, H. M.,Grundt, J. H.,Juliusson, P. B.,Eide, G. E.,Hurum, J.,Bjerknes, R.,Markestad, T. (2017). Social and somatic determinants of underweight, overweight and obesity at 5 years of age: a Norwegian regional cohort study BMJ Open, $7(8)$, e014548

120 Duff, Elizabeth (2016). Infants born to obese women and fed with breast milk gain less weight than those fed with powdered milk within their first 6 months of life Midwifery, 43(\#issue\#), A4-A5

121 Dugas, C.,Kearney, M.,Mercier, R.,Perron, J.,Tchernof, A.,Marc, I.,Weisnagel, S. J.,Robitaille, J. (2018). Early life nutrition, glycemic Intervention/exposure and anthropometric profiles of children exposed to gestational diabetes mellitus in utero Early Hum Dev, 118(\#issue\#), 37-41

122 Eagleton, S. G.,Hohman, E. E.,Verdiglione, N.,Birch, L. L.,Paul, I. M.,Savage, J. S. (2019). INSIGHT Study Maternal Return to Work Intervention/exposure and Infant Weight Outcomes Acad Pediatr, 19(1), 67-73

123 Eastman, C. J. (2016). lodine in breastfeeding Aust Prescr, 39(1), 4

124 Edmonson, M. B.,Eickhoff, J. C. (2017). Weight Gain and Obesity in Infants and Young Children Exposed to Prolonged Antibiotic Prophylaxis JAMA Pediatr, 171(2), 150-156

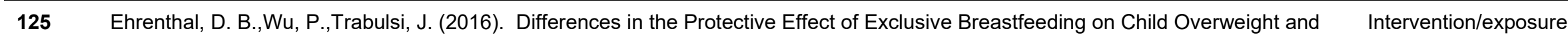
Obesity by Mother's Race Matern Child Health J, 20(9), 1971-9

126 Ek, W. E.,Karlsson, T.,Hernándes, C. A.,Rask-Andersen, M.,Johansson, (2018). Breast-feeding and risk of asthma, hay fever, and Publication status eczema Journal of Allergy and Clinical Immunology, 141(3), 1157-1159.e9

127 Elbert, N. J.,van Meel, E. R.,den Dekker, H. T.,de Jong, N. W.,Nijsten, T. E. C., Jaddoe, V. W. V.,de Jongste, J. C.,Pasmans, Sgma,Duijts, L. (2017). Duration and exclusiveness of breastfeeding and risk of childhood atopic diseases Allergy, 72(12), 19361943

128 Elbert, Niels J.,van Meel, Evelien R., den Dekker, H. T.,de Jong, Nicolette W.,Nijsten, Tamar E. C., Jaddoe, Vincent W. V.,de Jongste, Johan C.,Pasmans, Suzanne G. M. A.,Duijts, Liesbeth (2018). Duration and exclusiveness of breastfeeding and risk of childhood atopic diseases MIDIRS Midwifery Digest, 28(2), 234-234

129 El-Heneidy, A.,Abdel-Rahman, M. E.,Mihala, G.,Ross, L. J.,Comans, T. A. (2018). Milk Other Than Breast Milk and the Development Outcome of Asthma in Children 3 Years of Age. A Birth Cohort Study (2006(-)2011) Nutrients, 10(11), \#Pages\#

130 El-Heneidy, Asmaa,Abdel-Rahman, Manar E.,Mihala, Gabor,Ross, Lynda J.,Comans, Tracy A. (2018). Milk Other Than Breast Milk and the Development of Asthma in Children 3 Years of Age. A Birth Cohort Study (2006-2011) Nutrients, 10(11), 1798

131 Emmerson, A. J. B.,Dockery, K. E.,Mughal, M. Z., Roberts, S. A., Tower, C. L.,Berry, J. L. (2018). Vitamin D status of White pregnant Study design women and infants at birth and 4 months in North West England: A cohort study Matern Child Nutr, 14(1), \#Pages\#

132 Emmett, P. M. (2016). Dietary Patterns during Complementary Feeding and Later Outcomes Nestle Nutr Inst Workshop Ser, 85(\#issue\#), 145-54 
133 Eny, K. M.,Chen, S.,Anderson, L. N.,Chen, Y.,Lebovic, G.,Pullenayegum, E.,Parkin, P. C.,Maguire, J. L.,Birken, C. S. (2018). Breastfeeding duration, maternal body mass index, and birth weight are associated with differences in body mass index growth trajectories in early childhood Am J Clin Nutr, 107(4), 584-592

\section{Ercan, M.,Ozcetin, M.,Karaci, M.,Ozgurhan, G.,Yasar, A.,Guven, B. (2016). Relationship between newborn craniotabes and vitamin \\ Intervention/exposure} D status North Clin Istanb, 3(1), 15-21

135 Eroglu, C.,Demir, F.,Erge, D.,Uysal, P.,Kirdar, S.,Yilmaz, M.,Kurt Omurlu, I. (2019). The relation between serum vitamin D levels, viral infections and severity of attacks in children with recurrent wheezing Allergol Immunopathol (Madr), \#volume\#(\#issue\#), \#Pages\#

136 Escribano, J.,Ferre, N.,Gispert-Llaurado, M.,Luque, V.,Rubio-Torrents, C.,Zaragoza-Jordana, M.,Polanco, I.,Codoner, F. M.,Chenoll, E.,Morera, M.,Moreno-Munoz, J. A.,Rivero, M.,Closa-Monasterolo, R. (2018). Bifidobacterium longum subsp infantis CECT7210supplemented formula reduces diarrhea in healthy infants: a randomized controlled trial Pediatr Res, 83(6), 1120-1128

137 Escribano, J.,Luque, V.,Canals-Sans, J.,Ferre, N.,Koletzko, B.,Grote, V.,Weber, M.,Gruszfeld, D.,Szott, K.,Verduci, E.,Riva, E.,Brasselle, G.,Poncelet, P.,Closa-Monasterolo, R. (2016). Mental performance in 8-year-old children fed reduced protein content formula during the 1st year of life: safety analysis of a randomised clinical trial Br J Nutr, \#volume\#(\#issue\#), 1-9

138 Essau, C. A.,Sasagawa, S.,Lewinsohn, P. M.,Rohde, P. (2018). The impact of pre- and perinatal factors on psychopathology in adulthood J Affect Disord, 236(\#issue\#), 52-59

Study design

139 Estevez-Gonzalez, M. D.,Santana Del Pino, A.,Henriquez-Sanchez, P.,Pena-Quintana, L.,Saavedra-Santana, P. (2016) Participant age Breastfeeding during the first 6 months of life, adiposity rebound and overweight/obesity at 8 years of age Int J Obes (Lond), 40(1), $10-3$

140 Faith, M. S.,Hittner, J. B.,Hurston, S. R.,Yin, J.,Greenspan, L. C., Quesenberry, C. P., Jr.,Gunderson, E. P. (2019). Association of Infant Temperament With Subsequent Obesity in Young Children of Mothers With Gestational Diabetes Mellitus JAMA Pediatr, 173(5), 424-433

141 Fallah, R.,Kazemnejad, A.,Shoghli, A.,Vahabi, N. (2018). Growth velocity of children and its affective factors in northwestern Iran: A longitudinal study using marginal models Med J Islam Repub Iran, 32(\#issue\#), 72

142 Farahnak, Z., Yuan, Y.,Vanstone, C. A.,Weiler, H. A. (2019). Maternal and neonatal red blood cell n-3 polyunsaturated fatty acids inversely associate with infant whole body fat mass assessed by dual-energy x-ray absorptiometry Appl Physiol Nutr Metab, \#volume\#(\#issue\#), \#Pages\#

143 Farhangi, M. A. (2016). Nutritional status and feeding practices in pre-school children aged 1-5 years in rural and urban areas of East Azerbaijan- Iran Progress in Nutrition, 18(1), 16-21

144 Fatemi, M. J.,Fararouei, M.,Moravej, H.,Dianatinasab, M. (2018). Stunting and its associated factors among 6-7-year-old children in southern Iran: a nested case-control study Public Health Nutr, \#volume\#(\#issue\#), 1-8

Duplicate from 1980 to 2016 search

Intervention/exposure

Intervention/exposure

Study design

Intervention/exposure

Study design

Intervention/exposure

No key confounders accounted for

145 Fatemi, Mohammad Javad,Fararouei, Mohammad,Moravej, Hossein,Dianatinasab, Mostafa (2019). Stunting and its associated factors among 6-7-year-old children in southern Iran: a nested case-control study Public Health Nutrition, 22(1), 55-62 
146 Fields, D.,Czerkies, L.,Sun, S.,Storm, H.,Saavedra, J.,Sorensen, R. (2016). A Randomized Controlled Trial Assessing Growth of Infants Fed a 100\% Whey Extensively Hydrolyzed Formula Compared With a Casein-Based Extensively Hydrolyzed Formula Glob

Pediatr Health, 3(\#issue\#), 2333794x16636613

147

Fiocchi, A.,Fierro, V.,La Marra, F.,Dahdah, L. A. (2016 Allergy, Asthma and Immunology, 117(5), 465-467

148 Fisher, H. R.,Lack, G.,Du Toit, G. (2019). Solid foods should be introduced into susceptible infants' diets in early life-PRO Annals of Allergy, Asthma and Immunology, 122(6), 583-585

149 Flaherman, V. J.,Schaefer, E. W.,Kuzniewicz, M. K.,Li, S.,Walsh, E.,Paul, I. M. (2017). Newborn Weight Loss During Birth Hospitalization and Breastfeeding Outcomes Through Age 1 Month J Hum Lact, 33(1), 225-230

150 Fleddermann, M.,Demmelmair, H.,Grote, V.,Trisic, B.,Nikolic, T.,Koletzko, B. (2016). Growth during early infancy and anthropometry at 4 years of age: follow-up of the BeMIM study Journal of pediatric gastroenterology and nutrition. Conference: 49th annual meeting of the european society for paediatric gastroenterology, hepatology and nutrition, ESPGHAN 2016. Athens greece. Conference start: 20160525. Conference end: 20160528. Conference publication: (var.pagings), 62(\#issue\#), 680

151 Fleddermann, M.,Demmelmair, H.,Hellmuth, C.,Grote, V.,Trisic, B.,Nikolic, T.,Koletzko, B. (2018). Association of infant formula composition and anthropometry at 4 years: Follow-up of a randomized controlled trial (BeMIM study) PLoS One, 13(7), e0199859

152 Flensborg-Madsen, T.,Mortensen, E. L. (2017). Predictors of motor developmental milestones during the first year of life Eur J Pediatr, 176(1), 109-119

153 Flohr, C.,Henderson, A. J.,Kramer, M. S.,Patel, R.,Thompson, J.,Rifas-Shiman, S. L., Yang, S., Vilchuck, K.,Bogdanovich, N.,Hameza, M.,Martin, R. M.,Oken, E. (2018). Effect of an Intervention to Promote Breastfeeding on Asthma, Lung Function, and Atopic Eczema at Age 16 Years: Follow-up of the PROBIT Randomized Trial JAMA Pediatr, 172(1), e174064

154 Flohr, C.,Kramer, M. S.,Patel, R.,Thompson, J.,Rifas-Shiman, S. L.,Yang, S.,Vilchuk, K.,Bogdanovich, N.,Hameza, M.,Martin, R. M.,et al., (2017). Does prolonged and exclusive breastfeeding reduce the risk of atopic eczema in adolescence? the PROBIT clusterrandomized trial in the Republic of Belarus British journal of dermatology, 177(\#issue\#), 159-

155 Foiles, A. M.,Kerling, E. H.,Wick, J. A.,Scalabrin, D. M.,Colombo, J.,Carlson, S. E. (2016). Formula with long-chain polyunsaturated fatty acids reduces incidence of allergy in early childhood Pediatr Allergy Immunol, 27(2), 156-61

156 Fonolla, J.,Maldonado-Lobon, J. A.,Gil-Campo, M.,Maldonado, J.,Flores, K.,Benavides, M. R.,Jaldo, R.,Del Barco, I. J.,Valero, A. D.,Lara, F.,et al., (2017). An infant formula enriched with the human milk strain Lactobacillus fermentum CECT5716 is safe and reduces dhiarrea incidences during first year of life Journal of pediatric gastroenterology and nutrition, 64(\#issue\#), 933-

157 Fonseca, P. C. A.,Carvalho, C. A.,Ribeiro, S. A. V.,Nobre, L. N.,Pessoa, M. C.,Ribeiro, A. Q.,Priore, S. E.,Franceschini, Sdcc (2017). Determinants of the mean growth rate of children under the age of six months: a cohort study Cien Saude Colet, 22(8), 2713-2726 Forbes, J. D.,Azad, M. B.,Vehling, L.,Tun, H. M.,Konya, T. B.,Guttman, D. S.,Field, C. J.,Lefebvre, D.,Sears, M. R.,Becker, A. B.,Mandhane, P. J., Turvey, S. E.,Moraes, T. J.,Subbarao, P.,Scott, J. A.,Kozyrskyj, A. L. (2018). Association of Exposure to Formula in the Hospital and Subsequent Infant Feeding Practices With Gut Microbiota and Risk of Overweight in the First Year of Life JAMA Pediatr, 172(7), e181161
Publication status

Publication status, Outcome

Intervention/exposure

Publication status

Intervention/exposure

Outcome

Outcome

Publication status, Outcome

Intervention/exposure

Publication status

Intervention/exposure,

Outcome

Participant age 
159 Fortes, C.,Mastroeni, S.,Mannooranparampil, T. J.,Di Lallo, D. (2019). Pre-natal folic acid and iron supplementation and atopic dermatitis in the first 6 years of life Arch Dermatol Res, 311(5), 361-367

160 Foster, B. A.,Escaname, E.,Powell, T. L.,Larsen, B.,Siddiqui, S. K.,Menchaca, J.,Aquino, C.,Ramamurthy, R.,Hale, D. E. (2017). Randomized Controlled Trial of DHA Supplementation during Pregnancy: Child Adiposity Outcomes Nutrients, 9(6), \#Pages\#

161 Gaffney, K. F.,Brito, A. V.,Kitsantas, P.,Kermer, D. A. (2016). Early Feeding Practices and Weight Status at One Year of Age: A Comparison of Hispanic Immigrant Mother-Infant Dyads with Participants of the Infant Feeding Practices Study II Child Obes, 12(5), 384-91 Gahagan, S.,Delker, E.,Blanco, E.,Burrows, R.,Lozoff, B. (2019). Randomized Controlle Formula: Developmental Outcomes at 16 Years J Pediatr, 212(\#issue\#), 124-130.e1

163 Gahagan, S.,Delker, E.,Castillo, M.,Lozoff, B. (2017). Iron-fortified vs low-iron infant formula: cognitive outcomes at 10 and 16 years Publication status American journal of hematology, 92(8), E231-

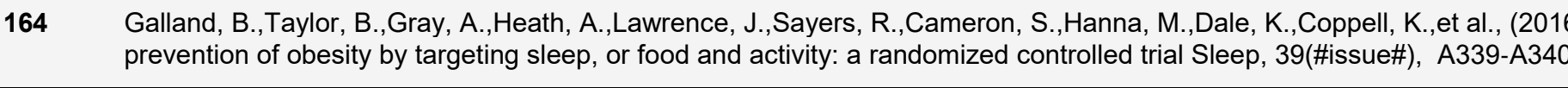

165 Gallier, S.,Xia, Y.,Rowan, A.,Wang, B. (2018). Milk fat globule membrane as a source of gangliosides and phospholipids support brain development and healthy growth Journal of pediatric gastroenterology and nutrition, 66(\#issue\#), 942-

166 Gallo, S.,Hazell, T.,Vanstone, C. A.,Agellon, S.,Jones, G.,L'Abbé, M.,Rodd, C.,Weiler, H. A. (2016). Vitamin D supplementation in breastfed infants from Montréal, Canada: 25-hydroxyvitamin $D$ and bone health effects from a follow-up study at 3 years of age Osteoporosis International, \#volume\#(\#issue\#), 1-8

167 Gallo, S.,Hazell, T.,Vanstone, C. A.,Agellon, S.,Jones, G.,L'Abbe, M.,Rodd, C.,Weiler, H. A. (2016). Vitamin D supplementation in breastfed infants from Montreal, Canada: 25-hydroxyvitamin D and bone health effects from a follow-up study at 3 years of age Osteoporos Int, 27(8), 2459-66

168 Gao, X.,Yan, Y.,Zeng, G.,Sha, T.,Liu, S.,He, Q.,Chen, C.,Li, L.,Xiang, S.,Li, H., Tan, S.,Yan, Q. (2019). Influence of prenatal and early-life exposures on food allergy and eczema in infancy: a birth cohort study BMC Pediatr, 19(1), 239

169 Geohagan, J.,de Gaston, D.,Sadler, A.,Palmer, P. (2018). Does oral maternal Vitamin D supplementation normalize the Vitamin D level in exclusively breastfed infants? J Okla State Med Assoc, 111(10), 870-871

170 Georgieva, M.,Manios, Y.,Rasheva, N.,Pancheva, R.,Dimitrova, E.,Stoeva, T. D.,Schaafsma, A. (2016). Effects of carob-bean gum thickened formulas on infants' reflux, growth and tolerance indices Journal of pediatric gastroenterology and nutrition. Conference: 49th annual meeting of the european society for paediatric gastroenterology, hepatology and nutrition, ESPGHAN 2016. Athens greece. Conference start: 20160525. Conference end: 20160528. Conference publication: (var.pagings), 62(\#issue\#), 468

171 Ghaemmaghami, P.,Ayatollahi, S. M. T.,Alinejad, V.,Sharafi, Z. (2018). Growth curves and their associated weight and height factors

171 Ghaemmaghami, P.,Ayatollahi, S. M. T.,Alinejad, V.,Sharafi, Z. (2018). Growth curves and their associated weil
in children from birth to 4 years old in West Azerbaijan Province, northwest Iran Arch Pediatr, 25(6), 389-393

Outcome

No key confounders accounted for

Intervention/exposure

Outcome

Intervention/exposure

Publication status

Publication status

Intervention/exposure,

Duplicate within 2016 to 2019 search

Intervention/exposure

Intervention/exposure

Study design

Intervention/exposure

Outcome 
172 Gianni, M. L.,Roggero, P.,Baudry, C.,Fressange-Mazda, C.,Galli, C.,Agostoni, C.,le Ruyet, P.,Mosca, F. (2018). An infant formula containing dairy lipids increased red blood cell membrane Omega 3 fatty acids in 4 month-old healthy newborns: a randomized controlled trial BMC Pediatr, 18(1), 53

173 Gianni, M. L.,Roggero, P.,Baudry, C.,Fressange-Mazda, C.,le Ruyet, P.,Mosca, F. (2018). No effect of adding dairy lipids or long chain polyunsaturated fatty acids on formula tolerance and growth in full term infants: a randomized controlled trial BMC Pediatr, 18(1), 10

174 Gianni, M. L.,Roggero, P.,Baudry, C.,Galli, C.,Le Ruyet, P.,Mosca, F. (2016). Dairy lipids in infant formula: impact on the Omega-3 fatty acid content in membrane phospholipids of red blood cells in healthy term infants Journal of pediatric gastroenterology and nutrition. Conference: 49th annual meeting of the european society for paediatric gastroenterology, hepatology and nutrition, ESPGHAN 2016. Athens greece. Conference start: 20160525. Conference end: 20160528. Conference publication: (var.pagings), 62(\#issue\#), 656

175 Gianni, M. L.,Roggero, P.,Baudry, C.,Le Ruyet, P.,Mosca, F. (2016). Dairy lipids in infant formula: impact on growth and gastrointestinal tolerance in healthy infants Journal of pediatric gastroenterology and nutrition. Conference: 49th annual meeting of the european society for paediatric gastroenterology, hepatology and nutrition, ESPGHAN 2016. Athens greece. Conference start: 20160525. Conference end: 20160528. Conference publication: (var.pagings), 62(\#issue\#), 831

\section{6 Gibbs, B.} $579-588$

\section{Gibson, L. A.,Hernandez Alava, M.,Kelly, M. P.,Campbell, M. J. (2017). The effects of breastfeeding on childhood BMl: a propensity Outcome} score matching approach J Public Health (Oxf), 39(4), e152-e160

178 Gibson, L.,Porter, M. (2018). Drinking or Smoking While Breastfeeding and Later Cognition in Children Pediatrics, 142(2), \#Pages\# Outcome

179 Gillette, M. T.,Lohman, B. J.,Neppl, T. K. (2017). Lower levels of maternal capital in early life predict offspring obesity in adulthood Intervention/exposure Ann Hum Biol, 44(3), 252-260

180 Girard, L. C.,Doyle, O.,Tremblay, R. E. (2017). Breastfeeding, Cognitive and Noncognitive Development in Early Childhood: A Population Study Pediatrics, 139(4), \#Pages\#

181 Girard, L. C.,Farkas, C. (2019). Breastfeeding and behavioural problems: Propensity score matching with a national cohort of infants Outcome in Chile BMJ Open, 9(2), e025058

182 Girard, L. C.,Tremblay, R. E.,Nagin, D.,Cote, S. M. (2019). Development of Aggression Subtypes from Childhood to Adolescence: a Group-Based Multi-Trajectory Modelling Perspective J Abnorm Child Psychol, 47(5), 825-838

Intervention/exposure, Outcome

183 Girard, Lisa-Christine,Doyle, Orla,Tremblay, Richard E. (2018). Breastfeeding and externalising problems: a quasi-experimental design with a national cohort European Child \& Adolescent Psychiatry, 27(7), 877-884

Outcome

Godleski, S. A.,Shisler, S.,Eiden, R. D.,Huestis, M. A. (2018). Co-use of tobacco and marijuana during pregnancy: Pathways to

Study design,

externalizing behavior problems in early childhood Neurotoxicol Teratol, 69(\#issue\#), 39-48

Intervention/exposure 
185 Goetz, A. R.,Mara, C. A.,Stark, L. J. (2018). Greater Breastfeeding in Early Infancy Is Associated with Slower Weight Gain among High Birth Weight Infants J Pediatr, 201(\#issue\#), 27-33.e4

186 Golding, J.,Gregory, S.,Ellis, G.,Nunes, T.,Bryant, P.,lles-Caven, Y.,Nowicki, S. (2019). Maternal Prenatal External Locus of Control and Reduced Mathematical and Science Abilities in Their Offspring: A Longitudinal Birth Cohort Study Front Psychol, 10(\#issue\#), 194

187 Golding, J.,Iles-Caven, Y.,Ellis, G.,Gregory, S.,Nowicki, S. (2019). The relationship between parental locus of control and adolescent Intervention/exposure obesity: a longitudinal pre-birth cohort Int J Obes (Lond), 43(4), 724-734

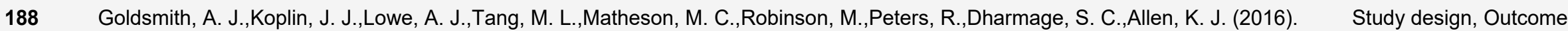
Formula and breast feeding in infant food allergy: A population-based study J Paediatr Child Health, 52(4), 377-84

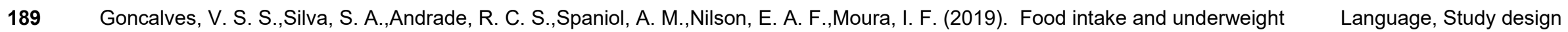
markers in children under 6 months old monitored via the Food and Nutrition Surveillance System, Brazil, 2015 Epidemiol Serv Saude, 28(2), e2018358

190 Gorohi, F.,Shiemorteza, M.,Nori, M. M. (2018). Comparison of height, weight and head circumference index and the incidence of infectious and gastrointestinal diseases in breast-fed and formula-fed infants at 0 to 1 year old in Bu-Ali Sina Hospital Biomedical and Pharmacology Journal, 11(3), 1717-1730

191 Grace, T.,Oddy, W.,Bulsara, M.,Hands, B. (2017). Breastfeeding and motor development: A longitudinal cohort study Hum Mov Sci, 51(\#issue\#), 9-16

192 Graulau, R. E.,Banna, J.,Campos, M.,Gibby, C. L. K.,Palacios, C. (2019). Amount, Preparation and Type of Formula Consumed and Its Association with Weight Gain in Infants Participating in the WIC Program in Hawaii and Puerto Rico Nutrients, 11(3), \#Pages\#

Study design, Outcome

193 Gridneva, Z.,Hepworth, A. R.,Ward, L. C.,Lai, C. T.,Hartmann, P. E.,Geddes, D. T. (2017). Determinants of body composition in breastfed infants using bioimpedance spectroscopy and ultrasound skinfolds-methods comparison Pediatr Res, 81(3), 423-433

194 Gridneva, Z., Kugananthan, S.,Rea, A.,Lai, C. T.,Ward, L. C.,Murray, K.,Hartmann, P. E., Geddes, D. T. (2018). Human Milk Adiponectin and Leptin and Infant Body Composition over the First 12 Months of Lactation Nutrients, 10(8), \#Pages\#

195 Gridneva, Z.,Rea, A.,Hepworth, A. R.,Ward, L. C.,Lai, C. T.,Hartmann, P. E.,Geddes, D. T. (2018). Relationships between Breastfeeding Patterns and Maternal and Infant Body Composition over the First 12 Months of Lactation Nutrients, 10(1), \#Pages\#

196 Gridneva, Z.,Rea, A., Tie, W. J.,Lai, C. T.,Kugananthan, S.,Ward, L. C.,Murray, K.,Hartmann, P. E., Geddes, D. T. (2019). Carbohydrates in Human Milk and Body Composition of Term Infants during the First 12 Months of Lactation Nutrients, 11(7), \#Pages\#

197 Grillo, L. P.,Gigante, D. P.,Horta, B. L.,de Barros, F. C. (2016). Childhood stunting and the metabolic syndrome components in young Intervention/exposure adults from a Brazilian birth cohort study Eur J Clin Nutr, $70(5), \quad 548-53$

198 Grimshaw, K. E.,Bryant, T.,Oliver, E. M.,Martin, J.,Maskell, J.,Kemp, T.,Clare Mills, E. N.,Foote, K. D.,Margetts, B. M.,Beyer, K.,Roberts, G. (2015). Incidence and risk factors for food hypersensitivity in UK infants: results from a birth cohort study Clin Transl Allergy, 6(\#issue\#), 1 
Grip, T.,Dyrlund, T. F.,Ahonen, L.,Domellof, M.,Hernell, O.,Hyotylainen, T.,Knip, M.,Lonnerdal, B.,Oresic, M.,Timby, N. (2016). Serum lipid profile in infants fed formula supplemented with a bovine milk fat globule membrane fraction Journal of pediatric gastroenterology and nutrition. Conference: 49th annual meeting of the european society for paediatric gastroenterology, hepatology and nutrition, ESPGHAN 2016. Athens greece. Conference start: 20160525. Conference end: 20160528. Conference publication: (var.pagings), 62(\#issue\#), 676 Pediatr Res, 84(5), 726-732

201 Grip, T.,Hernell, O.,Lonnerdal, B.,Domellof, M.,Timby, N. (2016). Plasma metabolome in infants fed formula supplemented with milk fat globule membranes Journal of pediatric gastroenterology and nutrition. Conference: 49th annual meeting of the european society for paediatric gastroenterology, hepatology and nutrition, ESPGHAN 2016. Athens greece. Conference start: 20160525. Conference end: 20160528. Conference publication: (var.pagings), 62(\#issue\#), 660

202 Grote, V.,Theurich, M.,Luque, V.,Gruszfeld, D.,Verduci, E.,Xhonneux, A.,Koletzko, B. (2018). Complementary Feeding, Infant Growth, and Obesity Risk: Timing, Composition, and Mode of Feeding Nestle Nutr Inst Workshop Ser, 89(\#issue\#), 93-103

203 Gruszfeld, D.,Weber, M.,Gradowska, K.,Socha, P.,Grote, V.,Xhonneux, A.,Dain, E.,Verduci, E.,Riva, E.,Closa-Monasterolo, R.,Escribano, J.,Koletzko, B. (2016). Association of early protein intake and pre-peritoneal fat at five years of age: Follow-up of a randomized clinical trial Nutr Metab Cardiovasc Dis, 26(9), 824-32

Guerrero, A. D.,Mao, C.,Fuller, B.,Bridges, M.,Franke, T.,Kuo, A. A. (2016). Racial and Ethnic Disparities in Early Childhood Obesity: Growth Trajectories in Body Mass Index J Racial Ethn Health Disparities, 3(1), 129-37

205 Gunderson, E. P.,Greenspan, L. C.,Faith, M. S.,Hurston, S. R., Quesenberry, C. P., Jr. (2018). Breastfeeding and growth during infancy among offspring of mothers with gestational diabetes mellitus: a prospective cohort study Pediatr Obes, 13(8), 492-504

\section{Publication status}

Publication status

Intervention/exposure

Gunnarsdottir, J.,Cnattingius, S.,Lundgren, M.,Selling, K.,Hogberg, U.,Wikstrom, A. K. (2018). Prenatal exposure to preeclampsia is Intervention/exposure associated with accelerated height gain in early childhood PLoS One, 13(2), e0192514

207 Gunnell, L.,Neher, J.,Safranek, S.,Guthmann, R. (2016). Does breastfeeding affect the risk of childhood obesity? Journal of Family Publication status Practice, 65(12), 931-932

208 Gunnell, Lindsay,Neher, Jon,Safranek, Sarah (2016). Q / Does breastfeeding affect the risk of childhood obesity? Journal of Family Publication status Practice, 65(12), 931-932 Infant Feeding in Relation to the Risk of Advanced Islet Autoimmunity and Type 1 Diabetes in Children With Increased Genetic

Outcome Susceptibility: A Cohort Study Am J Epidemiol, 187(1), 34-44 
Han, D. H.,Shin, J. M.,An, S.,Kim, J. S.,Kim, D. Y.,Moon, S.,Kim, J. S., Cho, J. S.,Kim, S. W.,Kim, Y. H., Roh, H. J., Shim, W. S., Rha, K

212 Hand, S.,Jones, K.,Doull, I. (2016). Age of weaning and asthma and atopy in young adults European respiratory journal. Conference: european respiratory society annual congress 2016. United kingdom. Conference start: 20160903. Conference end: 20160907, 48(no pagination), \#Pages\#

213 Hara, K., Ikeda, K.,Hasegawa, T.,Koyama, Y.,Wada, Y. (2017). Serum 25-Hydroxyvitamin D3 levels of one-month-old term infants in Tokyo using liquid chromatography tandem mass spectrometry International journal of pediatric endocrinology. Conference: 9th biennial scientific meeting of the asia pacific paediatric endocrine society, APPES and the 50th annual meeting of the japanese society for pediatric endocrinology, JSPE. Japan, 2017(Supplement 1) (no pagination), \#Pages\#

214 Hara, K., Ikeda, K.,Koyama, Y.,Wada, Y.,Hasegawa, T. (2018). Serum 25-hydroxyvitamin D3 levels of one-month-old term infants in Tokyo using liquid chromatography tandem mass spectrometry Acta Paediatrica, International Journal of Paediatrics, 107(3), 532533

215 Harding, K. L.,Aguayo, V. M.,Webb, P. (2018). Birthweight and feeding practices are associated with child growth outcomes in South Asia Matern Child Nutr, 14 Suppl 4(\#issue\#), e12650

216 Harrison, Michelle,Brodribb, Wendy,Davies, Peter S. W.,Hepworth, Julie (2019). Relationships between parental feeding practices, infant weight concern, infant dietary behaviour and body weight: Findings from the Feeding A Baby (FAB) Study Obesity Research \& Clinical Practice, 13(1), 86-86

217 Hazell, T. J.,Gallo, S.,Vanstone, C. A.,Agellon, S.,Rodd, C.,Weiler, H. A. (2017). Vitamin D supplementation trial in infancy: body composition effects at 3 years of age in a prospective follow-up study from Montreal Pediatr Obes, 12(1), 38-47

218 Hazrati, S.,Khan, F.,Huddleston, K.,De La Cruz, F.,Deeken, J. F.,Fuller, A.,Wong, W. S. W.,Niederhuber, J. E.,Hourigan, S. K. (2019). Clinical and social factors associated with excess weight in Hispanic and non-Hispanic White children Pediatr Res, 85(3), 256-261

Publication status

Publication status

Hellmuth, C.,Uhl, O.,Demmelmair, H.,Grunewald, M.,Auricchio, R.,Castillejo, G.,Korponay-Szabo, I. R.,Polanco, I.,Roca, M.,Vriezinga S. L.,Werkstetter, K. J.,Koletzko, B.,Mearin, M. L.,Kirchberg, F. F. (2018). The impact of human breast milk components on the infant metabolism PLoS One, 13(6), e0197713

220 Herberth, G.,Pierzchalski, A.,Feltens, R.,Bauer, M.,Röder, S.,Olek, S.,Hinz, D.,Borte, M.,von Bergen, M.,Lehmann, I. (2017). Prenatal phthalate exposure associates with low regulatory T-cell numbers and atopic dermatitis in early childhood: Results from the LINA mother-child study Journal of Allergy and Clinical Immunology, 139(4), 1376-1379.e8

Hewison, Martin, Wagner, Carol L.,Hollis, Bruce W.,Roth, Daniel E.,Gernand, Alison D.,Al Mahmud, Abdullah (2018). Supplementation in Pregnancy and Lactation and Infant Growth \#journal\#, 379(\#issue\#), 1880-1881

Study design

Study design, Country

Publication status

Intervention/exposure

Study design,

Intervention/exposure

ntervention/exposure

Outcome

222 Hirata, M.,Kusakawa, I.,Ohde, S., Yamanaka, M.,Yoda, H. (2017). Risk factors of infant anemia in the perinatal period Pediatr Int, 59(4), 447-451 
Hisada, A.,Yoshinaga, J.,Zhang, J.,Kato, T.,Shiraishi, H.,Shimodaira, K.,Okai, T.,Ariki, N.,Komine, Y.,Shirakawa, M.,Noda, Y.,Kato, N

(2017). Maternal Exposure to Pyrethroid Insecticides during Pregnancy and Infant Development at 18 Months of Age Int J Environ

Res Public Health, 14(1), \#Pages\#

224

Hoeke, H.,Roeder, S.,Mueller, A.,Bertsche, T.,Borte, M.,Rolle-Kampczyk, U.,von Bergen, M.,Wissenbach, D. K. (2016) Biomonitoring of prenatal analgesic intake and correlation with infantile anti-aeroallergens IgE Allergy, 71(6), 901-6

225 Hoffman, D. R.,Harris, C. L.,Wampler, J. L.,Patterson, A. C.,Berseth, C. L. (2019). Growth, tolerance, and DHA and ARA status of healthy term infants receiving formula with two different ARA concentrations: Double-blind, randomized, controlled trial Prostaglandins Leukotrienes and Essential Fatty Acids, 146(\#issue\#), 19-27

226

Hohman, E. E.,Savage, J. S.,Birch, L. L.,Beiler, J. S.,Paul, I. M. (2018). Pacifier Use and Early Life Weight Outcomes in the Intervention Nurses Start Infants Growing on Healthy Trajectories Study Child Obes, 14(1), 58-66

227 Hohman, E. E.,Savage, J. S.,Paul, I. M.,Birch, L. L. (2016). INSIGHT study parenting intervention to prevent childhood obesity improves patterns of dietary exposures in infants FASEB journal, 30(\#issue\#), \#Pages\# Hojat, M.,Mogarab, V.,Jahromi, H. K. (2016). The study of growth differences of infants less than 6 months which have used breast milk and infant formula along with breast milk International Journal of Pharmaceutical Research and Allied Sciences, 5(4), 108-119

Holmsen, S. T.,Bakkebo, T.,Seferowicz, M.,Retterstol, K. (2017). Statins and breastfeeding in familial hypercholesterolaemia Tidsskr Nor Laegeforen, 137(10), 686-687

230 Horodynski, M. A.,Pierce, S. J.,Reyes-Gastelum, D.,Olson, B.,Shattuck, M. (2017). Feeding Practices and Infant Growth: Quantifying the Effects of Breastfeeding Termination and Complementary Food Introduction on BMI z-Score Growth Velocity through Growth Curve Models Child Obes, 13(6), 490-498

231 Horta, B. L.,Victora, C. G.,Franca, G. V. A.,Hartwig, F. P.,Ong, K. K.,Rolfe, E. L.,Magalhaes, E. I. S.,Lima, N. P.,Barros, F. C. (2018). Breastfeeding moderates FTO related adiposity: a birth cohort study with 30 years of follow-up Sci Rep, 8(1), 2530

232 Hu, C.,Duijts, L.,Erler, N. S.,Elbert, N. J.,Piketty, C.,Bourdes, V.,Blanchet-Rethore, S.,de Jongste, J. C.,Pasmans, Sgma,Felix, J. F.,Nijsten, T. (2019). Most associations of early-life environmental exposures and genetic risk factors poorly differentiate between eczema phenotypes: the Generation R Study Br J Dermatol, \#volume\#(\#issue\#), \#Pages\#

233 Huang, J. G.,Chan, S. H.,Lee, L. Y. (2018). The Influence of Ethnicity on Exclusively Breast-Fed Infants' Anthropometry in a Multiethnic Asian Population Ann Acad Med Singapore, 47(6), 208-215

234 Huang, J.,Vaughn, M. G.,Kremer, K. P. (2016). Breastfeeding and child development outcomes: an investigation of the nurturing hypothesis Matern Child Nutr, 12(4), 757-67

235 Huang, J.,Zhang, Z.,Wu, Y.,Wang, Y.,Wang, J.,Zhou, L.,Ni, Z.,Hao, L.,Yang, N.,Yang, X. (2018). Early feeding of larger volumes of formula milk is associated with greater body weight or overweight in later infancy Nutr J, 17(1), 12

236 Huang, T.,Yue, Y.,Wang, H.,Zheng, J.,Chen, Z.,Chen, T.,Zhang, M.,Wang, S. (2019). Infant Breastfeeding and Behavioral Disorders in School-Age Children Breastfeed Med, 14(2), 115-120

Outcome

Intervention/exposure

Intervention/exposure

Publication status

Study design

Publication status

No key confounders

accounted for

Intervention/exposure

Outcome

\section{Outcome}

Outcome

Intervention/exposure

Study design

dy design

331 

Fermented Infant Formulae With Specific Oligosaccharides Support Adequate Infant Growth and Are Well-Tolerated J Pediatr Gastroenterol Nutr, 63(4), e43-53

239 Hui, L. L.,Kwok, M. K.,Nelson, E. A. S.,Lee, S. L.,Leung, G. M.,Schooling, C. M. (2018). The association of breastfeeding with insulin resistance at 17 years: Prospective observations from Hong Kong's "Children of 1997" birth cohort Matern Child Nutr, 14(1), \#Pages\#

Hui, L. Kwok, M. K. Nelson, E. A S.,Lee, S. L.,Leung, G. M.,Schooling, C. M. (2019). Breastfeeding in Infancy and Lipid Profile in Adolescence Pediatrics, 143(5), \#Pages\# Hui, L. L.,Lam, H. S.,Lau, E. Y. Y.,Nelson, E. A. S.,Wong, T. W.,Fielding, R. (2016). Prenatal dioxin exposure and neurocognitive development in Hong Kong 11-year-old children Environ Res, 150(\#issue\#), 205-212

242 Hui, L. L.,Lee, S. L.,Kwok, M. K.,Yu, C. W.,Schooling, C. M. (2018). Formula-feeding and the risk of type-2 diabetes mellitus among Outcome Hong Kong adolescents Hong Kong Med J, 24 Suppl 4(4), 20-23

243 Hui, L. L.,Li, A. M.,Nelson, E. A. S.,Leung, G. M.,Lee, S. L.,Schooling, C. M. (2018). In utero exposure to gestational diabetes and adiposity: does breastfeeding make a difference? Int J Obes (Lond), 42(7), 1317-1325

244 Hummel, S.,Beyerlein, A., Tamura, R.,Uusitalo, U.,Andren Aronsson, C.,Yang, J.,Riikonen, A.,Lernmark, A.,Rewers, M. J.,Hagopian, W. A.,She, J. X.,Simell, O. G.,Toppari, J.,Ziegler, A. G.,Akolkar, B.,Krischer, J. P., Virtanen, S. M.,Norris, J. M. (2017). First Infant Formula Type and Risk of Islet Autoimmunity in The Environmental Determinants of Diabetes in the Young (TEDDY) Study Diabetes Care, 40(3), 398-404

245 Huynh, D.,Condo, D.,Gibson, R.,Muhlhausler, B.,Ryan, P.,Skeaff, S.,Makrides, M.,Zhou, S. J. (2017). lodine status of postpartum women and their infants in Australia after the introduction of mandatory iodine fortification Br J Nutr, 117(12), 1656-1662

Intervention/exposure

246 Iguacel, I.,Chung, A.,Gearon, E.,Moreno, L. A.,Peeters, A.,Backholer, K. (2018). Influence of early-life risk factors on socioeconomic Intervention/exposure inequalities in weight gain J Public Health (Oxf), 40(4), e447-e455 factors and their cumulative effects as predictors of overweight in Spanish children Int J Public Health, 63(4), 501-512

248 Iguacel, I.,Fernandez-Alvira, J. M.,Labayen, I.,Moreno, L. A.,Samper, M. P.,Rodriguez, G. (2018). Social vulnerabilities as determinants of overweight in 2-, 4- and 6-year-old Spanish children Eur J Public Health, 28(2), 289-295

249 Iguacel, I.,Monje, L.,Cabero, M. J.,Moreno Aznar, L. A.,Samper, M. P.,Rodriguez-Palmero, M.,Rivero, M.,Rodriguez, G. (2019). Feeding patterns and growth trajectories in breast-fed and formula-fed infants during the introduction of complementary food Nutr Hosp, 36(4), 777-785 

Perinatal exposure to dioxins and dioxin-like compounds and infant growth and body mass index at seven years: A pooled analysis of size/power three European birth cohorts Environ Int, 94(\#issue\#), 399-407 106-114 166(\#issue\#), 128-136 Jackson, D. B. Beaver, K. M. (2016). The Association Between Breastfeeding Exposure and Duration, Neuropsychological Deficits,

Outcome and Psychopathic Personality Traits in Offspring: The Moderating Role of 5HTTLPR Psychiatr Q, 87(1), 107-27

255 Jansson, L. M.,Jordan, C. J.,Velez, M. L. (2018). Perinatal Marijuana Use and the Developing Child Jama, 320(6), 545-546

Publication status Jardi, C.,Aranda, N.,Bedmar, C.,Arija, V. (2019). Excess nutritional risk in infants and toddlers in a Spanish city Int J Vitam Nutr Res, \#volume\#(\#issue\#), 1-11

257 Jardi, C.,Hernandez-Martinez, C.,Canals, J.,Arija, V.,Bedmar, C.,Voltas, N.,Aranda, N. (2018). Influence of breastfeeding and iron status on mental and psychomotor development during the first year of life Infant Behav Dev, 50(\#issue\#), 300-310 Jarvinen, K. M. (2018). Variations in Human Milk Composition: Impact on Immune Development and Allergic Disease Susceptibility Breastfeed Med, 13(S1), S11-s13

259 Jess, T.,Morgen, C. S.,Harpsoe, M. C.,Sorensen, T. I. A.,Ajslev, T. A.,Antvorskov, J. C.,Allin, K. H. (2019). Antibiotic use during pregnancy and childhood overweight: A population-based nationwide cohort study Sci Rep, 9(1), 11528

Study design

Intervention/exposure

Study design

260 Jia, N.,Gu, G.,Zhao, L.,He, S.,Xiong, F.,Chai, Y.,Quan, L.,Hou, H.,Dai, Y. (2018). Longitudinal study of breastfeeding and growth in 0- Intervention/exposure

6 month infants Asia Pac J Clin Nutr, 27(6), 1294-1301

Johansson, E. K.,Bergstrom, A.,Kull, I.,Lind, T.,Soderhall, C.,Melen, E.,Asad, S.,Bradley, M.,Lieden, A.,Ballardini, N.,Wahl (2018). Prognosis of Preschool Eczema and Factors of Importance for Remission Acta Derm Venereol, 98(7), 630-635

262 Johansson, U.,Öhlund, I.,Hernell, O.,Lönnerdal, B.,Lindberg, L.,Lind, T. (2019). Protein-reduced complementary foods based on nordic ingredients combined with systematic introduction of taste portions increase intake of fruits and vegetables in 9 month old infants: A randomised controlled trial Nutrients, 11(6), \#Pages\# eggs is associated with increased risk of allergy development - results from the FARMFLORA birth cohort Food Nutr Res, 61(1) 1393306 

infants, reflecting family fish consumption, were inversely associated with allergy development but not related to farm residence Acta Paediatr, 105(12), 1462-1471

266 JoO

267

Jose, Am- L.,Federico, L. V.,Gil-Campos, M.,Maldonado, J.,Flores, K.,Benavides, R.,Jaldo, R.,Jimenez, I.,Fonolla, J.,Olivares, M. (2016). Consumption of the human milk strain bifidobacterium breve cect7263 might improve symptoms of infant colic Journal of clinical gastroenterology, 50(\#issue\#), S226-

268 Kain, J.,Leyton, B.,Baur, L.,Lira, M.,Corvalán, C. (2019). Demographic, social and health-related variables that predict normal-weight preschool children having overweight or obesity when entering primary education in Chile Nutrients, 11(6), \#Pages\#

269 Kajzer, J.,Oliver, J.,Marriage, B. (2016). Gastrointestinal tolerance of formula supplemented with oligosaccharides FASEB journal Conference: experimental biology 2016, EB. San diego, CA united states. Conference start: 20160402. Conference end: 20160406 Conference publication: (var.pagings), 30(no pagination), \#Pages\#

270 Kalhoff, H.,Kersting, M. (2016). Adequate iron supply in infants fed according to dietary guidelines? Journal of pediatric gastroenterology and nutrition, 62(\#issue\#), 873-

271 Kalhoff, Hermann,Kersting, Mathilde (2017). Breastfeeding or formula feeding and iron status in the second 6 months of life: A critica role for complementary feeding \#journal\#, 187(\#issue\#), 333-333

272 Kampouri, M.,Kyriklaki, A.,Roumeliotaki, T.,Koutra, K.,Anousaki, D.,Sarri, K.,Vassilaki, M.,Kogevinas, M.,Chatzi, L. (2018). Patterns of Early-Life Social and Environmental Exposures and Child Cognitive Development, Rhea Birth Cohort, Crete, Greece Child Dev, 89(4), 1063-1073 Kanazawa, S.,Segal, N. L. (2017). Same-sex twins are taller and heavier than opposite-sex twins (but only if breastfed): Possible evidence for sex bias in human breast milk J Exp Child Psychol, 156(\#issue\#), 186-191

274 Kapoor, M.,Bird, J. A. (2017). Cow's milk protein is often tolerated by children with oat-induced FPIES Journal of Allergy and Clinical Immunology: In Practice, 5(2), 496-497

275 Katsuragi, S.,Okamura, T.,Kokubo, Y.,Watanabe, M.,Higashiyama, A.,Ikeda, T.,Miyamoto, Y. (2019). The Perinatal Condition Around Birth and Cardiovascular Risk Factors in the Japanese General Population: The Suita Study Journal of atherosclerosis and thrombosis, \#volume\#(\#issue\#), \#Pages\# Kaul, P.,Bowker, S. L.,Savu, A.,Yeung, R. O.,Donovan, L. E.,Ryan, E. A. (2019). Association between maternal diabetes, being large for gestational age and breast-feeding on being overweight or obese in childhood Diabetologia, 62(2), 249-258

277 Kawai, E.,Takagai, S.,Takei, N.,Itoh, H.,Kanayama, N.,Tsuchiya, K. J. (2017). Maternal postpartum depressive symptoms predict delay in non-verbal communication in 14-month-old infants Infant Behav Dev, 46(\#issue\#), 33-45
Outcome

Intervention/exposure, Participant health

Publication status

No key confounders accounted for, Intervention/exposure

Publication status

Publication status

Publication status

Intervention/exposure

Outcome

\section{Outcome}

Intervention/exposure

ntervention/exposure

Intervention/exposure 
279 Kerr, J. A.,Long, C.,Clifford, S. A.,Muller, J.,Gillespie, A. N.,Donath, S.,Wake, M. (2017). Early-life exposures predicting onset and resol of childhood overweight or obesity Archives of Disease in Childhood, 102(10), 922-929

No key confounders accounted for, Intervention/exposure

280 Kerr, J. A.,Long, C.,Clifford, S. A.,Muller, J.,Gillespie, A. N.,Donath, S.,Wake, M. (2017). Early-life exposures predicting onset and resolution of childhood overweight or obesity Arch Dis Child, 102(10), 915-922

No key confounders accounted for

281 Kesztyüs, D.,Traub, M.,Lauer, R.,Kesztyüs, T.,Steinacker, J. M. (2016). Correlates of longitudinal changes in the waist-to-height ratio of primary school children: Implications for prevention Preventive Medicine Reports, 3(\#issue\#), 1-6

No key confounders accounted for

282 Khatiwada, A.,Shoaibi, A.,Neelon, B.,Emond, J. A.,Benjamin-Neelon, S. E. (2018). Household chaos during infancy and infant weight status at 12 months Pediatr Obes, 13(10), 607-613

Intervention/exposure,

Outcome

283 Khatun, M.,Al Mamun, A.,Scott, J.,William, G. M.,Clavarino, A.,Najman, J. M. (2017). Do children born to teenage parents have lower Intervention/exposure adult intelligence? A prospective birth cohort study PLoS One, 12(3), e0167395

284 Khodabakhshi, A.,Mehrad-Majd, H.,Vahid, F.,Safarian, M. (2018). Association of maternal breast milk and serum levels of macronutrients, hormones, and maternal body composition with infant's body weight Eur J Clin Nutr, 72(3), 394-400

Intervention/exposure

285 Kim, H.,Kim, H.,Lee, E.,Kim, Y.,Ha, E. H.,Chang, N. (2017). Association between maternal intake of n-6 to n-3 fatty acid ratio during Intervention/exposure pregnancy and infant neurodevelopment at 6 months of age: results of the MOCEH cohort study Nutr J, 16(1), 23

286 Kim, Y. H.,Kim, K. W.,Lee, S. Y.,Koo, K. O.,Kwon, S. O.,Seo, J. H.,Suh, D. I.,Shin, Y. H.,Ahn, K.,Oh, S. Y.,Lee, S.,Sohn, M. H.,Hong, Outcome S. J. (2019). Maternal Perinatal Dietary Patterns Affect Food Allergy Development in Susceptible Infants J Allergy Clin Immunol Pract, \#volume\#(\#issue\#), \#Pages\#

287 Kimura, Masahiko,Kurozawa, Youichi,Saito, Yumi,Watanabe, Hiroshi,Kobayashi, Ayame,Taketani, Takeshi (2018). High prevalence of anemia in 10-month-old breast-fed Japanese infants Pediatrics International, 60(7), 651-655

288 Kirchberg, F. F.,Hellmuth, C.,Totzauer, M.,Uhl, O.,Closa-Monasterolo, R.,Escribano, J.,Gruszfeld, D.,Gradowska, K.,Verduci, E.,Mariani, B.,Moretti, M.,Rousseaux, D.,Koletzko, B. (2019). Impact of infant protein supply and other early life factors on plasma metabolome at 5.5 and 8 years of age: a randomized trial Int $\mathrm{J}$ Obes (Lond), \#volume\#(\#issue\#), \#Pages\#

289 Kishi, R.,Araki, A.,Minatoya, M.,Hanaoka, T.,Miyashita, C.,Itoh, S.,Kobayashi, S.,Ait Bamai, Y.,Yamazaki, K.,Miura, R.,Tamura, N.,Ito, K.,Goudarzi, H. (2017). The Hokkaido Birth Cohort Study on Environment and Children's Health: cohort profile-updated 2017 Environ Health Prev Med, 22(1), 46

290 Kjaer, T. W.,Faurholt-Jepsen, D.,Medrano, R.,Elwan, D.,Mehta, K.,Christensen, V. B.,Wojcicki, J. M. (2019). Higher Birthweight and Maternal Pre-pregnancy BMI Persist with Obesity Association at Age 9 in High Risk Latino Children J Immigr Minor Health, 21(1), 8997 
292 Klingberg, S.,Brekke, H. K.,Ludvigsson, J. (2019). Introduction of fish and other foods during infancy and risk of asthma in the All Babies In Southeast Sweden cohort study Eur J Pediatr, 178(3), 395-402

293 Klopp, A.,Vehling, L.,Becker, A. B.,Subbarao, P.,Mandhane, P. J.,Turvey, S. E.,Lefebvre, D. L.,Sears, M. R.,Azad, M. B. (2017)

Modes of Infant Feeding and the Risk of Childhood Asthma: A Prospective Birth Cohort Study J Pediatr, 190(\#issue\#), 192-199.e2
294 Knip, M.,Akerblom, H. K.,Al Taji, E.,Becker, D.,Bruining, J.,Castano, L.,Danne, T.,de Beaufort, C.,Dosch, H. M.,Dupre, J.,Fraser, W. D.,Howard, N.,Ilonen, J.,Konrad, D.,Kordonouri, O.,Krischer, J. P.,Lawson, M. L.,Ludvigsson, J.,Madacsy, L., Mahon, J. L., Ormisson, A.,Palmer, J. P.,Pozzilli, P.,Savilahti, E.,Serrano-Rios, M.,Songini, M.,Taback, S.,Vaarala, O.,White, N. H.,Virtanen, S. M.,Wasikowa, R. (2018). Effect of Hydrolyzed Infant Formula vs Conventional Formula on Risk of Type 1 Diabetes: The TRIGR Randomized Clinical Trial Jama, 319(1), 38-48

295 Koh, K. (2017). Maternal breastfeeding and children's cognitive development Soc Sci Med, 187(\#issue\#), 101-108

Study design Intervention/exposure Effects of Breastfeeding: Role of the Intestinal Microbiota JAMA Pediatr, 170(8), 750-7

297 Kouwenhoven, S.,Antl, N.,Finken, M.,Van Der Beek, E.,Koletzko, B.,Van Goudoever, J. (2018). Safety of a modified, low protein infant formula in term infants; An RCT with a reference breastfed group Journal of pediatric gastroenterology and nutrition, 66(\#issue\#), 915-916 before the cart Am J Clin Nutr, 107(4), 635-639

299 Kuniyoshi, Y.,Kikuya, M.,Matsubara, H.,Ishikuro, M.,Obara, T.,Kure, S.,Kuriyama, S. (2019). Association of Feeding Practice with Childhood Overweight and/or Obesity in Affected Areas Before and After the Great East Japan Earthquake Breastfeed Med, 14(6) 382-389 300 Kwok, M. K.,Schooling, C. M.,Subramanian, S. V.,Leung, G. M.,Kawachi, I. (2016). Pathways from parental educational attainment to adolescent blood pressure J Hypertens, 34(9), 1787-95

302 Lambidou, M.,Alteheld, B.,Jochum, F.,Nomayo, A.,Stehle, P. (2016). Effect of high beta-palmitate infant formula supplemented with galacto-oligosaccharides on stool fatty acid soaps Journal of pediatric gastroenterology and nutrition. Conference: 49th annual meeting of the european society for paediatric gastroenterology, hepatology and nutrition, ESPGHAN 2016. Athens greece. Conference start: 20160525. Conference end: 20160528. Conference publication: (var.pagings), 62(\#issue\#), 879 
304 Larsson, M. W.,Lind, M. V.,Larnkjaer, A.,Due, A. P.,Blom, I. C.,Wells, J.,Lai, C. T.,Molgaard, C., Geddes, D. T.,Michaelsen, K. F. (2018). Excessive Weight Gain Followed by Catch-Down in Exclusively Breastfed Infants: An Exploratory Study Nutrients, 10(9), \#Pages\# PPARG2 Single Nucleotide Polymorphisms are Associated with Plasma Lipids in 9-Mo-Old Infants J Nutr, 149(5), 708-715

Lauritzen, L.,Eriksen, S. E.,Hjorth, M. F.,Nielsen, M. S.,Olsen, S. F.,Stark, K. D.,Michaelsen, K. F.,Damsgaard, C. T. (2016). Materna fish oil supplementation during lactation is associated with reduced height at 13 years of age and higher blood pressure in boys only British Journal of Nutrition, 116(12), 2082-2090

307 Laws, R. A.,Denney-Wilson, E. A.,Taki, S.,Russell, C. G.,Zheng, M.,Litterbach, E. K.,Ong, K. L.,Lymer, S. J.,Elliott, R.,Campbell, K. J. Intervention/exposure (2018). Key Lessons and Impact of the Growing Healthy mHealth Program on Milk Feeding, Timing of Introduction of Solids, and Infant Growth: Quasi-Experimental Study JMIR Mhealth Uhealth, 6(4), e78

308 Laws, Rachel,Litterbach, Eloise-Kate,Taki, Sarah,Russell, Georgina,Denney-Wilson, Elizabeth,Campbell, Karen (2019). Obesity prevention in infants: A qualitative study exploring the influence of the Growing healthy program on infant feeding behaviours Obesity Research \& Clinical Practice, 13(1), 92-92

309 Lee, H. R., Shin, S.,Yoon, J. H.,Roh, E. Y.,Chang, J. Y. (2016). Reference Intervals of Hematology and Clinical Chemistry Analytes for 1-Year-Old Korean Children Ann Lab Med, 36(5), 481-8

310 Lee, H.,Park, H.,Ha, E.,Hong, Y. C.,Ha, M.,Park, H.,Kim, B. N.,Lee, B.,Lee, S. J.,Lee, K. Y.,Kim, J. H.,Jeong, K. S.,Kim, Y. (2016). Effect of Breastfeeding Duration on Cognitive Development in Infants: 3-Year Follow-up Study J Korean Med Sci, 31(4), 579-84

Study design

ntervention/exposure

311 Lee, I.,Bang, K. S.,Moon, H.,Kim, J. (2019). Risk Factors for Obesity Among Children Aged 24 to 80 months in Korea: A Decision Tree Analysis J Pediatr Nurs, 46(\#issue\#), e15-e23 Lee, M. T.,Wu, C. C.,Ou, C. Y.,Chang, J. C.,Liu, C. A.,Wang, C. L.,Chuang, H.,Kuo, H. C.,Hsu, T. Y.,Chen, C. P.,Yang, K. D. (2017).

Publication status A prospective birth cohort study of different risk factors for development of allergic diseases in offspring of non-atopic parents Oncotarget, 8(7), 10858-10870

313 Lee, M.,Ha, M.,Hong, Y. C.,Park, H.,Kim, Y.,Kim, E. J.,Kim, Y.,Ha, E. (2019). Exposure to prenatal secondhand smoke and early neurodevelopment: Mothers and Children's Environmental Health (MOCEH) study Environ Health, 18(1), 22

314 Lee, S. H.,Weerasinghe, Wmsp, van der Werf, J. H. J. (2017). Genotype-environment interaction on human cognitive function conditioned on the status of breastfeeding and maternal smoking around birth Sci Rep, 7(1), 6087

315 Lee, Y. A.,Cho, S. W.,Sung, H. K.,Kim, K.,Song, Y. S.,Moon, S. J.,Oh, J. W.,Ju, D. L., Choi, S., Song, S. H.,Cheon, G. J.,Park, Y. J.,Shin, C. H.,Park, S. K.,Jun, J. K.,Chung, J. K. (2018). Effects of Maternal lodine Status during Pregnancy and Lactation on Maternal Thyroid Function and Offspring Growth and Development: A Prospective Study Protocol for the Ideal Breast Milk Cohort Endocrinol Metab (Seoul), 33(3), 395-402

316 Lee-Sarwar, K. A.,Kelly, R. S.,Lasky-Su, J.,Zeiger, R. S.,O'Connor, G. T.,Sandel, M. T.,Bacharier, L. B.,Beigelman, A.,Laranjo N.,Gold, D. R.,Weiss, S. T.,Litonjua, A. A. (2019). Integrative analysis of the intestinal metabolome of childhood asthma J Allergy Clin Immunol, 144(2), 442-454 
317 Lemcke, S.,Parner, E. T.,Bjerrum, M.,Thomsen, P. H.,Lauritsen, M. B. (2016). Early development in children that are later diagnosed with disorders of attention and activity: a longitudinal study in the Danish National Birth Cohort Eur Child Adolesc Psychiatry, 25(10), 1055-66

318 Lemcke, S.,Parner, E. T.,Bjerrum, M.,Thomsen, P. H.,Lauritsen, M. B. (2018). EARLY REGULATION IN CHILDREN WHO ARE LATER DIAGNOSED WITH AUTISM SPECTRUM DISORDER. A LONGITUDINAL STUDY WITHIN THE DANISH NATIONAL BIRTH COHORT Infant Ment Health J, 39(2), 170-182

319 Lentferink, Yvette E. Elst, Marieke A J. Knibbe Catherijne A. J.,van der Vorst, Marja M. J. (2017). Predictors of Insulin Resistance in Children versus Adolescents with Obesity Journal of Obesity, \#volume\#(\#issue\#), 1-7

320 Lepping, R. J.,Honea, R. A.,Martin, L. E.,Liao, K.,Choi, I. Y.,Lee, P.,Papa, V. B.,Brooks, W. M.,Shaddy, D. J.,Carlson, S. E.,Colombo, J.,Gustafson, K. M. (2019). Long-chain polyunsaturated fatty acid supplementation in the first year of life affects brain function, structure, and metabolism at age nine years Dev Psychobiol, 61(1), 5-16

321 Lertxundi, A.,Andiarena, A.,Martinez, M. D.,Ayerdi, M.,Murcia, M.,Estarlich, M.,Guxens, M.,Sunyer, J.,Julvez, J.,Ibarluzea, J. (2019) Prenatal exposure to PM2.5 and NO2 and sex-dependent infant cognitive and motor development Environ Res, 174(\#issue\#), 114121

322 Leung, J. Y.,Kwok, M. K.,Leung, G. M.,Schooling, C. M. (2016). Breastfeeding and childhood hospitalizations for asthma and other wheezing disorders Ann Epidemiol, 26(1), 21-7.e1-3

$323 \mathrm{Li}$, Y.,Mu, Z.,Wang, H.,Liu, J.,Jiang, F. (2018). The role of particulate matters on methylation of IFN-gamma and IL-4 promoter genes Outcome in pediatric allergic rhinitis Oncotarget, 9(25), 17406-17419

324 Liao, K.,McCandliss, B. D.,Carlson, S. E.,Colombo, J.,Shaddy, D. J.,Kerling, E. H.,Lepping, R. J.,Sittiprapaporn, W.,Cheatham, C. L.,Gustafson, K. M. (2017). Event-related potential differences in children supplemented with long-chain polyunsaturated fatty acids during infancy Dev Sci, 20(5), \#Pages\#

325 Libuda, L.,Hilbig, A.,Berber-Al-Tawil, S.,Kalhoff, H.,Kersting, M. (2018). Association between full breastfeeding, timing of complementary food introduction, and iron status in infancy in Germany: results of a secondary analysis of a randomized trial Eur $\mathrm{J}$ Nutr, 57(2), 523-531

326 Lichtman-Sadot, S.,Bell, N. P. (2017). Child Health in Elementary School Following California's Paid Family Leave Program J Policy Anal Manage, 36(4), 790-827 327 Lifschitz, C. (2016). Can we do something in early life to reduce the risk of obesity? Iranian journal of neonatology, 7(2), 16-19

Intervention/exposure

328 Lind, Mads V.,Larnkjær, Anni,Mølgaard, Christian,Michaelsen, Kim F. (2017). Early Nutrition and Its Effect on Growth, Body Composition, and Later Obesity World Review of Nutrition \& Dietetics, 116(\#issue\#), 118-133

Publication status

329 Liotto, N.,Orsi, A.,Menis, C.,Piemontese, P.,Morlacchi, L.,Condello, C. C.,Gianni, M. L.,Roggero, P.,Mosca, F. (2018). Clinical evaluation of two different protein content formulas fed to full-term healthy infants: a randomized controlled trial BMC Pediatr, 18(1), 
331 Liu, J. X.,Xu, X.,Liu, J. H.,Hardin, J. W.,Li, R. (2018). Association of maternal gestational weight gain with their offspring's anthropometric outcomes at late infancy and 6 years old: mediating roles of birth weight and breastfeeding duration Int $\mathrm{J}$ Obes (Lond), 42(1), 8-14

\section{Liu, J.,Liu, J.,Frongillo, E. A., Jr.,Boghossian, N. S.,Cai, B.,Zhou, H.,Hazlett, L. J. (2019). Body mass index trajectories during the first Participant age} year of life and their determining factors Am J Hum Biol, 31(1), e23188

333 Liu, Q.,Wang, W.,Jing, W. (2019). Indoor air pollution aggravates asthma in Chinese children and induces the changes in serum level Outcome of miR-155 Int J Environ Health Res, 29(1), 22-30

334 Logan, C. A.,Brandt, S.,Wabitsch, M.,Brenner, H.,Wiens, F.,Stahl, B.,Marosvolgyi, T.,Decsi, T.,Rothenbacher, D.,Genuneit, J. (2017). New approach shows no association between maternal milk fatty acid composition and childhood wheeze or asthma Allergy, 72(9), 1374-1383 concentration in human breast milk and its potential role in child atopic dermatitis: Results of the Ulm Birth Cohort Studies Clin Exp Allergy, 49(2), 199-206

336 Lonnerdal, B.,Kvistgaard, A. S.,Peerson, J. M.,Donovan, S. M.,Peng, Y. M. (2016). Growth, Nutrition, and Cytokine Response of Breast-fed Infants and Infants Fed Formula With Added Bovine Osteopontin J Pediatr Gastroenterol Nutr, 62(4), 650-7

337 Lossius, A. K.,Magnus, M. C.,Lunde, J.,Stordal, K. (2018). Prospective Cohort Study of Breastfeeding and the Risk of Childhood Asthma J Pediatr, 195(\#issue\#), 182-189.e2

338 Love, T. M. T.,Thurston, S. W.,Davidson, P. W. (2017). Finding vulnerable subpopulations in the Seychelles Child Developmen Study: Effect modification with latent groups Statistical Methods in Medical Research, 26(2), 809-822 Matter Volume J Am Acad Child Adolesc Psychiatry, 55(5), 367-75

340 Luecken, L. J.,Jewell, S. L.,MacKinnon, D. P. (2017). Maternal acculturation and the growth of impoverished Mexican American infants Obesity (Silver Spring), 25(2), 445-451

341 Lund-Blix, N. A.,Dydensborg Sander, S.,Stordal, K.,Nybo Andersen, A. M.,Ronningen, K. S.,Joner, G.,Skrivarhaug, T.,Njolstad, P. R.,Husby, S.,Stene, L. C. (2017). Infant Feeding and Risk of Type 1 Diabetes in Two Large Scandinavian Birth Cohorts Diabetes Care, 40(7), 920-927

\section{M. R, Perkin,K, Logan,A, Tseng (2016). Randomized Trial of Introduction of Allergenic Food in Breast-Fed Infants Journal of Clinical Outcome} Chiropractic Pediatrics, 15(3), 1331-1332

343 Mannan, H. (2018). Early Infant Feeding of Formula or Solid Foods and Risk of Childhood Overweight or Obesity in a Socioeconomically Disadvantaged Region of Australia: A Longitudinal Cohort Analysis Int J Environ Res Public Health, 15(8), \#Pages\# 
344 Marell Hesla, H.,Stenius, F.,Järnbert-Pettersson, H.,Alm, J. (2017). Allergy-related disease in relation to early life exposures-the ALADDIN birth cohort Journal of Allergy and Clinical Immunology, 139(2), 686-688

345 Marques, R. C.,Abreu, L.,Bernardi, J. V.,Dorea, J. G. (2016). Traditional living in the Amazon: Extended breastfeeding, fish consumption, mercury exposure and neurodevelopment Ann Hum Biol, 43(4), 360-70

346 Martens, P. J.,Shafer, L. A.,Dean, H. J.,Sellers, E. A.,Yamamoto, J.,Ludwig, S.,Heaman, M.,Phillips-Beck, W.,Prior, H. J.,Morris, M.,McGavock, J.,Dart, A. B.,Shen, G. X. (2016). Breastfeeding Initiation Associated With Reduced Incidence of Diabetes in Mothers and Offspring Obstet Gynecol, 128(5), 1095-1104 S18

348 Martin, C. R. (2019). Breast Milk Lipidomics: Insights to Infant Health Requirements and Targeted Strategies for the Vulnerable Breastfeed Med, 14(S1), S13-s14

349 Mascheretti, S.,Trezzi, V.,Giorda, R.,Boivin, M.,Plourde, V.,Vitaro, F.,Brendgen, M.,Dionne, G.,Marino, C. (2017). Complex effects of dyslexia risk factors account for ADHD traits: evidence from two independent samples J Child Psychol Psychiatry, 58(1), 75-82

350 Mastroeni, M. F.,Mastroeni, Ssbs,Czarnobay, S. A.,Ekwaru, J. P.,Loehr, S. A., Veugelers, P. J. (2017). Breast-feeding duration for the prevention of excess body weight of mother-child pairs concurrently: a 2-year cohort study Public Health Nutr, 20(14), 2537-2548

351 Matro, R.,Martin, C. F.,Wolf, D.,Shah, S. A.,Mahadevan, U. (2018). Exposure Concentrations of Infants Breastfed by Women Receiving Biologic Therapies for Inflammatory Bowel Diseases and Effects of Breastfeeding on Infections and Development Gastroenterology, 155(3), 696-704

352 McCallister, M.,Medrano, R.,Wojcicki, J. (2018). Early life obesity increases the risk for asthma in San Francisco born Latina girls Allergy Asthma Proc, 39(4), 273-280

353 McCarthy, E. K.,ní Chaoimh, C.,Hourihane, J. O. B.,Kenny, L. C.,Irvine, A. D.,Murray, D. M.,Kiely, M. (2017). Iron intakes and status of 2-year-old children in the Cork BASELINE Birth Cohort Study Maternal and Child Nutrition, 13(3), \#Pages\#

Outcome

\section{Outcome}

\section{Outcome}

Study design

Intervention/exposure

Outcome

No key confounders

accounted for,

Intervention/exposure

Intervention/exposure

Study design,

Intervention/exposure

\begin{tabular}{ll}
\hline 354 & Mclntyre, L. M.,Griffen, A. M.,BrintzenhofeSzoc, K. (2018). Breast Is Best . . . Except When It's Not J Hum Lact, 34(3), 575-580 \\
\hline 355 & $\begin{array}{l}\text { McKinlay, C.,Okesene-Gafa, K., Taylor, R.,Wall, C., Rush, E.,McCowan, M., Thompson, J., Crowther, C.,McCowan, L. (2019). Dietary } \\
\text { intervention and/or probiotic capsules in obese pregnant women and infant growth and feeding at } 5 \text { months: healthy mums and } \\
\text { babies (humba) trial Journal of paediatrics and child health, 55(\#issue\#), 35- }\end{array}$
\end{tabular}

Study design

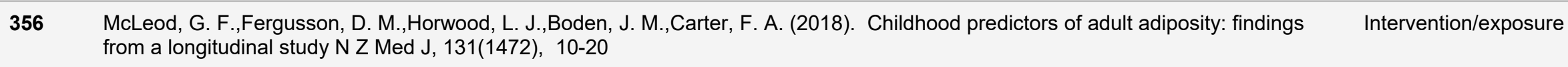

357 Mennella, J. A.,Inamdar, L.,Pressman, N.,Schall, J. I.,Papas, M. A.,Schoeller, D.,Stallings, V. A., Trabulsi, J. C. (2018). Type of infant Intervention/exposure formula increases early weight gain and impacts energy balance: a randomized controlled trial Am J Clin Nutr, 108(5), 1015-1025 
359 Meyer, D. M.,Brei, C.,Stecher, L.,Much, D.,Brunner, S.,Hauner, H. (2019). Associations between long-chain PUFAs in maternal blood, cord blood, and breast milk and offspring body composition up to 5 years: follow-up from the INFAT study Eur J Clin Nutr, 73(3), 458-464

360 Michael, N.,Gupta, V.,Sadananthan, S. A.,Sampathkumar, A.,Chen, L.,Pan, H.,Tint, M. T.,Lee, K. J.,Loy, S. L.,Aris, I. M.,Shek, L. P.,Yap, F. K. P.,Godfrey, K. M.,Leow, M. K.,Lee, Y. S.,Kramer, M. S.,Henry, C. J.,Fortier, M. V.,Seng Chong, Y.,Gluckman, P. D.,Karnani, N.,Velan, S. S. (2019). Determinants of intramyocellular lipid accumulation in early childhood Int J Obes (Lond),

\#volume\#(\#issue\#), \#Pages\#

361 Michaliszyn, S. F.,Sjaarda, L. A.,Scifres, C.,Simhan, H.,Arslanian, S. A. (2017). Maternal excess gestational weight gain and infant waist circumference: a 2-y observational study Pediatr Res, 81(1-1), 63-67

No key confounders accounted for,

Intervention/exposure

362 Michaliszyn, Sara F.,Sjaarda, Lindsey A.,Scifres, Christina,Simhan, Hyagriv,Arslanian, Silva A. (2016). Maternal excess gestational Intervention/exposure weight gain and infant waist circumference: a 2-y observational study Pediatric Research, \#volume\#(\#issue\#), N.PAG-N.PAG

363 Michels, K. A.,Ghassabian, A.,Mumford, S. L.,Sundaram, R.,Bell, E. M. (2018). Breastfeeding and motor development in term and preterm infants in a longitudinal US cohort Journal of Clinical Chiropractic Pediatrics, 17(2), 1467-1467

364 Michels, K. A.,Ghassabian, A.,Mumford, S. L.,Sundaram, R.,Bell, E. M.,Bello, S. C., Yeung, E. H. (2017). Breastfeeding and motor development in term and preterm infants in a longitudinal US cohort Am J Clin Nutr, 106(6), 1456-1462

365 Michie, C. (2016). How to reduce the risks associated with Vitamin D self-supplementation Clinical Pharmacist, 8(5), \#Pages\#

Publication status

366 Mihalopoulos, N. L.,Urban, B. M.,Metos, J. M.,Balch, A. H.,Young, P. C.,Jordan, K. C. (2017). Breast-feeding, Leptin:Adiponectin Ratio, and Metabolic Dysfunction in Adolescents with Obesity South Med J, 110(5), 347-352

Study design, Participant health

367 Miklavcic, J. J.,Larsen, B. M.,Mazurak, V. C.,Scalabrin, D. M.,MacDonald, I. M.,Shoemaker, G. K.,Casey, L., Van Aerde, J. E.,Clandinin, M. T. (2017). Reduction of Arachidonate Is Associated With Increase in B-Cell Activation Marker in Infants: A Intervention/exposure Randomized Trial J Pediatr Gastroenterol Nutr, 64(3), 446-453

368 Minchin, M. (2016). Still LEAPing to wrong conclusions? Breastfeed Rev, 24(2), 7-10

Publication status

369 Mohamad, M.,Loy, S. L.,Lim, P. Y.,Wang, Y.,Soo, K. L.,Mohamed, H. J. J. (2018). Maternal Serum and Breast Milk Adiponectin: The Intervention/exposure Association with Infant Adiposity Development Int J Environ Res Public Health, 15(6), \#Pages\#

370 Moore, Alison (2019). The role of breastmilk in body composition World of Irish Nursing \& Midwifery, 27(5), 59-59

Publication status

371 Moore, B. F.,Sauder, K. A.,Starling, A. P.,Ringham, B. M.,Glueck, D. H.,Dabelea, D. (2017). Exposure to secondhand smoke, exclusive breastfeeding and infant adiposity at age 5 months in the Healthy Start study Pediatr Obes, 12 Suppl 1(\#issue\#), 111-119

Study design Intervention/exposure

372 Morris, Alan (2018). Risk factors: Breastfeeding reduces risk of type 2 diabetes mellitus Nature Reviews Endocrinology, 14(3), 128128

Publication status 
373 Mukheriee, N.,Sutter, T. R.,Arshad, S. H.,Holloway, J. W.,Zhang, H.,Karmaus, W. (2018). Breastfeeding duration modifies the effect of smoking during pregnancy on eczema from early childhood to adolescence Clinical and Experimental Allergy, 48(12), 1688-1697

374 Munhoz, T. N.,Santos, I. S.,Karam, S. M.,Martines, J.,Pelto, G.,Barcelos, R.,,Goncalves, H., Valle, N. C.,Anselmi, L.,Matijasevich, A (2017). Effect of childhood nutrition counselling on intelligence in adolescence: a 15-year follow-up of a cluster-randomised trial Public Health Nutr, 20(11), 2034-2041

375 Musaad, S. M.,Donovan, S. M.,Fiese, B. H. (2016). The Independent and Cumulative Effect of Early Life Risk Factors on Child Growth: A Preliminary Report Child Obes, 12(3), 193-201

376 Naik, P.,Faridi, M. M. A.,Batra, P.,Madhu, S. V. (2017). Oral Supplementation of Parturient Mothers with Vitamin D and Its Effect on 25OHD Status of Exclusively Breastfed Infants at 6 Months of Age: A Double-Blind Randomized Placebo Controlled Trial Breastfeed Med, 12(10), 621-628

377 Nakano, S.,Suzuki, M.,Minowa, K.,Hirai, S.,Takubo, N.,Sakamoto, Y.,Ishijima, M.,Hoshino, E.,Tokita, A.,Shimizu, T. (2018). Current Vitamin D Status in Healthy Japanese Infants and Young Children J Nutr Sci Vitaminol (Tokyo), 64(2), 99-105

378 Nascimento, Jxpt,Ribeiro, C. C. C.,Batista, R. F. L.,de Britto Alves, Mtss,Simoes, V. M. F.,Padilha, L. L., Cardoso, V. C., Vianna, E. O.,Bettiol, H.,Barbieri, M. A.,Silva, Aamd (2017). The First 1000 Days of Life Factors Associated with "Childhood Asthma Symptoms": Brisa Cohort, Brazil Sci Rep, 7(1), 16028

379 Navarrete, M. A.,Silva, J. R.,Van ljzendoorn, M. H.,Carcamo, R. A. (2018). Physical and psychosocial development of Mapuche and nonindigenous Chilean toddlers: A modest role of ethnicity Dev Psychopathol, 30(5), 1959-1976

380 Nazeri, P. (2018). Lactating mothers and infants residing in an area with effective salt iodization program have no need for iodine supplements: results from a doubleblind, placebo-controlled, randomized clinical trial Breastfeeding medicine. Conference: 19th international society for research in human milk and lactation conference, ISRHML 2018. Japan, 13(7), A12-A13

381 Nazeri, P.,Mirmiran, P.,Tahmasebinejad, Z.,Hedayati, M.,Delshad, H.,Azizi, F. (2017). The Effects of lodine Fortified Milk on the lodine Status of Lactating Mothers and Infants in an Area with a Successful Salt lodization Program: A Randomized Controlled Trial Nutrients, 9(2), \#Pages\#

382 Nazeri, P.,Tahmasebinejad, Z.,Mehrabi, Y.,Hedayati, M.,Mirmiran, P.,Azizi, F. (2018). Lactating Mothers and Infants Residing in an Area with an Effective Salt lodization Program Have No Need for lodine Supplements: Results from a Double-Blind, PlaceboControlled, Randomized Controlled Trial Thyroid, 28(11), 1547-1558

383 Newman, K.,O'Donovan, K.,Bear, N.,Robertson, A.,Mutch, R.,Cherian, S. (2019). Nutritional assessment of resettled paediatric refugees in Western Australia J Paediatr Child Health, 55(5), 574-581

384 Niinisto, S.,Takkinen, H. M.,Erlund, I.,Ahonen, S.,Toppari, J.,Ilonen, J.,Veijola, R.,Knip, M.,Vaarala, O.,Virtanen, S. M. (2017). Fatty acid status in infancy is associated with the risk of type 1 diabetes-associated autoimmunity Diabetologia, 60(7), 1223-1233

385 Nobre, L. N.,Lessa, A. D. (2016). Influence of breastfeeding in the first months of life on blood pressure levels of preschool children J Outcome Pediatr (Rio J), 92(6), 588-594

386 Norman, M. (2017). Breastfeeding and outcome Acta Paediatrica, International Journal of Paediatrics, 106(3), 516 

Practices, and Subsequent Weight Matern Child Health J, 22(12), 1805-1814

389

O'Donovan, S. M.,O'B Hourihane J,Murray, D. M.,Kenny, L. C.,Khashan, A. S.,Chaoimh, C. N.,Irvine, A. D.,Kiely, M. (2016) Neonatal adiposity increases the risk of atopic dermatitis during the first year of life J Allergy Clin Immunol, 137(1), 108-117

390 Ohlendorf, J. M.,Robinson, K.,Garnier-Villarreal, M. (2019). The impact of maternal BMI, gestational weight gain, and breastfeeding on early childhood weight: Analysis of a statewide WIC dataset Prev Med, 118(\#issue\#), 210-215

391 Olaya, G. A.,Lawson, M.,Fewtrell, M. (2017). Iron Status at Age 6 Months in Colombian Infants Exclusively Breast-fed for 4 to 5 Versus 6 Months J Pediatr Gastroenterol Nutr, 64(3), 465-471

392 Olaya, G.,Buitrago, M. F.,Fewtrell, M. (2018). Randomised trial testing new complementary feeding guidelines: effects on food consumption and growth at 6 years of age Journal of pediatric gastroenterology and nutrition, 66(\#issue\#), 1160393 Olson, J. S.,Hayward, M. D. (2017). Breastfeeding, overweight status, and inflammation Soc Sci Res, 64(\#issue\#), 226-236

394 Oppenheimer, J. J.,Marshall, G. D. (2017). Increasing our knowledge base of asthma Annals of Allergy, Asthma and Immunology, $119(6), 476-479$ Orengul, A. C.,Tarakcioglu, M. C.,Gormez, V.,Akkoyun, S.,Zorlu, A.,Aliyeva, N.,Uzuner, S.,Caliskan, Y.,Bikmazer, A. (2019). Duration of Breastfeeding, Bottle-Feeding, and Parafunctional Oral Habits in Relation to Anxiety Disorders Among Children Breastfeed Med, 14(1), 57-62

396 Oropeza-Ceja, L. G.,Rosado, J. L.,Ronquillo, D.,Garcia, O. P.,Caamano, M. D. C.,Garcia-Ugalde, C.,Viveros-Contreras, R.,DuarteVazquez, M. A. (2018). Lower Protein Intake Supports Normal Growth of Full-Term Infants Fed Formula: A Randomized Controlled Trial Nutrients, 10(7), \#Pages\#

397 Ortelan, N.,Augusto, R. A.,Souza, J. M. P. (2019). Factors associated with the evolution of weight of children in a supplementary feeding program Rev Bras Epidemiol, 22(\#issue\#), e190002 O'Sullivan, Siobhan (2018). Breastfeeding infants with type 1 diabetes World of Irish Nursing \& Midwifery, 26(6), 63-64

399 Ou, X.,Andres, A.,Pivik, R. T.,Cleves, M. A.,Snow, J. H.,Ding, Z.,Badger, T. M. (2016). Voxel-Based Morphometry and fMRI Revealed Differences in Brain Gray Matter in Breastfed and Milk Formula-Fed Children AJNR Am J Neuroradiol, 37(4), 713-9

Duplicate from 1980 to 2016 search Outcome

Publication status Study design Intervention/exposure

Intervention/exposure

Intervention/exposure

Intervention/exposure

Publication status

tervention/exposure,

Owen, C. G.,Oken, E.,Rudnicka, A. R.,Patel, R.,Thompson, J.,Rifas-Shiman, S. L.,Vilchuck, K.,Bogdanovich, N.,Hameza, M.,Kramer, Outcome M. S.,Martin, R. M. (2018). The effect of longer-term and exclusive breastfeeding promotion on visual outcome in adolescence Investigative Ophthalmology and Visual Science, 59(7), 2670-2678 trajectories are modifiable through early-life intervention and predict asthma in adolescence Pediatr Allergy Immunol, 29(6), 612-621
Outcome 
402 Ozcan, A.,Kendirci, M.,Kondolot, M.,Kardas, F.,Akin, L. (2017). Evaluation of vitamin D prophylaxis in 3-36-month-old infants and children J Pediatr Endocrinol Metab, 30(5), 543-549

403 Panagiotopoulos, C.,Hadjiyannakis, S.,Henderson, M. (2018). Type 2 Diabetes in Children and Adolescents Canadian Journal of Diabetes, 42(\#issue\#), S247-S254

404 Pang, W. W.,Tan, P. T.,Cai, S.,Fok, D.,Chua, M. C.,Lim, S. B.,Shek, L. P.,Chan, S. Y.,Tan, K. H.,Yap, F.,Gluckman, P. D.,Godfrey, K M.,Meaney, M. J.,Broekman, B. F. P.,Kramer, M. S.,Chong, Y. S.,Rifkin-Graboi, A. (2019). Nutrients or nursing? Understanding how breast milk feeding affects child cognition Eur J Nutr, \#volume\#(\#issue\#), \#Pages\#

405 Paolella, Giulia,Vajro, Pietro (2016). Childhood Obesity, Breastfeeding, Intestinal Microbiota, and Early Exposure to Antibiotics JAMA Pediatrics, 170(8), 735-737

406 Park, A. L.,Tu, K.,Ray, J. G. (2017). Differences in growth of Canadian children compared to the WHO 2006 Child Growth Standards Paediatr Perinat Epidemiol, 31(5), 452-462

407 Park, S. H.,Ha, E.,Hong, Y. S.,Park, H. (2016). Serum Levels of Persistent Organic Pollutants and Insulin Secretion among Children Age 7-9 Years: A Prospective Cohort Study Environ Health Perspect, 124(12), 1924-1930

408 Park, S. H.,Ha, E.,Hong, Y. S.,Park, H. (2016). Serum levels of persistent organic pollutants and insulin secretion among children age 7-9 years: A prospective cohort study Environmental Health Perspectives, 124(12), 1924-1930

409 Park, S. J.,Lee, H. J. (2018). Exclusive breastfeeding and partial breastfeeding reduce the risk of overweight in childhood: A nationwide longitudinal study in Korea Obes Res Clin Pract, 12(2), 222-228

410 Parkin, P. C.,DeGroot, J.,Maguire, J. L.,Birken, C. S.,Zlotkin, S. (2016). Severe iron-deficiency anaemia and feeding practices in young children Public Health Nutr, 19(4), 716-22

411 Parrino, C.,Vinciguerra, F.,La Spina, N.,Romeo, L.,Tumminia, A.,Baratta, R.,Squatrito, S.,Vigneri, R.,Frittitta, L. (2016). Influence of early-life and parental factors on childhood overweight and obesity J Endocrinol Invest, 39(11), 1315-1321

412 Patel, N.,Dalrymple, K. V.,Briley, A. L.,Pasupathy, D.,Seed, P. T.,Flynn, A. C.,Poston, L. (2018). Mode of infant feeding, eating behaviour and anthropometry in infants at 6-months of age born to obese women - a secondary analysis of the UPBEAT trial BMC Pregnancy Childbirth, 18(1), 355

413 Patel, N.,Godfrey, K. M.,Pasupathy, D.,Levin, J.,Flynn, A. C.,Hayes, L.,Briley, A. L.,Bell, R.,Lawlor, D. A.,Oteng-Ntim, E.,Nelson, S. M.,Robson, S. C.,Sattar, N.,Singh, C.,Wardle, J.,White, S. L.,Seed, P. T.,Poston, L. (2017). Infant adiposity following a randomised controlled trial of a behavioural intervention in obese pregnancy International Journal of Obesity, 41(7), $1018-1026$

414 Pattemore, P. K.,Silvers, K. M.,Frampton, C. M.,Wickens, K.,Ingham, T.,Fishwick, D.,Crane, J.,Town, G. I.,Epton, M. J. (2018). Hair nicotine at 15 months old, tobacco exposure and wheeze or asthma from 15 months to 6 years old Pediatr Pulmonol, 53(4), 443-451

415 Patterson, A. C.,Maditz, K. H.,Harris, C.,Wampler, J.,Kirchoff, A.,Zissman, E.,Berseth, C. L. (2016). Growth and tolerance of a routine infant formula with an alternative DHA source fed to term infants FASEB journal. Conference: experimental biology 2016, EB. San diego, CA united states. Conference start: 20160402. Conference end: 20160406. Conference publication: (var.pagings), 30(no pagination), \#Pages\# 
416 Pattison, Krista L.,Kraschnewski, Jennifer L.,Lehman, Erik,Savage, Jennifer S.,Downs, Danielle Symons,Leonard, Krista S.,Adams, Elizabeth L.,Paul, Ian M.,Kjerulff, Kristen H. (2018). Breastfeeding initiation and duration and child health outcomes in the first baby study Preventive Medicine, 115(\#issue\#), N.PAG-N.PAG

417 Pauwels, S.,Symons, L., Vanautgaerden, E. L.,Ghosh, M.,Duca, R. C.,Bekaert, B.,Freson, K.,Huybrechts, I.,Langie, S. A. S.,Koppen G.,Devlieger, R.,Godderis, L. (2019). The Influence of the Duration of Breastfeeding on the Infant's Metabolic Epigenome Nutrients, 11(6), \#Pages\#

418 Pennestri, M. H.,Laganiere, C.,Bouvette-Turcot, A. A.,Pokhvisneva, I.,Steiner, M.,Meaney, M. J.,Gaudreau, H. (2018). Uninterrupted Infant Sleep, Development, and Maternal Mood Pediatrics, 142(6), \#Pages\#

419 Penny, M. E.,Jimenez, M. M.,Marin, R. M. (2016). Early rapid weight gain and subsequent overweight and obesity in middle childhood in Peru BMC Obes, 3(\#issue\#), 55

420 Perez-Gaxiola, G. (2016). Increased bottle size was associated with increased weight gain in infants Arch Dis Child Educ Pract Ed, 101(5), 280

421 Perkin, M. R.,Logan, K.,Tseng, A.,Raji, B.,Ayis, S.,Peacock, J.,Brough, H.,Marrs, T.,,Radulovic, S.,Craven, J.,Flohr, C.,Lack, G. (2016). Randomized Trial of Introduction of Allergenic Foods in Breast-Fed Infants N Engl J Med, 374(18), 1733-43

Duplicate within 2016 to 2019 search

No key confounders accounted for

Intervention/exposure

Intervention/exposure

Study design

Intervention/exposure

Outcome

422 Peters, R. L., Koplin, J. J.,Dharmage, S. C., Tang, M. L. K.,McWilliam, V. L., Gurrin, L. C.,Neeland, M. R.,Lowe, A. J.,Ponsonby, A L.,Allen, K. J. (2019). Early Exposure to Cow's Milk Protein Is Associated with a Reduced Risk of Cow's Milk Allergic Outcomes J Allergy Clin Immunol Pract, 7(2), 462-470.e1

423 Phelan, S.,Hagobian, T. A.,Ventura, A.,Brannen, A.,Erickson-Hatley, K.,Schaffner, A.,Muñoz-Christian, K.,Mercado, A.,Tate, D. F. (2019). 'Ripple' effect on infant zBMI trajectory of an internet-based weight loss program for low-income postpartum women Pediatric Obesity, 14(1), N.PAG-N.PAG

424 Philpott, L. (2017). Allergy aware Australian Journal of Pharmacy, 98(1165), 46-50

425 Pihl, Andreas Friis,Fonvig, Cilius Esmann,Stjernholm, Theresa,Hansen, Torben,Pedersen, Oluf,Holm, Jens-Christian (2016). The Role of the Gut Microbiota in Childhood Obesity Childhood Obesity, 12(4), 292-299

426 Pivik, R. T.,Andres, A.,Bai, S.,Cleves, M. A.,Tennal, K. B.,Gu, Y.,Badger, T. M. (2016). Infant Diet-Related Changes in Syllable Processing Between 4 and 5 Months: Implications for Developing Native Language Sensitivity Dev Neuropsychol, 41(4), 215-230

Study design

Intervention/exposure

427 Pivik, R. T.,Andres, A., Tennal, K. B.,Gu, Y.,Downs, H.,Bellando, B. J.,Jarratt, K.,Cleves, M. A.,Badger, T. M. (2019). Resting gamma power during the postnatal critical period for GABAergic system development is modulated by infant diet and sex Int J Psychophysiol, 135(\#issue\#), 73-94 polyunsaturated fatty acids in infant formula and cardiovascular markers in childhood Matern Child Nutr, 14(2), e12523

429 Pluymen, L. P. M.,Wijga, A. H.,Gehring, U.,Koppelman, G. H.,Smit, H. A.,van Rossem, L. (2018). Early introduction of complementary foods and childhood overweight in breastfed and formula-fed infants in the Netherlands: the PIAMA birth cohort study Eur J Nutr, 57(5), 1985-1993 
430 Pluymen, Linda P. M.,Dalmeijer, Geertje W.,Smit, Henriëtte A.,Uiterwaal, Cuno S. P. M.,van Rossem, Lenie,van der Ent, Cornelis K. (2018). Long-chain polyunsaturated fatty acids in infant formula and cardiovascular markers in childhood Maternal \& Child Nutrition, $14(2), 1-1$

431 Polidano, C.,Zhu, A.,Bornstein, J. C. (2017). The relation between cesarean birth and child cognitive development Sci Rep, 7(1) 11483

432 Prpic, I.,Milardovic, A.,Vlasic-Cicvaric, I.,Spiric, Z.,Radic Nisevic, J.,Vukelic, P.,Snoj Tratnik, J.,Mazej, D.,Horvat, M. (2017). Prenata exposure to low-level methylmercury alters the child's fine motor skills at the age of 18 months Environ Res, 152(\#issue\#), 369-374

433 Pruszkowska-Przybylska, P.,Sitek, A.,Rosset, I.,Sobalska-Kwapis, M.,Słomka, M.,Strapagiel, D.,Ż (2018). Association of the 2D:4D digit ratio with body composition among the Polish children aged 6-13years Early Human Development, 124(\#issue\#), 26-32

434 Puccio, G.,Alliet, P.,Cajozzo, C.,Janssens, E.,Corsello, G.,Sprenger, N.,Wernimont, S.,Egli, D.,Gosoniu, L.,Steenhout, P. (2017) Effects of Infant Formula With Human Milk Oligosaccharides on Growth and Morbidity: A Randomized Multicenter Trial J Pediatr Gastroenterol Nutr, 64(4), 624-631

435 Putet, G.,Labaune, J. M.,Mace, K.,Steenhout, P.,Grathwohl, D.,Raverot, V.,Morel, Y.,Picaud, J. C. (2016). Effect of dietary protein on plasma insulin-like growth factor-1, growth, and body composition in healthy term infants: a randomised, double-blind, controlled trial (Early Protein and Obesity in Childhood (EPOCH) study) Br J Nutr, 115(2), 271-84

436 Radke, M. Picaud, J. C.,Loui, A Cambonie, G.,Faas, D. Lafeber, H. N., de Groot, N.,Pecquet, S. S., Steenhout, P. G.,Hascoet, J. M. (2017). Starter formula enriched in prebiotics and probiotics ensures normal growth of infants and promotes gut health: a randomized clinical trial Pediatr Res, 81(4), 622-631

437 Radke, Michael,Picaud, Jean-Charles,Loui, Andrea,Cambonie, Gilles,Faas, Dirk,Lafeber, Harry N.,de Groot, Nanda,Pecquet, Sophie S.,Steenhout, Philippe G.,Hascoet, Jean-Michel (2016). Starter formula enriched in prebiotics and probiotics ensures normal growth of infants and promotes gut health: a randomized clinical trial Pediatric Research, \#volume\#(\#issue\#), N.PAG-N.PAG search

438 Ramos, Jose Geraldo,Strapasson, Márcia Rejane,Ferreira, Charles Francisco (2018). 26. Breastfeeding practices in the first 6 months after delivery: Effects of arterial hypertension Pregnancy Hypertension, 13(\#issue\#), S57-S57

439 Ranucci, G.,Buccigrossi, V.,Borgia, E.,Piacentini, D.,Visentin, F.,Cantarutti, L.,Baiardi, P.,Felisi, M. G.,Spagnuolo, M. I.,Zanconato, S.,et al., (2018). Association between environmental determinants and intestinal microbiota structure in the development of atopic dermatitis in infants at high risk of atopy Journal of pediatric gastroenterology and nutrition, 66(\#issue\#), 1162-

440 Ranucci, G.,Buccigrossi, V.,Borgia, E.,Piacentini, D.,Visentin, F.,Cantarutti, L.,Baiardi, P.,Felisi, M.,Spagnuolo, M. I.,Zanconato,

Outcome S.,Baraldi, E., Giaquinto, C., Guarino, A. (2018). Galacto-Oligosaccharide/Polidextrose Enriched Formula Protects against Respiratory Infections in Infants at High Risk of Atopy: A Randomized Clinical Trial Nutrients, 10(3), \#Pages\#

441 Rao, D. P.,Kropac, E.,Do, M. T.,Roberts, K. C.,Jayaraman, G. C. (2017). Status report -- Childhood overweight and obesity in Canada: an integrative assessment Health Promot Chronic Dis Prev Can, 37(3), 87-93

442 Rauschert, S.,Mori, T. A.,Beilin, L. J.,Jacoby, P.,Uhl, O.,Koletzko, B.,Oddy, W. H.,Hellmuth, C. (2017). Early Life Factors, Obesity Risk, and the Metabolome of Young Adults Obesity (Silver Spring), 25(9), 1549-1555 
443 Rautava, S. (2018). Probiotic Intervention Through the Pregnant and Breastfeeding Mother to Reduce Disease Risk in the Child Breastfeed Med, 13(S1), S14-s15

444 Ray, S.,Seth, A.,Baijal, N.,Singh, S.,Sharma, G.,Kumar, P.,Chandra, J. (2019). Comparison of Feeding Options for HIV-Exposed Infants: A Retrospective Cohort Study Indian Pediatr, 56(6), 476-480

445 Reifsnider, E.,McCormick, D. P.,Cullen, K. W.,Todd, M.,Moramarco, M. W.,Gallagher, M. R.,Reyna, L. (2018). Randomized Controlled Trial to Prevent Infant Overweight in a High-Risk Population Acad Pediatr, 18(3), 324-333

Study design

Country

Country

Intervention/exposure

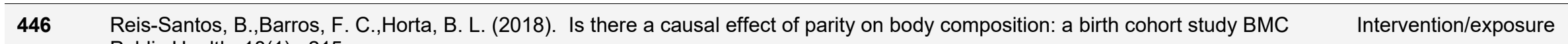
Public Health, 18(1), 215

447 Rejali, M.,Pahlavni, S.,Hassanzadeh, A. (2017). Evaluation of 1-year-old children development in Isfahan City and its effective factors using ages and stages questionnaire, in 2014 J Educ Health Promot, 6(\#issue\#), 57

448 Rendina, D. N.,Blohowiak, S. E.,Coe, C. L.,Kling, P. J. (2018). Maternal Perceived Stress during Pregnancy Increases Risk for Low Neonatal Iron at Delivery and Depletion of Storage Iron at One Year J Pediatr, 200(\#issue\#), 166-173.e2 449 Riano-Galan, I.,Fernandez-Somoano, A., Rodriguez-Dehli, C.,Valvi, D.,Vrijheid, M.,Tardon, A. (2017). Proatherogenic Lipid Profile in
Early Childhood: Association with Weight Status at 4 Years and Parental Obesity J Pediatr, 187(\#issue\#), 153-157.e2

450 Robbins, K. A.,Uygungil, B. (2017). Nutritional Deficiencies and Food Allergy J Allergy Clin Immunol Pract, 5(2), 528-529

Study design

451 Rodriguez-Cano, A. M.,Mier-Cabrera, J.,Allegre-Davalos, A. L.,Munoz-Manrique, C.,Perichart-Perera, O. (2019). Higher fat mass and fat mass accretion during the first six months of life in exclusively breastfed infants Pediatr Res, \#volume\#(\#issue\#), \#Pages\#

No key confounders accounted for

452 Rodriguez-Cano, A. M.,Mier-Cabrera, J.,Munoz-Manrique, C.,Cardona-Perez, A.,Villalobos-Alcazar, G.,Perichart-Perera, O. (2019). Study design Anthropometric and clinical correlates of fat mass in healthy term infants at 6 months of age BMC Pediatr, 19(1), 60

453 Rodríguez-Cano, Ameyalli M.,Mier-Cabrera, Jennifer,Muñoz-Manrique, Cinthya,Cardona-Pérez, Arturo,Villalobos-Alcázar Gicela,Perichart-Perera, Otilia (2019). Anthropometric and clinical correlates of fat mass in healthy term infants at 6 months of age BMC Pediatrics, 19(1), N.PAG-N.PAG

454 Rodriguez-Herrera, A.,Abrahamse-Berkeveld, M.,Alles, M.,Bouritius, H.,Rubio, R. P.,Munoz, A.,Agosti, M.,Lista, G.,Corvaglia, L. T.,Navero, J. L. P. (2016). A partly fermented infant formula containing scGOS/lcFOS supports adequate growth in healthy, term infants: the life study Journal of pediatric gastroenterology and nutrition. Conference: 49th annual meeting of the european society for paediatric gastroenterology, hepatology and nutrition, ESPGHAN 2016. Athens greece. Conference start: 20160525. Conference end: 20160528. Conference publication: (var.pagings), 62(\#issue\#), 658-659

455 Rodriguez-Herrera, A.,Mulder, K.,Bouritius, H.,Rubio, R.,Muñoz, A.,Agosti, M.,Lista, G.,Corvaglia, L.,Ludwig, T.,AbrahamseBerkeveld, M.,Perez-Navero, J. L. (2019). Gastrointestinal tolerance, growth and safety of a partly fermented formula with specific prebiotics in healthy infants: A double-blind, randomized, controlled trial Nutrients, 11(7), \#Pages\#

456 Rodriguez-Lopez, M.,Osorio, L.,Acosta-Rojas, R.,Figueras, J.,Cruz-Lemini, M.,Figueras, F.,Bijnens, B.,Gratacos, E.,Crispi, F. (2016). Influence of breastfeeding and postnatal nutrition on cardiovascular remodeling induced by fetal growth restriction Pediatr Res, 79(11), $100-6$ 

feeding practices in infancy Appetite, 108(\#issue\#), 399-406

458 Rohan, Annie J. (2017). Breastfeeding, Cognitive and Non-Cognitive Development in Early Childhood: A Population Study MCN: The American Journal of Maternal Child Nursing, 42(5), 302-302

459 Rose, C. M.,Savage, J. S.,Birch, L. L. (2016). Patterns of early dietary exposures have implications for maternal and child weight outcomes Obesity (Silver Spring), 24(2), 430-8

460 Ruiz, A. N.,Herrmann, F.,Valbuena, N. S.,Miranda, M. T.,Morera, M.,Folgoso, C. C. (2017). Association of linear growth velocity and Publication status behavior at 18 months of life in healthy children Journal of pediatric gastroenterology and nutrition, 64(\#issue\#), 923-

461 Rzehak, P.,Oddy, W. H.,Mearin, M. L.,Grote, V.,Mori, T. A.,Szajewska, H.,Shamir, R.,Koletzko, S.,Weber, M.,Beilin, L. J.,Huang, R. C.,Koletzko, B. (2017). Infant feeding and growth trajectory patterns in childhood and body composition in young adulthood Am $\mathrm{J}$ Clin Nutr, 106(2), 568-580 Sakihara, T.,Sugiura, S.,Ito, K. (2016). The ingestion of cow's milk formula in the first 3 months of life prevents the development of cow's milk allergy Asia Pac Allergy, 6(4), 207-212

463 Salahuddin, M.,Perez, A., Ranjit, N.,Hoelscher, D. M.,Kelder, S. H. (2017). The associations of large-for-gestational-age and infant feeding practices with children's body mass index z-score trajectories: the Early Childhood Longitudinal Study, Birth Cohort Clin Obes, 7(5), 307-315 Salameh, K.,Dawodu, A. H. (2018). Randomized controlled study of effectiveness and safety of high dose vitamine D supplementation on breast milk v D limited sun Journal of pediatric gastroenterology and nutrition, 66(\#issue\#), 1097-

Publication status

Intervention/exposure

Outcome

(1)

Outcome

Intervention/exposure

Publication status

465 Salas Lorenzo, I.,Chisaguano Tonato, A. M.,de la Garza Puentes, A., Nieto, A., Herrmann, F.,Dieguez, E.,Castellote, A. I.,LopezSabater, M. C.,Rodriguez-Palmero, M.,Campoy, C. (2019). The Effect of an Infant Formula Supplemented with AA and DHA on Fatty Acid Levels of Infants with Different FADS Genotypes: The COGNIS Study Nutrients, 11(3), \#Pages\#

Salimar,,Irawati, A.,Besral, (2019). Maternal height as an determinan
Journal of Public Health Research and Development, 10(3), 765-771

467 Salo, H. M.,Koponen, J.,Kiviranta, H.,Rantakokko, P.,Honkanen, J.,Härkönen, T.,Ilonen, J.,Virtanen, S. M.,Tillmann, V.,Knip, M.,Vaarala, O. (2019). No evidence of the role of early chemical exposure in the development of $\beta$-cell autoimmunity Environmental science and pollution research international, 26(2), 1370-1378

468 Sanchez-Valverde, F.,Etayo, V.,Gil, F.,Aznal, E.,Martinez, D.,Amezqueta, A.,Mendizabal, M.,Galbete, A.,Pastor, N.,,Vanderhoof, J. 179(4), 290-296

469 Santos, I. S.,Barros, F. C.,Munhoz, T.,Matijasevich, A. (2017). Gestational age at birth and behavioral problems from four to 11 years Intervention/exposure of age: birth cohort study BMC Pediatr, 17(1), 184 
470 Santos, L. P.,Assuncao, M. C. F.,Matijasevich, A.,Santos, I. S.,Barros, A. J. D. (2016). Dietary intake patterns of children aged 6 years and their association with socioeconomic and demographic characteristics, early feeding practices and body mass index BMC Public Health, 16(1), 1055

471 Santos, L. P.,Ong, K. K.,Santos, I. S.,Matijasevich, A.,Barros, A. J. D. (2019). Effects of dietary intake patterns from 1 to 4 years on BMI z-score and body shape at age of 6 years: a prospective birth cohort study from Brazil Eur J Nutr, 58(4), 1723-1734

472 Sardecka, I.,Los-Rycharska, E.,Ludwig, H.,Gawryjolek, J.,Krogulska, A. (2018). Early risk factors for cow's milk allergy in children in the first year of life Allergy Asthma Proc, 39(6), e44-e54

473 Sauder, K. A.,Bekelman, T. A.,Harrall, K. K.,Glueck, D. H.,Dabelea, D. (2019). Gestational diabetes exposure and adiposity outcomes in childhood and adolescence: An analysis of effect modification by breastfeeding, diet quality, and physical activity in the EPOCH study Pediatr Obes, \#volume\#(\#issue\#), \#Pages\#

474 Sauder, K. A.,Kaar, J. L.,Starling, A. P.,Ringham, B. M.,Glueck, D. H.,Dabelea, D. (2017). Predictors of Infant Body Composition at 5 Study design Months of Age: The Healthy Start Study J Pediatr, 183(\#issue\#), 94-99.e1

475 Sauder, K. A.,Starling, A. P.,Shapiro, A. L.,Kaar, J. L.,Ringham, B. M.,Glueck, D. H.,Dabelea, D. (2016). Exploring the association between maternal prenatal multivitamin use and early infant growth: The Healthy Start Study Pediatr Obes, 11(5), 434-41

476 Savage, J. S.,Birch, L. L.,Marini, M.,Anzman-Frasca, S.,Paul, I. M. (2016). Effect of the INSIGHT Responsive Parenting Intervention Intervention/exposure on Rapid Infant Weight Gain and Overweight Status at Age 1 Year: A Randomized Clinical Trial JAMA Pediatr, 170(8), 742-9

477 Sbihi, H.,Koehoorn, M.,Tamburic, L.,Brauer, M. (2017). Asthma Trajectories in a Population-based Birth Cohort. Impacts of Air Pollution and Greenness Am J Respir Crit Care Med, 195(5), 607-613

478 Scalabrin, D.,Harris, C.,Johnston, W. H.,Berseth, C. L. (2017). Long-term safety assessment in children who received hydrolyzed protein formulas with Lactobacillus rhamnosus GG: a 5-year follow-up Eur J Pediatr, 176(2), 217-224

479 Schwenke, E.,Fasching, P. A.,Faschingbauer, F.,Pretscher, J.,Kehl, S.,Peretz, R.,Keller, A.,Haberle, L.,Eichler, A.,Irlbauer-Muller V.,Dammer, U.,Beckmann, M. W.,Schneider, M. (2018). Predicting attention deficit hyperactivity disorder using pregnancy and birth characteristics Arch Gynecol Obstet, 298(5), 889-895

480 Scott-Jupp, R. (2017). Breastfeeding and obesity Arch Dis Child, 102(7), 616

481 Sekhobo, J. P. (2017). Estimation of WIC effects in multilevel, cross-sector obesity prevention interventions Obesity, 25(7), 11571158 (2018). Prevalence estimates and risk factors for early childhood wheeze across Europe: the EuroPrevall birth cohort Thorax, 73(11), 1049-1061

483 Sen, S.,Penfield-Cyr, A.,Hollis, B. W.,Wagner, C. L. (2017). Maternal Obesity, 25-Hydroxy Vitamin D Concentration, and Bone Density in Breastfeeding Dyads J Pediatr, 187(\#issue\#), 147-152.e1

Publication status

\section{Outcome}

Intervention/exposure

Outcome

Publication status

\section{Outcome}

Intervention/exposure


484 Seo, S.,Yoon, W. S.,Cho, Y.,Park, S. H.,Choung, J. T.,Yoo, Y. (2016). Leptin and Atopic Dermatitis in Korean Elementary School Children Iran J Allergy Asthma Immunol, 15(2), 138-44

485 Seppo, A. E.,Autran, C. A.,Bode, L.,Järvinen, K. M. (2017). Human milk oligosaccharides and development of cow's milk allergy in infants Journal of Allergy and Clinical Immunology, 139(2), 708-711.e5

486 Shahramian, I.,Kalvandi, G.,Javaherizadeh, H.,Khalili, M.,Noori, N. M.,Delaramnasab, M.,Bazi, A. (2018). The effects of prebiotic supplementation on weight gain, diarrhoea, constipation, fever and respiratory tract infections in the first year of life Journal of Paediatrics and Child Health, 54(8), 875-880

487 Shalitin, Shlomit,Battelino, Tadej,Moreno, Luis A. (2017). Obesity, Metabolic Syndrome, and Nutrition World Review of Nutrition \& Dietetics, 116(\#issue\#), 16-51

488 Sharma, A. K.,Gallo, S.,Vanstone, C. A.,Agellon, S.,L'Abbe, M.,Khamessan, A.,Comeau, K.,Weiler, H. A.,Rodd, C. (2016). Parathyroid hormone-ionized calcium dynamics over the first year of life J Pediatr Endocrinol Metab, 29(6), 709-14

490 Sherwood, W. B.,Bion, V.,Lockett, G. A.,Ziyab, A. H.,Soto-Ramirez, N.,Mukherjee, N.,Kurukulaaratchy, R. J.,Ewart, S.,Zhang, H.,Arshad, S. H.,Karmaus, W.,Holloway, J. W.,Rezwan, F. I. (2019). Duration of breastfeeding is associated with leptin (LEP) DNA methylation profiles and BMI in 10-year-old children Clin Epigenetics, 11(1), 128

491 Shi, J.,Tan, D.,Xie, H.,Yang, B.,Liu, R.,Yu, D.,Lu, Y.,Mei, B.,Wang, Z. (2017). Unequal Distribution of Overweight Adolescents in Immigrant-Rich Areas: Analysis of Disparities among Public and Private School Students in Shanghai, China Int J Environ Res Public Health, 14(3), \#Pages\#

492 Shinn, L. M.,Tangney, C. C.,Busche, C.,Sharp, C. M.,Mullen, M. C. (2018). Demographic Correlates of Infant Feeding Practices and Growth Performance in the First Year of Life Int J Pediatr, 2018(\#issue\#), 6569204

493 Shoaibi, A.,Neelon, B.,Ostbye, T.,Benjamin-Neelon, S. E. (2019). Longitudinal associations of gross motor development, motor milestone achievement and weight-for-length z score in a racially diverse cohort of US infants BMJ Open, 9(1), e024440

Publication status

Intervention/exposure

Study design

No key confounders

accounted for

Study design

Intervention/exposure

Intervention/exposure

494 Sicherer, S. H.,Wood, R. A.,Perry, T. T.,Jones, S. M.,Leung, D. Y. M.,Henning, A. K.,Dawson, P.,Burks, A. W.,Lindblad, R.,Sampson, Outcome H. A. (2019). Clinical factors associated with peanut allergy in a high-risk infant cohort Allergy, \#volume\#(\#issue\#), \#Pages\#

495 Singhal, A. (2019). The Impact of Human Milk Feeding on Long-Term Risk of Obesity and Cardiovascular Disease Breastfeed Med, 14(S1), S9-s10

496 Sinno, D.,Tamim, H.,Faytrouni, F.,Mikati, M. A.,Charafeddine, L. (2018). Factors affecting child development assessed by the Ages and Stages Questionnaire (ASQ) in an Arabic speaking population Early Hum Dev, 120(\#issue\#), 61-66

Study design

Study design Intervention/exposure

497 Sirkka, O.,Vrijkotte, T.,Halberstadt, J.,Abrahamse-Berkeveld, M.,Hoekstra, T.,Seidell, J.,Olthof, M. (2018). Prospective associations of age at complementary feeding and exclusive breastfeeding duration with body mass index at 5-6 years within different risk groups Pediatr Obes, 13(8), 522-529 
498 Sitarik, A. R.,Kasmikha, N. S.,Kim, H.,Wegienka, G.,Havstad, S.,Ownby, D.,Zoratti, E.,Johnson, C. C. (2018). Breast-feeding and Sitarik, A. R.,Kasmikha, N. S.,Kim, H.,Wegienka, G.,Havstad, S.,Ownby, D.,Zoratti, E.,Johnson, C. C. (2018). Breast-feeding
delivery mode modify the association between maternal atopy and childhood allergic outcomes Journal of Allergy and Clinical Immunology, 142(6), 2002-2004.e2

499

500 Slomski, A. (2018). Neurocognitive Benefits from Breastfeeding May Not Endure Jama, 319(24), 2470

Slupsky, C. M.,He, X.,Hernell, O.,Andersson, Y.,Rudolph, C.,Lonnerdal, B.,West, C. E. (2017). Postprandial metabolic response of breast-fed infants and infants fed lactose-free vs regular infant formula: A randomized controlled trial Sci Rep, 7(1), 3640

\section{Publication status}

Intervention/exposure, Group size/power

\section{Outcome}

Soto-Ramirez, N.,Kar, S.,Zhang,
Exp Allergy, 47(10), 1285-1298

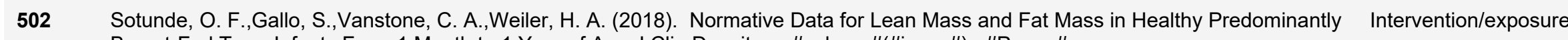
Breast-Fed Term Infants From 1 Month to 1 Year of Age J Clin Densitom, \#volume\#(\#issue\#), \#Pages\#

503 Souza, C. O.,Leite, M. E. Q.,Lasekan, J.,Baggs, G.,Pinho, L. S.,Druzian, J. I.,Ribeiro, T. C. M.,Mattos, A. P.,Menezes-Filho, J. A.,Costa-Ribeiro, H. (2017). Milk protein-based formulas containing different oils affect fatty acids balance in term infants: $A$ randomized blinded crossover clinical trial Lipids Health Dis, 16(1), 78

504 Spalinger, J.,Nydegger, A.,Belli, D.,Furlano, R. I.,Yan, J.,Tanguy, J.,Pecquet, S.,Destaillats, F.,Egli, D., Steenhout, P. (2017). Growth of infants fed formula with evolving nutrition composition: A single-arm non-inferiority study Nutrients, 9(3), \#Pages\#

505 St John, A. M.,Kao, K.,Liederman, J.,Grieve, P. G.,Tarullo, A. R. (2017). Maternal cortisol slope at 6 months predicts infant cortiso slope and EEG power at 12 months Developmental psychobiology, 59(6), 787-801

506 Standl, M.,Schulte-Korne, G.,Heinrich, J. (2016). Breastfeeding and symptoms of dyslexia in children and adolescents European journal of epidemiology, Conference: Health - Exploring Complexity: An Interdisciplinary Systems Approach, HEC 2016. Germany. Conference Start: 20160828. Conference End: 20160902. 31(\#issue\#), S193-S194

507 Stanford, F. C. (2016). Obesity and Breastfeeding: Exploring the Relationship Breastfeed Med, 11(\#issue\#), 411-2

508 Stelmach, I.,Kwarta, P.,Jerzynska, J.,Stelmach, W.,Krakowiak, J.,Karbownik, M.,Podlecka, D.,Hanke, W.,Polanska, K. (2019). Duration of breastfeeding and psychomotor development in 1-year-old children - Polish Mother and Child Cohort Study Int J Occup Med Environ Health, 32(2), 175-184

509 Stemeseder, T.,Klinglmayr, E.,Moser, S.,Lang, R.,Himly, M.,Oostingh, G. J.,Zumbach, J.,Bathke, A. C.,Hawranek, T.,Gadermaier, G. (2017). Influence of Intrinsic and Lifestyle Factors on the Development of IgE Sensitization Int Arch Allergy Immunol, 173(2), 99-104

510 Stergiakouli, E.,Martin, J.,Hamshere, M.,St Pourcain, B.,Timpson, N.,Thapar, A.,Smith, G. D. (2017). Shared genetic effects between clinical ADHD and smoking, alcohol and breastfeeding in mothers from the general population European neuropsychopharmacology, 27(\#issue\#), S141-

511 Stranak, Z.,Feyereislova, S.,Cerna, M.,Kollarova, J.,Feyereisl, J. (2016). Limited Amount of Formula May Facilitate Breastfeeding: Randomized, Controlled Trial to Compare Standard Clinical Practice versus Limited Supplemental Feeding PLoS One, 11(2), e0150053
Intervention/exposure, Group size/power

Intervention/exposure

Intervention/exposure

Publication status

Study design

Study design

\section{Outcome}

Outcome
Publication status 
Straub, N.,Grunert, P.,Northstone, K.,Emmett, P. (2016). Economic impact of breast-feeding-associated improvements of childhood cognitive development, based on data from the ALSPAC Br J Nutr, \#volume\#(\#issue\#), 1-6

513 Strom, M.,Mortensen, E. L.,Kesmodel, U. S.,Halldorsson, T.,Olsen, J.,Olsen, S. F. (2019). Is breast feeding associated with offspring Outcome IQ at age 5? Findings from prospective cohort: Lifestyle During Pregnancy Study BMJ Open, 9(5), e023134

514 Stromberg Celind, F.,Wennergren, G.,Vasileiadou, S.,Alm, B.,Goksor, E. (2018). Antibiotics in the first week of life were associated with atopic asthma at 12 years of age Acta Paediatr, 107(10), 1798-1804

Intervention/exposure,

Outcome

515 Stuart, B.,Panico, L. (2016). Early-childhood BMI trajectories: evidence from a prospective, nationally representative British cohort Outcome study Nutr Diabetes, 6(\#issue\#), e198

516 Subhan, F. B.,Colman, I.,McCargar, L.,Bell, R. C. (2017). Higher Pre-pregnancy BMl and Excessive Gestational Weight Gain are Risk Factors for Rapid Weight Gain in Infants Matern Child Health J, 21(6), 1396-1407

No key confounders accounted for,

Intervention/exposure

517 Sukumar, N.,Saravanan, P. (2019). Investigating vitamin B12 deficiency BMJ (Online), 365(\#issue\#), \#Pages\# Study design

518 Sutin, A. R.,Stephan, Y.,Terracciano, A. (2016). Breastfeeding and Adult Personality Eur J Pers, 30(5), 484-491

Outcome

519 Szabelska-Zakrzewska, K.,Durko, A.,Socha-Banasiak, A.,Majewska, M.,Kolejwa, M.,Kazanek-Zasada, J.,Czkwianianc, E. (2018). Metabolic syndrome in overweight or obese children and adolescents based on own material Abstract Key words Dev Period Med, 22(4), 351-357

520 Szajewska, H.,Ruszczynski, M.,Szymanski, H.,Sadowska-Krawczenko, I.,Piwowarczyk, A.,Rasmussen, P. B.,Kristensen, M. B.,West, C. E.,Hernell, O. (2017). Effects of infant formula supplemented with prebiotics compared with synbiotics on growth up to the age of 12 mo: a randomized controlled trial Pediatr Res, 81(5), 752-758

521 Szymlek-Gay, E. A.,Domellof, M.,Hernell, O.,Hurrell, R. F.,Lind, T.,Lonnerdal, B.,Zeder, C.,Egli, I. M. (2016). Mode of oral iron administration and the amount of iron habitually consumed do not affect iron absorption, systemic iron utilisation or zinc absorption in iron-sufficient infants: a randomised trial Br J Nutr, 116(6), 1046-60

522 Tambalis, K. D.,Mourtakos, S.,Panagiotakos, D. B.,Sidossis, L. S. (2018). Association of Exclusive Breastfeeding with Risk of Obesity in Childhood and Early Adulthood Breastfeed Med, \#volume\#(\#issue\#), \#Pages\#

523 Tanaka, K.,Miyake, Y.,Furukawa, S.,Arakawa, M. (2016). Perinatal smoking exposure and behavioral problems in Japanese children Intervention/exposure aged 5 years: The Kyushu Okinawa Maternal and Child Health Study Environmental Research, 151(\#issue\#), 383-388

524 Tang, M.,Griese, K. E.,Krebs, N. F. (2016). Dietary intakes of formula-fed infants consuming a meat-or dairy-based complementary Publication status diet: a semi-controlled feeding trial FASEB journal. Conference: experimental biology 2016, EB. San diego, CA united states. Conference start: 20160402. Conference end: 20160406. Conference publication: (var.pagings), 30(no pagination), \#Pages\#

525 Tang, M.,Rijnierse, A.,Nauta, A.,Boyle, R.,Hourihane, J.,Chiang, W.,Chua, M.,Smith, P.,Gold, M.,Ziegler, J.,et al., (2017). Influence of Publication status early feeding patterns on eczema development in high-risk infants Allergy, 72(\#issue\#), 15- 
527 Taylor-Robinson, D. C.,Williams, H.,Pearce, A.,Law, C.,Hope, S. (2016). Do early-life exposures explain why more advantaged children get eczema? Findings from the U.K. Millennium Cohort Study Br J Dermatol, 174(3), 569-78

\section{Outcome}

Terashita, S.,Nakamura, T.,Igarashi, N. (2017). Longitudinal study on the effectiveness of vitamin D supplements in exclusively breast-fed infants Clin Pediatr Endocrinol, 26(4), 215-222

529 Tham, E. H.,Lee, B. W.,Chan, Y. H.,Loo, E. X. L., Toh, J. Y.,Goh, A., Teoh, O. H., Yap, F., Tan, K. H.,Godfrey, K. M.,Chong, M. F. F.,Van Bever, H. P. S.,Chong, Y. S.,Shek, L. P. (2018). Low Food Allergy Prevalence Despite Delayed Introduction of Allergenic Foods-Data from the GUSTO Cohort J Allergy Clin Immunol Pract, 6(2), 466-475.e1

530 Thorisdottir, B.,Gunnarsdottir, I.,Steingrimsdottir, L.,Palsson, G. I.,Birgisdottir, B. E., Thorsdottir, I. (2016). Vitamin D Intake and Status Outcome in 6-Year-Old Icelandic Children Followed up from Infancy Nutrients, 8(2), 75

531 Thorisdottir, B.,Gunnarsdottir, I.,Vidarsdottir, A. G.,Sigurdardottir, S.,Birgisdottir, B. E., Thorsdottir, I. (2019). Infant Feeding, Vitamin D Intervention/exposure and IgE Sensitization to Food Allergens at 6 Years in a Longitudinal Icelandic Cohort Nutrients, 11(7), \#Pages\#

\begin{tabular}{ll}
532 & Tobolic, T. J. (2019). Primum Non Nocere Breastfeeding Breastfeed Med, 14(1), 77-78 \\
\hline 533 & Totzauer, M.,Luque, V.,Escribano, J.,Closa-Monasterolo, R., Verduci, E., ReDionigi, A.,Hoyos, J.,Langhendries, J. P.,Gruszfeld, \\
& $\begin{array}{l}\text { D.,Socha, P.,Koletzko, B.,Grote, V. (2018). Effect of Lower Versus Higher Protein Content in Infant Formula Through the First Year } \\
\text { on Body Composition from } 1 \text { to } 6 \text { Years: Follow-Up of a Randomized Clinical Trial Obesity (Silver Spring), 26(7), 1203-1210 }\end{array}$
\end{tabular}

534 Troesch, B.,Demmelmair, J.,Gimpfl, M.,Hecht, C.,Lakovic, G.,Roehle, R.,Sipka, L.,Trisic, B.,Vusurovic, M.,Schoop, R.,Zdjelar, S.,Koletzko, B. (2019). Suitability and safety of L-5-methyltetrahydrofolate as a folate source in infant formula: A randomizedcontrolled trial PLoS One, 14(8), e0216790

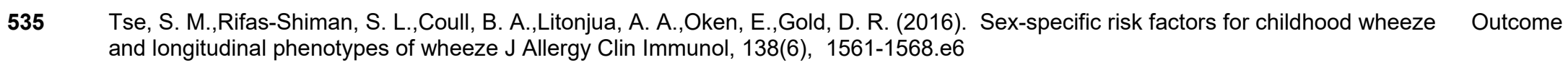
536 Tsoucalas, G.,Sgantzos, M. (2017). Oribasius-Pediatric Skin Eruptions and the Origins of the Allergic Reaction to Breast Milk JAMA Outcome
Dermatol, 153(4), 303

537 Uğraş Dikmen, A.,Konşuk Ünlü, H.,Özcebe, L. H. (2019). Evaluation of being overweight/obese and related sociodemographic factors in 0-5 year age group in Turkey: Turkey demographic health survey 2013 advanced analysis Turkish Journal of Medical Sciences, 49(3), 879-887

538 Uhl, O.,Fleddermann, M.,Hellmuth, C.,Demmelmair, H.,Koletzko, B. (2016). Phospholipid Species in Newborn and 4 Month Old Infants after Consumption of Different Formulas or Breast Milk PLoS One, 11(8), e0162040

539 Umer, A.,Hamilton, C.,Edwards, R. A.,Cottrell, L., Giacobbi, P., Jr.,Innes, K.,John, C.,Kelley, G. A.,Neal, W.,Lilly, C. (2019) Association Between Breastfeeding and Childhood Cardiovascular Disease Risk Factors Matern Child Health J, 23(2), 228-239

Intervention/exposure

Intervention/exposure

Study design

Intervention/exposure

Study design

Study design
Intervention/exposure, Group size/power

Outcome 
Uusitalo, U.,Lee, H. S.,Andren Aronsson, C.,Vehik, K.,Yang, J.,Hummel, S.,Silvis, K.,Lernmark, A., Rewers, M.,Hagopian, W.,She, J.

541 van der Willik, Esmee,Vrijkotte, Tanja G. M.,Altenburg, Teatske M.,Gademan, Maaike G. J.,Kist-van Holthe, Joana (2016). Exclusively breastfed overweight infants are at the same risk of childhood overweight as formula fed overweight infants MIDIRS Midwifery Digest, 26(1), 101-102

542 van der Wurff, I. S.,Bakker, E. C.,Hornstra, G.,Kirschner, P. A., Gielen, M.,Godschalk, R. W.,Kremers, S.,Zeegers, M. P.,de Groot, R. H. (2016). Association between prenatal and current exposure to selected LCPUFAs and school performance at age 7 Prostaglandins Leukot Essent Fatty Acids, 108(\#issue\#), 22-9

543 van Ginkel, C. D.,van der Meulen, G. N.,Bak, E.,Flokstra-de Blok, B. M. J.,Kollen, B. J.,Koppelman, G. H.,Dubois, A. E. J. (2018). Retrospective observational cohort study regarding the effect of breastfeeding on challenge-proven food allergy Eur $\mathrm{J}$ Clin Nutr, 72(4), $557-563$

544 van Meel, E. R., de Jong, M.,Elbert, N. J.,den Dekker, H. T.,Reiss, I. K., de Jongste, J. C.,Jaddoe, V. W. V.,Duijts, L. (2017). Duration and exclusiveness of breastfeeding and school-age lung function and asthma Ann Allergy Asthma Immunol, 119(1), 21-26.e2

545 van Rossem, L.,Smit, H. A.,Lentjes, Egwm,Maitimu-Smeele, I.,Brunekreef, B.,Koppelman, G. H.,Wijga, A. H. (2019). Does breast milk adiponectin affect BMI and cardio-metabolic markers in childhood? Br J Nutr, 121(8), 905-913

546 van Steenkiste, K. (2016). [Not Available] Kinderkrankenschwester, 35(7), 248-249

547 van Wouwe, Jacobus P.,Lanting, Caren I.,Akkermans, Marjolijn D.,Eussen, Simone R. B. M.,van der Horst-Graat, Judith M.,van Elburg, Ruurd M.,van Goudoever, Johannes B.,Brus, Frank (2017). More ways to successfully supplement vitamin D...Akkermans MD, Eussen SRBM, van der Horst-Graat JM, van Elburg RM, van Goudoever JB, Brus F. A micronutrient-fortified young-child formula improves the iron and vitamin D status of healthy young European children: a randomized, double-blind controlled trial. Am $\mathrm{J}$ Clin Nutr 2017;105:391-9 \#journal\#, 105(\#issue\#), 1564-1566

548 Vandyousefi, S.,Goran, M. I.,Gunderson, E. P.,Khazaee, E.,Landry, M. J.,Ghaddar, R.,Asigbee, F. M.,Davis, J. N. (2019). Association of breastfeeding and gestational diabetes mellitus with the prevalence of prediabetes and the metabolic syndrome in offspring of Hispanic mothers Pediatr Obes, 14(7), e12515

549 Varsi, Kristin,Bolann, Bjørn,Torsvik, Ingrid,Rosvold Eik, Tina Constanse,Høl, Paul Johan,Bjørke-Monsen, Anne-Lise (2017). Impact of Maternal Selenium Status on Infant Outcome during the First 6 Months of Life Nutrients, 9(5), 486

550 Vehapoglu, A., Goknar, N.,Turel, O.,Torun, E.,Ozgurhan, G. (2017). Risk factors for childhood obesity: Do the birth weight, type of delivery, and mother's overweight have an implication on current weight status? World J Pediatr, 13(5), 457-464

Publication status, Intervention/exposure

Study design, Intervention/exposure

Outcome

\section{Outcome}

Intervention/exposure

Publication status

Publication status

Intervention/exposure

Intervention/exposure

Study design,

Intervention/exposure

551 Veile, A.,Faria, A. A.,Rivera, S.,Tuller, S. M.,Kramer, K. L. (2019). Birth mode, breastfeeding and childhood infectious morbidity in the Outcome Yucatec Maya Am J Hum Biol, \#volume\#(\#issue\#), e23218

552 Venter, C.,Maslin, K.,Dean, T.,Arshad, S. H. (2016). Does concurrent breastfeeding alongside the introduction of solid food prevent the development of food allergy? J Nutr Sci, 5(\#issue\#), e40

\section{Outcome}

(1)



Behav Pediatr, 38(2), 109-119 Ventura, A. K., T 27(1), 130-136

$555 \quad$ Vergara Perez, Ines, Vila Sexto, Leticia (2018). Suspected severe acute food protein-induced enterocolitis syndrome caused by cow's Study design milk through breast milk Annals of Allergy, Asthma \& Immunology, 121(2), 245-246

556 Vianna, C. A.,Horta, B. L.,Gigante, D. P.,de Barros, F. C. (2016). Pulse Wave Velocity at Early Adulthood: Breastfeeding and Outcome Nutrition during Pregnancy and Childhood PLoS One, 11(4), e0152501

557 Viljoen, K.,Segurado, R.,O'Brien, J.,Murrin, C.,Mehegan, J.,Kelleher, C. C. (2018). Pregnancy diet and offspring asthma risk over a Outcome 10-year period: the Lifeways Cross Generation Cohort Study, Ireland BMJ Open, 8(2), e017013

558 Viner, R. M.,Costa, S.,Johnson, W. (2019). Patterns of BMI development between 10 and 42 years of age and their determinants in Intervention/exposure the 1970 British Cohort Study J Epidemiol Community Health, 73(1), 79-85

559 Visentin, S.,Vicentin, D.,Magrini, G.,Santandreu, F.,Disalvo, L.,Sala, M.,Fasano, V.,Gonzalez, H. F. (2016). Red blood cell membrane Outcome fatty acid composition in infants fed formulas with different lipid profiles Early Hum Dev, 100(\#issue\#), 11-5

560 Vogelezang, S.,Santos, S.,van der Beek, E. M.,Abrahamse-Berkeveld, M.,Duijts, L.,van der Lugt, A.,Felix, J. F.,Jaddoe, V. W. V. (2018). Infant breastfeeding and childhood general, visceral, liver, and pericardial fat measures assessed by magnetic resonance imaging Am J Clin Nutr, 108(4), 722-729

561 von Berg, A.,Filipiak-Pittroff, B.,Kramer, U.,Link, E.,Heinrich, J.,Koletzko, S.,Grubl, A.,Hoffmann, U.,Beckmann, C.,Reinhardt, D.,Bauer, C. P.,Wichmann, E.,Berdel, D. (2017). The German Infant Nutritional Intervention Study (GINI) for the preventive effect of hydrolyzed infant formulas in infants at high risk for allergic diseases. Design and selected results Allergol Select, 1(1), 28-38

562 von Berg, A.,Filipiak-Pittroff, B.,Schulz, H.,Hoffmann, U.,Link, E.,Sussmann, M.,Schnappinger, M.,Bruske, I.,Standl, M.,Kramer, U.,Hoffmann, B.,Heinrich, J.,Bauer, C. P.,Koletzko, S.,Berdel, D. (2016). Allergic manifestation 15 years after early intervention with hydrolyzed formulas--the GINI Study Allergy, 71(2), 210-9

563 Von Berg, A.,Filipiak-Pittroff, B.,Schulz, H.,Hoffmann, U.,Link, E.,Sußmann, M.,Schnappinger, M.,Brüske, I.,Standl, M.,Krämer U.,Hoffmann, B.,Heinrich, J.,Bauer, C. P.,Koletzko, S.,Berdel, D. (2016). Allergic manifestation 15 years after early intervention with hydrolyzed formulas - The GINI Study Allergy: European Journal of Allergy and Clinical Immunology, 71(2), 210-219

564 von Weikersthal, G. F. (2016). New chances in allergy risk: prevention by breastfeeding, HA food and care from the beginning Kinderkrankenschwester : organ der sektion kinderkrankenpflege, 35(5), 165-168

565 Wagner, C. L.,Eidelman, Arthur I. (2018). The Impact of Vitamin D on the Maternal and Infant Epigenome: The Role of Pregnancy and Breastfeeding Breastfeeding Medicine, 13(5), 305-306

566 Wallby, T.,Lagerberg, D.,Magnusson, M. (2017). Relationship Between Breastfeeding and Early Childhood Obesity: Results of a Prospective Longitudinal Study from Birth to 4 Years Breastfeed Med, 12(\#issue\#), 48-53 
Wang, A. H.,Fitzpatrick, C. (20 Behav Pediatr, 40(1), 40-48

\section{8}

Wang, F.,Liu, H.,Wan, Y.,Li, J.,Chen, Y.,Zheng, J.,Huang, T.,Li, D. (2016). Prolonged Exclusive Breastfeeding Duration Is Positively Associated with Risk of Anemia in Infants Aged 12 Months J Nutr, 146(9), 1707-13

569

Wang, H.,Mueller, N. T.,Li, J.,Sun, N.,Huo, Y.,Ren, F.,Wang, X. (2017). Association of Maternal Plasma Folate and Cardiometabolic Risk Factors in Pregnancy with Elevated Blood Pressure of Offspring in Childhood Am J Hypertens, 30(5), 532-540

570 Wang, I. J.,Wen, H. J.,Chiang, T. L.,Lin, S. J., Guo, Y. L. (2016). Maternal psychologic problems increased the risk of childhood atopic Outcome dermatitis Pediatr Allergy Immunol, 27(2), 169-76

571 Wang, J.,Groetch, M. (2017). Preventing food allergies with tweaks to the infant diet: A practical reality? Annals of Allergy, Asthma Publication status and Immunology, 118(4), 385-388

572 Wang, J.,Ramette, A.,Jurca, M.,Goutaki, M.,Beardsmore, C. S.,Kuehni, C. E. (2017). Association between breastfeeding and eczema Outcome during childhood and adolescence: A cohort study PLoS One, 12(9), e0185066

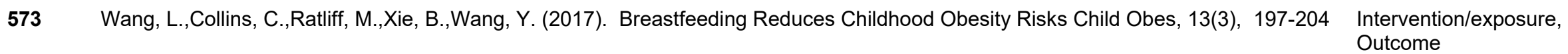

574 Wang, L.,van Grieken, A.,Yang-Huang, J.,Vlasblom, E.,L'Hoir, M. P.,Boere-Boonekamp, M. M.,Raat, H. (2018). Relationship between socioeconomic status and weight gain during infancy: The BeeBOFT study PLoS One, 13(11), e0205734

575 Wang, P.,Hao, M.,Han, W.,Yamauchi, T. (2019). Factors associated with nutritional status and motor development among young Study design children Nurs Health Sci, \#volume\#(\#issue\#), \#Pages\#

576 Wang, W.,Sun, Y.,Zhang, M.,Zhang, Y.,Chen, W.,Tan, L.,Shen, J.,Zhao, Z.,Lan, S.,Zhang, W. (2018). Breast milk and infant iodine status during the first 12 weeks of lactation in Tianjin City, China Asia Pac J Clin Nutr, 27(2), 393-398

Study design,

Intervention/exposure

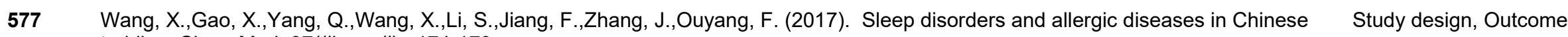
toddlers Sleep Med, 37(\#issue\#), 174-179

578 Warstedt, K.,Furuhjelm, C.,Falth-Magnusson, K.,Fageras, M.,Duchen, K. (2016). High levels of omega-3 fatty acids in milk from omega-3 fatty acid-supplemented mothers are related to less immunoglobulin E-associated disease in infancy Acta Paediatr, 105(11) $1337-1347$

579 Weber, M.,Luque, V.,Escribano, J.,Closa, R.,Verduci, E.,ReDionigi, A.,Hoyos, J.,Langhendries, J. P.,Gruszfeld, D.,Socha, P.,et al., (2016). Effect of early protein supply on body fat deposition during infancy and childhood: a randomized trial Journal of pediatric gastroenterology and nutrition. Conference: 49th annual meeting of the european society for paediatric gastroenterology, hepatology and nutrition, ESPGHAN 2016. Athens greece. Conference start: 20160525. Conference end: 20160528. Conference publication: (var.pagings), 62(\#issue\#), 668-669

580 Whaley, S. E.,Koleilat, M.,Leonard, S.,Whaley, M. (2017). Breastfeeding Is Associated With Reduced Obesity in Hispanic 2- to 5-

Year-Olds Served by WIC J Nutr Educ Behav, 49(7 Suppl 2), S144-S150.e1

\section{Outcome}

Publication status 
581 Wheeler, B. J.,Taylor, B. J.,de Lange, M.,Harper, M. J.,Jones, S.,Mekhail, A.,Houghton, L. A. (2018). A Longitudinal Study of 25Hydroxy Vitamin D and Parathyroid Hormone Status throughout Pregnancy and Exclusive Lactation in New Zealand Mothers and Their Infants at 45 degrees S Nutrients, 10(1), \#Pages\#

582 Wheeler, B. J.,Taylor, B. J.,Herbison, P.,Haszard, J. J.,Mikhail, A.,Jones, S.,Harper, M. J.,Houghton, L. A. (2016). High-Dose Monthly Maternal Cholecalciferol Supplementation during Breastfeeding Affects Maternal and Infant Vitamin D Status at 5 Months Postpartum: A Randomized Controlled Trial J Nutr, 146(10), 1999-2006

583 Wheeler, B. J.,Taylor, B. J.,Herbison, P.,Haszard, J. J.,Mikhail, A.,Jones, S.,Harper, M. J.,Houghton, L. A. (2017). Effect of high dose monthly maternal cholecalciferol supplementation during breastfeeding on infant and maternal vitamin d status at 5 months postpartum: a randomized controlled trial International journal of pediatric endocrinology, 2017(\#issue\#), \#Pages\#

584 Wickens, K.,Barthow, C.,Mitchell, E. A.,Kang, J.,van Zyl, N.,Purdie, G.,Stanley, T.,Fitzharris, P.,Murphy, R.,Crane, J. (2018). Effects of Lactobacillus rhamnosus HN001 in early life on the cumulative prevalence of allergic disease to 11 years Pediatr Allergy Immunol, 29(8), 808-814

585 Wicklow, B., Gallo, S.,Majnemer, A., Vanstone, C.,Comeau, K.,Jones, G.,L'Abbe, M.,Khamessan, A., Sharma, A., Weiler, H.,Rodd, C. (2016). Impact of Vitamin D Supplementation on Gross Motor Development of Healthy Term Infants: A Randomized Dose-Response Trial Phys Occup Ther Pediatr, 36(3), 330-42 Wojcicki, J. M.,Heyman, M. B.,Elwan, D.,Lin, J.,Blackburn, E.,Epel, E. (2016). Early exclusive breastfeeding is associated with longer telomeres in Latino preschool children Am J Clin Nutr, 104(2), 397-405

587 Wong, V. C. H.,Maguire, J. L.,Omand, J. A.,Dai, D. W. H.,Lebovic, G.,Parkin, P. C.,O'Connor, D. L.,Birken, C. S.,Cohn, R.,Lau, E.,Laupacis, A.,Salter, M.,Szatmari, P.,Weir, S.,Anderson, L. N.,Borkhoff, C. M.,Keown-Stoneman, C.,Kowal, C.,Mason,

D.,Abdurrahman, M.,Anderson, K.,Arbess, G.,Baker, J.,Barozzino, T.,Bergeron, S.,Bhagat, D.,Bloch, G.,Bonifacio, J.,Bowry,

A.,Calpin, C.,Campbell, D.,Cheema, S.,Cheng, E.,Chisamore, B.,Constantin, E.,Danayan, K.,Das, P.,Derocher, M. B.,Do, A.,Doukas, K.,Egger, A.,Farber, A.,Freedman, A.,Freeman, S., Gazeley, S.,Guiang, C.,Ha, D.,Handford, C.,Hanson, L.,Harrington, L., Jacobson,

S.,Jagiello, L.,Jansz, G.,Kadar, P.,Kim, F.,Kiran, T.,Knowles, H.,Kwok, B.,Lakhoo, S.,Lam-Antoniades, M.,Leduc, D.,Leung, F. H.,Li, A.,Li, P.,Malach, J.,Male, R.,Mascoll, V.,Meret, A.,Mok, E.,Moodie, R.,Nader, M.,Nash, K.,Naymark, S.,Owen, J.,Peer, M.,Pena, K.,Perlmutar, M.,Persaud, N.,Pinto, A.,Porepa, M.,Qi, V.,Ramji, N.,Ramji, N.,Raza, D.,Rosenthal, A., Rouleau, K.,Ruderman, C.,Saunderson, J.,Schiralli, V.,Sgro, M.,Shuja, H.,Shepherd, S.,Smiltnieks, B.,Srikanthan, C.,Taylor, C., Treherne, S., Turner, S.,Uddin, F.,van den Heuvel, M.,Vaughan, J.,Weisdorf, T.,Wijayasinghe, S.,Wong, P.,Yaremko, J.,Ying, E.,Young, E.,Zajdman, M.,Bazeghi, F.,Bouchard, V.,Bustos, M.,Camacho, C.,Dalwadi, D.,Koroshegyi, C.,Malhi, T., Thadani, S.,Thompson, J., Thompson, L.,Aglipay, M.,Bayoumi, I.,Carsley, S.,Cost, K.,Eny, K.,Kim, T.,Kinlin, L.,Omand, J.,Vanderhout, S., Vanderloo, L.,Allen, C.,Boodhoo, B., Chan O.,Hall, J.,Juni, P.,Pope, K.,Thorpe, K.,Kandel, R.,Rodrigues, M.,Vandenberghe, H. (2019). A Positive Association Between Dietary Intake of Higher Cow's Milk-Fat Percentage and Non-High-Density Lipoprotein Cholesterol in Young Children Journal of Pediatrics, 211(\#issue\#), 105-111.e2 Modeled Using Superimposition by Translation and Rotation Are Differentially Associated with Body Composition Components at 3 and 7 Years of Age J Pediatr, 196(\#issue\#), 182-188.e1

589 Wopereis, H.,Sim, K.,Shaw, A.,Warner, J. O.,Knol, J.,Kroll, J. S. (2018). Intestinal microbiota in infants at high risk for allergy: Effects of prebiotics and role in eczema development J Allergy Clin Immunol, 141(4), 1334-1342.e5

No key confounders accounted for, Outcome 

Stool Characteristics of Healthy Term Infants Fed an Infant Formula Containing Hydrolyzed Whey Protein (63\%) and Intact Casein (37\%): A Randomized Clinical Trial Nutrients, 9(11), \#Pages\#

591 Wu, Y. Y.,Lye, S.,Briollais, L. (2017). The role of early life growth development, the FTO gene and exclusive breastfeeding on child BMI trajectories Int J Epidemiol, 46(5), 1512-1522

592 Xinias, I.,Cassimos, D.,Trypsianis, G.,Nivatsi, M.,Mavroudi, A. (2019). Immediate vs delayed cow's milk protein allergy in terms of tolerance at year 1 Annals of Allergy, Asthma and Immunology, 123(3), 304-306 Month-Old Infants with Different Feeding Practices J Nutr Sci Vitaminol (Tokyo), 62(4), 235-239

594 Yang, M.,Tan, M.,Wu, J.,Chen, Z.,Long, X.,Zeng, Y.,Cai, H.,Zhang, Y.,Geng, L.,Xiao, Y.,Ke, H.,Liu, Y.,Rong, L.,Fu, S.,Wang, H.,Wang, Y.,Li, X.,Chen, P.,Li, K.,Xie, J.,Chen, H.,Li, H.,Wang, H.,Li, D. Y.,Gong, S. (2019). Prevalence, Characteristics, and Outcome of Cow's Milk Protein Allergy in Chinese Infants: A Population-Based Survey JPEN J Parenter Enteral Nutr, 43(6), 803-808 up of the PROBIT cluster-randomized trial PLoS Med, 15(4), e1002554 infants fed formula with a new algal source of docosahexaenoic acid: Double-blind, randomized, controlled trial Prostaglandins Leukot Essent Fatty Acids, 115(\#issue\#), 89-96

597 Yeung, H.,Leff, M.,Rhee, K. E. (2017). Effect of Exclusive Breastfeeding Among Overweight and Obese Mothers on Infant Weightfor-Length Percentile at 1 Year Breastfeed Med, 12(\#issue\#), 39-47

598 Yorulmaz, A.,Sert, S.,Yilmaz, F. H.,Kara, F.,Cinarlidere, S. (2018). The evaluation of primary school readiness levels of the children aged 66 - 72 months with the denver II test Iranian Journal of Pediatrics, 28(5), \#Pages\#

599 Young, B. E.,Levek, C.,Reynolds, R. M.,Rudolph, M. C.,MacLean, P.,Hernandez, T. L.,Friedman, J. E.,Krebs, N. F. (2018). Bioactive components in human milk are differentially associated with rates of lean and fat mass deposition in infants of mothers with normal vs. elevated BMI Pediatr Obes, 13(10), 598-606

600 Zamora-Kapoor, A.,Omidpanah, A.,Nelson, L. A.,Kuo, A. A.,Harris, R.,Buchwald, D. S. (2017). Breastfeeding in Infancy Is Associated with Body Mass Index in Adolescence: A Retrospective Cohort Study Comparing American Indians/Alaska Natives and Non-Hispanic Whites J Acad Nutr Diet, 117(7), 1049-1056

601 Zaragoza Cortes, J.,Trejo Osti, L. E.,Ocampo Torres, M.,Maldonado Vargas, L.,Ortiz Gress, A. A. (2018). Poor breastfeeding complementary feeding and dietary diversity in children and their relationship with stunting in rural communities Nutr Hosp, 35(2), 271-278

602 Zavareh, M. S. A.,Hasani, M.,Darabi, M.,Mirzaei, A.,Khorshidi, A.,Saeidi, A.,Momeni, K.,Jalilian, M. (2018). Growth indicators and nutritional supplement evaluation in 6-12 months year old children's: A perspective from Ilam Electronic Journal of General Medicine, 15(3), \#Pages\#

No key confounders accounted fo

Publication status, Participant health

Study design

Intervention/exposure,

Outcome

Outcome

Intervention/exposure

Intervention/exposure

Study design

ntervention/exposure

Outcome

Study design

Study design

Intervention/exposure 
603 Zhong, H.,Penders, J.,Shi, Z.,Ren, H.,Cai, K.,Fang, C.,Ding, Q., Thijs, C.,Blaak, E. E.,Stehouwer, C. D. A.,Xu, X.,Yang, H.,Wang J.,Wang, J.,Jonkers, Dmae,Masclee, A. A. M.,Brix, S.,Li, J.,Arts, I. C. W.,Kristiansen, K. (2019). Impact of early events and lifestyle on the gut microbiota and metabolic phenotypes in young school-age children Microbiome, 7(1), 2

604 Zhu, Y.,Olsen, S. F.,Mendola, P.,Halldorsson, T. I.,Yeung, E. H.,Granstrom, C.,Bjerregaard, A. A.,Wu, J.,Rawal, S.,Chavarro, J. E.,Hu, F. B.,Zhang, C. (2017). Maternal dietary intakes of refined grains during pregnancy and growth through the first $7 \mathrm{y}$ of life among children born to women with gestational diabetes Am J Clin Nutr, 106(1), 96-104

605 Zhuang, J.,Bei, F.,Qin, Y.,Sun, J.,Wu, S. (2018). Effect of high sn-2 palmitate infant formula on the excretion of fatty acids, calcium and magnesium in infants Chinese journal of clinical nutrition, 26(4), 214-220

606 Zielinska, M. A.,Hamulka, J.,Grabowicz-Chadrzynska, I.,Brys, J.,Wesolowska, A. (2019). Association between Breastmilk LC PUFA, Intervention/exposure Carotenoids and Psychomotor Development of Exclusively Breastfed Infants Int J Environ Res Public Health, 16(7), \#Pages\#

607 (2016). Five-Year Follow-Up of High-Risk Infants with Family History of Allergy Who Were Exclusively Breast-Fed or Fed Partia

Publication status Whey Hydrolysate, Soy, and Conventional Cow's Milk Formulas: Expression of Serious Concern J Pediatr Gastroenterol Nutr, 63(2), 307

608 (2017). Breast really is best World of Irish Nursing \& Midwifery, 25(5), 63-63

Publication status

609 (2017). Further evidence that breastfeeding reduces ill health Practising Midwife, 20(6), 1-2

Publication status

610 (2017). Relationship between growth and illness, enteropathogens and dietary intakes in the first 2 years of life: findings from the MAL-ED birth cohort study BMJ Glob Health, 2(4), e000370

Intervention/exposure, Country

611 (2018). Breastfeeding Prevents Diabetes Diabetes Self-Management, 35(5), 6-6

Publication status

612 (2018). The effects of feeding practices and appetitive traits on infant anthropometry in 6-month infants born to obese women-a secondary analysis of the UPBEAT trial Reproductive sciences (thousand oaks, calif.), Conference: 65th Annual Scientific Meeting of the Society for Gynecologic Investigation, SGI 2018. United States. 25(1), 268A 